Keywords: SB7b, Frit 418, Frit 702, variability, durability

Retention: Permanent

\title{
Sludge Batch 7b Glass Variability Study
}

F.C. Johnson
T.B. Edwards

October 2011

Savannah River National Laboratory Savannah River Nuclear Solutions, LLC Aiken, SC 29808

Prepared for the U.S. Department of Energy under contract number DE-AC09-08SR22470.

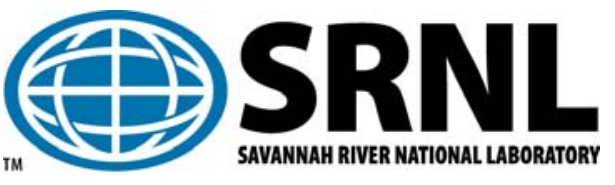


SRNL-STI-2011-00440

Revision 0

\section{DISCLAIMER}

This work was prepared under an agreement with and funded by the U.S. Government. Neither the U.S. Government or its employees, nor any of its contractors, subcontractors or their employees, makes any express or implied:

1. warranty or assumes any legal liability for the accuracy, completeness, or for the use or results of such use of any information, product, or process disclosed; or

2. representation that such use or results of such use would not infringe privately owned rights; or

3. endorsement or recommendation of any specifically identified commercial product, process, or service.

Any views and opinions of authors expressed in this work do not necessarily state or reflect those of the United States Government, or its contractors, or subcontractors.

\section{Printed in the United States of America \\ Prepared for \\ U.S. Department of Energy}




\section{REVIEWS AND APPROVALS}

AUTHORS:

F.C. Johnson, Process Technology Programs

Date

T.B. Edwards, Applied Computational Engineering and Statistics

Date

TECHNICAL REVIEW:

D.K. Peeler, Process Technology Programs

Date

APPROVALS:

C.C. Herman, Manager

Date

Process Technology Programs

S.L. Marra, Manager

Date

Environmental \& Chemical Process Technology Research Programs

J.E. Occhipinti, Manager

Date

Waste Solidification Engineering 


\section{ACKNOWLEDGEMENTS}

The authors would like to acknowledge the technical support provided by Phyllis Workman, Pat Simmons and Kevin Kalbaugh for glass fabrication and the physical property measurements as well as SRNL Analytical Development personnel (Damon Click, Boyd Wiedenman, David Missimer, Ronnie Rutherford, Beverly Burch, and Loretta Farrow) for the chemical analysis and X-ray diffraction data. 


\section{EXECUTIVE SUMMARY}

The Defense Waste Processing Facility (DWPF) is preparing to initiate processing Sludge Batch $7 \mathrm{~b}$ (SB7b). In support of the upcoming processing, the Savannah River National Laboratory (SRNL) provided a recommendation to utilize Frits 418 with a $6 \% \mathrm{Na}_{2} \mathrm{O}$ addition $\left(26 \mathrm{wt} \% \mathrm{Na}_{2} \mathrm{O}\right.$ in sludge) and 702 with a $4 \% \mathrm{Na}_{2} \mathrm{O}$ addition $\left(24 \mathrm{wt} \% \mathrm{Na}_{2} \mathrm{O}\right.$ in sludge) to process $\mathrm{SB} 7 \mathrm{~b}$. This recommendation was based on assessments of the compositional projections for SB7b available at the time from the Savannah River Remediation (SRR). To support qualification of SB7b, SRNL executed a variability study to assess the applicability of the current durability models for SB7b. The durability models were assessed over the expected composition range of SB7b, including potential caustic additions, combined with Frits 702 and 418 over a 32-40\% waste loading (WL) range.

Thirty four glasses were selected based on Frits 418 and 702 coupled with the sludge projections with an additional $4-6 \% \mathrm{Na}_{2} \mathrm{O}$ to reflect the potential caustic addition. Six of these glasses, based on average nominal sludge compositions including the appropriate caustic addition, were developed for both Frit 418 and Frit 702 at 32, 36 and 40\% WL to provide coverage in the center of the anticipated SB7b glass region. All glasses were fabricated and characterized using chemical composition analysis, X-ray diffraction (XRD) and the Product Consistency Test (PCT).

To comply with the DWPF Glass Product Control Program, a total of thirty four glasses were fabricated to assess the applicability of the current DWPF PCCS durability models. Based on the measured PCT response, all of the glasses were acceptable with respect to the Environmental Assessment (EA) benchmark glass regardless of thermal history. The NL[B] values of the SB7b variability study glasses were less than $1.99 \mathrm{~g} / \mathrm{L}$ as compared to $16.695 \mathrm{~g} / \mathrm{L}$ for EA. A small number of the D-optimally selected "outer layer" extreme vertices (EV) glasses were not predictable using the current Product Composition Control System (PCCS) models for durability, but were acceptable compared to the EA glass when tested. These glasses fell outside of the lower $95 \%$ confidence band, which demonstrates conservatism in the model. A few of the glasses fell outside of the upper 95\% confidence band; however, these particular glasses have normalized release values that were much lower than the values of EA and should be of no practical concern.

Per the requirements of the DWPF Glass Product Control Program, the PCCS durability models have been shown to be applicable to the SB7b sludge system with a range of $\mathrm{Na}_{2} \mathrm{O}$ concentrations blended with Frits 418 or 702 . PCT results from the glasses fabricated as part of the variability study were shown to be predictable by the current DWPF PCCS models and/or acceptable with respect to the EA benchmark glass regardless of thermal history or compositional view. 


\section{TABLE OF CONTENTS}

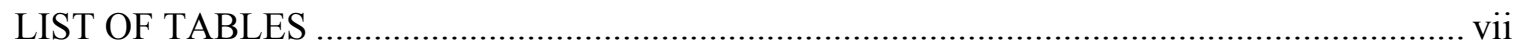

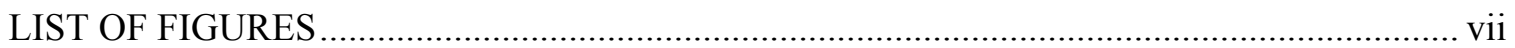

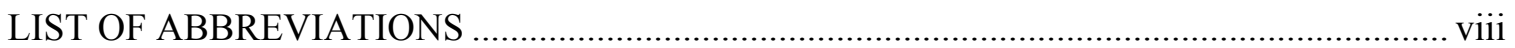

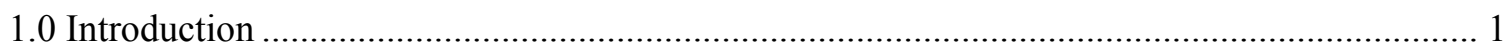

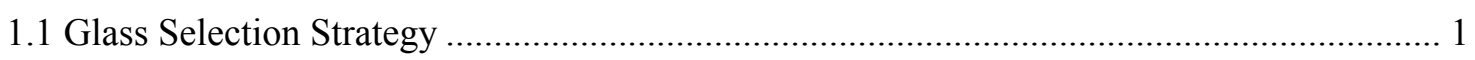

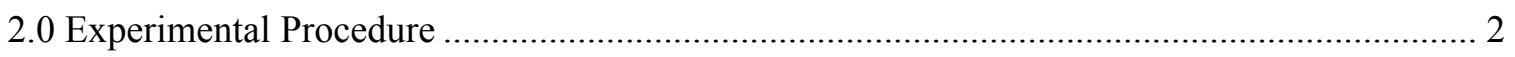

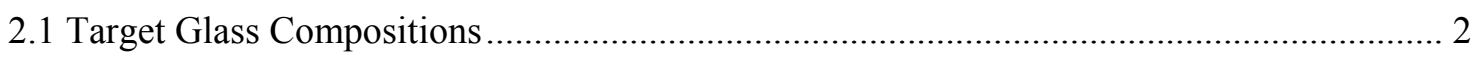

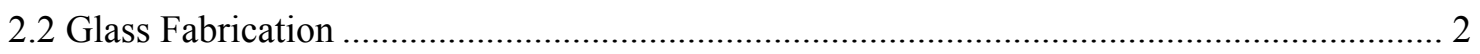

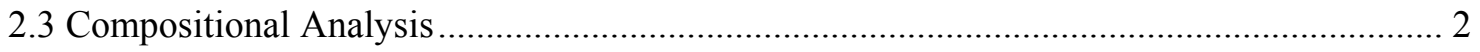

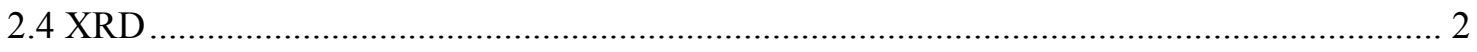

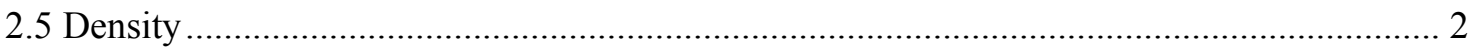

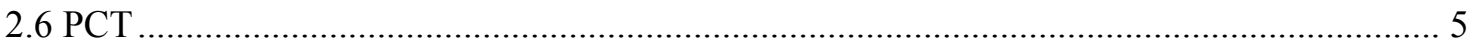

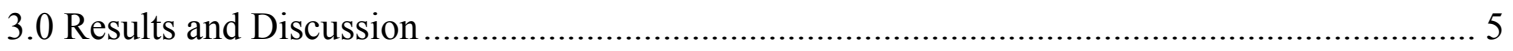

3.1 Chemical Composition Measurements ....................................................................... 5

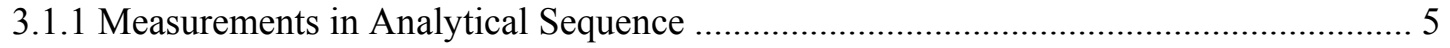

3.1.2 Composition Measurements by Glass Identifier ........................................................... 5

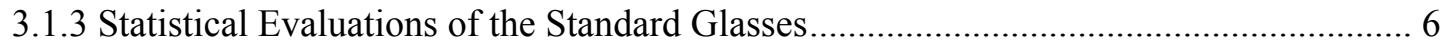

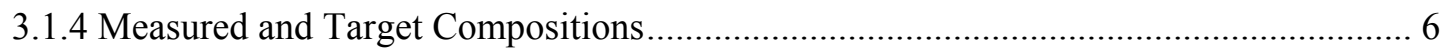

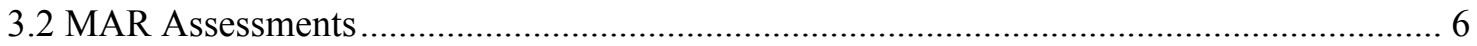

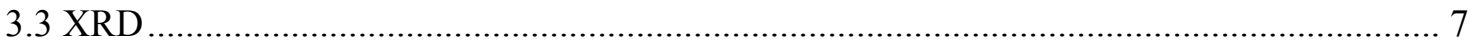

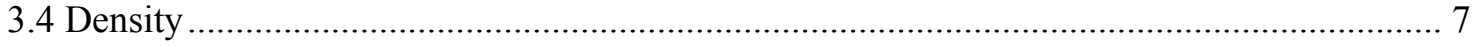

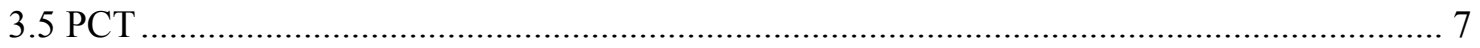

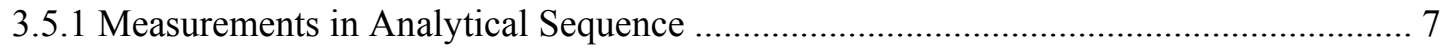

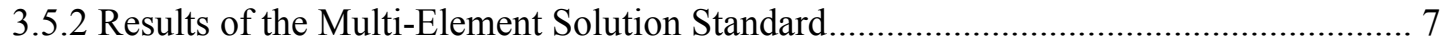

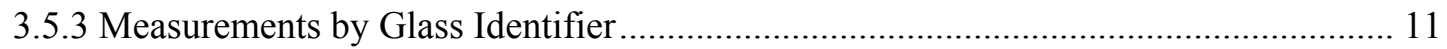

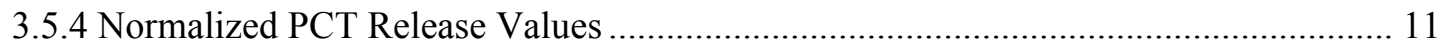

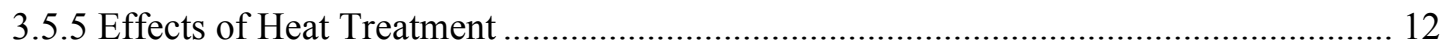

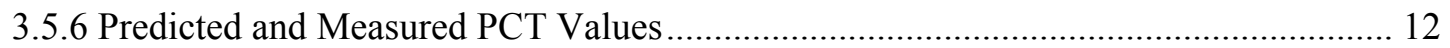

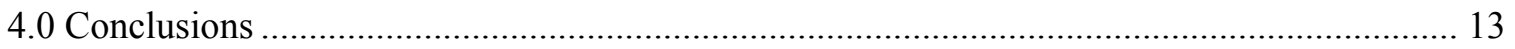

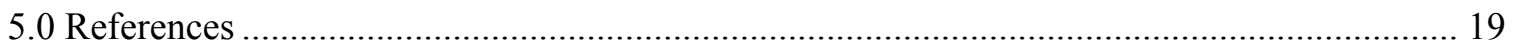




\section{LIST OF TABLES}

Table 1-1. Bounds of SB7b Projected Glass Region................................................................. 3

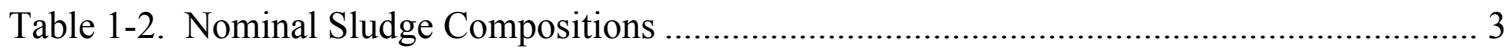

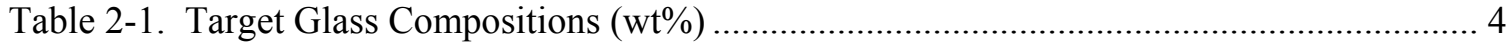

Table 3-1. Results of the PCCS Assessment at the MAR (Target Compositions) ........................ 8

Table 3-2. Results of the PCCS Assessment at the MAR (Measured Compositions)................... 9

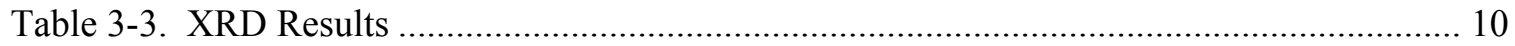

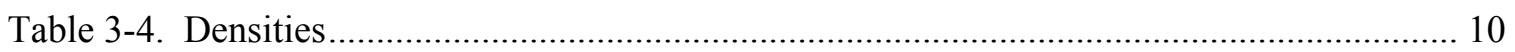

Table 3-5. Results from Samples of the Multi-Element Solution Standard (ppm) ...................... 11

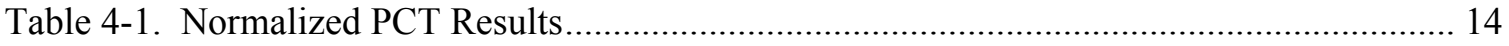

\section{LIST OF FIGURES}

Figure 3-1. Del $\mathrm{G}_{\mathrm{p}}$ versus $\log$ NL [B] values based on measured compositions....................... 13 


\section{LIST OF ABBREVIATIONS}

\begin{tabular}{|c|c|}
\hline $\mathrm{AD}$ & Analytical Development \\
\hline ANOVA & Analysis of Variance \\
\hline AR & Aqua Regia \\
\hline ARM & Approved Reference Material \\
\hline ARP & Actinide Removal Process \\
\hline $\mathrm{CCC}$ & Centerline Canister Cooling \\
\hline DWPF & Defense Waste Processing Facility \\
\hline EA & Environmental Assessment \\
\hline EV & Extreme Vertices \\
\hline ICP-AES & Inductively Coupled Plasma-Atomic Emission Spectroscopy \\
\hline MAR & Measurement Acceptability Region \\
\hline NL & Normalized Leachate \\
\hline PCCS & Product Composition Control System \\
\hline PCT & Product Consistency Test \\
\hline $\mathrm{PF}$ & Peroxide Fusion \\
\hline SB & Sludge Batch \\
\hline $\mathrm{SB} 7_{\text {ref }}$ & SB7 Reference \\
\hline SRNL & Savannah River National Laboratory \\
\hline SRR & Savannah River Remediation \\
\hline TL & Liquidus Temperature \\
\hline TTQAP & Task Technical And Quality Assurance Plan \\
\hline TTR & Technical Task Request \\
\hline $\mathrm{U}_{\text {std }}$ & Uranium Standard \\
\hline WL & Waste Loading \\
\hline XRD & X-ray Diffraction \\
\hline
\end{tabular}




\subsection{Introduction}

The Defense Waste Processing Facility (DWPF) is preparing to initiate processing Sludge Batch $7 \mathrm{~b}$ (SB7b). In support of the upcoming processing, the Savannah River National Laboratory (SRNL) provided a recommendation ${ }^{\mathrm{a}}$ to utilize Frits 418 with a $6 \% \mathrm{Na}_{2} \mathrm{O}$ addition $\left(26 \mathrm{wt} \% \mathrm{Na}_{2} \mathrm{O}\right.$ in sludge), 702 with a $4 \% \mathrm{Na}_{2} \mathrm{O}$ addition (24 wt $\% \mathrm{Na}_{2} \mathrm{O}$ in sludge) and 425 with a $2 \% \mathrm{Na}_{2} \mathrm{O}$ addition (22 $\mathrm{wt} \% \mathrm{Na}_{2} \mathrm{O}$ in sludge) to process SB7b. This recommendation was based on assessments of the compositional projections ${ }^{\mathrm{b}}$ for $\mathrm{SB} 7 \mathrm{~b}$ available at the time from the Savannah River Remediation (SRR) The $\mathrm{Na}_{2} \mathrm{O}$ concentration in the sludge projection was low, so in order to improve sulfur retention in the glass, all frits were evaluated with a caustic addition. These particular caustic additions were shown to yield the best operating windows in terms of waste loading. Based on the balance of sulfur solubility and caustic addition, the selection of frits was narrowed down to Frits 418 and $702 .^{\circ}$

To support qualification of SB7b, SRNL executed a variability study to assess the applicability of the current durability models for SB7b. The durability models were assessed over the expected composition range of SB7b, including potential caustic additions, combined with Frits 702 and 418 over a $32-40 \%$ waste loading (WL) range. The experimental portion of the variability study was initiated by a DWPF Technical Task Request ${ }^{1}$ (TTR) and was performed under a SRNL Task Technical and Quality Assurance Plan ${ }^{2}$ (TT\&QAP).

\subsection{Glass Selection Strategy}

For a complete discussion of the sludge projections and assessments leading up to the frit selection see SRNL-L3100-2011-00160. ${ }^{3}$ Only a brief summary will be provided in this section.

In order to develop glasses for the variability study, one sludge region was created from the combination of the 4 and $6 \% \mathrm{Na}_{2} \mathrm{O}$ addition sludges. ${ }^{\mathrm{d}, \mathrm{e}}$ Extreme vertices (EVs) for this single sludge region were generated and bounds were created using 32 and $40 \%$ WL. Both Frits 418 and 702 were combined with the minimum concentrations of sludge components at $32 \% \mathrm{WL}$ and the maximum concentration of sludge components at $40 \% \mathrm{WL}$ to create one glass region. The bounds of each component for the glass region are shown in Table 2-1. EVs were then generated based on the bounds of this glass region and an initial set of 14 "outer layer" EV glasses were Doptimally selected using the Custom Design feature of the Design of Experiments in JMP ${ }^{\mathrm{TM}}{ }^{4}$ An additional set of $14 \mathrm{EV}$ glasses were D-optimally selected to provide "inner layer" coverage. Using average nominal sludge compositions shown in Table $2-2^{\mathrm{f}}$, a third set of 6 glasses was developed for both Frit 418 and Frit 702 at 32, 36 and 40\% WL to provide coverage in the center of the anticipated SB7b region.

\footnotetext{
${ }^{a}$ D.K. Peeler and T.B. Edwards, "MAR Assessments on SB7b Projections: 5/25/2011," Savannah River National Laboratory, Oral Presentation at the SRR-SRNL Plan of the Month Meeting, May 26, 2011.

${ }^{\mathrm{b}}$ SB7b projections received from H.B. Shah on May 25, 2011 via email. See SRNL-NB-2010-00108, page 134 for more details.

${ }^{\mathrm{c}}$ Using the $5 / 25 / 2011 \mathrm{SB} 7 \mathrm{~b}$ projections, Frit 418 with a $6 \% \mathrm{Na}_{2} \mathrm{O}$ addition yielded an operating window of $25-39 \%$ in the variation stage of the Measurement Acceptability Region (MAR) assessments, whereas Frit 702 with a $4 \% \mathrm{Na}_{2} \mathrm{O}$ addition yielded an operating window of $25-37 \%$. Frit 418 offered a slightly bigger operating window, but Frit 702 was shown to have better sulfur retention.

${ }^{d}$ Extreme vertices (EVs) and subsequently centroids for both the $4 \% \mathrm{Na}_{2} \mathrm{O}$ and $6 \% \mathrm{Na}_{2} \mathrm{O}$ sludges were previously defined by Peeler and Edwards for the frit recommendation and take into account with/without Actinide Removal Process (ARP) stream and two Tank 40 blending scenarios.

${ }^{\mathrm{e}}$ Nominal coupled operations projections are based on introduction of the ARP stream from S.G. Subosits, "Actinide Removal Process Material Balance Calculation with Low Curie Salt Feed," X-CLC-S-00113 Rev. 0, Appendix J, September 24, 2004.

${ }^{\mathrm{f}}$ The average nominal sludge compositions (with $4 \%$ and $6 \% \mathrm{Na}_{2} \mathrm{O}$ additions) took into account with/without ARP and two blending scenarios.
} 
Thirty four glasses were fabricated and characterized using chemical composition analysis, X-ray diffraction (XRD) and the Product Consistency Test (PCT).

\subsection{Experimental Procedure}

\subsection{Target Glass Compositions}

Target glass compositions of the variability study glasses are shown in Table 2-3. Glasses SB7b01 through -14 were based on the "outer layer" EVs, SB7b-15 through -28 were based on the "inner layer" EVs and SB7b-29 through -34 were based on the nominal sludge compositions.

\subsection{Glass Fabrication}

Each variability study glass was prepared from the proper proportions of reagent-grade metal oxides, carbonates, $\mathrm{H}_{3} \mathrm{BO}_{3}$, and salts in $150 \mathrm{~g}$ batches. The raw materials were thoroughly mixed and placed into a platinum alloy, $250 \mathrm{ml}$ crucible. Batched materials were placed into a hightemperature furnace at the target melt temperature of $1150^{\circ} \mathrm{C}$. The crucible was removed from the furnace after an isothermal hold at $1150^{\circ} \mathrm{C}$ for 1 hour. The molten glass was quenched by pouring the liquid onto a clean, stainless steel plate. The glass pour patty was used as a sampling stock for the various property measurements (i.e., chemical composition, durability testing and XRD).

Approximately $25 \mathrm{~g}$ of each glass was heat-treated to simulate cooling along the centerline of a DWPF-type canister to gauge the effects of thermal history on the product performance. ${ }^{5}$ This cooling schedule is referred to as the centerline canister cooling (ccc) curve.

\subsection{Compositional Analysis}

A representative sample from each glass was submitted to Analytical Development (AD) and analyzed under the auspices of an analytical plan. ${ }^{6} \quad$ Two dissolution methods were utilized to allow measurement of the compositions: aqua regia (AR) and sodium peroxide fusion (PF). For each study glass, measurements were obtained from samples prepared in duplicate by each of these dissolution methods. All of the prepared samples were analyzed (twice for each element of interest) by Inductively Coupled Plasma-Atomic Emission Spectroscopy (ICP-AES). Between the duplicate analyses, the instrument was recalibrated. The analytical plan was developed in such a way as to provide the opportunity to evaluate potential sources of bias and error. Glass standards were also intermittently measured to assess the performance of the ICP-AES instrument over the course of these analyses.

\subsection{XRD}

Select pairs of quenched and ccc glasses were submitted to AD for XRD analysis based on PCT response. $^{\mathrm{g}}$ Samples were analyzed under conditions providing a detection limit of approximately $0.5 \mathrm{vol} \%$, i.e. no crystals can be detected if the amount in the sample is less than $\sim 0.5 \mathrm{vol} \%$.

\subsection{Density}

The density of representative samples of each of the as-fabricated glasses was measured in duplicate via the Archimedes method. ${ }^{7}$ A NIST standard glass was measured intermittently throughout the samples as an internal check of the measured density value.

\footnotetext{
${ }^{\mathrm{g}}$ Both the quenched and ccc version of a glass were submitted if the difference between the normalized PCT response was greater than $0.2 \mathrm{~g} / \mathrm{L}$.
} 
Table 2-1. Bounds of SB7b Projected Glass Region

\begin{tabular}{|c|c|c|}
\hline Component & Minimum $(w t \%)$ & Maximum (wt $\%)$ \\
\hline $\mathrm{Al}_{2} \mathrm{O}_{3}$ & 6.353 & 9.900 \\
\hline $\mathrm{B}_{2} \mathrm{O}_{3}$ & 4.800 & 5.440 \\
\hline $\mathrm{BaO}$ & 0.014 & 0.066 \\
\hline $\mathrm{CaO}$ & 0.204 & 0.674 \\
\hline $\mathrm{Ce}_{2} \mathrm{O}_{3}$ & 0.027 & 0.132 \\
\hline $\mathrm{Cr}_{2} \mathrm{O}_{3}$ & 0.018 & 0.087 \\
\hline $\mathrm{CuO}$ & 0.008 & 0.037 \\
\hline $\mathrm{Fe}_{2} \mathrm{O}_{3}$ & 7.474 & 11.600 \\
\hline $\mathrm{K}_{2} \mathrm{O}$ & 0.018 & 0.089 \\
\hline $\mathrm{La}_{2} \mathrm{O}_{3}$ & 0.004 & 0.021 \\
\hline $\mathrm{Li}_{2} \mathrm{O}$ & 4.800 & 6.800 \\
\hline $\mathrm{MgO}$ & 0.018 & 0.440 \\
\hline $\mathrm{MnO}$ & 1.503 & 2.365 \\
\hline $\mathrm{Na}_{2} \mathrm{O}$ & 11.067 & 16.036 \\
\hline $\mathrm{NiO}$ & 1.178 & 2.023 \\
\hline $\mathrm{PbO}$ & 0.004 & 0.021 \\
\hline $\mathrm{SO}_{4}$ & 0.518 & 0.784 \\
\hline $\mathrm{SiO}_{2}$ & 46.460 & 52.751 \\
\hline $\mathrm{ThO}_{2}$ & 0.327 & 0.862 \\
\hline $\mathrm{TiO}_{2}$ & 0.000 & 0.723 \\
\hline $\mathrm{U}_{3} \mathrm{O}_{8}$ & 2.141 & 3.329 \\
\hline $\mathrm{ZnO}$ & 0.003 & 0.016 \\
\hline $\mathrm{ZrO}_{2}$ & 0.020 & 0.097 \\
\hline
\end{tabular}

Table 2-2. Nominal Sludge Compositions

\begin{tabular}{|c|c|c|}
\hline \multirow{2}{*}{ Component } & \multicolumn{2}{|c|}{ Nominal Sludge Compostions (wt\%) } \\
\cline { 2 - 3 } & $+4 \% \mathrm{Na}_{2} \mathrm{O}$ & $+6 \% \mathrm{Na}_{2} \mathrm{O}$ \\
\hline $\mathrm{Al}_{2} \mathrm{O}_{3}$ & 22.502 & 21.917 \\
\hline $\mathrm{BaO}$ & 0.106 & 0.103 \\
\hline $\mathrm{CaO}$ & 1.179 & 1.149 \\
\hline $\mathrm{Ce}_{2} \mathrm{O}_{3}$ & 0.210 & 0.205 \\
\hline $\mathrm{Cr}_{2} \mathrm{O}_{3}$ & 0.138 & 0.135 \\
\hline $\mathrm{CuO}$ & 0.059 & 0.058 \\
\hline $\mathrm{Fe}_{2} \mathrm{O}_{3}$ & 26.540 & 25.853 \\
\hline $\mathrm{K}_{2} \mathrm{O}$ & 0.142 & 0.139 \\
\hline $\mathrm{La}_{2} \mathrm{O}_{3}$ & 0.034 & 0.033 \\
\hline $\mathrm{MgO}_{2} \mathrm{O}$ & 0.564 \\
\hline $\mathrm{MnO}_{\mathrm{Nay}}$ & 0.579 & 5.229 \\
\hline $\mathrm{Na}_{2} \mathrm{O}$ & 5.367 & 25.973 \\
\hline $\mathrm{NiO}^{2}$ & 24.022 & 4.324 \\
\hline $\mathrm{PbO}^{2}$ & 4.439 & 0.032 \\
\hline $\mathrm{SO}_{4}$ & 0.033 & 1.775 \\
\hline $\mathrm{SiO}_{2}$ & 1.821 & 2.701 \\
\hline $\mathrm{ThO}_{2}$ & 2.773 & 1.556 \\
\hline $\mathrm{TiO}_{2}$ & 1.598 & 0.666 \\
\hline $\mathrm{U}_{3} \mathrm{O}_{8}$ & 0.666 & 7.414 \\
\hline $\mathrm{ZnO}_{\mathrm{ZrO}}$ & 7.611 & 0.024 \\
\hline & 0.025 & 0.151 \\
\hline
\end{tabular}




\section{Table 2-3. Target Glass Compositions (wt \%)}

\begin{tabular}{|c|c|c|c|c|c|c|c|c|c|c|c|c|c|c|c|c|c|c|c|c|c|c|c|c|}
\hline Glass ID & & $\mathrm{Al}_{2} \mathrm{O}_{3}$ & $\mathrm{~B}_{2} \mathrm{O}_{3}$ & $\mathrm{BaO}$ & $\mathrm{CaO}$ & $\mathrm{Ce}_{2} \mathrm{O}_{3}$ & $\mathrm{Cr}_{2} \mathrm{O}_{3}$ & $\mathrm{CuO}$ & $\mathrm{Fe}_{2} \mathrm{O}_{3}$ & $\mathrm{~K}_{2} \mathrm{O}$ & $\mathrm{La}_{2} \mathrm{O}_{3}$ & $\mathrm{Li}_{2} \mathrm{O}$ & $\mathrm{MgO}$ & $\mathrm{MnO}$ & $\mathrm{Na}_{2} \mathrm{O}$ & $\mathrm{NiO}$ & $\mathrm{PbO}$ & $\mathrm{SO}_{4}$ & $\mathrm{SiO}_{2}$ & $\mathrm{ThO}_{2}$ & $\mathrm{TiO}_{2} \mathrm{~L}$ & $\mathrm{U}_{3} \mathrm{O}_{8}$ & $\mathrm{ZnO}$ & $\mathrm{ZrO}_{2}$ \\
\hline SB7b-01 & \multirow{14}{*}{ Outer Layer EVs } & 6.353 & 5.440 & 0.005 & 0.204 & 0.019 & 0.012 & 0.005 & 11.600 & 0.013 & 0.003 & 4.800 & 0.053 & 1.503 & 16.036 & 2.023 & 0.003 & 0.784 & 48.121 & 0.862 & 0.0002 & 2.141 & 0.002 & 0.014 \\
\hline SB7b-02 & & 6.353 & 5.440 & 0.070 & 0.204 & 0.140 & 0.092 & 0.039 & 11.600 & 0.095 & 0.023 & 4.800 & 0.404 & 2.365 & 15.162 & 1.178 & 0.022 & .518 & 46.460 & 0.862 & 0.7233 & 3.329 & 0.017 & 0.103 \\
\hline SB7b-03 & & 8.841 & 4.800 & 0.070 & 0.674 & 0.140 & 0.092 & 0.039 & 7.474 & 0.095 & 0.023 & 6.800 & 0.404 & 2.365 & 16.036 & 2.023 & 0.022 & .518 & 46.460 & 0.862 & 0.0002 & 2.141 & 0.017 & 0.103 \\
\hline SB7b-04 & & 9.900 & 4.800 & 0.009 & 0.204 & 0.019 & 0.012 & 0.005 & 7.474 & 0.013 & 0.003 & $4.800 \mathrm{C}$ & 0.053 & 2.365 & 11.420 & 1.178 & 0.003 & 0.784 & 52.751 & 0.862 & 0.0003 & 329 & 0.002 & 0.014 \\
\hline SB7b-05 & & 6.353 & 5.440 & 0.070 & 0.674 & 0.140 & 0.092 & 0.039 & 7.474 & 0.095 & 0.023 & 4.800 & 0.404 & 1.503 & 15.204 & 1.178 & 0.022 & 0.518 & 52.194 & 0.327 & 0.0003 & 3.329 & 0.017 & 0.103 \\
\hline SB7b-06 & & 9.900 & 5.440 & 0.070 & 0.204 & 0.140 & 0.092 & 0.039 & 11.600 & 0.095 & 0.023 & 6.800 & 0.404 & 2.365 & 11.067 & 1.906 & 0.022 & 0.784 & 46.460 & 0.327 & 0.0002 & 2.141 & 0.017 & 0.103 \\
\hline SB7b-07 & & 9.900 & 4.800 & 0.009 & 0.204 & 0.019 & 0.012 & 0.005 & 7.474 & 0.013 & 0.003 & 6.800 & 0.053 & 1.503 & 16.036 & 1.178 & 0.003 & 0.518 & 48.263 & 0.327 & \begin{tabular}{|l|l}
0.723 & \\
\end{tabular} & 2.141 & 0.002 & 0.014 \\
\hline SB7b-08 & & 6.353 & 4.800 & 0.009 & 0.674 & 0.019 & 0.012 & 0.005 & 11.474 & 0.013 & 0.003 & 6.800 & 0.053 & 2.365 & 15.323 & 1.178 & 0.003 & 0.784 & 46.460 & $|0.327|$ & 0.0003 & 329 & 0.002 & 0.014 \\
\hline SB7b-09 & & 9.900 & 5.440 & 0.009 & 0.674 & 0.019 & 0.012 & 0.005 & 11.291 & 0.013 & 0.003 & 6.800 & 0.053 & 1.503 & 11.067 & 2.023 & 0.003 & 0.518 & 46.460 & 0.862 & $0.000\}$ & 329 & 0.002 & 0.014 \\
\hline SB7b-10 & & 9.900 & 5.440 & 0.009 & 0.674 & 0.019 & 0.012 & 0.005 & 7.474 & 0.013 & 0.003 & 4.800 & 0.053 & 2.365 & 15.568 & 2.023 & 0.003 & 0.784 & 46.460 & 0.327 & 0.723 & 3.329 & 0.002 & 0.014 \\
\hline SB7b-11 & & 9.900 & 4.800 & 0.070 & 0.674 & 0.140 & 0.092 & 0.039 & 11.600 & 0.095 & 0.023 & 4.800 & 0.404 & 1.503 & .569 & 1.178 & 0.022 & 0.784 & 46.460 & 0.862 & 0.723 & 2.141 & 0.017 & 0.103 \\
\hline SB7b-12 & & 6.353 & 4.800 & 0.009 & 0.674 & 0.019 & 0.012 & 0.005 & 11.600 & 0.013 & 0.003 & $4.800 \mathrm{C}$ & 0.053 & 2.365 & 11.067 & 2.023 & 0.003 & 0.518 & 52.476 & 0.327 & \begin{tabular}{|l|l|}
0.723 \\
\end{tabular} & 2.141 & 0.002 & 0.014 \\
\hline SB7b-13 & & 6.353 & 4.800 & 0.070 & 0.204 & 0.140 & 0.092 & 0.039 & 8.114 & 0.095 & 0.023 & $6.800 \mathrm{C}$ & 0.404 & 1.503 & .067 & 2.023 & 0.022 & 0.784 & 52.432 & 0.862 & \begin{tabular}{|l|l}
0.723 \\
\end{tabular} & 3.329 & 0.017 & 0.103 \\
\hline SB7b-14 & & 6.353 & \begin{tabular}{|l|l|}
5.440 \\
\end{tabular} & 0.009 & 0.674 & 0.019 & 0.012 & 0.005 & 7.474 & 0.013 & 0.003 & $6.800 \mathrm{C}$ & 0.053 & 2.365 & 12.322 & 1.178 & 0.003 & 0.784 & 52.751 & 0.862 & \begin{tabular}{|l|l|}
0.7232 \\
\end{tabular} & 2.141 & 0.002 & 0.014 \\
\hline SB7b-15 & \multirow{14}{*}{ Inner Layer EVs } & 7.240 & 4.960 & 0.025 & 0.557 & \begin{tabular}{|c|}
0.049 \\
\end{tabular} & 0.032 & 0.014 & 8.506 & 0.033 & 0.008 & $5.300 \mathrm{C}$ & 0.141 & 1.719 & 14.794 & 1.389 & 0.008 & 0.718 & 50.431 & 0.461 & 0.5423 & .032 & .006 & 0.036 \\
\hline SB7b-16 & & 9.013 & 4.960 & 0.055 & 0.322 & \begin{tabular}{|l|l|}
0.110 \\
\end{tabular} & 0.072 & 0.031 & 10.414 & 0.075 & 0.018 & 6.300 & 0.316 & 1.719 & 2.309 & 1.389 & 0.017 & 0.718 & 48.033 & 0.461 & \begin{tabular}{|l|l|}
0.542 \\
\end{tabular} & 3.032 & 0.013 & 0.081 \\
\hline SB7b-17 & & 7.240 & \begin{tabular}{|l|l|}
5.280 \\
\end{tabular} & 0.055 & 0.322 & 0.110 & 0.072 & 0.031 & 8.506 & 0.075 & 0.018 & 6.300 & 0.316 & 1.719 & 12.829 & 1.812 & 17 & 0.585 & \begin{tabular}{|l|l|}
51.178 \\
\end{tabular} & 0.461 & \begin{tabular}{|l|l|}
0.542 \\
\end{tabular} & 2.438 & 0.013 & 0.081 \\
\hline SB7b-18 & & 7.240 & 4.960 & 0.055 & 0.557 & 0.110 & 0.072 & 0.031 & 10.569 & 0.075 & 0.018 & 5.300 & 0.316 & 2.150 & 14.165 & 1.812 & 177 & 0.718 & 48.033 & 0.728 & 0.5422 & 438 & 0.013 & 0.081 \\
\hline SB7b-19 & & 9.013 & 4.960 & 0.055 & 0.322 & 0.110 & 0.072 & 0.031 & 8.506 & 0.075 & 0.018 & 5.300 & 0.316 & \begin{tabular}{|l|}
1.719 \\
\end{tabular} & .794 & 1.389 & 0.017 & 0.585 & 49.277 & 0.728 & 0.1812 & 2.438 & 0.013 & 0.081 \\
\hline SB7b-20 & & 9.013 & 4.960 & 0.025 & 0.322 & 0.049 & \begin{tabular}{|l|l|}
0.032 \\
\end{tabular} & 0.014 & 8.807 & 0.033 & 0.008 & $5.300 \mathrm{C}$ & 0.141 & 2.150 & 12.309 & 1.812 & 0.008 & 0.718 & 51.178 & 0.461 & 0.1812 & 2.438 & 0.006 & 0.036 \\
\hline SB7b-21 & & 7.240 & \begin{tabular}{|l|}
5.280 \\
\end{tabular} & 0.055 & 0.557 & 0.110 & 0.072 & 0.031 & 8.506 & 0.075 & 0.018 & 6.300 & 0.316 & 2.150 & 12.309 & 1.389 & 0.017 & 0.718 & 50.822 & 0.728 & 0.1813 & 3.032 & 0.013 & 0.081 \\
\hline SB $7 b-22$ & & 7.240 & \begin{tabular}{|l|}
4.960 \\
\end{tabular} & 0.025 & 0.557 & 0.049 & 0.032 & 0.014 & 10.569 & 0.033 & 0.008 & 6.300 & 0.141 & 1.719 & 12.309 & 1.389 & 0.008 & 0.585 & 50.941 & 0.461 & 0.1812 & 2.438 & \begin{tabular}{|l|}
0.006 \\
\end{tabular} & 0.036 \\
\hline SB7b-23 & & 9.013 & 4.960 & 0.025 & \begin{tabular}{|l|}
0.557 \\
\end{tabular} & 0.049 & 0.032 & 0.014 & 8.506 & 0.033 & 0.008 & 6.300 & 0.141 & \begin{tabular}{|l|}
1.719 \\
\end{tabular} & 12.309 & 1.812 & 0.008 & 0.585 & 49.586 & 0.728 & 0.5423 & 3.032 & 0.006 & 0.036 \\
\hline SB7b-24 & & 9.013 & 5.280 & 0.025 & 0.557 & 0.049 & 032 & 0.014 & .506 & 0.033 & 0.008 & 6.300 & 0.141 & 2.150 & 14.395 & 1.38 & 0.008 & 0.585 & 48.0 & 0.461 & 0.542 & 2.43 & 0.0 & 0.036 \\
\hline SB7b-25 & & 7.240 & 4.960 & 0.055 & 0.322 & 0.110 & 0.072 & 0.031 & 9.342 & 0.075 & 0.018 & $6.300 \mathrm{C}$ & 0.316 & 2.150 & 14.794 & 1.812 & 0.017 & 0.585 & 48.033 & 0.461 & 0.1813 & 3.032 & 0.0 & 0.081 \\
\hline SB7b-26 & & 9.013 & 5.280 & 0.055 & 0.557 & 0.110 & 0.072 & 0.031 & 10.569 & 0.075 & 0.018 & $5.300 \mathrm{C}$ & 0.316 & 1.719 & 12.309 & 1.812 & 0.017 & 0.585 & 48.394 & 0.461 & 0.1813 & 3.032 & 0.01 & 0.081 \\
\hline SB7b-27 & & 7.240 & 5.280 & 0.025 & 0.322 & 0.049 & 0.032 & 0.014 & 10.569 & 0.033 & 0.008 & 6.3000 & 0.141 & 1.719 & 14.309 & 1.812 & 0.008 & 0.718 & 48.033 & $0.728 \mathrm{C}$ & 0.181 & 2.438 & 0.006 & 0.036 \\
\hline SB7b-28 & & 7.240 & 5.280 & 0.025 & 0.322 & 0.049 & 0.032 & 0.014 & 10.569 & 0.033 & 0.008 & 5.300 & 0.141 & 2.150 & 12.309 & 1.389 & 0.008 & 0.585 & \begin{tabular}{|l|}
50.203 \\
\end{tabular} & $0.728 \mathrm{C}$ & 0.542 & 3.032 & 0.006 & 0.036 \\
\hline SB7b-29 & ominal $\left(+6 \% \mathrm{Na}_{2} \mathrm{O}\right)$-Frit 418 at $32 \% \mathrm{WL}$ & 7.013 & 5.440 & 0.033 & 0.368 & 0.066 & 0.043 & 0.018 & 8.273 & 0.044 & 0.011 & 5.440 & 0.180 & 1.673 & 13.751 & 1.384 & 0.010 & 0.568 & 52.544 & 0.498 & 0.213 & 2.372 & 0.008 & 0.048 \\
\hline SB7b-30 & Nominal $(+6 \%$ Na2O)-Frit 418 at $36 \%$ WL & 7.890 & 5.120 & 0.037 & 0.413 & 0.074 & 0.048 & 0.021 & 9.307 & 0.050 & 0.012 & 5.120 & 0.203 & 1.882 & 14.470 & 1.557 & 0.012 & 0.639 & 49.612 & $0.560 \mid \mathrm{C}$ & 0.240 & 2.669 & 0.009 & 0.054 \\
\hline SB7b-31 & Nominal $(+6 \%$ Na2O)-Frit 418 at $40 \% \mathrm{WL}$ & 8.767 & 4.800 & 0.041 & 0.459 & 0.082 & 0.054 & 0.023 & 10.341 & 0.056 & 0.013 & 4.800 & 0.226 & 2.092 & 15.189 & 1.730 & 0.013 & 0.710 & 46.680 & $0.622 \mathrm{C}$ & 0.266 & 2.965 & 0.010 & 0.061 \\
\hline SB7b-32 & Nominal $(+4 \%$ Na2O)-Frit 702 at $32 \%$ WL & 7.201 & 5.440 & 0.034 & 0.377 & 0.067 & 0.044 & 0.019 & 8.493 & 0.046 & 0.011 & 6.800 & 0.185 & 1.717 & 11.767 & 1.421 & 0.011 & 0.583 & 52.567 & $0.511 \mid \mathrm{C}$ & 0.213 & 2.435 & 0.008 & 0.050 \\
\hline SB7b-33 & Nominal $(+4 \% \mathrm{Na} 2 \mathrm{O})$-Frit 702 at $36 \% \mathrm{WL}$ & 8.101 & 5.120 & 0.038 & 0.424 & 0.076 & 0.050 & 0.021 & 9.554 & 0.051 & 0.012 & $6.400 \mathrm{C}$ & 0.208 & 1.932 & 12.488 & 1.598 & 0.012 & 0.655 & 49.638 & 0.575 & 0.240 & 2.740 & 0.00 & 0.056 \\
\hline SB7b-34 & Nominal $(+4 \% \mathrm{Na} 2 \mathrm{O})$-Frit 702 at $40 \% \mathrm{WL}$ & 9.001 & 4.800 & 0.042 & 0.471 & 0.084 & 0.055 & 0.024 & 10.616 & 0.057 & 0.014 & 6.000 & 0.232 & 2.147 & 13.209 & $\mid 1.776$ & 0.013 & 0.728 & 46.709 & 0.639 & 0.267 & 3.044 & 0.010 & 0.062 \\
\hline
\end{tabular}




\section{6 $\underline{\mathrm{PCT}}$}

The PCT was performed in triplicate on each quenched and ccc glass to assess chemical durability using Method A of the procedure. ${ }^{8}$ Also included in the experimental test matrix was the Environmental Assessment (EA) glass, the Approved Reference Material (ARM) glass, and blanks from the sample cleaning batch. Samples were ground, washed, and prepared according to the standard procedure. The resulting solutions were sampled (filtered and acidified) and analyzed by AD under the auspices of an analytical plan. ${ }^{9}$ Samples of a multi-element, standard solution were also included in the analytical plan (as a check on the accuracy of the ICP-AES instrument). Normalized release rates were calculated based on target and measured compositional views using the average of the leachate concentrations.

\subsection{Results and Discussion}

\subsection{Chemical Composition Measurements}

Tables A-1 and A-2 in Appendix A provide the elemental concentration measurements from the glasses that were prepared using AR, while the measurements using PF are shown in Table A-3 and Table A-4. Measurements for the Batch 1, uranium standard ( $\left.\mathrm{U}_{\text {std }}\right)$ glass and SB7 reference glass $\left(\mathrm{SB} 7_{\text {ref }}\right)^{8}$ are also provided in the tables of Appendix A.

Elemental concentrations were converted to oxide concentrations by multiplying the values for each element by the gravimetric factor for the corresponding oxide. During this process, an elemental concentration that was determined to be below the detection limit of the analytical procedures used was reduced to half of that detection limit as the oxide concentration was determined. ${ }^{10}$

\subsubsection{Measurements in Analytical Sequence}

Exhibit A-1 in Appendix A provides plots in analytical sequence of the sample measurements generated for each oxide by preparation method (i.e., AQ and PF) for analytical block 1, 2 and 3. Exhibit A-2 in Appendix A provides the same plots for analytical blocks 4 and 5. These plots include all of the measurement data, and in addition to analytical sequence of the measurements, the laboratory identifiers of each sample are shown. These plots provide the reader with an opportunity to perform a high-level review of the data; however, the data reported in these exhibits are presented in analytical sequence and it may be difficult to ascertain specific issues with the measured data for an individual glass. In the next section, the measured data are compiled and presented to provide assessments of reproducibility and comparisons between target and measured compositions. Specific and/or general issues with the compositional measurements are discussed.

\subsubsection{Composition Measurements by Glass Identifier}

Exhibit A-3 in Appendix A provides plots of the oxide concentration measurements by Glass $\mathrm{ID} / \mathrm{Lab}$ ID (including Batch $1, \mathrm{U}_{\text {std }}$ and $\mathrm{SB} 7_{\text {ref }}$ ). These plots demonstrate the individual measurements across the duplicates of each preparation method and the two ICP-AES calibrations for each glass.

In general, there appears to be good repeatability amongst the measurements for each of the major oxides except for $\mathrm{SiO}_{2}$. Some scatter is also observed in the minor components, including $\mathrm{Ce}_{2} \mathrm{O}_{3}$,

\footnotetext{
${ }^{8}$ The SB7 ref glass was developed as a non-radioactive internal standard representing Frit 418 coupled with the Tank 40 60 " heel nominal projection (after removing $\mathrm{U}_{3} \mathrm{O}_{8}$ and $\mathrm{ThO}_{2}$ and renormalizing) at $36 \% \mathrm{WL}$. This glass was not only submitted to AD but to the Process Science Analytical Laboratory (PSAL) for compositional analysis. For more information on the SB7 $7_{\text {ref }}$ glass see SRNL-NB-2010-00162 pages $31-32$.
} 
$\mathrm{Cr}_{2} \mathrm{O}_{3}, \mathrm{CuO}, \mathrm{La}_{2} \mathrm{O}_{3}, \mathrm{PbO}, \mathrm{ZnO}$ and $\mathrm{ZrO}_{2}$. While the reference value for $\mathrm{Cr}_{2} \mathrm{O}_{3}$ is $0 \mathrm{wt} \%$ in the $\mathrm{U}_{\text {std }}$ glass, the measured values are on the order of $0.25 \mathrm{wt} \%$. These measured values are consistent with previous measurements and indicate that the $\mathrm{U}_{\text {std }}$ glass does contain a small fraction of $\mathrm{Cr}_{2} \mathrm{O}_{3}$, which was not report in the reference composition.

Exhibit A-4 in Appendix A provides a comparison of the oxide concentration measurements for $\mathrm{AR}$ and PF. Based on a review of these plots, a decision was made by the authors to use the AR rather than the $\mathrm{PF}$ measurements for $\mathrm{PbO}$ and $\mathrm{SO}_{4}$. There appears to be a detection limit issue with the PF measurements for these oxides. For the remainder of the oxides, an average of the AR and PF values (when applicable) was taken in order to determine an average measured composition. ${ }^{9}$

\subsubsection{Statistical Evaluations of the Standard Glasses}

Exhibit A-5 in Appendix A provides statistical analyses of the $\mathrm{SB} 7_{\text {ref }}$ and $\mathrm{U}_{\text {std }}$ results by calibration block for each oxide of interest for both the AR and PF preparation methods. The results include analysis of variance (ANOVA) investigations, which determine statistically significant differences among the means of these groups for each of the oxides for each of the standards. The following components indicate a significant ICP-AES calibration effect on the block averages at the 5\% significance level:

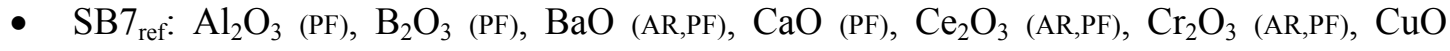
(AR,PF), $\mathrm{Fe}_{2} \mathrm{O}_{3}$ (AR,PF), $\mathrm{K}_{2} \mathrm{O}$ (AR,PF), $\mathrm{La}_{2} \mathrm{O}_{3}$ (AR,PF), $\mathrm{Li}_{2} \mathrm{O}$ (PF), $\mathrm{MgO}$ (AR,PF), $\mathrm{MnO}$ (AR,PF), $\mathrm{NiO}$ (AR,PF), $\mathrm{PbO}$ (AR,PF), $\mathrm{SiO}_{2}$ (PF), $\mathrm{SO}_{4}$ (AR), $\mathrm{TiO}_{2}$ (AR,PF), $\mathrm{ZnO}$ (AR) and $\mathrm{ZrO}_{2}$ (AR)

- $\mathrm{U}_{\text {std }}: \mathrm{Al}_{2} \mathrm{O}_{3}$ (AR,PF), $\mathrm{B}_{2} \mathrm{O}_{3}$ (AR), $\mathrm{CaO}$ (AR), $\mathrm{Fe}_{2} \mathrm{O}_{3}$ (AR), $\mathrm{Li}_{2} \mathrm{O}$ (AR), $\mathrm{MgO}$ (AR), $\mathrm{MnO}$ (AR), $\mathrm{Na}_{2} \mathrm{O}$ (AR), $\mathrm{NiO}$ (AR), $\mathrm{SiO}_{2}(\mathrm{PF}), \mathrm{TiO}_{2}(\mathrm{AR}, \mathrm{PF})$ and $\mathrm{U}_{3} \mathrm{O}_{8}(\mathrm{PF})$

Reference values for the oxide concentrations of the standards are given in the header for each set of measurements in Exhibit A-5. While there is a calibration effect, these results do not impact the outcome of this study.

\subsubsection{Measured and Target Compositions}

Table A-5 in Appendix A provides a summary of the average measured and target compositions, as well as some associated and relative differences. Exhibit A-6 in Appendix A provides plots showing a comparison of the target and average measured compositions of each oxide for all of the glasses.

In general, the average measured values were consistent with the target values for each oxide of interest. Of the major oxides, there is some variability in the average measured $\mathrm{SiO}_{2}$; however, these differences do not impact the conclusions drawn from this study. Again, some variation is observed in the minor components, including $\mathrm{Ce}_{2} \mathrm{O}_{3}, \mathrm{Cr}_{2} \mathrm{O}_{3}, \mathrm{CuO}, \mathrm{La}_{2} \mathrm{O}_{3}, \mathrm{PbO}, \mathrm{ZnO}$ and $\mathrm{ZrO}_{2}$. The measured sums of oxides for all of the glass fall within the interval of 95 to $105 \mathrm{wt} \%$.

\subsection{MAR Assessments}

Results from the PCCS assessment at the Measurement Acceptability Region (MAR) based on target and measured compositions are shown in Table 3-1 and Table 3-2. The columns in the table list the glass identifier with $\mathrm{Al}_{2} \mathrm{O}_{3}$ content, $\mathrm{TiO}_{2}$ content, sum of alkali $\left(\mathrm{R}_{2} \mathrm{O}\right)$, nepheline discriminator value (Neph. Discr.), the overall PCCS assessment at the MAR and the predicted values for: $\Delta \mathrm{G}_{\mathrm{p}}$ value for boron (B Del $\mathrm{G}_{\mathrm{p}}$ value), normalized release for boron in grams/liter (NL

\footnotetext{
${ }^{9}$ AR was the only digestion used for $\mathrm{Ca}, \mathrm{Na}$ and $\mathrm{Zr}$. PF was the only digestion used for Si.
} 
[B $(\mathrm{g} / \mathrm{L})])$, liquidus temperature in degrees Celsius $\left(\mathrm{T}_{\mathrm{L}}\right.$ Pred. $\left.\left({ }^{\circ} \mathrm{C}\right)\right)$ and viscosity in Poise (Visc Pred (P)). Glasses SB7b-03 and -08 were intentionally chosen to fail the PCCS constraint for $\Delta \mathrm{G}_{\mathrm{p}}$ at the MAR, which is shown in both compositional views. Slight differences in predicted property values are observed for glasses based on target and measured composition as compared to its measured composition.

\section{$3.3 \underline{\text { XRD }}$}

Table 3-3 summarizes the results of the XRD analysis of the quenched and heat treated (ccc) glasses. ${ }^{10}$ No crystals were detected in any of the analyzed quenched glasses. Some of the "outer" layer EV ccc glasses exhibited a small degree of crystallization. Trevorite (a spinel $\mathrm{NiFe}_{2} \mathrm{O}_{4}$ ) was detected in SB7b-03ccc, $-06 \mathrm{ccc}$ and $-09 \mathrm{ccc}$, which is a typical crystal found in slowly cooled, higher WL DWPF-like glasses. Hematite $\left(\mathrm{Fe}_{2} \mathrm{O}_{3}\right)$ was also observed in some of the glasses as well as lithium iron oxide $\left(\mathrm{LiFeO}_{2}\right)$; however, neither of these crystal types is known to have a significant impact on durability.

\subsection{Density}

Average densities ${ }^{11}$ of the variability study glasses are shown in Table 3-4. Values were in the range of $2.65-2.75 \mathrm{~g} / \mathrm{cc}$.

\section{$3.5 \underline{\mathrm{PCT}}$}

Table B-1 in Appendix B provides the elemental leachate concentration measurements for the solution samples generated by the PCTs. Any measurement below the detection limit of the analytical procedure (indicated by a " $<$ ") was replaced by $1 / 2$ of the detection limit in subsequent analyses. ${ }^{10}$ In addition to adjustments for detection limits, the values were adjusted for the dilution factors: study glasses, blanks and the ARM glass were multiplied by 1.6667 to determine the values in parts per million (ppm) and the values for EA were multiplied by 16.6667. Over the course of the 7-day test, there were no water loss issues.

\subsubsection{Measurements in Analytical Sequence}

Exhibits B-1 through B-4 in Appendix B provides plots of the common logarithms of the leachate (ppm) concentrations in analytical sequence. No issues were observed in these plots.

\subsubsection{Results of the Multi-Element Solution Standard}

Exhibit B-5 in Appendix B provides analyses of measurements of the multi-element solution standard by analytical set/ICP-AES calibration block, and ANOVA investigations for each element of interest. A statistically significant difference (at a 5\% level) among the averages of these measurements was indicated for B, Na and $\mathrm{Si}$ in Set 1 and $\mathrm{Li}$ in Set 2.

Table 3-5 summarizes the average measurements and the reference values for the 4 elements of interest for both sets of PCTs. The results indicate consistent and accurate measurements throughout the measurement process and none of the differences are greater than $5 \%$. Averaging the ppm values for each set of triplicates helps to minimize the impact of any potential instrumentation effects.

\footnotetext{
${ }^{10}$ XRD patterns are shown in SRNL-NB-2010-00162, pages 115-119 and pages 144-145.

${ }^{11}$ All density values are shown in SRNL-NB-2010-00162, pages 124-125.
} 
Table 3-1. Results of the PCCS Assessment at the MAR (Target Compositions)

\begin{tabular}{|c|c|c|c|c|c|c|c|c|c|}
\hline Glass ID & B Del Gp & $\mathrm{NL}[\mathrm{B}(\mathrm{g} / \mathrm{L})]$ & $\mathrm{T}_{\mathrm{L}}$ Pred $\left({ }^{\circ} \mathrm{C}\right)$ & Visc Pred $(\mathrm{P})$ & $\mathrm{Al}_{2} \mathrm{O}_{3}(\mathrm{wt} \%)$ & $\mathrm{TiO}_{2}(\mathrm{wt} \%)$ & $\mathrm{R}_{2} \mathrm{O}(\mathrm{wt} \%)$ & Neph Discr. & MAR Status \\
\hline SB7b-01 & -12.34 & 2.16 & 916 & 24 & \begin{tabular}{|l|}
6.353 \\
\end{tabular} & 0.000 & \begin{tabular}{l|l}
20.849 \\
\end{tabular} & 0.68 & low viscosity \\
\hline SB7b-02 & -12.03 & 1.89 & 910 & 23 & 6.353 & 0.723 & 20.057 & 0.68 & low viscosity \\
\hline SB7b-03 & -13.97 & 4.26 & 878 & 18 & 8.841 & 0.000 & 22.931 & 0.65 & DGp low viscosity \\
\hline SB7b-04 & -7.38 & 0.27 & 897 & 122 & 9.900 & 0.000 & 16.233 & 0.71 & high viscosity \\
\hline SB7b-05 & -12.06 & 1.92 & 818 & 48 & 6.353 & 0.000 & 20.099 & 0.71 & acceptable \\
\hline SB7b-06 & -8.97 & 0.53 & 1048 & 32 & 9.900 & 0.000 & 17.962 & 0.69 & TL \\
\hline SB7b-07 & -12.81 & 2.62 & 809 & 24 & 9.900 & 0.723 & 22.849 & 0.65 & low viscosity \\
\hline SB7b-08 & -13.86 & 4.07 & 831 & 12 & 6.353 & 0.000 & 22.136 & 0.68 & DGp low viscosity \\
\hline SB7b-09 & -8.67 & 0.47 & 1015 & 33 & 9.900 & 0.000 & 17.880 & 0.69 & $\mathrm{TL}$ \\
\hline SB7b-10 & -11.45 & 1.49 & 905 & 40 & 9.900 & 0.723 & 20.381 & 0.65 & acceptable \\
\hline SB7b-11 & -9.04 & 0.55 & 982 & 44 & 9.900 & 0.723 & 18.464 & 0.66 & acceptable \\
\hline SB7b-12 & -8.00 & 0.35 & 1012 & 73 & 6.353 & 0.723 & 15.880 & 0.75 & acceptable \\
\hline SB7b-13 & -9.65 & 0.70 & 947 & 49 & 6.353 & 0.723 & 17.962 & 0.75 & acceptable \\
\hline SB7b-14 & -11.10 & 1.29 & 798 & 42 & 6.353 & 0.723 & 19.135 & 0.74 & acceptable \\
\hline SB7b-15 & -11.55 & 1.55 & 849 & 40 & 7.240 & 0.542 & 20.127 & 0.70 & acceptable \\
\hline SB7b-16 & -9.57 & 0.68 & 957 & 37 & 9.013 & 0.542 & 18.684 & 0.69 & acceptable \\
\hline SB7b-17 & -10.66 & 1.07 & 916 & 41 & 7.240 & 0.542 & 19.204 & 0.72 & acceptable \\
\hline SB7b-18 & -11.16 & 1.32 & 950 & 32 & 7.240 & 0.542 & 19.540 & 0.69 & acceptable \\
\hline SB7b-19 & -11.02 & 1.24 & 891 & 43 & 9.013 & 0.181 & 20.169 & 0.67 & acceptable \\
\hline SB7b-20 & -8.83 & 0.50 & 952 & 71 & 9.013 & 0.181 & 17.642 & 0.71 & acceptable \\
\hline SB7b-21 & -10.51 & 1.01 & 889 & 43 & 7.240 & 0.181 & 18.684 & 0.72 & acceptable \\
\hline SB7b-22 & -10.06 & 0.83 & 912 & 39 & 7.240 & 0.181 & 18.642 & 0.72 & acceptable \\
\hline SB7b-23 & -9.60 & 0.69 & 934 & 47 & 9.013 & 0.542 & 18.642 & 0.70 & acceptable \\
\hline SB7b-24 & -11.70 & 1.65 & 868 & 29 & 9.013 & 0.542 & 20.728 & 0.67 & acceptable \\
\hline SB7b-25 & -12.67 & 2.48 & 896 & 22 & 7.240 & 0.181 & 21.169 & 0.69 & low viscosity \\
\hline SB7b-26 & -8.90 & 0.51 & 1007 & 51 & 9.013 & 0.181 & 17.684 & 0.69 & acceptable \\
\hline SB7b-27 & -11.94 & 1.83 & 911 & 22 & 7.240 & 0.181 & 20.642 & 0.69 & low viscosity \\
\hline SB7b-28 & -9.37 & 0.62 & 933 & 50 & 7.240 & 0.542 & 17.642 & 0.72 & acceptable \\
\hline SB7b-29 & -10.81 & 1.14 & 855 & 51 & 7.013 & 0.213 & 19.236 & 0.72 & acceptable \\
\hline SB7b-30 & -10.99 & 1.23 & 901 & 42 & 7.890 & 0.240 & 19.640 & 0.69 & acceptable \\
\hline SB7b-31 & -11.17 & 1.33 & 943 & 34 & 8.767 & 0.266 & 20.045 & 0.66 & acceptable \\
\hline SB7b-32 & -10.13 & 0.86 & 877 & 45 & 7.201 & 0.213 & 18.613 & 0.73 & acceptable \\
\hline SB7b-33 & -10.24 & 0.90 & 926 & 38 & 8.101 & 0.240 & 18.939 & 0.71 & acceptable \\
\hline SB7b-34 & -10.35 & 0.94 & 970 & 31 & 9.001 & 0.267 & 19.266 & 0.68 & acceptable \\
\hline
\end{tabular}


Table 3-2. Results of the PCCS Assessment at the MAR (Measured Compositions)

\begin{tabular}{|c|c|c|c|c|c|c|c|c|c|}
\hline Glass ID & B Del Gp & $\mathrm{NL}[\mathrm{B}(\mathrm{g} / \mathrm{L})]$ & $\mathrm{T}_{\mathrm{L}}$ Pred $\left({ }^{\circ} \mathrm{C}\right)$ & Visc Pred $(\mathrm{P})$ & $\mathrm{Al}_{2} \mathrm{O}_{3}(\mathrm{wt} \%)$ & $\mathrm{TiO}_{2}(\mathrm{wt} \%)$ & $\mathrm{R}_{2} \mathrm{O}(\mathrm{wt} \%)$ & Neph Discr. & MAR Status \\
\hline SB7b-01 & \begin{tabular}{|c|}
-12.28 \\
\end{tabular} & 2.10 & 915 & 26 & 6.443 & 0.010 & 20.9124 & 0.68 & acceptable \\
\hline SB7b-02 & -11.84 & 1.75 & 908 & 23 & 6.382 & 0.690 & 19.8259 & 0.68 & acceptable \\
\hline SB7b-03 & -13.79 & 3.96 & 895 & 17 & 8.867 & 0.009 & 22.6788 & 0.65 & DGp low viscosity \\
\hline SB7b-04 & -7.42 & 0.28 & 892 & 131 & 10.147 & 0.012 & 16.4141 & 0.71 & high viscosity \\
\hline SB7b-05 & -11.95 & 1.84 & 817 & 49 & 6.481 & 0.009 & 20.0551 & 0.71 & acceptable \\
\hline SB7b-06 & -8.89 & 0.51 & 1036 & 33 & 10.038 & 0.008 & 17.8762 & 0.69 & $\mathrm{TL}$ \\
\hline SB7b-07 & -12.44 & 2.26 & 812 & 27 & 9.913 & 0.684 & 22.4911 & 0.65 & acceptable \\
\hline SB7b-08 & -13.69 & 3.80 & 835 & 13 & 6.436 & 0.011 & 22.057 & 0.68 & DGp low viscosity \\
\hline SB7b-09 & -8.74 & 0.48 & 1002 & 36 & 10.026 & 0.011 & 18.0949 & 0.69 & acceptable \\
\hline SB7b-10 & -11.42 & 1.47 & 898 & 44 & 9.946 & 0.691 & 20.4461 & 0.65 & acceptable \\
\hline SB7b-11 & -8.81 & 0.50 & 977 & 48 & 9.993 & 0.683 & 18.2424 & 0.67 & acceptable \\
\hline SB7b-12 & -7.96 & 0.35 & 1009 & 77 & 6.533 & 0.668 & 15.9094 & 0.75 & acceptable \\
\hline SB7b-13 & -9.41 & 0.64 & 947 & 49 & 6.462 & 0.680 & 17.6233 & 0.75 & acceptable \\
\hline SB7b-14 & -11.11 & 1.29 & 801 & 43 & 6.507 & 0.676 & 19.2258 & 0.74 & acceptable \\
\hline SB7b-15 & -11.68 & 1.64 & 853 & 35 & 7.303 & 0.510 & 20.2122 & 0.69 & acceptable \\
\hline SB7b-16 & -9.38 & 0.63 & 956 & 39 & 9.041 & 0.520 & 18.4957 & 0.69 & acceptable \\
\hline SB7b-17 & -10.40 & 0.96 & 918 & 46 & 7.315 & 0.510 & 19.0121 & 0.72 & acceptable \\
\hline SB7b-18 & -11.18 & 1.33 & 952 & 32 & 7.383 & 0.520 & 19.6463 & 0.69 & acceptable \\
\hline SB7b-19 & -11.37 & 1.44 & 883 & 39 & 9.150 & 0.184 & 20.5862 & 0.67 & acceptable \\
\hline SB7b-20 & -8.51 & 0.44 & 945 & 81 & 9.070 & 0.174 & 17.3447 & 0.71 & acceptable \\
\hline SB7b-21 & -10.77 & 1.12 & 886 & 35 & 7.376 & 0.181 & 18.8744 & 0.71 & acceptable \\
\hline SB7b-22 & -10.34 & 0.94 & 896 & 37 & 7.398 & 0.182 & 18.8992 & 0.72 & acceptable \\
\hline SB7b-23 & -9.45 & 0.65 & 926 & 51 & 9.164 & 0.530 & 18.5687 & 0.70 & acceptable \\
\hline SB7b-24 & -11.69 & 1.65 & 863 & 28 & 8.987 & 0.509 & 20.6465 & 0.67 & acceptable \\
\hline SB7b-25 & -12.72 & 2.53 & 889 & 22 & 7.450 & 0.180 & 21.3621 & 0.68 & low viscosity \\
\hline SB7b-26 & -8.85 & 0.50 & 990 & 52 & 9.115 & 0.174 & 17.6005 & 0.69 & acceptable \\
\hline SB7b-27 & -11.92 & 1.81 & 918 & 20 & 7.362 & 0.176 & 20.6172 & 0.68 & low viscosity \\
\hline SB7b-28 & -9.17 & 0.58 & 935 & 52 & 7.272 & 0.515 & 17.4306 & 0.72 & acceptable \\
\hline SB7b-29 & -10.97 & 1.22 & 856 & 47 & 7.090 & 0.199 & 19.3881 & 0.71 & acceptable \\
\hline SB7b-30 & -10.83 & 1.15 & 901 & 47 & 8.033 & 0.227 & 19.6336 & 0.69 & acceptable \\
\hline SB7b-31 & -9.05 & 0.55 & 985 & 53 & 7.686 & 0.213 & 17.4244 & 0.71 & acceptable \\
\hline SB7b-32 & -11.91 & 1.81 & 844 & 33 & 8.330 & 0.252 & 20.9378 & 0.69 & acceptable \\
\hline SB7b-33 & -10.38 & 0.95 & 924 & 34 & 8.238 & 0.233 & 19.0711 & 0.70 & acceptable \\
\hline SB7b-34 & -10.30 & 0.92 & 964 & 33 & 9.056 & 0.258 & 19.2668 & 0.68 & acceptable \\
\hline
\end{tabular}


Table 3-3. XRD Results

\begin{tabular}{|c|c|c|c|}
\hline Glass ID & Sludge Description & Quenched & ccc \\
\hline SB7b-03ccc & \multirow{7}{*}{ Outer layer EV } & amorphous & trevorite $\left(\mathrm{NiFe}_{2} \mathrm{O}_{4}\right)$, hematite $\left(\mathrm{Fe}_{2} \mathrm{O}_{3}\right)$ \\
\hline SB7b-06ccc & & amorphous & trevorite $\left(\mathrm{NiFe}_{2} \mathrm{O}_{4}\right)$ \\
\hline SB7b-07ccc & & amorphous & hematite $\left(\mathrm{Fe}_{2} \mathrm{O}_{3}\right)$ \\
\hline SB7b-08cec & & amorphous & hematite $\left(\mathrm{Fe}_{2} \mathrm{O}_{3}\right)$ \\
\hline SB7b-09cec & & amorphous & lithium iron oxide $\left(\mathrm{LiFeO}_{2}\right)$, trevorite $\left(\mathrm{NiFe}_{2} \mathrm{O} 4\right)$ \\
\hline SB7b-13ccc & & amorphous & amorphous \\
\hline SB7b-14ccc & & amorphous & amorphous \\
\hline SB7b-24ccc & \multirow{3}{*}{ Inner layer EV } & amorphous & amorphous \\
\hline SB7b-25ccc & & amorphous & amorphous \\
\hline SB7b-27ccc & & amorphous & possibly hematite $\left(\mathrm{Fe}_{2} \mathrm{O}_{3}\right)$ \\
\hline SB7b-32ccc & Nominal $(+4 \% \mathrm{Na} 2 \mathrm{O})$-Frit 702 at $32 \% \mathrm{WL}$ & amorphous & amorphous \\
\hline SB7b-33ccc & Nominal $(+4 \% \mathrm{Na} 2 \mathrm{O})$-Frit 702 at $36 \% \mathrm{WL}$ & amorphous & amorphous \\
\hline SB7b-34ccc & Nominal $(+4 \% \mathrm{Na} 2 \mathrm{O})$-Frit 702 at $40 \% \mathrm{WL}$ & amorphous & amorphous \\
\hline
\end{tabular}

Table 3-4. Densities

\begin{tabular}{|c|c|c|c|}
\hline Glass ID & Density (g/cc) & Glass ID & Density $(\mathrm{g} / \mathrm{cc})$ \\
\hline SB7b-01 & 2.72 & SB7b-18 & 2.72 \\
\hline SB7b-02 & 2.75 & SB7b-19 & 2.67 \\
\hline SB7b-03 & 2.69 & SB7b-20 & 2.66 \\
\hline SB7b-04 & 2.65 & SB7b-21 & 2.69 \\
\hline SB7b-05 & 2.67 & SB7b-22 & 2.67 \\
\hline SB7b-06 & 2.70 & SB7b-23 & 2.68 \\
\hline SB7b-07 & 2.65 & SB7b-24 & 2.68 \\
\hline SB7b-08 & 2.73 & SB7b-25 & 2.71 \\
\hline SB7b-09 & 2.72 & SB7b-26 & 2.70 \\
\hline SB7b-10 & 2.70 & SB7b-27 & 2.71 \\
\hline SB7b-11 & 2.71 & SB7b-28 & 2.71 \\
\hline SB7b-12 & 2.70 & SB7b-29 & 2.66 \\
\hline SB7b-13 & 2.69 & SB7b-30 & 2.69 \\
\hline SB7b-14 & 2.66 & SB7b-31 & 2.71 \\
\hline SB7b-15 & 2.69 & SB7b-32 & 2.67 \\
\hline SB7b-16 & 2.75 & SB7b-33 & 2.69 \\
\hline SB7b-17 & 2.68 & SB7b-34 & 2.72 \\
\hline
\end{tabular}


Table 3-5. Results from Samples of the Multi-Element Solution Standard (ppm)

\begin{tabular}{|c|c|c|c|c|}
\hline Analytical Set/Block & Average B & Average Li & Average Na & Average Si \\
\hline $1 / 1$ & 19.8 & 9.9 & 82.6 & 52.5 \\
\hline $1 / 2$ & 19.8 & 9.9 & 83.3 & 52.2 \\
\hline $1 / 3$ & 19.8 & 9.9 & 83.6 & 52.2 \\
\hline $1 / 4$ & 19.6 & 10.0 & 82.6 & 51.3 \\
\hline $1 / 5$ & 19.7 & 9.8 & 83.1 & 52.3 \\
\hline $1 / 6$ & 19.7 & 9.8 & 82.9 & 52.1 \\
\hline $2 / 1$ & 19.8 & 10.1 & 83.3 & 52.3 \\
\hline $2 / 2$ & 20.1 & 10.1 & 84.1 & 52.8 \\
\hline $2 / 3$ & 19.9 & 10.1 & 83.2 & 52.3 \\
\hline $2 / 4$ & 19.7 & 10.0 & 83.8 & 52.2 \\
\hline $2 / 5$ & 19.7 & 9.9 & 83.0 & 52.0 \\
\hline $2 / 6$ & 19.8 & 9.9 & 83.0 & 52.2 \\
\hline Average & 19.8 & 9.9 & 83.2 & 52.2 \\
\hline Reference Value & 20 & 10 & 81 & 50 \\
\hline$\%$ Difference & -1.0 & -0.6 & 2.7 & 4.4 \\
\hline
\end{tabular}

\subsubsection{Measurements by Glass Identifier}

Exhibit B-6 in Appendix B provides plots of the leachate concentrations for both the quenched and ccc versions of each glass, as well as the standards (EA, $\mathrm{ARM}^{12}$, multi-element solution standard and blanks). ${ }^{11}$ Two units of measure are used in these plots: ppm and the common logarithms of the ppm values. The common logarithm plots allow for the assessment of the repeatability of the measurements and any differences between the quenched and ccc version of a given glass. In general, the most noticeable differences are seen in the glasses with identified crystallization (SB7b-03ccc, $-06 \mathrm{ccc},-07 \mathrm{ccc},-08 \mathrm{ccc},-09 \mathrm{ccc},-13 \mathrm{ccc}$ and $-27 \mathrm{ccc}$ ), especially in Li.

\subsubsection{Normalized PCT Release Values}

PCT leachate concentrations were normalized using the target and measured cation compositions $(\mathrm{wt} \%)$ in the glass to obtain a grams-per-liter $(\mathrm{g} / \mathrm{L})$ leachate concentration.

Exhibit B-7 in Appendix B provides scatter plots that contain normalized released rates for both the quenched and ccc versions of the glasses based on the target and measured compositions. These plots offer an opportunity to investigate the consistency in the leaching across the elements for the glasses of this study. Consistency in the leaching across the elements is typically demonstrated by a high degree of linear correlation among the values for pairs of these elements. The smallest correlation in this plot is for $\mathrm{Na}$ and $\mathrm{Li}$, with a value of $\sim 86.2 \%$, which demonstrates the consistency of the results among the 4 analytes.

Table 4-1 summarizes the normalized PCTs for all of the glasses of this study, which are listed by heat treatment and compositional view for each glass. A review of the data shows that all of the glasses were acceptable relative to the EA glass regardless of thermal history (quenched or CCC) or compositional view (target or measured). It is noted that the NL [B] release for the EA glass from this study was relatively low (i.e., $13.8 \mathrm{~g} / \mathrm{L}$ in set 1 and $9.3 \mathrm{~g} / \mathrm{L}$ in set 2) as compared to the

\footnotetext{
${ }^{12}$ The concentrations of each element of interest for ARM are within the controls limits in THERMO ${ }^{\mathrm{TM}}$.
} 
reported values of $16.695 \mathrm{~g} / \mathrm{L}$. Variations in the EA glass response have been observed in previous studies, which is why ARM is used as the PCT standard and EA is used as a benchmark. $^{12,13}$ All of the SB7b variability study glasses were acceptable relative to the EA glass regardless of the historical release value $(16.695 \mathrm{~g} / \mathrm{L})$ or the values observed in this study.

\subsubsection{Effects of Heat Treatment}

Exhibit B-8 in Appendix B provides plots of the normalized PCT responses both the quenched and CCC glasses. These plots provide a basis for judging the practical impact of differences in the PCT response due to the heat treatment of the glass. In general, the ccc versions were relatively consistent with the quenched versions (within experimental error). As stated in Section 3.5.3, the most noticeable differences are seen in the glasses with identified crystallization (SB7b$03 c c c,-06 c c c,-07 c c c,-08 c c c,-09 c c c,-13 c c c$ and $-27 c c c)$, especially in Li. In these particular glasses, the PCT releases of the ccc version of the glasses was slightly elevated as compared to the quenched version of the glass; however, the releases of each compositional view were well below EA and were acceptable. One of the glasses (SB7b-25) has lower PCT releases in the ccc version of the glass rather than the quenched version of the glass.

\subsubsection{Predicted and Measured PCT Values}

Exhibits B-9 and B-10 in Appendix B provide plots of the DWPF models for B, Li, Na, and Si that relate the logarithm of the normalized PCT (for each element of interest) to a linear function of a free energy of hydration term $\left(\Delta \mathrm{G}_{\mathrm{p}}, \mathrm{kcal} / 100 \mathrm{~g}\right.$ glass $)$ derived for the target and measured compositional views, respectively. The results for both heat treatments are shown on each plot. Prediction limits at a 95\% confidence level for an individual PCT result are also plotted along with the linear fit. The EA and ARM results are also indicated on these plots. Figure 3-1 provides a closer look at the boron PCT responses.

All of the glasses, both quenched and ccc, were acceptable relative to the EA glass. Each of the nominal glasses, as well as the "inner layer" EV glasses, were predictable with respect to the PCCS durability models for durability. In Figure 3-1, as well as the plots in Exhibit B-9, there were a small number of the "outer layer" EV quenched glasses that were unpredictable and fall outside of the lower 95\% confidence band. Of these glasses, two glasses (SB7b-03 and -08) failed the PCCS MAR constraint for $\Delta \mathrm{G}_{\mathrm{p}}$; however, the normalized boron releases values were only in the range of 1.12-1.99 $\mathrm{g} / \mathrm{L}$ regardless of heat treatment and/or compositional view. Glasses outside of the lower $95 \%$ confidence band at more negative values of $\Delta \mathrm{G}_{\mathrm{p}}$ demonstrate the conservatism of the model as the measured normalized boron release values were lower than the predicted values.

In Exhibits B-9 and B-10, a few of the "outer layer" ccc glasses also fall above the upper 95\% confidence band. These points have more positive $\Delta \mathrm{G}_{\mathrm{p}}$ and were of little practical concern. The normalized PCT values were acceptable when compared to EA and this type of behavior for $\Delta \mathrm{G}_{\mathrm{p}^{-}}$ model predictions has been observed in previous variability studies supporting DWPF. ${ }^{14-16}$ Of these glasses, SB7b-06ccc and -09ccc contained lithium iron oxide and/or trevorite. The current models for durability are only valid for homogeneous glasses and would not be expected to predict the PCT response of glasses containing crystals. 


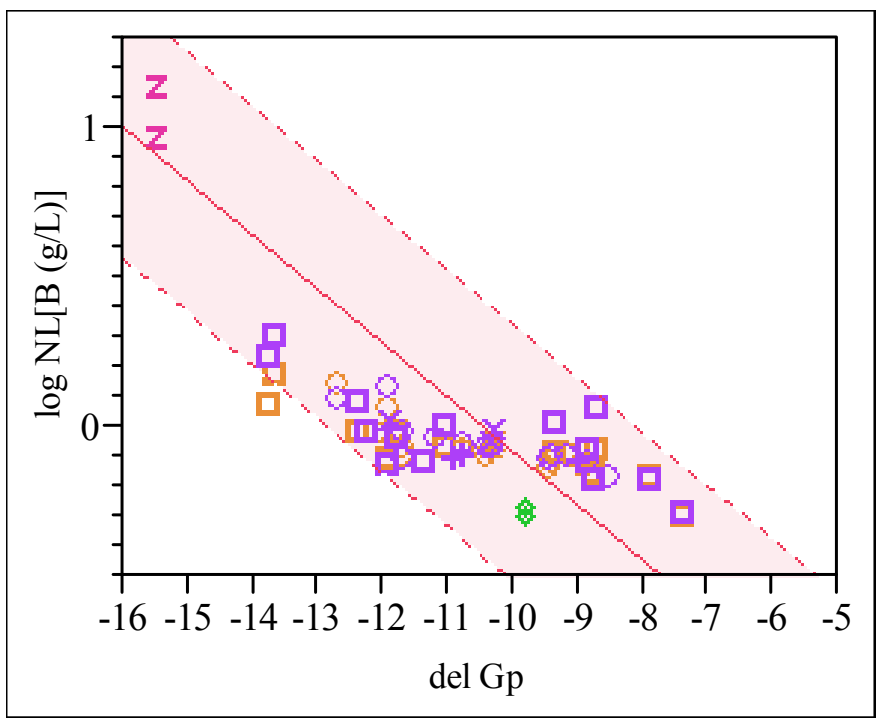

Legend

\begin{tabular}{|c|l|c|c|l|c|}
\hline$\diamond$ & ARM & --- & $\square$ & D-Opt EV Outer Layer & quenched \\
\hline $\mathrm{z}$ & EA & --- & + & Nominal-Frit 418 & ccc \\
\hline 0 & D-Opt EV Inner Layer & $\mathrm{ccc}$ & + & Nominal-Frit 418 & quenched \\
\hline 0 & D-Opt EV Inner Layer & quenched & $\times$ & Nominal-Frit 702 & ccc \\
\hline$\square$ & D-Opt EV Outer Layer & $\mathrm{ccc}$ & $\times$ & Nominal-Frit 702 & quenched \\
\hline
\end{tabular}

Figure 3-1. Del $G_{p}$ versus $\log$ NL [B] values based on measured compositions.

\subsection{Conclusions}

To comply with the DWPF Glass Product Control Program, a total of thirty four glasses were fabricated to assess the applicability of the current DWPF PCCS durability models for the SB7b sludge system (plus caustic addition) blended with Frits 418 or 702 . Based on the measured PCT response, all of the glasses were acceptable with respect to the Environmental Assessment (EA) benchmark glass regardless of thermal history. The NL[B] values of the SB7b variability study glasses were less than $1.99 \mathrm{~g} / \mathrm{L}$. A small number of the D-optimally selected "outer layer" EV glasses were not predictable using the current PCCS models for durability, but were acceptable compared to the EA glass when tested. These glasses fell outside of the lower $95 \%$ confidence band, which demonstrates conservatism in the model. A few of the glasses fell outside of the upper $95 \%$ confidence band; however, these particular glasses have normalized release values that were much lower than the values of EA and should be of no practical concern.

Per the requirements of the DWPF Glass Product Control Program, the PCCS durability models have been shown to be applicable to the SB7b sludge system with a range of $\mathrm{Na}_{2} \mathrm{O}$ concentrations blended with Frits 418 or 702 . PCT results from the glasses fabricated as part of the variability study were shown to be predictable by the current DWPF PCCS models and/or acceptable with respect to the EA benchmark glass regardless of thermal history or compositional view. 
Table 4-1. Normalized PCT Results

\begin{tabular}{|c|c|c|c|c|c|c|c|c|c|c|}
\hline Glass ID & Heat Treatment & Comp View & $\begin{array}{c}\log \mathrm{NL} \\
{[\mathrm{B}(\mathrm{g} / \mathrm{L})]}\end{array}$ & $\begin{array}{c}\log \mathrm{NL} \\
{[\mathrm{Li}(\mathrm{g} / \mathrm{L})]}\end{array}$ & $\begin{array}{c}\log \mathrm{NL} \\
{[\mathrm{Na}(\mathrm{g} / \mathrm{L})]}\end{array}$ & $\begin{array}{c}\log \mathrm{NL} \\
{[\mathrm{Si}(\mathrm{g} / \mathrm{L})]}\end{array}$ & NL B $(g / L)$ & NL Li (g/L) & $\mathrm{NL} \mathrm{Na}(\mathrm{g} / \mathrm{L})$ & NL Si $(\mathrm{g} / \mathrm{L})$ \\
\hline \multirow{2}{*}{ ARM } & \multirow{2}{*}{ reference } & set 1 & -0.30 & -0.22 & -0.28 & -0.53 & 0.50 & 0.60 & 0.52 & 0.30 \\
\hline & & set 2 & -0.27 & -0.20 & -0.25 & -0.51 & 0.53 & 0.64 & 0.56 & 0.31 \\
\hline \multirow{2}{*}{ EA } & \multirow{2}{*}{ reference } & set 1 & 1.14 & 0.90 & 1.05 & 0.56 & 13.82 & 7.97 & 11.20 & 3.63 \\
\hline & & set 2 & 0.97 & 0.77 & 0.89 & 0.45 & 9.27 & 5.95 & 7.78 & 2.80 \\
\hline \multirow{4}{*}{ SB7b-01 } & \multirow{2}{*}{ quenched } & target & -0.03 & -0.04 & 0.10 & -0.16 & 0.92 & 0.91 & 1.25 & 0.70 \\
\hline & & measured & -0.02 & -0.04 & 0.10 & -0.16 & 0.96 & 0.92 & 1.25 & 0.69 \\
\hline & \multirow{2}{*}{$\operatorname{ccc}$} & target & -0.04 & -0.01 & 0.07 & -0.15 & 0.92 & 0.98 & 1.18 & 0.70 \\
\hline & & measured & -0.02 & 0.00 & 0.07 & -0.16 & 0.96 & 0.99 & 1.18 & 0.69 \\
\hline \multirow{4}{*}{ SB7b-02 } & \multirow{2}{*}{ quenched } & target & -0.03 & -0.02 & 0.07 & -0.19 & 0.93 & 0.94 & 1.16 & 0.65 \\
\hline & & measured & -0.02 & -0.02 & 0.07 & -0.18 & 0.96 & 0.95 & 1.19 & 0.66 \\
\hline & \multirow{2}{*}{$\operatorname{ccc}$} & target & -0.05 & -0.03 & 0.02 & -0.20 & 0.89 & 0.94 & 1.05 & 0.63 \\
\hline & & measured & -0.04 & -0.02 & 0.03 & -0.20 & 0.91 & 0.94 & 1.07 & 0.63 \\
\hline \multirow{4}{*}{ SB7b-03 } & \multirow{2}{*}{ quenched } & target & 0.05 & 0.11 & 0.24 & -0.09 & 1.12 & 1.29 & 1.73 & 0.81 \\
\hline & & measured & 0.07 & 0.12 & 0.24 & -0.08 & 1.19 & 1.31 & 1.75 & 0.83 \\
\hline & \multirow{2}{*}{$\operatorname{ccc}$} & target & 0.21 & 0.39 & 0.26 & 0.07 & 1.62 & 2.43 & 1.80 & 1.17 \\
\hline & & measured & 0.23 & 0.39 & 0.26 & 0.08 & 1.72 & 2.47 & 1.82 & 1.20 \\
\hline \multirow{4}{*}{ SB7b-04 } & \multirow{2}{*}{ quenched } & target & -0.31 & -0.16 & -0.30 & -0.34 & 0.49 & 0.69 & 0.50 & 0.46 \\
\hline & & measured & -0.30 & -0.16 & -0.31 & -0.35 & 0.51 & 0.69 & 0.50 & 0.45 \\
\hline & \multirow{2}{*}{$\mathrm{ccc}$} & target & -0.31 & -0.17 & -0.30 & -0.34 & 0.49 & 0.67 & 0.51 & 0.46 \\
\hline & & measured & -0.29 & -0.17 & -0.30 & -0.35 & 0.51 & 0.68 & 0.50 & 0.45 \\
\hline \multirow{4}{*}{ SB7b-05 } & \multirow{2}{*}{ quenched } & target & -0.14 & -0.07 & 0.02 & -0.24 & 0.73 & 0.84 & 1.05 & 0.58 \\
\hline & & measured & -0.11 & -0.07 & 0.02 & -0.23 & 0.77 & 0.86 & 1.05 & 0.58 \\
\hline & \multirow{2}{*}{$\operatorname{ccc}$} & target & -0.15 & -0.08 & -0.03 & -0.26 & 0.70 & 0.83 & 0.94 & 0.55 \\
\hline & & measured & -0.13 & -0.07 & -0.02 & -0.26 & 0.74 & 0.85 & 0.95 & 0.56 \\
\hline \multirow{4}{*}{ SB7b-06 } & \multirow{2}{*}{ quenched } & target & -0.09 & -0.06 & -0.08 & -0.22 & 0.81 & 0.88 & 0.83 & 0.60 \\
\hline & & measured & -0.08 & -0.05 & -0.08 & -0.22 & 0.84 & 0.88 & 0.84 & 0.61 \\
\hline & \multirow{2}{*}{$\operatorname{ccc}$} & target & -0.09 & 0.08 & -0.04 & -0.13 & 0.81 & 1.20 & 0.90 & 0.74 \\
\hline & & measured & -0.08 & 0.08 & -0.04 & -0.13 & 0.84 & 1.21 & 0.91 & 0.74 \\
\hline
\end{tabular}


Table 3-5 cont. Normalized PCT Results

\begin{tabular}{|c|c|c|c|c|c|c|c|c|c|c|}
\hline Glass ID & Heat Treatment & Comp View & $\begin{array}{c}\log \mathrm{NL} \\
{[\mathrm{B}(\mathrm{g} / \mathrm{L})]}\end{array}$ & $\begin{array}{c}\log \mathrm{NL} \\
{[\mathrm{Li}(\mathrm{g} / \mathrm{L})]}\end{array}$ & $\begin{array}{c}\log \mathrm{NL} \\
{[\mathrm{Na}(\mathrm{g} / \mathrm{L})]}\end{array}$ & $\begin{array}{c}\log \mathrm{NL} \\
{[\mathrm{Si}(\mathrm{g} / \mathrm{L})]}\end{array}$ & NL B (g/L) & NL Li $(g / L)$ & $\mathrm{NL} \mathrm{Na}(\mathrm{g} / \mathrm{L})$ & NL Si (g/L) \\
\hline \multirow{4}{*}{ SB7b-07 } & \multirow{2}{*}{ quenched } & target & -0.04 & 0.03 & 0.17 & -0.10 & 0.90 & 1.08 & 1.49 & 0.79 \\
\hline & & measured & -0.02 & 0.04 & 0.18 & -0.11 & 0.95 & 1.10 & 1.53 & 0.78 \\
\hline & \multirow{2}{*}{$\operatorname{ccc}$} & target & 0.06 & 0.33 & 0.19 & 0.08 & 1.15 & 2.12 & 1.53 & 1.19 \\
\hline & & measured & 0.08 & 0.33 & 0.19 & 0.07 & 1.21 & 2.15 & 1.56 & 1.18 \\
\hline \multirow{4}{*}{ SB7b-08 } & \multirow{2}{*}{ quenched } & target & 0.15 & 0.16 & 0.25 & -0.02 & 1.42 & 1.46 & 1.80 & 0.96 \\
\hline & & measured & 0.17 & 0.17 & 0.26 & -0.02 & 1.50 & 1.48 & 1.81 & 0.95 \\
\hline & \multirow{2}{*}{$\operatorname{ccc}$} & target & 0.28 & 0.44 & 0.31 & 0.17 & 1.89 & 2.73 & 2.04 & 1.47 \\
\hline & & measured & 0.30 & 0.44 & 0.31 & 0.17 & 1.99 & 2.76 & 2.06 & 1.47 \\
\hline \multirow{4}{*}{ SB7b-09 } & \multirow{2}{*}{ quenched } & target & -0.10 & -0.05 & -0.09 & -0.23 & 0.80 & 0.89 & 0.82 & 0.59 \\
\hline & & measured & -0.08 & -0.05 & -0.09 & -0.24 & 0.83 & 0.89 & 0.81 & 0.58 \\
\hline & \multirow{2}{*}{$\operatorname{ccc}$} & target & 0.04 & 0.28 & 0.06 & 0.02 & 1.09 & 1.92 & 1.16 & 1.04 \\
\hline & & measured & 0.06 & 0.28 & 0.06 & 0.01 & 1.14 & 1.91 & 1.14 & 1.02 \\
\hline \multirow{4}{*}{ SB7b-10 } & \multirow{2}{*}{ quenched } & target & -0.15 & -0.12 & 0.01 & -0.24 & 0.72 & 0.76 & 1.02 & 0.57 \\
\hline & & measured & -0.12 & -0.11 & 0.01 & -0.25 & 0.76 & 0.77 & 1.02 & 0.56 \\
\hline & \multirow{2}{*}{$\mathrm{ccc}$} & target & -0.14 & -0.08 & -0.01 & -0.24 & 0.72 & 0.82 & 0.97 & 0.57 \\
\hline & & measured & -0.12 & -0.08 & -0.02 & -0.25 & 0.76 & 0.83 & 0.97 & 0.56 \\
\hline \multirow{4}{*}{ SB7b-11 } & \multirow{2}{*}{ quenched } & target & -0.18 & -0.13 & -0.10 & -0.30 & 0.67 & 0.75 & 0.79 & 0.50 \\
\hline & & measured & -0.16 & -0.12 & -0.10 & -0.30 & 0.70 & 0.76 & 0.80 & 0.50 \\
\hline & \multirow{2}{*}{$\mathrm{ccc}$} & target & -0.20 & -0.15 & -0.14 & -0.31 & 0.63 & 0.71 & 0.73 & 0.48 \\
\hline & & measured & -0.18 & -0.14 & -0.13 & -0.31 & 0.66 & 0.73 & 0.74 & 0.49 \\
\hline \multirow{4}{*}{ SB7b-12 } & \multirow{2}{*}{ quenched } & target & -0.19 & -0.11 & -0.18 & -0.30 & 0.64 & 0.77 & 0.66 & 0.50 \\
\hline & & measured & -0.17 & -0.11 & -0.18 & -0.30 & 0.67 & 0.78 & 0.66 & 0.50 \\
\hline & \multirow{2}{*}{$\mathrm{ccc}$} & target & -0.20 & -0.12 & -0.19 & -0.30 & 0.64 & 0.76 & 0.65 & 0.50 \\
\hline & & measured & -0.18 & -0.11 & -0.18 & -0.30 & 0.67 & 0.77 & 0.65 & 0.50 \\
\hline \multirow{4}{*}{ SB7b-13 } & \multirow{2}{*}{ quenched } & target & -0.11 & -0.04 & -0.07 & -0.22 & 0.77 & 0.92 & 0.85 & 0.60 \\
\hline & & measured & -0.09 & -0.02 & -0.06 & -0.21 & 0.82 & 0.94 & 0.86 & 0.62 \\
\hline & \multirow{2}{*}{$\operatorname{ccc}$} & target & -0.02 & 0.16 & 0.00 & -0.10 & 0.96 & 1.44 & 1.00 & 0.80 \\
\hline & & measured & 0.01 & 0.17 & 0.01 & -0.08 & 1.01 & 1.47 & 1.02 & 0.82 \\
\hline
\end{tabular}


Table 3-5 cont. Normalized PCT Results

\begin{tabular}{|c|c|c|c|c|c|c|c|c|c|c|}
\hline Glass ID & Heat Treatment & Comp View & $\begin{array}{c}\log \mathrm{NL} \\
{[\mathrm{B}(\mathrm{g} / \mathrm{L})]}\end{array}$ & $\begin{array}{c}\log \mathrm{NL} \\
{[\mathrm{Li}(\mathrm{g} / \mathrm{L})]}\end{array}$ & $\begin{array}{c}\log \mathrm{NL} \\
{[\mathrm{Na}(\mathrm{g} / \mathrm{L})]}\end{array}$ & $\begin{array}{c}\log \mathrm{NL} \\
{[\mathrm{Si}(\mathrm{g} / \mathrm{L})]}\end{array}$ & NL B $(g / L)$ & NL Li (g/L) & $\mathrm{NL} \mathrm{Na}(\mathrm{g} / \mathrm{L})$ & NL Si $(g / L)$ \\
\hline \multirow{4}{*}{ SB7b-14 } & \multirow{2}{*}{ quenched } & target & -0.09 & -0.01 & 0.00 & -0.20 & 0.81 & 0.99 & 0.99 & 0.63 \\
\hline & & measured & -0.07 & 0.00 & -0.01 & -0.20 & 0.85 & 1.00 & 0.99 & 0.63 \\
\hline & \multirow{2}{*}{$\mathrm{ccc}$} & target & -0.03 & 0.20 & 0.04 & -0.04 & 0.94 & 1.59 & 1.08 & 0.90 \\
\hline & & measured & 0.00 & 0.21 & 0.03 & -0.04 & 0.99 & 1.61 & 1.08 & 0.90 \\
\hline \multirow{4}{*}{ SB7b-15 } & \multirow{2}{*}{ quenched } & target & -0.12 & -0.05 & 0.03 & -0.20 & 0.76 & 0.90 & 1.08 & 0.63 \\
\hline & & measured & -0.10 & -0.04 & 0.03 & -0.19 & 0.79 & 0.91 & 1.08 & 0.65 \\
\hline & \multirow{2}{*}{$\mathrm{ccc}$} & target & -0.13 & -0.04 & -0.01 & -0.19 & 0.75 & 0.91 & 0.99 & 0.64 \\
\hline & & measured & -0.11 & -0.04 & -0.01 & -0.18 & 0.78 & 0.92 & 0.98 & 0.67 \\
\hline \multirow{4}{*}{ SB7b-16 } & \multirow{2}{*}{ quenched } & target & -0.15 & -0.10 & -0.09 & -0.24 & 0.72 & 0.80 & 0.82 & 0.58 \\
\hline & & measured & -0.12 & -0.09 & -0.08 & -0.24 & 0.75 & 0.82 & 0.83 & 0.58 \\
\hline & \multirow{2}{*}{$\operatorname{ccc}$} & target & -0.12 & -0.04 & -0.08 & -0.22 & 0.75 & 0.92 & 0.83 & 0.61 \\
\hline & & measured & -0.10 & -0.03 & -0.07 & -0.22 & 0.79 & 0.93 & 0.84 & 0.61 \\
\hline \multirow{4}{*}{ SB7b-17 } & \multirow{2}{*}{ quenched } & target & -0.13 & -0.06 & -0.03 & -0.22 & 0.75 & 0.86 & 0.93 & 0.60 \\
\hline & & measured & -0.10 & -0.06 & -0.02 & -0.22 & 0.79 & 0.87 & 0.94 & 0.60 \\
\hline & \multirow{2}{*}{$\operatorname{ccc}$} & target & -0.09 & 0.02 & -0.03 & -0.18 & 0.81 & 1.05 & 0.93 & 0.66 \\
\hline & & measured & -0.07 & 0.03 & -0.03 & -0.19 & 0.85 & 1.06 & 0.94 & 0.65 \\
\hline \multirow{4}{*}{ SB7b-18 } & \multirow{2}{*}{ quenched } & target & -0.05 & -0.03 & 0.05 & -0.20 & 0.88 & 0.94 & 1.12 & 0.62 \\
\hline & & measured & -0.04 & -0.03 & 0.05 & -0.20 & 0.92 & 0.94 & 1.11 & 0.63 \\
\hline & \multirow{2}{*}{$\operatorname{ccc}$} & target & -0.05 & -0.02 & 0.02 & -0.20 & 0.88 & 0.96 & 1.06 & 0.63 \\
\hline & & measured & -0.04 & -0.01 & 0.02 & -0.20 & 0.92 & 0.97 & 1.05 & 0.63 \\
\hline \multirow{4}{*}{ SB7b-19 } & \multirow{2}{*}{ quenched } & target & -0.13 & -0.06 & 0.03 & -0.22 & 0.74 & 0.86 & 1.08 & 0.60 \\
\hline & & measured & -0.12 & -0.06 & 0.02 & -0.21 & 0.77 & 0.86 & 1.05 & 0.61 \\
\hline & \multirow{2}{*}{$\mathrm{ccc}$} & target & -0.14 & -0.05 & -0.01 & -0.22 & 0.73 & 0.88 & 0.98 & 0.61 \\
\hline & & measured & -0.12 & -0.06 & -0.02 & -0.21 & 0.75 & 0.88 & 0.96 & 0.62 \\
\hline \multirow{4}{*}{ SB $7 b-20$} & \multirow{2}{*}{ quenched } & target & -0.20 & -0.09 & -0.12 & -0.27 & 0.64 & 0.82 & 0.76 & 0.54 \\
\hline & & measured & -0.17 & -0.08 & -0.11 & -0.27 & 0.67 & 0.84 & 0.77 & 0.54 \\
\hline & \multirow{2}{*}{$\operatorname{ccc}$} & target & -0.20 & -0.08 & -0.14 & -0.26 & 0.63 & 0.83 & 0.73 & 0.55 \\
\hline & & measured & -0.17 & -0.07 & -0.13 & -0.27 & 0.67 & 0.85 & 0.75 & 0.54 \\
\hline
\end{tabular}


Table 3-5 cont. Normalized PCT Results

\begin{tabular}{|c|c|c|c|c|c|c|c|c|c|c|}
\hline Glass ID & Heat Treatment & Comp View & $\begin{array}{c}\log \mathrm{NL} \\
{[\mathrm{B}(\mathrm{g} / \mathrm{L})]}\end{array}$ & $\begin{array}{c}\log \mathrm{NL} \\
{[\mathrm{Li}(\mathrm{g} / \mathrm{L})]}\end{array}$ & $\begin{array}{c}\log \mathrm{NL} \\
{[\mathrm{Na}(\mathrm{g} / \mathrm{L})]}\end{array}$ & $\begin{array}{c}\log \mathrm{NL} \\
{[\mathrm{Si}(\mathrm{g} / \mathrm{L})]}\end{array}$ & NL B $(g / L)$ & NL Li (g/L) & $\mathrm{NL} \mathrm{Na}(\mathrm{g} / \mathrm{L})$ & NL Si $(g / L)$ \\
\hline \multirow{4}{*}{ SB7b-21 } & \multirow{2}{*}{ quenched } & target & -0.10 & -0.02 & -0.02 & -0.22 & 0.80 & 0.95 & 0.96 & 0.60 \\
\hline & & measured & -0.08 & -0.02 & -0.02 & -0.20 & 0.83 & 0.95 & 0.95 & 0.63 \\
\hline & \multirow{2}{*}{$\mathrm{ccc}$} & target & -0.08 & 0.04 & -0.03 & -0.19 & 0.83 & 1.10 & 0.94 & 0.65 \\
\hline & & measured & -0.06 & 0.04 & -0.03 & -0.16 & 0.86 & 1.10 & 0.93 & 0.69 \\
\hline \multirow{4}{*}{ SB7b-22 } & \multirow{2}{*}{ quenched } & target & -0.09 & 0.00 & 0.00 & -0.19 & 0.81 & 1.00 & 0.99 & 0.65 \\
\hline & & measured & -0.08 & 0.00 & -0.01 & -0.18 & 0.83 & 1.00 & 0.98 & 0.67 \\
\hline & \multirow{2}{*}{$\mathrm{ccc}$} & target & -0.08 & 0.06 & -0.02 & -0.17 & 0.82 & 1.14 & 0.95 & 0.68 \\
\hline & & measured & -0.07 & 0.05 & -0.03 & -0.16 & 0.85 & 1.13 & 0.94 & 0.69 \\
\hline \multirow{4}{*}{ SB7b-23 } & \multirow{2}{*}{ quenched } & target & -0.16 & -0.07 & -0.07 & -0.25 & 0.70 & 0.84 & 0.84 & 0.57 \\
\hline & & measured & -0.14 & -0.07 & -0.07 & -0.25 & 0.73 & 0.85 & 0.85 & 0.56 \\
\hline & \multirow{2}{*}{$\mathrm{ccc}$} & target & -0.13 & 0.01 & -0.07 & -0.19 & 0.75 & 1.02 & 0.86 & 0.64 \\
\hline & & measured & -0.11 & 0.01 & -0.06 & -0.19 & 0.78 & 1.02 & 0.87 & 0.64 \\
\hline \multirow{4}{*}{ SB7b-24 } & \multirow{2}{*}{ quenched } & target & -0.09 & -0.03 & 0.05 & -0.19 & 0.80 & 0.93 & 1.12 & 0.65 \\
\hline & & measured & -0.07 & -0.02 & 0.05 & -0.18 & 0.86 & 0.96 & 1.13 & 0.67 \\
\hline & \multirow{2}{*}{$\mathrm{ccc}$} & target & -0.04 & 0.12 & 0.06 & -0.11 & 0.90 & 1.33 & 1.14 & 0.77 \\
\hline & & measured & -0.02 & 0.13 & 0.06 & -0.10 & 0.96 & 1.36 & 1.15 & 0.79 \\
\hline \multirow{4}{*}{ SB7b-25 } & \multirow{2}{*}{ quenched } & target & 0.06 & 0.36 & 0.20 & 0.04 & 1.16 & 2.31 & 1.58 & 1.09 \\
\hline & & measured & 0.14 & 0.37 & 0.19 & 0.05 & 1.37 & 2.32 & 1.57 & 1.12 \\
\hline & \multirow{2}{*}{$\operatorname{ccc}$} & target & 0.01 & 0.12 & 0.20 & -0.07 & 1.03 & 1.31 & 1.60 & 0.85 \\
\hline & & measured & 0.09 & 0.12 & 0.20 & -0.06 & 1.22 & 1.32 & 1.58 & 0.87 \\
\hline \multirow{4}{*}{ SB7b-26 } & \multirow{2}{*}{ quenched } & target & -0.15 & -0.10 & -0.10 & -0.28 & 0.71 & 0.80 & 0.79 & 0.53 \\
\hline & & measured & -0.13 & -0.09 & -0.10 & -0.27 & 0.75 & 0.81 & 0.80 & 0.54 \\
\hline & \multirow{2}{*}{$\mathrm{ccc}$} & target & -0.14 & -0.07 & -0.10 & -0.26 & 0.73 & 0.86 & 0.79 & 0.55 \\
\hline & & measured & -0.12 & -0.06 & -0.10 & -0.25 & 0.76 & 0.88 & 0.79 & 0.56 \\
\hline \multirow{4}{*}{ SB $7 b-27$} & \multirow{2}{*}{ quenched } & target & 0.04 & 0.07 & 0.16 & -0.11 & 1.10 & 1.18 & 1.44 & 0.78 \\
\hline & & measured & 0.06 & 0.08 & 0.16 & -0.09 & 1.15 & 1.19 & 1.44 & 0.81 \\
\hline & \multirow{2}{*}{$\operatorname{ccc}$} & target & 0.11 & 0.33 & 0.18 & 0.03 & 1.29 & 2.15 & 1.53 & 1.07 \\
\hline & & measured & 0.13 & 0.34 & 0.18 & 0.04 & 1.35 & 2.19 & 1.53 & 1.10 \\
\hline
\end{tabular}


Table 3-5 cont. Normalized PCT Results

\begin{tabular}{|c|c|c|c|c|c|c|c|c|c|c|}
\hline Glass ID & Heat Treatment & Comp View & $\begin{array}{c}\log \mathrm{NL} \\
{[\mathrm{B}(\mathrm{g} / \mathrm{L})]}\end{array}$ & $\begin{array}{c}\log \mathrm{NL} \\
{[\mathrm{Li}(\mathrm{g} / \mathrm{L})]}\end{array}$ & $\begin{array}{c}\log \mathrm{NL} \\
{[\mathrm{Na}(\mathrm{g} / \mathrm{L})]}\end{array}$ & $\begin{array}{c}\log \mathrm{NL} \\
{[\mathrm{Si}(\mathrm{g} / \mathrm{L})]}\end{array}$ & NL B (g/L) & NL Li $(g / L)$ & $\mathrm{NL} \mathrm{Na}(\mathrm{g} / \mathrm{L})$ & NL Si (g/L) \\
\hline \multirow{4}{*}{ SB7b-28 } & \multirow{2}{*}{ quenched } & target & -0.11 & -0.06 & -0.07 & -0.25 & 0.77 & 0.87 & 0.85 & 0.57 \\
\hline & & measured & -0.09 & -0.06 & -0.06 & -0.25 & 0.81 & 0.88 & 0.87 & 0.57 \\
\hline & \multirow{2}{*}{$\operatorname{ccc}$} & target & -0.12 & -0.03 & -0.09 & -0.23 & 0.75 & 0.94 & 0.82 & 0.58 \\
\hline & & measured & -0.10 & -0.02 & -0.08 & -0.23 & 0.79 & 0.95 & 0.84 & 0.59 \\
\hline \multirow{4}{*}{ SB7b-29 } & \multirow{2}{*}{ quenched } & target & -0.14 & -0.05 & 0.00 & -0.22 & 0.73 & 0.90 & 1.00 & 0.60 \\
\hline & & measured & -0.11 & -0.04 & 0.00 & -0.21 & 0.77 & 0.91 & 0.99 & 0.62 \\
\hline & \multirow{2}{*}{$\operatorname{ccc}$} & target & -0.14 & -0.03 & -0.03 & -0.21 & 0.73 & 0.94 & 0.94 & 0.61 \\
\hline & & measured & -0.11 & -0.02 & -0.03 & -0.20 & 0.77 & 0.95 & 0.93 & 0.63 \\
\hline \multirow{4}{*}{ SB7b-30 } & \multirow{2}{*}{ quenched } & target & -0.11 & -0.04 & 0.03 & -0.20 & 0.78 & 0.90 & 1.07 & 0.63 \\
\hline & & measured & -0.09 & -0.04 & 0.03 & -0.21 & 0.82 & 0.91 & 1.07 & 0.61 \\
\hline & \multirow{2}{*}{$\operatorname{ccc}$} & target & -0.12 & -0.04 & -0.02 & -0.23 & 0.76 & 0.90 & 0.96 & 0.59 \\
\hline & & measured & -0.10 & -0.04 & -0.02 & -0.24 & 0.79 & 0.91 & 0.96 & 0.58 \\
\hline \multirow{4}{*}{ SB7b-31 } & \multirow{2}{*}{ quenched } & target & -0.07 & -0.06 & -0.19 & -0.25 & 0.85 & 0.86 & 0.64 & 0.56 \\
\hline & & measured & -0.13 & -0.08 & -0.10 & -0.28 & 0.75 & 0.83 & 0.79 & 0.52 \\
\hline & \multirow{2}{*}{$\mathrm{ccc}$} & target & -0.07 & -0.06 & -0.21 & -0.25 & 0.85 & 0.88 & 0.62 & 0.56 \\
\hline & & measured & -0.13 & -0.07 & -0.12 & -0.28 & 0.75 & 0.84 & 0.77 & 0.53 \\
\hline \multirow{4}{*}{ SB7b-32 } & \multirow{2}{*}{ quenched } & target & -0.12 & 0.03 & 0.21 & -0.16 & 0.76 & 1.08 & 1.64 & 0.70 \\
\hline & & measured & -0.02 & 0.06 & 0.13 & -0.13 & 0.95 & 1.15 & 1.34 & 0.74 \\
\hline & \multirow{2}{*}{$\mathrm{ccc}$} & target & -0.08 & 0.07 & 0.02 & -0.17 & 0.84 & 1.16 & 1.05 & 0.67 \\
\hline & & measured & 0.02 & 0.09 & -0.07 & -0.15 & 1.04 & 1.24 & 0.86 & 0.72 \\
\hline \multirow{4}{*}{ SB7b-33 } & \multirow{2}{*}{ quenched } & target & -0.09 & -0.01 & 0.00 & -0.21 & 0.81 & 0.97 & 1.00 & 0.62 \\
\hline & & measured & -0.07 & -0.01 & -0.01 & -0.19 & 0.85 & 0.98 & 0.98 & 0.64 \\
\hline & \multirow{2}{*}{$\mathrm{ccc}$} & target & -0.07 & 0.08 & 0.08 & -0.16 & 0.84 & 1.21 & 1.21 & 0.70 \\
\hline & & measured & -0.05 & 0.09 & 0.08 & -0.14 & 0.88 & 1.22 & 1.20 & 0.72 \\
\hline \multirow{4}{*}{ SB7b-34 } & \multirow{2}{*}{ quenched } & target & -0.07 & -0.02 & 0.00 & -0.20 & 0.85 & 0.96 & 0.99 & 0.63 \\
\hline & & measured & -0.05 & -0.01 & 0.00 & -0.20 & 0.89 & 0.97 & 0.99 & 0.63 \\
\hline & \multirow{2}{*}{$\operatorname{ccc}$} & target & -0.03 & 0.23 & 0.12 & -0.06 & 0.94 & 1.70 & 1.31 & 0.87 \\
\hline & & measured & -0.01 & 0.24 & 0.12 & -0.06 & 0.99 & 1.72 & 1.31 & 0.87 \\
\hline
\end{tabular}




\subsection{References}

1. J.M. Bricker, "Sludge Batch 7 and $7 \mathrm{~b}$ - Glass Variability Studies," Savannah River Remediation, Aiken, SC, HLW-DWPF-TTR-2010-0038, 2010.

2. D.K. Peeler, "Task Technical and Quality Assurance Plan for Sludge Batch 7 Glass Variability Study," Savannah River National Laboratory, Aiken, SC, SRNL-RP-201001455, 2010.

3. D.K. Peeler and T.B. Edwards, "SB7b Measurement Acceptability Region (MAR) Assessments Based on Compositional Projections from 5-25-11," Savannah River National Laboratory, Aiken, SC, SRNL-L3100-2011-00160, 2011.

4. $\quad \mathrm{JMP}^{\mathrm{TM}}$ Version 7.0.2, SAS Institute Inc., Cary, NC, 2008.

5. S.L. Marra and C.M. Jantzen, "Characterization of Projected DWPF Glass Heat Treated to Simulate Canister Centerline Cooling," Westinghouse Savannah River Company, Aiken, SC, WSRC-TR-92-142, Rev. 1, 1993.

6. T.B. Edwards, "An Analytical Plan for Measuring the Chemical Compositions of the SB7b Variability Study Glasses," Savannah River National Laboratory, Aiken, SC, SRNL-L4221-2011-00010, 2011.

7. "Glass Density Using the Mettler AT400 (or Equivalent) Balance," Savannah River National Laboratory, Aiken, SC, ITS-0057, Latest Revision.

8. "Standard Test Methods for Determining Chemical Durability of Nuclear, Hazardous, and Mixed Waste Glasses and Multiphase Glass Ceramics: The Product Consistency Test (PCT)," ASTM International, West Conshohocken, PA, ASTM C 1285-02, 2002.

9. T.B. Edwards, "Analytical Plans for Measuring the PCT Solutions for the SB7b Variability Study Glasses," Savannah River National Laboratory, Aiken, SC, SRNLL4221-2011-00011, 2011.

10. "Statistical Analysis of Ground-Water Monitoring Data at RCRA Facilities," United States Environmental Protection Agency, Washington, D.C., 530-SW-89-026, 1989.

11. C.M. Jantzen, J.B. Pickett, K.G. Brown, T.B. Edwards, and D.C. Beam, "Process/Product Models for the Defense Waste Processing Facility (DWPF): Part I. Predicting Glass Durability from Composition Using a Thermodynamic Hydration Energy Reaction Model (Thermo)," Westinghouse Savannah River Company, Aiken, SC, WSRC-TR-93672, Rev. 1, 1995.

12. F.C. Johnson and T.B. Edwards, "Sludge Batch 6 Variability Study with Frit 418," Savannah River National Laboratory, Aiken, SC, SRNL-STI-2010-00242, 2010.

13. D.K. Peeler and T.B. Edwards, "The Sludge Batch 7a Glass Variability Study with Frit 418 and Frit 702," Savannah River National Laboratory, Aiken, SC, SRNL-STI-201100063, 2011. 
14. J.R. Harbour, T.B. Edwards, and R.J. Workman, "Summary of Results for Macrobatch 3 Variability Study," Westinghouse Savannah River Company, Aiken, SC, WSRC-TR2000-00351, Revision 0, 2000.

15. T.H. Lorier, T.B. Edwards, I.A. Reamer, D.R. Best, and D.K. Peeler, "SB3 Phase 2 Variability Study: The Impact of Redox on Durability for the Frit 418 - SB2/3 System," Westinghouse Savannah River Company, Aiken, SC, WSRC-TR-2003-00539, 2003.

16. D.K. Peeler, T.B. Edwards, C.C. Herman, T.H. Lorier, D.R. Best, I.A. Reamer, and R.J. Workman, "Sludge Batch 3 Phase 1 Variability Study," Westinghouse Savannah River Company, Aiken, SC, WSRC-TR-2002-00549, 2003. 
SRNL-STI-2011-00440

Revision 0

Appendix A. Tables and Exhibits Supporting the Analysis of the Chemical Composition Measurements 
RTable A-1. Measured Elemental Concentrations (ug/g) for the Study Glasses Prepared Using Aqua Regia (part 1)

\begin{tabular}{|c|c|c|c|c|c|c|c|c|c|c|c|c|c|c|c|c|c|}
\hline Glass ID & Lab ID - ck & Block & Sub-Blk & Sequence & Lab ID & Al & B & Ba & $\begin{array}{l}\mathbf{C a} \\
\end{array}$ & $\mathrm{Ce}$ & $\mathrm{Cr}$ & $\mathbf{C u}$ & $\mathrm{Fe}$ & $\mathbf{K}$ & $\mathbf{L a}$ & $\mathbf{L i}$ & $\mathrm{Mg}$ \\
\hline SB7Ref & SB7Ref & 1 & 1 & 1 & SB7REFAR11 & 47500 & 15900 & 168 & 3470 & 184 & 829 & 383 & 61500 & 2380 & 113 & 24100 & 1640 \\
\hline Ustd & Ustd & 1 & 1 & 2 & \begin{tabular}{|l|} 
USTDAR11 \\
\end{tabular} & 20000 & 26900 & 13.9 & 8880 & 71.1 & 1570 & 47.7 & 91700 & 23300 & 13.8 & 13700 & 6930 \\
\hline SB7b-19 & A27 & 1 & 1 & 3 & A27AR2 & 49400 & 15500 & 521 & 2490 & 902 & 351 & 300 & 61100 & 627 & 171 & 25300 & 2020 \\
\hline SB7b-33 & A10 & 1 & 1 & 4 & A10AR1 & 44300 & 15900 & 347 & 3090 & 610 & 300 & 197 & 68500 & 447 & 111 & 30100 & 1330 \\
\hline SB7b-14 & A09 & 1 & 1 & 5 & A09AR2 & 34700 & 16700 & 103 & 4900 & 142 & 81.7 & 487 & 53700 & $<203$ & 56.6 & 31900 & 372 \\
\hline SB7b-26 & A05 & 1 & 1 & 6 & A05AR2 & 47200 & 15700 & 498 & 3860 & 834 & 288 & 240 & 70000 & 636 & 155 & 24000 & 1910 \\
\hline SB7b-25 & A18 & 1 & 1 & 7 & A18AR1 & 39000 & 13200 & 497 & 2320 & 883 & 379 & 282 & 65200 & 601 & 139 & 29200 & 1950 \\
\hline SB7b-26 & A05 & 1 & 1 & 8 & A05AR1 & 48000 & 15900 & 501 & 3900 & 849 & 288 & 249 & 71000 & 626 & 155 & 24200 & 1920 \\
\hline SB7b-13 & A07 & 1 & 1 & 9 & A07AR1 & 34600 & 14600 & 634 & 1490 & 1120 & 520 & 1080 & 57300 & 823 & 190 & 31400 & 2480 \\
\hline SB7Ref & SB7Ref & 1 & 1 & 10 & SB7REFAR12 & 47000 & 15900 & 168 & 3470 & 181 & 828 & 381 & 61500 & 2320 & 114 & 23900 & 1640 \\
\hline Ustd & Ustd & 1 & 1 & 11 & USTDAR12 & 20100 & 26800 & 13.9 & 8970 & 74.2 & 1570 & 48.1 & 92100 & 23100 & 14.8 & 13700 & 6920 \\
\hline SB7b-13 & A07 & 1 & 1 & 12 & A07AR2 & 32300 & 13800 & 589 & 1410 & 1060 & 484 & 1010 & 53600 & 746 & 178 & 29300 & 2330 \\
\hline SB7b-29 & A21 & 1 & 1 & 13 & A21AR2 & 37100 & 16400 & 301 & 2650 & 512 & 264 & 183 & 57600 & 413 & 99 & 25000 & 1150 \\
\hline SB7b-19 & A27 & 1 & 1 & 14 & A27AR1 & 47700 & 15000 & 505 & 2360 & 869 & 338 & 282 & 59600 & 607 & 165 & 24500 & 1960 \\
\hline SB7b-25 & A18 & 1 & 1 & 15 & A18AR2 & 38700 & 13200 & 491 & 2300 & 868 & 374 & 280 & 65200 & 620 & 136 & 28900 & 1950 \\
\hline SB7b-29 & A21 & 1 & 1 & 16 & A21AR1 & 37700 & 16500 & 304 & 2670 & 512 & 267 & 187 & 58400 & 385 & 100 & 25400 & 1160 \\
\hline SB7b-14 & A09 & 1 & 1 & 17 & A09AR1 & 33500 & 16200 & 99.7 & 4780 & 148 & 78.5 & 503 & 52000 & $<200$ & 55.1 & 31100 & 364 \\
\hline SB7b-33 & A10 & 1 & 1 & 18 & A10AR2 & 42500 & 15200 & 332 & 2940 & 580 & 286 & 188 & 65700 & 453 & 106 & 29100 & 1280 \\
\hline blank & blank & 1 & 1 & 19 & BLANKAR1 & $<266$ & $<162$ & $<0.48$ & 43.5 & $<67.9$ & $<8.16$ & $<5.04$ & 83.2 & $<205$ & $<4.32$ & $<117$ & 4.4 \\
\hline SB7Ref & SB7Ref & 1 & 1 & 20 & SB7REFAR13 & 47100 & 15900 & 167 & 3450 & 180 & 822 & 382 & 61100 & 2340 & 112 & 24200 & 1630 \\
\hline Ustd & Ustd & 1 & 1 & 21 & \begin{tabular}{|l|} 
USTDAR13 \\
\end{tabular} & 19200 & 25700 & 13.4 & 8570 & $<67.1$ & 1520 & 47.5 & 88100 & 22700 & 13.6 & 13300 & 6720 \\
\hline SB7Ref & SB7Ref & 1 & 2 & 1 & SB7REFAR11 & 48300 & 16100 & 177 & 3460 & 198 & 845 & 389 & 61600 & 2400 & 119 & 24100 & 1630 \\
\hline Ustd & Ustd & 1 & 2 & 2 & \begin{tabular}{|l|} 
USTDAR11 \\
\end{tabular} & 20400 & 27200 & 15.5 & 8910 & 67.9 & 1600 & 47.2 & 92600 & 24000 & 16 & 13800 & 6900 \\
\hline SB7b-25 & A18 & 1 & 2 & 3 & A18AR2 & 39600 & 13300 & 521 & 2320 & 923 & 387 & 282 & 65600 & 666 & 143 & 29100 & 1940 \\
\hline SB7b-14 & A09 & 1 & 2 & 4 & A09AR2 & 35500 & 16800 & 109 & 4940 & 162 & 85 & 491 & 53900 & $<203$ & 62.2 & 31900 & 372 \\
\hline SB7b-33 & A10 & 1 & 2 & 5 & A10AR2 & 43600 & 15300 & 352 & 2950 & 619 & 295 & 191 & 66400 & 515 & 111 & 29200 & 1270 \\
\hline SB7b-14 & A09 & 1 & 2 & 6 & A09AR1 & 34400 & 16300 & 106 & 4790 & 155 & 82.2 & 512 & 52300 & 207 & 59.7 & 31100 & 364 \\
\hline SB7b-33 & A10 & 1 & 2 & 7 & A10AR1 & 45200 & 15900 & 368 & 3110 & 642 & 307 & 201 & 68600 & 506 & 117 & 30200 & 1320 \\
\hline SB7b-29 & A21 & 1 & 2 & 8 & A21AR2 & 37900 & 16400 & 319 & 2660 & 548 & 272 & 185 & 57700 & 395 & 104 & 25100 & 1140 \\
\hline SB7b-26 & A05 & 1 & 2 & 9 & A05AR1 & 48900 & 16000 & 528 & 3910 & 896 & 293 & 250 & 71300 & 670 & 163 & 24200 & 1920 \\
\hline SB7Ref & SB7Ref & 1 & 2 & 10 & SB7REFAR12 & 48200 & 16000 & 178 & 3490 & 198 & 849 & 394 & 61700 & 2420 & 119 & 24100 & 1640 \\
\hline Ustd & Ustd & 1 & 2 & 11 & USTDAR12 & 20500 & 27000 & 15.7 & 8940 & 74.6 & 1600 & 49.5 & 92200 & 23200 & 17.4 & 13800 & 6890 \\
\hline SB7b-13 & A07 & 1 & 2 & 12 & A07AR1 & 35300 & 14700 & 667 & 1490 & 1190 & 528 & 1100 & 57400 & 862 & 198 & 31700 & 2470 \\
\hline SB7b-19 & A27 & 1 & 2 & 13 & A27AR2 & 50400 & 15500 & 551 & 2520 & 941 & 361 & 307 & 61500 & 628 & 178 & 25400 & 2030 \\
\hline SB7b-19 & A27 & 1 & 2 & 14 & A27AR1 & 48900 & 15100 & 532 & 2460 & 913 & 347 & 299 & 59800 & 658 & 172 & 24700 & 1960 \\
\hline SB7b-29 & A21 & 1 & 2 & 15 & A21AR1 & 38700 & 16700 & 323 & 2680 & 538 & 274 & 192 & 58700 & 436 & 105 & 25600 & 1150 \\
\hline SB7b-26 & A05 & 1 & 2 & 16 & A05AR2 & 49000 & 16000 & 524 & 3880 & 881 & 296 & 244 & 71300 & 713 & 160 & 24300 & 1910 \\
\hline SB7b-13 & A07 & 1 & 2 & 17 & A07AR2 & 33100 & 13800 & 622 & 1460 & 1100 & 493 & 1030 & 53900 & 761 & 187 & 29600 & 2320 \\
\hline SB7b-25 & A18 & 1 & 2 & 18 & A18AR1 & 39900 & 13300 & 526 & 2330 & 926 & 388 & 285 & 65700 & 632 & 144 & 29200 & 1950 \\
\hline blank & blank & 1 & 2 & 19 & BLANKAR1 & $<266$ & $<162$ & $<0.48$ & 44 & $<67.9$ & $<8.16$ & $<5.04$ & $\begin{array}{l}84.8 \\
\end{array}$ & $<205$ & $<4.32$ & $<117$ & 5.48 \\
\hline SB7Ref & SB7Ref & 1 & 2 & 20 & SB7REFAR13 & 48700 & 16000 & 177 & 3470 & 203 & 844 & 387 & 62100 & 2390 & 120 & 24400 & 1630 \\
\hline Ustd & Ustd & 1 & 2 & 21 & \begin{tabular}{|l|} 
USTDAR13 \\
\end{tabular} & 19900 & 26100 & 15 & 8670 & 68.6 & 1550 & 47.4 & 89700 & 23000 & 16.2 & 13500 & 6680 \\
\hline SB7Ref & SB7Ref & 2 & 1 & 1 & SB7REFAR21 & 48000 & 16000 & 169 & 3490 & 189 & 833 & 373 & 61700 & 2330 & 117 & 24300 & 1640 \\
\hline Ustd & Ustd & 2 & 1 & 2 & USTDAR21 & 20900 & 27800 & 14.3 & 9260 & 72.9 & 1640 & 63.6 & 95000 & 24200 & 18.4 & 14300 & 7190 \\
\hline SB7b-05 & A11 & 2 & 1 & 3 & A11AR1 & 34700 & 16500 & 643 & 4790 & 1120 & 546 & 347 & 52800 & 785 & 188 & 22300 & 2460 \\
\hline SB7b-30 & A13 & 2 & 1 & 4 & A13AR2 & 41900 & 15300 & 340 & 2910 & 592 & 282 & 168 & 64600 & 442 & 121 & 23400 & 1270 \\
\hline SB7b-03 & A17 & 2 & 1 & 5 & A17AR1 & 46700 & 14300 & 639 & 4780 & 1090 & 1000 & 283 & 51900 & 741 & 186 & 31100 & 2460 \\
\hline SB7b-31 & A02 & 2 & 1 & 6 & A02AR1 & 40700 & 17200 & 333 & 2840 & 578 & 298 & 174 & 75600 & 414 & 123 & 23200 & 1200 \\
\hline
\end{tabular}


RTable A-1. Measured Elemental Concentrations (ug/g) for the Study Glasses Prepared Using Aqua Regia (part 1)

\begin{tabular}{|c|c|c|c|c|c|c|c|c|c|c|c|c|c|c|c|c|c|}
\hline Glass ID & Lab ID - ck & Block & Sub-Blk & Sequence & Lab ID & Al & B & Ba & $\begin{array}{l}\mathbf{C a} \\
\end{array}$ & $\mathrm{Ce}$ & $\mathrm{Cr}$ & $\mathbf{C u}$ & $\mathrm{Fe}$ & $\mathbf{K}$ & La & $\mathbf{L i}$ & Mg \\
\hline SB7b-20 & A03 & 2 & 1 & 7 & A03AR1 & 47900 & 14800 & 238 & 2300 & 391 & 123 & 165 & 60200 & 271 & 93.9 & 24200 & 876 \\
\hline SB7b-31 & A02 & 2 & 1 & 8 & A02AR2 & 40400 & 17200 & 329 & 2850 & 571 & 292 & 172 & 75700 & 370 & 122 & 23300 & 1190 \\
\hline SB7b-06 & A19 & 2 & 1 & 9 & A19AR2 & 52700 & 16600 & 617 & 1490 & 1130 & 455 & 331 & 78300 & 724 & 195 & 31600 & 2490 \\
\hline SB7Ref & SB7Ref & 2 & 1 & 10 & SB7REFAR22 & 48300 & 16200 & 171 & 3540 & 193 & 842 & 370 & 62600 & 2380 & 118 & 24600 & 1660 \\
\hline Ustd & Ustd & 2 & 1 & 11 & USTDAR22 & 20600 & 27600 & 14.1 & 9170 & 81.3 & 1630 & 62.9 & 94900 & 23500 & 17.3 & 14200 & 7130 \\
\hline SB7b-20 & A03 & 2 & 1 & 12 & A03AR2 & 47500 & 14700 & 237 & 2280 & 396 & 122 & 158 & 59900 & 275 & 93 & 23900 & 868 \\
\hline SB7b-17 & A25 & 2 & 1 & 13 & A25AR2 & 38300 & 15700 & 509 & 2320 & 882 & 444 & 363 & 59700 & 599 & 156 & 28700 & 1930 \\
\hline SB7b-30 & A13 & 2 & 1 & 14 & A13AR1 & 42300 & 15500 & 345 & 2980 & 595 & 287 & 171 & 65800 & 390 & 121 & 23800 & 1280 \\
\hline SB7b-05 & A11 & 2 & 1 & 15 & A11AR2 & 34000 & 16300 & 651 & 4820 & 1120 & 553 & 353 & 52600 & 783 & 190 & 22200 & 2490 \\
\hline SB7b-06 & A19 & 2 & 1 & 16 & A19AR1 & 52200 & 16400 & 631 & 1470 & 1130 & 449 & 332 & 78100 & 720 & 193 & 31200 & 2450 \\
\hline SB7b-03 & A17 & 2 & 1 & 17 & A17AR2 & 46500 & 14300 & 648 & 4770 & 1130 & 1010 & 291 & 52200 & 759 & 187 & 31200 & 2470 \\
\hline SB7b-17 & A25 & 2 & 1 & 18 & A25AR1 & 38000 & 15600 & 513 & 2310 & 876 & 443 & 371 & 59400 & 598 & 154 & 28700 & 1930 \\
\hline blank & blank & 2 & 1 & 19 & BLANKAR2 & $<266$ & $<162$ & $<0.48$ & 34.8 & $<67.9$ & $<8.16$ & $<5.04$ & $<76.8$ & $<205$ & $<4.32$ & $<117$ & 6.76 \\
\hline SB7Ref & SB7Ref & 2 & 1 & 20 & SB7REFAR23 & 47800 & 16000 & 175 & 3540 & 181 & 849 & 378 & 62200 & 2350 & 119 & 24500 & 1670 \\
\hline Ustd & Ustd & 2 & 1 & 21 & \begin{tabular}{|l|} 
USTDAR23 \\
\end{tabular} & 20200 & 27100 & 14.1 & 9140 & 70.9 & 1620 & 63.1 & 93500 & 23300 & 18 & 13900 & 7100 \\
\hline SB7Ref & SB7Ref & 2 & 2 & 1 & SB7REFAR21 & 47800 & 16000 & 168 & 3470 & 194 & 828 & 375 & 61400 & 2370 & 115 & 24100 & 1630 \\
\hline Ustd & Ustd & 2 & 2 & 2 & USTDAR21 & 20500 & 27600 & 14.1 & 9270 & 80.3 & 1640 & 64.4 & 93700 & 24400 & 17.6 & 14000 & 7180 \\
\hline SB7b-30 & A13 & 2 & 2 & 3 & A13AR1 & 42500 & 15600 & 342 & 2950 & 594 & 286 & 171 & 65300 & 461 & 121 & 23600 & 1280 \\
\hline SB7b-17 & A25 & 2 & 2 & 4 & A25AR1 & 38400 & 15800 & 508 & 2320 & 879 & 443 & 370 & 59400 & 639 & 156 & 28600 & 1940 \\
\hline SB7b-06 & A19 & 2 & 2 & 5 & A19AR2 & 53300 & 16700 & 614 & 1490 & 1140 & 455 & 328 & 78800 & 793 & 195 & 31500 & 2490 \\
\hline SB7b-05 & A11 & 2 & 2 & 6 & A11AR2 & 34200 & 16400 & 645 & 4820 & 1120 & 550 & 354 & 52300 & 814 & 189 & 22000 & 2470 \\
\hline SB7b-05 & A11 & 2 & 2 & 7 & A11AR1 & 33900 & 16300 & 639 & 4770 & 1110 & 545 & 348 & 51900 & 815 & 188 & 21900 & 2460 \\
\hline SB7b-31 & A02 & 2 & 2 & 8 & A02AR2 & 40300 & 17200 & 328 & 2830 & 563 & 291 & 174 & 75400 & 450 & 122 & 23000 & 1180 \\
\hline SB7b-03 & A17 & 2 & 2 & 9 & A17AR2 & 46600 & 14400 & 638 & 4760 & 1110 & 999 & 290 & 52000 & 796 & 186 & 31000 & 2460 \\
\hline SB7Ref & SB7Ref & 2 & 2 & 10 & SB7REFAR22 & 48100 & 16300 & 171 & 3550 & 198 & 844 & 379 & 62300 & 2350 & 117 & 24500 & 1660 \\
\hline Ustd & Ustd & 2 & 2 & 11 & USTDAR22 & 20700 & 27700 & 14.1 & 9190 & 74.7 & 1620 & 64.5 & 94500 & 24000 & 17.6 & 14100 & 7120 \\
\hline SB7b-06 & A19 & 2 & 2 & 12 & A19AR1 & 52200 & 16400 & 626 & 1470 & 1120 & 446 & 331 & 77500 & 831 & 192 & 31100 & 2450 \\
\hline SB7b-20 & A03 & 2 & 2 & 13 & A03AR1 & 47600 & 14800 & 238 & 2310 & 402 & 126 & 167 & 59800 & 349 & 93.4 & 23900 & 880 \\
\hline SB7b-17 & A25 & 2 & 2 & 14 & A25AR2 & 38200 & 15800 & 506 & 2320 & 878 & 437 & 362 & 59400 & 668 & 155 & 28700 & 1930 \\
\hline SB7b-20 & A03 & 2 & 2 & 15 & A03AR2 & 47200 & 14800 & 236 & 2280 & 398 & 123 & 160 & 59500 & 371 & 92.2 & 23800 & 864 \\
\hline SB7b-31 & A02 & 2 & 2 & 16 & A02AR1 & 40400 & 17200 & 333 & 2890 & 576 & 298 & 179 & 75700 & 433 & 122 & 23200 & 1200 \\
\hline SB7b-30 & A13 & 2 & 2 & 17 & A13AR2 & 41300 & 15300 & 340 & 2910 & 593 & 283 & 170 & 64100 & 478 & 120 & 23100 & 1260 \\
\hline SB7b-03 & A17 & 2 & 2 & 18 & A17AR1 & 45900 & 14200 & 640 & 4770 & 1110 & 991 & 294 & 51400 & 788 & 186 & 30600 & 2450 \\
\hline blank & blank & 2 & 2 & 19 & BLANKAR2 & $<266$ & $<162$ & $<0.48$ & 36.1 & $<67.9$ & $<8.16$ & $<5.04$ & $<76.8$ & $<205$ & $<4.32$ & $<117$ & 6.72 \\
\hline SB7Ref & SB7Ref & 2 & 2 & 20 & SB7REFAR23 & 47800 & 16100 & 172 & 3540 & 190 & 842 & 377 & 61900 & 2390 & 118 & 24200 & 1670 \\
\hline Ustd & Ustd & 2 & 2 & 21 & \begin{tabular}{|l|} 
USTDAR23 \\
\end{tabular} & 20200 & 27200 & 13.9 & 9100 & 77.1 & 1600 & 64.6 & 93100 & 23300 & 16.6 & 13800 & 7060 \\
\hline SB7Ref & SB7Ref & 3 & 1 & 1 & SB7REFAR31 & 49900 & 16800 & 178 & 3670 & 190 & 876 & 400 & 64700 & 2530 & 122 & 25100 & 1720 \\
\hline Ustd & Ustd & 3 & 1 & 2 & USTDAR31 & 20900 & 27900 & 14.2 & 9290 & 71.1 & 1640 & 48.5 & 95600 & 23900 & 19.7 & 14300 & 7180 \\
\hline SB7b-04 & A32 & 3 & 1 & 3 & A32AR1 & 53900 & 14800 & 99.5 & 1510 & 238 & 74.7 & 243 & 50800 & $<199$ & 80.9 & 22200 & 361 \\
\hline SB7b-15 & A16 & 3 & 1 & 4 & A16AR2 & 39000 & 15100 & 230 & 3990 & 364 & 209 & 367 & 60900 & 364 & 82.5 & 24400 & 894 \\
\hline SB7b-22 & A14 & 3 & 1 & 5 & A14AR2 & 39500 & 15300 & 238 & 4080 & 406 & 99.1 & 189 & 70700 & 327 & 88.2 & 29300 & 913 \\
\hline SB7b-27 & A34 & 3 & 1 & 6 & A34AR2 & 39000 & 16100 & 229 & 2390 & 378 & 225 & 181 & 76200 & 402 & 149 & 29000 & 900 \\
\hline SB7b-16 & A15 & 3 & 1 & 7 & A15AR1 & 48400 & 15200 & 511 & 2350 & 897 & 385 & 338 & 74300 & 707 & 170 & 29100 & 1950 \\
\hline SB7b-22 & A14 & 3 & 1 & 8 & A14AR1 & 39800 & 15500 & 241 & 4090 & 409 & 96.6 & 189 & 71500 & 409 & 88.6 & 29700 & 917 \\
\hline SB7b-04 & A32 & 3 & 1 & 9 & A32AR2 & 53800 & 14800 & 99.2 & 1510 & 242 & 76.1 & 252 & 51100 & 230 & 80.5 & 22100 & 361 \\
\hline SB7Ref & SB7Ref & 3 & 1 & 10 & SB7REFAR32 & 49500 & 16700 & 177 & 3650 & 185 & 870 & 403 & 65000 & 2560 & 120 & 25000 & 1710 \\
\hline Ustd & Ustd & 3 & 1 & 11 & USTDAR32 & 20700 & 27900 & 14 & 9260 & 83.2 & 1640 & 45.9 & 95800 & 24300 & 19.6 & 14200 & 7170 \\
\hline SB7b-32 & A29 & 3 & 1 & 12 & A29AR2 & 44500 & 14000 & 373 & 3180 & 621 & 280 & 193 & 57300 & 505 & 133 & 29700 & 1340 \\
\hline
\end{tabular}


RTable A-1. Measured Elemental Concentrations (ug/g) for the Study Glasses Prepared Using Aqua Regia (part 1)

\begin{tabular}{|c|c|c|c|c|c|c|c|c|c|c|c|c|c|c|c|c|c|}
\hline Glass ID & Lab ID - ck & Block & Sub-Blk & Sequence & Lab ID & Al & B & Ba & $\begin{array}{l}\mathbf{C a} \\
\end{array}$ & $\mathrm{Ce}$ & $\mathrm{Cr}$ & $\mathbf{C u}$ & $\mathrm{Fe}$ & $\mathbf{K}$ & $\mathbf{L a}$ & $\mathbf{L i}$ & Mg \\
\hline SB7b-16 & A15 & 3 & 1 & 13 & A15AR2 & 47000 & 14700 & 492 & 2290 & 877 & 370 & 348 & 72000 & 663 & 166 & 28300 & 1900 \\
\hline SB7b-27 & A34 & 3 & 1 & 14 & A34AR1 & 38700 & 15900 & 226 & 2390 & 376 & 244 & 186 & 75400 & 346 & 146 & 28800 & 893 \\
\hline SB7b-10 & A06 & 3 & 1 & 15 & A06AR1 & 52500 & 16200 & 99.3 & 4800 & 239 & 87.6 & 142 & 52100 & $<204$ & 75.2 & 21900 & 362 \\
\hline SB7b-10 & A06 & 3 & 1 & 16 & A06AR2 & 53800 & 16500 & 102 & 4880 & 244 & 89.9 & 123 & 53000 & $<201$ & 73.8 & 22400 & 364 \\
\hline SB7b-15 & A16 & 3 & 1 & 17 & A16AR1 & 39000 & 15100 & 230 & 4000 & 368 & 208 & 385 & 61100 & 381 & 83.4 & 24600 & 896 \\
\hline SB7b-32 & A29 & 3 & 1 & 18 & A29AR1 & 44300 & 13800 & 370 & 3150 & 615 & 277 & 196 & 56900 & 524 & 130 & 29700 & 1330 \\
\hline blank & blank & 3 & 1 & 19 & BLANKAR3 & $<266$ & $<162$ & $<0.48$ & 27.6 & $<67.9$ & $<8.16$ & $<5.04$ & $<76.8$ & $<205$ & $<4.32$ & $<117$ & 3.36 \\
\hline SB7Ref & SB7Ref & 3 & 1 & 20 & SB7REFAR33 & 48100 & 16300 & 173 & 3560 & 174 & 855 & 393 & 63000 & 2460 & 119 & 24500 & 1680 \\
\hline Ustd & Ustd & 3 & 1 & 21 & \begin{tabular}{|l|} 
USTDAR33 \\
\end{tabular} & 20300 & 27300 & 13.8 & 9160 & 66.1 & 1620 & 48.3 & 93800 & 24200 & 20.7 & 13900 & 7080 \\
\hline SB7Ref & SB7Ref & 3 & 2 & 1 & SB7REFAR31 & 49400 & 16700 & 176 & 3640 & 189 & 868 & 407 & 64200 & 2490 & 119 & 25100 & 1720 \\
\hline Ustd & Ustd & 3 & 2 & 2 & USTDAR31 & 20700 & 27900 & 14.1 & 9260 & 80.9 & 1630 & 48.1 & 95600 & 24100 & 16.2 & 14300 & 7200 \\
\hline SB7b-10 & A06 & 3 & 2 & 3 & A06AR1 & 52700 & 16300 & 98.5 & 4800 & 233 & 85.6 & 143 & 52100 & $<204$ & 70.2 & 21900 & 363 \\
\hline SB7b-15 & A16 & 3 & 2 & 4 & A16AR1 & 38900 & 15200 & 229 & 4020 & 359 & 208 & 387 & 61100 & 277 & 79.2 & 24400 & 900 \\
\hline SB7b-27 & A34 & 3 & 2 & 5 & A34AR2 & 39200 & 16100 & 228 & 2380 & 370 & 222 & 180 & 76300 & 331 & 143 & 28900 & 904 \\
\hline SB7b-22 & A14 & 3 & 2 & 6 & A14AR1 & 39700 & 15500 & 239 & 4120 & 403 & 95.2 & 189 & 71400 & 290 & 86 & 29600 & 924 \\
\hline SB7b-04 & A32 & 3 & 2 & 7 & A32AR2 & 53700 & 14900 & 99.4 & 1510 & 255 & 74.1 & 251 & 51000 & $<200$ & 75.5 & 22100 & 365 \\
\hline SB7b-27 & A34 & 3 & 2 & 8 & A34AR1 & 38400 & 15900 & 225 & 2410 & 373 & 241 & 186 & 75300 & 269 & 142 & 28600 & 894 \\
\hline SB7b-16 & A15 & 3 & 2 & 9 & A15AR2 & 46900 & 14700 & 490 & 2290 & 858 & 366 & 348 & 71800 & 575 & 162 & 28200 & 1920 \\
\hline SB7Ref & SB7Ref & 3 & 2 & 10 & SB7REFAR32 & 48900 & 16700 & 176 & 3630 & 185 & 861 & 408 & 64400 & 2390 & 118 & 25000 & 1720 \\
\hline Ustd & Ustd & 3 & 2 & 11 & USTDAR32 & 20600 & 27800 & 14 & 9290 & 73.4 & 1630 & 47.8 & 95300 & 24000 & 17.6 & 14100 & 7200 \\
\hline SB7b-04 & A32 & 3 & 2 & 12 & A32AR1 & 53100 & 14700 & 98.5 & 1500 & 239 & 73.6 & 247 & 50500 & $<199$ & 76 & 22100 & 362 \\
\hline SB7b-32 & A29 & 3 & 2 & 13 & A29AR2 & 44100 & 14000 & 370 & 3190 & 617 & 277 & 197 & 56900 & 440 & 128 & 29900 & 1350 \\
\hline SB7b-10 & A06 & 3 & 2 & 14 & A06AR2 & 53100 & 16400 & 101 & 4890 & 253 & 88.7 & 122 & 52800 & $<201$ & 70.7 & 22200 & 367 \\
\hline SB7b-32 & A29 & 3 & 2 & 15 & A29AR1 & 44100 & 13900 & 367 & 3150 & 600 & 275 & 194 & 56800 & 503 & 127 & 29700 & 1340 \\
\hline SB7b-15 & A16 & 3 & 2 & 16 & A16AR2 & 38700 & 15100 & 228 & 3980 & 355 & 204 & 371 & 60900 & 319 & 79.3 & 24400 & 896 \\
\hline SB7b-22 & A14 & 3 & 2 & 17 & A14AR2 & 39100 & 15200 & 238 & 4070 & 391 & 98.2 & 190 & 70200 & 302 & 84.6 & 29200 & 918 \\
\hline SB7b-16 & A15 & 3 & 2 & 18 & A15AR1 & 48000 & 15100 & 506 & 2340 & 889 & 375 & 333 & 74100 & 612 & 168 & 29100 & 1960 \\
\hline blank & blank & 3 & 2 & 19 & BLANKAR3 & $<266$ & $<162$ & $<0.48$ & 25.8 & $<67.9$ & $<8.16$ & $<5.04$ & $<76.8$ & $<205$ & $<4.32$ & $<117$ & 3.28 \\
\hline SB7Ref & SB7Ref & 3 & 2 & 20 & SB7REFAR33 & 47600 & 16200 & 174 & 3570 & 185 & 845 & 399 & 63000 & 2400 & 117 & 24400 & 1690 \\
\hline Ustd & Ustd & 3 & 2 & 21 & USTDAR33 & 20100 & 27100 & 14 & 9130 & 76.9 & 1610 & 49.5 & 93500 & 23000 & 17 & 13800 & 7100 \\
\hline SB7Ref & SB7Ref & 4 & 1 & 1 & SB7REFAR41 & 48300 & 16300 & 163 & 3480 & 192 & 830 & 387 & 62700 & 2410 & 112 & 24700 & 1650 \\
\hline Ustd & Ustd & 4 & 1 & 2 & USTDAR41 & 20400 & 27300 & 12.4 & 8930 & $<67.1$ & 1560 & 45 & 93200 & 23700 & 23.3 & 14000 & 6980 \\
\hline SB7b-18 & A24 & 4 & 1 & 3 & A24AR1 & 38700 & 15100 & 482 & 4010 & 870 & 456 & 284 & 74800 & 662 & 165 & 24600 & 1950 \\
\hline SB7b-21 & A08 & 4 & 1 & 4 & A08AR1 & 38900 & 16000 & 487 & 3990 & 860 & 340 & 289 & 59600 & 682 & 163 & 29500 & 1960 \\
\hline SB7b-12 & A23 & 4 & 1 & 5 & A23AR2 & 34200 & 14500 & 97.7 & 4800 & 150 & 84.8 & 251 & 79900 & $<203$ & 50.5 & 22300 & 362 \\
\hline SB7b-34 & A30 & 4 & 1 & 6 & A30AR1 & 47700 & 14500 & 397 & 3380 & 650 & 337 & 201 & 74000 & 507 & 148 & 27800 & 1450 \\
\hline SB7b-12 & A23 & 4 & 1 & 7 & A23AR1 & 34200 & 14500 & 97.7 & 4830 & 157 & 103 & 238 & 80400 & $<202$ & 49.8 & 22400 & 362 \\
\hline SB7b-01 & A04 & 4 & 1 & 8 & A04AR2 & 32900 & 16100 & 89.3 & 1480 & 137 & 86.1 & 54.9 & 80600 & $<198$ & 55 & 21800 & 391 \\
\hline SB7b-21 & A08 & 4 & 1 & 9 & A08AR2 & 39200 & 16100 & 495 & 4030 & 865 & 345 & 303 & 60400 & 689 & 166 & 29700 & 1980 \\
\hline SB7Ref & SB7Ref & 4 & 1 & 10 & SB7REFAR42 & 47000 & 16100 & 163 & 3500 & 181 & 833 & 384 & 61900 & 2430 & 112 & 24500 & 1650 \\
\hline Ustd & Ustd & 4 & 1 & 11 & USTDAR42 & 19700 & 26600 & 12.2 & 8900 & 75.5 & 1550 & 44.1 & 91000 & 23300 & 22.3 & 13700 & 6930 \\
\hline SB7b-09 & A33 & 4 & 1 & 12 & A33AR2 & 52500 & 16600 & 76.7 & 4860 & 236 & 86.9 & 182 & 78000 & $<197$ & 76.8 & 32000 & 324 \\
\hline SB7b-18 & A24 & 4 & 1 & 13 & A24AR2 & 38700 & 15200 & 487 & 4040 & 874 & 462 & 270 & 75400 & 657 & 166 & 25000 & 1980 \\
\hline SB7b-09 & A33 & 4 & 1 & 14 & A33AR1 & 52400 & 16600 & 76.1 & 4820 & 236 & 90.5 & 170 & 78000 & $<196$ & 76.6 & 32000 & 324 \\
\hline SB7b-01 & A04 & 4 & 1 & 15 & A04AR1 & 33800 & 16600 & 91.6 & 1520 & 138 & 86.7 & 54.7 & 82600 & $<204$ & 57.5 & 22500 & 401 \\
\hline SB7b-08 & A22 & 4 & 1 & 16 & A22AR1 & 33400 & 14400 & 87.6 & 4780 & 182 & 89.5 & 63.7 & 80600 & $<196$ & 68.1 & 31700 & 369 \\
\hline SB7b-34 & A30 & 4 & 1 & 17 & A30AR2 & 46900 & 14500 & 398 & 3400 & 650 & 319 & 203 & 73500 & 547 & 149 & 27800 & 1440 \\
\hline SB7b-08 & A22 & 4 & 1 & 18 & A22AR2 & 32800 & 14300 & 86.6 & 4730 & 177 & 91.1 & 63.4 & 79700 & $<199$ & 66.5 & 31400 & 366 \\
\hline
\end{tabular}


RTable A-1. Measured Elemental Concentrations (ug/g) for the Study Glasses Prepared Using Aqua Regia (part 1)

\begin{tabular}{|c|c|c|c|c|c|c|c|c|c|c|c|c|c|c|c|c|c|}
\hline Glass ID & Lab ID - ck & Block & Sub-Blk & Sequence & Lab ID & Al & B & Ba & Ca & $\mathrm{Ce}$ & $\mathrm{Cr}$ & $\mathbf{C u}$ & $\mathrm{Fe}$ & $\mathbf{K}$ & La & $\mathbf{L i}$ & Mg \\
\hline blank & blank & 4 & 1 & 19 & BLANKAR4 & $<266$ & $<162$ & $<0.48$ & 35.6 & $<67.9$ & $<8.16$ & $<5.04$ & $<76.8$ & $<205$ & $<4.32$ & $<117$ & 4.44 \\
\hline SB7Ref & SB7Ref & 4 & 1 & 20 & SB7REFAR43 & 44600 & 15200 & 156 & 3360 & 172 & 802 & 372 & 59200 & 2350 & 110 & 23500 & 1600 \\
\hline Ustd & Ustd & 4 & 1 & 21 & USTDAR43 & 19800 & 27000 & 12.3 & 8980 & 80.4 & 1570 & 45.6 & 92300 & 24100 & 23.7 & 14000 & 7010 \\
\hline SB7Ref & SB7Ref & 4 & 2 & 1 & SB7REFAR41 & 48400 & 16400 & 173 & 3530 & 184 & 855 & 391 & 62600 & 2440 & 119 & 24600 & 1660 \\
\hline Ustd & Ustd & 4 & 2 & 2 & USTDAR41 & 20400 & 27400 & 13.7 & 9040 & 83.1 & 1600 & 47.8 & 93300 & 23600 & 22 & 13800 & 7000 \\
\hline SB7b-01 & A04 & 4 & 2 & 3 & A04AR2 & 33800 & 16300 & 95.1 & 1490 & 201 & 88.9 & 56 & 81500 & $<198$ & 56.7 & 21600 & 395 \\
\hline SB7b-21 & A08 & 4 & 2 & 4 & A08AR2 & 39900 & 16400 & 523 & 4060 & 898 & 354 & 307 & 61000 & 653 & 169 & 29600 & 2000 \\
\hline SB7b-09 & A33 & 4 & 2 & 5 & A33AR2 & 53700 & 16700 & 81.4 & 4890 & 244 & 90.6 & 185 & 78800 & $<197$ & 77.9 & 31700 & 327 \\
\hline SB7b-18 & A24 & 4 & 2 & 6 & A24AR1 & 39300 & 15200 & 509 & 4030 & 883 & 467 & 284 & 75700 & 615 & 170 & 24600 & 1960 \\
\hline SB7b-01 & A04 & 4 & 2 & 7 & A04AR1 & 34700 & 16800 & 98.4 & 1530 & 211 & 90.2 & 57.3 & 83700 & $<204$ & 58.4 & 22400 & 404 \\
\hline SB7b-09 & A33 & 4 & 2 & 8 & A33AR1 & 53800 & 16800 & 81.6 & 4860 & 239 & 92.9 & 176 & 79200 & $<196$ & 77.4 & 31800 & 325 \\
\hline SB7b-34 & A30 & 4 & 2 & 9 & A30AR1 & 48200 & 14600 & 419 & 3390 & 666 & 345 & 207 & 74500 & 487 & 150 & 27700 & 1450 \\
\hline SB7Ref & SB7Ref & 4 & 2 & 10 & \begin{tabular}{|l|} 
SB7REFAR42 \\
\end{tabular} & 48200 & 16200 & 172 & 3530 & 183 & 854 & 390 & 62600 & 2410 & 119 & 24500 & 1670 \\
\hline Ustd & Ustd & 4 & 2 & 11 & USTDAR42 & 20100 & 26900 & 13.5 & 8960 & 82.1 & 1600 & 47.3 & 91900 & 23200 & 22.6 & 13700 & 6950 \\
\hline SB7b-12 & A23 & 4 & 2 & 12 & A23AR1 & 34800 & 14700 & 105 & 4870 & 148 & 105 & 247 & 81100 & $<202$ & 51.1 & 22300 & 364 \\
\hline SB7b-12 & A23 & 4 & 2 & 13 & A23AR2 & 34500 & 14500 & 105 & 4880 & 153 & 85.7 & 262 & 80200 & $<203$ & 51.2 & 22200 & 366 \\
\hline SB7b-34 & A30 & 4 & 2 & 14 & A30AR2 & 48200 & 14700 & 425 & 3410 & 667 & 328 & 207 & 74600 & 524 & 151 & 28000 & 1450 \\
\hline SB7b-21 & A08 & 4 & 2 & 15 & A08AR1 & 39100 & 16000 & 520 & 4060 & 889 & 350 & 301 & 59900 & 648 & 166 & 29100 & 1970 \\
\hline SB7b-08 & A22 & 4 & 2 & 16 & A22AR1 & 34300 & 14600 & 94.2 & 4840 & 187 & 92.3 & 68.7 & 81500 & $<196$ & 67.5 & 31400 & 375 \\
\hline SB7b-18 & A24 & 4 & 2 & 17 & A24AR2 & 39500 & 15300 & 513 & 4080 & 889 & 474 & 276 & 76200 & 670 & 169 & 24800 & 1980 \\
\hline SB7b-08 & A22 & 4 & 2 & 18 & A22AR2 & 33900 & 14400 & 92.3 & 4800 & 178 & 93.7 & 66.4 & 80700 & $<199$ & 67.9 & 31100 & 371 \\
\hline blank & blank & 4 & 2 & 19 & \begin{tabular}{|l|} 
BLANKAR4 \\
\end{tabular} & $<266$ & $<162$ & $<0.48$ & 35.5 & $<67.9$ & $<8.16$ & $<5.04$ & $<76.8$ & $<205$ & $<4.32$ & $<117$ & 4.88 \\
\hline SB7Ref & SB7Ref & 4 & 2 & 20 & SB7REFAR43 & 46100 & 15400 & 165 & 3400 & 186 & 819 & 376 & 60000 & 2360 & 113 & 23400 & 1600 \\
\hline Ustd & Ustd & 4 & 2 & 21 & USTDAR43 & 20200 & 27100 & 13.5 & 9100 & 81 & 1610 & 46.6 & 93200 & 23500 & 22.5 & 13900 & 7030 \\
\hline SB7Ref & SB7Ref & 5 & 1 & 1 & SB7REFAR51 & 47800 & 16100 & 168 & 3460 & 179 & 836 & 381 & 61900 & 2410 & 115 & 24300 & 1640 \\
\hline Ustd & Ustd & 5 & 1 & 2 & USTDSR51 & 20500 & 27400 & 13.5 & 9050 & 73.4 & 1600 & 46.6 & 93800 & 23600 & 20.3 & 13900 & 7040 \\
\hline SB7b-23 & A31 & 5 & 1 & 3 & A31AR2 & 48700 & 15100 & 225 & 4050 & 392 & 156 & 220 & 58700 & 314 & 89.2 & 29300 & 907 \\
\hline SB7b-23 & A31 & 5 & 1 & 4 & A31AR1 & 47800 & 14800 & 220 & 3920 & 377 & 149 & 218 & 57700 & 277 & 87.3 & 28800 & 882 \\
\hline SB7b-11 & A20 & 5 & 1 & 5 & A20AR1 & 53500 & 14700 & 629 & 4850 & 1090 & 551 & 306 & 81100 & 767 & 190 & 22300 & 2470 \\
\hline SB7b-02 & A01 & 5 & 1 & 6 & A01AR2 & 33200 & 16400 & 587 & 1460 & 1080 & 511 & 217 & 79000 & 818 & 180 & 21700 & 2400 \\
\hline SB7b-28 & A28 & 5 & 1 & 7 & A28AR2 & 37700 & 15500 & 217 & 2290 & 377 & 197 & 185 & 71900 & 318 & 89.1 & 23800 & 864 \\
\hline SB7b-07 & A12 & 5 & 1 & 8 & A12AR1 & 52700 & 14500 & 82.1 & 1510 & 213 & 100 & 62.5 & 53100 & $<200$ & 54.2 & 31600 & 367 \\
\hline SB7b-11 & A20 & 5 & 1 & 9 & A20AR2 & 51400 & 14100 & 600 & 4660 & 1040 & 526 & 292 & 77900 & 730 & 182 & 21500 & 2380 \\
\hline SB7Ref & SB7Ref & 5 & 1 & 10 & SB7REFAR52 & 47800 & 16100 & 167 & 3490 & 186 & 838 & 382 & 62000 & 2360 & 114 & 24500 & 1650 \\
\hline Ustd & Ustd & 5 & 1 & 11 & USTDSR52 & 20800 & 27800 & 13.5 & 9250 & 75.4 & 1630 & 47 & 95400 & 23700 & 20.3 & 14200 & 7170 \\
\hline SB7b-24 & A26 & 5 & 1 & 12 & A26AR2 & 48100 & 16000 & 243 & 4050 & 389 & 205 & 180 & 59400 & 305 & 78.9 & 29200 & 902 \\
\hline SB7b-07 & A12 & 5 & 1 & 13 & A12AR2 & 51000 & 14100 & 79.8 & 1490 & 194 & 97.5 & 59.6 & 51600 & $<202$ & 53.1 & 30600 & 360 \\
\hline SB7b-24 & A26 & 5 & 1 & 14 & A26AR1 & 47700 & 15900 & 239 & 4000 & 388 & 204 & 178 & 58900 & 280 & 77.3 & 29200 & 891 \\
\hline SB7b-28 & A28 & 5 & 1 & 15 & A28AR1 & 39000 & 16100 & 224 & 2420 & 384 & 204 & 176 & 74300 & 296 & 92.2 & 24700 & 902 \\
\hline SB7b-02 & A01 & 5 & 1 & 16 & A01AR1 & 33900 & 16700 & 598 & 1490 & 1110 & 538 & 233 & 81300 & 849 & 184 & 22300 & 2480 \\
\hline blank & blank & 5 & 1 & 17 & BLANKAR5 & $<266$ & $<162$ & $<0.48$ & 30.9 & $<67.9$ & $<8.16$ & $<5.04$ & 117 & $<205$ & $<4.32$ & $<117$ & 4.32 \\
\hline SB7Ref & SB7Ref & 5 & 1 & 18 & SB7REFAR53 & 46900 & 15700 & 164 & 3450 & 175 & 829 & 376 & 61100 & 2350 & 113 & 24200 & 1620 \\
\hline Ustd & Ustd & 5 & 1 & 19 & USTDAR53 & 20500 & 27500 & 13.5 & 9260 & 75.4 & 1620 & 47.1 & 94700 & 24000 & 20.6 & 14100 & 7170 \\
\hline SB7Ref & SB7Ref & 5 & 2 & 1 & SB7REFAR51 & 48200 & 16200 & 168 & 3500 & 192 & 834 & 379 & 62000 & 2370 & 115 & 24400 & 1640 \\
\hline Ustd & Ustd & 5 & 2 & 2 & USTDSR51 & 20700 & 27600 & 13.1 & 9030 & 87.6 & 1590 & 46 & 93900 & 23400 & 21.2 & 14000 & 7000 \\
\hline SB7b-02 & A01 & 5 & 2 & 3 & A01AR1 & 34600 & 17100 & 609 & 1490 & 1150 & 538 & 233 & 82000 & 861 & 187 & 22400 & 2470 \\
\hline SB7b-07 & A12 & 5 & 2 & 4 & A12AR1 & 53400 & 14700 & 82.9 & 1520 & 210 & 99 & 61.4 & 53300 & $<200$ & 55 & 31600 & 369 \\
\hline SB7b-23 & A31 & 5 & 2 & 5 & A31AR2 & 49300 & 15200 & 227 & 4050 & 391 & 155 & 219 & 58900 & 330 & 93.4 & 29400 & 910 \\
\hline
\end{tabular}


RTable A-1. Measured Elemental Concentrations (ug/g) for the Study Glasses Prepared Using Aqua Regia (part 1)

\begin{tabular}{|c|c|c|c|c|c|c|c|c|c|c|c|c|c|c|c|c|c|}
\hline Glass ID & Lab ID - ck & Block & Sub-Blk & Sequence & Lab ID & Al & B & Ba & $\mathbf{C a}$ & Ce & $\mathrm{Cr}$ & $\mathbf{C u}$ & $\mathbf{F e}$ & $\mathbf{K}$ & La & $\mathbf{L i}$ & Mg \\
\hline SB7b-24 & A26 & 5 & 2 & 6 & A26AR2 & 48900 & 16100 & 246 & 4040 & 400 & 203 & 179 & 59600 & 303 & 80.5 & 29300 & 900 \\
\hline SB7b-24 & A26 & 5 & 2 & 7 & A26AR1 & 48300 & 16000 & 244 & 4000 & 394 & 202 & 179 & 59300 & 290 & 78.1 & 29100 & 888 \\
\hline SB7b-23 & A31 & 5 & 2 & 8 & A31AR1 & 47900 & 14900 & 222 & 3950 & 383 & 149 & 220 & 57900 & 328 & 89.8 & 29000 & 884 \\
\hline SB7b-28 & A28 & 5 & 2 & 9 & A28AR1 & 39300 & 16200 & 227 & 2380 & 397 & 203 & 176 & 74500 & 308 & 93.5 & 24600 & 898 \\
\hline SB7Ref & SB7Ref & 5 & 2 & 10 & SB7REFAR52 & 48000 & 16200 & 169 & 3500 & 192 & 839 & 383 & 62200 & 2420 & 116 & 24400 & 1650 \\
\hline Ustd & Ustd & 5 & 2 & 11 & \begin{tabular}{|l|} 
USTDSR52 \\
\end{tabular} & 20800 & 28000 & 13.4 & 9230 & 77.2 & 1630 & 46.2 & 95600 & 23900 & 20.4 & 14200 & 7140 \\
\hline SB7b-11 & A20 & 5 & 2 & 12 & A20AR2 & 51800 & 14300 & 612 & 4680 & 1060 & 529 & 299 & 78400 & 805 & 184 & 21600 & 2380 \\
\hline SB7b-28 & A28 & 5 & 2 & 13 & A28AR2 & 38000 & 15700 & 220 & 2290 & 393 & 196 & 186 & 71600 & 290 & 89.3 & 23900 & 869 \\
\hline SB7b-11 & A20 & 5 & 2 & 14 & A20AR1 & 53600 & 14900 & 635 & 4850 & 1110 & 548 & 303 & 81200 & 821 & 191 & 22400 & 2460 \\
\hline SB7b-07 & A12 & 5 & 2 & 15 & A12AR2 & 51400 & 14200 & 81.1 & 1490 & 212 & 96.4 & 61.2 & 51600 & $<202$ & 53.3 & 30700 & 359 \\
\hline SB7b-02 & $\mathrm{A} 01$ & 5 & 2 & 16 & A01AR2 & 33200 & 16500 & 595 & 1460 & 1100 & 511 & 218 & 79200 & 871 & 180 & 21800 & 2410 \\
\hline blank & blank & 5 & 2 & 17 & BLANKAR5 & $<266$ & $<162$ & $<0.48$ & 32.2 & $<67.9$ & $<8.16$ & $<5.04$ & 131 & $<205$ & $<4.32$ & $<117$ & 4.24 \\
\hline SB7Ref & SB7Ref & 5 & 2 & 18 & SB7REFAR53 & 47000 & 15800 & 168 & 3460 & 183 & 834 & 383 & 61100 & 2330 & 115 & 24000 & 1630 \\
\hline Ustd & Ustd & 5 & 2 & 19 & USTDAR53 & 20600 & 27800 & 13.5 & 9270 & 78.2 & 1640 & 45.9 & 95100 & 23700 & 21.9 & 14300 & 7180 \\
\hline
\end{tabular}


Table A-2. Measured Elemental Concentrations (ug/g) for the Study Glasses Prepared Using Aqua Regia (part 2)

\begin{tabular}{|c|c|c|c|c|c|c|c|c|c|c|c|c|c|c|}
\hline Glass ID & Block & Sub-Blk & Sequence & Lab ID & Mn & $\mathrm{Na}$ & $\mathrm{Ni}$ & $\mathbf{P b}$ & $\mathrm{S}$ & Th & Ti & $\mathbf{U}$ & Zn & $\mathrm{Zr}$ \\
\hline SB7Ref & 1 & 1 & 1 & SB7REFAR11 & 16300 & 109000 & 9610 & $<55.6$ & 2020 & 69.8 & 2630 & $<2530$ & 178 & 626 \\
\hline Ustd & 1 & 1 & 2 & USTDAR11 & 21000 & 84700 & 8380 & $<56.6$ & $<593$ & 395 & 5070 & 18200 & 81.1 & 11.6 \\
\hline SB7b-19 & 1 & 1 & 3 & A27AR2 & 14000 & 114000 & 11100 & 152 & 1990 & 6900 & 1090 & 20600 & 193 & 387 \\
\hline SB7b-33 & 1 & 1 & 4 & A10AR1 & 15400 & 95100 & 12700 & 84.1 & 2210 & 5380 & 1350 & 22600 & 169 & 413 \\
\hline SB7b-14 & 1 & 1 & 5 & A09AR2 & 18800 & 92900 & 9510 & $<56.5$ & 2290 & 7330 & 3590 & 18200 & 171 & 241 \\
\hline SB7b-26 & 1 & 1 & 6 & A05AR2 & 13100 & 89900 & 13500 & 169 & 1760 & 4280 & 992 & 23900 & 193 & 545 \\
\hline SB7b-25 & 1 & 1 & 7 & A18AR1 & 16900 & 111000 & 13900 & 152 & 2270 & 4460 & 1040 & 25200 & 213 & 519 \\
\hline SB7b-26 & 1 & 1 & 8 & A05AR1 & 13200 & 90500 & 13700 & 169 & 1390 & 4300 & 990 & 24300 & 194 & 454 \\
\hline SB7b-13 & 1 & 1 & 9 & A07AR1 & 11800 & 82900 & 15900 & 152 & 2220 & 7850 & 3860 & 27300 & 388 & 687 \\
\hline SB7Ref & 1 & 1 & 10 & SB7REFAR12 & 16200 & 109000 & 9610 & $<55.6$ & 2000 & 72.7 & 2640 & $<2530$ & 178 & 604 \\
\hline Ustd & 1 & 1 & 11 & USTDAR12 & 21100 & 84900 & 8450 & $<56.6$ & $<593$ & 394 & 5000 & 18400 & 81 & 12.5 \\
\hline SB7b-13 & 1 & 1 & 12 & A07AR2 & 11100 & 77500 & 14900 & 150 & 1710 & 7320 & 3630 & 25500 & 361 & 650 \\
\hline SB7b-29 & 1 & 1 & 13 & A21AR2 & 13000 & 101000 & 11000 & 86.5 & 1890 & 4440 & 1120 & 19200 & 147 & 314 \\
\hline SB7b-19 & 1 & 1 & 14 & A27AR1 & 13500 & 111000 & 10800 & 135 & 2110 & 6670 & 1050 & 20100 & 185 & 616 \\
\hline SB7b-25 & 1 & 1 & 15 & A18AR2 & 16800 & 110000 & 13900 & 148 & 1990 & 4430 & 1040 & 24800 & 209 & 515 \\
\hline SB7b-29 & 1 & 1 & 16 & A21AR1 & 13200 & 104000 & 11100 & 86.2 & 1960 & 4500 & 1140 & 19600 & 149 & 309 \\
\hline SB7b-14 & 1 & 1 & 17 & A09AR1 & 18200 & 90500 & 9090 & $<55.9$ & 2110 & 7400 & 3790 & 17400 & 172 & 293 \\
\hline SB7b-33 & 1 & 1 & 18 & A10AR2 & 14800 & 91500 & 12200 & $\begin{array}{ll}77.3 \\
\end{array}$ & 2000 & 5140 & 1310 & 22100 & 162 & 437 \\
\hline blank & 1 & 1 & 19 & BLANKAR1 & $<42.4$ & $<5900$ & $<364$ & $<57.3$ & $<600$ & $<32.3$ & $<3.6$ & $<2610$ & $<5.28$ & $<4.4$ \\
\hline SB7Ref & 1 & 1 & 20 & SB7REFAR13 & 16100 & 109000 & 9620 & $<55.6$ & 1740 & 64.7 & 2610 & $<2530$ & 176 & 619 \\
\hline Ustd & 1 & 1 & 21 & USTDAR13 & 20200 & 81700 & 8050 & $<56.6$ & $<593$ & 380 & 4850 & 17900 & 79.2 & 11.8 \\
\hline SB7Ref & 1 & 2 & 1 & SB7REFAR11 & 16400 & 111000 & 9620 & $<55.6$ & 1610 & 66.3 & 2690 & $<2530$ & 180 & 628 \\
\hline Ustd & 1 & 2 & 2 & USTDAR11 & 21300 & 85600 & 8400 & $<56.6$ & $<593$ & 399 & 5210 & 18400 & 82.1 & 12.7 \\
\hline SB7b-25 & 1 & 2 & 3 & A18AR2 & 17000 & 111000 & 14000 & 167 & 1710 & 4470 & 1080 & 24900 & 214 & 529 \\
\hline SB7b-14 & 1 & 2 & 4 & A09AR2 & 19100 & 93600 & 9490 & $<56.5$ & 1890 & 7370 & 3690 & 18100 & 173 & 246 \\
\hline SB7b-33 & 1 & 2 & 5 & A10AR2 & 15100 & 92700 & 12200 & 85.5 & 1670 & 5200 & 1350 & 22200 & 165 & 451 \\
\hline SB7b-14 & 1 & 2 & 6 & A09AR1 & 18500 & 91400 & 9200 & $<55.9$ & 1560 & 7460 & 3910 & 17300 & 176 & 299 \\
\hline SB7b-33 & 1 & 2 & 7 & A10AR1 & 15600 & 95600 & 12700 & 101 & 1630 & 5410 & 1400 & 22700 & 171 & 424 \\
\hline SB7b-29 & 1 & 2 & 8 & A21AR2 & 13200 & 103000 & 10900 & 83.7 & 1560 & 4470 & 1170 & 19300 & 151 & 321 \\
\hline SB7b-26 & 1 & 2 & 9 & A05AR1 & 13400 & 91300 & 13600 & 183 & 1550 & 4340 & 1020 & 24200 & 198 & 460 \\
\hline SB7Ref & 1 & 2 & 10 & SB7REFAR12 & 16300 & 110000 & 9520 & $<55.6$ & 1660 & 67 & 2720 & $<2530$ & 181 & 611 \\
\hline Ustd & 1 & 2 & 11 & USTDAR12 & 21300 & 85200 & 8420 & $<56.6$ & $<593$ & 395 & 5170 & 18400 & 83.1 & 13.7 \\
\hline SB7b-13 & 1 & 2 & 12 & A07AR1 & 11900 & 83600 & 15900 & 173 & 1520 & 7880 & 3980 & 28000 & 395 & 702 \\
\hline SB7b-19 & 1 & 2 & 13 & A27AR2 & 14100 & 114000 & 11200 & 137 & 1830 & 6950 & 1130 & 20700 & 195 & 397 \\
\hline SB7b-19 & 1 & 2 & 14 & A27AR1 & 13700 & 111000 & 10800 & 125 & 1650 & 6730 & 1100 & 20100 & 190 & 633 \\
\hline SB7b-29 & 1 & 2 & 15 & A21AR1 & 13400 & 104000 & 11100 & 72.8 & 1870 & 4530 & 1180 & 19900 & 152 & 316 \\
\hline SB7b-26 & 1 & 2 & 16 & A05AR2 & 13400 & 91300 & 13600 & 184 & 1420 & 4320 & 1030 & 24200 & 196 & 560 \\
\hline SB7b-13 & 1 & 2 & 17 & A07AR2 & 11200 & 77900 & 14800 & 166 & 1250 & 7400 & 3760 & 26100 & 369 & 665 \\
\hline SB7b-25 & 1 & 2 & 18 & A18AR1 & 17000 & 112000 & 14000 & 145 & 2020 & 4510 & 1080 & 25100 & 216 & 534 \\
\hline blank & 1 & 2 & 19 & BLANKAR1 & $<42.4$ & $<5900$ & $<364$ & $<57.3$ & $<600$ & $<32.3$ & 4.88 & $<2610$ & $<5.28$ & $<4.4$ \\
\hline SB7Ref & 1 & 2 & 20 & SB7REFAR13 & 16500 & 111000 & 9660 & $<55.6$ & 1650 & 67 & 2710 & $<2530$ & 179 & 627 \\
\hline Ustd & 1 & 2 & 21 & USTDAR13 & 20700 & 83000 & 8200 & $<56.6$ & $<593$ & 384 & 5000 & 17500 & 81 & 12.3 \\
\hline SB7Ref & 2 & 1 & 1 & SB7REFAR21 & 16300 & 110000 & 9700 & $<56.2$ & 1680 & 63.3 & 2540 & $<2560$ & 176 & 229 \\
\hline Ustd & 2 & 1 & 2 & USTDAR21 & 21900 & 87600 & 8710 & $<55.6$ & $<583$ & 404 & 5260 & 19000 & 84.4 & 9.62 \\
\hline SB7b-05 & 2 & 1 & 3 & A11AR1 & 11700 & 113000 & 9300 & 225 & 2220 & 3290 & 40.7 & 27400 & 197 & 584 \\
\hline SB7b-30 & 2 & 1 & 4 & A13AR2 & 14600 & 107000 & 12100 & 103 & 2050 & 5140 & 1320 & 22200 & 151 & 420 \\
\hline SB7b-03 & 2 & 1 & 5 & A17AR1 & 18200 & 118000 & 15100 & 131 & 2170 & 7800 & 45.4 & 17800 & 320 & 401 \\
\hline
\end{tabular}


Table A-2. Measured Elemental Concentrations (ug/g) for the Study Glasses Prepared Using Aqua Regia (part 2)

\begin{tabular}{|c|c|c|c|c|c|c|c|c|c|c|c|c|c|c|}
\hline Glass ID & Block & Sub-Blk & Sequence & Lab ID & Mn & $\mathrm{Na}$ & $\mathrm{Ni}$ & $\mathbf{P b}$ & $\mathrm{S}$ & Th & Ti & $\mathbf{U}$ & Zn & $\mathrm{Zr}$ \\
\hline SB7b-31 & 2 & 1 & 6 & A02AR1 & 14100 & 91500 & 14100 & 83.9 & 1830 & 6000 & 1230 & 26000 & 187 & 384 \\
\hline SB7b-20 & 2 & 1 & 7 & A03AR1 & 16500 & 89700 & 13600 & $<56.1$ & 2100 & 4230 & 1000 & 19700 & 163 & 279 \\
\hline SB7b-31 & 2 & 1 & 8 & A02AR2 & 14000 & 91500 & 14000 & 91.9 & 1700 & 5900 & 1200 & 25500 & 185 & 407 \\
\hline SB7b-06 & 2 & 1 & 9 & A19AR2 & 18400 & 81900 & 14400 & 179 & 2380 & 3280 & 38.5 & 17300 & 246 & 449 \\
\hline SB7Ref & 2 & 1 & 10 & SB7REFAR22 & 16600 & 111000 & 9880 & $<56.2$ & 2010 & 65.4 & 2570 & $<2560$ & 179 & 242 \\
\hline Ustd & 2 & 1 & 11 & USTDAR22 & 21700 & 86300 & 8600 & $<55.6$ & $<583$ & 399 & 5150 & 19000 & 84.6 & 10.6 \\
\hline SB7b-20 & 2 & 1 & 12 & A03AR2 & 16500 & 88500 & 13500 & $<55.5$ & 1940 & 4210 & 996 & 19500 & 165 & 303 \\
\hline SB7b-17 & 2 & 1 & 13 & A25AR2 & 13400 & 93500 & 14100 & 94.7 & 1700 & 4150 & 2830 & 19500 & 227 & 241 \\
\hline SB7b-30 & 2 & 1 & 14 & A13AR1 & 14800 & 108000 & 12200 & 90.4 & 2150 & 5120 & 1280 & 22300 & 152 & 265 \\
\hline SB7b-05 & 2 & 1 & 15 & A11AR2 & 11700 & 112000 & 9350 & 230 & 1770 & 3300 & 40.8 & 27000 & 192 & 494 \\
\hline SB7b-06 & 2 & 1 & 16 & A19AR1 & 18300 & 80300 & 14400 & 174 & 2550 & 3230 & 38.6 & 17200 & 243 & 483 \\
\hline SB7b-03 & 2 & 1 & 17 & A17AR2 & 18300 & 118000 & 15100 & 142 & 1720 & 7860 & 48.3 & 17700 & 318 & 538 \\
\hline SB7b-17 & 2 & 1 & 18 & A25AR1 & 13200 & 93600 & 14000 & 118 & 1610 & 4120 & 2830 & 19300 & 227 & 460 \\
\hline blank & 2 & 1 & 19 & BLANKAR2 & $<42.4$ & $<5900$ & $<364$ & $<57.3$ & $<600$ & $<32.3$ & $<3.6$ & $<2610$ & $<5.28$ & $<4.4$ \\
\hline SB7Ref & 2 & 1 & 20 & SB7REFAR23 & 16400 & 110000 & 9680 & $<56.2$ & 1710 & 66.1 & 2630 & $<2560$ & 179 & 217 \\
\hline Ustd & 2 & 1 & 21 & USTDAR23 & 21400 & 85200 & 8630 & $<55.6$ & $<583$ & 406 & 5210 & 18500 & 83.8 & 10.2 \\
\hline SB7Ref & 2 & 2 & 1 & SB7REFAR21 & 16200 & 109000 & 9610 & $<56.2$ & 1810 & 66.1 & 2530 & $<2560$ & 175 & 226 \\
\hline Ustd & 2 & 2 & 2 & USTDAR21 & 21500 & 86400 & 8530 & $<55.6$ & $<583$ & 407 & 5250 & 18000 & 85.2 & 8.75 \\
\hline SB7b-30 & 2 & 2 & 3 & A13AR1 & 14700 & 108000 & 12100 & 107 & 2360 & 5090 & 1280 & 21300 & 151 & 266 \\
\hline SB7b-17 & 2 & 2 & 4 & A25AR1 & 13300 & 94200 & 13900 & 120 & 1800 & 4110 & 2820 & 19700 & 228 & 455 \\
\hline SB7b-06 & 2 & 2 & 5 & A19AR2 & 18400 & 82100 & 14300 & 187 & 2730 & 3260 & 40.1 & 17400 & 246 & 452 \\
\hline SB7b-05 & 2 & 2 & 6 & A11AR2 & 11700 & 113000 & 9200 & 209 & 2120 & 3290 & 41.9 & 26500 & 193 & 497 \\
\hline SB7b-05 & 2 & 2 & 7 & A11AR1 & 11600 & 112000 & 9190 & 220 & 2230 & 3270 & 41.5 & 26100 & 197 & 585 \\
\hline SB7b-31 & 2 & 2 & 8 & A02AR2 & 14000 & 91100 & 14000 & 96.4 & 1970 & 5850 & 1200 & 25000 & 184 & 409 \\
\hline SB7b-03 & 2 & 2 & 9 & A17AR2 & 18200 & 118000 & 15000 & 140 & 2010 & 7800 & 48.6 & 17600 & 317 & 524 \\
\hline SB7Ref & 2 & 2 & 10 & SB7REFAR22 & 16400 & 110000 & 9750 & 74.8 & 2170 & 67.2 & 2580 & $<2560$ & 180 & 240 \\
\hline Ustd & 2 & 2 & 11 & USTDAR22 & 21700 & 87100 & 8590 & $<55.6$ & $<583$ & 402 & 5190 & 18700 & 84.4 & 9.93 \\
\hline SB7b-06 & 2 & 2 & 12 & A19AR1 & 18100 & 80600 & 14200 & 183 & 2700 & 3210 & 40.5 & 16700 & 239 & 481 \\
\hline SB7b-20 & 2 & 2 & 13 & A03AR1 & 16400 & 90200 & 13500 & 65.4 & 1990 & 4230 & 1010 & 19300 & 162 & 280 \\
\hline SB7b-17 & 2 & 2 & 14 & A25AR2 & 13200 & 94000 & 13900 & 125 & 1990 & 4110 & 2830 & 19200 & 225 & 242 \\
\hline SB7b-20 & 2 & 2 & 15 & A03AR2 & 16400 & 89400 & 13400 & $<55.5$ & 2290 & 4180 & 1000 & 19500 & 163 & 306 \\
\hline SB7b-31 & 2 & 2 & 16 & A02AR1 & 14000 & 91600 & 14000 & 97.6 & 1910 & 5990 & 1230 & 24800 & 186 & 384 \\
\hline SB7b-30 & 2 & 2 & 17 & A13AR2 & 14400 & 105000 & 11900 & 97.7 & 2360 & 5140 & 1330 & 21000 & 150 & 422 \\
\hline SB7b-03 & 2 & 2 & 18 & A17AR1 & 18000 & 116000 & 15000 & 154 & 1990 & 7790 & 48.1 & 16600 & 319 & 403 \\
\hline blank & 2 & 2 & 19 & BLANKAR2 & $<42.4$ & $<5900$ & $<364$ & $<57.3$ & $<600$ & $<32.3$ & $<3.6$ & $<2610$ & $<5.28$ & $<4.4$ \\
\hline SB7Ref & 2 & 2 & 20 & SB7REFAR23 & 16300 & 110000 & 9670 & $<56.2$ & 2170 & 67.3 & 2610 & $<2560$ & 180 & 210 \\
\hline Ustd & 2 & 2 & 21 & USTDAR23 & 21300 & 85200 & 8490 & $<55.6$ & $<583$ & 399 & 5180 & 18300 & 84.1 & 9.72 \\
\hline SB7Ref & 3 & 1 & 1 & SB7REFAR31 & 17000 & 114000 & 10100 & $<54.9$ & 2770 & 71.9 & 2660 & $<2500$ & 189 & 684 \\
\hline Ustd & 3 & 1 & 2 & USTDAR31 & 21900 & 87200 & 8650 & $<54.9$ & 1210 & 400 & 5200 & 18800 & 80.9 & 11.1 \\
\hline SB7b-04 & 3 & 1 & 3 & A32AR1 & 18800 & 86000 & 9210 & 116 & 3990 & 7860 & 47.4 & 28100 & 131 & 290 \\
\hline SB7b-15 & 3 & 1 & 4 & A16AR2 & 13500 & 110000 & 11000 & $<54.6$ & 3060 & 4270 & 2850 & 24800 & 154 & 285 \\
\hline SB7b-22 & 3 & 1 & 5 & A14AR2 & 13700 & 91600 & 10900 & $<56.8$ & 3210 & 4230 & 1020 & 20100 & 135 & 182 \\
\hline SB7b-27 & 3 & 1 & 6 & A34AR2 & 13600 & 107000 & 14300 & $<57.2$ & 3410 & 6710 & 1060 & 19700 & 166 & 338 \\
\hline SB7b-16 & 3 & 1 & 7 & A15AR1 & 13600 & 92000 & 11000 & 112 & 2710 & 4460 & 3070 & 25000 & 212 & 556 \\
\hline SB7b-22 & 3 & 1 & 8 & A14AR1 & 13800 & 93400 & 11100 & $<56.6$ & 3420 & 4280 & 1050 & 20400 & 136 & 288 \\
\hline SB7b-04 & 3 & 1 & 9 & A32AR2 & 18800 & 85600 & 9330 & 90.8 & 4210 & 7890 & 47.2 & 28100 & 124 & 295 \\
\hline SB7Ref & 3 & 1 & 10 & SB7REFAR32 & 17100 & 114000 & 10100 & $<54.9$ & 3210 & 69.1 & 2660 & $<2500$ & 185 & 660 \\
\hline
\end{tabular}


Table A-2. Measured Elemental Concentrations (ug/g) for the Study Glasses Prepared Using Aqua Regia (part 2)

\begin{tabular}{|c|c|c|c|c|c|c|c|c|c|c|c|c|c|c|}
\hline Glass ID & Block & Sub-Blk & Sequence & Lab ID & Mn & $\mathrm{Na}$ & $\mathrm{Ni}$ & $\mathbf{P b}$ & $\mathrm{S}$ & Th & Ti & $\mathbf{U}$ & Zn & $\mathrm{Zr}$ \\
\hline Ustd & 3 & 1 & 11 & USTDAR32 & 21900 & 87300 & 8650 & $<54.9$ & 987 & 398 & 5230 & 18500 & 80.5 & 10.3 \\
\hline SB7b-32 & 3 & 1 & 12 & A29AR2 & 15700 & 107000 & 10600 & 86.4 & 3570 & 4570 & 1460 & 19000 & 157 & 394 \\
\hline SB7b-16 & 3 & 1 & 13 & A15AR2 & 13200 & 88900 & 10800 & 117 & 3470 & 4320 & 2970 & 24000 & 212 & 369 \\
\hline SB7b-27 & 3 & 1 & 14 & A34AR1 & 13400 & 106000 & 14100 & $<55.5$ & 3750 & 6650 & 1050 & 19600 & 168 & 364 \\
\hline SB7b-10 & 3 & 1 & 15 & A06AR1 & 18500 & 115000 & 15200 & $<56.9$ & 3440 & 3280 & 3990 & 27400 & 145 & 140 \\
\hline SB7b-10 & 3 & 1 & 16 & A06AR2 & 18800 & 117000 & 15600 & $<56$ & 3060 & 3330 & 4090 & 28100 & 140 & 156 \\
\hline SB7b-15 & 3 & 1 & 17 & A16AR1 & 13500 & 110000 & 11000 & $<54.8$ & 3220 & 4300 & 2910 & 24900 & 159 & 256 \\
\hline SB7b-32 & 3 & 1 & 18 & A29AR1 & 15700 & 107000 & 10600 & 83.8 & 3620 & 4530 & 1470 & 19100 & 154 & 455 \\
\hline blank & 3 & 1 & 19 & BLANKAR3 & $<42.4$ & $<5900$ & $<364$ & $<57.3$ & 1370 & $<32.3$ & $<3.6$ & $<2610$ & $<5.28$ & $<4.4$ \\
\hline SB7Ref & 3 & 1 & 20 & SB7REFAR33 & 16600 & 112000 & 9710 & $<54.9$ & 3170 & 69.1 & 2610 & $<2500$ & 183 & 645 \\
\hline Ustd & 3 & 1 & 21 & USTDAR33 & 21400 & 86000 & 8480 & $<54.9$ & 1310 & 395 & 5150 & 18500 & 80.9 & 7.8 \\
\hline SB7Ref & 3 & 2 & 1 & SB7REFAR31 & 16900 & 114000 & 9960 & 65.3 & 1850 & 68.1 & 2640 & $<2500$ & 188 & 638 \\
\hline Ustd & 3 & 2 & 2 & USTDAR31 & 21900 & 87400 & 8740 & $<54.9$ & $<575$ & 403 & 5170 & 18800 & 83.5 & 10.7 \\
\hline SB7b-10 & 3 & 2 & 3 & A06AR1 & 18500 & 115000 & 15300 & $<56.9$ & 1490 & 3270 & 3960 & 27600 & 146 & 137 \\
\hline SB7b-15 & 3 & 2 & 4 & A16AR1 & 13600 & 110000 & 11100 & $<54.8$ & 1730 & 4310 & 2890 & 24400 & 158 & 255 \\
\hline SB7b-27 & 3 & 2 & 5 & A34AR2 & 13600 & 107000 & 14400 & $<57.2$ & 2140 & 6730 & 1060 & 20000 & 162 & 333 \\
\hline SB7b-22 & 3 & 2 & 6 & A14AR1 & 13800 & 92600 & 11200 & $<56.6$ & 1570 & 4310 & 1040 & 20200 & 139 & 287 \\
\hline SB7b-04 & 3 & 2 & 7 & A32AR2 & 18800 & 85900 & 9280 & 109 & 2640 & 7940 & 48.3 & 27800 & 126 & 292 \\
\hline SB7b-27 & 3 & 2 & 8 & A34AR1 & 13400 & 105000 & 14100 & $<55.5$ & 2080 & 6650 & 1040 & 19600 & 167 & 358 \\
\hline SB7b-16 & 3 & 2 & 9 & A15AR2 & 13200 & 89100 & 10700 & 118 & 1730 & 4330 & 2970 & 23700 & 211 & 364 \\
\hline SB7Ref & 3 & 2 & 10 & SB7REFAR32 & 16900 & 113000 & 10000 & $<54.9$ & 2030 & 69.1 & 2640 & $<2500$ & 185 & 619 \\
\hline Ustd & 3 & 2 & 11 & USTDAR32 & 21800 & 87000 & 8730 & $<54.9$ & $<575$ & 408 & 5190 & 18600 & 83 & 10.6 \\
\hline SB7b-04 & 3 & 2 & 12 & A32AR1 & 18600 & 85400 & 9250 & 116 & 2400 & 7860 & 46.6 & 27800 & 130 & 285 \\
\hline SB7b-32 & 3 & 2 & 13 & A29AR2 & 15600 & 107000 & 10600 & 88 & 2420 & 4590 & 1460 & 18500 & 156 & 388 \\
\hline SB7b-10 & 3 & 2 & 14 & A06AR2 & 18700 & 116000 & 15500 & $<56$ & 2100 & 3330 & 4070 & 27700 & 141 & 153 \\
\hline SB7b-32 & 3 & 2 & 15 & A29AR1 & 15600 & 106000 & 10600 & 74.7 & 2190 & 4540 & 1460 & 18600 & 155 & 453 \\
\hline SB7b-15 & 3 & 2 & 16 & A16AR2 & 13500 & 110000 & 11000 & $<54.6$ & 1930 & 4270 & 2840 & 24800 & 156 & 284 \\
\hline SB7b-22 & 3 & 2 & 17 & A14AR2 & 13600 & 91500 & 11000 & 58.1 & 1810 & 4270 & 1020 & 19700 & 138 & 179 \\
\hline SB7b-16 & 3 & 2 & 18 & A15AR1 & 13500 & 91600 & 11000 & 106 & 1680 & 4470 & 3050 & 24800 & 212 & 550 \\
\hline blank & 3 & 2 & 19 & BLANKAR3 & $<42.4$ & $<5900$ & $<364$ & $<57.3$ & $<600$ & $<32.3$ & $<3.6$ & $<2610$ & $<5.28$ & $<4.4$ \\
\hline SB7Ref & 3 & 2 & 20 & SB7REFAR33 & 16600 & 110000 & 9830 & $<54.9$ & 2090 & 72.1 & 2600 & $<2500$ & 183 & 588 \\
\hline Ustd & 3 & 2 & 21 & USTDAR33 & 21300 & 84700 & 8630 & $<54.9$ & $<575$ & 406 & 5150 & 18300 & 81.4 & 10.3 \\
\hline SB7Ref & 4 & 1 & 1 & SB7REFAR41 & 16600 & 112000 & 9700 & 59.4 & 1530 & 64.9 & 2660 & $<2510$ & 179 & 717 \\
\hline Ustd & 4 & 1 & 2 & USTDAR41 & 21400 & 85800 & 8560 & $<56.6$ & $<593$ & 371 & 5000 & 18700 & 82.7 & 13 \\
\hline SB7b-18 & 4 & 1 & 3 & A24AR1 & 16800 & 105000 & 14300 & 138 & 1330 & 6650 & 2970 & 19800 & 195 & 479 \\
\hline SB7b-21 & 4 & 1 & 4 & A08AR1 & 16800 & 92100 & 10800 & 127 & 1680 & 6690 & 1020 & 24800 & 179 & 559 \\
\hline SB7b-12 & 4 & 1 & 5 & A23AR2 & 18300 & 81500 & 15400 & $<56.6$ & $<593$ & 3070 & 3610 & 17500 & 156 & 141 \\
\hline SB7b-34 & 4 & 1 & 6 & A30AR1 & 16700 & 98300 & 13700 & 86 & 2260 & 5970 & 1470 & 24700 & 183 & 349 \\
\hline SB7b-12 & 4 & 1 & 7 & A23AR1 & 18400 & 82100 & 15500 & $<56.4$ & 1030 & 3080 & 3650 & 17200 & 156 & 122 \\
\hline SB7b-01 & 4 & 1 & 8 & A04AR2 & 11400 & 117000 & 15300 & 72.5 & 2170 & 7620 & 43.3 & 17800 & 127 & 307 \\
\hline SB7b-21 & 4 & 1 & 9 & A08AR2 & 17000 & 92900 & 10900 & 144 & 1800 & 6780 & 1040 & 25000 & 187 & 631 \\
\hline SB7Ref & 4 & 1 & 10 & SB7REFAR42 & 16300 & 110000 & 9570 & $<55.1$ & 1560 & 64.5 & 2640 & $<2510$ & 179 & 747 \\
\hline Ustd & 4 & 1 & 11 & USTDAR42 & 20900 & 83500 & 8240 & $<56.6$ & $<593$ & 369 & 4980 & 17700 & 80.8 & 12.8 \\
\hline SB7b-09 & 4 & 1 & 12 & A33AR2 & 11800 & 83500 & 15100 & $<54.9$ & 1690 & 8000 & 46.6 & 27900 & 146 & 303 \\
\hline SB7b-18 & 4 & 1 & 13 & A24AR2 & 16900 & 106000 & 14300 & 132 & 1040 & 6740 & 3020 & 20000 & 194 & 546 \\
\hline SB7b-09 & 4 & 1 & 14 & A33AR1 & 11800 & 83000 & 15200 & 61.5 & 1720 & 7950 & 46.1 & 28200 & 143 & 327 \\
\hline SB7b-01 & 4 & 1 & 15 & A04AR1 & 11700 & 121000 & 15600 & 73.6 & 2490 & 7840 & 43.9 & 18100 & 131 & 276 \\
\hline
\end{tabular}


Table A-2. Measured Elemental Concentrations (ug/g) for the Study Glasses Prepared Using Aqua Regia (part 2)

\begin{tabular}{|c|c|c|c|c|c|c|c|c|c|c|c|c|c|c|}
\hline Glass ID & Block & Sub-Blk & Sequence & Lab ID & Mn & $\mathrm{Na}$ & $\mathrm{Ni}$ & $\mathbf{P b}$ & $\mathrm{S}$ & Th & Ti & $\mathbf{U}$ & Zn & $\mathrm{Zr}$ \\
\hline SB7b-08 & 4 & 1 & 16 & A22AR1 & 18300 & 113000 & 9050 & $<54.7$ & 2510 & 3320 & 48.1 & 27400 & 96.4 & 172 \\
\hline SB7b-34 & 4 & 1 & 17 & A30AR2 & 16600 & 97400 & 13600 & 83.1 & 2110 & 5990 & 1480 & 24600 & 182 & 518 \\
\hline SB7b-08 & 4 & 1 & 18 & A22AR2 & 18100 & 112000 & 9000 & $<55.6$ & 2310 & 3280 & 48 & 26500 & 95 & 180 \\
\hline blank & 4 & 1 & 19 & BLANKAR4 & $<42.4$ & $<5900$ & $<364$ & $<57.3$ & $<600$ & $<32.3$ & $<3.6$ & $<2610$ & $<5.28$ & $<4.4$ \\
\hline SB7Ref & 4 & 1 & 20 & SB7REFAR43 & 15500 & 105000 & 9110 & 55.4 & 1820 & 61.5 & 2530 & $<2510$ & 171 & 719 \\
\hline Ustd & 4 & 1 & 21 & USTDAR43 & 21100 & 84800 & 8340 & $<56.6$ & $<593$ & 372 & 5040 & 18700 & 81.7 & 11 \\
\hline SB7Ref & 4 & 2 & 1 & SB7REFAR41 & 16600 & 113000 & 9740 & 80.9 & 1300 & 60.1 & 2750 & $<2510$ & 183 & 722 \\
\hline Ustd & 4 & 2 & 2 & USTDAR41 & 21500 & 86600 & 8500 & $<56.6$ & $<593$ & 378 & 5160 & 19100 & 83.9 & 13.1 \\
\hline SB7b-01 & 4 & 2 & 3 & A04AR2 & 11600 & 118000 & 15500 & 89.4 & 2550 & 7680 & 48.1 & 18000 & 131 & 310 \\
\hline SB7b-21 & 4 & 2 & 4 & A08AR2 & 17200 & 93700 & 11100 & 152 & 2640 & 6820 & 1070 & 25300 & 192 & 641 \\
\hline SB7b-09 & 4 & 2 & 5 & A33AR2 & 12000 & 83000 & 15400 & 70.1 & 1510 & 8020 & 50 & 28100 & 149 & 306 \\
\hline SB7b-18 & 4 & 2 & 6 & A24AR1 & 17000 & 105000 & 14400 & 150 & 1260 & 6710 & 3080 & 20200 & 201 & 486 \\
\hline SB7b-01 & 4 & 2 & 7 & A04AR1 & 11900 & 121000 & 15800 & 92.6 & 2730 & 7910 & 49.3 & 18400 & 136 & 281 \\
\hline SB7b-09 & 4 & 2 & 8 & A33AR1 & 12100 & 82900 & 15400 & 66.3 & 1660 & 8010 & 51.8 & 28500 & 147 & 331 \\
\hline SB7b-34 & 4 & 2 & 9 & A30AR1 & 16900 & 97700 & 13700 & 113 & 2430 & 6020 & 1520 & 25400 & 188 & 355 \\
\hline SB7Ref & 4 & 2 & 10 & SB7REFAR42 & 16600 & 110000 & 9820 & 83 & 1710 & 65.3 & 2730 & $<2510$ & 184 & 751 \\
\hline Ustd & 4 & 2 & 11 & USTDAR42 & 21100 & 83700 & 8380 & $<56.6$ & $<593$ & 374 & 5140 & 18600 & 83.4 & 13.6 \\
\hline SB7b-12 & 4 & 2 & 12 & A23AR1 & 18600 & 81700 & 15800 & $<56.4$ & 925 & 3100 & 3780 & 17600 & 161 & 124 \\
\hline SB7b-12 & 4 & 2 & 13 & A23AR2 & 18400 & 81400 & 15700 & $<56.6$ & 968 & 3110 & 3770 & 17800 & 161 & 144 \\
\hline SB7b-34 & 4 & 2 & 14 & A30AR2 & 16900 & 98100 & 13800 & 97.7 & 2050 & 6050 & 1540 & 25200 & 187 & 530 \\
\hline SB7b-21 & 4 & 2 & 15 & A08AR1 & 16900 & 91100 & 10900 & 152 & 2260 & 6770 & 1070 & 25300 & 185 & 571 \\
\hline SB7b-08 & 4 & 2 & 16 & A22AR1 & 18600 & 114000 & 9200 & $<54.7$ & 2740 & 3360 & 54.3 & 27900 & 101 & 177 \\
\hline SB7b-18 & 4 & 2 & 17 & A24AR2 & 17100 & 106000 & 14400 & 139 & 1480 & 6770 & 3120 & 20700 & 200 & 554 \\
\hline SB7b-08 & 4 & 2 & 18 & A22AR2 & 18400 & 112000 & 9170 & $<55.6$ & 2950 & 3300 & 52.8 & 27600 & 99.1 & 184 \\
\hline blank & 4 & 2 & 19 & BLANKAR4 & $<42.4$ & $<5900$ & $<364$ & $<57.3$ & $<600$ & $<32.3$ & $<3.6$ & $<2610$ & $<5.28$ & $<4.4$ \\
\hline SB7Ref & 4 & 2 & 20 & SB7REFAR43 & 15800 & 106000 & 9390 & 56 & 1790 & 64.2 & 2610 & $<2510$ & 176 & 728 \\
\hline Ustd & 4 & 2 & 21 & USTDAR43 & 21400 & 83900 & 8530 & $<56.6$ & $<593$ & 381 & 5200 & 18800 & 83.5 & 11.3 \\
\hline SB7Ref & 5 & 1 & 1 & SB7REFAR51 & 16300 & 109000 & 9620 & 66.9 & 1900 & 68 & 2670 & $<2520$ & 287 & 684 \\
\hline Ustd & 5 & 1 & 2 & USTDSR51 & 21400 & 85400 & 8450 & $<56.3$ & $<590$ & 387 & 5140 & 18400 & 85.9 & 13.1 \\
\hline SB7b-23 & 5 & 1 & 3 & A31AR2 & 13500 & 90500 & 13900 & $<55.9$ & 1600 & 6760 & 3100 & 25000 & 178 & 375 \\
\hline SB7b-23 & 5 & 1 & 4 & A31AR1 & 13300 & 89100 & 13700 & 58 & 1490 & 6580 & 3030 & 25000 & 175 & 380 \\
\hline SB7b-11 & 5 & 1 & 5 & A20AR1 & 11700 & 99900 & 8930 & 188 & 1820 & 7820 & 4000 & 17400 & 180 & 803 \\
\hline SB7b-02 & 5 & 1 & 6 & A01AR2 & 17800 & 108000 & 8850 & 153 & 1150 & 7750 & 3890 & 26900 & 182 & 782 \\
\hline SB7b-28 & 5 & 1 & 7 & A28AR2 & 16300 & 87400 & 10600 & $<56.6$ & 1460 & 6430 & 2860 & 23800 & 147 & 358 \\
\hline SB7b-07 & 5 & 1 & 8 & A12AR1 & 11700 & 117000 & 9070 & $<55.7$ & 994 & 3130 & 3890 & 17400 & 88.6 & 162 \\
\hline SB7b-11 & 5 & 1 & 9 & A20AR2 & 11300 & 96700 & 8570 & 178 & 1550 & 7500 & 3840 & 17000 & 176 & 737 \\
\hline SB7Ref & 5 & 1 & 10 & SB7REFAR52 & 16400 & 108000 & 9550 & 57.8 & 1670 & 65.4 & 2660 & $<2520$ & 288 & 700 \\
\hline Ustd & 5 & 1 & 11 & USTDSR52 & 21800 & 86300 & 8630 & $<56.3$ & $<590$ & 391 & 5230 & 18900 & 87 & 14.1 \\
\hline SB7b-24 & 5 & 1 & 12 & A26AR2 & 16900 & 106000 & 10700 & $<55.5$ & 1570 & 4330 & 3020 & 19900 & 144 & 336 \\
\hline SB7b-07 & 5 & 1 & 13 & A12AR2 & 11300 & 114000 & 8770 & $<56.5$ & 1090 & 3060 & 3830 & 16900 & 86.9 & 179 \\
\hline SB7b-24 & 5 & 1 & 14 & A26AR1 & 16800 & 105000 & 10600 & $<57.8$ & 1620 & 4270 & 2970 & 19600 & 147 & 315 \\
\hline SB7b-28 & 5 & 1 & 15 & A28AR1 & 16800 & 90200 & 10800 & $<56.9$ & 1520 & 6690 & 2950 & 24900 & 148 & 343 \\
\hline SB7b-02 & 5 & 1 & 16 & A01AR1 & 18200 & 110000 & 9090 & 149 & 1320 & 7930 & 3980 & 27700 & 190 & 679 \\
\hline blank & 5 & 1 & 17 & BLANKAR5 & $<42.4$ & $<5900$ & $<364$ & $<57.3$ & $<600$ & $<32.3$ & $<3.6$ & $<2610$ & 6.96 & $<4.4$ \\
\hline SB7Ref & 5 & 1 & 18 & SB7REFAR53 & 16100 & 108000 & 9450 & $<55.3$ & 1730 & 65 & 2610 & $<2520$ & 285 & 661 \\
\hline Ustd & 5 & 1 & 19 & USTDAR53 & 21600 & 85800 & 8520 & $<56.3$ & $<590$ & 391 & 5190 & 18500 & 85.3 & 12.5 \\
\hline SB7Ref & 5 & 2 & 1 & SB7REFAR51 & 16400 & 113000 & 9680 & 59.9 & 1940 & 66.3 & 2680 & $<2520$ & 290 & 669 \\
\hline
\end{tabular}


Table A-2. Measured Elemental Concentrations (ug/g) for the Study Glasses Prepared Using Aqua Regia (part 2)

\begin{tabular}{|c|c|c|c|c|c|c|c|c|c|c|c|c|c|c|}
\hline Glass ID & Block & Sub-Blk & Sequence & Lab ID & Mn & $\mathrm{Na}$ & $\mathbf{N i}$ & $\mathbf{P b}$ & $\mathrm{S}$ & Th & Ti & $\mathbf{U}$ & Zn & $\mathrm{Zr}$ \\
\hline Ustd & 5 & 2 & 2 & USTDSR51 & 21600 & 87600 & 8510 & $<56.3$ & $<590$ & 383 & 5130 & 19000 & 87.3 & 12.9 \\
\hline SB7b-02 & 5 & 2 & 3 & A01AR1 & 18400 & 114000 & 9220 & 147 & 1140 & 7950 & 4010 & 27700 & 194 & 685 \\
\hline SB7b-07 & 5 & 2 & 4 & A12AR1 & 11800 & 120000 & 9090 & $<55.7$ & 962 & 3150 & 3930 & 17500 & 90 & 165 \\
\hline SB7b-23 & 5 & 2 & 5 & A31AR2 & 13600 & 92500 & 14000 & $<55.9$ & 1240 & 6770 & 3130 & 25500 & 182 & 379 \\
\hline SB7b-24 & 5 & 2 & 6 & A26AR2 & 17000 & 108000 & 10800 & $<55.5$ & 1370 & 4330 & 3060 & 19600 & 147 & 340 \\
\hline SB7b-24 & 5 & 2 & 7 & A26AR1 & 17000 & 107000 & 10800 & $<57.8$ & 1460 & 4280 & 3010 & 19700 & 149 & 319 \\
\hline SB7b-23 & 5 & 2 & 8 & A31AR1 & 13300 & 90100 & 13600 & $<55.9$ & 1230 & 6600 & 3050 & 24500 & 179 & 383 \\
\hline SB7b-28 & 5 & 2 & 9 & A28AR1 & 17000 & 91300 & 10900 & $<56.9$ & 1190 & 6670 & 2990 & 24900 & 150 & 348 \\
\hline SB7Ref & 5 & 2 & 10 & SB7REFAR52 & 16400 & 110000 & 9650 & 58.9 & 1590 & 68 & 2690 & $<2520$ & 292 & 684 \\
\hline Ustd & 5 & 2 & 11 & USTDSR52 & 21800 & 87600 & 8680 & $<56.3$ & $<590$ & 384 & 5260 & 18600 & 87.8 & 14.1 \\
\hline SB7b-11 & 5 & 2 & 12 & A20AR2 & 11400 & 98000 & 8610 & 161 & 1230 & 7550 & 3900 & 16900 & 179 & 745 \\
\hline SB7b-28 & 5 & 2 & 13 & A28AR2 & 16200 & 88700 & 10500 & $<56.6$ & 1090 & 6440 & 2880 & 24100 & 148 & 360 \\
\hline SB7b-11 & 5 & 2 & 14 & A20AR1 & 11800 & 102000 & 8950 & 172 & 1470 & 7820 & 4050 & 17800 & 181 & 809 \\
\hline SB7b-07 & 5 & 2 & 15 & A12AR2 & 11400 & 115000 & 8840 & $<56.5$ & 1020 & 3070 & 3890 & 17100 & 86.9 & 184 \\
\hline SB7b-02 & 5 & 2 & 16 & A01AR2 & 17900 & 110000 & 8900 & 156 & 1270 & 7740 & 3940 & 26700 & 183 & 787 \\
\hline blank & 5 & 2 & 17 & BLANKAR5 & $<42.4$ & $<5900$ & $<364$ & $<57.3$ & $<600$ & $<32.3$ & $<3.6$ & $<2610$ & 7.04 & 4.64 \\
\hline SB7Ref & 5 & 2 & 18 & SB7REFAR53 & 16100 & 108000 & 9470 & $<55.3$ & 1570 & 65.1 & 2670 & $<2520$ & 290 & 635 \\
\hline Ustd & 5 & 2 & 19 & USTDAR53 & 21700 & 86500 & 8590 & $<56.3$ & $<590$ & 394 & 5310 & 18700 & 88 & 13.3 \\
\hline
\end{tabular}


Table A-3. Measured Elemental Concentrations (ug/g) for the Study Glasses Prepared Using Peroxide Fusion (part 1)

\begin{tabular}{|c|c|c|c|c|c|c|c|c|c|c|c|c|c|c|}
\hline Glass ID & Block & Sub-Blk & Sequence & Lab ID & Al & B & Ba & $\mathrm{Ce}$ & $\mathrm{Cr}$ & $\mathbf{C u}$ & $\mathrm{Fe}$ & $\mathbf{K}$ & La & $\mathbf{L i}$ \\
\hline SB7Ref & 1 & 1 & 1 & SB7REFPF11 & 47900 & 15100 & 186 & $<478$ & 854 & 388 & 60800 & 2810 & 127 & 23800 \\
\hline Ustd & 1 & 1 & 2 & USTDPF11 & 21000 & 26200 & $<40.9$ & $<475$ & 1670 & $<49.6$ & 92300 & 25400 & $<42.5$ & 13900 \\
\hline SB7b-05 & 1 & 1 & 3 & A11PF1 & 34700 & 15500 & 650 & 961 & 562 & 344 & 51600 & $<2340$ & 187 & 22000 \\
\hline SB7b-18 & 1 & 1 & 4 & A24PF1 & 39400 & 14300 & 518 & 631 & 479 & 282 & 74000 & $<2340$ & 160 & 24300 \\
\hline SB7b-29 & 1 & 1 & 5 & A21PF2 & 37100 & 15300 & 321 & $<470$ & 420 & 201 & 56700 & $<2350$ & 108 & 24400 \\
\hline SB7b-12 & 1 & 1 & 6 & A23PF1 & 34600 & 13800 & 111 & $<478$ & 96.1 & 253 & 79500 & $<2380$ & 54.6 & 22000 \\
\hline SB7b-08 & 1 & 1 & 7 & A22PF2 & 34800 & 13800 & 120 & $<475$ & 98.4 & 72.4 & 80200 & $<2370$ & 63 & 31300 \\
\hline SB7b-12 & 1 & 1 & 8 & A23PF2 & 35200 & 13800 & 120 & $<460$ & 115 & 242 & 79900 & $<2300$ & 58.8 & 22100 \\
\hline SB7b-08 & 1 & 1 & 9 & A22PF1 & 34200 & 13800 & 103 & $<462$ & 103 & 58.2 & 80200 & $<2300$ & 75.9 & 31000 \\
\hline SB7Ref & 1 & 1 & 10 & SB7REFPF12 & 47900 & 15100 & 183 & $<478$ & 845 & 384 & 60800 & 3610 & 108 & 23700 \\
\hline Ustd & 1 & 1 & 11 & USTDPF12 & 20800 & 26100 & $<40.9$ & $<475$ & 1650 & $<49.6$ & 92100 & 25000 & $<42.5$ & 13700 \\
\hline SB7b-11 & 1 & 1 & 12 & A20PF1 & 53200 & 13800 & 639 & 1070 & 609 & 311 & 79200 & $<2380$ & 193 & 21800 \\
\hline SB7b-24 & 1 & 1 & 13 & A26PF2 & 45700 & 14300 & 245 & $<474$ & 190 & 160 & 55400 & $<2360$ & 67.6 & 27200 \\
\hline SB7b-11 & 1 & 1 & 14 & A20PF2 & 53100 & 13900 & 643 & 1010 & 595 & 314 & 79300 & $<2350$ & 183 & 21900 \\
\hline SB7b-05 & 1 & 1 & 15 & A11PF2 & 34400 & 15400 & 640 & 1220 & 554 & 349 & 51400 & $<2340$ & 194 & 21800 \\
\hline SB7b-29 & 1 & 1 & 16 & A21PF1 & 37400 & 15500 & 315 & $<479$ & 271 & 184 & 56700 & $<2390$ & 110 & 24900 \\
\hline SB7b-18 & 1 & 1 & 17 & A24PF2 & 38900 & 14200 & 508 & 795 & 478 & 265 & 73000 & $<2330$ & 181 & 24300 \\
\hline SB7b-24 & 1 & 1 & 18 & A26PF1 & 47900 & 15000 & 257 & $<479$ & 202 & 168 & 57500 & $<2390$ & 75.4 & 28600 \\
\hline blank & 1 & 1 & 19 & BLANKPF1 & 782 & $<162$ & 46.4 & $<482$ & $<81.6$ & $<50.4$ & 91.2 & $<2410$ & $<43.2$ & $<117$ \\
\hline SB7Ref & 1 & 1 & 20 & SB7REFPF13 & 48000 & 15100 & 181 & $<478$ & 852 & 384 & 61800 & 3460 & 110 & 23700 \\
\hline Ustd & 1 & 1 & 21 & USTDPF13 & 20900 & 26300 & $<40.9$ & $<475$ & 1690 & $<49.6$ & 94300 & 24800 & $<42.5$ & 13600 \\
\hline SB7Ref & 1 & 2 & 1 & SB7REFPF11 & 47800 & 15200 & 184 & $<478$ & 876 & 422 & 60200 & 4050 & 108 & 23700 \\
\hline Ustd & 1 & 2 & 2 & USTDPF11 & 21000 & 26400 & $<40.9$ & $<475$ & 1690 & 70.9 & 91600 & 25600 & $<42.5$ & 13700 \\
\hline SB7b-05 & 1 & 2 & 3 & A11PF2 & 34200 & 15700 & 646 & 1090 & 577 & 369 & 50800 & 2580 & 194 & 21500 \\
\hline SB7b-08 & 1 & 2 & 4 & A22PF2 & 34800 & 14000 & 118 & $<475$ & 135 & 89 & 79800 & 2940 & 44.1 & 31200 \\
\hline SB7b-18 & 1 & 2 & 5 & A24PF2 & 39000 & 14400 & 520 & 775 & 502 & 305 & 73500 & 2350 & 148 & 24100 \\
\hline SB7b-11 & 1 & 2 & 6 & A20PF2 & 53200 & 14100 & 647 & 929 & 616 & 336 & 78900 & 2630 & 171 & 21700 \\
\hline SB7b-24 & 1 & 2 & 7 & A26PF1 & 47900 & 15100 & 254 & $<479$ & 208 & 195 & 57200 & 2750 & 63.5 & 28400 \\
\hline SB7b-29 & 1 & 2 & 8 & A21PF2 & 36900 & 15300 & 317 & $<470$ & 434 & 198 & 55400 & 2460 & 95.2 & 24200 \\
\hline SB7b-08 & 1 & 2 & 9 & A22PF1 & 34300 & 13900 & 104 & $<462$ & 100 & 74.3 & 79200 & 2640 & 62.1 & 31100 \\
\hline SB7Ref & 1 & 2 & 10 & SB7REFPF12 & 47700 & 15300 & 180 & $<478$ & 883 & 403 & 60100 & 3950 & 105 & 23900 \\
\hline Ustd & 1 & 2 & 11 & USTDPF12 & 20800 & 26300 & $<40.9$ & $<475$ & 1700 & 61.4 & 91000 & 25500 & $<42.5$ & 13800 \\
\hline SB7b-12 & 1 & 2 & 12 & A23PF1 & 34300 & 13800 & 109 & $<478$ & 90.7 & 265 & 77800 & 3060 & 59.4 & 22000 \\
\hline SB7b-18 & 1 & 2 & 13 & A24PF1 & 39100 & 14400 & 519 & 670 & 503 & 302 & 73200 & 2870 & 163 & 24200 \\
\hline SB7b-24 & 1 & 2 & 14 & A26PF2 & 46000 & 14500 & 251 & $<474$ & 216 & 193 & 55000 & 3070 & 69.2 & 27500 \\
\hline SB7b-29 & 1 & 2 & 15 & A21PF1 & 37400 & 15700 & 316 & $<479$ & 297 & 209 & 56300 & 2680 & 98.5 & 24800 \\
\hline SB7b-12 & 1 & 2 & 16 & A23PF2 & 34800 & 14000 & 118 & $<460$ & 111 & 252 & 78800 & 2530 & $<41.2$ & 22100 \\
\hline SB7b-11 & 1 & 2 & 17 & A20PF1 & 53300 & 14000 & 645 & 1010 & 635 & 327 & 78700 & 2830 & 208 & 21900 \\
\hline SB7b-05 & 1 & 2 & 18 & A11PF1 & 34300 & 15600 & 646 & 951 & 582 & 359 & 50800 & 2990 & 168 & 21800 \\
\hline blank & 1 & 2 & 19 & BLANKPF1 & 912 & $<162$ & 46.4 & $<482$ & $<81.6$ & $<50.4$ & 118 & 2840 & $<43.2$ & $<117$ \\
\hline SB7Ref & 1 & 2 & 20 & SB7REFPF13 & 47500 & 15200 & 183 & $<478$ & 872 & 409 & 59700 & 4470 & 117 & 24000 \\
\hline Ustd & 1 & 2 & 21 & USTDPF13 & 20700 & 26200 & $<40.9$ & $<475$ & 1670 & $<49.6$ & 90700 & 26100 & $<42.5$ & 13800 \\
\hline SB7Ref & 2 & 1 & 1 & SB7REFPF21 & 48100 & 15500 & 198 & $<473$ & 889 & 402 & 61100 & 2820 & 98.2 & 23800 \\
\hline Ustd & 2 & 1 & 2 & USTDPF21 & 21200 & 26600 & 56.3 & $<465$ & 1710 & 70.2 & 92100 & 24500 & $<41.6$ & 13900 \\
\hline SB7b-20 & 2 & 1 & 3 & A03PF1 & 47700 & 14200 & 262 & 524 & 201 & 182 & 58800 & $<2290$ & 99.6 & 23500 \\
\hline SB7b-30 & 2 & 1 & 4 & A13PF2 & 42900 & 15000 & 379 & 567 & 315 & 186 & 64900 & $<2360$ & 114 & 23500 \\
\hline SB7b-01 & 2 & 1 & 5 & A04PF1 & 34200 & 15800 & 120 & $<465$ & 105 & 67.8 & 80600 & $<2320$ & 54 & 21900 \\
\hline
\end{tabular}


Table A-3. Measured Elemental Concentrations (ug/g) for the Study Glasses Prepared Using Peroxide Fusion (part 1)

\begin{tabular}{|c|c|c|c|c|c|c|c|c|c|c|c|c|c|c|}
\hline Glass ID & Block & Sub-Blk & Sequence & Lab ID & Al & B & Ba & $\mathrm{Ce}$ & $\mathrm{Cr}$ & $\mathbf{C u}$ & $\mathrm{Fe}$ & $\mathbf{K}$ & La & $\mathbf{L i}$ \\
\hline SB7b-30 & 2 & 1 & 6 & A13PF1 & 42800 & 15000 & 371 & 640 & 314 & 182 & 65100 & $<2340$ & 122 & 23600 \\
\hline SB7b-17 & 2 & 1 & 7 & A25PF2 & 39100 & 15300 & 538 & 765 & 481 & 394 & 59300 & $<2350$ & 143 & 28900 \\
\hline SB7b-14 & 2 & 1 & 8 & A09PF1 & 34200 & 15700 & 118 & $<462$ & 100 & 530 & 51500 & $<2300$ & 71.3 & 30900 \\
\hline SB7b-04 & 2 & 1 & 9 & A32PF1 & 53400 & 13900 & 116 & $<470$ & 123 & 253 & 49000 & $<2340$ & 74.8 & 21800 \\
\hline SB7Ref & 2 & 1 & 10 & SB7REFPF22 & 48600 & 15500 & 196 & $<473$ & 875 & 414 & 61400 & 3030 & 88.7 & 24200 \\
\hline Ustd & 2 & 1 & 11 & USTDPF22 & 21500 & 26800 & 56.3 & $<465$ & 1710 & 67.1 & 92400 & 25700 & $<41.6$ & 14200 \\
\hline SB7b-01 & 2 & 1 & 12 & A04PF2 & 34600 & 15900 & 115 & $<482$ & 99.1 & 65.5 & 80900 & $<2400$ & 64.7 & 22100 \\
\hline SB7b-14 & 2 & 1 & 13 & A09PF2 & 34500 & 15700 & 123 & $<469$ & 107 & 519 & 51400 & $<2340$ & 63.1 & 31300 \\
\hline SB7b-20 & 2 & 1 & 14 & A03PF2 & 48900 & 14500 & 259 & $<475$ & 195 & 178 & 60300 & $<2370$ & 93.8 & 24400 \\
\hline SB7b-34 & 2 & 1 & 15 & A30PF1 & 48200 & 14000 & 441 & 688 & 358 & 221 & 72300 & $<2340$ & 155 & 27800 \\
\hline SB7b-34 & 2 & 1 & 16 & A30PF2 & 47900 & 13900 & 441 & 599 & 348 & 234 & 71200 & $<2290$ & 143 & 27100 \\
\hline SB7b-04 & 2 & 1 & 17 & A32PF2 & 54200 & 14000 & 127 & $<483$ & 118 & 243 & 48800 & $<2410$ & 78.5 & 22100 \\
\hline SB7b-17 & 2 & 1 & 18 & A25PF1 & 39500 & 15400 & 542 & 900 & 488 & 384 & 59700 & $<2380$ & 155 & 29400 \\
\hline blank & 2 & 1 & 19 & BLANKPF2 & 1230 & $<162$ & 62.4 & $<482$ & $<81.6$ & $<50.4$ & 150 & $<2410$ & $<43.2$ & $<117$ \\
\hline SB7Ref & 2 & 1 & 20 & SB7REFPF23 & 48200 & 15300 & 198 & $<473$ & 882 & 408 & 60500 & 3110 & 103 & 24000 \\
\hline Ustd & 2 & 1 & 21 & USTDPF23 & 21600 & 26700 & 55.5 & $<465$ & 1720 & 60.1 & 92000 & 24900 & $<41.6$ & 14100 \\
\hline SB7Ref & 2 & 2 & 1 & SB7REFPF21 & 48200 & 15500 & 198 & $<473$ & 879 & 415 & 61400 & 3650 & 91.1 & 23900 \\
\hline Ustd & 2 & 2 & 2 & USTDPF21 & 21300 & 26600 & 57.1 & $<465$ & 1700 & 69.4 & 93000 & 25400 & $<41.6$ & 13900 \\
\hline SB7b-20 & 2 & 2 & 3 & A03PF1 & 48100 & 14200 & 264 & $<458$ & 181 & 174 & 59300 & 2520 & 77.5 & 23700 \\
\hline SB7b-14 & 2 & 2 & 4 & A09PF2 & 34400 & 15700 & 124 & $<469$ & 89.2 & 526 & 51700 & $<2340$ & $<42$ & 30900 \\
\hline SB7b-01 & 2 & 2 & 5 & A04PF2 & 34400 & 15900 & 113 & $<482$ & 85.5 & 63.9 & 81500 & $<2400$ & 46.3 & 21900 \\
\hline SB7b-14 & 2 & 2 & 6 & A09PF1 & 34300 & 15800 & 116 & $<462$ & 90.4 & 514 & 52000 & $<2300$ & 58.2 & 31100 \\
\hline SB7b-04 & 2 & 2 & 7 & A32PF2 & 53900 & 14000 & 125 & $<483$ & 96.3 & 254 & 49400 & 2530 & 51.2 & 21800 \\
\hline SB7b-34 & 2 & 2 & 8 & A30PF1 & 48200 & 14000 & 437 & 670 & 372 & 222 & 72900 & 2640 & 135 & 27300 \\
\hline SB7b-30 & 2 & 2 & 9 & A13PF1 & 43100 & 15100 & 377 & 637 & 310 & 171 & 65300 & 2970 & 101 & 23600 \\
\hline SB7Ref & 2 & 2 & 10 & SB7REFPF22 & 48400 & 15500 & 196 & $<473$ & 874 & 413 & 61900 & 3570 & 90.3 & 24000 \\
\hline Ustd & 2 & 2 & 11 & USTDPF22 & 21400 & 26800 & 57.1 & $<465$ & 1720 & 50.1 & 93900 & 25800 & $<41.6$ & 14000 \\
\hline SB7b-34 & 2 & 2 & 12 & A30PF2 & 48100 & 13900 & 441 & 574 & 357 & 221 & 71600 & 2410 & 119 & 26900 \\
\hline SB7b-01 & 2 & 2 & 13 & A04PF1 & 34400 & 15900 & 117 & $<465$ & 80.2 & 60.1 & 81100 & 2570 & $<41.6$ & 21900 \\
\hline SB7b-04 & 2 & 2 & 14 & A32PF1 & 53600 & 14100 & 116 & $<470$ & 118 & 244 & 49300 & 2490 & 62.3 & 21900 \\
\hline SB7b-30 & 2 & 2 & 15 & A13PF2 & 43300 & 15200 & 384 & 610 & 315 & 182 & 65600 & 2380 & 90.1 & 23700 \\
\hline SB7b-17 & 2 & 2 & 16 & A25PF2 & 38800 & 15300 & 540 & 794 & 482 & 395 & 59300 & 2450 & 141 & 28800 \\
\hline SB7b-20 & 2 & 2 & 17 & A03PF2 & 49100 & 14600 & 262 & $<475$ & 193 & 176 & 60900 & 2380 & 63.9 & 24300 \\
\hline SB7b-17 & 2 & 2 & 18 & A25PF1 & 39400 & 15500 & 546 & 997 & 486 & 384 & 60400 & 2690 & 150 & 29100 \\
\hline blank & 2 & 2 & 19 & BLANKPF2 & 1110 & $<162$ & 64 & $<482$ & $<81.6$ & $<50.4$ & 168 & $<2410$ & $<43.2$ & $<117$ \\
\hline SB7Ref & 2 & 2 & 20 & SB7REFPF23 & 48500 & 15500 & 198 & $<473$ & 869 & 404 & 61100 & 3680 & 84 & 23900 \\
\hline Ustd & 2 & 2 & 21 & USTDPF23 & 21600 & 26800 & 57.8 & $<465$ & 1730 & 51.7 & 93200 & 25900 & $<41.6$ & 14100 \\
\hline SB7Ref & 3 & 1 & 1 & SB7REFPF31 & 47800 & 15400 & 191 & $<472$ & 883 & 410 & 61200 & 3860 & 72 & 24100 \\
\hline Ustd & 3 & 1 & 2 & USTDPF31 & 20900 & 26800 & $<41$ & $<476$ & 1710 & 52.1 & 93500 & 25200 & $<42.6$ & 14000 \\
\hline SB7b-27 & 3 & 1 & 3 & A34PF2 & 39200 & 15300 & 261 & $<473$ & 256 & 200 & 74300 & $<2360$ & 108 & 28900 \\
\hline SB7b-09 & 3 & 1 & 4 & A33PF2 & 52800 & 15900 & 93.3 & $<477$ & 96.2 & 190 & 76500 & $<2380$ & 59.4 & 31200 \\
\hline SB7b-19 & 3 & 1 & 5 & A27PF1 & 47800 & 14300 & 518 & 894 & 504 & 321 & 58800 & $<2370$ & 159 & 24500 \\
\hline SB7b-16 & 3 & 1 & 6 & A15PF1 & 48000 & 14400 & 512 & 842 & 429 & 364 & 72400 & $<2350$ & 150 & 28700 \\
\hline SB7b-27 & 3 & 1 & 7 & A34PF1 & 38900 & 15100 & 238 & $<478$ & 236 & 192 & 73400 & $<2380$ & 122 & 28500 \\
\hline SB7b-21 & 3 & 1 & 8 & A08PF1 & 38800 & 15400 & 518 & 975 & 429 & 321 & 59100 & $<2310$ & 147 & 29000 \\
\hline SB7b-19 & 3 & 1 & 9 & A27PF2 & 47500 & 14200 & 522 & 860 & 501 & 286 & 58500 & $<2340$ & 139 & 24000 \\
\hline SB7Ref & 3 & 1 & 10 & SB7REFPF32 & 47800 & 15300 & 194 & $<472$ & 882 & 376 & 61400 & 3820 & 83.7 & 24000 \\
\hline
\end{tabular}


Table A-3. Measured Elemental Concentrations (ug/g) for the Study Glasses Prepared Using Peroxide Fusion (part 1)

\begin{tabular}{|c|c|c|c|c|c|c|c|c|c|c|c|c|c|c|}
\hline Glass ID & Block & Sub-Blk & Sequence & Lab ID & Al & B & Ba & $\mathrm{Ce}$ & $\mathrm{Cr}$ & $\mathbf{C u}$ & $\mathrm{Fe}$ & $\mathbf{K}$ & La & $\mathbf{L i}$ \\
\hline Ustd & 3 & 1 & 11 & USTDPF32 & 20800 & 26600 & $<41$ & $<476$ & 1690 & $<49.7$ & 93500 & 25800 & $<42.6$ & 13900 \\
\hline SB7b-21 & 3 & 1 & 12 & A08PF2 & 38600 & 15200 & 511 & 824 & 444 & 301 & 58700 & $<2290$ & 135 & 28700 \\
\hline SB7b-31 & 3 & 1 & 13 & A02PF1 & 40800 & 16500 & 340 & 630 & 356 & 183 & 75300 & $<2300$ & 113 & 23200 \\
\hline SB7b-16 & 3 & 1 & 14 & A15PF2 & 48000 & 14400 & 514 & 755 & 425 & 367 & 72000 & $<2360$ & 142 & 28900 \\
\hline SB7b-31 & 3 & 1 & 15 & A02PF2 & 40700 & 16600 & 345 & 612 & 340 & 183 & 75300 & $<2330$ & 85.9 & 23200 \\
\hline SB7b-23 & 3 & 1 & 16 & A31PF1 & 48500 & 14300 & 240 & 578 & 197 & 225 & 58100 & $<2360$ & 79.3 & 28800 \\
\hline SB7b-23 & 3 & 1 & 17 & A31PF2 & 47900 & 14200 & 234 & 588 & 189 & 225 & 57600 & $<2340$ & 83.4 & 28900 \\
\hline SB7b-09 & 3 & 1 & 18 & A33PF1 & 52700 & 15800 & 93.4 & $<460$ & 90.9 & 195 & 76700 & $<2300$ & 73.3 & 31300 \\
\hline blank & 3 & 1 & 19 & BLANKPF3 & 1160 & $<162$ & 59.2 & $<482$ & $<81.6$ & $<50.4$ & 118 & $<2410$ & $<43.2$ & $<117$ \\
\hline SB7Ref & 3 & 1 & 20 & SB7REFPF33 & 47300 & 15200 & 187 & $<472$ & 869 & 386 & 61100 & 3460 & 73.5 & 24000 \\
\hline Ustd & 3 & 1 & 21 & USTDPF33 & 20700 & 26500 & $<41$ & $<476$ & 1680 & $<49.7$ & 93100 & 25700 & $<42.6$ & 14000 \\
\hline SB7Ref & 3 & 2 & 1 & SB7REFPF31 & 47900 & 15400 & 192 & $<472$ & 885 & 403 & 60700 & 3150 & 65.7 & 24200 \\
\hline Ustd & 3 & 2 & 2 & USTDPF31 & 21000 & 26800 & 42.6 & $<476$ & 1700 & $<49.7$ & 92400 & 24900 & $<42.6$ & 14000 \\
\hline SB7b-27 & 3 & 2 & 3 & A34PF2 & 39300 & 15400 & 259 & $<473$ & 261 & 175 & 73600 & $<2360$ & 122 & 28900 \\
\hline SB7b-31 & 3 & 2 & 4 & A02PF2 & 41100 & 16700 & 359 & $<467$ & 362 & 194 & 74600 & $<2330$ & 113 & 23400 \\
\hline SB7b-19 & 3 & 2 & 5 & A27PF2 & 48100 & 14400 & 539 & 802 & 492 & 307 & 58100 & $<2340$ & 157 & 24400 \\
\hline SB7b-27 & 3 & 2 & 6 & A34PF1 & 39000 & 15300 & 243 & $<478$ & 235 & 172 & 72900 & $<2380$ & 124 & 28900 \\
\hline SB7b-16 & 3 & 2 & 7 & A15PF2 & 48400 & 14500 & 533 & 644 & 426 & 369 & 71800 & $<2360$ & 172 & 29000 \\
\hline SB7b-16 & 3 & 2 & 8 & A15PF1 & 48100 & 14500 & 530 & 875 & 430 & 371 & 72000 & $<2350$ & 162 & 29100 \\
\hline SB7b-19 & 3 & 2 & 9 & A27PF1 & 47600 & 14500 & 535 & 895 & 493 & 313 & 58200 & $<2370$ & 152 & 24600 \\
\hline SB7Ref & 3 & 2 & 10 & SB7REFPF32 & 48500 & 15500 & 200 & $<472$ & 881 & 397 & 60900 & 3120 & 85.3 & 24400 \\
\hline Ustd & 3 & 2 & 11 & USTDPF32 & 21000 & 26900 & 41.8 & $<476$ & 1700 & $<49.7$ & 92200 & 25100 & $<42.6$ & 14200 \\
\hline SB7b-31 & 3 & 2 & 12 & A02PF1 & 41000 & 16700 & 353 & 555 & 349 & 172 & 74300 & $<2300$ & 108 & 23500 \\
\hline SB7b-09 & 3 & 2 & 13 & A33PF2 & 53000 & 15900 & 91 & $<477$ & 93 & 161 & 75600 & $<2380$ & 65.7 & 31700 \\
\hline SB7b-23 & 3 & 2 & 14 & A31PF2 & 48700 & 14400 & 244 & $<470$ & 182 & 219 & 56900 & $<2340$ & 101 & 29300 \\
\hline SB7b-21 & 3 & 2 & 15 & A08PF2 & 38800 & 15400 & 532 & 754 & 439 & 295 & 57900 & $<2290$ & 151 & 29200 \\
\hline SB7b-09 & 3 & 2 & 16 & A33PF1 & 53600 & 16000 & 99.9 & $<460$ & 88.2 & 177 & 75800 & $<2300$ & 61.8 & 31600 \\
\hline SB7b-21 & 3 & 2 & 17 & A08PF1 & 39000 & 15500 & 538 & 942 & 439 & 307 & 58300 & $<2310$ & 160 & 29400 \\
\hline SB7b-23 & 3 & 2 & 18 & A31PF1 & 49200 & 14500 & 248 & $<473$ & 202 & 229 & 57400 & $<2360$ & 82.5 & 29600 \\
\hline blank & 3 & 2 & 19 & BLANKPF3 & 1040 & $<162$ & 63.2 & $<482$ & $<81.6$ & $<50.4$ & 102 & $<2410$ & $<43.2$ & $<117$ \\
\hline SB7Ref & 3 & 2 & 20 & SB7REFPF33 & 48800 & 15400 & 195 & $<472$ & 888 & 401 & 60500 & 2980 & 78.2 & 24500 \\
\hline Ustd & 3 & 2 & 21 & USTDPF33 & 21300 & 26800 & 42.6 & $<476$ & 1710 & $<49.7$ & 92000 & 24700 & $<42.6$ & 14100 \\
\hline SB7Ref & 4 & 1 & 1 & SB7REFPF41 & 47900 & 15400 & 182 & $<478$ & 857 & 403 & 60500 & 3290 & 103 & 23800 \\
\hline Ustd & 4 & 1 & 2 & USTDPF41 & 21100 & 26900 & $<40.5$ & $<469$ & 1700 & 69.3 & 93600 & 25700 & $<42$ & 14000 \\
\hline SB7b-25 & 4 & 1 & 3 & A18PF1 & 39900 & 12800 & 517 & 773 & 413 & 327 & 64900 & $<2350$ & 122 & 28800 \\
\hline SB7b-13 & 4 & 1 & 4 & A07PF1 & 34900 & 14000 & 650 & 1200 & 602 & 1090 & 56300 & $<2400$ & 180 & 31100 \\
\hline SB7b-07 & 4 & 1 & 5 & A12PF2 & 52800 & 13800 & 94.3 & $<481$ & 98 & 63.8 & 52000 & $<2400$ & $<43$ & 31000 \\
\hline SB7b-03 & 4 & 1 & 6 & A17PF1 & 47700 & 14000 & 668 & 1040 & 1150 & 312 & 51800 & $<2340$ & 163 & 31000 \\
\hline SB7b-25 & 4 & 1 & 7 & A18PF2 & 39400 & 12700 & 506 & 706 & 417 & 308 & 64700 & $<2370$ & 150 & 29200 \\
\hline SB7b-13 & 4 & 1 & 8 & A07PF2 & 34500 & 13800 & 639 & 1300 & 602 & 1070 & 55400 & $<2320$ & 191 & 30800 \\
\hline SB7b-33 & 4 & 1 & 9 & A10PF1 & 43300 & 14800 & 350 & 508 & 312 & 196 & 65800 & $<2370$ & 103 & 29000 \\
\hline SB7Ref & 4 & 1 & 10 & SB7REFPF42 & 47600 & 15300 & 175 & $<478$ & 870 & 384 & 60200 & 3120 & 97.5 & 23900 \\
\hline Ustd & 4 & 1 & 11 & USTDPF42 & 21100 & 26900 & $<40.5$ & $<469$ & 1690 & 53.7 & 93700 & 25300 & $<42$ & 14200 \\
\hline SB7b-06 & 4 & 1 & 12 & A19PF1 & 53700 & 16200 & 639 & 1040 & 570 & 350 & 78400 & $<2370$ & 172 & 31400 \\
\hline SB7b-06 & 4 & 1 & 13 & A19PF2 & 53400 & 16200 & 632 & 970 & 574 & 361 & 78100 & $<2370$ & 183 & 31500 \\
\hline SB7b-03 & 4 & 1 & 14 & A17PF2 & 47100 & 13800 & 659 & 1080 & 1130 & 328 & 51000 & $<2320$ & 183 & 30900 \\
\hline SB7b-07 & 4 & 1 & 15 & A12PF1 & 52700 & 13900 & 93.6 & $<475$ & 112 & 70.1 & 52000 & $<2370$ & 47.3 & 31100 \\
\hline
\end{tabular}


Table A-3. Measured Elemental Concentrations (ug/g) for the Study Glasses Prepared Using Peroxide Fusion (part 1)

\begin{tabular}{|c|c|c|c|c|c|c|c|c|c|c|c|c|c|c|}
\hline Glass ID & Block & Sub-Blk & Sequence & Lab ID & Al & B & Ba & $\mathrm{Ce}$ & $\mathrm{Cr}$ & $\mathbf{C u}$ & $\mathrm{Fe}$ & $\mathbf{K}$ & $\mathbf{L a}$ & $\mathbf{L i}$ \\
\hline SB7b-33 & 4 & 1 & 16 & A10PF2 & 43500 & 14900 & 354 & 482 & 320 & 201 & 65800 & $<2310$ & 97.4 & 29200 \\
\hline SB7b-26 & 4 & 1 & 17 & A05PF1 & 48300 & 15300 & 517 & 662 & 391 & 262 & 70200 & $<2360$ & 127 & 24000 \\
\hline SB7b-26 & 4 & 1 & 18 & A05PF2 & 47900 & 15300 & 513 & 681 & 380 & 268 & 69800 & 2690 & 149 & 24000 \\
\hline blank & 4 & 1 & 19 & BLANKPF4 & 691 & $<162$ & 43.2 & $<482$ & $<81.6$ & $<50.4$ & $<76.8$ & $<2410$ & $<43.2$ & $<117$ \\
\hline SB7Ref & 4 & 1 & 20 & SB7REFPF43 & 47300 & 15200 & 180 & $<478$ & 858 & 391 & 60000 & 3480 & 88 & 24000 \\
\hline Ustd & 4 & 1 & 21 & USTDPF43 & 20400 & 26000 & $<40.5$ & $<469$ & 1640 & 53.7 & 90300 & 25200 & $<42$ & 13700 \\
\hline SB7Ref & 4 & 2 & 1 & SB7REFPF41 & 47500 & 15300 & 185 & $<478$ & 878 & 418 & 60400 & 3400 & 106 & 23900 \\
\hline Ustd & 4 & 2 & 2 & USTDPF41 & 21200 & 26700 & $<40.5$ & $<469$ & 1710 & 73.2 & 93600 & 25200 & $<42$ & 14100 \\
\hline SB7b-33 & 4 & 2 & 3 & A10PF2 & 43300 & 14900 & 362 & 499 & 315 & 222 & 65900 & $<2310$ & 95.1 & 29500 \\
\hline SB7b-06 & 4 & 2 & 4 & A19PF1 & 53700 & 16000 & 652 & 1000 & 581 & 363 & 78000 & 2290 & 168 & 31400 \\
\hline SB7b-25 & 4 & 2 & 5 & A18PF1 & 39600 & 12600 & 524 & 797 & 428 & 316 & 64500 & $<2350$ & 129 & 29100 \\
\hline SB7b-26 & 4 & 2 & 6 & A05PF2 & 48100 & 15300 & 530 & 604 & 395 & 274 & 70100 & 2370 & 148 & 24000 \\
\hline SB7b-33 & 4 & 2 & 7 & A10PF1 & 43100 & 14700 & 360 & 532 & 321 & 211 & 65700 & $<2370$ & 118 & 29200 \\
\hline SB7b-13 & 4 & 2 & 8 & A07PF1 & 34600 & 14000 & 665 & 961 & 611 & 1090 & 55900 & 2700 & 203 & 31500 \\
\hline SB7b-07 & 4 & 2 & 9 & A12PF1 & 52900 & 13900 & 93 & $<475$ & 108 & 67 & 52000 & $<2370$ & $<42.6$ & 31400 \\
\hline SB7Ref & 4 & 2 & 10 & SB7REFPF42 & 48100 & 15300 & 184 & $<478$ & 884 & 391 & 60100 & 3700 & 91.9 & 24300 \\
\hline Ustd & 4 & 2 & 11 & USTDPF42 & 21100 & 26800 & $<40.5$ & $<469$ & 1730 & 64.6 & 93400 & 25900 & $<42$ & 14200 \\
\hline SB7b-03 & 4 & 2 & 12 & A17PF2 & 47300 & 13900 & 675 & 1210 & 1150 & 335 & 51000 & 2360 & 180 & 31300 \\
\hline SB7b-13 & 4 & 2 & 13 & A07PF2 & 34300 & 13800 & 655 & 1140 & 619 & 1080 & 55300 & $<2320$ & 201 & 31100 \\
\hline SB7b-26 & 4 & 2 & 14 & A05PF1 & 48500 & 15400 & 535 & 726 & 397 & 274 & 70500 & 2460 & 142 & 24400 \\
\hline SB7b-03 & 4 & 2 & 15 & A17PF1 & 47600 & 13900 & 681 & 1140 & 1150 & 325 & 51400 & 2350 & 179 & 31400 \\
\hline SB7b-25 & 4 & 2 & 16 & A18PF2 & 39300 & 12700 & 523 & 819 & 430 & 305 & 64300 & 2510 & 147 & 29500 \\
\hline SB7b-07 & 4 & 2 & 17 & A12PF2 & 52800 & 13700 & 92.2 & $<481$ & 116 & 71.7 & 51500 & $<2400$ & 53.4 & 31300 \\
\hline SB7b-06 & 4 & 2 & 18 & A19PF2 & 53800 & 16200 & 648 & 917 & 578 & 361 & 78400 & 2290 & 194 & 32100 \\
\hline blank & 4 & 2 & 19 & BLANKPF4 & 554 & $<162$ & 44.8 & $<482$ & $<81.6$ & $<50.4$ & $<76.8$ & $<2410$ & $<43.2$ & $<117$ \\
\hline SB7Ref & 4 & 2 & 20 & SB7REFPF43 & 47600 & 15200 & 185 & $<478$ & 880 & 398 & 59900 & 3510 & 102 & 24000 \\
\hline Ustd & 4 & 2 & 21 & USTDPF43 & 20500 & 25900 & $<40.5$ & $<469$ & 1660 & $<49$ & 90100 & 25500 & $<42$ & 13800 \\
\hline SB7Ref & 5 & 1 & 1 & SB7REFPF51 & 47000 & 15200 & 182 & $<478$ & 851 & 410 & 60300 & 3440 & 92 & 24100 \\
\hline Ustd & 5 & 1 & 2 & USTDPF51 & 20800 & 26600 & $<40.6$ & $<471$ & 1700 & 56.2 & 93000 & 25300 & $<42.2$ & 14100 \\
\hline SB7b-15 & 5 & 1 & 3 & A16PF1 & 38200 & 14400 & 238 & $<471$ & 221 & 382 & 59500 & 2420 & 80.5 & 24500 \\
\hline SB7b-10 & 5 & 1 & 4 & A06PF1 & 51900 & 15600 & 114 & $<470$ & 97.9 & 139 & 50900 & 2450 & 75.7 & 22100 \\
\hline SB7b-10 & 5 & 1 & 5 & A06PF2 & 51800 & 15600 & 109 & $<468$ & 87.3 & 118 & 50900 & $<2340$ & 66.8 & 22000 \\
\hline SB7b-32 & 5 & 1 & 6 & A29PF2 & 43200 & 13100 & 377 & 485 & 279 & 195 & 55000 & 2830 & 111 & 29600 \\
\hline SB7b-02 & 5 & 1 & 7 & A01PF2 & 33500 & 16200 & 622 & 668 & 559 & 245 & 79800 & 2610 & 157 & 22300 \\
\hline SB7b-02 & 5 & 1 & 8 & A01PF1 & 33600 & 16200 & 632 & 1100 & 562 & 266 & 80100 & 2380 & 185 & 22400 \\
\hline SB7b-22 & 5 & 1 & 9 & A14PF2 & 38600 & 14400 & 248 & $<480$ & 92 & 213 & 68300 & 2970 & 73.2 & 29400 \\
\hline SB7Ref & 5 & 1 & 10 & SB7REFPF52 & 47000 & 15200 & 187 & $<478$ & 850 & 390 & 60500 & 3820 & 87.3 & 24300 \\
\hline Ustd & 5 & 1 & 11 & USTDPF52 & 20500 & 26100 & $<40.6$ & $<471$ & 1640 & $<49.2$ & 91600 & 25200 & $<42.2$ & 13900 \\
\hline SB7b-28 & 5 & 1 & 12 & A28PF1 & 37900 & 15300 & 233 & $<463$ & 213 & 176 & 72400 & 2770 & 74.4 & 24500 \\
\hline SB7b-22 & 5 & 1 & 13 & A14PF1 & 38300 & 14400 & 246 & $<470$ & 106 & 190 & 68500 & 2560 & 88.1 & 29400 \\
\hline SB7b-28 & 5 & 1 & 14 & A28PF2 & 38300 & 15300 & 235 & $<468$ & 207 & 182 & 73200 & 2630 & 75.3 & 24700 \\
\hline SB7b-32 & 5 & 1 & 15 & A29PF1 & 43800 & 13200 & 381 & $<466$ & 283 & 191 & 55200 & 2380 & 114 & 30100 \\
\hline SB7b-15 & 5 & 1 & 16 & A16PF2 & 38100 & 14200 & 238 & $<481$ & 198 & 371 & 58600 & 2570 & 61.5 & 24400 \\
\hline blank & 5 & 1 & 17 & BLANKPF5 & 754 & $<162$ & 52.8 & $<482$ & $<81.6$ & $<50.4$ & $<76.8$ & $<2410$ & $<43.2$ & $<117$ \\
\hline SB7Ref & 5 & 1 & 18 & SB7REFPF53 & 47100 & 15000 & 181 & $<478$ & 828 & 373 & 59800 & 3790 & 92.8 & 24300 \\
\hline Ustd & 5 & 1 & 19 & USTDPF53 & 20700 & 26600 & $<40.6$ & $<471$ & 1680 & $<49.2$ & 92600 & 25700 & $<42.2$ & 14400 \\
\hline SB7Ref & 5 & 2 & 1 & SB7REFPF51 & 47800 & 15300 & 184 & $<478$ & 851 & 414 & 60300 & 3400 & 94.4 & 23900 \\
\hline
\end{tabular}


Table A-3. Measured Elemental Concentrations (ug/g) for the Study Glasses Prepared Using Peroxide Fusion (part 1)

\begin{tabular}{|c|c|c|c|c|c|c|c|c|c|c|c|c|c|c|}
\hline Glass ID & Block & Sub-Blk & Sequence & Lab ID & Al & B & $\mathbf{B a}$ & $\mathbf{C e}$ & $\mathrm{Cr}$ & $\mathbf{C u}$ & $\mathrm{Fe}$ & $\mathbf{K}$ & $\mathbf{L a}$ & $\mathbf{L i}$ \\
\hline Ustd & 5 & 2 & 2 & USTDPF51 & 21100 & 26900 & $<40.6$ & $<471$ & 1710 & 65.6 & 93300 & 25500 & $<42.2$ & 14000 \\
\hline SB7b-15 & 5 & 2 & 3 & A16PF2 & 38500 & 14500 & 244 & $<481$ & 210 & 398 & 58700 & $<2400$ & 75.8 & 24200 \\
\hline SB7b-22 & 5 & 2 & 4 & A14PF2 & 39200 & 14500 & 254 & $<480$ & 104 & 214 & 68400 & $<2390$ & 70.9 & 29200 \\
\hline SB7b-10 & 5 & 2 & 5 & A06PF1 & 52500 & 15700 & 112 & $<470$ & 101 & 148 & 50800 & $<2350$ & 79.6 & 22000 \\
\hline SB7b-28 & 5 & 2 & 6 & A28PF2 & 39000 & 15500 & 239 & $<468$ & 235 & 206 & 72800 & $<2330$ & 81.5 & 24500 \\
\hline SB7b-02 & 5 & 2 & 7 & A01PF2 & 34100 & 16300 & 634 & 812 & 555 & 260 & 80100 & $<2350$ & 178 & 22100 \\
\hline SB7b-32 & 5 & 2 & 8 & A29PF1 & 44800 & 13400 & 389 & 497 & 283 & 207 & 56400 & $<2320$ & 112 & 29800 \\
\hline SB7b-15 & 5 & 2 & 9 & A16PF1 & 38800 & 14500 & 244 & $<471$ & 215 & 392 & 59700 & $<2350$ & 85.9 & 24300 \\
\hline SB7Ref & 5 & 2 & 10 & SB7REFPF52 & 47600 & 15300 & 184 & $<478$ & 862 & 409 & 60200 & 3360 & 93.6 & 23900 \\
\hline Ustd & 5 & 2 & 11 & USTDPF52 & 20600 & 26500 & $<40.6$ & $<471$ & 1680 & 53.9 & 92500 & 25200 & $<42.2$ & 13800 \\
\hline SB7b-28 & 5 & 2 & 12 & A28PF1 & 38700 & 15500 & 237 & $<463$ & 208 & 193 & 72900 & $<2310$ & 79.8 & 24200 \\
\hline SB7b-02 & 5 & 2 & 13 & A01PF1 & 34100 & 16500 & 643 & 1030 & 554 & 271 & 80400 & $<2360$ & 169 & 22200 \\
\hline SB7b-22 & 5 & 2 & 14 & A14PF1 & 39000 & 14600 & 245 & $<470$ & 97.3 & 182 & 68900 & $<2350$ & 84.2 & 29100 \\
\hline SB7b-32 & 5 & 2 & 15 & A29PF2 & 43900 & 13200 & 379 & $<462$ & 286 & 192 & 55300 & $<2310$ & 112 & 29500 \\
\hline SB7b-10 & 5 & 2 & 16 & A06PF2 & 52800 & 15700 & 106 & $<468$ & 91.6 & 110 & 50900 & $<2340$ & 75.3 & 21900 \\
\hline blank & 5 & 2 & 17 & BLANKPF5 & 958 & $<162$ & 52.8 & $<482$ & $<81.6$ & $<50.4$ & $<76.8$ & $<2410$ & $<43.2$ & $<117$ \\
\hline SB7Ref & 5 & 2 & 18 & SB7REFPF53 & 47700 & 15200 & 183 & $<478$ & 853 & 405 & 60200 & 3520 & 102 & 24000 \\
\hline Ustd & 5 & 2 & 19 & USTDPF53 & 21000 & 26800 & $<40.6$ & $<471$ & 1690 & $<49.2$ & 93100 & 25000 & $<42.2$ & 14200 \\
\hline
\end{tabular}


Table A-4. Measured Elemental Concentrations (ug/g) for the Study Glasses Prepared Using Peroxide Fusion (part 2)

\begin{tabular}{|c|c|c|c|c|c|c|c|c|c|c|c|c|c|c|}
\hline Glass ID & Block & Sub-Blk & Sequence & Lab ID & Mg & Mn & $\mathrm{Ni}$ & $\mathbf{P b}$ & $\mathrm{S}$ & $\mathrm{Si}$ & Th & Ti & $\mathbf{U}$ & $\mathrm{Zn}$ \\
\hline SB7Ref & 1 & 1 & 1 & SB7REFPF11 & 1580 & 16000 & 9540 & $<568$ & $<5950$ & 216000 & $<434$ & 2820 & $<2590$ & 197 \\
\hline Ustd & 1 & 1 & 2 & USTDPF11 & 7190 & 21200 & 8550 & $<564$ & $<5910$ & 208000 & $<431$ & 5610 & 19000 & 110 \\
\hline SB7b-05 & 1 & 1 & 3 & A11PF1 & 2390 & 11500 & 9210 & $<557$ & $<5830$ & 245000 & 2770 & 80.8 & 26300 & 206 \\
\hline SB7b-18 & 1 & 1 & 4 & A24PF1 & 1900 & 16600 & 14100 & $<557$ & $<5840$ & 226000 & 5880 & 3190 & 19700 & 215 \\
\hline SB7b-29 & 1 & 1 & 5 & A21PF2 & 1140 & 12700 & 10800 & $<558$ & $<5850$ & 238000 & 4260 & 1200 & 19600 & 154 \\
\hline SB7b-12 & 1 & 1 & 6 & A23PF1 & 366 & 18200 & 15400 & $<567$ & $<5940$ & 248000 & 2800 & 4310 & 17200 & 170 \\
\hline SB7b-08 & 1 & 1 & 7 & A22PF2 & 383 & 18200 & 9180 & $<564$ & $<5900$ & 219000 & 2880 & 83.9 & 27300 & 117 \\
\hline SB7b-12 & 1 & 1 & 8 & A23PF2 & 371 & 18300 & 15600 & $<547$ & $<5730$ & 246000 & 2880 & 4310 & 17900 & 183 \\
\hline SB7b-08 & 1 & 1 & 9 & A22PF1 & 355 & 18100 & 9130 & $<549$ & $<5750$ & 220000 & 2520 & 93.9 & 26200 & 123 \\
\hline SB7Ref & 1 & 1 & 10 & SB7REFPF12 & 1580 & 16000 & 9550 & $<568$ & $<5950$ & 239000 & $<434$ & 2790 & $<2590$ & 188 \\
\hline Ustd & 1 & 1 & 11 & USTDPF12 & 7160 & 21000 & 8490 & $<564$ & $<5910$ & 211000 & $<431$ & 5550 & 18800 & 89 \\
\hline SB7b-11 & 1 & 1 & 12 & A20PF1 & 2460 & 11500 & 8850 & $<567$ & $<5940$ & 217000 & 7260 & 4230 & 17300 & 198 \\
\hline SB7b-24 & 1 & 1 & 13 & A26PF2 & 844 & 15800 & 10100 & $<563$ & $<5900$ & 215000 & 3520 & 2980 & 19000 & 145 \\
\hline SB7b-11 & 1 & 1 & 14 & A20PF2 & 2390 & 11400 & 8870 & $<559$ & $<5850$ & 215000 & 6710 & 4200 & 17100 & 204 \\
\hline SB7b-05 & 1 & 1 & 15 & A11PF2 & 2450 & 11400 & 9170 & $<556$ & $<5830$ & 243000 & 2940 & 70.7 & 27200 & 203 \\
\hline SB7b-29 & 1 & 1 & 16 & A21PF1 & 1120 & 12900 & 10800 & $<569$ & $<5960$ & 243000 & 4170 & 1270 & 19200 & 167 \\
\hline SB7b-18 & 1 & 1 & 17 & A24PF2 & 1910 & 16400 & 14000 & $<554$ & $<5800$ & 222000 & 6050 & 3180 & 19700 & 213 \\
\hline SB7b-24 & 1 & 1 & 18 & A26PF1 & 835 & 16400 & 10500 & $<569$ & $<5960$ & 222000 & 3550 & 3160 & 19100 & 154 \\
\hline blank & 1 & 1 & 19 & BLANKPF1 & 63.2 & $<42.4$ & $<364$ & $<573$ & $<6000$ & 904 & $<438$ & 80 & $<2610$ & $<52.8$ \\
\hline SB7Ref & 1 & 1 & 20 & SB7REFPF13 & 1590 & 16200 & 9730 & $<568$ & $<5950$ & 238000 & $<434$ & 2820 & $<2590$ & 205 \\
\hline Ustd & 1 & 1 & 21 & USTDPF13 & 7310 & 21600 & 8670 & $<564$ & $<5910$ & 215000 & $<431$ & 5660 & 18100 & 104 \\
\hline SB7Ref & 1 & 2 & 1 & SB7REFPF11 & 1580 & 16000 & 9570 & $<568$ & $<5950$ & 227000 & $<434$ & 2830 & $<2590$ & 201 \\
\hline Ustd & 1 & 2 & 2 & USTDPF11 & 7210 & 21200 & 8610 & $<564$ & $<5910$ & 204000 & $<431$ & 5680 & 18200 & 101 \\
\hline SB7b-05 & 1 & 2 & 3 & A11PF2 & 2450 & 11500 & 9240 & $<556$ & $<5830$ & 239000 & 2920 & 61 & 26300 & 200 \\
\hline SB7b-08 & 1 & 2 & 4 & A22PF2 & 390 & 18300 & 9230 & $<564$ & $<5900$ & 217000 & 2760 & 66.1 & 26700 & 116 \\
\hline SB7b-18 & 1 & 2 & 5 & A24PF2 & 1920 & 16600 & 14200 & $<554$ & $<5800$ & 222000 & 5930 & 3220 & 19200 & 211 \\
\hline SB7b-11 & 1 & 2 & 6 & A20PF2 & 2410 & 11500 & 8950 & $<559$ & $<5850$ & 217000 & 6690 & 4280 & 16500 & 194 \\
\hline SB7b-24 & 1 & 2 & 7 & A26PF1 & 843 & 16500 & 10600 & $<569$ & $<5960$ & 222000 & 3530 & 3170 & 18400 & 151 \\
\hline SB7b-29 & 1 & 2 & 8 & A21PF2 & 1130 & 12600 & 10700 & $<558$ & $<5850$ & 234000 & 4120 & 1210 & 18900 & 155 \\
\hline SB7b-08 & 1 & 2 & 9 & A22PF1 & 358 & 18100 & 9200 & $<549$ & $<5750$ & 217000 & 2480 & 61.7 & 25700 & 115 \\
\hline SB7Ref & 1 & 2 & 10 & SB7REFPF12 & 1570 & 16000 & 9610 & $<568$ & $<5950$ & 235000 & $<434$ & 2820 & $<2590$ & 194 \\
\hline Ustd & 1 & 2 & 11 & USTDPF12 & 7190 & 21100 & 8540 & $<564$ & $<5910$ & 211000 & $<431$ & 5660 & 18500 & 103 \\
\hline SB7b-12 & 1 & 2 & 12 & A23PF1 & 369 & 18100 & 15500 & $<567$ & $<5940$ & 244000 & 2820 & 4310 & 16900 & 166 \\
\hline SB7b-18 & 1 & 2 & 13 & A24PF1 & 1900 & 16500 & 14100 & $<557$ & $<5840$ & 224000 & 5790 & 3180 & 19300 & 214 \\
\hline SB7b-24 & 1 & 2 & 14 & A26PF2 & 858 & 15800 & 10200 & $<563$ & $<5900$ & 216000 & 3480 & 3050 & 17700 & 158 \\
\hline SB7b-29 & 1 & 2 & 15 & A21PF1 & 1140 & 12800 & 10900 & $<569$ & $<5960$ & 241000 & 4200 & 1270 & 19300 & 164 \\
\hline SB7b-12 & 1 & 2 & 16 & A23PF2 & 373 & 18200 & 15600 & $<547$ & $<5730$ & 242000 & 2820 & 4320 & 17700 & 171 \\
\hline SB7b-11 & 1 & 2 & 17 & A20PF1 & 2470 & 11500 & 9020 & $<567$ & $<5940$ & 218000 & 7300 & 4290 & 17200 & 190 \\
\hline SB7b-05 & 1 & 2 & 18 & A11PF1 & 2390 & 11400 & 9170 & $<557$ & $<5830$ & 241000 & 2660 & 47.8 & 25600 & 207 \\
\hline blank & 1 & 2 & 19 & BLANKPF1 & 76.8 & $<42.4$ & $<364$ & $<573$ & $<6000$ & 999 & $<438$ & $<36$ & $<2610$ & $<52.8$ \\
\hline SB7Ref & 1 & 2 & 20 & SB7REFPF13 & 1570 & 15900 & 9630 & $<568$ & $<5950$ & 233000 & $<434$ & 2820 & $<2590$ & 187 \\
\hline Ustd & 1 & 2 & 21 & USTDPF13 & 7150 & 21000 & 8420 & $<564$ & $<5910$ & 209000 & $<431$ & 5610 & 18300 & 93.7 \\
\hline SB7Ref & 2 & 1 & 1 & SB7REFPF21 & 1560 & 16300 & 9650 & $<562$ & $<5890$ & 223000 & $<430$ & 2840 & $<2560$ & 196 \\
\hline Ustd & 2 & 1 & $\frac{1}{2}$ & USTDPF21 & 7170 & 21500 & 8580 & $<552$ & $<5780$ & 212000 & $<423$ & 5760 & 17700 & 94.8 \\
\hline SB7b-20 & 2 & 1 & 3 & A03PF1 & 845 & 16300 & 13400 & $<544$ & $<5700$ & 235000 & 3830 & 1030 & 18800 & 173 \\
\hline SB7b-30 & 2 & 1 & 4 & A13PF2 & 1230 & 14700 & 12200 & $<561$ & $<5880$ & 235000 & 4050 & 1400 & 19700 & 164 \\
\hline SB7b-01 & 2 & 1 & 5 & A04PF1 & 382 & 11600 & 15500 & $<552$ & $<5780$ & 226000 & 6840 & 71.7 & 17000 & 143 \\
\hline
\end{tabular}


Table A-4. Measured Elemental Concentrations (ug/g) for the Study Glasses Prepared Using Peroxide Fusion (part 2)

\begin{tabular}{|c|c|c|c|c|c|c|c|c|c|c|c|c|c|c|}
\hline Glass ID & Block & Sub-Blk & Sequence & Lab ID & Mg & Mn & $\mathrm{Ni}$ & $\mathbf{P b}$ & $\mathrm{S}$ & $\mathrm{Si}$ & Th & $\mathrm{Ti}$ & $\mathbf{U}$ & Zn \\
\hline SB7b-30 & 2 & 1 & 6 & A13PF1 & 1250 & 14700 & 12200 & $<558$ & $<5840$ & 237000 & 4700 & 1410 & 21400 & 166 \\
\hline SB7b-17 & 2 & 1 & 7 & A25PF2 & 1900 & 13300 & 14100 & $<559$ & $<5850$ & 244000 & 3570 & 3260 & 18600 & 252 \\
\hline SB7b-14 & 2 & 1 & 8 & A09PF1 & 353 & 18400 & 9260 & $<549$ & $<5750$ & 246000 & 6960 & 4380 & 17100 & 195 \\
\hline SB7b-04 & 2 & 1 & 9 & A32PF1 & 346 & 18400 & 9140 & $<558$ & $<5840$ & 246000 & 6890 & 88.6 & 25900 & 139 \\
\hline SB7Ref & 2 & 1 & 10 & SB7REFPF22 & 1550 & 16200 & 9700 & $<562$ & $<5890$ & 244000 & $<430$ & 2850 & $<2560$ & 194 \\
\hline Ustd & 2 & 1 & 11 & USTDPF22 & 7180 & 21500 & 8600 & $<552$ & $<5780$ & 217000 & $<423$ & 5830 & 17700 & 103 \\
\hline SB7b-01 & 2 & 1 & 12 & A04PF2 & 384 & 11700 & 15600 & $<572$ & $<5990$ & 230000 & 6670 & 76.3 & 16600 & 141 \\
\hline SB7b-14 & 2 & 1 & 13 & A09PF2 & 375 & 18300 & 9240 & $<557$ & $<5840$ & 248000 & 6240 & 4370 & 16400 & 189 \\
\hline SB7b-20 & 2 & 1 & 14 & A03PF2 & 868 & 16700 & 13700 & $<564$ & $<5910$ & 246000 & 3550 & 1120 & 18400 & 176 \\
\hline SB7b-34 & 2 & 1 & 15 & A30PF1 & 1380 & 16600 & 13600 & $<557$ & $<5830$ & 221000 & 5220 & 1620 & 23800 & 194 \\
\hline SB7b-34 & 2 & 1 & 16 & A30PF2 & 1400 & 16300 & 13400 & $<544$ & $<5700$ & 217000 & 4600 & 1550 & 22400 & 204 \\
\hline SB7b-04 & 2 & 1 & 17 & A32PF2 & 416 & 18400 & 9100 & $<573$ & $<6000$ & 251000 & 6640 & 97.2 & 25800 & 138 \\
\hline SB7b-17 & 2 & 1 & 18 & A25PF1 & 1910 & 13400 & 14200 & $<567$ & $<5940$ & 241000 & 3820 & 3290 & 19500 & 252 \\
\hline blank & 2 & 1 & 19 & BLANKPF2 & 68.8 & $<42.4$ & $<364$ & $<573$ & $<6000$ & 966 & $<438$ & 50.4 & $<2610$ & $<52.8$ \\
\hline SB7Ref & 2 & 1 & 20 & SB7REFPF23 & 1540 & 16100 & 9570 & $<562$ & $<5890$ & 241000 & $<430$ & 2870 & $<2560$ & 191 \\
\hline Ustd & 2 & 1 & 21 & USTDPF23 & 7170 & 21500 & 8570 & $<552$ & $<5780$ & 216000 & $<423$ & 5820 & 17700 & 94.8 \\
\hline SB7Ref & 2 & 2 & 1 & SB7REFPF21 & 1560 & 16300 & 9680 & $<562$ & $<5890$ & 227000 & $<430$ & 2820 & $<2560$ & 192 \\
\hline Ustd & 2 & 2 & 2 & USTDPF21 & 7170 & 21600 & 8690 & $<552$ & $<5780$ & 213000 & $<423$ & 5750 & 17800 & 103 \\
\hline SB7b-20 & 2 & 2 & 3 & A03PF1 & 848 & 16400 & 13500 & $<544$ & 7440 & 235000 & 3780 & 1060 & 19600 & 170 \\
\hline SB7b-14 & 2 & 2 & 4 & A09PF2 & 375 & 18400 & 9320 & $<557$ & $<5840$ & 246000 & 6290 & 4330 & 16500 & 186 \\
\hline SB7b-01 & 2 & 2 & 5 & A04PF2 & 385 & 11800 & 15700 & $<572$ & $<5990$ & 229000 & 6540 & 80.6 & 16300 & 151 \\
\hline SB7b-14 & 2 & 2 & 6 & A09PF1 & 355 & 18600 & 9350 & $<549$ & $<5750$ & 246000 & 6840 & 4380 & 17300 & 197 \\
\hline SB7b-04 & 2 & 2 & 7 & A32PF2 & 420 & 18600 & 9320 & $<573$ & $<6000$ & 253000 & 6590 & 95.1 & 25500 & 143 \\
\hline SB7b-34 & 2 & 2 & 8 & A30PF1 & 1390 & 16800 & 13800 & $<557$ & $<5830$ & 221000 & 5200 & 1630 & 24300 & 208 \\
\hline SB7b-30 & 2 & 2 & 9 & A13PF1 & 1250 & 14800 & 12300 & $<558$ & $<5840$ & 237000 & 4590 & 1430 & 21700 & 175 \\
\hline SB7Ref & 2 & 2 & 10 & SB7REFPF22 & 1560 & 16400 & 9760 & $<562$ & $<5890$ & 243000 & $<430$ & 2860 & $<2560$ & 194 \\
\hline Ustd & 2 & 2 & 11 & USTDPF22 & 7190 & 21700 & 8700 & $<552$ & $<5780$ & 215000 & $<423$ & 5830 & 18300 & 102 \\
\hline SB7b-34 & 2 & 2 & 12 & A30PF2 & 1420 & 16500 & 13500 & $<544$ & $<5700$ & 216000 & 4520 & 1560 & 22600 & 211 \\
\hline SB7b-01 & 2 & 2 & 13 & A04PF1 & 384 & 11700 & 15600 & $<552$ & 6360 & 227000 & 6670 & 85.1 & 17000 & 147 \\
\hline SB7b-04 & 2 & 2 & 14 & A32PF1 & 350 & 18600 & 9240 & $<558$ & $<5840$ & 247000 & 6780 & 101 & 26800 & 146 \\
\hline SB7b-30 & 2 & 2 & 15 & A13PF2 & 1230 & 14900 & 12400 & $<561$ & $<5880$ & 237000 & 4030 & 1430 & 19900 & 176 \\
\hline SB7b-17 & 2 & 2 & 16 & A25PF2 & 1900 & 13400 & 14200 & $<559$ & $<5850$ & 242000 & 3500 & 3280 & 18700 & 249 \\
\hline SB7b-20 & 2 & 2 & 17 & A03PF2 & 873 & 16900 & 13900 & $<564$ & $<5910$ & 248000 & 3450 & 1130 & 18500 & 180 \\
\hline SB7b-17 & 2 & 2 & 18 & A25PF1 & 1920 & 13600 & 14300 & $<567$ & $<5940$ & 241000 & 3750 & 3310 & 19100 & 264 \\
\hline blank & 2 & 2 & 19 & BLANKPF2 & 69.6 & $<42.4$ & $<364$ & $<573$ & $<6000$ & 1090 & $<438$ & 101 & $<2610$ & $<52.8$ \\
\hline SB7Ref & 2 & 2 & 20 & SB7REFPF23 & 1550 & 16300 & 9730 & $<562$ & 7140 & 239000 & $<430$ & 2870 & $<2560$ & 199 \\
\hline Ustd & 2 & 2 & 21 & USTDPF23 & 7190 & 21700 & 8710 & $<552$ & $<5780$ & 215000 & $<423$ & 5840 & 17700 & 96.4 \\
\hline SB7Ref & 3 & 1 & 1 & SB7REFPF31 & 1510 & 16100 & 9610 & $<560$ & $<5870$ & 231000 & $<429$ & 2810 & $<2550$ & 196 \\
\hline Ustd & 3 & 1 & 2 & USTDPF31 & 7290 & 21500 & 8570 & $<565$ & $<5920$ & 213000 & $<433$ & 5670 & 18000 & 87.6 \\
\hline SB7b-27 & 3 & 1 & 3 & A34PF2 & 890 & 13300 & 14100 & $<562$ & $<5890$ & 216000 & 5880 & 1020 & 19100 & 192 \\
\hline SB7b-09 & 3 & 1 & 4 & A33PF2 & 346 & 11600 & 15100 & $<567$ & $<5940$ & 223000 & 7120 & 78.8 & 26500 & 155 \\
\hline SB7b-19 & 3 & 1 & 5 & A27PF1 & 1940 & 13400 & 10800 & $<565$ & $<5920$ & 226000 & 5980 & 1120 & 19200 & 193 \\
\hline SB7b-16 & 3 & 1 & 6 & A15PF1 & 1910 & 13200 & 10900 & $<559$ & $<5850$ & 224000 & 3760 & 3180 & 23700 & 235 \\
\hline SB7b-27 & 3 & 1 & 7 & A34PF1 & 817 & 13100 & 14000 & $<567$ & $<5940$ & 221000 & 4980 & 1060 & 17700 & 181 \\
\hline SB7b-21 & 3 & 1 & 8 & A08PF1 & 1920 & 16600 & 10800 & $<549$ & $<5750$ & 230000 & 5980 & 1110 & 24100 & 191 \\
\hline SB7b-19 & 3 & 1 & 9 & A27PF2 & 1880 & 13300 & 10700 & $<557$ & $<5840$ & 228000 & 5200 & 1070 & 18500 & 198 \\
\hline SB7Ref & 3 & 1 & 10 & SB7REFPF32 & 1520 & 16100 & 9600 & $<560$ & $<5870$ & 242000 & $<429$ & 2780 & $<2550$ & 195 \\
\hline
\end{tabular}


Table A-4. Measured Elemental Concentrations (ug/g) for the Study Glasses Prepared Using Peroxide Fusion (part 2)

\begin{tabular}{|c|c|c|c|c|c|c|c|c|c|c|c|c|c|c|}
\hline Glass ID & Block & Sub-Blk & Sequence & Lab ID & Mg & Mn & $\mathbf{N i}$ & $\mathbf{P b}$ & $\mathrm{S}$ & $\mathrm{Si}$ & Th & Ti & $\mathbf{U}$ & Zn \\
\hline Ustd & 3 & 1 & 11 & USTDPF32 & 7270 & 21400 & 8580 & $<565$ & $<5920$ & 213000 & $<433$ & 5650 & 17600 & 88.4 \\
\hline SB7b-21 & 3 & 1 & 12 & A08PF2 & 1890 & 16400 & 10700 & $<546$ & $<5720$ & 223000 & 5440 & 1090 & 22600 & 210 \\
\hline SB7b-31 & 3 & 1 & 13 & A02PF1 & 1210 & 13900 & 14100 & $<546$ & $<5720$ & 230000 & 4940 & 1340 & 24800 & 193 \\
\hline SB7b-16 & 3 & 1 & 14 & A15PF2 & 1880 & 13100 & 10900 & $<562$ & $<5890$ & 223000 & 3470 & 3170 & 22300 & 239 \\
\hline SB7b-31 & 3 & 1 & 15 & A02PF2 & 1150 & 13900 & 14000 & $<554$ & $<5810$ & 234000 & 4980 & 1300 & 24100 & 213 \\
\hline SB7b-23 & 3 & 1 & 16 & A31PF1 & 899 & 13300 & 13800 & $<562$ & $<5890$ & 230000 & 6050 & 3220 & 24200 & 183 \\
\hline SB7b-23 & 3 & 1 & 17 & A31PF2 & 865 & 13200 & 13700 & $<558$ & $<5850$ & 235000 & 5420 & 3210 & 22900 & 181 \\
\hline SB7b-09 & 3 & 1 & 18 & A33PF1 & 325 & 11600 & 15100 & $<547$ & $<5730$ & 219000 & 6970 & 71 & 26600 & 144 \\
\hline blank & 3 & 1 & 19 & BLANKPF3 & 14.4 & $<42.4$ & $<364$ & $<573$ & $<6000$ & $<782$ & $<438$ & 60 & $<2610$ & $<52.8$ \\
\hline SB7Ref & 3 & 1 & 20 & SB7REFPF33 & 1510 & 16000 & 9560 & $<560$ & $<5870$ & 239000 & $<429$ & 2770 & $<2550$ & 176 \\
\hline Ustd & 3 & 1 & 21 & USTDPF33 & 7300 & 21300 & 8560 & $<565$ & $<5920$ & 211000 & $<433$ & 5620 & 17700 & 88.4 \\
\hline SB7Ref & 3 & 2 & 1 & SB7REFPF31 & 1480 & 16200 & 9550 & $<560$ & $<5870$ & 231000 & $<429$ & 2840 & $<2550$ & 189 \\
\hline Ustd & 3 & 2 & 2 & USTDPF31 & 7230 & 21600 & 8520 & $<565$ & $<5920$ & 210000 & $<433$ & 5780 & 18300 & 93.9 \\
\hline SB7b-27 & 3 & 2 & 3 & A34PF2 & 879 & 13400 & 14100 & $<562$ & $<5890$ & 215000 & 5920 & 1040 & 20100 & 192 \\
\hline SB7b-31 & 3 & 2 & 4 & A02PF2 & 1130 & 14100 & 14100 & $<554$ & $<5810$ & 236000 & 5000 & 1340 & 24700 & 217 \\
\hline SB7b-19 & 3 & 2 & 5 & A27PF2 & 1860 & 13500 & 10700 & $<557$ & $<5840$ & 229000 & 5210 & 1110 & 18500 & 195 \\
\hline SB7b-27 & 3 & 2 & 6 & A34PF1 & 813 & 13300 & 14000 & $<567$ & $<5940$ & 222000 & 5020 & 1110 & 18300 & 189 \\
\hline SB7b-16 & 3 & 2 & 7 & A15PF2 & 1860 & 13400 & 10800 & $<562$ & $<5890$ & 222000 & 3480 & 3270 & 22900 & 239 \\
\hline SB7b-16 & 3 & 2 & 8 & A15PF1 & 1890 & 13400 & 10800 & $<559$ & $<5850$ & 227000 & 3790 & 3280 & 24400 & 230 \\
\hline SB7b-19 & 3 & 2 & 9 & A27PF1 & 1920 & 13500 & 10700 & $<565$ & $<5920$ & 224000 & 5990 & 1140 & 19700 & 200 \\
\hline SB7Ref & 3 & 2 & 10 & SB7REFPF32 & 1500 & 16300 & 9660 & $<560$ & $<5870$ & 242000 & $<429$ & 2890 & $<2550$ & 210 \\
\hline Ustd & 3 & 2 & 11 & USTDPF32 & 7210 & 21600 & 8580 & $<565$ & $<5920$ & 213000 & $<433$ & 5830 & 18600 & 82.9 \\
\hline SB7b-31 & 3 & 2 & 12 & A02PF1 & 1190 & 14100 & 14100 & $<546$ & $<5720$ & 230000 & 5010 & 1380 & 26000 & 198 \\
\hline SB7b-09 & 3 & 2 & 13 & A33PF2 & 342 & 11700 & 15100 & $<567$ & $<5940$ & 224000 & 7180 & 90.5 & 27700 & 155 \\
\hline SB7b-23 & 3 & 2 & 14 & A31PF2 & 851 & 13400 & 13700 & $<558$ & $<5850$ & 237000 & 5490 & 3350 & 23500 & 192 \\
\hline SB7b-21 & 3 & 2 & 15 & A08PF2 & 1860 & 16600 & 10700 & $<546$ & $<5720$ & 223000 & 5570 & 1150 & 23700 & 201 \\
\hline SB7b-09 & 3 & 2 & 16 & A33PF1 & 323 & 11700 & 15100 & $<547$ & $<5730$ & 219000 & 6940 & 90.1 & 27200 & 149 \\
\hline SB7b-21 & 3 & 2 & 17 & A08PF1 & 1890 & 16700 & 10800 & $<549$ & $<5750$ & 227000 & 5990 & 1150 & 24500 & 188 \\
\hline SB7b-23 & 3 & 2 & 18 & A31PF1 & 891 & 13400 & 13800 & $<562$ & $<5890$ & 231000 & 6200 & 3360 & 25100 & 184 \\
\hline blank & 3 & 2 & 19 & BLANKPF3 & 15.2 & $<42.4$ & $<364$ & $<573$ & $<6000$ & $<782$ & $<438$ & 92.8 & $<2610$ & $<52.8$ \\
\hline SB7Ref & 3 & 2 & 20 & SB7REFPF33 & 1480 & 16300 & 9590 & $<560$ & $<5870$ & 241000 & $<429$ & 2910 & $<2550$ & 181 \\
\hline Ustd & 3 & 2 & 21 & USTDPF33 & 7160 & 21600 & 8520 & $<565$ & $<5920$ & 212000 & $<433$ & 5870 & 18900 & 90 \\
\hline SB7Ref & 4 & 1 & 1 & SB7REFPF41 & 1500 & 16100 & 9520 & $<567$ & $<5940$ & 190000 & $<434$ & 2850 & $<2590$ & 191 \\
\hline Ustd & 4 & 1 & 2 & USTDPF41 & 7230 & 21500 & 8610 & $<557$ & $<5840$ & 198000 & $<426$ & 5800 & 17800 & 91.1 \\
\hline SB7b-25 & 4 & 1 & 3 & A18PF1 & 1890 & 16800 & 13900 & $<560$ & $<5870$ & 220000 & 3680 & 1080 & 23200 & 237 \\
\hline SB7b-13 & 4 & 1 & 4 & A07PF1 & 2470 & 11700 & 15700 & $<571$ & $<5980$ & 249000 & 7320 & 4340 & 26900 & 414 \\
\hline SB7b-07 & 4 & 1 & 5 & A12PF2 & 360 & 11600 & 9160 & $<571$ & $<5980$ & 226000 & 2630 & 4300 & 16800 & 100 \\
\hline SB7b-03 & 4 & 1 & 6 & A17PF1 & 2430 & 18300 & 15200 & $<556$ & $<5820$ & 218000 & 6820 & 47 & 16900 & 339 \\
\hline SB7b-25 & 4 & 1 & 7 & A18PF2 & 1940 & 16800 & 13900 & $<564$ & $<5900$ & 220000 & 3970 & 1090 & 24400 & 231 \\
\hline SB7b-13 & 4 & 1 & 8 & A07PF2 & 2420 & 11500 & 15500 & $<552$ & $<5780$ & 228000 & 7260 & 4290 & 26600 & 403 \\
\hline SB7b-33 & 4 & 1 & 9 & A10PF1 & 1230 & 14900 & 12200 & $<565$ & $<5920$ & 230000 & 4440 & 1420 & 20700 & 174 \\
\hline SB7Ref & 4 & 1 & 10 & SB7REFPF42 & 1510 & 16000 & 9500 & $<567$ & $<5940$ & 210000 & $<434$ & 2820 & $<2590$ & 187 \\
\hline Ustd & 4 & 1 & 11 & USTDPF42 & 7270 & 21500 & 8640 & $<557$ & $<5840$ & 199000 & $<426$ & 5770 & 18200 & 93.4 \\
\hline SB7b-06 & 4 & 1 & 12 & A19PF1 & 2460 & 18400 & 14700 & $<563$ & $<5900$ & 221000 & 2730 & 48.8 & 17100 & 263 \\
\hline SB7b-06 & 4 & 1 & 13 & A19PF2 & 2430 & 18400 & 14700 & $<564$ & $<5910$ & 210000 & 2650 & 48.8 & 16500 & 258 \\
\hline SB7b-03 & 4 & 1 & 14 & A17PF2 & 2420 & 18100 & 15100 & $<552$ & $<5780$ & 205000 & 7040 & 52.4 & 16800 & 329 \\
\hline SB7b-07 & 4 & 1 & 15 & A12PF1 & 352 & 11500 & 9110 & $<564$ & $<5910$ & 228000 & 2710 & 4270 & 16700 & 89.8 \\
\hline
\end{tabular}


Table A-4. Measured Elemental Concentrations (ug/g) for the Study Glasses Prepared Using Peroxide Fusion (part 2)

\begin{tabular}{|c|c|c|c|c|c|c|c|c|c|c|c|c|c|c|}
\hline Glass ID & Block & Sub-Blk & Sequence & Lab ID & Mg & Mn & $\mathrm{Ni}$ & $\mathbf{P b}$ & $\mathrm{S}$ & $\mathrm{Si}$ & Th & $\mathrm{Ti}$ & $\mathbf{U}$ & Zn \\
\hline SB7b-33 & 4 & 1 & 16 & A10PF2 & 1240 & 14900 & 12300 & $<549$ & $<5750$ & 220000 & 4170 & 1420 & 20600 & 173 \\
\hline SB7b-26 & 4 & 1 & 17 & A05PF1 & 1840 & 13100 & 13700 & $<561$ & $<5880$ & 225000 & 3380 & 1060 & 22700 & 205 \\
\hline SB7b-26 & 4 & 1 & 18 & A05PF2 & 1850 & 13000 & 13600 & $<555$ & $<5810$ & 218000 & 3410 & 1060 & 22200 & 206 \\
\hline blank & 4 & 1 & 19 & BLANKPF4 & $<12$ & $<42.4$ & $<364$ & $<573$ & $<6000$ & $<>782$ & $<438$ & $<36$ & $<2610$ & $<52.8$ \\
\hline SB7Ref & 4 & 1 & 20 & SB7REFPF43 & 1500 & 15900 & 9470 & $<567$ & $<5940$ & 204000 & $<434$ & 2810 & $<2590$ & 185 \\
\hline Ustd & 4 & 1 & 21 & USTDPF43 & 7020 & 20800 & 8370 & $<557$ & $<5840$ & 193000 & $<426$ & 5580 & 17600 & 87.9 \\
\hline SB7Ref & 4 & 2 & 1 & SB7REFPF41 & 1510 & 16200 & 9620 & $<567$ & $<5940$ & 198000 & $<434$ & 2890 & $<2590$ & 192 \\
\hline Ustd & 4 & 2 & 2 & USTDPF41 & 7190 & 21800 & 8610 & $<557$ & $<5840$ & 198000 & $<426$ & 5860 & 17600 & 97.3 \\
\hline SB7b-33 & 4 & 2 & 3 & A10PF2 & 1240 & 15100 & 12300 & $<549$ & $<5750$ & 219000 & 4300 & 1470 & 20300 & 180 \\
\hline SB7b-06 & 4 & 2 & 4 & A19PF1 & 2420 & 18500 & 14700 & $<563$ & $<5900$ & 219000 & 2740 & 56.3 & 17000 & 270 \\
\hline SB7b-25 & 4 & 2 & 5 & A18PF1 & 1880 & 16900 & 13900 & $<560$ & $<5870$ & 220000 & 3750 & 1080 & 23400 & 248 \\
\hline SB7b-26 & 4 & 2 & 6 & A05PF2 & 1850 & 13300 & 13600 & $<555$ & $<5810$ & 219000 & 3440 & 1090 & 22800 & 208 \\
\hline SB7b-33 & 4 & 2 & 7 & A10PF1 & 1230 & 15000 & 12400 & $<565$ & $<5920$ & 229000 & 4500 & 1450 & 21000 & 183 \\
\hline SB7b-13 & 4 & 2 & 8 & A07PF1 & 2460 & 11700 & 15600 & $<571$ & $<5980$ & 248000 & 7440 & 4390 & 26900 & 413 \\
\hline SB7b-07 & 4 & 2 & 9 & A12PF1 & 351 & 11700 & 9130 & $<564$ & $<5910$ & 228000 & 2740 & 4360 & 16100 & 95.4 \\
\hline SB7Ref & 4 & 2 & 10 & SB7REFPF42 & 1510 & 16200 & 9550 & $<567$ & $<5940$ & 209000 & $<434$ & 2890 & $<2590$ & 197 \\
\hline Ustd & 4 & 2 & 11 & USTDPF42 & 7210 & 21800 & 8630 & $<557$ & $<5840$ & 199000 & $<426$ & 5880 & 18000 & 92.6 \\
\hline SB7b-03 & 4 & 2 & 12 & A17PF2 & 2410 & 18300 & 15100 & $<552$ & $<5780$ & 204000 & 7150 & 58.2 & 17400 & 345 \\
\hline SB7b-13 & 4 & 2 & 13 & A07PF2 & 2400 & 11600 & 15500 & $<552$ & $<5780$ & 227000 & 7280 & 4360 & 26500 & 413 \\
\hline SB7b-26 & 4 & 2 & 14 & A05PF1 & 1830 & 13400 & 13600 & $<561$ & $<5880$ & 226000 & 3480 & 1110 & 22600 & 216 \\
\hline SB7b-03 & 4 & 2 & 15 & A17PF1 & 2400 & 18400 & 15200 & $<556$ & $<5820$ & 219000 & 6970 & 73 & 17100 & 345 \\
\hline SB7b-25 & 4 & 2 & 16 & A18PF2 & 1920 & 17000 & 13900 & $<564$ & $<5900$ & 220000 & 3990 & 1130 & 24400 & 227 \\
\hline SB7b-07 & 4 & 2 & 17 & A12PF2 & 356 & 11600 & 9020 & $<571$ & $<5980$ & 226000 & 2600 & 4350 & 16700 & 95.7 \\
\hline SB7b-06 & 4 & 2 & 18 & A19PF2 & 2410 & 18700 & 14700 & $<564$ & $<5910$ & 210000 & 2720 & 59.5 & 16200 & 273 \\
\hline blank & 4 & 2 & 19 & BLANKPF4 & $<12$ & $<42.4$ & $<364$ & $<573$ & $<6000$ & $<782$ & $<438$ & 54.4 & $<2610$ & $<52.8$ \\
\hline SB7Ref & 4 & 2 & 20 & SB7REFPF43 & 1500 & 16100 & 9500 & $<567$ & $<5940$ & 207000 & $<434$ & 2880 & $<2590$ & 195 \\
\hline Ustd & 4 & 2 & 21 & USTDPF43 & 6950 & 21100 & 8290 & $<557$ & $<5840$ & 193000 & $<426$ & 5710 & 17500 & 94.9 \\
\hline SB7Ref & 5 & 1 & 1 & SB7REFPF51 & 1470 & 16000 & 9470 & $<568$ & $<5950$ & 206000 & $<435$ & 2830 & $<2590$ & 215 \\
\hline Ustd & 5 & 1 & 2 & USTDPF51 & 7250 & 21500 & 8650 & $<559$ & $<5850$ & 205000 & $<428$ & 5770 & 17200 & 82.7 \\
\hline SB7b-15 & 5 & 1 & 3 & A16PF1 & 878 & 13300 & 10900 & $<559$ & $<5860$ & 227000 & 3800 & 3230 & 24200 & 171 \\
\hline SB7b-10 & 5 & 1 & 4 & A06PF1 & 350 & 18300 & 15200 & $<559$ & $<5850$ & 219000 & 2620 & 4200 & 25700 & 154 \\
\hline SB7b-10 & 5 & 1 & 5 & A06PF2 & 342 & 18300 & 15300 & $<556$ & $<5820$ & 221000 & 2560 & 4230 & 25100 & 140 \\
\hline SB7b-32 & 5 & 1 & 6 & A29PF2 & 1250 & 15300 & 10300 & $<549$ & $<5750$ & 233000 & 3500 & 1530 & 17100 & 166 \\
\hline SB7b-02 & 5 & 1 & 7 & A01PF2 & 2400 & 18100 & 9180 & $<559$ & $<5850$ & 211000 & 6260 & 4280 & 24900 & 222 \\
\hline SB7b-02 & 5 & 1 & 8 & A01PF1 & 2420 & 18200 & 9210 & $<563$ & $<5890$ & 217000 & 6930 & 4260 & 26500 & 206 \\
\hline SB7b-22 & 5 & 1 & 9 & A14PF2 & 851 & 13200 & 10800 & $<570$ & $<5970$ & 231000 & 3300 & 1130 & 17900 & 143 \\
\hline SB7Ref & 5 & 1 & 10 & SB7REFPF52 & 1480 & 16000 & 9580 & $<568$ & $<5950$ & 219000 & $<435$ & 2830 & $<2590$ & 186 \\
\hline Ustd & 5 & 1 & 11 & USTDPF52 & 7110 & 21100 & 8480 & $<559$ & $<5850$ & 204000 & $<428$ & 5650 & 17200 & 89 \\
\hline SB7b-28 & 5 & 1 & 12 & A28PF1 & 806 & 16500 & 10800 & $<549$ & $<5750$ & 226000 & 4640 & 3210 & 21600 & 150 \\
\hline SB7b-22 & 5 & 1 & 13 & A14PF1 & 880 & 13300 & 10800 & $<558$ & $<5850$ & 233000 & 3880 & 1130 & 19400 & 140 \\
\hline SB7b-28 & 5 & 1 & 14 & A28PF2 & 845 & 16700 & 11000 & $<556$ & $<5820$ & 240000 & 5260 & 3230 & 22800 & 140 \\
\hline SB7b-32 & 5 & 1 & 15 & A29PF1 & 1230 & 15400 & 10500 & $<553$ & $<5800$ & 227000 & 3230 & 1530 & 16800 & 158 \\
\hline SB7b-15 & 5 & 1 & 16 & A16PF2 & 834 & 13100 & 10700 & $<572$ & $<5990$ & 227000 & 3270 & 3190 & 22500 & 166 \\
\hline blank & 5 & 1 & 17 & BLANKPF5 & $<12$ & $<42.4$ & $<364$ & $<573$ & $<6000$ & $<782$ & $<438$ & $<36$ & $<2610$ & $<52.8$ \\
\hline SB7Ref & 5 & 1 & 18 & SB7REFPF53 & 1470 & 15900 & 9380 & $<568$ & $<5950$ & 215000 & $<435$ & 2820 & $<2590$ & 291 \\
\hline Ustd & 5 & 1 & 19 & USTDPF53 & 7220 & 21400 & 8540 & $<559$ & $<5850$ & 208000 & $<428$ & 5770 & 17700 & 82 \\
\hline SB7Ref & 5 & 2 & 1 & SB7REFPF51 & 1470 & 16100 & 9430 & $<568$ & $<5950$ & 206000 & $<435$ & 2890 & $<2590$ & 225 \\
\hline
\end{tabular}


Table A-4. Measured Elemental Concentrations (ug/g) for the Study Glasses Prepared Using Peroxide Fusion (part 2)

\begin{tabular}{|c|c|c|c|c|c|c|c|c|c|c|c|c|c|c|}
\hline Glass ID & Block & Sub-Blk & Sequence & Lab ID & Mg & Mn & $\mathrm{Ni}$ & $\mathbf{P b}$ & $\mathrm{S}$ & $\mathrm{Si}$ & Th & Ti & $\mathbf{U}$ & $\mathbf{Z n}$ \\
\hline Ustd & 5 & 2 & 2 & USTDPF51 & 7190 & 21700 & 8550 & $<559$ & $<5850$ & 206000 & $<428$ & 5870 & 17500 & 95.2 \\
\hline SB7b-15 & 5 & 2 & 3 & A16PF2 & 832 & 13300 & 10800 & $<572$ & $<5990$ & 228000 & 3370 & 3260 & 22200 & 177 \\
\hline SB7b-22 & 5 & 2 & 4 & A14PF2 & 846 & 13400 & 10600 & $<570$ & $<5970$ & 233000 & 3350 & 1170 & 18600 & 148 \\
\hline SB7b-10 & 5 & 2 & 5 & A06PF1 & 346 & 18400 & 15100 & $<559$ & $<5850$ & 220000 & 2720 & 4280 & 26000 & 155 \\
\hline SB7b-28 & 5 & 2 & 6 & A28PF2 & 834 & 16800 & 10900 & $<556$ & $<5820$ & 239000 & 5310 & 3310 & 22700 & 154 \\
\hline SB7b-02 & 5 & 2 & 7 & A01PF2 & 2410 & 18300 & 9120 & $<559$ & $<5850$ & 211000 & 6310 & 4360 & 24900 & 239 \\
\hline SB7b-32 & 5 & 2 & 8 & A29PF1 & 1240 & 15700 & 10500 & $<553$ & $<5800$ & 230000 & 3320 & 1600 & 16700 & 177 \\
\hline SB7b-15 & 5 & 2 & 9 & A16PF1 & 873 & 13500 & 10900 & $<559$ & $<5860$ & 228000 & 3810 & 3310 & 24200 & 172 \\
\hline SB7Ref & 5 & 2 & 10 & SB7REFPF52 & 1470 & 16200 & 9460 & $<568$ & $<5950$ & 219000 & $<435$ & 2880 & $<2590$ & 194 \\
\hline Ustd & 5 & 2 & 11 & USTDPF52 & 7110 & 21400 & 8510 & $<559$ & $<5850$ & 207000 & $<428$ & 5800 & 17300 & 92.9 \\
\hline SB7b-28 & 5 & 2 & 12 & A28PF1 & 807 & 16700 & 10800 & $<549$ & $<5750$ & 228000 & 4700 & 3290 & 22100 & 154 \\
\hline SB7b-02 & 5 & 2 & 13 & A01PF1 & 2410 & 18400 & 9180 & $<563$ & $<5890$ & 220000 & 7000 & 4370 & 26300 & 210 \\
\hline SB7b-22 & 5 & 2 & 14 & A14PF1 & 884 & 13500 & 10800 & $<558$ & $<5850$ & 234000 & 3850 & 1170 & 19600 & 151 \\
\hline SB7b-32 & 5 & 2 & 15 & A29PF2 & 1240 & 15400 & 10400 & $<549$ & $<5750$ & 235000 & 3590 & 1560 & 17400 & 166 \\
\hline SB7b-10 & 5 & 2 & 16 & A06PF2 & 341 & 18500 & 15300 & $<556$ & $<5820$ & 223000 & 2580 & 4310 & 25900 & 158 \\
\hline blank & 5 & 2 & 17 & BLANKPF5 & $<12$ & $<42.4$ & $<364$ & $<573$ & $<6000$ & $<782$ & $<438$ & 44 & $<2610$ & $<52.8$ \\
\hline SB7Ref & 5 & 2 & 18 & SB7REFPF53 & 1460 & 16100 & 9390 & $<568$ & $<5950$ & 216000 & $<435$ & 2870 & $<2590$ & 293 \\
\hline Ustd & 5 & 2 & 19 & USTDPF53 & 7200 & 21700 & 8510 & $<559$ & $<5850$ & 208000 & $<428$ & 5870 & 18100 & 94.5 \\
\hline
\end{tabular}


SRNL-STI-2011-00440

Revision 0

Table A-5. Average Measured Versus Target Compositions by Oxide by Glass ID

\begin{tabular}{|c|c|c|c|c|c|}
\hline & & Measured & Target & Difference of & \% Difference of \\
\hline & & & & Measuserd versus & Measuserd versus \\
\hline Glass ID & Oxide & $(\mathrm{wt} \%)$ & (wt\%) & Target & Target \\
\hline SB7b-01 & $\mathrm{Al}_{2} \mathrm{O}_{3}$ & 6.4432 & 6.3530 & 0.0902 & $1.4 \%$ \\
\hline SB7b-01 & $\mathrm{B}_{2} \mathrm{O}_{3}$ & 5.2042 & 5.4400 & -0.2358 & $-4.3 \%$ \\
\hline SB7b-01 & $\mathrm{BaO}$ & 0.0117 & 0.0090 & 0.0027 & $30.2 \%$ \\
\hline SB7b-01 & $\mathrm{CaO}$ & 0.2106 & 0.2040 & 0.0066 & $3.2 \%$ \\
\hline SB7b-01 & $\mathrm{Ce}_{2} \mathrm{O}_{3}$ & 0.0239 & 0.0190 & 0.0049 & $25.9 \%$ \\
\hline SB7b-01 & $\mathrm{Cr}_{2} \mathrm{O}_{3}$ & 0.0132 & 0.0120 & 0.0012 & $9.9 \%$ \\
\hline SB7b-01 & $\mathrm{CuO}$ & 0.0075 & 0.0050 & 0.0025 & $50.3 \%$ \\
\hline SB7b-01 & $\mathrm{Fe}_{2} \mathrm{O}_{3}$ & 11.6610 & 11.6000 & 0.0610 & $0.5 \%$ \\
\hline SB7b-01 & $\mathrm{K}_{2} \mathrm{O}$ & 0.0984 & 0.0130 & 0.0854 & $656.6 \%$ \\
\hline SB7b-01 & $\mathrm{La}_{2} \mathrm{O}_{3}$ & 0.0061 & 0.0030 & 0.0031 & $102.0 \%$ \\
\hline SB7b-01 & $\mathrm{Li}_{2} \mathrm{O}$ & 4.7391 & 4.8000 & -0.0609 & $-1.3 \%$ \\
\hline SB7b-01 & $\mathrm{MgO}$ & 0.0648 & 0.0530 & 0.0118 & $22.3 \%$ \\
\hline SB7b-01 & $\mathrm{MnO}$ & 1.5075 & 1.5030 & 0.0045 & $0.3 \%$ \\
\hline SB7b-01 & $\mathrm{Na}_{2} \mathrm{O}$ & 16.0749 & 16.0360 & 0.0389 & $0.2 \%$ \\
\hline SB7b-01 & $\mathrm{NiO}$ & 1.9819 & 2.0230 & -0.0411 & $-2.0 \%$ \\
\hline SB7b-01 & $\mathrm{PbO}$ & 0.0088 & 0.0030 & 0.0058 & $194.5 \%$ \\
\hline SB7b-01 & $\mathrm{SiO}_{2}$ & 48.7760 & 48.1210 & 0.6550 & $1.4 \%$ \\
\hline SB7b-01 & $\mathrm{SO}_{4}$ & 0.7445 & 0.7840 & -0.0395 & $-5.0 \%$ \\
\hline SB7b-01 & $\mathrm{ThO}_{2}$ & 0.8217 & 0.8620 & -0.0403 & $-4.7 \%$ \\
\hline SB7b-01 & $\mathrm{TiO}_{2}$ & 0.0104 & 0.0000 & 0.0104 & \\
\hline SB7b-01 & $\mathrm{U}_{3} \mathrm{O}_{8}$ & 2.0518 & 2.1410 & -0.0892 & $-4.2 \%$ \\
\hline SB7b-01 & $\mathrm{ZnO}$ & 0.0172 & 0.0020 & 0.0152 & $761.2 \%$ \\
\hline SB7b-01 & $\mathrm{ZrO}_{2}$ & 0.0396 & 0.0140 & 0.0256 & $183.2 \%$ \\
\hline SB7b-01 & Sum & 100.5180 & 100.0000 & 0.5180 & $0.5 \%$ \\
\hline SB7b-02 & $\mathrm{Al}_{2} \mathrm{O}_{3}$ & 6.3818 & 6.3530 & 0.0288 & $0.5 \%$ \\
\hline SB7b-02 & $\mathrm{B}_{2} \mathrm{O}_{3}$ & 5.3088 & 5.4400 & -0.1312 & $-2.4 \%$ \\
\hline SB7b-02 & $\mathrm{BaO}$ & 0.0687 & 0.0700 & -0.0013 & $-1.9 \%$ \\
\hline SB7b-02 & $\mathrm{CaO}$ & 0.2064 & 0.2040 & 0.0024 & $1.2 \%$ \\
\hline SB7b-02 & $\mathrm{Ce}_{2} \mathrm{O}_{3}$ & 0.1179 & 0.1400 & -0.0221 & $-15.8 \%$ \\
\hline SB7b-02 & $\mathrm{Cr}_{2} \mathrm{O}_{3}$ & 0.0791 & 0.0920 & -0.0129 & $-14.1 \%$ \\
\hline SB7b-02 & $\mathrm{CuO}$ & 0.0304 & 0.0390 & -0.0086 & $-22.0 \%$ \\
\hline SB7b-02 & $\mathrm{Fe}_{2} \mathrm{O}_{3}$ & 11.4716 & 11.6000 & -0.1284 & $-1.1 \%$ \\
\hline SB7b-02 & $\mathrm{K}_{2} \mathrm{O}$ & 0.1618 & 0.0950 & 0.0668 & $70.3 \%$ \\
\hline SB7b-02 & $\mathrm{La}_{2} \mathrm{O}_{3}$ & 0.0208 & 0.0230 & -0.0022 & $-9.5 \%$ \\
\hline SB7b-02 & $\mathrm{Li}_{2} \mathrm{O}$ & 4.7687 & 4.8000 & -0.0313 & $-0.7 \%$ \\
\hline SB7b-02 & $\mathrm{MgO}$ & 0.4021 & 0.4040 & -0.0019 & $-0.5 \%$ \\
\hline SB7b-02 & $\mathrm{MnO}$ & 2.3451 & 2.3650 & -0.0199 & $-0.8 \%$ \\
\hline SB7b-02 & $\mathrm{Na}_{2} \mathrm{O}$ & 14.8954 & 15.1620 & -0.2666 & $-1.8 \%$ \\
\hline SB7b-02 & $\mathrm{NiO}$ & 1.1572 & 1.1780 & -0.0208 & $-1.8 \%$ \\
\hline SB7b-02 & $\mathrm{PbO}$ & 0.0163 & 0.0220 & -0.0057 & $-25.9 \%$ \\
\hline SB7b-02 & $\mathrm{SiO}_{2}$ & 45.9415 & 46.4600 & -0.5185 & $-1.1 \%$ \\
\hline SB7b-02 & $\mathrm{SO}_{4}$ & 0.3655 & 0.5180 & -0.1525 & $-29.4 \%$ \\
\hline SB7b-02 & $\mathrm{ThO}_{2}$ & 0.8231 & 0.8620 & -0.0389 & $-4.5 \%$ \\
\hline SB7b-02 & $\mathrm{TiO}_{2}$ & 0.6899 & 0.7230 & -0.0331 & $-4.6 \%$ \\
\hline SB7b-02 & $\mathrm{U}_{3} \mathrm{O}_{8}$ & 3.1190 & 3.3290 & -0.2100 & $-6.3 \%$ \\
\hline SB7b-02 & $\mathrm{ZnO}$ & 0.0253 & 0.0170 & 0.0083 & $48.8 \%$ \\
\hline SB7b-02 & $\mathrm{ZrO}_{2}$ & 0.0990 & 0.1030 & -0.0040 & $-3.8 \%$ \\
\hline SB7b-02 & Sum & 98.4953 & 99.9990 & -1.5037 & $-1.5 \%$ \\
\hline SB7b-03 & $\mathrm{Al}_{2} \mathrm{O}_{3}$ & 8.8665 & 8.8410 & 0.0255 & $0.3 \%$ \\
\hline SB7b-03 & $\mathrm{B}_{2} \mathrm{O}_{3}$ & 4.5401 & 4.8000 & -0.2599 & $-5.4 \%$ \\
\hline SB7b-03 & $\mathrm{BaO}$ & 0.0732 & 0.0700 & 0.0032 & $4.6 \%$ \\
\hline SB7b-03 & $\mathrm{CaO}$ & 0.6674 & 0.6740 & -0.0066 & $-1.0 \%$ \\
\hline SB7b-03 & $\mathrm{Ce}_{2} \mathrm{O}_{3}$ & 0.1305 & 0.1400 & -0.0095 & $-6.8 \%$ \\
\hline SB7b-03 & $\mathrm{Cr}_{2} \mathrm{O}_{3}$ & 0.1568 & 0.0920 & 0.0648 & $70.4 \%$ \\
\hline SB7b-03 & $\mathrm{CuO}$ & 0.0385 & 0.0390 & -0.0005 & $-1.4 \%$ \\
\hline SB7b-03 & $\mathrm{Fe}_{2} \mathrm{O}_{3}$ & 7.3755 & 7.4740 & -0.0985 & $-1.3 \%$ \\
\hline SB7b-03 & $\mathrm{K}_{2} \mathrm{O}$ & 0.1524 & 0.0950 & 0.0574 & $60.5 \%$ \\
\hline SB7b-03 & $\mathrm{La}_{2} \mathrm{O}_{3}$ & 0.0213 & 0.0230 & -0.0017 & $-7.6 \%$ \\
\hline SB7b-03 & $\mathrm{Li}_{2} \mathrm{O}$ & 6.6874 & 6.8000 & -0.1126 & $-1.7 \%$ \\
\hline SB7b-03 & $\mathrm{MgO}$ & 0.4042 & 0.4040 & 0.0002 & $0.1 \%$ \\
\hline SB7b-03 & $\mathrm{MnO}$ & 2.3532 & 2.3650 & -0.0118 & $-0.5 \%$ \\
\hline
\end{tabular}


SRNL-STI-2011-00440

Revision 0

Table A-5. Average Measured Versus Target Compositions by Oxide by Glass ID

\begin{tabular}{|c|c|c|c|c|c|}
\hline & & Measured & Target & Difference of & \% Difference of \\
\hline & & & & Measuserd versus & Measuserd versus \\
\hline Glass ID & Oxide & (wt\%) & (wt\%) & Target & Target \\
\hline SB7b-03 & $\mathrm{Na}_{2} \mathrm{O}$ & 15.8390 & 16.0360 & -0.1970 & $-1.2 \%$ \\
\hline SB7b-03 & $\mathrm{NiO}$ & 1.9215 & 2.0230 & -0.1015 & $-5.0 \%$ \\
\hline SB7b-03 & $\mathrm{PbO}$ & 0.0153 & 0.0220 & -0.0067 & $-30.6 \%$ \\
\hline SB7b-03 & $\mathrm{SiO}_{2}$ & 45.2462 & 46.4600 & -1.2138 & $-2.6 \%$ \\
\hline SB7b-03 & $\mathrm{SO}_{4}$ & 0.5909 & 0.5180 & 0.0729 & $14.1 \%$ \\
\hline SB7b-03 & $\mathrm{ThO}_{2}$ & 0.8425 & 0.8620 & -0.0195 & $-2.3 \%$ \\
\hline SB7b-03 & $\mathrm{TiO}_{2}$ & 0.0088 & 0.0000 & 0.0088 & \\
\hline SB7b-03 & $\mathrm{U}_{3} \mathrm{O}_{8}$ & 2.0326 & 2.1410 & -0.1084 & $-5.1 \%$ \\
\hline SB7b-03 & $\mathrm{ZnO}$ & 0.0410 & 0.0170 & 0.0240 & $140.9 \%$ \\
\hline SB7b-03 & $\mathrm{ZrO}_{2}$ & 0.0630 & 0.1030 & -0.0400 & $-38.8 \%$ \\
\hline SB7b-03 & Sum & 98.0676 & 99.9990 & -1.9314 & $-1.9 \%$ \\
\hline SB7b-04 & $\mathrm{Al}_{2} \mathrm{O}_{3}$ & 10.1466 & 9.9000 & 0.2466 & $2.5 \%$ \\
\hline SB7b-04 & $\mathrm{B}_{2} \mathrm{O}_{3}$ & 4.6367 & 4.8000 & -0.1633 & $-3.4 \%$ \\
\hline SB7b-04 & $\mathrm{BaO}$ & 0.0123 & 0.0090 & 0.0033 & $36.6 \%$ \\
\hline SB7b-04 & $\mathrm{CaO}$ & 0.2109 & 0.2040 & 0.0069 & $3.4 \%$ \\
\hline SB7b-04 & $\mathrm{Ce}_{2} \mathrm{O}_{3}$ & 0.0282 & 0.0190 & 0.0092 & $48.5 \%$ \\
\hline SB7b-04 & $\mathrm{Cr}_{2} \mathrm{O}_{3}$ & 0.0138 & 0.0120 & 0.0018 & $14.8 \%$ \\
\hline SB7b-04 & $\mathrm{CuO}$ & 0.0311 & 0.0050 & 0.0261 & $521.8 \%$ \\
\hline SB7b-04 & $\mathrm{Fe}_{2} \mathrm{O}_{3}$ & 7.1467 & 7.4740 & -0.3273 & $-4.4 \%$ \\
\hline SB7b-04 & $\mathrm{K}_{2} \mathrm{O}$ & 0.1193 & 0.0130 & 0.1063 & $817.8 \%$ \\
\hline SB7b-04 & $\mathrm{La}_{2} \mathrm{O}_{3}$ & 0.0085 & 0.0030 & 0.0055 & $183.3 \%$ \\
\hline SB7b-04 & $\mathrm{Li}_{2} \mathrm{O}$ & 4.7391 & 4.8000 & -0.0609 & $-1.3 \%$ \\
\hline SB7b-04 & $\mathrm{MgO}$ & 0.0618 & 0.0530 & 0.0088 & $16.6 \%$ \\
\hline SB7b-04 & $\mathrm{MnO}$ & 2.4049 & 2.3650 & 0.0399 & $1.7 \%$ \\
\hline SB7b-04 & $\mathrm{Na}_{2} \mathrm{O}$ & 11.5557 & 11.4200 & 0.1357 & $1.2 \%$ \\
\hline SB7b-04 & $\mathrm{NiO}$ & 1.1750 & 1.1780 & -0.0030 & $-0.3 \%$ \\
\hline SB7b-04 & $\mathrm{PbO}$ & 0.0116 & 0.0030 & 0.0086 & $287.6 \%$ \\
\hline SB7b-04 & $\mathrm{SiO}_{2}$ & 53.3221 & 52.7510 & 0.5711 & $1.1 \%$ \\
\hline SB7b-04 & $\mathrm{SO}_{4}$ & 0.9916 & 0.7840 & 0.2076 & $26.5 \%$ \\
\hline SB7b-04 & $\mathrm{ThO}_{2}$ & 0.8314 & 0.8620 & -0.0306 & $-3.6 \%$ \\
\hline SB7b-04 & $\mathrm{TiO}_{2}$ & 0.0119 & 0.0000 & 0.0119 & \\
\hline SB7b-04 & $\mathrm{U}_{3} \mathrm{O}_{8}$ & 3.1809 & 3.3290 & -0.1481 & $-4.4 \%$ \\
\hline SB7b-04 & $\mathrm{ZnO}$ & 0.0168 & 0.0020 & 0.0148 & $737.9 \%$ \\
\hline SB7b-04 & $\mathrm{ZrO}_{2}$ & 0.0392 & 0.0140 & 0.0252 & $180.3 \%$ \\
\hline SB7b-04 & Sum & 100.6960 & 100.0000 & 0.6960 & $0.7 \%$ \\
\hline SB7b-05 & $\mathrm{Al}_{2} \mathrm{O}_{3}$ & 6.4810 & 6.3530 & 0.1280 & $2.0 \%$ \\
\hline SB7b-05 & $\mathrm{B}_{2} \mathrm{O}_{3}$ & 5.1398 & 5.4400 & -0.3002 & $-5.5 \%$ \\
\hline SB7b-05 & $\mathrm{BaO}$ & 0.0720 & 0.0700 & 0.0020 & $2.9 \%$ \\
\hline SB7b-05 & $\mathrm{CaO}$ & 0.6716 & 0.6740 & -0.0024 & $-0.4 \%$ \\
\hline SB7b-05 & $\mathrm{Ce}_{2} \mathrm{O}_{3}$ & 0.1273 & 0.1400 & -0.0127 & $-9.1 \%$ \\
\hline SB7b-05 & $\mathrm{Cr}_{2} \mathrm{O}_{3}$ & 0.0816 & 0.0920 & -0.0104 & $-11.3 \%$ \\
\hline SB7b-05 & $\mathrm{CuO}$ & 0.0442 & 0.0390 & 0.0052 & $13.3 \%$ \\
\hline SB7b-05 & $\mathrm{Fe}_{2} \mathrm{O}_{3}$ & 7.4023 & 7.4740 & -0.0717 & $-1.0 \%$ \\
\hline SB7b-05 & $\mathrm{K}_{2} \mathrm{O}$ & 0.1672 & 0.0950 & 0.0722 & $76.0 \%$ \\
\hline SB7b-05 & $\mathrm{La}_{2} \mathrm{O}_{3}$ & 0.0220 & 0.0230 & -0.0010 & $-4.5 \%$ \\
\hline SB7b-05 & $\mathrm{Li}_{2} \mathrm{O}$ & 4.7229 & 4.8000 & -0.0771 & $-1.6 \%$ \\
\hline SB7b-05 & $\mathrm{MgO}$ & 0.4055 & 0.4040 & 0.0015 & $0.4 \%$ \\
\hline SB7b-05 & $\mathrm{MnO}$ & 1.4930 & 1.5030 & -0.0100 & $-0.7 \%$ \\
\hline SB7b-05 & $\mathrm{Na}_{2} \mathrm{O}$ & 15.1650 & 15.2040 & -0.0390 & $-0.3 \%$ \\
\hline SB7b-05 & $\mathrm{NiO}$ & 1.1744 & 1.1780 & -0.0036 & $-0.3 \%$ \\
\hline SB7b-05 & $\mathrm{PbO}$ & 0.0238 & 0.0220 & 0.0018 & $8.2 \%$ \\
\hline SB7b-05 & $\mathrm{SiO}_{2}$ & 51.7711 & 52.1940 & -0.4229 & $-0.8 \%$ \\
\hline SB7b-05 & $\mathrm{SO}_{4}$ & 0.6246 & 0.5180 & 0.1066 & $20.6 \%$ \\
\hline SB7b-05 & $\mathrm{ThO}_{2}$ & 0.3476 & 0.3270 & 0.0206 & $6.3 \%$ \\
\hline SB7b-05 & $\mathrm{TiO}_{2}$ & 0.0089 & 0.0000 & 0.0089 & \\
\hline SB7b-05 & $\mathrm{U}_{3} \mathrm{O}_{8}$ & 3.1308 & 3.3290 & -0.1982 & $-6.0 \%$ \\
\hline SB7b-05 & $\mathrm{ZnO}$ & 0.0248 & 0.0170 & 0.0078 & $46.0 \%$ \\
\hline SB7b-05 & $\mathrm{ZrO}_{2}$ & 0.0729 & 0.1030 & -0.0301 & $-29.2 \%$ \\
\hline SB7b-05 & Sum & 99.1742 & 99.9990 & -0.8248 & $-0.8 \%$ \\
\hline SB7b-06 & $\mathrm{Al}_{2} \mathrm{O}_{3}$ & 10.0380 & 9.9000 & 0.1380 & $1.4 \%$ \\
\hline SB7b-06 & $\mathrm{B}_{2} \mathrm{O}_{3}$ & 5.2605 & 5.4400 & -0.1795 & $-3.3 \%$ \\
\hline
\end{tabular}


SRNL-STI-2011-00440

Revision 0

Table A-5. Average Measured Versus Target Compositions by Oxide by Glass ID

\begin{tabular}{|c|c|c|c|c|c|}
\hline & & Measured & Target & Difference of & \% Difference of \\
\hline & & & & Measuserd versus & Measuserd versus \\
\hline Glass ID & Oxide & $(\mathrm{wt} \%)$ & (wt\%) & Target & Target \\
\hline SB7b-06 & $\mathrm{BaO}$ & 0.0706 & 0.0700 & 0.0006 & $0.9 \%$ \\
\hline SB7b-06 & $\mathrm{CaO}$ & 0.2071 & 0.2040 & 0.0031 & $1.5 \%$ \\
\hline SB7b-06 & $\mathrm{Ce}_{2} \mathrm{O}_{3}$ & 0.1237 & 0.1400 & -0.0163 & $-11.7 \%$ \\
\hline SB7b-06 & $\mathrm{Cr}_{2} \mathrm{O}_{3}$ & 0.0751 & 0.0920 & -0.0169 & $-18.4 \%$ \\
\hline SB7b-06 & $\mathrm{CuO}$ & 0.0431 & 0.0390 & 0.0041 & $10.6 \%$ \\
\hline SB7b-06 & $\mathrm{Fe}_{2} \mathrm{O}_{3}$ & 11.1803 & 11.6000 & -0.4197 & $-3.6 \%$ \\
\hline SB7b-06 & $\mathrm{K}_{2} \mathrm{O}$ & 0.1508 & 0.0950 & 0.0558 & $58.8 \%$ \\
\hline SB7b-06 & $\mathrm{La}_{2} \mathrm{O}_{3}$ & 0.0219 & 0.0230 & -0.0011 & $-4.9 \%$ \\
\hline SB7b-06 & $\mathrm{Li}_{2} \mathrm{O}$ & 6.7763 & 6.8000 & -0.0237 & $-0.3 \%$ \\
\hline SB7b-06 & $\mathrm{MgO}$ & 0.4063 & 0.4040 & 0.0023 & $0.6 \%$ \\
\hline SB7b-06 & $\mathrm{MnO}$ & 2.3758 & 2.3650 & 0.0108 & $0.5 \%$ \\
\hline SB7b-06 & $\mathrm{Na}_{2} \mathrm{O}$ & 10.9491 & 11.0670 & -0.1179 & $-1.1 \%$ \\
\hline SB7b-06 & $\mathrm{NiO}$ & 1.8467 & 1.9060 & -0.0593 & $-3.1 \%$ \\
\hline SB7b-06 & $\mathrm{PbO}$ & 0.0195 & 0.0220 & -0.0025 & $-11.5 \%$ \\
\hline SB7b-06 & $\mathrm{SiO}_{2}$ & 45.9950 & 46.4600 & -0.4650 & $-1.0 \%$ \\
\hline SB7b-06 & $\mathrm{SO}_{4}$ & 0.7759 & 0.7840 & -0.0081 & $-1.0 \%$ \\
\hline SB7b-06 & $\mathrm{ThO}_{2}$ & 0.3388 & 0.3270 & 0.0118 & $3.6 \%$ \\
\hline SB7b-06 & $\mathrm{TiO}_{2}$ & 0.0077 & 0.0000 & 0.0077 & \\
\hline SB7b-06 & $\mathrm{U}_{3} \mathrm{O}_{8}$ & 1.9958 & 2.1410 & -0.1452 & $-6.8 \%$ \\
\hline SB7b-06 & $\mathrm{ZnO}$ & 0.0317 & 0.0170 & 0.0147 & $86.5 \%$ \\
\hline SB7b-06 & $\mathrm{ZrO}_{2}$ & 0.0630 & 0.1030 & -0.0400 & $-38.9 \%$ \\
\hline SB7b-06 & Sum & 98.7526 & 99.9990 & -1.2464 & $-1.2 \%$ \\
\hline SB7b-07 & $\mathrm{Al}_{2} \mathrm{O}_{3}$ & 9.9128 & 9.9000 & 0.0128 & $0.1 \%$ \\
\hline SB7b-07 & $\mathrm{B}_{2} \mathrm{O}_{3}$ & 4.5401 & 4.8000 & -0.2599 & $-5.4 \%$ \\
\hline SB7b-07 & $\mathrm{BaO}$ & 0.0098 & 0.0090 & 0.0008 & $8.4 \%$ \\
\hline SB7b-07 & $\mathrm{CaO}$ & 0.2102 & 0.2040 & 0.0062 & $3.1 \%$ \\
\hline SB7b-07 & $\mathrm{Ce}_{2} \mathrm{O}_{3}$ & 0.0261 & 0.0190 & 0.0071 & $37.6 \%$ \\
\hline SB7b-07 & $\mathrm{Cr}_{2} \mathrm{O}_{3}$ & 0.0151 & 0.0120 & 0.0031 & $25.9 \%$ \\
\hline SB7b-07 & $\mathrm{CuO}$ & 0.0081 & 0.0050 & 0.0031 & $61.9 \%$ \\
\hline SB7b-07 & $\mathrm{Fe}_{2} \mathrm{O}_{3}$ & 7.4541 & 7.4740 & -0.0199 & $-0.3 \%$ \\
\hline SB7b-07 & $\mathrm{K}_{2} \mathrm{O}$ & 0.0779 & 0.0130 & 0.0649 & $499.1 \%$ \\
\hline SB7b-07 & $\mathrm{La}_{2} \mathrm{O}_{3}$ & 0.0053 & 0.0030 & 0.0023 & $75.5 \%$ \\
\hline SB7b-07 & $\mathrm{Li}_{2} \mathrm{O}$ & 6.7090 & 6.8000 & -0.0910 & $-1.3 \%$ \\
\hline SB7b-07 & $\mathrm{MgO}$ & 0.0596 & 0.0530 & 0.0066 & $12.4 \%$ \\
\hline SB7b-07 & $\mathrm{MnO}$ & 1.4946 & 1.5030 & -0.0084 & $-0.6 \%$ \\
\hline SB7b-07 & $\mathrm{Na}_{2} \mathrm{O}$ & 15.7042 & 16.0360 & -0.3318 & $-2.1 \%$ \\
\hline SB7b-07 & $\mathrm{NiO}$ & 1.1483 & 1.1780 & -0.0297 & $-2.5 \%$ \\
\hline SB7b-07 & $\mathrm{PbO}$ & 0.0030 & 0.0030 & 0.0000 & $0.7 \%$ \\
\hline SB7b-07 & $\mathrm{SiO}_{2}$ & 48.5621 & 48.2630 & 0.2991 & $0.6 \%$ \\
\hline SB7b-07 & $\mathrm{SO}_{4}$ & 0.3045 & 0.5180 & -0.2135 & $-41.2 \%$ \\
\hline SB7b-07 & $\mathrm{ThO}_{2}$ & 0.3284 & 0.3270 & 0.0014 & $0.4 \%$ \\
\hline SB7b-07 & $\mathrm{TiO}_{2}$ & 0.6843 & 0.7230 & -0.0387 & $-5.4 \%$ \\
\hline SB7b-07 & $\mathrm{U}_{3} \mathrm{O}_{8}$ & 1.9928 & 2.1410 & -0.1482 & $-6.9 \%$ \\
\hline SB7b-07 & $\mathrm{ZnO}$ & 0.0114 & 0.0020 & 0.0094 & $470.5 \%$ \\
\hline SB7b-07 & $\mathrm{ZrO}_{2}$ & 0.0233 & 0.0140 & 0.0093 & $66.4 \%$ \\
\hline SB7b-07 & Sum & 99.2849 & 100.0000 & -0.7151 & $-0.7 \%$ \\
\hline SB7b-08 & $\mathrm{Al}_{2} \mathrm{O}_{3}$ & 6.4361 & 6.3530 & 0.0831 & $1.3 \%$ \\
\hline SB7b-08 & $\mathrm{B}_{2} \mathrm{O}_{3}$ & 4.5562 & 4.8000 & -0.2438 & $-5.1 \%$ \\
\hline SB7b-08 & $\mathrm{BaO}$ & 0.0112 & 0.0090 & 0.0022 & $24.9 \%$ \\
\hline SB7b-08 & $\mathrm{CaO}$ & 0.6699 & 0.6740 & -0.0041 & $-0.6 \%$ \\
\hline SB7b-08 & $\mathrm{Ce}_{2} \mathrm{O}_{3}$ & 0.0243 & 0.0190 & 0.0053 & $28.0 \%$ \\
\hline SB7b-08 & $\mathrm{Cr}_{2} \mathrm{O}_{3}$ & 0.0147 & 0.0120 & 0.0027 & $22.3 \%$ \\
\hline SB7b-08 & $\mathrm{CuO}$ & 0.0087 & 0.0050 & 0.0037 & $74.0 \%$ \\
\hline SB7b-08 & $\mathrm{Fe}_{2} \mathrm{O}_{3}$ & 11.4716 & 11.4740 & -0.0024 & $0.0 \%$ \\
\hline SB7b-08 & $\mathrm{K}_{2} \mathrm{O}$ & 0.1251 & 0.0130 & 0.1121 & $862.5 \%$ \\
\hline SB7b-08 & $\mathrm{La}_{2} \mathrm{O}_{3}$ & 0.0076 & 0.0030 & 0.0046 & $151.7 \%$ \\
\hline SB7b-08 & $\mathrm{Li}_{2} \mathrm{O}$ & 6.7332 & 6.8000 & -0.0668 & $-1.0 \%$ \\
\hline SB7b-08 & $\mathrm{MgO}$ & 0.0615 & 0.0530 & 0.0085 & $16.0 \%$ \\
\hline SB7b-08 & $\mathrm{MnO}$ & 2.3581 & 2.3650 & -0.0069 & $-0.3 \%$ \\
\hline SB7b-08 & $\mathrm{Na}_{2} \mathrm{O}$ & 15.1987 & 15.3230 & -0.1243 & $-0.8 \%$ \\
\hline SB7b-08 & $\mathrm{NiO}$ & 1.1637 & 1.1780 & -0.0143 & $-1.2 \%$ \\
\hline
\end{tabular}


SRNL-STI-2011-00440

Revision 0

Table A-5. Average Measured Versus Target Compositions by Oxide by Glass ID

\begin{tabular}{|c|c|c|c|c|c|}
\hline & & Measured & Target & Difference of & \% Difference of \\
\hline & & & & Measuserd versus & Measuserd versus \\
\hline Glass ID & Oxide & (wt\%) & (wt\%) & Target & Target \\
\hline SB7b-08 & $\mathrm{PbO}$ & 0.0030 & 0.0030 & 0.0000 & $-1.0 \%$ \\
\hline SB7b-08 & $\mathrm{SiO}_{2}$ & 46.6902 & 46.4600 & 0.2302 & $0.5 \%$ \\
\hline SB7b-08 & $\mathrm{SO}_{4}$ & 0.7872 & 0.7840 & 0.0032 & $0.4 \%$ \\
\hline SB7b-08 & $\mathrm{ThO}_{2}$ & 0.3399 & 0.3270 & 0.0129 & $4.0 \%$ \\
\hline SB7b-08 & $\mathrm{TiO}_{2}$ & 0.0106 & 0.0000 & 0.0106 & \\
\hline SB7b-08 & $\mathrm{U}_{3} \mathrm{O}_{8}$ & 3.1735 & 3.3290 & -0.1555 & $-4.7 \%$ \\
\hline SB7b-08 & $\mathrm{ZnO}$ & 0.0134 & 0.0020 & 0.0114 & $571.0 \%$ \\
\hline SB7b-08 & $\mathrm{ZrO}_{2}$ & 0.0241 & 0.0140 & 0.0101 & $72.0 \%$ \\
\hline SB7b-08 & Sum & 99.8824 & 100.0000 & -0.1176 & $-0.1 \%$ \\
\hline SB7b-09 & $\mathrm{Al}_{2} \mathrm{O}_{3}$ & 10.0262 & 9.9000 & 0.1262 & $1.3 \%$ \\
\hline SB7b-09 & $\mathrm{B}_{2} \mathrm{O}_{3}$ & 5.2444 & 5.4400 & -0.1956 & $-3.6 \%$ \\
\hline SB7b-09 & $\mathrm{BaO}$ & 0.0097 & 0.0090 & 0.0007 & $7.5 \%$ \\
\hline SB7b-09 & $\mathrm{CaO}$ & 0.6797 & 0.6740 & 0.0057 & $0.8 \%$ \\
\hline SB7b-09 & $\mathrm{Ce}_{2} \mathrm{O}_{3}$ & 0.0277 & 0.0190 & 0.0087 & $45.8 \%$ \\
\hline SB7b-09 & $\mathrm{Cr}_{2} \mathrm{O}_{3}$ & 0.0133 & 0.0120 & 0.0013 & $11.0 \%$ \\
\hline SB7b-09 & $\mathrm{CuO}$ & 0.0225 & 0.0050 & 0.0175 & $349.4 \%$ \\
\hline SB7b-09 & $\mathrm{Fe}_{2} \mathrm{O}_{3}$ & 11.0552 & 11.2910 & -0.2358 & $-2.1 \%$ \\
\hline SB7b-09 & $\mathrm{K}_{2} \mathrm{O}$ & 0.0764 & 0.0130 & 0.0634 & $487.6 \%$ \\
\hline SB7b-09 & $\mathrm{La}_{2} \mathrm{O}_{3}$ & 0.0083 & 0.0030 & 0.0053 & $178.0 \%$ \\
\hline SB7b-09 & $\mathrm{Li}_{2} \mathrm{O}$ & 6.8166 & 6.8000 & 0.0166 & $0.2 \%$ \\
\hline SB7b-09 & $\mathrm{MgO}$ & 0.0546 & 0.0530 & 0.0016 & $3.1 \%$ \\
\hline SB7b-09 & $\mathrm{MnO}$ & 1.5220 & 1.5030 & 0.0190 & $1.3 \%$ \\
\hline SB7b-09 & $\mathrm{Na}_{2} \mathrm{O}$ & 11.2019 & 11.0670 & 0.1349 & $1.2 \%$ \\
\hline SB7b-09 & $\mathrm{NiO}$ & 1.9326 & 2.0230 & -0.0904 & $-4.5 \%$ \\
\hline SB7b-09 & $\mathrm{PbO}$ & 0.0061 & 0.0030 & 0.0031 & $102.3 \%$ \\
\hline SB7b-09 & $\mathrm{SiO}_{2}$ & 47.3320 & 46.4600 & 0.8720 & $1.9 \%$ \\
\hline SB7b-09 & $\mathrm{SO}_{4}$ & 0.4928 & 0.5180 & -0.0252 & $-4.9 \%$ \\
\hline SB7b-09 & $\mathrm{ThO}_{2}$ & 0.8561 & 0.8620 & -0.0059 & $-0.7 \%$ \\
\hline SB7b-09 & $\mathrm{TiO}_{2}$ & 0.0109 & 0.0000 & 0.0109 & \\
\hline SB7b-09 & $\mathrm{U}_{3} \mathrm{O}_{8}$ & 3.2531 & 3.3290 & -0.0759 & $-2.3 \%$ \\
\hline SB7b-09 & $\mathrm{ZnO}$ & 0.0185 & 0.0020 & 0.0165 & $824.3 \%$ \\
\hline SB7b-09 & $\mathrm{ZrO}_{2}$ & 0.0428 & 0.0140 & 0.0288 & $205.6 \%$ \\
\hline SB7b-09 & Sum & 100.7034 & 100.0000 & 0.7034 & $0.7 \%$ \\
\hline SB7b-10 & $\mathrm{Al}_{2} \mathrm{O}_{3}$ & 9.9459 & 9.9000 & 0.0459 & $0.5 \%$ \\
\hline SB7b-10 & $\mathrm{B}_{2} \mathrm{O}_{3}$ & 5.1518 & 5.4400 & -0.2882 & $-5.3 \%$ \\
\hline SB7b-10 & $\mathrm{BaO}$ & 0.0117 & 0.0090 & 0.0027 & $30.5 \%$ \\
\hline SB7b-10 & $\mathrm{CaO}$ & 0.6776 & 0.6740 & 0.0036 & $0.5 \%$ \\
\hline SB7b-10 & $\mathrm{Ce}_{2} \mathrm{O}_{3}$ & 0.0279 & 0.0190 & 0.0089 & $47.0 \%$ \\
\hline SB7b-10 & $\mathrm{Cr}_{2} \mathrm{O}_{3}$ & 0.0133 & 0.0120 & 0.0013 & $11.1 \%$ \\
\hline SB7b-10 & $\mathrm{CuO}$ & 0.0164 & 0.0050 & 0.0114 & $227.0 \%$ \\
\hline SB7b-10 & $\mathrm{Fe}_{2} \mathrm{O}_{3}$ & 7.3898 & 7.4740 & -0.0842 & $-1.1 \%$ \\
\hline SB7b-10 & $\mathrm{K}_{2} \mathrm{O}$ & 0.0959 & 0.0130 & 0.0829 & $637.8 \%$ \\
\hline SB7b-10 & $\mathrm{La}_{2} \mathrm{O}_{3}$ & 0.0086 & 0.0030 & 0.0056 & $187.0 \%$ \\
\hline SB7b-10 & $\mathrm{Li}_{2} \mathrm{O}$ & 4.7471 & 4.8000 & -0.0529 & $-1.1 \%$ \\
\hline SB7b-10 & $\mathrm{MgO}$ & 0.0588 & 0.0530 & 0.0058 & $10.9 \%$ \\
\hline SB7b-10 & $\mathrm{MnO}$ & 2.3887 & 2.3650 & 0.0237 & $1.0 \%$ \\
\hline SB7b-10 & $\mathrm{Na}_{2} \mathrm{O}$ & 15.6031 & 15.5680 & 0.0351 & $0.2 \%$ \\
\hline SB7b-10 & $\mathrm{NiO}$ & 1.9485 & 2.0230 & -0.0745 & $-3.7 \%$ \\
\hline SB7b-10 & $\mathrm{PbO}$ & 0.0030 & 0.0030 & 0.0000 & $1.3 \%$ \\
\hline SB7b-10 & $\mathrm{SiO}_{2}$ & 47.2250 & 46.4600 & 0.7650 & $1.6 \%$ \\
\hline SB7b-10 & $\mathrm{SO}_{4}$ & 0.7557 & 0.7840 & -0.0283 & $-3.6 \%$ \\
\hline SB7b-10 & $\mathrm{ThO}_{2}$ & 0.3370 & 0.3270 & 0.0100 & $3.0 \%$ \\
\hline SB7b-10 & $\mathrm{TiO}_{2}$ & 0.6908 & 0.7230 & -0.0322 & $-4.5 \%$ \\
\hline SB7b-10 & $\mathrm{U}_{3} \mathrm{O}_{8}$ & 3.1470 & 3.3290 & -0.1820 & $-5.5 \%$ \\
\hline SB7b-10 & $\mathrm{ZnO}$ & 0.0183 & 0.0020 & 0.0163 & $817.3 \%$ \\
\hline SB7b-10 & $\mathrm{ZrO}_{2}$ & 0.0198 & 0.0140 & 0.0058 & $41.4 \%$ \\
\hline SB7b-10 & Sum & 100.2818 & 100.0000 & 0.2818 & $0.3 \%$ \\
\hline SB7b-11 & $\mathrm{Al}_{2} \mathrm{O}_{3}$ & 9.9931 & 9.9000 & 0.0931 & $0.9 \%$ \\
\hline SB7b-11 & $\mathrm{B}_{2} \mathrm{O}_{3}$ & 4.5803 & 4.8000 & -0.2197 & $-4.6 \%$ \\
\hline SB7b-11 & $\mathrm{BaO}$ & 0.0705 & 0.0700 & 0.0005 & $0.7 \%$ \\
\hline SB7b-11 & $\mathrm{CaO}$ & 0.6660 & 0.6740 & -0.0080 & $-1.2 \%$ \\
\hline
\end{tabular}


SRNL-STI-2011-00440

Revision 0

Table A-5. Average Measured Versus Target Compositions by Oxide by Glass ID

\begin{tabular}{|c|c|c|c|c|c|}
\hline & & Measured & Target & Difference of & \% Difference of \\
\hline & & & & Measuserd versus & Measuserd versus \\
\hline Glass ID & Oxide & $(\mathrm{wt} \%)$ & (wt\%) & Target & Target \\
\hline SB7b-11 & $\mathrm{Ce}_{2} \mathrm{O}_{3}$ & 0.1218 & 0.1400 & -0.0182 & $-13.0 \%$ \\
\hline SB7b-11 & $\mathrm{Cr}_{2} \mathrm{O}_{3}$ & 0.0842 & 0.0920 & -0.0078 & $-8.5 \%$ \\
\hline SB7b-11 & $\mathrm{CuO}$ & 0.0389 & 0.0390 & -0.0001 & $-0.2 \%$ \\
\hline SB7b-11 & $\mathrm{Fe}_{2} \mathrm{O}_{3}$ & 11.3429 & 11.6000 & -0.2571 & $-2.2 \%$ \\
\hline SB7b-11 & $\mathrm{K}_{2} \mathrm{O}$ & 0.1648 & 0.0950 & 0.0698 & $73.5 \%$ \\
\hline SB7b-11 & $\mathrm{La}_{2} \mathrm{O}_{3}$ & 0.0220 & 0.0230 & -0.0010 & $-4.3 \%$ \\
\hline SB7b-11 & $\mathrm{Li}_{2} \mathrm{O}$ & 4.7122 & 4.8000 & -0.0878 & $-1.8 \%$ \\
\hline SB7b-11 & $\mathrm{MgO}$ & 0.4026 & 0.4040 & -0.0014 & $-0.4 \%$ \\
\hline SB7b-11 & $\mathrm{MnO}$ & 1.4865 & 1.5030 & -0.0165 & $-1.1 \%$ \\
\hline SB7b-11 & $\mathrm{Na}_{2} \mathrm{O}$ & 13.3654 & 13.5690 & -0.2036 & $-1.5 \%$ \\
\hline SB7b-11 & $\mathrm{NiO}$ & 1.1254 & 1.1780 & -0.0526 & $-4.5 \%$ \\
\hline SB7b-11 & $\mathrm{PbO}$ & 0.0188 & 0.0220 & -0.0032 & $-14.4 \%$ \\
\hline SB7b-11 & $\mathrm{SiO}_{2}$ & 46.3693 & 46.4600 & -0.0907 & $-0.2 \%$ \\
\hline SB7b-11 & $\mathrm{SO}_{4}$ & 0.4546 & 0.7840 & -0.3294 & $-42.0 \%$ \\
\hline SB7b-11 & $\mathrm{ThO}_{2}$ & 0.8342 & 0.8620 & -0.0278 & $-3.2 \%$ \\
\hline SB7b-11 & $\mathrm{TiO}_{2}$ & 0.6837 & 0.7230 & -0.0393 & $-5.4 \%$ \\
\hline SB7b-11 & $\mathrm{U}_{3} \mathrm{O}_{8}$ & 2.0223 & 2.1410 & -0.1187 & $-5.5 \%$ \\
\hline SB7b-11 & $\mathrm{ZnO}$ & 0.0234 & 0.0170 & 0.0064 & $37.5 \%$ \\
\hline SB7b-11 & $\mathrm{ZrO}_{2}$ & 0.1045 & 0.1030 & 0.0015 & $1.4 \%$ \\
\hline SB7b-11 & Sum & 98.6874 & 99.9990 & -1.3116 & $-1.3 \%$ \\
\hline SB7b-12 & $\mathrm{Al}_{2} \mathrm{O}_{3}$ & 6.5329 & 6.3530 & 0.1799 & $2.8 \%$ \\
\hline SB7b-12 & $\mathrm{B}_{2} \mathrm{O}_{3}$ & 4.5723 & 4.8000 & -0.2277 & $-4.7 \%$ \\
\hline SB7b-12 & $\mathrm{BaO}$ & 0.0120 & 0.0090 & 0.0030 & $33.9 \%$ \\
\hline SB7b-12 & $\mathrm{CaO}$ & 0.6779 & 0.6740 & 0.0039 & $0.6 \%$ \\
\hline SB7b-12 & $\mathrm{Ce}_{2} \mathrm{O}_{3}$ & 0.0226 & 0.0190 & 0.0036 & $19.1 \%$ \\
\hline SB7b-12 & $\mathrm{Cr}_{2} \mathrm{O}_{3}$ & 0.0145 & 0.0120 & 0.0025 & $20.5 \%$ \\
\hline SB7b-12 & $\mathrm{CuO}$ & 0.0315 & 0.0050 & 0.0265 & $529.0 \%$ \\
\hline SB7b-12 & $\mathrm{Fe}_{2} \mathrm{O}_{3}$ & 11.3947 & 11.6000 & -0.2053 & $-1.8 \%$ \\
\hline SB7b-12 & $\mathrm{K}_{2} \mathrm{O}$ & 0.1255 & 0.0130 & 0.1125 & $865.4 \%$ \\
\hline SB7b-12 & $\mathrm{La}_{2} \mathrm{O}_{3}$ & 0.0058 & 0.0030 & 0.0028 & $93.5 \%$ \\
\hline SB7b-12 & $\mathrm{Li}_{2} \mathrm{O}$ & 4.7741 & 4.8000 & -0.0259 & $-0.5 \%$ \\
\hline SB7b-12 & $\mathrm{MgO}$ & 0.0608 & 0.0530 & 0.0078 & $14.7 \%$ \\
\hline SB7b-12 & $\mathrm{MnO}$ & 2.3645 & 2.3650 & -0.0005 & $0.0 \%$ \\
\hline SB7b-12 & $\mathrm{Na}_{2} \mathrm{O}$ & 11.0098 & 11.0670 & -0.0572 & $-0.5 \%$ \\
\hline SB7b-12 & $\mathrm{NiO}$ & 1.9803 & 2.0230 & -0.0427 & $-2.1 \%$ \\
\hline SB7b-12 & $\mathrm{PbO}$ & 0.0030 & 0.0030 & 0.0000 & $1.4 \%$ \\
\hline SB7b-12 & $\mathrm{SiO}_{2}$ & 52.4129 & 52.4760 & -0.0632 & $-0.1 \%$ \\
\hline SB7b-12 & $\mathrm{SO}_{4}$ & 0.2411 & 0.5180 & -0.2769 & $-53.4 \%$ \\
\hline SB7b-12 & $\mathrm{ThO}_{2}$ & 0.3368 & 0.3270 & 0.0098 & $3.0 \%$ \\
\hline SB7b-12 & $\mathrm{TiO}_{2}$ & 0.6685 & 0.7230 & -0.0545 & $-7.5 \%$ \\
\hline SB7b-12 & $\mathrm{U}_{3} \mathrm{O}_{8}$ & 2.0607 & 2.1410 & -0.0803 & $-3.8 \%$ \\
\hline SB7b-12 & $\mathrm{ZnO}$ & 0.0206 & 0.0020 & 0.0186 & $930.1 \%$ \\
\hline SB7b-12 & $\mathrm{ZrO}_{2}$ & 0.0179 & 0.0140 & 0.0039 & $28.1 \%$ \\
\hline SB7b-12 & Sum & 99.3407 & 100.0000 & -0.6593 & $-0.7 \%$ \\
\hline SB7b-13 & $\mathrm{Al}_{2} \mathrm{O}_{3}$ & 6.4621 & 6.3530 & 0.1091 & $1.7 \%$ \\
\hline SB7b-13 & $\mathrm{B}_{2} \mathrm{O}_{3}$ & 4.5280 & 4.8000 & -0.2720 & $-5.7 \%$ \\
\hline SB7b-13 & $\mathrm{BaO}$ & 0.0715 & 0.0700 & 0.0015 & $2.1 \%$ \\
\hline SB7b-13 & $\mathrm{CaO}$ & 0.2046 & 0.2040 & 0.0006 & $0.3 \%$ \\
\hline SB7b-13 & $\mathrm{Ce}_{2} \mathrm{O}_{3}$ & 0.1328 & 0.1400 & -0.0072 & $-5.1 \%$ \\
\hline SB7b-13 & $\mathrm{Cr}_{2} \mathrm{O}_{3}$ & 0.0815 & 0.0920 & -0.0105 & $-11.5 \%$ \\
\hline SB7b-13 & $\mathrm{CuO}$ & 0.1338 & 0.0390 & 0.0948 & $243.0 \%$ \\
\hline SB7b-13 & $\mathrm{Fe}_{2} \mathrm{O}_{3}$ & 7.9545 & 8.1140 & -0.1595 & $-2.0 \%$ \\
\hline SB7b-13 & $\mathrm{K}_{2} \mathrm{O}$ & 0.1417 & 0.0950 & 0.0467 & $49.2 \%$ \\
\hline SB7b-13 & $\mathrm{La}_{2} \mathrm{O}_{3}$ & 0.0224 & 0.0230 & -0.0006 & $-2.6 \%$ \\
\hline SB7b-13 & $\mathrm{Li}_{2} \mathrm{O}$ & 6.6336 & 6.8000 & -0.1664 & $-2.4 \%$ \\
\hline SB7b-13 & $\mathrm{MgO}$ & 0.4011 & 0.4040 & -0.0029 & $-0.7 \%$ \\
\hline SB7b-13 & $\mathrm{MnO}$ & 1.4930 & 1.5030 & -0.0100 & $-0.7 \%$ \\
\hline SB7b-13 & $\mathrm{Na}_{2} \mathrm{O}$ & 10.8480 & 11.0670 & -0.2190 & $-2.0 \%$ \\
\hline SB7b-13 & $\mathrm{NiO}$ & 1.9692 & 2.0230 & -0.0538 & $-2.7 \%$ \\
\hline SB7b-13 & $\mathrm{PbO}$ & 0.0173 & 0.0220 & -0.0047 & $-21.5 \%$ \\
\hline SB7b-13 & $\mathrm{SiO}_{2}$ & 50.9153 & 52.4320 & -1.5167 & $-2.9 \%$ \\
\hline
\end{tabular}


Table A-5. Average Measured Versus Target Compositions by Oxide by Glass ID

\begin{tabular}{|c|c|c|c|c|c|}
\hline & & Measured & Target & Difference of & \% Difference of \\
\hline & & & & Measuserd versus & Measuserd versus \\
\hline Glass ID & Oxide & (wt\%) & (wt\%) & Target & Target \\
\hline SB7b-13 & $\mathrm{SO}_{4}$ & 0.5018 & 0.7840 & -0.2822 & $-36.0 \%$ \\
\hline SB7b-13 & $\mathrm{ThO}_{2}$ & 0.8499 & 0.8620 & -0.0121 & $-1.4 \%$ \\
\hline SB7b-13 & $\mathrm{TiO}_{2}$ & 0.6799 & 0.7230 & -0.0431 & $-6.0 \%$ \\
\hline SB7b-13 & $\mathrm{U}_{3} \mathrm{O}_{8}$ & 3.1514 & 3.3290 & -0.1776 & $-5.3 \%$ \\
\hline SB7b-13 & $\mathrm{ZnO}$ & 0.0491 & 0.0170 & 0.0321 & $188.9 \%$ \\
\hline SB7b-13 & $\mathrm{ZrO}_{2}$ & 0.0913 & 0.1030 & -0.0117 & $-11.3 \%$ \\
\hline SB7b-13 & Sum & 97.3338 & 99.9990 & -2.6652 & $-2.7 \%$ \\
\hline SB7b-14 & $\mathrm{Al}_{2} \mathrm{O}_{3}$ & 6.5070 & 6.3530 & 0.1540 & $2.4 \%$ \\
\hline SB7b-14 & $\mathrm{B}_{2} \mathrm{O}_{3}$ & 5.1881 & 5.4400 & -0.2519 & $-4.6 \%$ \\
\hline SB7b-14 & $\mathrm{BaO}$ & 0.0125 & 0.0090 & 0.0035 & $39.4 \%$ \\
\hline SB7b-14 & $\mathrm{CaO}$ & 0.6790 & 0.6740 & 0.0050 & $0.7 \%$ \\
\hline SB7b-14 & $\mathrm{Ce}_{2} \mathrm{O}_{3}$ & 0.0225 & 0.0190 & 0.0035 & $18.5 \%$ \\
\hline SB7b-14 & $\mathrm{Cr}_{2} \mathrm{O}_{3}$ & 0.0130 & 0.0120 & 0.0010 & $8.7 \%$ \\
\hline SB7b-14 & $\mathrm{CuO}$ & 0.0639 & 0.0050 & 0.0589 & $1177.5 \%$ \\
\hline SB7b-14 & $\mathrm{Fe}_{2} \mathrm{O}_{3}$ & 7.4791 & 7.4740 & 0.0051 & $0.1 \%$ \\
\hline SB7b-14 & $\mathrm{K}_{2} \mathrm{O}$ & 0.0775 & 0.0130 & 0.0645 & $496.5 \%$ \\
\hline SB7b-14 & $\mathrm{La}_{2} \mathrm{O}_{3}$ & 0.0066 & 0.0030 & 0.0036 & $118.5 \%$ \\
\hline SB7b-14 & $\mathrm{Li}_{2} \mathrm{O}$ & 6.7332 & 6.8000 & -0.0668 & $-1.0 \%$ \\
\hline SB7b-14 & $\mathrm{MgO}$ & 0.0607 & 0.0530 & 0.0077 & $14.6 \%$ \\
\hline SB7b-14 & $\mathrm{MnO}$ & 2.3936 & 2.3650 & 0.0286 & $1.2 \%$ \\
\hline SB7b-14 & $\mathrm{Na}_{2} \mathrm{O}$ & 12.4151 & 12.3220 & 0.0931 & $0.8 \%$ \\
\hline SB7b-14 & $\mathrm{NiO}$ & 1.1844 & 1.1780 & 0.0064 & $0.5 \%$ \\
\hline SB7b-14 & $\mathrm{PbO}$ & 0.0030 & 0.0030 & 0.0000 & $0.9 \%$ \\
\hline SB7b-14 & $\mathrm{SiO}_{2}$ & 52.7337 & 52.7510 & -0.0173 & $0.0 \%$ \\
\hline SB7b-14 & $\mathrm{SO}_{4}$ & 0.5879 & 0.7840 & -0.1961 & $-25.0 \%$ \\
\hline SB7b-14 & $\mathrm{ThO}_{2}$ & 0.7950 & 0.8620 & -0.0670 & $-7.8 \%$ \\
\hline SB7b-14 & $\mathrm{TiO}_{2}$ & 0.6764 & 0.7230 & -0.0466 & $-6.4 \%$ \\
\hline SB7b-14 & $\mathrm{U}_{3} \mathrm{O}_{8}$ & 2.0385 & 2.1410 & -0.1025 & $-4.8 \%$ \\
\hline SB7b-14 & $\mathrm{ZnO}$ & 0.0227 & 0.0020 & 0.0207 & $1035.1 \%$ \\
\hline SB7b-14 & $\mathrm{ZrO}_{2}$ & 0.0364 & 0.0140 & 0.0224 & $160.3 \%$ \\
\hline SB7b-14 & Sum & 99.7299 & 100.0000 & -0.2701 & $-0.3 \%$ \\
\hline SB7b-15 & $\mathrm{Al}_{2} \mathrm{O}_{3}$ & 7.3029 & 7.2400 & 0.0629 & $0.9 \%$ \\
\hline SB7b-15 & $\mathrm{B}_{2} \mathrm{O}_{3}$ & 4.7534 & 4.9600 & -0.2066 & $-4.2 \%$ \\
\hline SB7b-15 & $\mathrm{BaO}$ & 0.0263 & 0.0250 & 0.0013 & $5.0 \%$ \\
\hline SB7b-15 & $\mathrm{CaO}$ & 0.5593 & 0.5570 & 0.0023 & $0.4 \%$ \\
\hline SB7b-15 & $\mathrm{Ce}_{2} \mathrm{O}_{3}$ & 0.0351 & 0.0490 & -0.0139 & $-28.3 \%$ \\
\hline SB7b-15 & $\mathrm{Cr}_{2} \mathrm{O}_{3}$ & 0.0306 & 0.0320 & -0.0014 & $-4.5 \%$ \\
\hline SB7b-15 & $\mathrm{CuO}$ & 0.0478 & 0.0140 & 0.0338 & $241.2 \%$ \\
\hline SB7b-15 & $\mathrm{Fe}_{2} \mathrm{O}_{3}$ & 8.5871 & 8.5060 & 0.0811 & $1.0 \%$ \\
\hline SB7b-15 & $\mathrm{K}_{2} \mathrm{O}$ & 0.1311 & 0.0330 & 0.0981 & $297.2 \%$ \\
\hline SB7b-15 & $\mathrm{La}_{2} \mathrm{O}_{3}$ & 0.0092 & 0.0080 & 0.0012 & $15.1 \%$ \\
\hline SB7b-15 & $\mathrm{Li}_{2} \mathrm{O}$ & 5.2531 & 5.3000 & -0.0469 & $-0.9 \%$ \\
\hline SB7b-15 & $\mathrm{MgO}$ & 0.1452 & 0.1410 & 0.0042 & $3.0 \%$ \\
\hline SB7b-15 & $\mathrm{MnO}$ & 1.7318 & 1.7190 & 0.0128 & $0.7 \%$ \\
\hline SB7b-15 & $\mathrm{Na}_{2} \mathrm{O}$ & 14.8280 & 14.7940 & 0.0340 & $0.2 \%$ \\
\hline SB7b-15 & $\mathrm{NiO}$ & 1.3902 & 1.3890 & 0.0012 & $0.1 \%$ \\
\hline SB7b-15 & $\mathrm{PbO}$ & 0.0029 & 0.0080 & -0.0051 & $-63.2 \%$ \\
\hline SB7b-15 & $\mathrm{SiO}_{2}$ & 48.6691 & 50.4310 & -1.7619 & $-3.5 \%$ \\
\hline SB7b-15 & $\mathrm{SO}_{4}$ & 0.7445 & 0.7180 & 0.0265 & $3.7 \%$ \\
\hline SB7b-15 & $\mathrm{ThO}_{2}$ & 0.4466 & 0.4610 & -0.0144 & $-3.1 \%$ \\
\hline SB7b-15 & $\mathrm{TiO}_{2}$ & 0.5104 & 0.5420 & -0.0316 & $-5.8 \%$ \\
\hline SB7b-15 & $\mathrm{U}_{3} \mathrm{O}_{8}$ & 2.8301 & 3.0320 & -0.2019 & $-6.7 \%$ \\
\hline SB7b-15 & $\mathrm{ZnO}$ & 0.0204 & 0.0060 & 0.0144 & $240.5 \%$ \\
\hline SB7b-15 & $\mathrm{ZrO}_{2}$ & 0.0365 & 0.0360 & 0.0005 & $1.3 \%$ \\
\hline SB7b-15 & Sum & 98.0915 & 100.0010 & -1.9095 & $-1.9 \%$ \\
\hline SB7b-16 & $\mathrm{Al}_{2} \mathrm{O}_{3}$ & 9.0413 & 9.0130 & 0.0283 & $0.3 \%$ \\
\hline SB7b-16 & $\mathrm{B}_{2} \mathrm{O}_{3}$ & 4.7292 & 4.9600 & -0.2308 & $-4.7 \%$ \\
\hline SB7b-16 & $\mathrm{BaO}$ & 0.0571 & 0.0550 & 0.0021 & $3.7 \%$ \\
\hline SB7b-16 & $\mathrm{CaO}$ & 0.3243 & 0.3220 & 0.0023 & $0.7 \%$ \\
\hline SB7b-16 & $\mathrm{Ce}_{2} \mathrm{O}_{3}$ & 0.0972 & 0.1100 & -0.0128 & $-11.7 \%$ \\
\hline SB7b-16 & $\mathrm{Cr}_{2} \mathrm{O}_{3}$ & 0.0586 & 0.0720 & -0.0134 & $-18.6 \%$ \\
\hline
\end{tabular}


SRNL-STI-2011-00440

Revision 0

Table A-5. Average Measured Versus Target Compositions by Oxide by Glass ID

\begin{tabular}{|c|c|c|c|c|c|}
\hline & & Measured & Target & Difference of & \% Difference of \\
\hline & & & & Measuserd versus & Measuserd versus \\
\hline Glass ID & Oxide & $(\mathrm{wt} \%)$ & (wt\%) & Target & Target \\
\hline SB7b-16 & $\mathrm{CuO}$ & 0.0444 & 0.0310 & 0.0134 & $43.3 \%$ \\
\hline SB7b-16 & $\mathrm{Fe}_{2} \mathrm{O}_{3}$ & 10.3725 & 10.4140 & -0.0415 & $-0.4 \%$ \\
\hline SB7b-16 & $\mathrm{K}_{2} \mathrm{O}$ & 0.1094 & 0.0750 & 0.0344 & $45.9 \%$ \\
\hline SB7b-16 & $\mathrm{La}_{2} \mathrm{O}_{3}$ & 0.0189 & 0.0180 & 0.0009 & $5.2 \%$ \\
\hline SB7b-16 & $\mathrm{Li}_{2} \mathrm{O}$ & 6.2004 & 6.3000 & -0.0996 & $-1.6 \%$ \\
\hline SB7b-16 & $\mathrm{MgO}$ & 0.3165 & 0.3160 & 0.0005 & $0.2 \%$ \\
\hline SB7b-16 & $\mathrm{MnO}$ & 1.7205 & 1.7190 & 0.0015 & $0.1 \%$ \\
\hline SB7b-16 & $\mathrm{Na}_{2} \mathrm{O}$ & 12.1859 & 12.3090 & -0.1231 & $-1.0 \%$ \\
\hline SB7b-16 & $\mathrm{NiO}$ & 1.3823 & 1.3890 & -0.0067 & $-0.5 \%$ \\
\hline SB7b-16 & $\mathrm{PbO}$ & 0.0122 & 0.0170 & -0.0048 & $-28.2 \%$ \\
\hline SB7b-16 & $\mathrm{SiO}_{2}$ & 47.9203 & 48.0330 & -0.1127 & $-0.2 \%$ \\
\hline SB7b-16 & $\mathrm{SO}_{4}$ & 0.7183 & 0.7180 & 0.0003 & $0.0 \%$ \\
\hline SB7b-16 & $\mathrm{ThO}_{2}$ & 0.4563 & 0.4610 & -0.0047 & $-1.0 \%$ \\
\hline SB7b-16 & $\mathrm{TiO}_{2}$ & 0.5204 & 0.5420 & -0.0216 & $-4.0 \%$ \\
\hline SB7b-16 & $\mathrm{U}_{3} \mathrm{O}_{8}$ & 2.8124 & 3.0320 & -0.2196 & $-7.2 \%$ \\
\hline SB7b-16 & $\mathrm{ZnO}$ & 0.0279 & 0.0130 & 0.0149 & $114.2 \%$ \\
\hline SB7b-16 & $\mathrm{ZrO}_{2}$ & 0.0621 & 0.0810 & -0.0189 & $-23.3 \%$ \\
\hline SB7b-16 & Sum & 99.1882 & 100.0000 & -0.8118 & $-0.8 \%$ \\
\hline SB7b-17 & $\mathrm{Al}_{2} \mathrm{O}_{3}$ & 7.3147 & 7.2400 & 0.0747 & $1.0 \%$ \\
\hline SB7b-17 & $\mathrm{B}_{2} \mathrm{O}_{3}$ & 5.0069 & 5.2800 & -0.2731 & $-5.2 \%$ \\
\hline SB7b-17 & $\mathrm{BaO}$ & 0.0586 & 0.0550 & 0.0036 & $6.6 \%$ \\
\hline SB7b-17 & $\mathrm{CaO}$ & 0.3243 & 0.3220 & 0.0023 & $0.7 \%$ \\
\hline SB7b-17 & $\mathrm{Ce}_{2} \mathrm{O}_{3}$ & 0.1021 & 0.1100 & -0.0079 & $-7.2 \%$ \\
\hline SB7b-17 & $\mathrm{Cr}_{2} \mathrm{O}_{3}$ & 0.0677 & 0.0720 & -0.0043 & $-6.0 \%$ \\
\hline SB7b-17 & $\mathrm{CuO}$ & 0.0473 & 0.0310 & 0.0163 & $52.6 \%$ \\
\hline SB7b-17 & $\mathrm{Fe}_{2} \mathrm{O}_{3}$ & 8.5174 & 8.5060 & 0.0114 & $0.1 \%$ \\
\hline SB7b-17 & $\mathrm{K}_{2} \mathrm{O}$ & 0.1507 & 0.0750 & 0.0757 & $100.9 \%$ \\
\hline SB7b-17 & $\mathrm{La}_{2} \mathrm{O}_{3}$ & 0.0177 & 0.0180 & -0.0003 & $-1.5 \%$ \\
\hline SB7b-17 & $\mathrm{Li}_{2} \mathrm{O}$ & 6.2138 & 6.3000 & -0.0862 & $-1.4 \%$ \\
\hline SB7b-17 & $\mathrm{MgO}$ & 0.3184 & 0.3160 & 0.0024 & $0.8 \%$ \\
\hline SB7b-17 & $\mathrm{MnO}$ & 1.7238 & 1.7190 & 0.0048 & $0.3 \%$ \\
\hline SB7b-17 & $\mathrm{Na}_{2} \mathrm{O}$ & 12.6476 & 12.8290 & -0.1814 & $-1.4 \%$ \\
\hline SB7b-17 & $\mathrm{NiO}$ & 1.7926 & 1.8120 & -0.0194 & $-1.1 \%$ \\
\hline SB7b-17 & $\mathrm{PbO}$ & 0.0123 & 0.0170 & -0.0047 & $-27.5 \%$ \\
\hline SB7b-17 & $\mathrm{SiO}_{2}$ & 51.7711 & 51.1780 & 0.5931 & $1.2 \%$ \\
\hline SB7b-17 & $\mathrm{SO}_{4}$ & 0.5318 & 0.5850 & -0.0532 & $-9.1 \%$ \\
\hline SB7b-17 & $\mathrm{ThO}_{2}$ & 0.4428 & 0.4610 & -0.0182 & $-4.0 \%$ \\
\hline SB7b-17 & $\mathrm{TiO}_{2}$ & 0.5098 & 0.5420 & -0.0322 & $-5.9 \%$ \\
\hline SB7b-17 & $\mathrm{U}_{3} \mathrm{O}_{8}$ & 2.2641 & 2.4380 & -0.1739 & $-7.1 \%$ \\
\hline SB7b-17 & $\mathrm{ZnO}$ & 0.0299 & 0.0130 & 0.0169 & $130.3 \%$ \\
\hline SB7b-17 & $\mathrm{ZrO}_{2}$ & 0.0472 & 0.0810 & -0.0338 & $-41.7 \%$ \\
\hline SB7b-17 & Sum & 99.9126 & 100.0000 & -0.0874 & $-0.1 \%$ \\
\hline SB7b-18 & $\mathrm{Al}_{2} \mathrm{O}_{3}$ & 7.3832 & 7.2400 & 0.1432 & $2.0 \%$ \\
\hline SB7b-18 & $\mathrm{B}_{2} \mathrm{O}_{3}$ & 4.7534 & 4.9600 & -0.2066 & $-4.2 \%$ \\
\hline SB7b-18 & $\mathrm{BaO}$ & 0.0566 & 0.0550 & 0.0016 & $2.9 \%$ \\
\hline SB7b-18 & $\mathrm{CaO}$ & 0.5653 & 0.5570 & 0.0083 & $1.5 \%$ \\
\hline SB7b-18 & $\mathrm{Ce}_{2} \mathrm{O}_{3}$ & 0.0935 & 0.1100 & -0.0165 & $-15.0 \%$ \\
\hline SB7b-18 & $\mathrm{Cr}_{2} \mathrm{O}_{3}$ & 0.0698 & 0.0720 & -0.0022 & $-3.0 \%$ \\
\hline SB7b-18 & $\mathrm{CuO}$ & 0.0355 & 0.0310 & 0.0045 & $14.5 \%$ \\
\hline SB7b-18 & $\mathrm{Fe}_{2} \mathrm{O}_{3}$ & 10.6477 & 10.5690 & 0.0787 & $0.7 \%$ \\
\hline SB7b-18 & $\mathrm{K}_{2} \mathrm{O}$ & 0.1530 & 0.0750 & 0.0780 & $104.0 \%$ \\
\hline SB7b-18 & $\mathrm{La}_{2} \mathrm{O}_{3}$ & 0.0194 & 0.0180 & 0.0014 & $7.7 \%$ \\
\hline SB7b-18 & $\mathrm{Li}_{2} \mathrm{O}$ & 5.2719 & 5.3000 & -0.0281 & $-0.5 \%$ \\
\hline SB7b-18 & $\mathrm{MgO}$ & 0.3213 & 0.3160 & 0.0053 & $1.7 \%$ \\
\hline SB7b-18 & $\mathrm{MnO}$ & 2.1611 & 2.1500 & 0.0111 & $0.5 \%$ \\
\hline SB7b-18 & $\mathrm{Na}_{2} \mathrm{O}$ & 14.2214 & 14.1650 & 0.0564 & $0.4 \%$ \\
\hline SB7b-18 & $\mathrm{NiO}$ & 1.8101 & 1.8120 & -0.0019 & $-0.1 \%$ \\
\hline SB7b-18 & $\mathrm{PbO}$ & 0.0151 & 0.0170 & -0.0019 & $-11.4 \%$ \\
\hline SB7b-18 & $\mathrm{SiO}_{2}$ & 47.8134 & 48.0330 & -0.2196 & $-0.5 \%$ \\
\hline SB7b-18 & $\mathrm{SO}_{4}$ & 0.3827 & 0.7180 & -0.3353 & $-46.7 \%$ \\
\hline SB7b-18 & $\mathrm{ThO}_{2}$ & 0.7186 & 0.7280 & -0.0094 & $-1.3 \%$ \\
\hline
\end{tabular}


SRNL-STI-2011-00440

Revision 0

Table A-5. Average Measured Versus Target Compositions by Oxide by Glass ID

\begin{tabular}{|c|c|c|c|c|c|}
\hline & & Measured & Target & Difference of & \% Difference of \\
\hline & & & & Measuserd versus & Measuserd versus \\
\hline Glass ID & Oxide & $(\mathrm{wt} \%)$ & (wt\%) & Target & Target \\
\hline SB7b-18 & $\mathrm{TiO}_{2}$ & 0.5204 & 0.5420 & -0.0216 & $-4.0 \%$ \\
\hline SB7b-18 & $\mathrm{U}_{3} \mathrm{O}_{8}$ & 2.3378 & 2.4380 & -0.1002 & $-4.1 \%$ \\
\hline SB7b-18 & $\mathrm{ZnO}$ & 0.0256 & 0.0130 & 0.0126 & $96.7 \%$ \\
\hline SB7b-18 & $\mathrm{ZrO}_{2}$ & 0.0697 & 0.0810 & -0.0113 & $-13.9 \%$ \\
\hline SB7b-18 & Sum & 99.4464 & 100.0000 & -0.5536 & $-0.6 \%$ \\
\hline SB7b-19 & $\mathrm{Al}_{2} \mathrm{O}_{3}$ & 9.1499 & 9.0130 & 0.1369 & $1.5 \%$ \\
\hline SB7b-19 & $\mathrm{B}_{2} \mathrm{O}_{3}$ & 4.7695 & 4.9600 & -0.1905 & $-3.8 \%$ \\
\hline SB7b-19 & $\mathrm{BaO}$ & 0.0589 & 0.0550 & 0.0039 & $7.2 \%$ \\
\hline SB7b-19 & $\mathrm{CaO}$ & 0.3439 & 0.3220 & 0.0219 & $6.8 \%$ \\
\hline SB7b-19 & $\mathrm{Ce}_{2} \mathrm{O}_{3}$ & 0.1036 & 0.1100 & -0.0064 & $-5.8 \%$ \\
\hline SB7b-19 & $\mathrm{Cr}_{2} \mathrm{O}_{3}$ & 0.0619 & 0.0720 & -0.0101 & $-14.1 \%$ \\
\hline SB7b-19 & $\mathrm{CuO}$ & 0.0378 & 0.0310 & 0.0068 & $21.9 \%$ \\
\hline SB7b-19 & $\mathrm{Fe}_{2} \mathrm{O}_{3}$ & 8.4996 & 8.5060 & -0.0064 & $-0.1 \%$ \\
\hline SB7b-19 & $\mathrm{K}_{2} \mathrm{O}$ & 0.1089 & 0.0750 & 0.0339 & $45.2 \%$ \\
\hline SB7b-19 & $\mathrm{La}_{2} \mathrm{O}_{3}$ & 0.0190 & 0.0180 & 0.0010 & $5.3 \%$ \\
\hline SB7b-19 & $\mathrm{Li}_{2} \mathrm{O}$ & 5.3123 & 5.3000 & 0.0123 & $0.2 \%$ \\
\hline SB7b-19 & $\mathrm{MgO}$ & 0.3227 & 0.3160 & 0.0067 & $2.1 \%$ \\
\hline SB7b-19 & $\mathrm{MnO}$ & 1.7593 & 1.7190 & 0.0403 & $2.3 \%$ \\
\hline SB7b-19 & $\mathrm{Na}_{2} \mathrm{O}$ & 15.1650 & 14.7940 & 0.3710 & $2.5 \%$ \\
\hline SB7b-19 & $\mathrm{NiO}$ & 1.3807 & 1.3890 & -0.0083 & $-0.6 \%$ \\
\hline SB7b-19 & $\mathrm{PbO}$ & 0.0148 & 0.0170 & -0.0022 & $-13.0 \%$ \\
\hline SB7b-19 & $\mathrm{SiO}_{2}$ & 48.5086 & 49.2770 & -0.7684 & $-1.6 \%$ \\
\hline SB7b-19 & $\mathrm{SO}_{4}$ & 0.5677 & 0.5850 & -0.0173 & $-3.0 \%$ \\
\hline SB7b-19 & $\mathrm{ThO}_{2}$ & 0.7059 & 0.7280 & -0.0221 & $-3.0 \%$ \\
\hline SB7b-19 & $\mathrm{TiO}_{2}$ & 0.1837 & 0.1810 & 0.0027 & $1.5 \%$ \\
\hline SB7b-19 & $\mathrm{U}_{3} \mathrm{O}_{8}$ & 2.3201 & 2.4380 & -0.1179 & $-4.8 \%$ \\
\hline SB7b-19 & $\mathrm{ZnO}$ & 0.0241 & 0.0130 & 0.0111 & $85.4 \%$ \\
\hline SB7b-19 & $\mathrm{ZrO}_{2}$ & 0.0687 & 0.0810 & -0.0123 & $-15.2 \%$ \\
\hline SB7b-19 & Sum & 99.4864 & 100.0000 & -0.5136 & $-0.5 \%$ \\
\hline SB7b-20 & $\mathrm{Al}_{2} \mathrm{O}_{3}$ & 9.0696 & 9.0130 & 0.0566 & $0.6 \%$ \\
\hline SB7b-20 & $\mathrm{B}_{2} \mathrm{O}_{3}$ & 4.6930 & 4.9600 & -0.2670 & $-5.4 \%$ \\
\hline SB7b-20 & $\mathrm{BaO}$ & 0.0279 & 0.0250 & 0.0029 & $11.4 \%$ \\
\hline SB7b-20 & $\mathrm{CaO}$ & 0.3208 & 0.3220 & -0.0012 & $-0.4 \%$ \\
\hline SB7b-20 & $\mathrm{Ce}_{2} \mathrm{O}_{3}$ & 0.0412 & 0.0490 & -0.0078 & $-15.9 \%$ \\
\hline SB7b-20 & $\mathrm{Cr}_{2} \mathrm{O}_{3}$ & 0.0231 & 0.0320 & -0.0089 & $-27.8 \%$ \\
\hline SB7b-20 & $\mathrm{CuO}$ & 0.0213 & 0.0140 & 0.0073 & $52.0 \%$ \\
\hline SB7b-20 & $\mathrm{Fe}_{2} \mathrm{O}_{3}$ & 8.5550 & 8.8070 & -0.2520 & $-2.9 \%$ \\
\hline SB7b-20 & $\mathrm{K}_{2} \mathrm{O}$ & 0.1279 & 0.0330 & 0.0949 & $287.7 \%$ \\
\hline SB7b-20 & $\mathrm{La}_{2} \mathrm{O}_{3}$ & 0.0104 & 0.0080 & 0.0024 & $29.6 \%$ \\
\hline SB7b-20 & $\mathrm{Li}_{2} \mathrm{O}$ & 5.1589 & 5.3000 & -0.1411 & $-2.7 \%$ \\
\hline SB7b-20 & $\mathrm{MgO}$ & 0.1435 & 0.1410 & 0.0025 & $1.8 \%$ \\
\hline SB7b-20 & $\mathrm{MnO}$ & 2.1321 & 2.1500 & -0.0179 & $-0.8 \%$ \\
\hline SB7b-20 & $\mathrm{Na}_{2} \mathrm{O}$ & 12.0579 & 12.3090 & -0.2511 & $-2.0 \%$ \\
\hline SB7b-20 & $\mathrm{NiO}$ & 1.7258 & 1.8120 & -0.0862 & $-4.8 \%$ \\
\hline SB7b-20 & $\mathrm{PbO}$ & 0.0040 & 0.0080 & -0.0040 & $-49.9 \%$ \\
\hline SB7b-20 & $\mathrm{SiO}_{2}$ & 51.5571 & 51.1780 & 0.3791 & $0.7 \%$ \\
\hline SB7b-20 & $\mathrm{SO}_{4}$ & 0.6231 & 0.7180 & -0.0949 & $-13.2 \%$ \\
\hline SB7b-20 & $\mathrm{ThO}_{2}$ & 0.4475 & 0.4610 & -0.0135 & $-2.9 \%$ \\
\hline SB7b-20 & $\mathrm{TiO}_{2}$ & 0.1740 & 0.1810 & -0.0070 & $-3.9 \%$ \\
\hline SB7b-20 & $\mathrm{U}_{3} \mathrm{O}_{8}$ & 2.2596 & 2.4380 & -0.1784 & $-7.3 \%$ \\
\hline SB7b-20 & $\mathrm{ZnO}$ & 0.0210 & 0.0060 & 0.0150 & $250.6 \%$ \\
\hline SB7b-20 & $\mathrm{ZrO}_{2}$ & 0.0394 & 0.0360 & 0.0034 & $9.6 \%$ \\
\hline SB7b-20 & Sum & 99.2341 & 100.0010 & -0.7669 & $-0.8 \%$ \\
\hline SB7b-21 & $\mathrm{Al}_{2} \mathrm{O}_{3}$ & 7.3761 & 7.2400 & 0.1361 & $1.9 \%$ \\
\hline SB7b-21 & $\mathrm{B}_{2} \mathrm{O}_{3}$ & 5.0713 & 5.2800 & -0.2087 & $-4.0 \%$ \\
\hline SB7b-21 & $\mathrm{BaO}$ & 0.0576 & 0.0550 & 0.0026 & $4.6 \%$ \\
\hline SB7b-21 & $\mathrm{CaO}$ & 0.5646 & 0.5570 & 0.0076 & $1.4 \%$ \\
\hline SB7b-21 & $\mathrm{Ce}_{2} \mathrm{O}_{3}$ & 0.1026 & 0.1100 & -0.0074 & $-6.7 \%$ \\
\hline SB7b-21 & $\mathrm{Cr}_{2} \mathrm{O}_{3}$ & 0.0574 & 0.0720 & -0.0146 & $-20.3 \%$ \\
\hline SB7b-21 & $\mathrm{CuO}$ & 0.0379 & 0.0310 & 0.0069 & $22.4 \%$ \\
\hline SB7b-21 & $\mathrm{Fe}_{2} \mathrm{O}_{3}$ & 8.4871 & 8.5060 & -0.0189 & $-0.2 \%$ \\
\hline
\end{tabular}


SRNL-STI-2011-00440

Revision 0

Table A-5. Average Measured Versus Target Compositions by Oxide by Glass ID

\begin{tabular}{|c|c|c|c|c|c|}
\hline & & Measured & Target & Difference of & \% Difference of \\
\hline & & & & Measuserd versus & Measuserd versus \\
\hline Glass ID & Oxide & (wt\%) & (wt\%) & Target & Target \\
\hline SB7b-21 & $\mathrm{K}_{2} \mathrm{O}$ & 0.1095 & 0.0750 & 0.0345 & $46.0 \%$ \\
\hline SB7b-21 & $\mathrm{La}_{2} \mathrm{O}_{3}$ & 0.0184 & 0.0180 & 0.0004 & $2.4 \%$ \\
\hline SB7b-21 & $\mathrm{Li}_{2} \mathrm{O}$ & 6.3026 & 6.3000 & 0.0026 & $0.0 \%$ \\
\hline SB7b-21 & $\mathrm{MgO}$ & 0.3207 & 0.3160 & 0.0047 & $1.5 \%$ \\
\hline SB7b-21 & $\mathrm{MnO}$ & 2.1660 & 2.1500 & 0.0160 & $0.7 \%$ \\
\hline SB7b-21 & $\mathrm{Na}_{2} \mathrm{O}$ & 12.4623 & 12.3090 & 0.1533 & $1.2 \%$ \\
\hline SB7b-21 & $\mathrm{NiO}$ & 1.3791 & 1.3890 & -0.0099 & $-0.7 \%$ \\
\hline SB7b-21 & $\mathrm{PbO}$ & 0.0155 & 0.0170 & -0.0015 & $-8.9 \%$ \\
\hline SB7b-21 & $\mathrm{SiO}_{2}$ & 48.2947 & 50.8220 & -2.5273 & $-5.0 \%$ \\
\hline SB7b-21 & $\mathrm{SO}_{4}$ & 0.6276 & 0.7180 & -0.0904 & $-12.6 \%$ \\
\hline SB7b-21 & $\mathrm{ThO}_{2}$ & 0.7118 & 0.7280 & -0.0162 & $-2.2 \%$ \\
\hline SB7b-21 & $\mathrm{TiO}_{2}$ & 0.1814 & 0.1810 & 0.0004 & $0.2 \%$ \\
\hline SB7b-21 & $\mathrm{U}_{3} \mathrm{O}_{8}$ & 2.8787 & 3.0320 & -0.1533 & $-5.1 \%$ \\
\hline SB7b-21 & $\mathrm{ZnO}$ & 0.0239 & 0.0130 & 0.0109 & $83.5 \%$ \\
\hline SB7b-21 & $\mathrm{ZrO}_{2}$ & 0.0811 & 0.0810 & 0.0001 & $0.1 \%$ \\
\hline SB7b-21 & Sum & 97.3278 & 100.0000 & -2.6722 & $-2.7 \%$ \\
\hline SB7b-22 & $\mathrm{Al}_{2} \mathrm{O}_{3}$ & 7.3974 & 7.2400 & 0.1574 & $2.2 \%$ \\
\hline SB7b-22 & $\mathrm{B}_{2} \mathrm{O}_{3}$ & 4.8057 & 4.9600 & -0.1543 & $-3.1 \%$ \\
\hline SB7b-22 & $\mathrm{BaO}$ & 0.0272 & 0.0250 & 0.0022 & $8.8 \%$ \\
\hline SB7b-22 & $\mathrm{CaO}$ & 0.5723 & 0.5570 & 0.0153 & $2.7 \%$ \\
\hline SB7b-22 & $\mathrm{Ce}_{2} \mathrm{O}_{3}$ & 0.0375 & 0.0490 & -0.0115 & $-23.5 \%$ \\
\hline SB7b-22 & $\mathrm{Cr}_{2} \mathrm{O}_{3}$ & 0.0144 & 0.0320 & -0.0176 & $-55.0 \%$ \\
\hline SB7b-22 & $\mathrm{CuO}$ & 0.0243 & 0.0140 & 0.0103 & $73.9 \%$ \\
\hline SB7b-22 & $\mathrm{Fe}_{2} \mathrm{O}_{3}$ & 9.9704 & 10.5690 & -0.5986 & $-5.7 \%$ \\
\hline SB7b-22 & $\mathrm{K}_{2} \mathrm{O}$ & 0.1390 & 0.0330 & 0.1060 & $321.1 \%$ \\
\hline SB7b-22 & $\mathrm{La}_{2} \mathrm{O}_{3}$ & 0.0097 & 0.0080 & 0.0017 & $21.6 \%$ \\
\hline SB7b-22 & $\mathrm{Li}_{2} \mathrm{O}$ & 6.3215 & 6.3000 & 0.0215 & $0.3 \%$ \\
\hline SB7b-22 & $\mathrm{MgO}$ & 0.1479 & 0.1410 & 0.0069 & $4.9 \%$ \\
\hline SB7b-22 & $\mathrm{MnO}$ & 1.7480 & 1.7190 & 0.0290 & $1.7 \%$ \\
\hline SB7b-22 & $\mathrm{Na}_{2} \mathrm{O}$ & 12.4387 & 12.3090 & 0.1297 & $1.1 \%$ \\
\hline SB7b-22 & $\mathrm{NiO}$ & 1.3870 & 1.3890 & -0.0020 & $-0.1 \%$ \\
\hline SB7b-22 & $\mathrm{PbO}$ & 0.0039 & 0.0080 & -0.0041 & $-51.8 \%$ \\
\hline SB7b-22 & $\mathrm{SiO}_{2}$ & 49.7922 & 50.9410 & -1.1488 & $-2.3 \%$ \\
\hline SB7b-22 & $\mathrm{SO}_{4}$ & 0.7497 & 0.5850 & 0.1647 & $28.2 \%$ \\
\hline SB7b-22 & $\mathrm{ThO}_{2}$ & 0.4476 & 0.4610 & -0.0134 & $-2.9 \%$ \\
\hline SB7b-22 & $\mathrm{TiO}_{2}$ & 0.1820 & 0.1810 & 0.0010 & $0.6 \%$ \\
\hline SB7b-22 & $\mathrm{U}_{3} \mathrm{O}_{8}$ & 2.2980 & 2.4380 & -0.1400 & $-5.7 \%$ \\
\hline SB7b-22 & $\mathrm{ZnO}$ & 0.0176 & 0.0060 & 0.0116 & $193.0 \%$ \\
\hline SB7b-22 & $\mathrm{ZrO}_{2}$ & 0.0316 & 0.0360 & -0.0044 & $-12.2 \%$ \\
\hline SB7b-22 & Sum & 98.5634 & 100.0010 & -1.4376 & $-1.4 \%$ \\
\hline SB7b-23 & $\mathrm{Al}_{2} \mathrm{O}_{3}$ & 9.1641 & 9.0130 & 0.1511 & $1.7 \%$ \\
\hline SB7b-23 & $\mathrm{B}_{2} \mathrm{O}_{3}$ & 4.7252 & 4.9600 & -0.2348 & $-4.7 \%$ \\
\hline SB7b-23 & $\mathrm{BaO}$ & 0.0260 & 0.0250 & 0.0010 & $3.8 \%$ \\
\hline SB7b-23 & $\mathrm{CaO}$ & 0.5586 & 0.5570 & 0.0016 & $0.3 \%$ \\
\hline SB7b-23 & $\mathrm{Ce}_{2} \mathrm{O}_{3}$ & 0.0466 & 0.0490 & -0.0024 & $-5.0 \%$ \\
\hline SB7b-23 & $\mathrm{Cr}_{2} \mathrm{O}_{3}$ & 0.0252 & 0.0320 & -0.0068 & $-21.3 \%$ \\
\hline SB7b-23 & $\mathrm{CuO}$ & 0.0278 & 0.0140 & 0.0138 & $98.4 \%$ \\
\hline SB7b-23 & $\mathrm{Fe}_{2} \mathrm{O}_{3}$ & 8.2780 & 8.5060 & -0.2280 & $-2.7 \%$ \\
\hline SB7b-23 & $\mathrm{K}_{2} \mathrm{O}$ & 0.0896 & 0.0330 & 0.0566 & $171.4 \%$ \\
\hline SB7b-23 & $\mathrm{La}_{2} \mathrm{O}_{3}$ & 0.0103 & 0.0080 & 0.0023 & $29.4 \%$ \\
\hline SB7b-23 & $\mathrm{Li}_{2} \mathrm{O}$ & 6.2730 & 6.3000 & -0.0270 & $-0.4 \%$ \\
\hline SB7b-23 & $\mathrm{MgO}$ & 0.1469 & 0.1410 & 0.0059 & $4.2 \%$ \\
\hline SB7b-23 & $\mathrm{MnO}$ & 1.7270 & 1.7190 & 0.0080 & $0.5 \%$ \\
\hline SB7b-23 & $\mathrm{Na}_{2} \mathrm{O}$ & 12.2061 & 12.3090 & -0.1029 & $-0.8 \%$ \\
\hline SB7b-23 & $\mathrm{NiO}$ & 1.7529 & 1.8120 & -0.0591 & $-3.3 \%$ \\
\hline SB7b-23 & $\mathrm{PbO}$ & 0.0038 & 0.0080 & -0.0042 & $-52.2 \%$ \\
\hline SB7b-23 & $\mathrm{SiO}_{2}$ & 49.8992 & 49.5860 & 0.3132 & $0.6 \%$ \\
\hline SB7b-23 & $\mathrm{SO}_{4}$ & 0.4164 & 0.5850 & -0.1686 & $-28.8 \%$ \\
\hline SB7b-23 & $\mathrm{ThO}_{2}$ & 0.7093 & 0.7280 & -0.0187 & $-2.6 \%$ \\
\hline SB7b-23 & $\mathrm{TiO}_{2}$ & 0.5306 & 0.5420 & -0.0114 & $-2.1 \%$ \\
\hline SB7b-23 & $\mathrm{U}_{3} \mathrm{O}_{8}$ & 2.8846 & 3.0320 & -0.1474 & $-4.9 \%$ \\
\hline
\end{tabular}


SRNL-STI-2011-00440

Revision 0

Table A-5. Average Measured Versus Target Compositions by Oxide by Glass ID

\begin{tabular}{|c|c|c|c|c|c|}
\hline & & Measured & Target & Difference of & \% Difference of \\
\hline & & & & Measuserd versus & Measuserd versus \\
\hline Glass ID & Oxide & (wt\%) & (wt\%) & Target & Target \\
\hline SB7b-23 & $\mathrm{ZnO}$ & 0.0226 & 0.0060 & 0.0166 & $277.1 \%$ \\
\hline SB7b-23 & $\mathrm{ZrO}_{2}$ & 0.0512 & 0.0360 & 0.0152 & $42.3 \%$ \\
\hline SB7b-23 & Sum & 99.5751 & 100.0010 & -0.4259 & $-0.4 \%$ \\
\hline SB7b-24 & $\mathrm{Al}_{2} \mathrm{O}_{3}$ & 8.9869 & 9.0130 & -0.0261 & $-0.3 \%$ \\
\hline SB7b-24 & $\mathrm{B}_{2} \mathrm{O}_{3}$ & 4.9466 & 5.2800 & -0.3334 & $-6.3 \%$ \\
\hline SB7b-24 & $\mathrm{BaO}$ & 0.0276 & 0.0250 & 0.0026 & $10.5 \%$ \\
\hline SB7b-24 & $\mathrm{CaO}$ & 0.5628 & 0.5570 & 0.0058 & $1.0 \%$ \\
\hline SB7b-24 & $\mathrm{Ce}_{2} \mathrm{O}_{3}$ & 0.0370 & 0.0490 & -0.0120 & $-24.6 \%$ \\
\hline SB7b-24 & $\mathrm{Cr}_{2} \mathrm{O}_{3}$ & 0.0298 & 0.0320 & -0.0022 & $-6.9 \%$ \\
\hline SB7b-24 & $\mathrm{CuO}$ & 0.0224 & 0.0140 & 0.0084 & $60.1 \%$ \\
\hline SB7b-24 & $\mathrm{Fe}_{2} \mathrm{O}_{3}$ & 8.2619 & 8.5060 & -0.2441 & $-2.9 \%$ \\
\hline SB7b-24 & $\mathrm{K}_{2} \mathrm{O}$ & 0.1411 & 0.0330 & 0.1081 & $327.7 \%$ \\
\hline SB7b-24 & $\mathrm{La}_{2} \mathrm{O}_{3}$ & 0.0087 & 0.0080 & 0.0007 & $8.2 \%$ \\
\hline SB7b-24 & $\mathrm{Li}_{2} \mathrm{O}$ & 6.1492 & 6.3000 & -0.1508 & $-2.4 \%$ \\
\hline SB7b-24 & $\mathrm{MgO}$ & 0.1443 & 0.1410 & 0.0033 & $2.3 \%$ \\
\hline SB7b-24 & $\mathrm{MnO}$ & 2.1337 & 2.1500 & -0.0163 & $-0.8 \%$ \\
\hline SB7b-24 & $\mathrm{Na}_{2} \mathrm{O}$ & 14.3562 & 14.3950 & -0.0388 & $-0.3 \%$ \\
\hline SB7b-24 & $\mathrm{NiO}$ & 1.3409 & 1.3890 & -0.0481 & $-3.5 \%$ \\
\hline SB7b-24 & $\mathrm{PbO}$ & 0.0031 & 0.0080 & -0.0049 & $-61.9 \%$ \\
\hline SB7b-24 & $\mathrm{SiO}_{2}$ & 46.7972 & 48.0330 & -1.2358 & $-2.6 \%$ \\
\hline SB7b-24 & $\mathrm{SO}_{4}$ & 0.4509 & 0.5850 & -0.1341 & $-22.9 \%$ \\
\hline SB7b-24 & $\mathrm{ThO}_{2}$ & 0.4451 & 0.4610 & -0.0159 & $-3.5 \%$ \\
\hline SB7b-24 & $\mathrm{TiO}_{2}$ & 0.5092 & 0.5420 & -0.0328 & $-6.1 \%$ \\
\hline SB7b-24 & $\mathrm{U}_{3} \mathrm{O}_{8}$ & 2.2552 & 2.4380 & -0.1828 & $-7.5 \%$ \\
\hline SB7b-24 & $\mathrm{ZnO}$ & 0.0186 & 0.0060 & 0.0126 & $209.9 \%$ \\
\hline SB7b-24 & $\mathrm{ZrO}_{2}$ & 0.0442 & 0.0360 & 0.0082 & $22.9 \%$ \\
\hline SB7b-24 & Sum & 97.6725 & 100.0010 & -2.3285 & $-2.3 \%$ \\
\hline SB7b-25 & $\mathrm{Al}_{2} \mathrm{O}_{3}$ & 7.4494 & 7.2400 & 0.2094 & $2.9 \%$ \\
\hline SB7b-25 & $\mathrm{B}_{2} \mathrm{O}_{3}$ & 4.1778 & 4.9600 & -0.7822 & $-15.8 \%$ \\
\hline SB7b-25 & $\mathrm{BaO}$ & 0.0573 & 0.0550 & 0.0023 & $4.2 \%$ \\
\hline SB7b-25 & $\mathrm{CaO}$ & 0.3243 & 0.3220 & 0.0023 & $0.7 \%$ \\
\hline SB7b-25 & $\mathrm{Ce}_{2} \mathrm{O}_{3}$ & 0.0980 & 0.1100 & -0.0120 & $-10.9 \%$ \\
\hline SB7b-25 & $\mathrm{Cr}_{2} \mathrm{O}_{3}$ & 0.0588 & 0.0720 & -0.0132 & $-18.4 \%$ \\
\hline SB7b-25 & $\mathrm{CuO}$ & 0.0373 & 0.0310 & 0.0063 & $20.4 \%$ \\
\hline SB7b-25 & $\mathrm{Fe}_{2} \mathrm{O}_{3}$ & 9.2948 & 9.3420 & -0.0472 & $-0.5 \%$ \\
\hline SB7b-25 & $\mathrm{K}_{2} \mathrm{O}$ & 0.1290 & 0.0750 & 0.0540 & $71.9 \%$ \\
\hline SB7b-25 & $\mathrm{La}_{2} \mathrm{O}_{3}$ & 0.0163 & 0.0180 & -0.0017 & $-9.6 \%$ \\
\hline SB7b-25 & $\mathrm{Li}_{2} \mathrm{O}$ & 6.2703 & 6.3000 & -0.0297 & $-0.5 \%$ \\
\hline SB7b-25 & $\mathrm{MgO}$ & 0.3196 & 0.3160 & 0.0036 & $1.2 \%$ \\
\hline SB7b-25 & $\mathrm{MnO}$ & 2.1821 & 2.1500 & 0.0321 & $1.5 \%$ \\
\hline SB7b-25 & $\mathrm{Na}_{2} \mathrm{O}$ & 14.9628 & 14.7940 & 0.1688 & $1.1 \%$ \\
\hline SB7b-25 & $\mathrm{NiO}$ & 1.7720 & 1.8120 & -0.0400 & $-2.2 \%$ \\
\hline SB7b-25 & $\mathrm{PbO}$ & 0.0165 & 0.0170 & -0.0005 & $-3.1 \%$ \\
\hline SB7b-25 & $\mathrm{SiO}_{2}$ & 47.0646 & 48.0330 & -0.9684 & $-2.0 \%$ \\
\hline SB7b-25 & $\mathrm{SO}_{4}$ & 0.5984 & 0.5850 & 0.0134 & $2.3 \%$ \\
\hline SB7b-25 & $\mathrm{ThO}_{2}$ & 0.4731 & 0.4610 & 0.0121 & $2.6 \%$ \\
\hline SB7b-25 & $\mathrm{TiO}_{2}$ & 0.1797 & 0.1810 & -0.0013 & $-0.7 \%$ \\
\hline SB7b-25 & $\mathrm{U}_{3} \mathrm{O}_{8}$ & 2.8802 & 3.0320 & -0.1518 & $-5.0 \%$ \\
\hline SB7b-25 & $\mathrm{ZnO}$ & 0.0279 & 0.0130 & 0.0149 & $114.8 \%$ \\
\hline SB7b-25 & $\mathrm{ZrO}_{2}$ & 0.0708 & 0.0810 & -0.0102 & $-12.6 \%$ \\
\hline SB7b-25 & Sum & 98.4610 & 100.0000 & -1.5390 & $-1.5 \%$ \\
\hline SB7b-26 & $\mathrm{Al}_{2} \mathrm{O}_{3}$ & 9.1145 & 9.0130 & 0.1015 & $1.1 \%$ \\
\hline SB7b-26 & $\mathrm{B}_{2} \mathrm{O}_{3}$ & 5.0271 & 5.2800 & -0.2529 & $-4.8 \%$ \\
\hline SB7b-26 & $\mathrm{BaO}$ & 0.0579 & 0.0550 & 0.0029 & $5.2 \%$ \\
\hline SB7b-26 & $\mathrm{CaO}$ & 0.5439 & 0.5570 & -0.0131 & $-2.3 \%$ \\
\hline SB7b-26 & $\mathrm{Ce}_{2} \mathrm{O}_{3}$ & 0.0898 & 0.1100 & -0.0202 & $-18.4 \%$ \\
\hline SB7b-26 & $\mathrm{Cr}_{2} \mathrm{O}_{3}$ & 0.0498 & 0.0720 & -0.0222 & $-30.8 \%$ \\
\hline SB7b-26 & $\mathrm{CuO}$ & 0.0322 & 0.0310 & 0.0012 & $4.0 \%$ \\
\hline SB7b-26 & $\mathrm{Fe}_{2} \mathrm{O}_{3}$ & 10.0830 & 10.5690 & -0.4860 & $-4.6 \%$ \\
\hline SB7b-26 & $\mathrm{K}_{2} \mathrm{O}$ & 0.1708 & 0.0750 & 0.0958 & $127.8 \%$ \\
\hline SB7b-26 & $\mathrm{La}_{2} \mathrm{O}_{3}$ & 0.0176 & 0.0180 & -0.0004 & $-2.3 \%$ \\
\hline
\end{tabular}


SRNL-STI-2011-00440

Revision 0

Table A-5. Average Measured Versus Target Compositions by Oxide by Glass ID

\begin{tabular}{|c|c|c|c|c|c|}
\hline & & Measured & Target & Difference of & \% Difference of \\
\hline & & & & Measuserd versus & Measuserd versus \\
\hline Glass ID & Oxide & (wt\%) & (wt\%) & Target & Target \\
\hline SB7b-26 & $\mathrm{Li}_{2} \mathrm{O}$ & 5.1966 & 5.3000 & -0.1034 & $-2.0 \%$ \\
\hline SB7b-26 & $\mathrm{MgO}$ & 0.3116 & 0.3160 & -0.0044 & $-1.4 \%$ \\
\hline SB7b-26 & $\mathrm{MnO}$ & 1.7092 & 1.7190 & -0.0098 & $-0.6 \%$ \\
\hline SB7b-26 & $\mathrm{Na}_{2} \mathrm{O}$ & 12.2331 & 12.3090 & -0.0759 & $-0.6 \%$ \\
\hline SB7b-26 & $\mathrm{NiO}$ & 1.7322 & 1.8120 & -0.0798 & $-4.4 \%$ \\
\hline SB7b-26 & $\mathrm{PbO}$ & 0.0190 & 0.0170 & 0.0020 & $11.7 \%$ \\
\hline SB7b-26 & $\mathrm{SiO}_{2}$ & 47.4925 & 48.3940 & -0.9015 & $-1.9 \%$ \\
\hline SB7b-26 & $\mathrm{SO}_{4}$ & 0.4584 & 0.5850 & -0.1266 & $-21.6 \%$ \\
\hline SB7b-26 & $\mathrm{ThO}_{2}$ & 0.4402 & 0.4610 & -0.0208 & $-4.5 \%$ \\
\hline SB7b-26 & $\mathrm{TiO}_{2}$ & 0.1741 & 0.1810 & -0.0069 & $-3.8 \%$ \\
\hline SB7b-26 & $\mathrm{U}_{3} \mathrm{O}_{8}$ & 2.7549 & 3.0320 & -0.2771 & $-9.1 \%$ \\
\hline SB7b-26 & $\mathrm{ZnO}$ & 0.0251 & 0.0130 & 0.0121 & $93.4 \%$ \\
\hline SB7b-26 & $\mathrm{ZrO}_{2}$ & 0.0682 & 0.0810 & -0.0128 & $-15.8 \%$ \\
\hline SB7b-26 & Sum & 97.8016 & 100.0000 & -2.1984 & $-2.2 \%$ \\
\hline SB7b-27 & $\mathrm{Al}_{2} \mathrm{O}_{3}$ & 7.3620 & 7.2400 & 0.1220 & $1.7 \%$ \\
\hline SB7b-27 & $\mathrm{B}_{2} \mathrm{O}_{3}$ & 5.0351 & 5.2800 & -0.2449 & $-4.6 \%$ \\
\hline SB7b-27 & $\mathrm{BaO}$ & 0.0266 & 0.0250 & 0.0016 & $6.6 \%$ \\
\hline SB7b-27 & $\mathrm{CaO}$ & 0.3348 & 0.3220 & 0.0128 & $4.0 \%$ \\
\hline SB7b-27 & $\mathrm{Ce}_{2} \mathrm{O}_{3}$ & 0.0358 & 0.0490 & -0.0132 & $-26.9 \%$ \\
\hline SB7b-27 & $\mathrm{Cr}_{2} \mathrm{O}_{3}$ & 0.0351 & 0.0320 & 0.0031 & $9.6 \%$ \\
\hline SB7b-27 & $\mathrm{CuO}$ & 0.0230 & 0.0140 & 0.0090 & $64.5 \%$ \\
\hline SB7b-27 & $\mathrm{Fe}_{2} \mathrm{O}_{3}$ & 10.6763 & 10.5690 & 0.1073 & $1.0 \%$ \\
\hline SB7b-27 & $\mathrm{K}_{2} \mathrm{O}$ & 0.0917 & 0.0330 & 0.0587 & $177.8 \%$ \\
\hline SB7b-27 & $\mathrm{La}_{2} \mathrm{O}_{3}$ & 0.0155 & 0.0080 & 0.0075 & $93.5 \%$ \\
\hline SB7b-27 & $\mathrm{Li}_{2} \mathrm{O}$ & 6.2030 & 6.3000 & -0.0970 & $-1.5 \%$ \\
\hline SB7b-27 & $\mathrm{MgO}$ & 0.1449 & 0.1410 & 0.0039 & $2.8 \%$ \\
\hline SB7b-27 & $\mathrm{MnO}$ & 1.7286 & 1.7190 & 0.0096 & $0.6 \%$ \\
\hline SB7b-27 & $\mathrm{Na}_{2} \mathrm{O}$ & 14.3225 & 14.3090 & 0.0135 & $0.1 \%$ \\
\hline SB7b-27 & $\mathrm{NiO}$ & 1.7990 & 1.8120 & -0.0130 & $-0.7 \%$ \\
\hline SB7b-27 & $\mathrm{PbO}$ & 0.0030 & 0.0080 & -0.0050 & $-62.1 \%$ \\
\hline SB7b-27 & $\mathrm{SiO}_{2}$ & 46.7437 & 48.0330 & -1.2893 & $-2.7 \%$ \\
\hline SB7b-27 & $\mathrm{SO}_{4}$ & 0.8523 & 0.7180 & 0.1343 & $18.7 \%$ \\
\hline SB7b-27 & $\mathrm{ThO}_{2}$ & 0.6904 & 0.7280 & -0.0376 & $-5.2 \%$ \\
\hline SB7b-27 & $\mathrm{TiO}_{2}$ & 0.1760 & 0.1810 & -0.0050 & $-2.8 \%$ \\
\hline SB7b-27 & $\mathrm{U}_{3} \mathrm{O}_{8}$ & 2.2714 & 2.4380 & -0.1666 & $-6.8 \%$ \\
\hline SB7b-27 & $\mathrm{ZnO}$ & 0.0220 & 0.0060 & 0.0160 & $267.5 \%$ \\
\hline SB7b-27 & $\mathrm{ZrO}_{2}$ & 0.0470 & 0.0360 & 0.0110 & $30.7 \%$ \\
\hline SB7b-27 & Sum & 98.6399 & 100.0010 & -1.3611 & $-1.4 \%$ \\
\hline SB7b-28 & $\mathrm{Al}_{2} \mathrm{O}_{3}$ & 7.2722 & 7.2400 & 0.0322 & $0.4 \%$ \\
\hline SB7b-28 & $\mathrm{B}_{2} \mathrm{O}_{3}$ & 5.0351 & 5.2800 & -0.2449 & $-4.6 \%$ \\
\hline SB7b-28 & $\mathrm{BaO}$ & 0.0256 & 0.0250 & 0.0006 & $2.3 \%$ \\
\hline SB7b-28 & $\mathrm{CaO}$ & 0.3281 & 0.3220 & 0.0061 & $1.9 \%$ \\
\hline SB7b-28 & $\mathrm{Ce}_{2} \mathrm{O}_{3}$ & 0.0363 & 0.0490 & -0.0127 & $-25.8 \%$ \\
\hline SB7b-28 & $\mathrm{Cr}_{2} \mathrm{O}_{3}$ & 0.0304 & 0.0320 & -0.0016 & $-5.1 \%$ \\
\hline SB7b-28 & $\mathrm{CuO}$ & 0.0232 & 0.0140 & 0.0092 & $65.4 \%$ \\
\hline SB7b-28 & $\mathrm{Fe}_{2} \mathrm{O}_{3}$ & 10.4297 & 10.5690 & -0.1393 & $-1.3 \%$ \\
\hline SB7b-28 & $\mathrm{K}_{2} \mathrm{O}$ & 0.1345 & 0.0330 & 0.1015 & $307.6 \%$ \\
\hline SB7b-28 & $\mathrm{La}_{2} \mathrm{O}_{3}$ & 0.0099 & 0.0080 & 0.0019 & $23.7 \%$ \\
\hline SB7b-28 & $\mathrm{Li}_{2} \mathrm{O}$ & 5.2450 & 5.3000 & -0.0550 & $-1.0 \%$ \\
\hline SB7b-28 & $\mathrm{MgO}$ & 0.1415 & 0.1410 & 0.0005 & $0.3 \%$ \\
\hline SB7b-28 & $\mathrm{MnO}$ & 2.1466 & 2.1500 & -0.0034 & $-0.2 \%$ \\
\hline SB7b-28 & $\mathrm{Na}_{2} \mathrm{O}$ & 12.0511 & 12.3090 & -0.2579 & $-2.1 \%$ \\
\hline SB7b-28 & $\mathrm{NiO}$ & 1.3727 & 1.3890 & -0.0163 & $-1.2 \%$ \\
\hline SB7b-28 & $\mathrm{PbO}$ & 0.0031 & 0.0080 & -0.0049 & $-61.8 \%$ \\
\hline SB7b-28 & $\mathrm{SiO}_{2}$ & 49.8992 & 50.2030 & -0.3038 & $-0.6 \%$ \\
\hline SB7b-28 & $\mathrm{SO}_{4}$ & 0.3940 & 0.5850 & -0.1910 & $-32.7 \%$ \\
\hline SB7b-28 & $\mathrm{ThO}_{2}$ & 0.6563 & 0.7280 & -0.0717 & $-9.9 \%$ \\
\hline SB7b-28 & $\mathrm{TiO}_{2}$ & 0.5154 & 0.5420 & -0.0266 & $-4.9 \%$ \\
\hline SB7b-28 & $\mathrm{U}_{3} \mathrm{O}_{8}$ & 2.7549 & 3.0320 & -0.2771 & $-9.1 \%$ \\
\hline SB7b-28 & $\mathrm{ZnO}$ & 0.0185 & 0.0060 & 0.0125 & $208.9 \%$ \\
\hline SB7b-28 & $\mathrm{ZrO}_{2}$ & 0.0476 & 0.0360 & 0.0116 & $32.2 \%$ \\
\hline
\end{tabular}


SRNL-STI-2011-00440

Revision 0

Table A-5. Average Measured Versus Target Compositions by Oxide by Glass ID

\begin{tabular}{|c|c|c|c|c|c|}
\hline & & Measured & Target & Difference of & \% Difference of \\
\hline & & & & Measuserd versus & Measuserd versus \\
\hline Glass ID & Oxide & $(\mathrm{wt} \%)$ & (wt\%) & Target & Target \\
\hline SB7b-28 & Sum & 98.5708 & 100.0010 & -1.4302 & $-1.4 \%$ \\
\hline SB7b-29 & $\mathrm{Al}_{2} \mathrm{O}_{3}$ & 7.0903 & 7.0130 & 0.0773 & $1.1 \%$ \\
\hline SB7b-29 & $\mathrm{B}_{2} \mathrm{O}_{3}$ & 5.1438 & 5.4400 & -0.2962 & $-5.4 \%$ \\
\hline SB7b-29 & $\mathrm{BaO}$ & 0.0351 & 0.0330 & 0.0021 & $6.4 \%$ \\
\hline SB7b-29 & $\mathrm{CaO}$ & 0.3729 & 0.3680 & 0.0049 & $1.3 \%$ \\
\hline SB7b-29 & $\mathrm{Ce}_{2} \mathrm{O}_{3}$ & 0.0448 & 0.0660 & -0.0212 & $-32.1 \%$ \\
\hline SB7b-29 & $\mathrm{Cr}_{2} \mathrm{O}_{3}$ & 0.0457 & 0.0430 & 0.0027 & $6.2 \%$ \\
\hline SB7b-29 & $\mathrm{CuO}$ & 0.0241 & 0.0180 & 0.0061 & $33.8 \%$ \\
\hline SB7b-29 & $\mathrm{Fe}_{2} \mathrm{O}_{3}$ & 8.1761 & 8.2730 & -0.0969 & $-1.2 \%$ \\
\hline SB7b-29 & $\mathrm{K}_{2} \mathrm{O}$ & 0.1376 & 0.0440 & 0.0936 & $212.8 \%$ \\
\hline SB7b-29 & $\mathrm{La}_{2} \mathrm{O}_{3}$ & 0.0120 & 0.0110 & 0.0010 & $9.2 \%$ \\
\hline SB7b-29 & $\mathrm{Li}_{2} \mathrm{O}$ & 5.3661 & 5.4400 & -0.0739 & $-1.4 \%$ \\
\hline SB7b-29 & $\mathrm{MgO}$ & 0.1893 & 0.1800 & 0.0093 & $5.1 \%$ \\
\hline SB7b-29 & $\mathrm{MnO}$ & 1.6753 & 1.6730 & 0.0023 & $0.1 \%$ \\
\hline SB7b-29 & $\mathrm{Na}_{2} \mathrm{O}$ & 13.8844 & 13.7510 & 0.1334 & $1.0 \%$ \\
\hline SB7b-29 & $\mathrm{NiO}$ & 1.3886 & 1.3840 & 0.0046 & $0.3 \%$ \\
\hline SB7b-29 & $\mathrm{PbO}$ & 0.0089 & 0.0100 & -0.0011 & $-11.3 \%$ \\
\hline SB7b-29 & $\mathrm{SiO}_{2}$ & 51.1293 & 52.5440 & -1.4147 & $-2.7 \%$ \\
\hline SB7b-29 & $\mathrm{SO}_{4}$ & 0.5453 & 0.5680 & -0.0227 & $-4.0 \%$ \\
\hline SB7b-29 & $\mathrm{ThO}_{2}$ & 0.4934 & 0.4980 & -0.0046 & $-0.9 \%$ \\
\hline SB7b-29 & $\mathrm{TiO}_{2}$ & 0.1993 & 0.2130 & -0.0137 & $-6.4 \%$ \\
\hline SB7b-29 & $\mathrm{U}_{3} \mathrm{O}_{8}$ & 2.2847 & 2.3720 & -0.0873 & $-3.7 \%$ \\
\hline SB7b-29 & $\mathrm{ZnO}$ & 0.0193 & 0.0080 & 0.0113 & $141.0 \%$ \\
\hline SB7b-29 & $\mathrm{ZrO}_{2}$ & 0.0426 & 0.0480 & -0.0054 & $-11.4 \%$ \\
\hline SB7b-29 & Sum & 98.3088 & 99.9980 & -1.6892 & $-1.7 \%$ \\
\hline SB7b-30 & $\mathrm{Al}_{2} \mathrm{O}_{3}$ & 8.0327 & 7.8900 & 0.1427 & $1.8 \%$ \\
\hline SB7b-30 & $\mathrm{B}_{2} \mathrm{O}_{3}$ & 4.9103 & 5.1200 & -0.2097 & $-4.1 \%$ \\
\hline SB7b-30 & $\mathrm{BaO}$ & 0.0402 & 0.0370 & 0.0032 & $8.6 \%$ \\
\hline SB7b-30 & $\mathrm{CaO}$ & 0.4110 & 0.4130 & -0.0020 & $-0.5 \%$ \\
\hline SB7b-30 & $\mathrm{Ce}_{2} \mathrm{O}_{3}$ & 0.0707 & 0.0740 & -0.0033 & $-4.5 \%$ \\
\hline SB7b-30 & $\mathrm{Cr}_{2} \mathrm{O}_{3}$ & 0.0437 & 0.0480 & -0.0043 & $-9.0 \%$ \\
\hline SB7b-30 & $\mathrm{CuO}$ & 0.0219 & 0.0210 & 0.0009 & $4.4 \%$ \\
\hline SB7b-30 & $\mathrm{Fe}_{2} \mathrm{O}_{3}$ & 9.3056 & 9.3070 & -0.0014 & $0.0 \%$ \\
\hline SB7b-30 & $\mathrm{K}_{2} \mathrm{O}$ & 0.1426 & 0.0500 & 0.0926 & $185.2 \%$ \\
\hline SB7b-30 & $\mathrm{La}_{2} \mathrm{O}_{3}$ & 0.0133 & 0.0120 & 0.0013 & $11.2 \%$ \\
\hline SB7b-30 & $\mathrm{Li}_{2} \mathrm{O}$ & 5.0674 & 5.1200 & -0.0526 & $-1.0 \%$ \\
\hline SB7b-30 & $\mathrm{MgO}$ & 0.2083 & 0.2030 & 0.0053 & $2.6 \%$ \\
\hline SB7b-30 & $\mathrm{MnO}$ & 1.8981 & 1.8820 & 0.0161 & $0.9 \%$ \\
\hline SB7b-30 & $\mathrm{Na}_{2} \mathrm{O}$ & 14.4236 & 14.4700 & -0.0464 & $-0.3 \%$ \\
\hline SB7b-30 & $\mathrm{NiO}$ & 1.5493 & 1.5570 & -0.0077 & $-0.5 \%$ \\
\hline SB7b-30 & $\mathrm{PbO}$ & 0.0107 & 0.0120 & -0.0013 & $-10.7 \%$ \\
\hline SB7b-30 & $\mathrm{SiO}_{2}$ & 50.5944 & 49.6120 & 0.9824 & $2.0 \%$ \\
\hline SB7b-30 & $\mathrm{SO}_{4}$ & 0.6681 & 0.6390 & 0.0291 & $4.6 \%$ \\
\hline SB7b-30 & $\mathrm{ThO}_{2}$ & 0.5385 & 0.5600 & -0.0215 & $-3.8 \%$ \\
\hline SB7b-30 & $\mathrm{TiO}_{2}$ & 0.2268 & 0.2400 & -0.0132 & $-5.5 \%$ \\
\hline SB7b-30 & $\mathrm{U}_{3} \mathrm{O}_{8}$ & 2.4984 & 2.6690 & -0.1706 & $-6.4 \%$ \\
\hline SB7b-30 & $\mathrm{ZnO}$ & 0.0200 & 0.0090 & 0.0110 & $122.2 \%$ \\
\hline SB7b-30 & $\mathrm{ZrO}_{2}$ & 0.0464 & 0.0540 & -0.0076 & $-14.1 \%$ \\
\hline SB7b-30 & Sum & 100.7421 & 99.9990 & 0.7431 & $0.7 \%$ \\
\hline SB7b-31 & $\mathrm{Al}_{2} \mathrm{O}_{3}$ & 7.6855 & 8.7670 & -1.0815 & $-12.3 \%$ \\
\hline SB7b-31 & $\mathrm{B}_{2} \mathrm{O}_{3}$ & 5.4457 & 4.8000 & 0.6457 & $13.5 \%$ \\
\hline SB7b-31 & $\mathrm{BaO}$ & 0.0380 & 0.0410 & -0.0030 & $-7.4 \%$ \\
\hline SB7b-31 & $\mathrm{CaO}$ & 0.3991 & 0.4590 & -0.0599 & $-13.0 \%$ \\
\hline SB7b-31 & $\mathrm{Ce}_{2} \mathrm{O}_{3}$ & 0.0632 & 0.0820 & -0.0188 & $-22.9 \%$ \\
\hline SB7b-31 & $\mathrm{Cr}_{2} \mathrm{O}_{3}$ & 0.0472 & 0.0540 & -0.0068 & $-12.5 \%$ \\
\hline SB7b-31 & $\mathrm{CuO}$ & 0.0224 & 0.0230 & -0.0006 & $-2.6 \%$ \\
\hline SB7b-31 & $\mathrm{Fe}_{2} \mathrm{O}_{3}$ & 10.7567 & 10.3410 & 0.4157 & $4.0 \%$ \\
\hline SB7b-31 & $\mathrm{K}_{2} \mathrm{O}$ & 0.0948 & 0.0560 & 0.0388 & $69.3 \%$ \\
\hline SB7b-31 & $\mathrm{La}_{2} \mathrm{O}_{3}$ & 0.0133 & 0.0130 & 0.0003 & $2.5 \%$ \\
\hline SB7b-31 & $\mathrm{Li}_{2} \mathrm{O}$ & 5.0055 & 4.8000 & 0.2055 & $4.3 \%$ \\
\hline SB7b-31 & $\mathrm{MgO}$ & 0.1959 & 0.2260 & -0.0301 & $-13.3 \%$ \\
\hline
\end{tabular}


Table A-5. Average Measured Versus Target Compositions by Oxide by Glass ID

\begin{tabular}{|c|c|c|c|c|c|}
\hline & & Measured & Target & Difference of & \% Difference of \\
\hline & & & & Measuserd versus & Measuserd versus \\
\hline Glass ID & Oxide & (wt\%) & (wt\%) & Target & Target \\
\hline SB7b-31 & $\mathrm{MnO}$ & 1.8093 & 2.0920 & -0.2827 & $-13.5 \%$ \\
\hline SB7b-31 & $\mathrm{Na}_{2} \mathrm{O}$ & 12.3241 & 15.1890 & -2.8649 & $-18.9 \%$ \\
\hline SB7b-31 & $\mathrm{NiO}$ & 1.7879 & 1.7300 & 0.0579 & $3.3 \%$ \\
\hline SB7b-31 & $\mathrm{PbO}$ & 0.0100 & 0.0130 & -0.0030 & $-23.4 \%$ \\
\hline SB7b-31 & $\mathrm{SiO}_{2}$ & 49.7387 & 46.6800 & 3.0587 & $6.6 \%$ \\
\hline SB7b-31 & $\mathrm{SO}_{4}$ & 0.5550 & 0.7100 & -0.1550 & $-21.8 \%$ \\
\hline SB7b-31 & $\mathrm{ThO}_{2}$ & 0.6212 & 0.6220 & -0.0008 & $-0.1 \%$ \\
\hline SB7b-31 & $\mathrm{TiO}_{2}$ & 0.2131 & 0.2660 & -0.0529 & $-19.9 \%$ \\
\hline SB7b-31 & $\mathrm{U}_{3} \mathrm{O}_{8}$ & 2.9613 & 2.9650 & -0.0037 & $-0.1 \%$ \\
\hline SB7b-31 & $\mathrm{ZnO}$ & 0.0243 & 0.0100 & 0.0143 & $143.2 \%$ \\
\hline SB7b-31 & $\mathrm{ZrO}_{2}$ & 0.0535 & 0.0610 & -0.0075 & $-12.3 \%$ \\
\hline SB7b-31 & Sum & 99.8656 & 100.0000 & -0.1344 & $-0.1 \%$ \\
\hline SB7b-32 & $\mathrm{Al}_{2} \mathrm{O}_{3}$ & 8.3303 & 7.2010 & 1.1293 & $15.7 \%$ \\
\hline SB7b-32 & $\mathrm{B}_{2} \mathrm{O}_{3}$ & 4.3710 & 5.4400 & -1.0690 & $-19.7 \%$ \\
\hline SB7b-32 & $\mathrm{BaO}$ & 0.0420 & 0.0340 & 0.0080 & $23.4 \%$ \\
\hline SB7b-32 & $\mathrm{CaO}$ & 0.4432 & 0.3770 & 0.0662 & $17.6 \%$ \\
\hline SB7b-32 & $\mathrm{Ce}_{2} \mathrm{O}_{3}$ & 0.0571 & 0.0670 & -0.0099 & $-14.8 \%$ \\
\hline SB7b-32 & $\mathrm{Cr}_{2} \mathrm{O}_{3}$ & 0.0409 & 0.0440 & -0.0031 & $-7.0 \%$ \\
\hline SB7b-32 & $\mathrm{CuO}$ & 0.0245 & 0.0190 & 0.0055 & $28.9 \%$ \\
\hline SB7b-32 & $\mathrm{Fe}_{2} \mathrm{O}_{3}$ & 8.0385 & 8.4930 & -0.4545 & $-5.4 \%$ \\
\hline SB7b-32 & $\mathrm{K}_{2} \mathrm{O}$ & 0.1430 & 0.0460 & 0.0970 & $210.9 \%$ \\
\hline SB7b-32 & $\mathrm{La}_{2} \mathrm{O}_{3}$ & 0.0142 & 0.0110 & 0.0032 & $28.9 \%$ \\
\hline SB7b-32 & $\mathrm{Li}_{2} \mathrm{O}$ & 6.4049 & 6.8000 & -0.3951 & $-5.8 \%$ \\
\hline SB7b-32 & $\mathrm{MgO}$ & 0.2139 & 0.1850 & 0.0289 & $15.6 \%$ \\
\hline SB7b-32 & $\mathrm{MnO}$ & 2.0078 & 1.7170 & 0.2908 & $16.9 \%$ \\
\hline SB7b-32 & $\mathrm{Na}_{2} \mathrm{O}$ & 14.3899 & 11.7670 & 2.6229 & $22.3 \%$ \\
\hline SB7b-32 & $\mathrm{NiO}$ & 1.3377 & 1.4210 & -0.0833 & $-5.9 \%$ \\
\hline SB7b-32 & $\mathrm{PbO}$ & 0.0090 & 0.0110 & -0.0020 & $-18.5 \%$ \\
\hline SB7b-32 & $\mathrm{SiO}_{2}$ & 49.4713 & 52.5670 & -3.0957 & $-5.9 \%$ \\
\hline SB7b-32 & $\mathrm{SO}_{4}$ & 0.8838 & 0.5830 & 0.3008 & $51.6 \%$ \\
\hline SB7b-32 & $\mathrm{ThO}_{2}$ & 0.4533 & 0.5110 & -0.0577 & $-11.3 \%$ \\
\hline SB7b-32 & $\mathrm{TiO}_{2}$ & 0.2517 & 0.2130 & 0.0387 & $18.2 \%$ \\
\hline SB7b-32 & $\mathrm{U}_{3} \mathrm{O}_{8}$ & 2.1108 & 2.4350 & -0.3242 & $-13.3 \%$ \\
\hline SB7b-32 & $\mathrm{ZnO}$ & 0.0201 & 0.0080 & 0.0121 & $150.7 \%$ \\
\hline SB7b-32 & $\mathrm{ZrO}_{2}$ & 0.0571 & 0.0500 & 0.0071 & $14.1 \%$ \\
\hline SB7b-32 & Sum & 99.1158 & 100.0000 & -0.8842 & $-0.9 \%$ \\
\hline SB7b-33 & $\mathrm{Al}_{2} \mathrm{O}_{3}$ & 8.2382 & 8.1010 & 0.1372 & $1.7 \%$ \\
\hline SB7b-33 & $\mathrm{B}_{2} \mathrm{O}_{3}$ & 4.8942 & 5.1200 & -0.2258 & $-4.4 \%$ \\
\hline SB7b-33 & $\mathrm{BaO}$ & 0.0394 & 0.0380 & 0.0014 & $3.8 \%$ \\
\hline SB7b-33 & $\mathrm{CaO}$ & 0.4229 & 0.4240 & -0.0011 & $-0.3 \%$ \\
\hline SB7b-33 & $\mathrm{Ce}_{2} \mathrm{O}_{3}$ & 0.0655 & 0.0760 & -0.0105 & $-13.8 \%$ \\
\hline SB7b-33 & $\mathrm{Cr}_{2} \mathrm{O}_{3}$ & 0.0449 & 0.0500 & -0.0051 & $-10.3 \%$ \\
\hline SB7b-33 & $\mathrm{CuO}$ & 0.0251 & 0.0210 & 0.0041 & $19.7 \%$ \\
\hline SB7b-33 & $\mathrm{Fe}_{2} \mathrm{O}_{3}$ & 9.5147 & 9.5540 & -0.0393 & $-0.4 \%$ \\
\hline SB7b-33 & $\mathrm{K}_{2} \mathrm{O}$ & 0.0994 & 0.0510 & 0.0484 & $94.9 \%$ \\
\hline SB7b-33 & $\mathrm{La}_{2} \mathrm{O}_{3}$ & 0.0126 & 0.0120 & 0.0006 & $4.9 \%$ \\
\hline SB7b-33 & $\mathrm{Li}_{2} \mathrm{O}$ & 6.3376 & 6.4000 & -0.0624 & $-1.0 \%$ \\
\hline SB7b-33 & $\mathrm{MgO}$ & 0.2102 & 0.2080 & 0.0022 & $1.1 \%$ \\
\hline SB7b-33 & $\mathrm{MnO}$ & 1.9497 & 1.9320 & 0.0177 & $0.9 \%$ \\
\hline SB7b-33 & $\mathrm{Na}_{2} \mathrm{O}$ & 12.6341 & 12.4880 & 0.1461 & $1.2 \%$ \\
\hline SB7b-33 & $\mathrm{NiO}$ & 1.5747 & 1.5980 & -0.0233 & $-1.5 \%$ \\
\hline SB7b-33 & $\mathrm{PbO}$ & 0.0094 & 0.0120 & -0.0026 & $-21.9 \%$ \\
\hline SB7b-33 & $\mathrm{SiO}_{2}$ & 48.0273 & 49.6380 & -1.6107 & $-3.2 \%$ \\
\hline SB7b-33 & $\mathrm{SO}_{4}$ & 0.5625 & 0.6550 & -0.0925 & $-14.1 \%$ \\
\hline SB7b-33 & $\mathrm{ThO}_{2}$ & 0.5482 & 0.5750 & -0.0268 & $-4.7 \%$ \\
\hline SB7b-33 & $\mathrm{TiO}_{2}$ & 0.2329 & 0.2400 & -0.0071 & $-3.0 \%$ \\
\hline SB7b-33 & $\mathrm{U}_{3} \mathrm{O}_{8}$ & 2.5382 & 2.7400 & -0.2018 & $-7.4 \%$ \\
\hline SB7b-33 & $\mathrm{ZnO}$ & 0.0214 & 0.0090 & 0.0124 & $138.1 \%$ \\
\hline SB7b-33 & $\mathrm{ZrO}_{2}$ & 0.0583 & 0.0560 & 0.0023 & $4.0 \%$ \\
\hline SB7b-33 & Sum & 98.0614 & 99.9980 & -1.9366 & $-1.9 \%$ \\
\hline SB7b-34 & $\mathrm{Al}_{2} \mathrm{O}_{3}$ & 9.0554 & 9.0010 & 0.0544 & $0.6 \%$ \\
\hline
\end{tabular}


SRNL-STI-2011-00440

Revision 0

Table A-5. Average Measured Versus Target Compositions by Oxide by Glass ID

\begin{tabular}{|c|c|c|c|c|c|}
\hline & & Measured & Target & Difference of & \% Difference of \\
\hline & & & & Measuserd versus & Measuserd versus \\
\hline Glass ID & Oxide & $(\mathrm{wt} \%)$ & (wt\%) & Target & Target \\
\hline SB7b-34 & $\mathrm{B}_{2} \mathrm{O}_{3}$ & 4.5924 & 4.8000 & -0.2076 & $-4.3 \%$ \\
\hline SB7b-34 & $\mathrm{BaO}$ & 0.0474 & 0.0420 & 0.0054 & $12.9 \%$ \\
\hline SB7b-34 & $\mathrm{CaO}$ & 0.4750 & 0.4710 & 0.0040 & $0.9 \%$ \\
\hline SB7b-34 & $\mathrm{Ce}_{2} \mathrm{O}_{3}$ & 0.0756 & 0.0840 & -0.0084 & $-10.0 \%$ \\
\hline SB7b-34 & $\mathrm{Cr}_{2} \mathrm{O}_{3}$ & 0.0505 & 0.0550 & -0.0045 & $-8.2 \%$ \\
\hline SB7b-34 & $\mathrm{CuO}$ & 0.0269 & 0.0240 & 0.0029 & $11.9 \%$ \\
\hline SB7b-34 & $\mathrm{Fe}_{2} \mathrm{O}_{3}$ & 10.4475 & 10.6160 & -0.1685 & $-1.6 \%$ \\
\hline SB7b-34 & $\mathrm{K}_{2} \mathrm{O}$ & 0.1420 & 0.0570 & 0.0850 & $149.1 \%$ \\
\hline SB7b-34 & $\mathrm{La}_{2} \mathrm{O}_{3}$ & 0.0169 & 0.0140 & 0.0029 & $20.4 \%$ \\
\hline SB7b-34 & $\mathrm{Li}_{2} \mathrm{O}$ & 5.9312 & 6.0000 & -0.0688 & $-1.1 \%$ \\
\hline SB7b-34 & $\mathrm{MgO}$ & 0.2359 & 0.2320 & 0.0039 & $1.7 \%$ \\
\hline SB7b-34 & $\mathrm{MnO}$ & 2.1515 & 2.1470 & 0.0045 & $0.2 \%$ \\
\hline SB7b-34 & $\mathrm{Na}_{2} \mathrm{O}$ & 13.1936 & 13.2090 & -0.0154 & $-0.1 \%$ \\
\hline SB7b-34 & $\mathrm{NiO}$ & 1.7354 & 1.7760 & -0.0406 & $-2.3 \%$ \\
\hline SB7b-34 & $\mathrm{PbO}$ & 0.0102 & 0.0130 & -0.0028 & $-21.3 \%$ \\
\hline SB7b-34 & $\mathrm{SiO}_{2}$ & 46.7972 & 46.7090 & 0.0882 & $0.2 \%$ \\
\hline SB7b-34 & $\mathrm{SO}_{4}$ & 0.6628 & 0.7280 & -0.0652 & $-9.0 \%$ \\
\hline SB7b-34 & $\mathrm{ThO}_{2}$ & 0.6197 & 0.6390 & -0.0193 & $-3.0 \%$ \\
\hline SB7b-34 & $\mathrm{TiO}_{2}$ & 0.2579 & 0.2670 & -0.0091 & $-3.4 \%$ \\
\hline SB7b-34 & $\mathrm{U}_{3} \mathrm{O}_{8}$ & 2.8448 & 3.0440 & -0.1992 & $-6.5 \%$ \\
\hline SB7b-34 & $\mathrm{ZnO}$ & 0.0242 & 0.0100 & 0.0142 & $142.3 \%$ \\
\hline SB7b-34 & $\mathrm{ZrO}_{2}$ & 0.0592 & 0.0620 & -0.0028 & $-4.6 \%$ \\
\hline SB7b-34 & Sum & 99.4532 & 100.0000 & -0.5468 & $-0.5 \%$ \\
\hline SB7Ref & $\mathrm{Al}_{2} \mathrm{O}_{3}$ & 9.0425 & 8.8920 & 0.1505 & $1.7 \%$ \\
\hline SB7Ref & $\mathrm{B}_{2} \mathrm{O}_{3}$ & 5.0574 & 5.2610 & -0.2036 & $-3.9 \%$ \\
\hline SB7Ref & $\mathrm{BaO}$ & 0.0200 & 0.0170 & 0.0030 & $17.6 \%$ \\
\hline SB7Ref & $\mathrm{CaO}$ & 0.4912 & 0.4760 & 0.0152 & $3.2 \%$ \\
\hline SB7Ref & $\mathrm{Ce}_{2} \mathrm{O}_{3}$ & 0.0249 & 0.0240 & 0.0009 & $3.6 \%$ \\
\hline SB7Ref & $\mathrm{Cr}_{2} \mathrm{O}_{3}$ & 0.1249 & 0.1240 & 0.0009 & $0.8 \%$ \\
\hline SB7Ref & $\mathrm{CuO}$ & 0.0492 & 0.0330 & 0.0162 & $49.0 \%$ \\
\hline SB7Ref & $\mathrm{Fe}_{2} \mathrm{O}_{3}$ & 8.7788 & 8.6680 & 0.1108 & $1.3 \%$ \\
\hline SB7Ref & $\mathrm{K}_{2} \mathrm{O}$ & 0.3540 & 0.2120 & 0.1420 & $67.0 \%$ \\
\hline SB7Ref & $\mathrm{La}_{2} \mathrm{O}_{3}$ & 0.0124 & 0.0140 & -0.0016 & $-11.6 \%$ \\
\hline SB7Ref & $\mathrm{Li}_{2} \mathrm{O}$ & 5.2075 & 5.2610 & -0.0535 & $-1.0 \%$ \\
\hline SB7Ref & $\mathrm{MgO}$ & 0.2633 & 0.0270 & 0.2363 & $875.3 \%$ \\
\hline SB7Ref & $\mathrm{MnO}$ & 2.0997 & 2.0740 & 0.0257 & $1.2 \%$ \\
\hline SB7Ref & $\mathrm{Na}_{2} \mathrm{O}$ & 14.8639 & 14.6820 & 0.1819 & $1.2 \%$ \\
\hline SB7Ref & $\mathrm{NiO}$ & 1.2249 & 1.2230 & 0.0019 & $0.2 \%$ \\
\hline SB7Ref & $\mathrm{PbO}$ & 0.0045 & 0.0060 & -0.0015 & $-25.5 \%$ \\
\hline SB7Ref & $\mathrm{SiO}_{2}$ & 47.9916 & 51.5360 & -3.5444 & $-6.9 \%$ \\
\hline SB7Ref & $\mathrm{SO}_{4}$ & 0.5738 & 0.5860 & -0.0122 & $-2.1 \%$ \\
\hline SB7Ref & $\mathrm{ThO}_{2}$ & 0.0161 & 0.0000 & 0.0161 & \\
\hline SB7Ref & $\mathrm{TiO}_{2}$ & 0.4571 & 0.4860 & -0.0289 & $-5.9 \%$ \\
\hline SB7Ref & $\mathrm{U}_{3} \mathrm{O}_{8}$ & 0.1503 & 0.0000 & 0.1503 & \\
\hline SB7Ref & $\mathrm{ZnO}$ & 0.0251 & 0.0100 & 0.0151 & $150.9 \%$ \\
\hline SB7Ref & $\mathrm{ZrO}_{2}$ & 0.0780 & 0.1460 & -0.0680 & $-46.6 \%$ \\
\hline SB7Ref & Sum & 96.9113 & 99.7580 & -2.8467 & $-2.9 \%$ \\
\hline Ustd & $\mathrm{Al}_{2} \mathrm{O}_{3}$ & 3.9069 & 4.1000 & -0.1931 & $-4.7 \%$ \\
\hline Ustd & $\mathrm{B}_{2} \mathrm{O}_{3}$ & 8.6717 & 9.2090 & -0.5373 & $-5.8 \%$ \\
\hline Ustd & $\mathrm{BaO}$ & 0.0024 & 0.0000 & 0.0024 & \\
\hline Ustd & $\mathrm{CaO}$ & 1.2708 & 1.3010 & -0.0302 & $-2.3 \%$ \\
\hline Ustd & $\mathrm{Ce}_{2} \mathrm{O}_{3}$ & 0.0181 & 0.0000 & 0.0181 & \\
\hline Ustd & $\mathrm{Cr}_{2} \mathrm{O}_{3}$ & 0.2409 & 0.0000 & 0.2409 & \\
\hline Ustd & $\mathrm{CuO}$ & 0.0060 & 0.0000 & 0.0060 & \\
\hline Ustd & $\mathrm{Fe}_{2} \mathrm{O}_{3}$ & 13.2967 & 13.1960 & 0.1007 & $0.8 \%$ \\
\hline Ustd & $\mathrm{K}_{2} \mathrm{O}$ & 2.9511 & 2.9990 & -0.0479 & $-1.6 \%$ \\
\hline Ustd & $\mathrm{La}_{2} \mathrm{O}_{3}$ & 0.0023 & 0.0000 & 0.0023 & \\
\hline Ustd & $\mathrm{Li}_{2} \mathrm{O}$ & 3.0062 & 3.0570 & -0.0508 & $-1.7 \%$ \\
\hline Ustd & $\mathrm{MgO}$ & 1.1803 & 1.2100 & -0.0297 & $-2.5 \%$ \\
\hline Ustd & $\mathrm{MnO}$ & 2.7664 & 2.8920 & -0.1256 & $-4.3 \%$ \\
\hline Ustd & $\mathrm{Na}_{2} \mathrm{O}$ & 11.5479 & 11.7950 & -0.2471 & $-2.1 \%$ \\
\hline
\end{tabular}


SRNL-STI-2011-00440

Revision 0

Table A-5. Average Measured Versus Target Compositions by Oxide by Glass ID

\begin{tabular}{|c|c|c|c|c|c|}
\hline & & Measured & Target & Difference of & \% Difference of \\
\hline & & & & Measuserd versus & Measuserd versus \\
\hline Glass ID & Oxide & (wt\%) & (wt\%) & Target & Target \\
\hline Ustd & $\mathrm{NiO}$ & 1.0861 & 1.1200 & -0.0339 & $-3.0 \%$ \\
\hline Ustd & $\mathrm{PbO}$ & 0.0030 & 0.0000 & 0.0030 & \\
\hline Ustd & $\mathrm{SiO}_{2}$ & 44.4689 & 45.3530 & -0.8841 & $-1.9 \%$ \\
\hline Ustd & $\mathrm{SO}_{4}$ & 0.1143 & 0.0000 & 0.1143 & \\
\hline Ustd & $\mathrm{ThO}_{2}$ & 0.0345 & 0.0000 & 0.0345 & \\
\hline Ustd & $\mathrm{TiO}_{2}$ & 0.9084 & 1.0490 & -0.1406 & $-13.4 \%$ \\
\hline Ustd & $\mathrm{U}_{3} \mathrm{O}_{8}$ & 2.1503 & 2.4060 & -0.2557 & $-10.6 \%$ \\
\hline Ustd & $\mathrm{ZnO}$ & 0.0110 & 0.0000 & 0.0110 & \\
\hline Ustd & $\mathrm{ZrO}_{2}$ & 0.0016 & 0.0000 & 0.0016 & \\
\hline Ustd & Sum & 97.6457 & 99.6870 & -2.0413 & $-2.0 \%$ \\
\hline
\end{tabular}




\section{Exhibit A-1. Measurements of Study Glasses by Lab ID in Analytical Sequence for Analytical Blocks 1, 2, and 3 by Oxide and Preparation Method} Oxide=Al2O3 (wt\%), Prep Method=AR

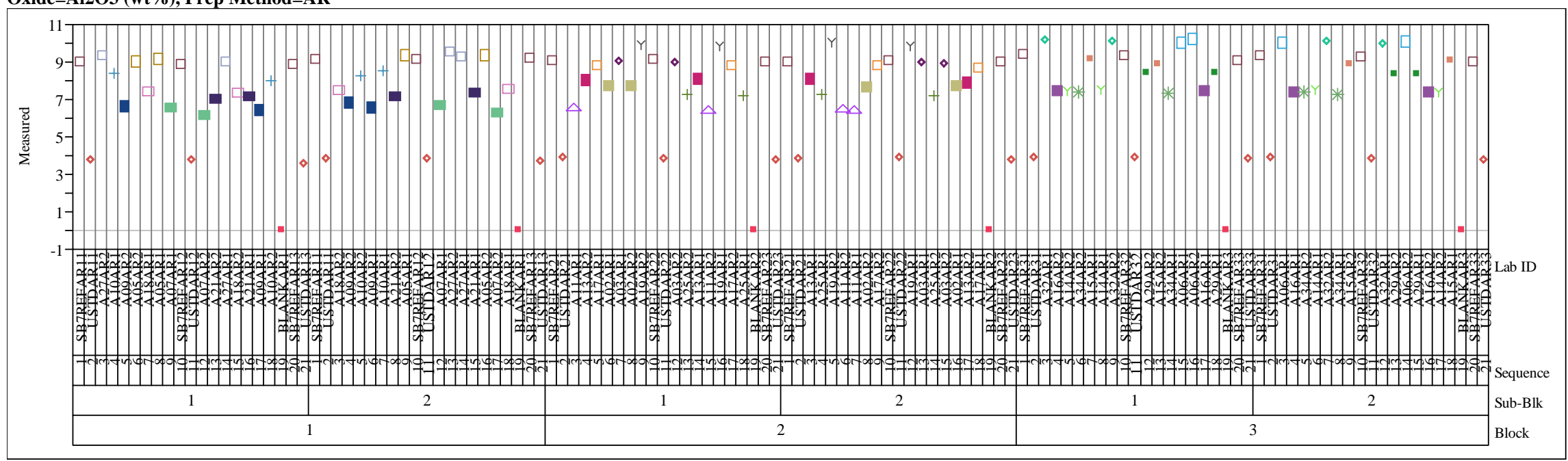

Oxide=Al2O3 (wt\%), Prep Method=PF

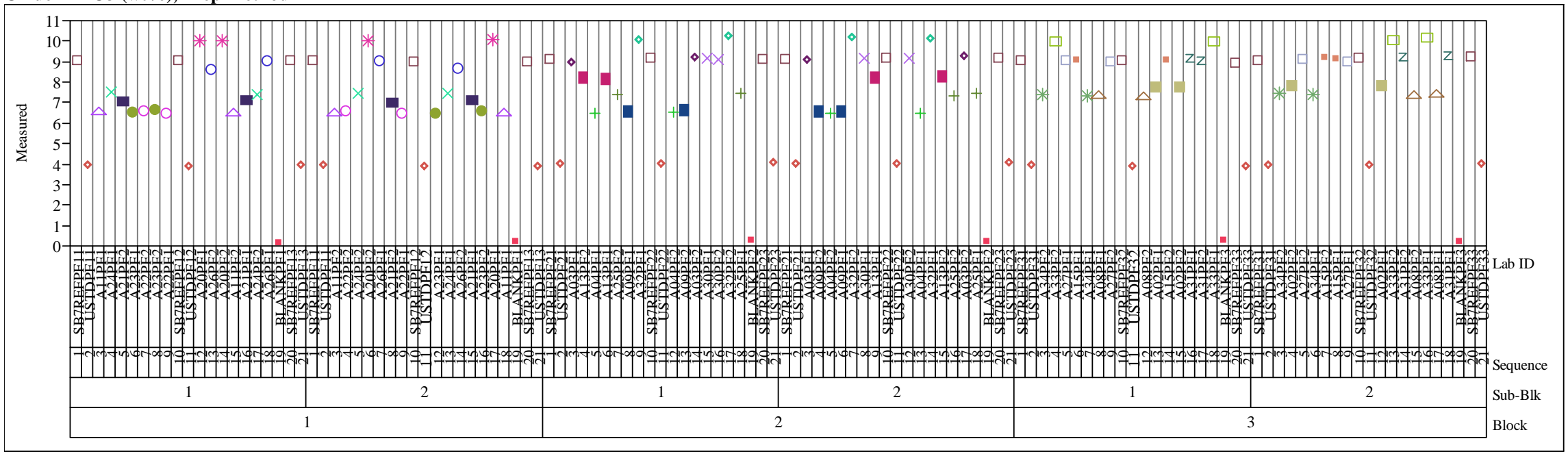


Exhibit A-1. Measurements of Study Glasses by Lab ID in Analytical Sequence for Analytical Blocks 1, 2, and 3 by Oxide and Preparation Method Oxide $=\mathbf{B 2 O} 3$ (wt\%), Prep Method $=$ AR

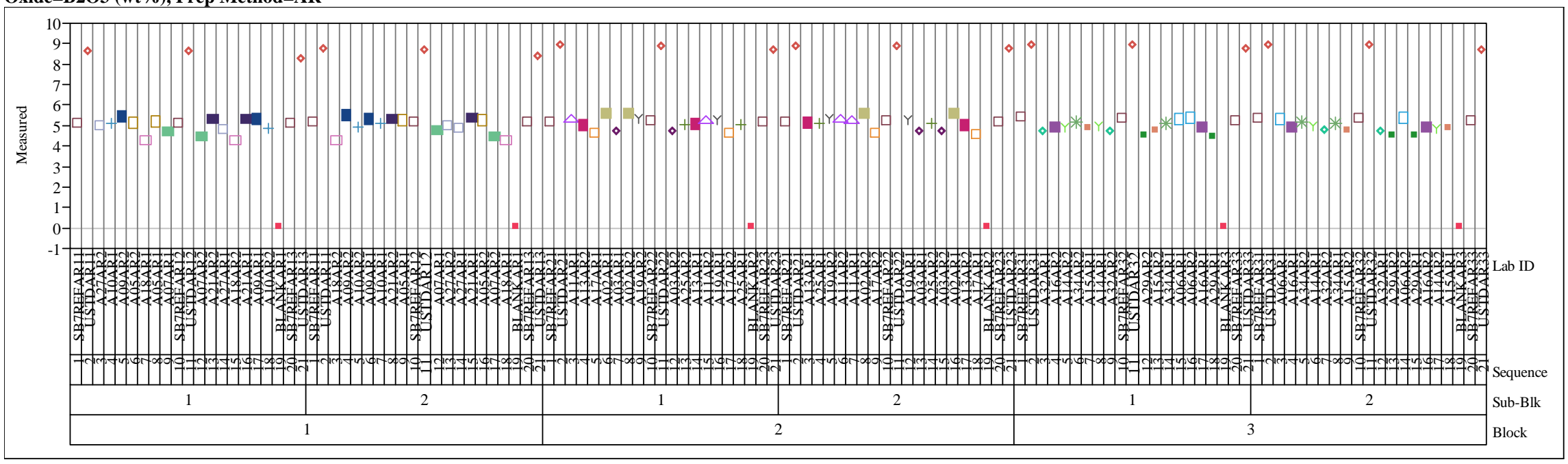

Oxide=B2O3 (wt\%), Prep Method=PF

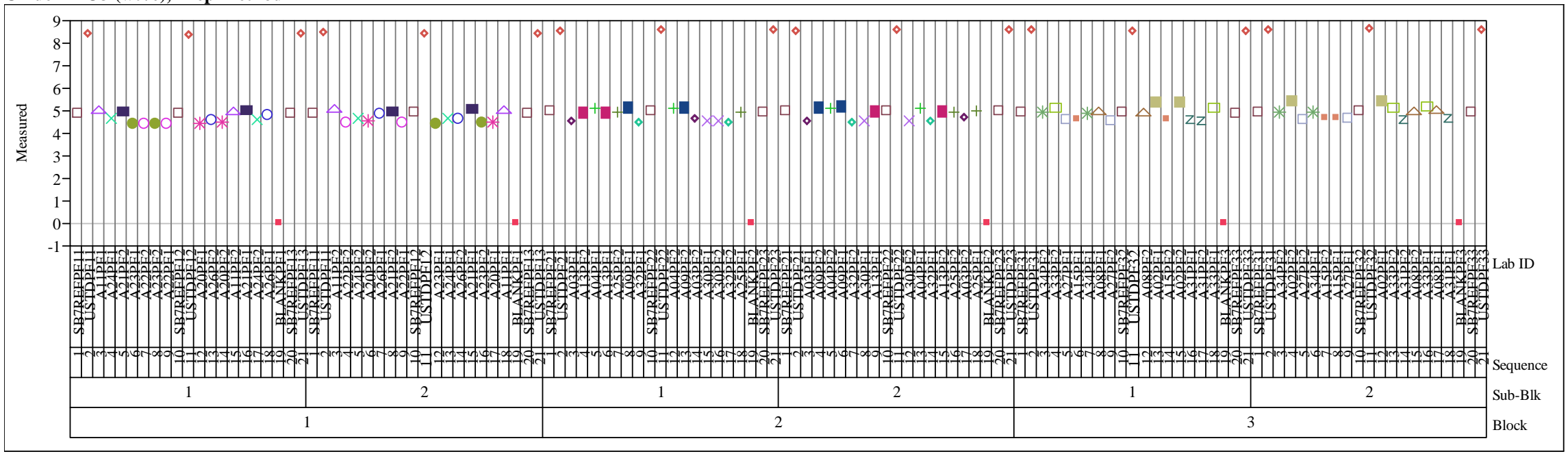


Exhibit A-1. Measurements of Study Glasses by Lab ID in Analytical Sequence for Analytical Blocks 1, 2, and 3 by Oxide and Preparation Method Oxide $=$ BaO (wt $\%)$, Prep Method $=$ AR

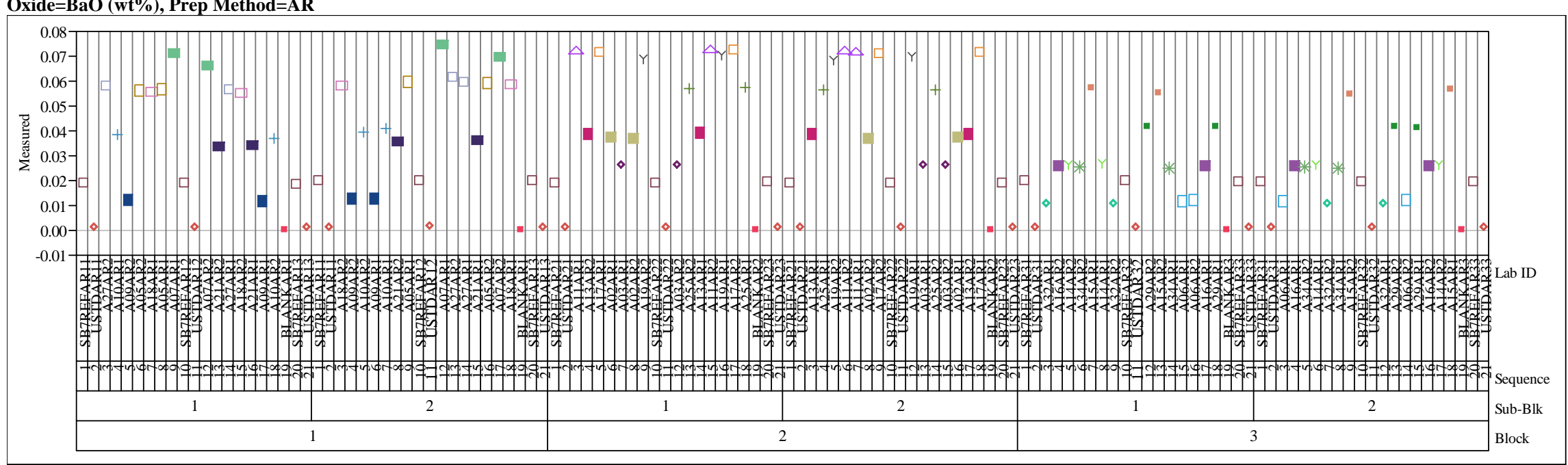

Oxide=BaO (wt\%), Prep Method=PF

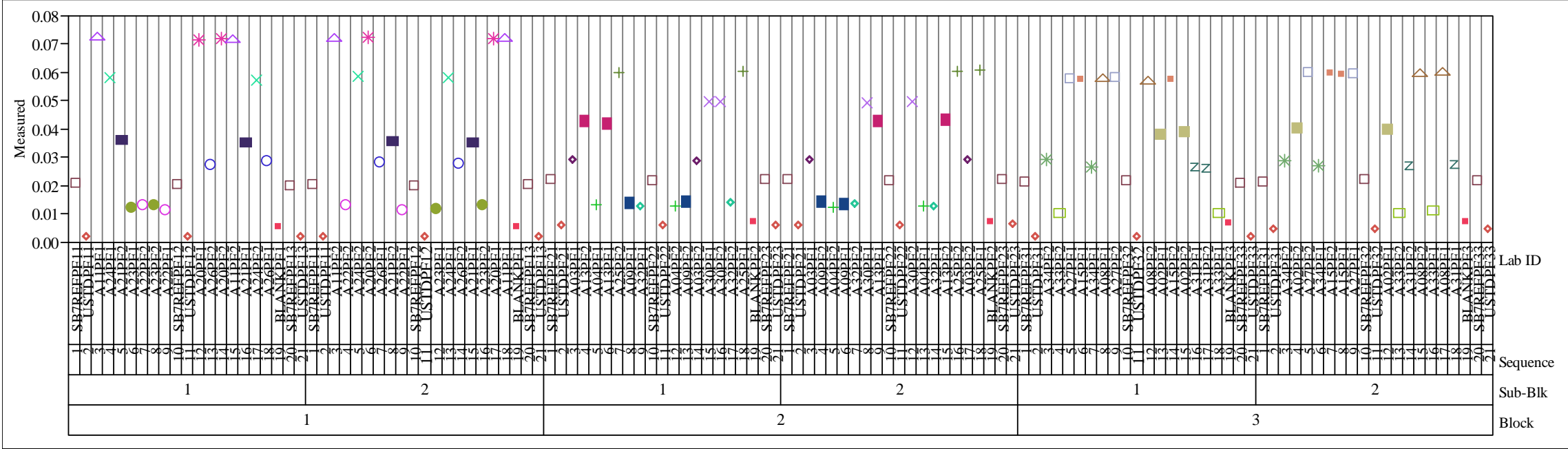




\section{Exhibit A-1. Measurements of Study Glasses by Lab ID in Analytical Sequence for Analytical Blocks 1, 2, and 3 by Oxide and Preparation Method} Oxide $=\mathrm{CaO}($ wt $\%)$, Prep Method $=$ AR

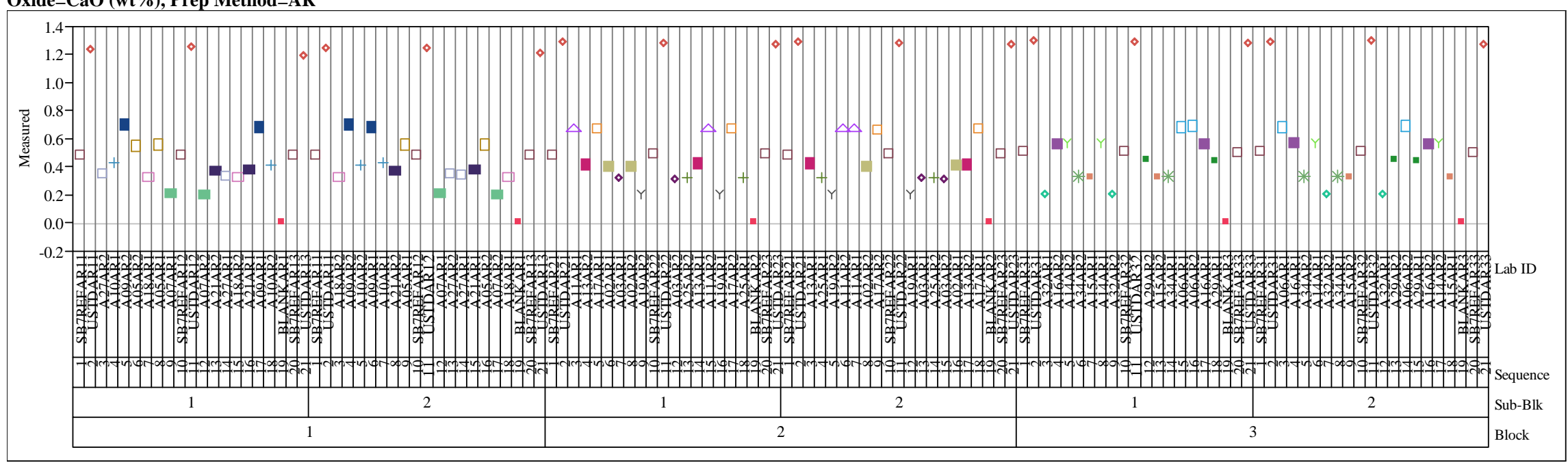

Oxide $=\mathrm{Ce} 2 \mathrm{O} 3($ wt $\%)$, Prep Method $=$ AR

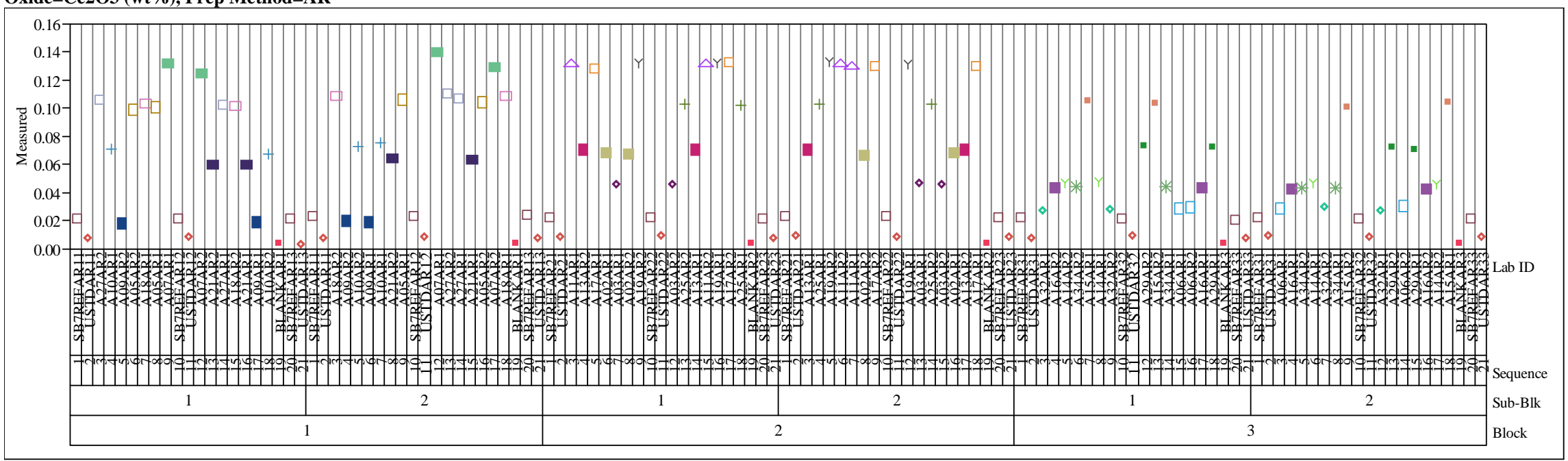


Exhibit A-1. Measurements of Study Glasses by Lab ID in Analytical Sequence for Analytical Blocks 1, 2, and 3 by Oxide and Preparation Method Oxide=Ce2O3 (wt\%), Prep Method=PF

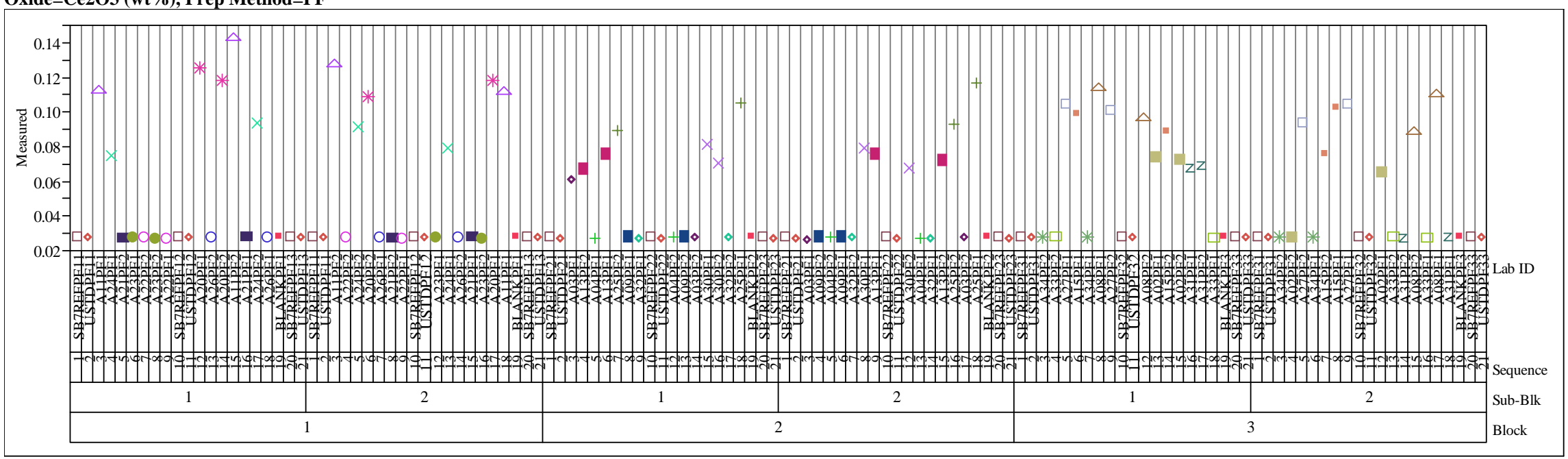

Oxide=Cr2O3 (wt \%), Prep Method $=$ AR

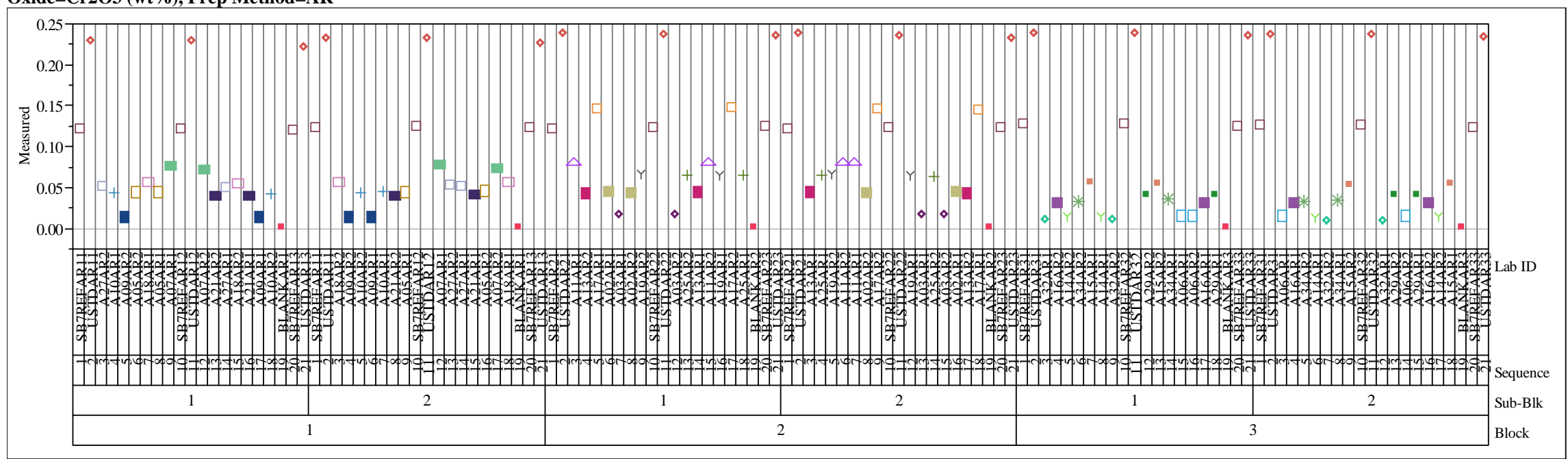


Exhibit A-1. Measurements of Study Glasses by Lab ID in Analytical Sequence for Analytical Blocks 1, 2, and 3 by Oxide and Preparation Method Oxide $=\mathrm{Cr} 2 \mathrm{O} 3(\mathrm{wt} \%)$, Prep Method $=\mathbf{P F}$

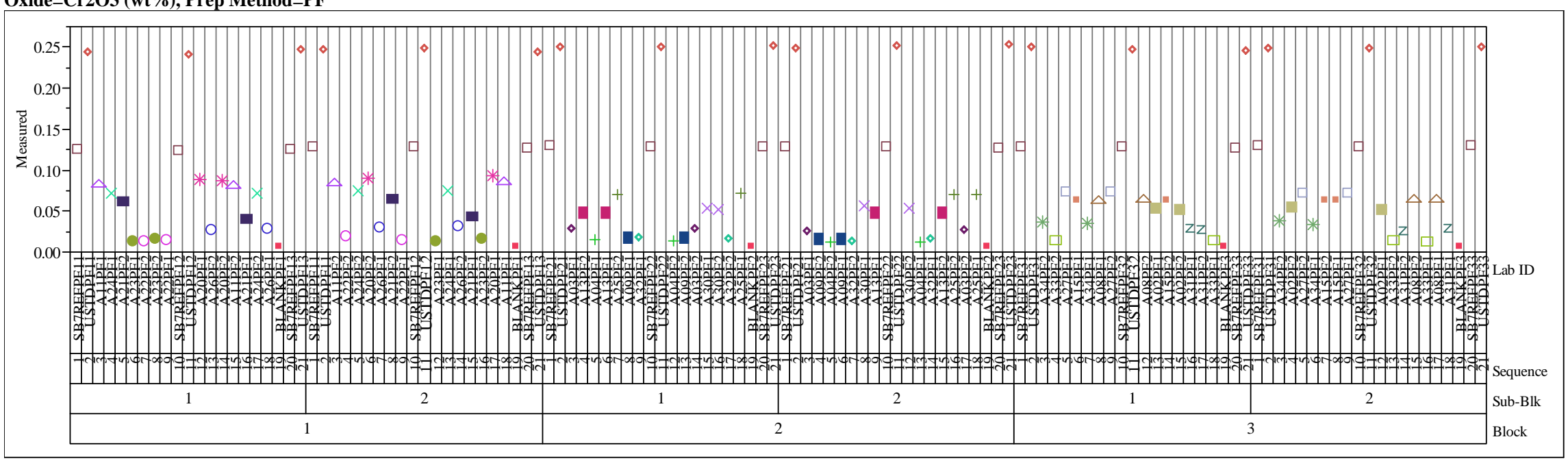

Oxide $=\mathrm{CuO}(w \mathrm{t} \%)$, Prep Method $=\mathrm{AR}$

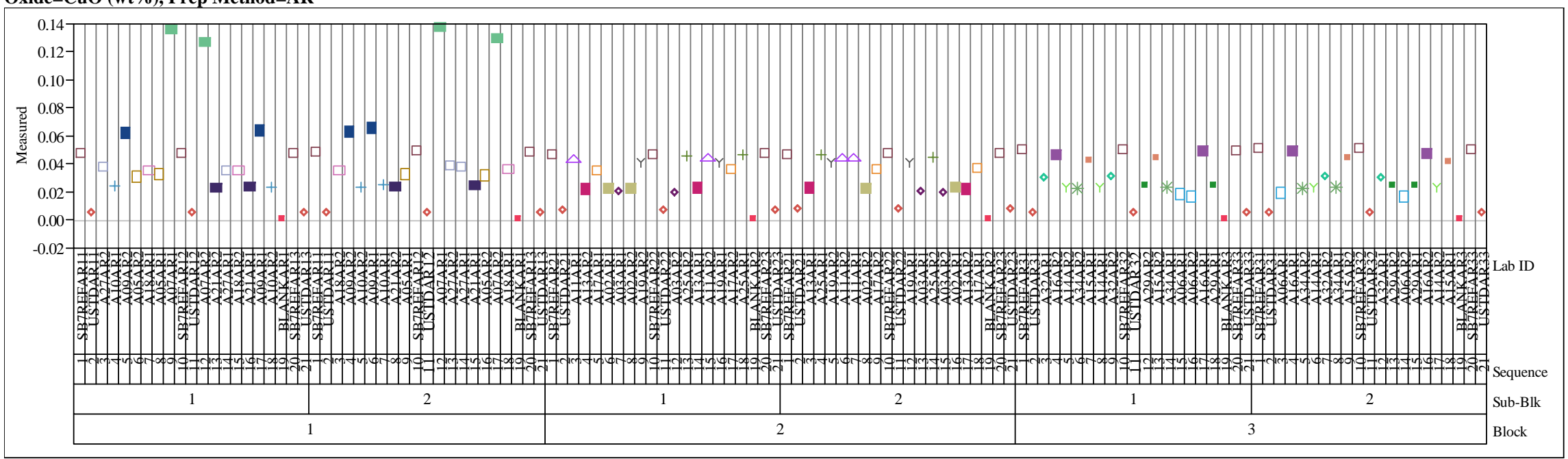


Exhibit A-1. Measurements of Study Glasses by Lab ID in Analytical Sequence for Analytical Blocks 1, 2, and 3 by Oxide and Preparation Method Oxide $=\mathbf{C u O}($ wt $\%)$, Prep Method $=\mathbf{P F}$

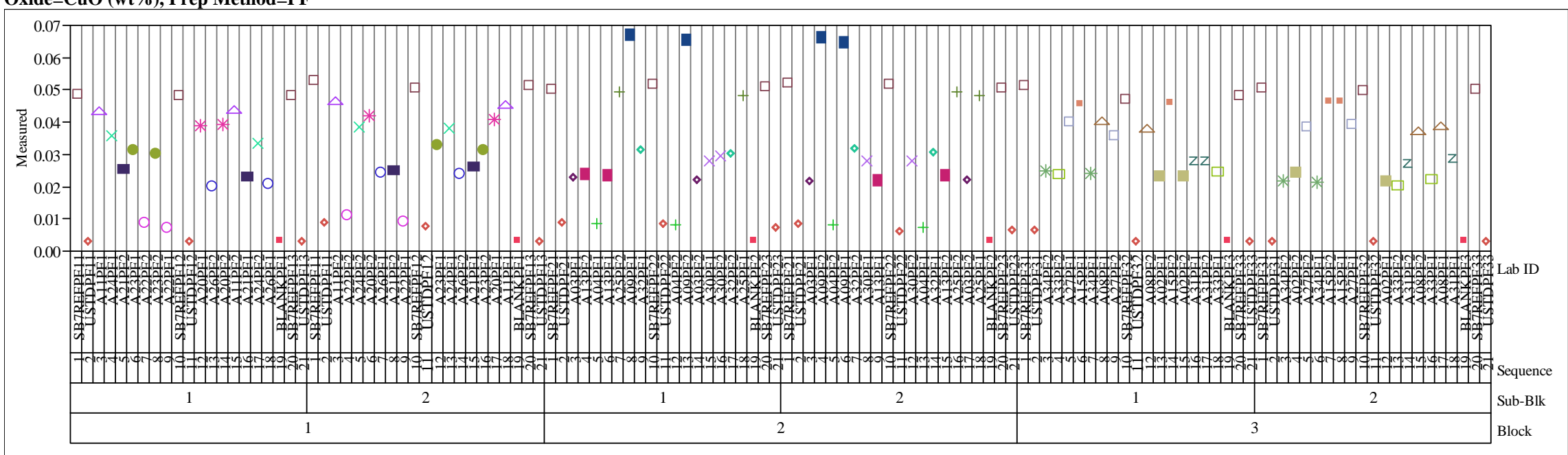

Oxide=Fe2O3 (wt\%), Prep Method=AR

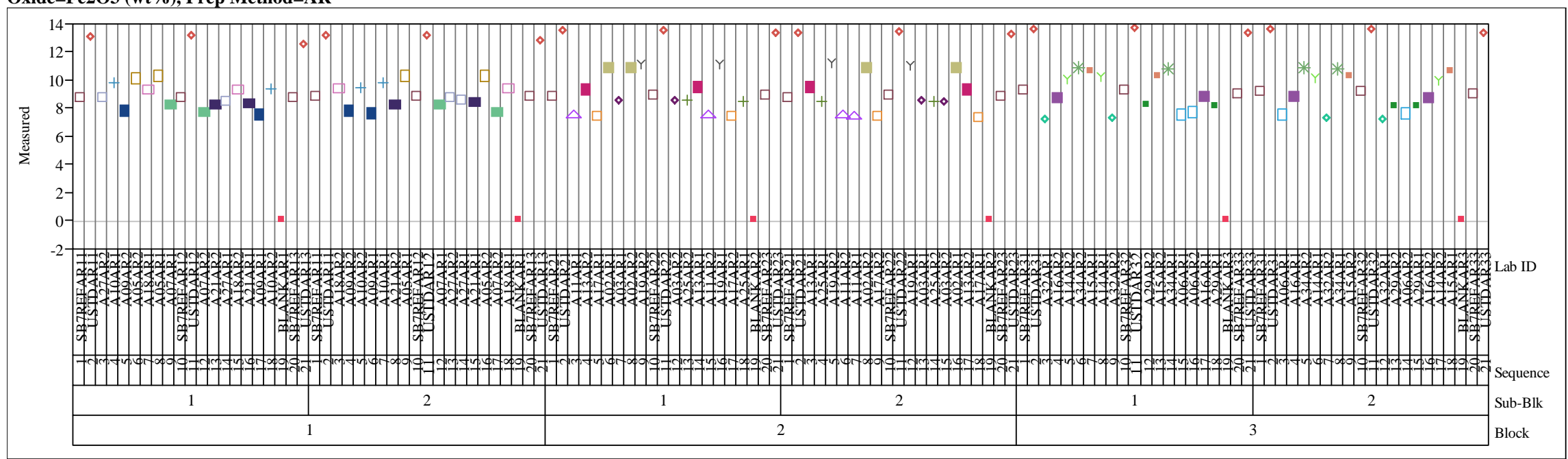


Exhibit A-1. Measurements of Study Glasses by Lab ID in Analytical Sequence for Analytical Blocks 1, 2, and 3 by Oxide and Preparation Method Oxide=Fe2O3 (wt\%), Prep Method=PF

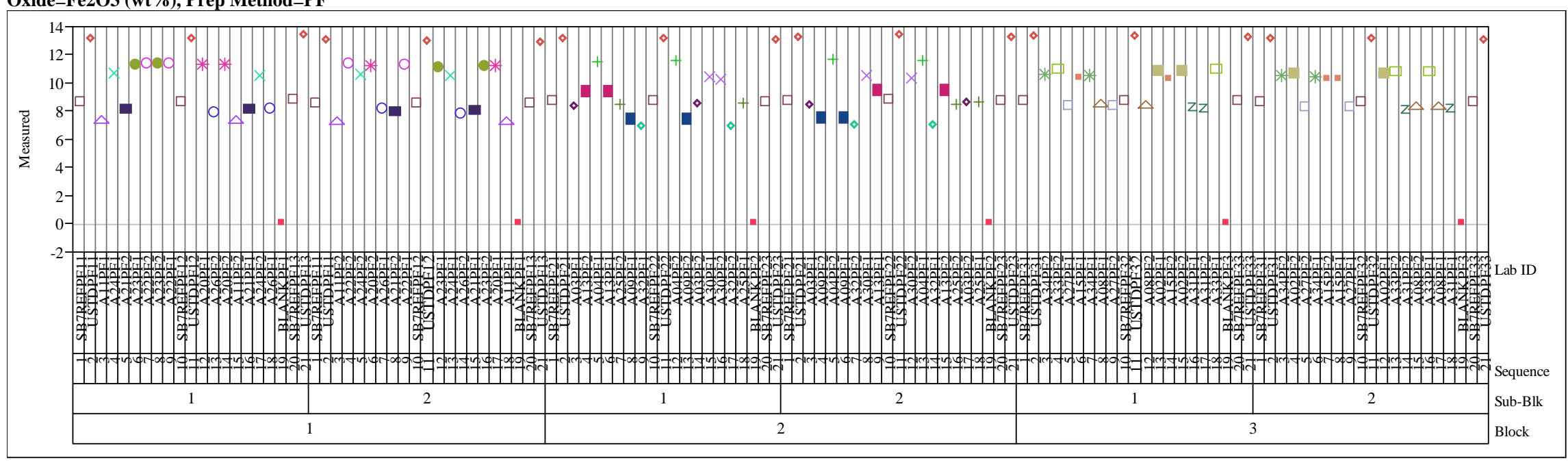

Oxide=K2O (wt \%), Prep Method=AR

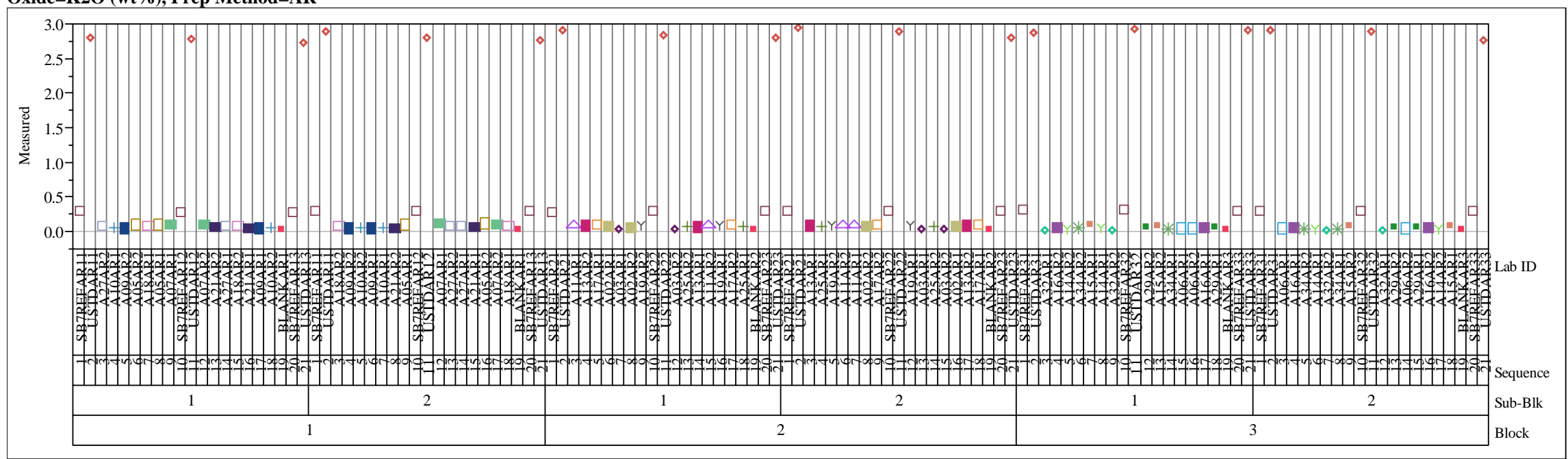




\section{Exhibit A-1. Measurements of Study Glasses by Lab ID in Analytical Sequence for Analytical Blocks 1, 2, and 3 by Oxide and Preparation Method} Oxide=K2O (wt \%), Prep Method=PF

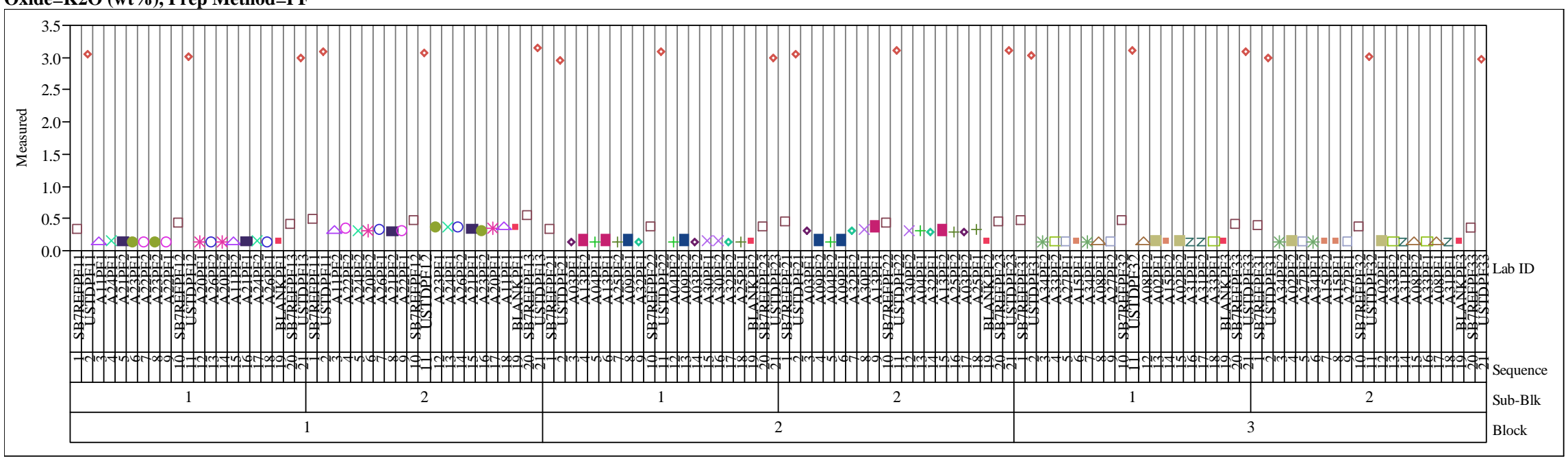

Oxide=La2O3 (wt\%), Prep Method=AR

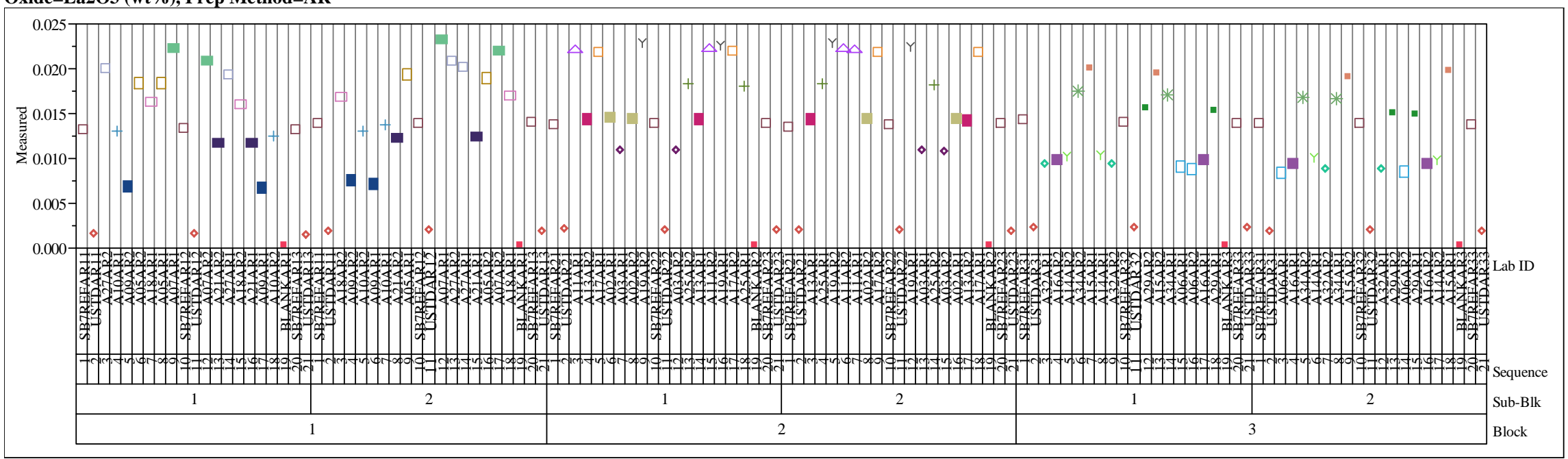


Exhibit A-1. Measurements of Study Glasses by Lab ID in Analytical Sequence for Analytical Blocks 1, 2, and 3 by Oxide and Preparation Method Oxide=La2O3 (wt \%), Prep Method=PF

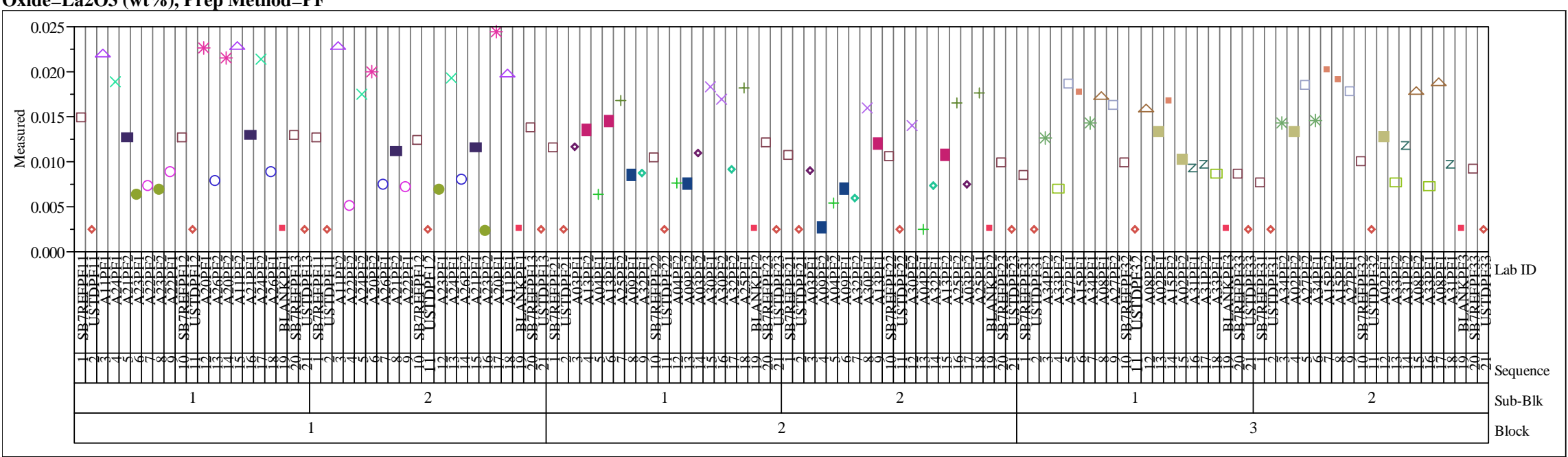

Oxide $=$ Li2O (wt \%), Prep Method $=$ AR

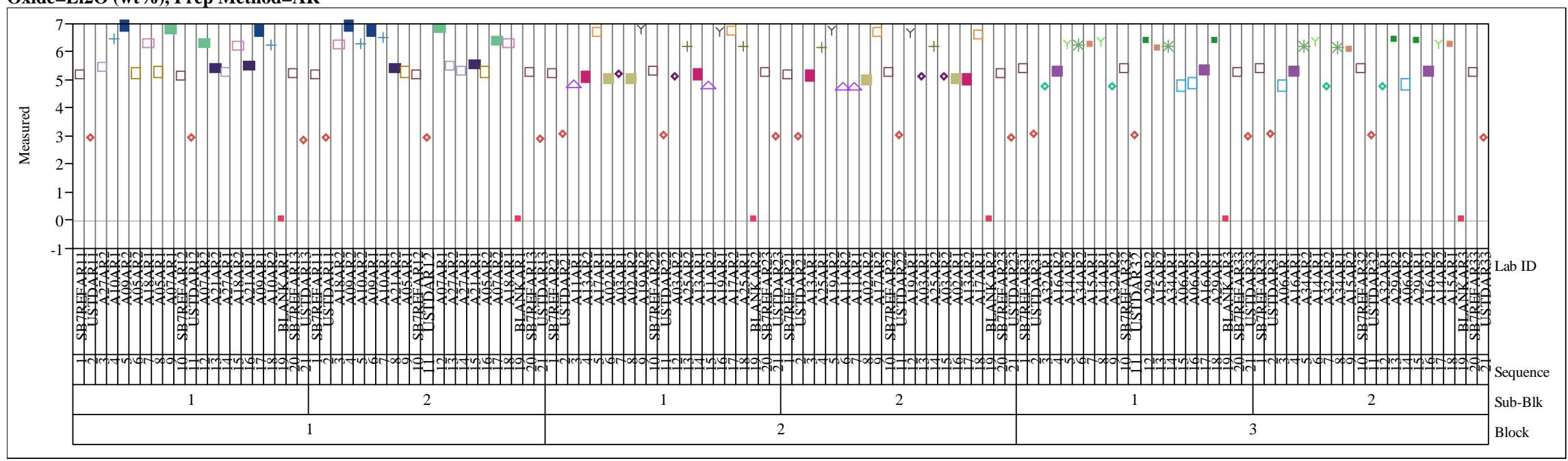




\section{Exhibit A-1. Measurements of Study Glasses by Lab ID in Analytical Sequence for Analytical Blocks 1, 2, and 3 by Oxide and Preparation Method} Oxide $=$ Li2O ( $w$ t $\%)$, Prep Method=PF

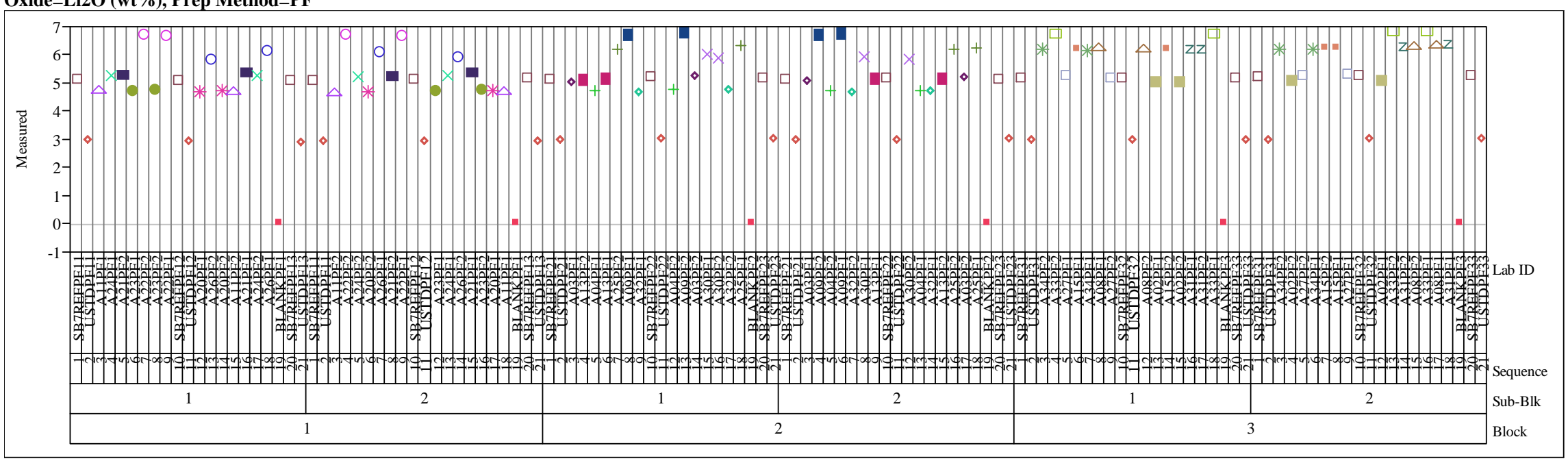

Oxide=MgO (wt \%), Prep Method=AR

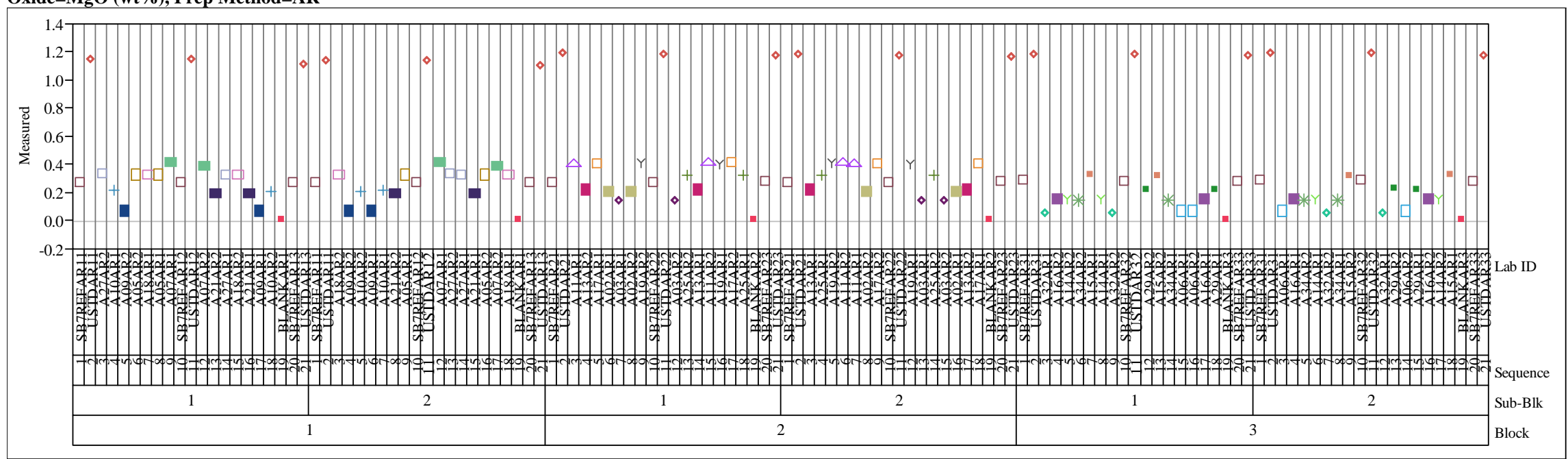




\section{Exhibit A-1. Measurements of Study Glasses by Lab ID in Analytical Sequence for Analytical Blocks 1, 2, and 3 by Oxide and Preparation Method} Oxide $=$ MgO (wt\%), Prep Method $=$ PF

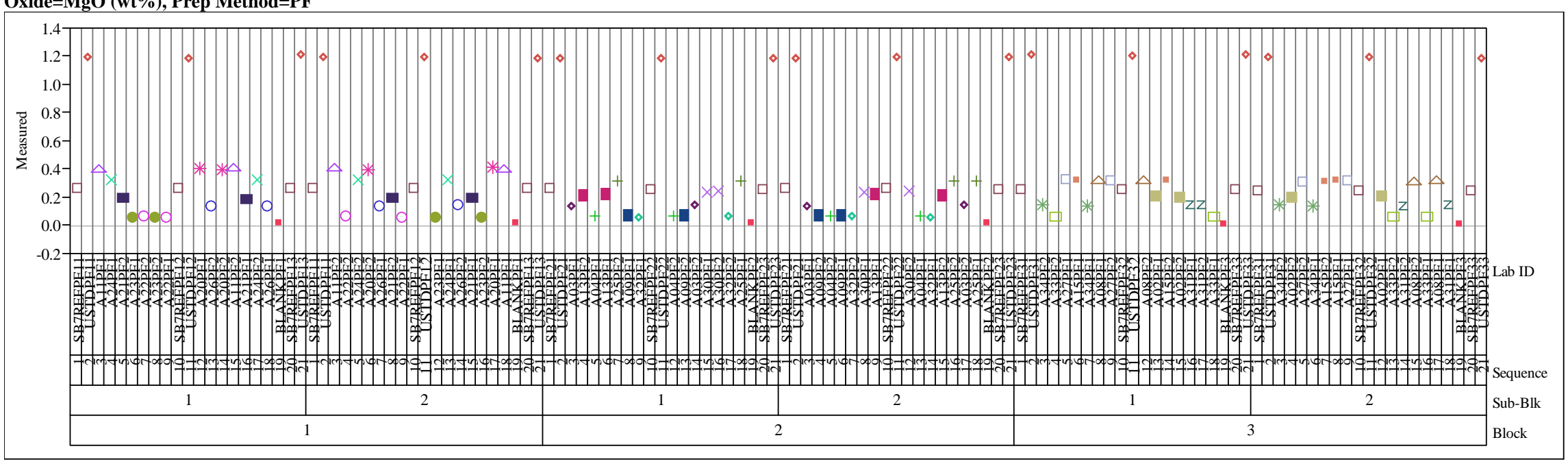

Oxide=MnO (wt\%), Prep Method=AR

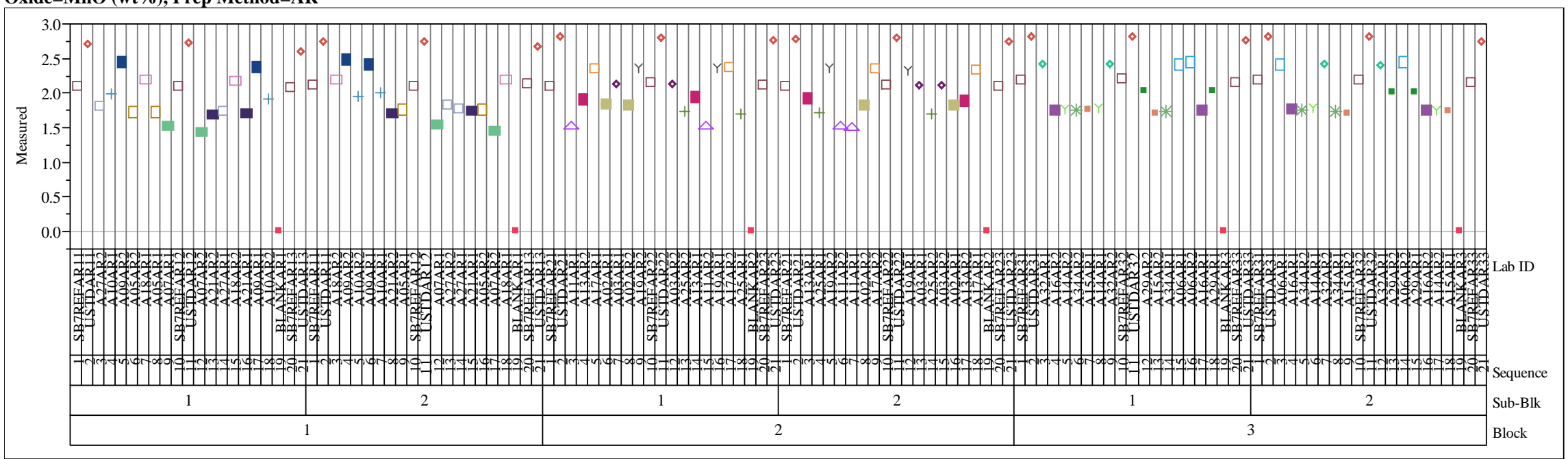


Exhibit A-1. Measurements of Study Glasses by Lab ID in Analytical Sequence for Analytical Blocks 1, 2, and 3 by Oxide and Preparation Method Oxide=MnO (wt\%), Prep Method=PF

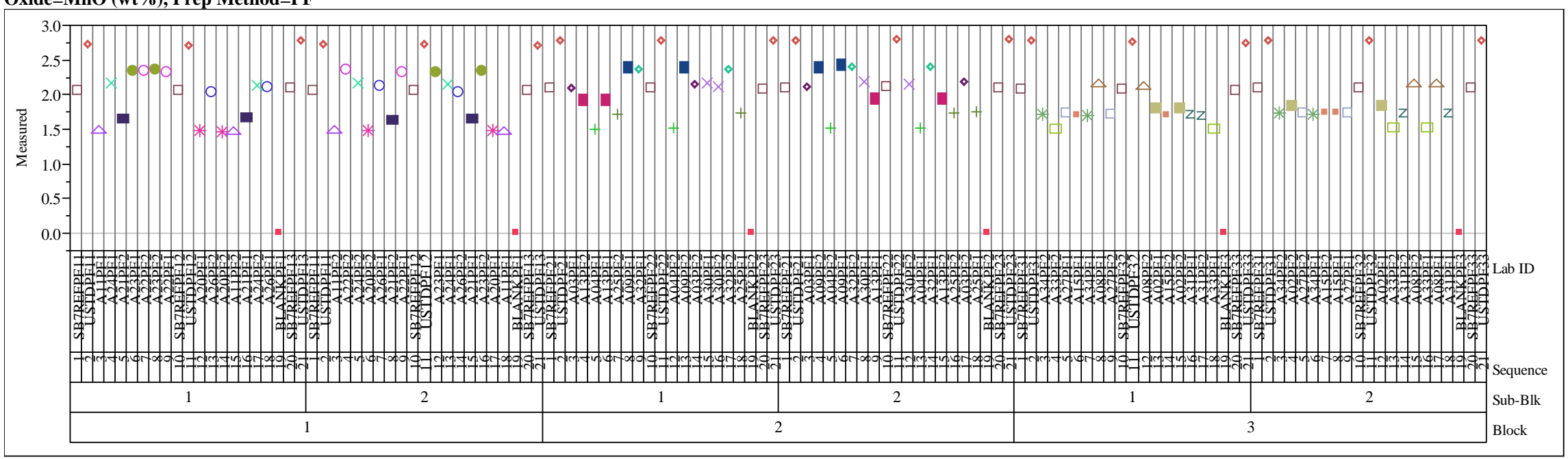

Oxide=Na2O (wt\%), Prep Method=AR

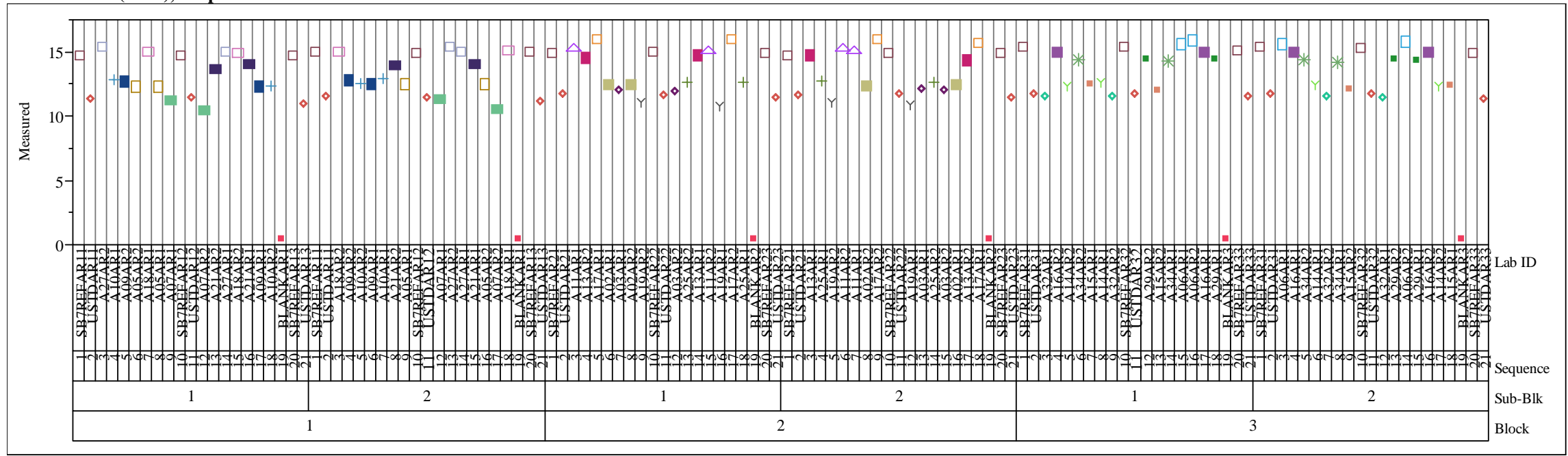


Exhibit A-1. Measurements of Study Glasses by Lab ID in Analytical Sequence for Analytical Blocks 1, 2, and 3 by Oxide and Preparation Method Oxide=NiO (wt \%), Prep Method $=$ AR

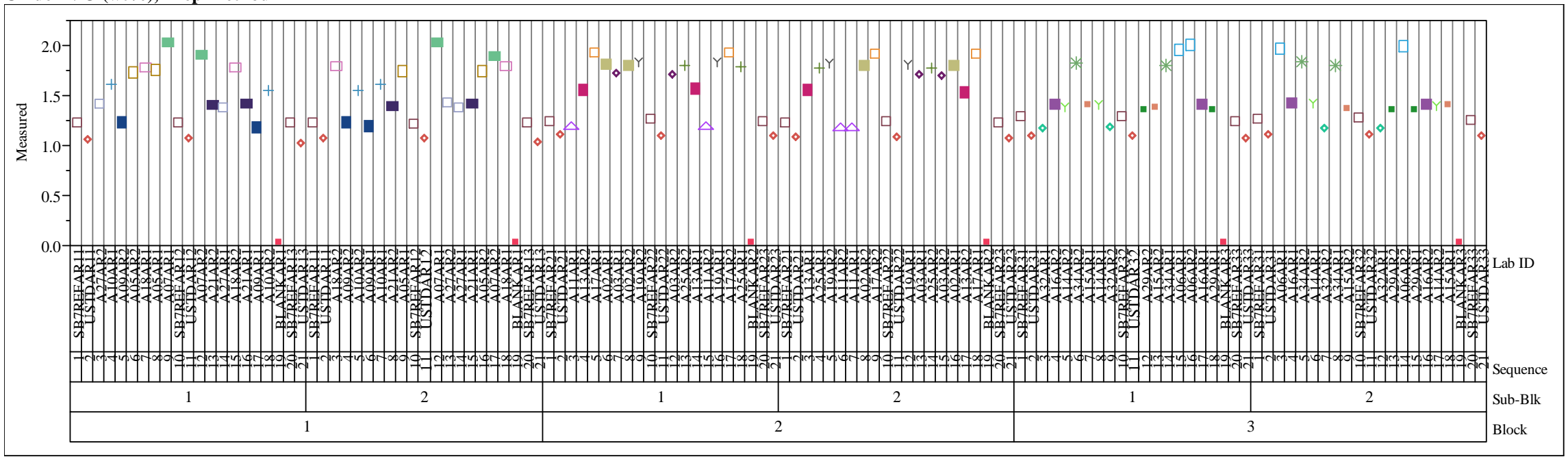

Oxide=NiO (wt\%), Prep Method=PF

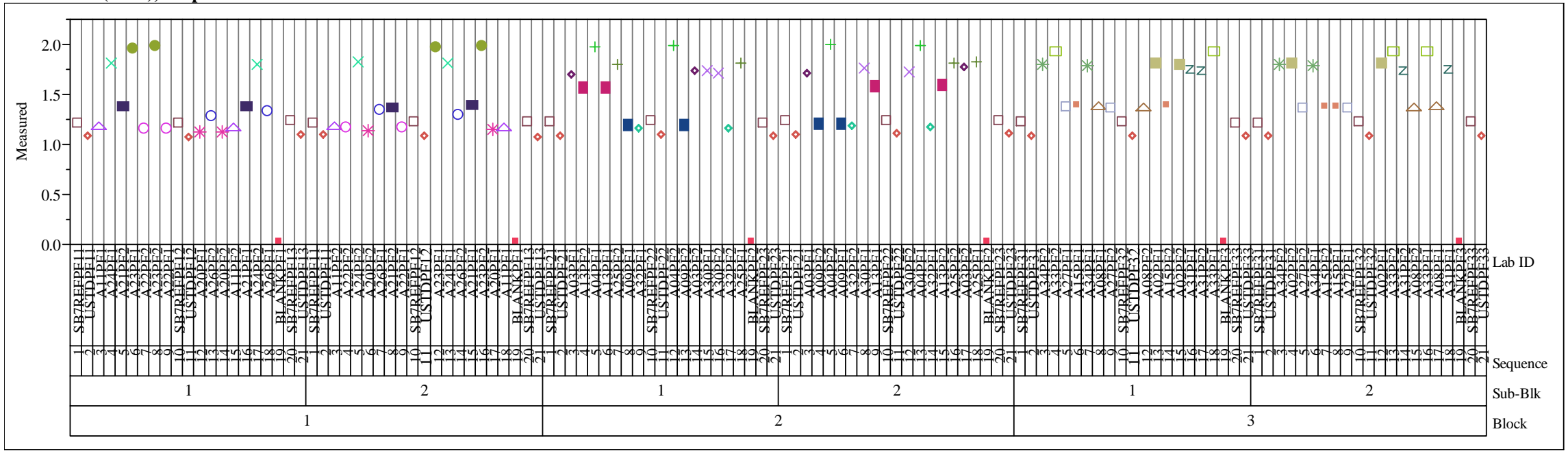


Exhibit A-1. Measurements of Study Glasses by Lab ID in Analytical Sequence for Analytical Blocks 1, 2, and 3 by Oxide and Preparation Method Oxide $=$ PbO $(w t \%)$, Prep Method $=A R$

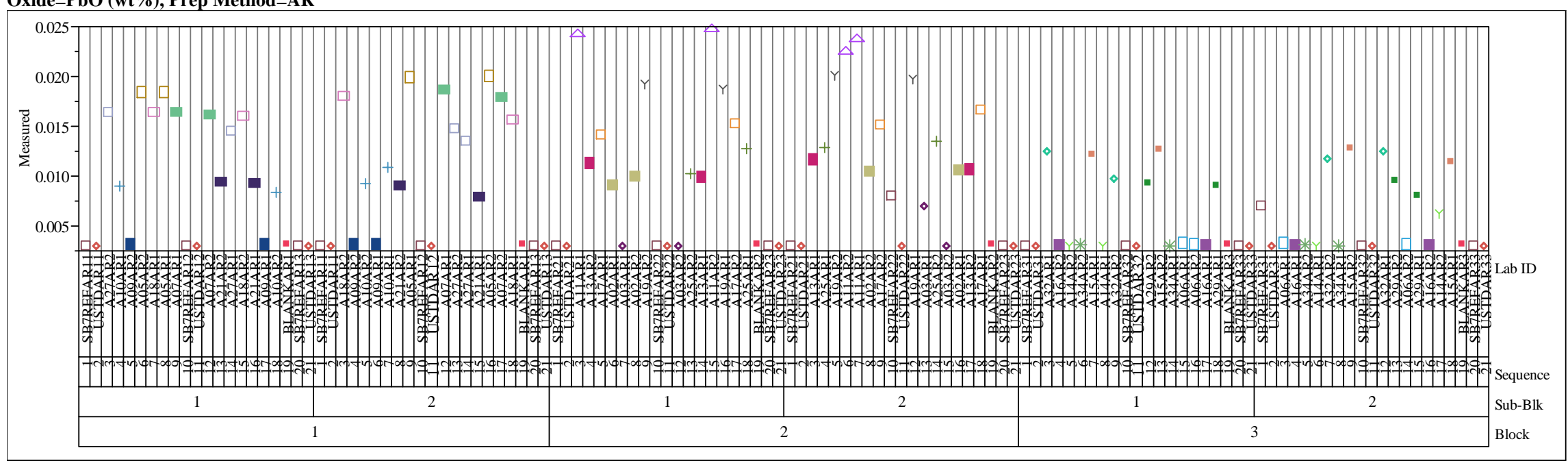

Oxide=PbO (wt\%), Prep Method=PF

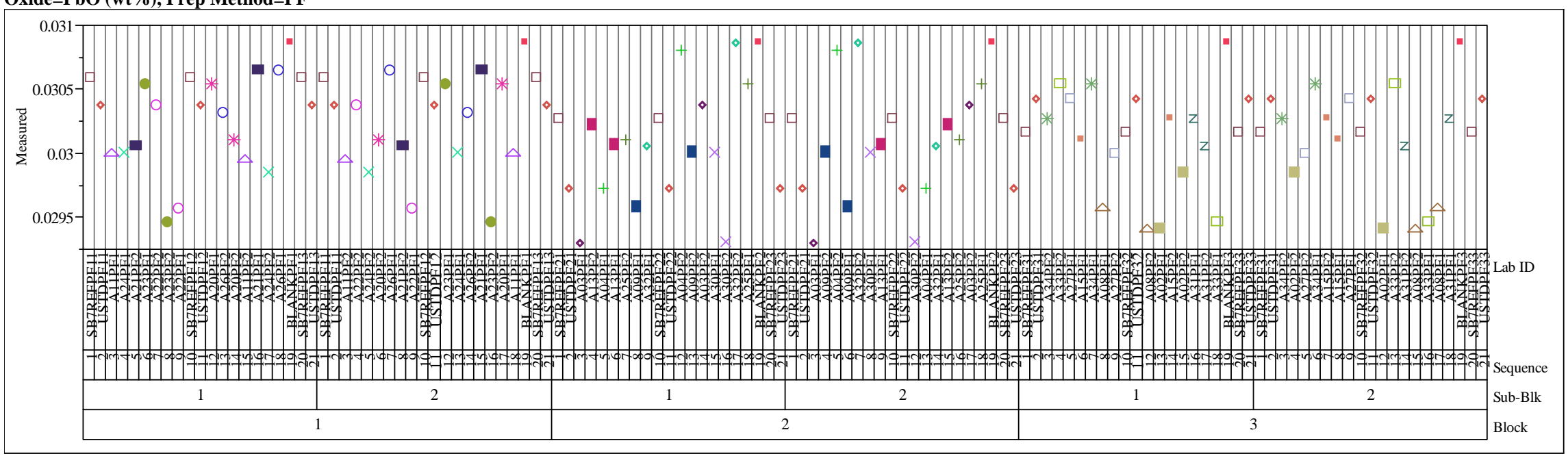


Exhibit A-1. Measurements of Study Glasses by Lab ID in Analytical Sequence for Analytical Blocks 1, 2, and 3 by Oxide and Preparation Method Oxide=SiO2 (wt \%), Prep Method=PF

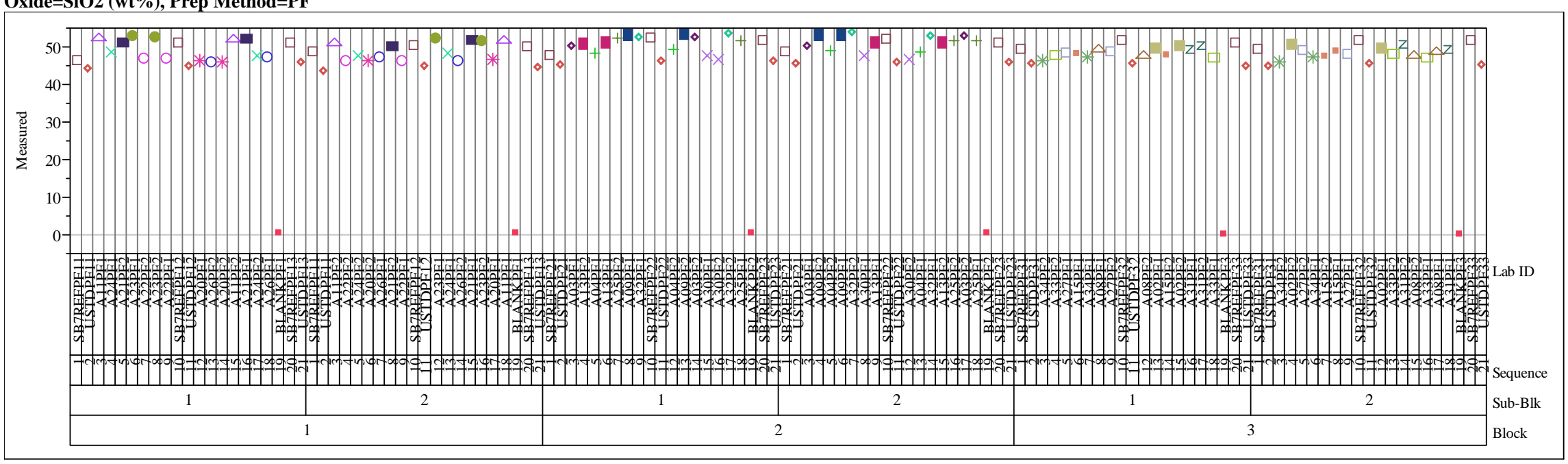

Oxide=SO4 (wt\%), Prep Method=AR

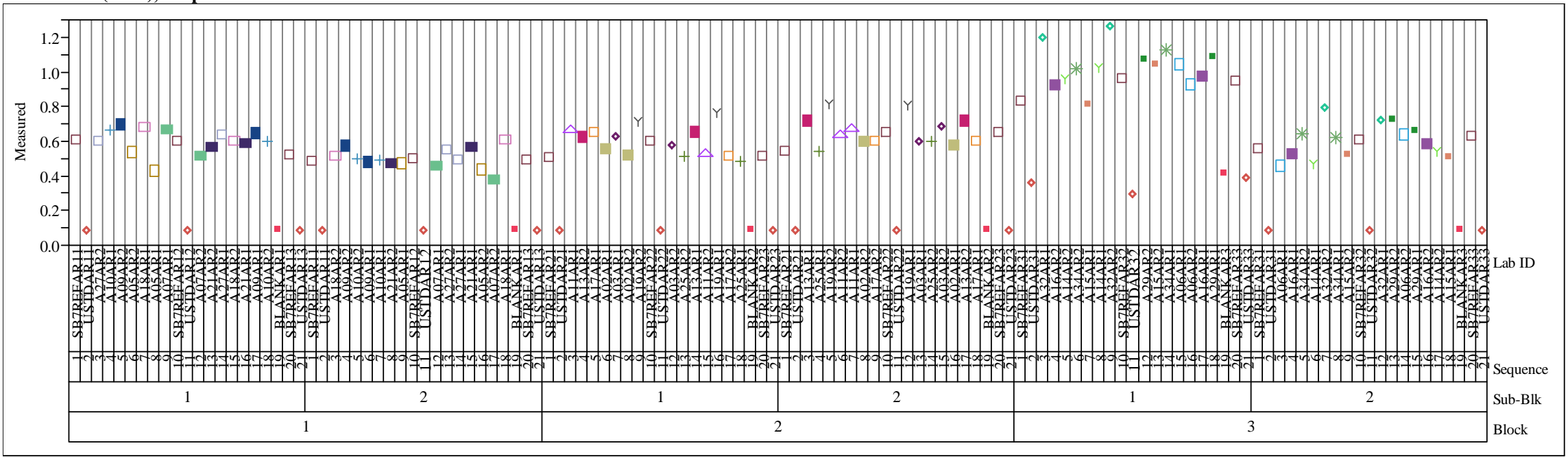


Exhibit A-1. Measurements of Study Glasses by Lab ID in Analytical Sequence for Analytical Blocks 1, 2, and 3 by Oxide and Preparation Method Oxide=SO4 (wt\%), Prep Method=PF

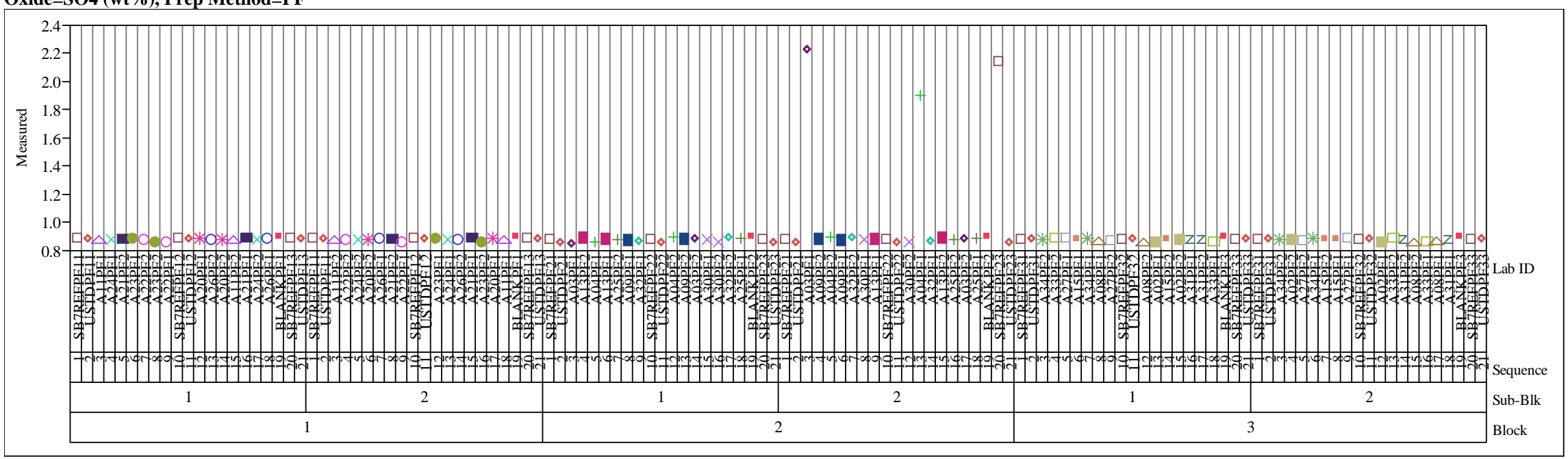

Oxide=ThO2 (wt\%), Prep Method=AR

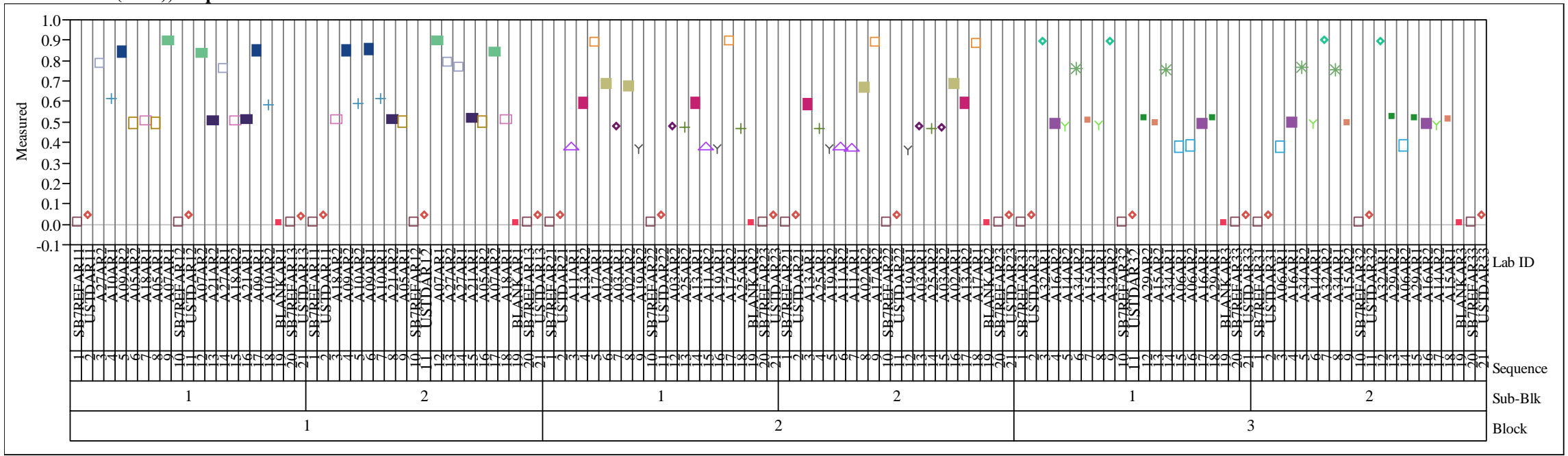


Exhibit A-1. Measurements of Study Glasses by Lab ID in Analytical Sequence for Analytical Blocks 1, 2, and 3 by Oxide and Preparation Method Oxide=ThO2 (wt \%), Prep Method=PF

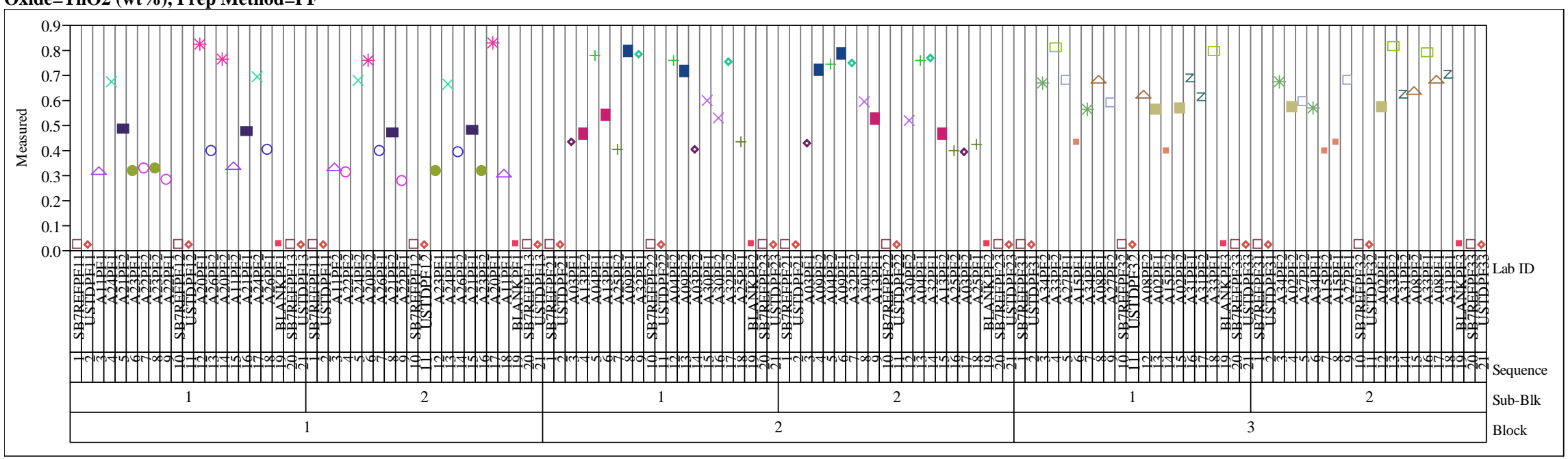

Oxide=TiO2 ( $w t \%)$, Prep Method=AR

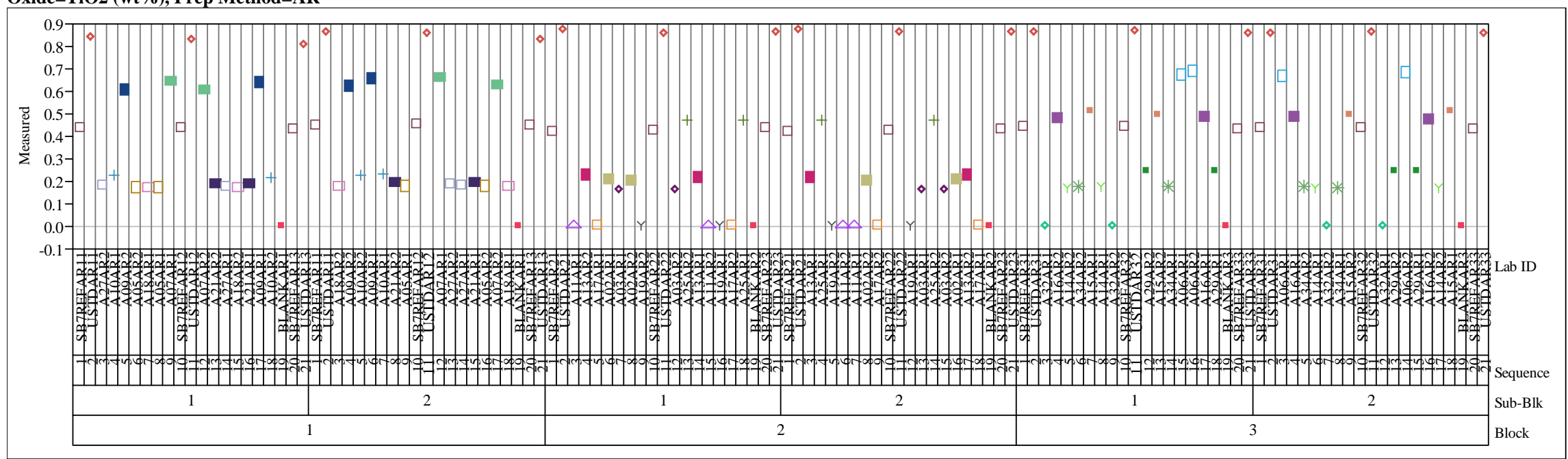


Exhibit A-1. Measurements of Study Glasses by Lab ID in Analytical Sequence for Analytical Blocks 1, 2, and 3 by Oxide and Preparation Method Oxide $=$ TiO2 ( $w$ t $\%)$, Prep Method=PF

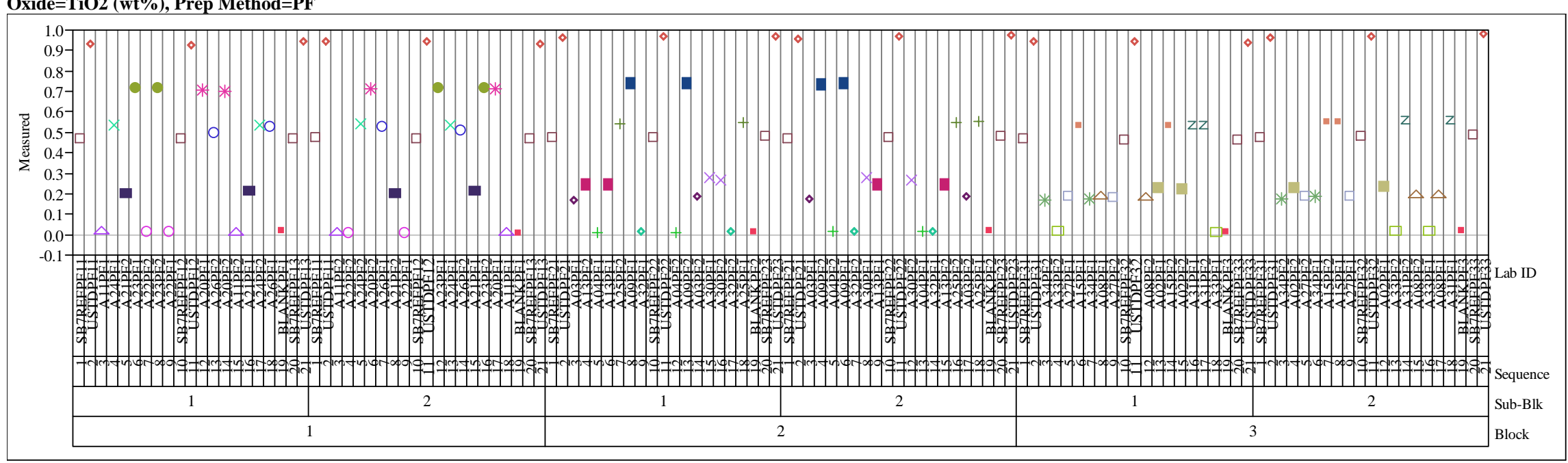

Oxide=U3O8 (wt\%), Prep Method=AR

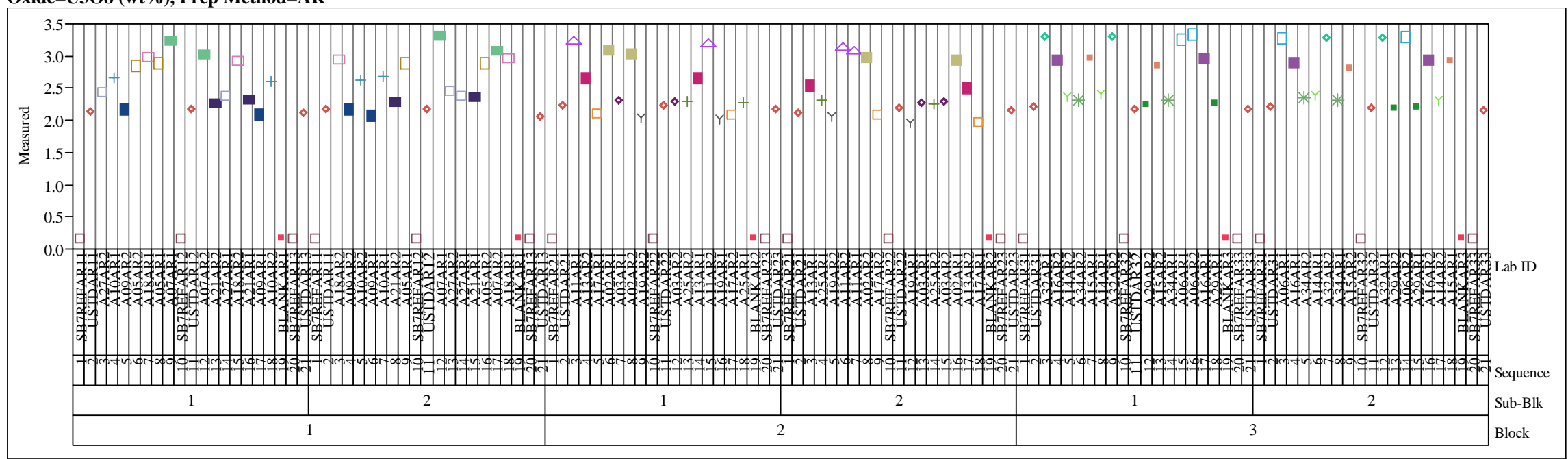


Exhibit A-1. Measurements of Study Glasses by Lab ID in Analytical Sequence for Analytical Blocks 1, 2, and 3 by Oxide and Preparation Method Oxide=U3O8 (wt\%), Prep Method=PF

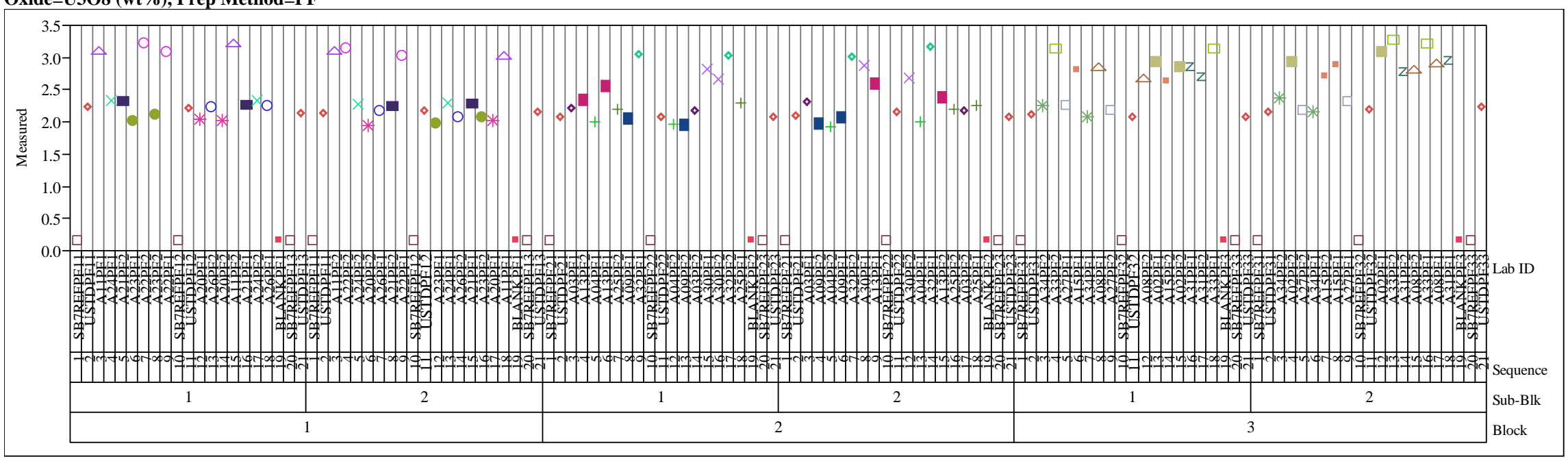

Oxide $=\mathrm{ZnO}$ (wt \%), Prep Method=AR

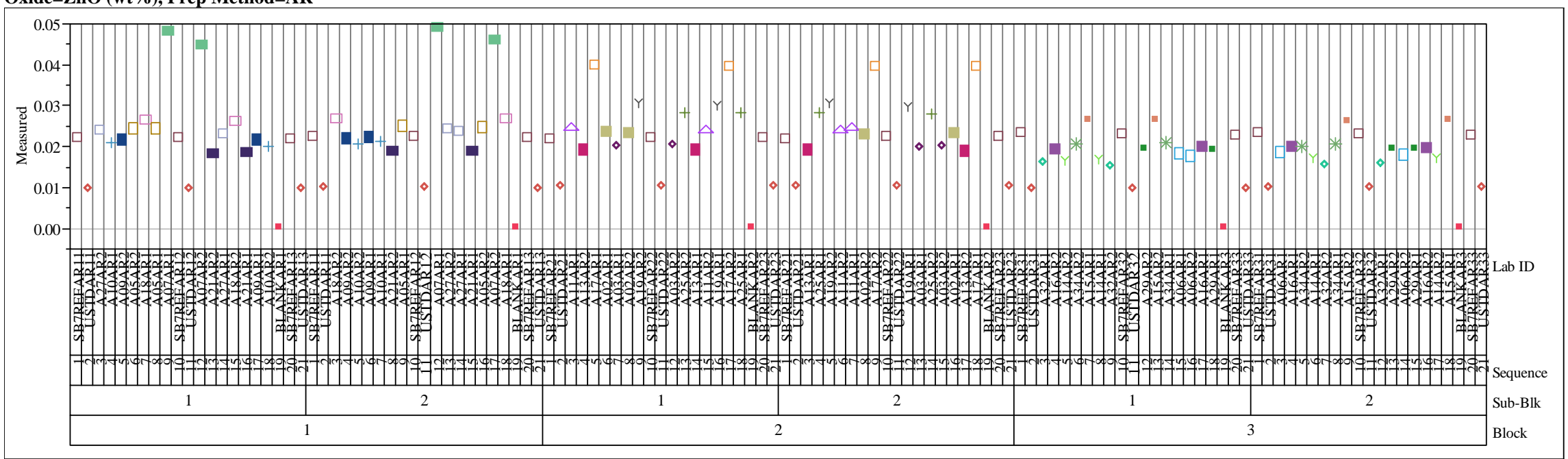


Exhibit A-1. Measurements of Study Glasses by Lab ID in Analytical Sequence for Analytical Blocks 1, 2, and 3 by Oxide and Preparation Method Oxide $=\mathrm{ZnO}$ (wt\%), Prep Method=PF

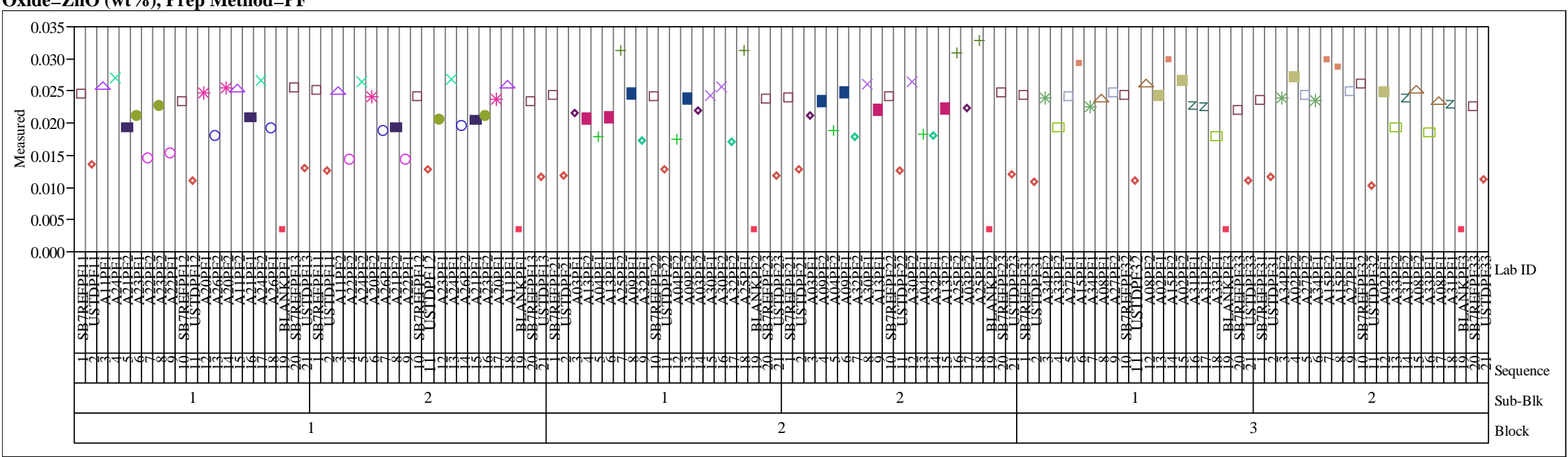

Oxide=ZrO2 (wt \%), Prep Method=AR

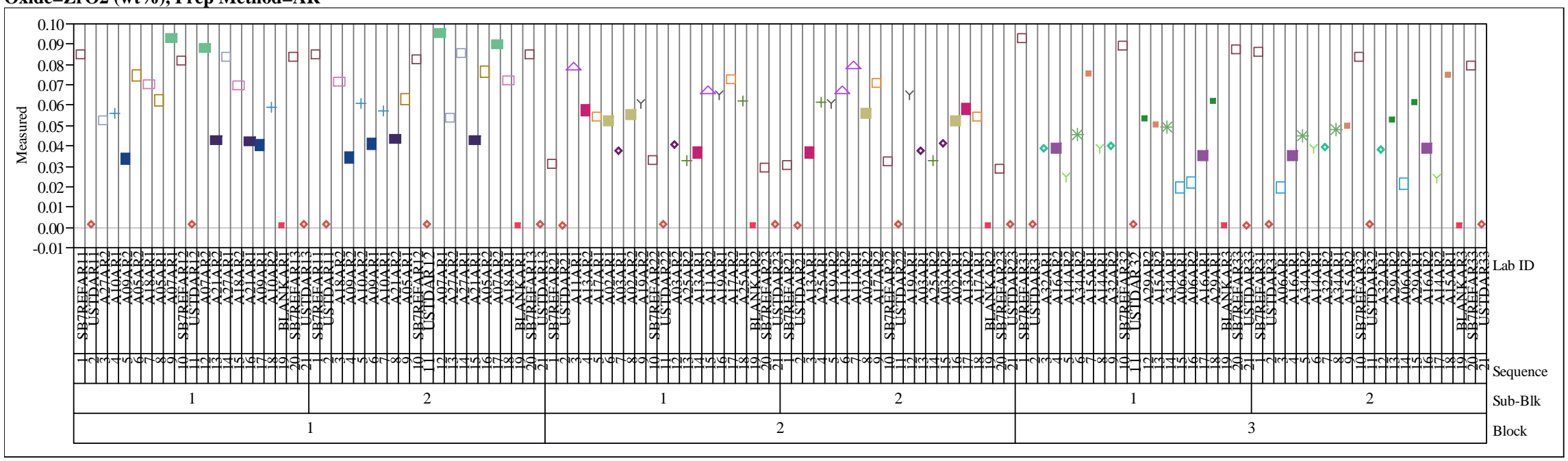


Exhibit A-2. Measurements of Initial Glasses in Analytical Sequence for Samples by Oxide and Preparation Method for Analytical Blocks 4 and 5

Oxide=Al2O3 (wt \%), Prep Method=AR

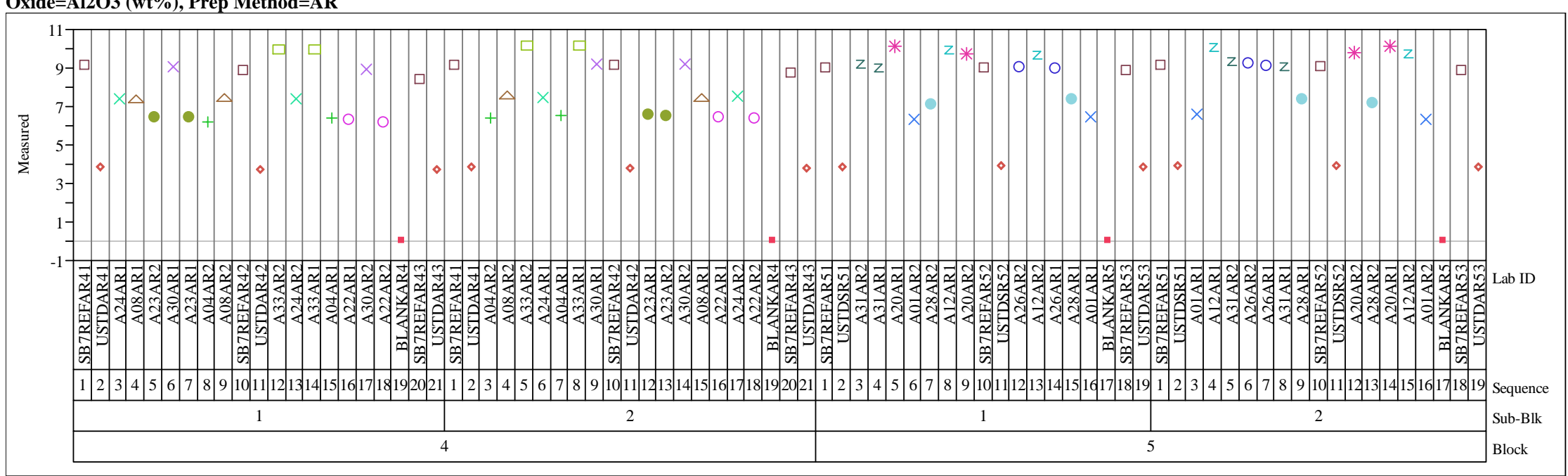

Oxide=Al2O3 (wt \%), Prep Method=PF

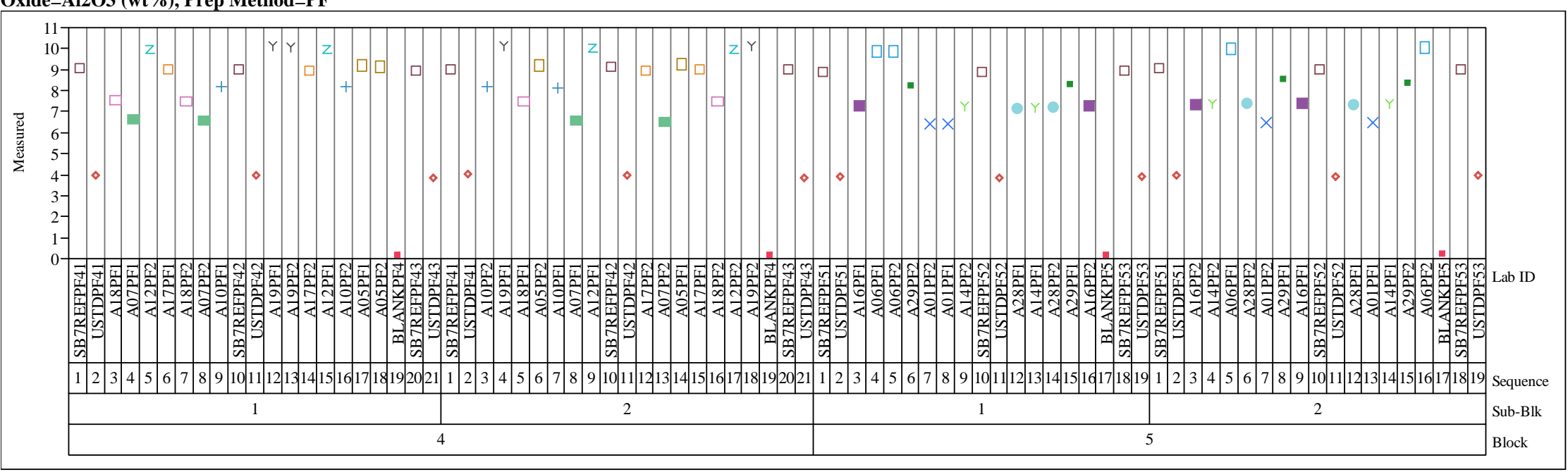


Exhibit A-2. Measurements of Initial Glasses in Analytical Sequence for Samples by Oxide and Preparation Method for Analytical Blocks 4 and 5

Oxide=B2O3 (wt \%), Prep Method=AR

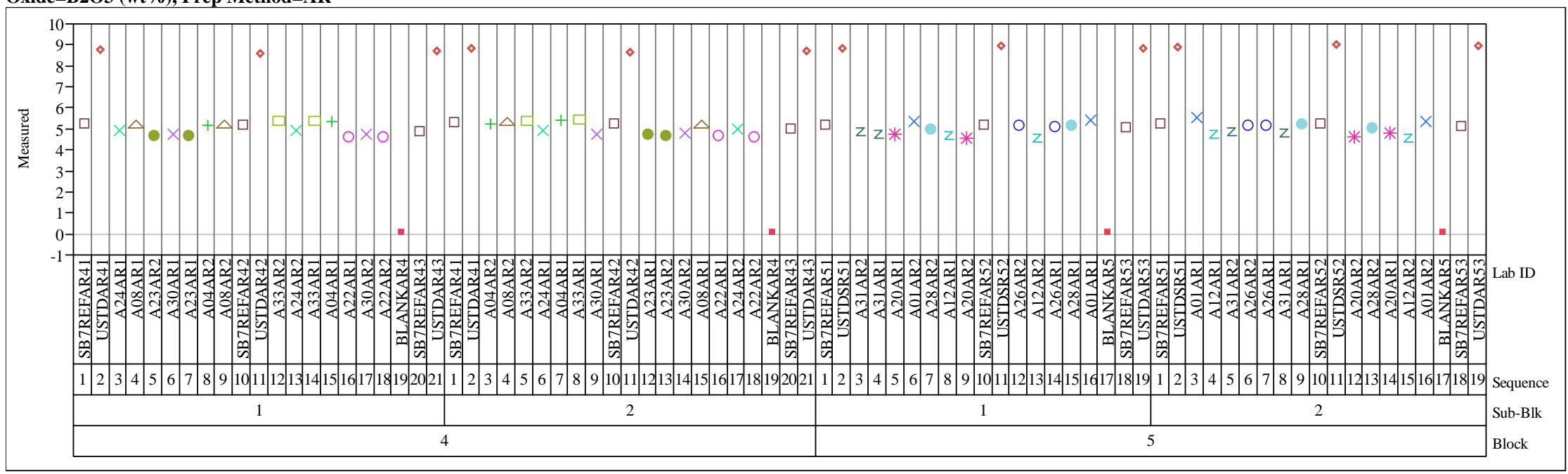

Oxide=B2O3 (wt\%), Prep Method=PF

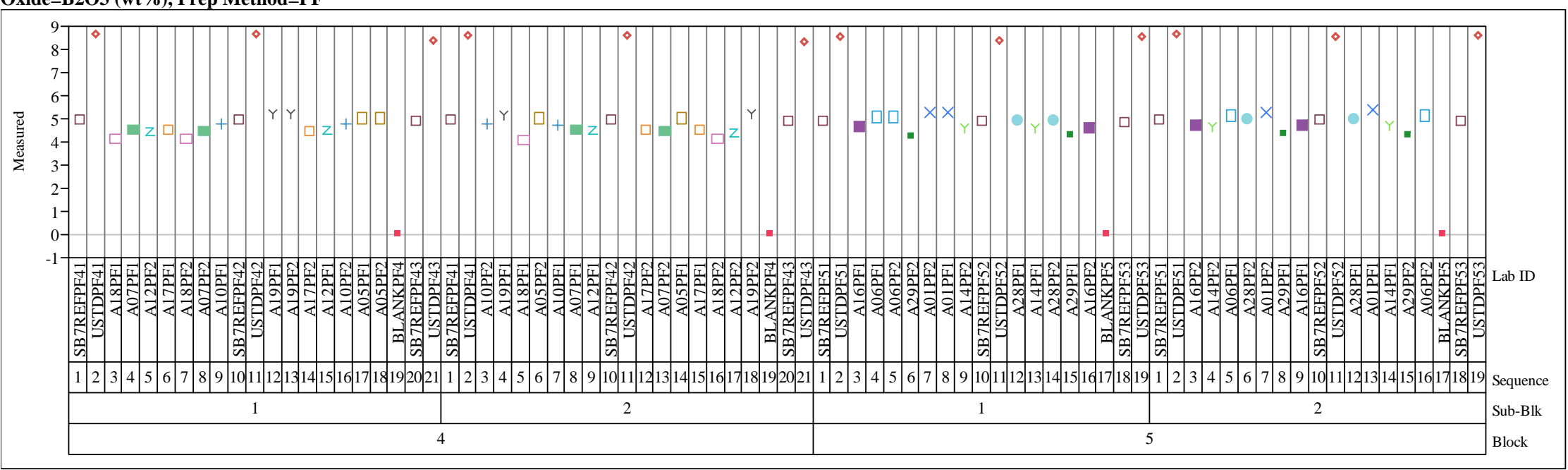


Exhibit A-2. Measurements of Initial Glasses in Analytical Sequence for Samples by Oxide and Preparation Method for Analytical Blocks 4 and 5

Oxide= $\mathrm{BaO}(\mathrm{wt} \%)$, Prep Method $=\mathrm{AR}$

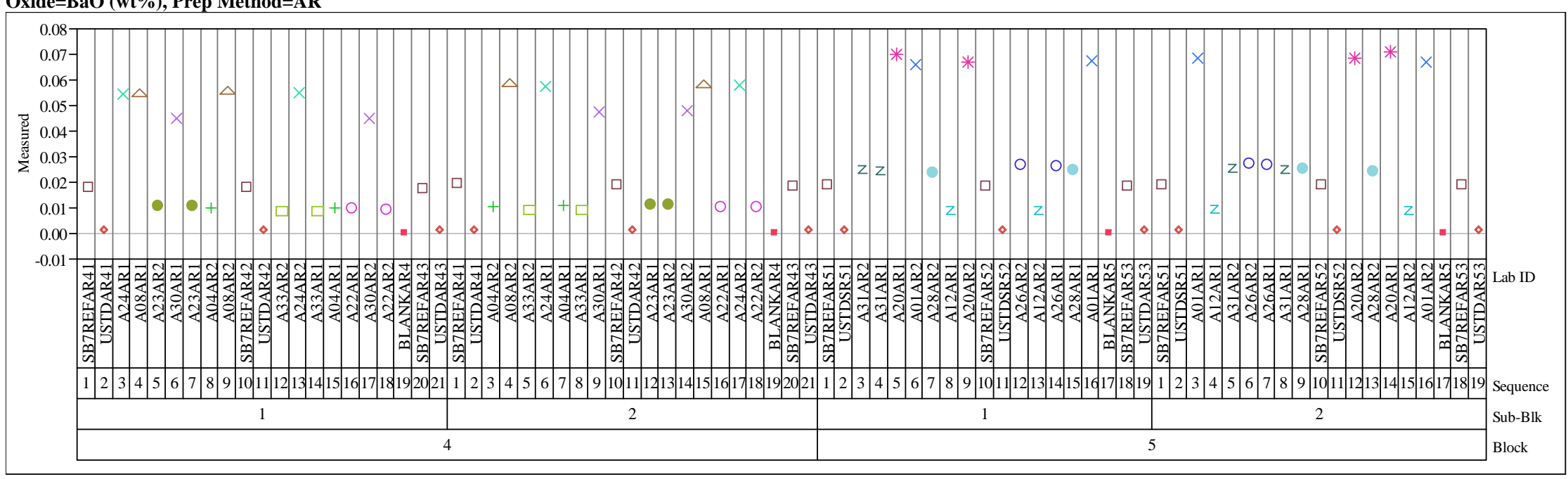

Oxide=BaO (wt \%), Prep Method=PF

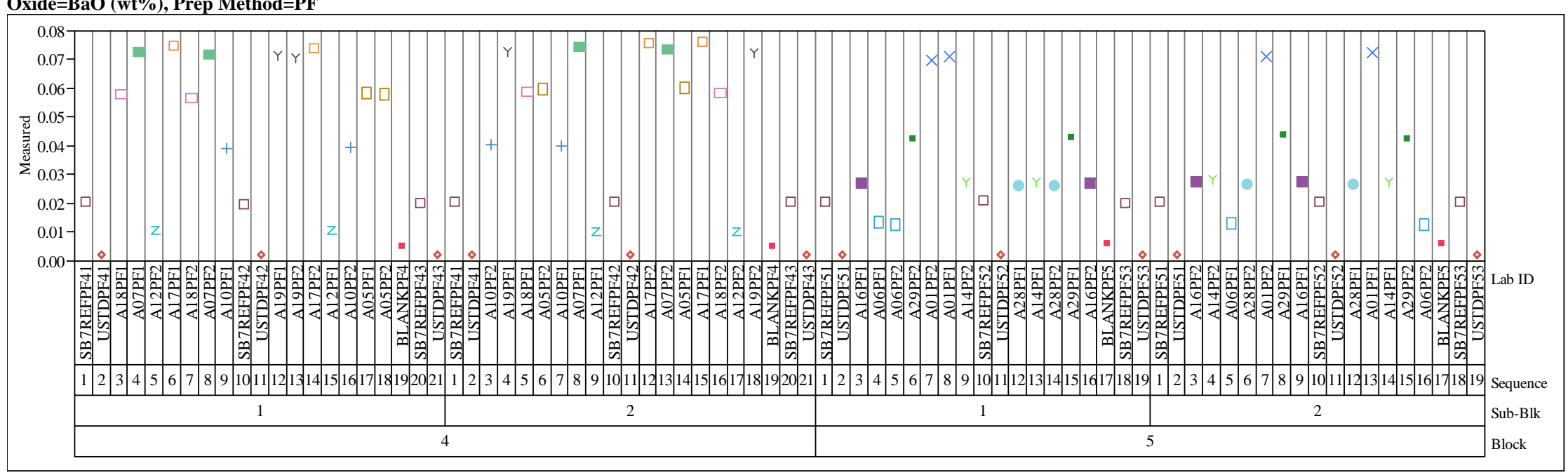


Exhibit A-2. Measurements of Initial Glasses in Analytical Sequence for Samples by Oxide and Preparation Method for Analytical Blocks 4 and 5

Oxide $=\mathrm{CaO}($ wt $\%)$, Prep Method=AR

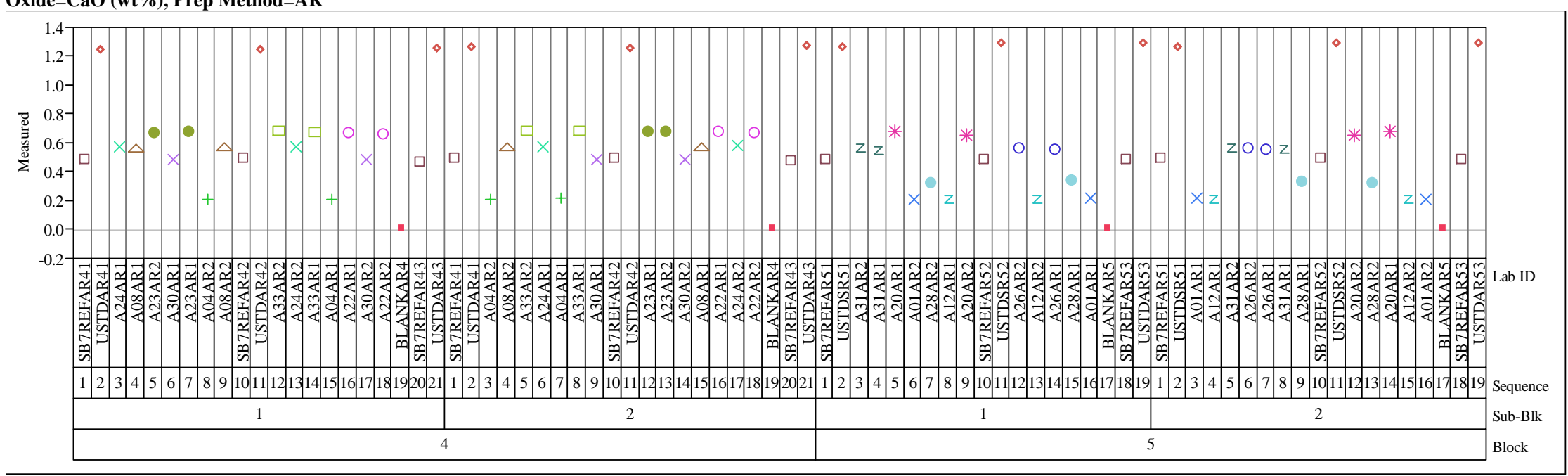

Oxide=Ce2O3 (wt \%), Prep Method=AR

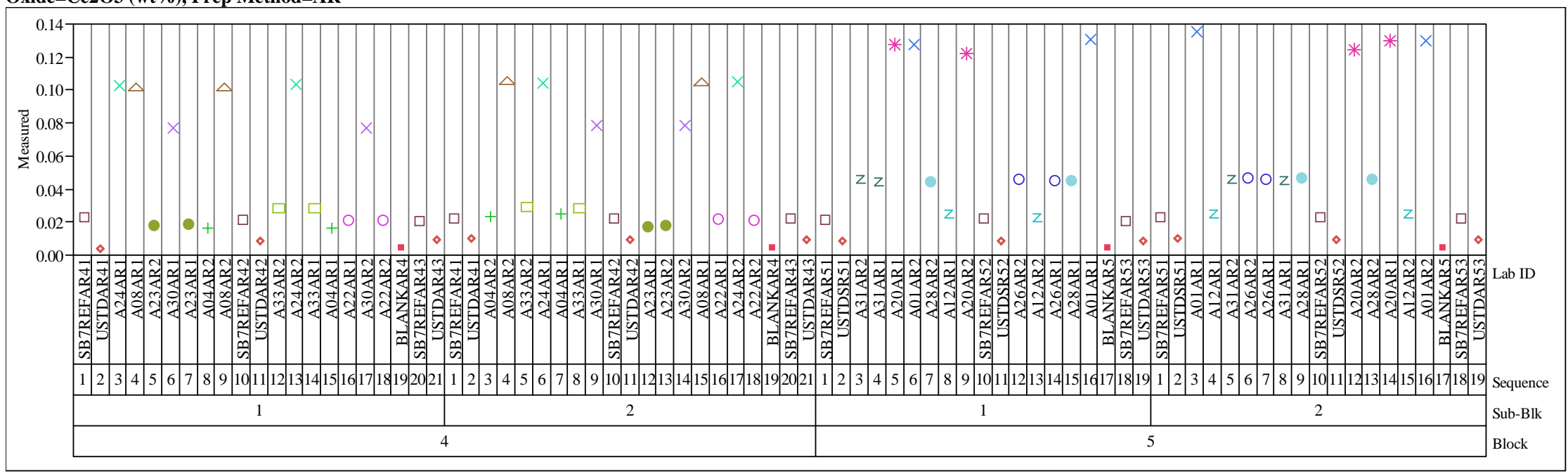


Exhibit A-2. Measurements of Initial Glasses in Analytical Sequence for Samples by Oxide and Preparation Method for Analytical Blocks 4 and 5

Oxide $=\mathrm{Ce} 2 \mathrm{O} 3(\mathrm{wt} \%)$, Prep Method=PF

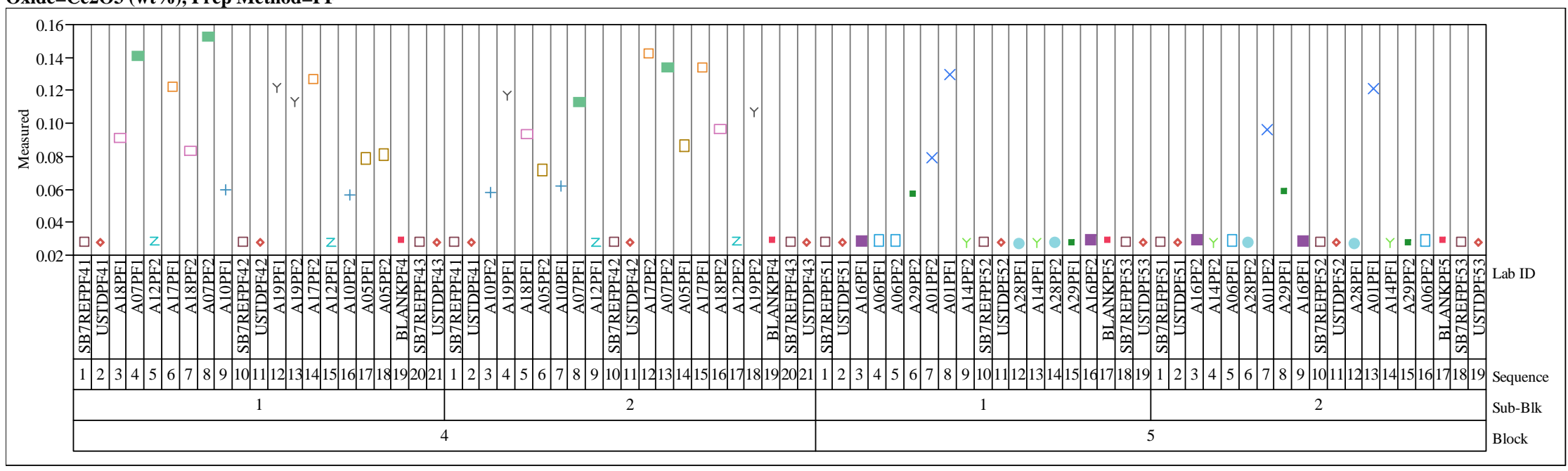

Oxide $=\mathrm{Cr} 2 \mathrm{O} 3(\mathrm{wt} \%)$, Prep Method $=\mathrm{AR}$

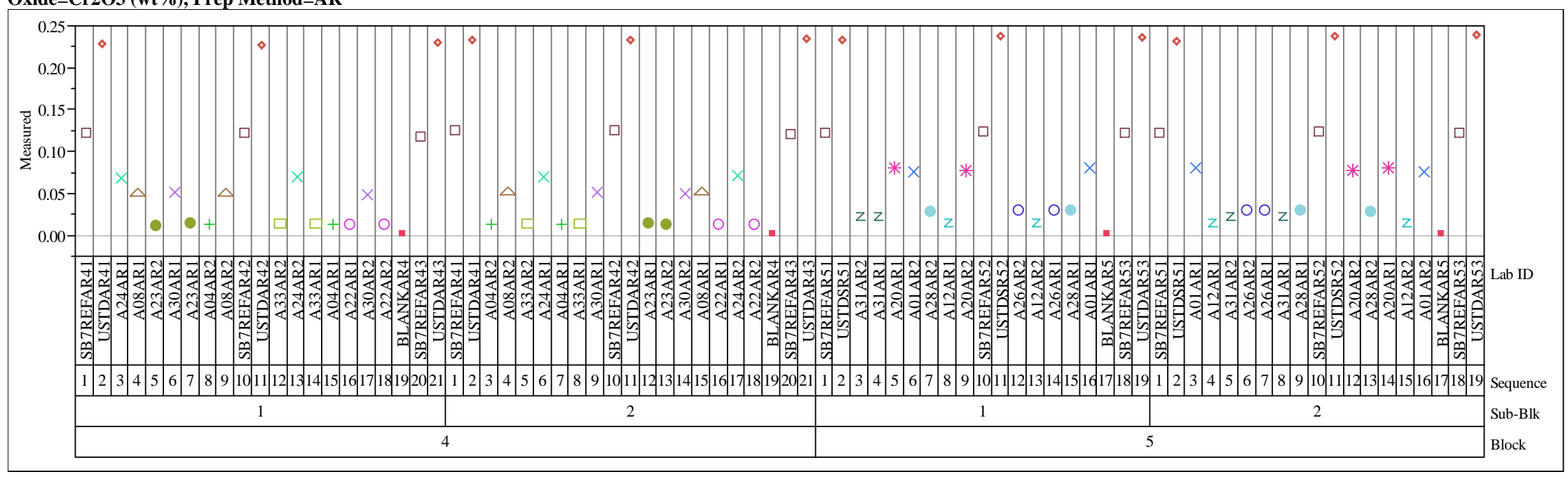


Exhibit A-2. Measurements of Initial Glasses in Analytical Sequence for Samples by Oxide and Preparation Method for Analytical Blocks 4 and 5

Oxide=Cr2O3 (wt \%), Prep Method=PF

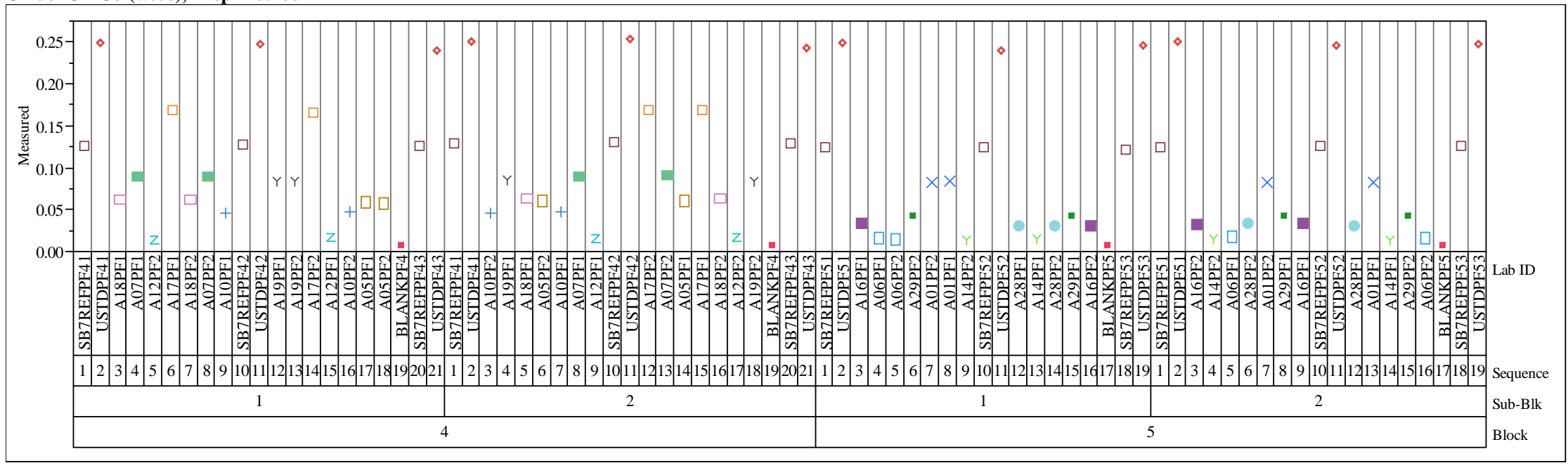

Oxide $=\mathrm{CuO}$ (wt \%), Prep Method $=$ AR

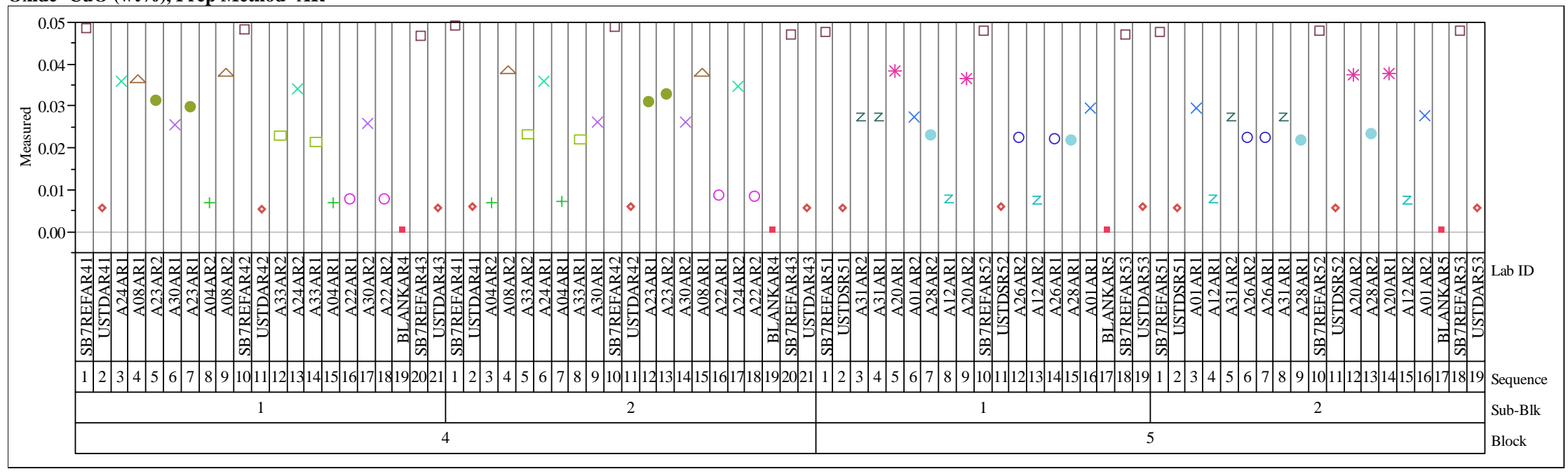


Exhibit A-2. Measurements of Initial Glasses in Analytical Sequence for Samples by Oxide and Preparation Method for Analytical Blocks 4 and 5

Oxide $=\mathrm{CuO}($ wt $\%)$, Prep Method=PF

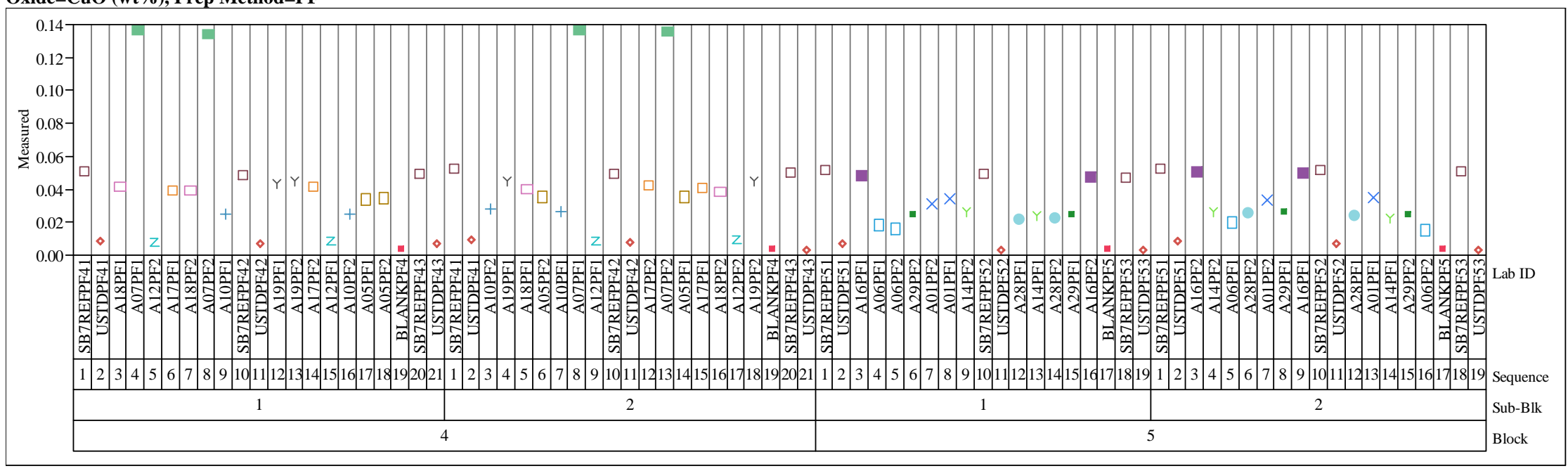

Oxide=Fe2O3 (wt\%), Prep Method=AR

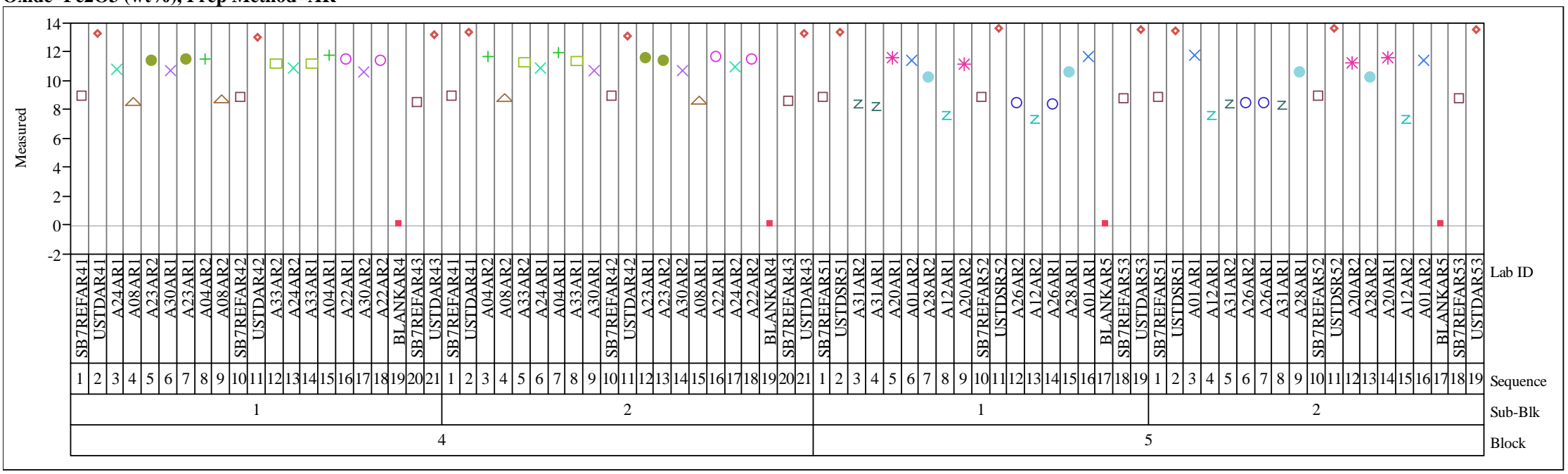


Exhibit A-2. Measurements of Initial Glasses in Analytical Sequence for Samples by Oxide and Preparation Method for Analytical Blocks 4 and 5

Oxide=Fe2O3 (wt\%), Prep Method=PF

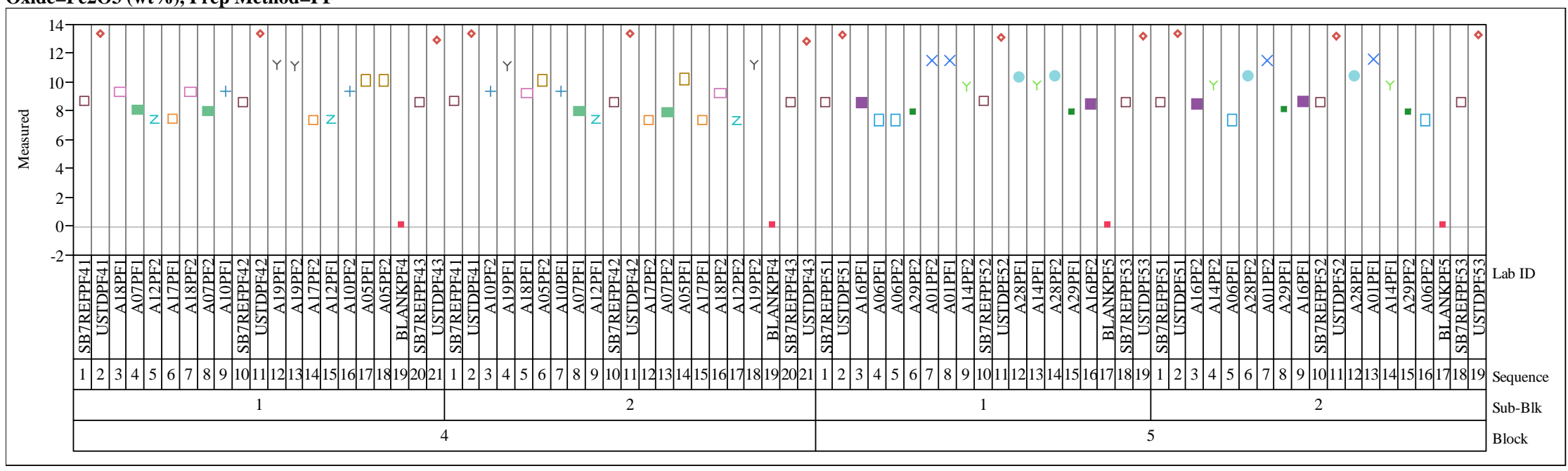

Oxide=K2O (wt\%), Prep Method=AR

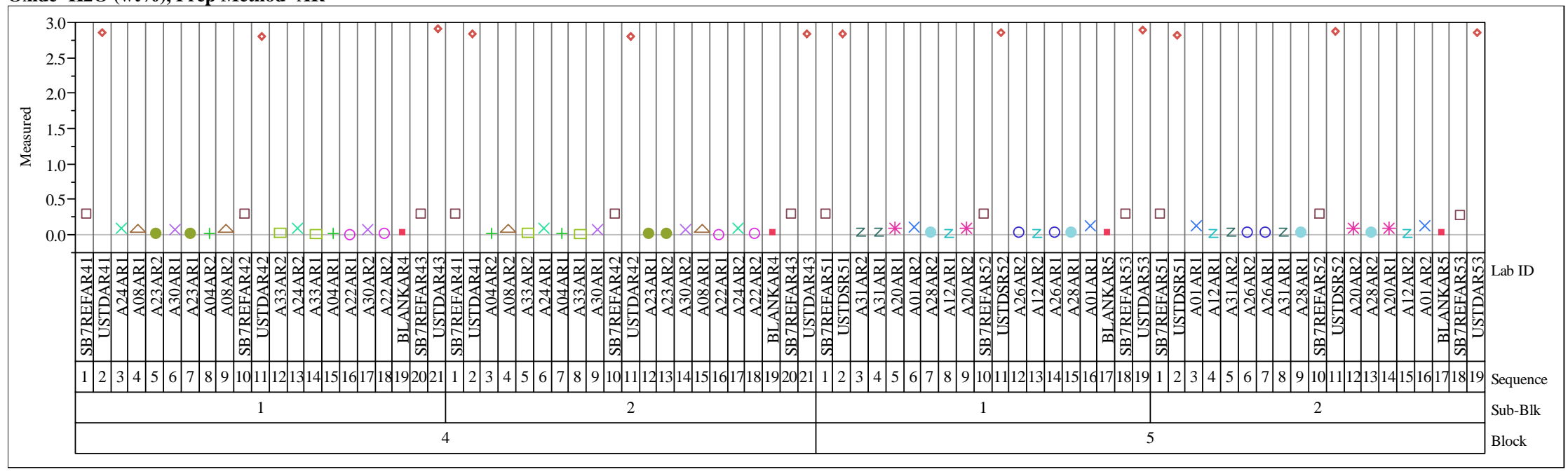


Exhibit A-2. Measurements of Initial Glasses in Analytical Sequence for Samples by Oxide and Preparation Method for Analytical Blocks 4 and 5

Oxide=K2O (wt\%), Prep Method=PF

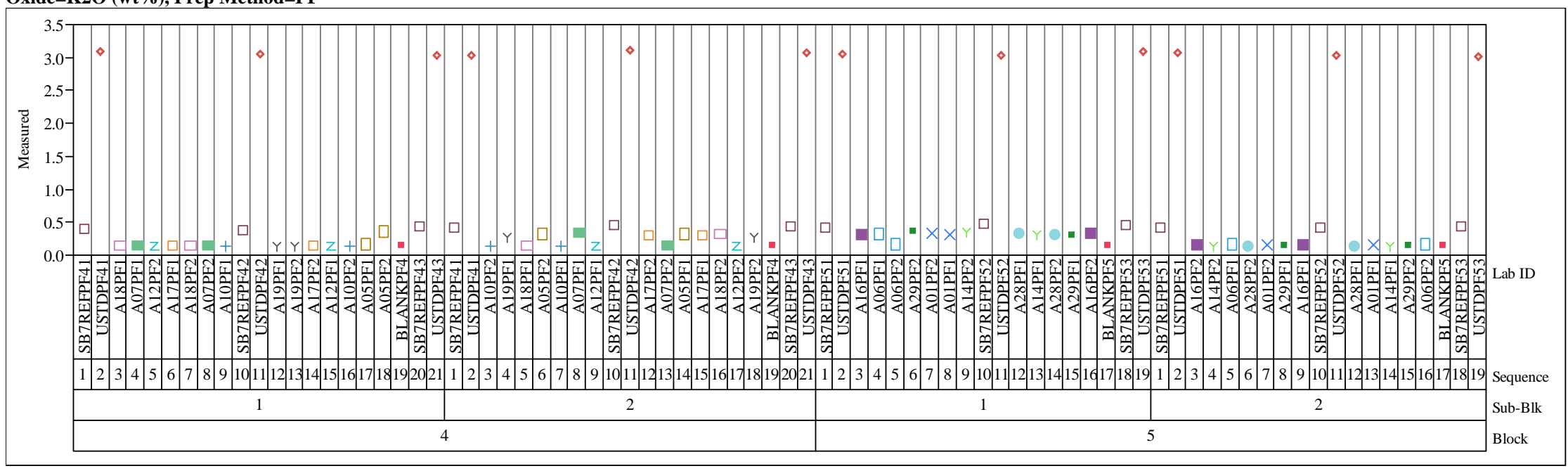

Oxide $=$ La2O3 (wt \%), Prep Method=AR

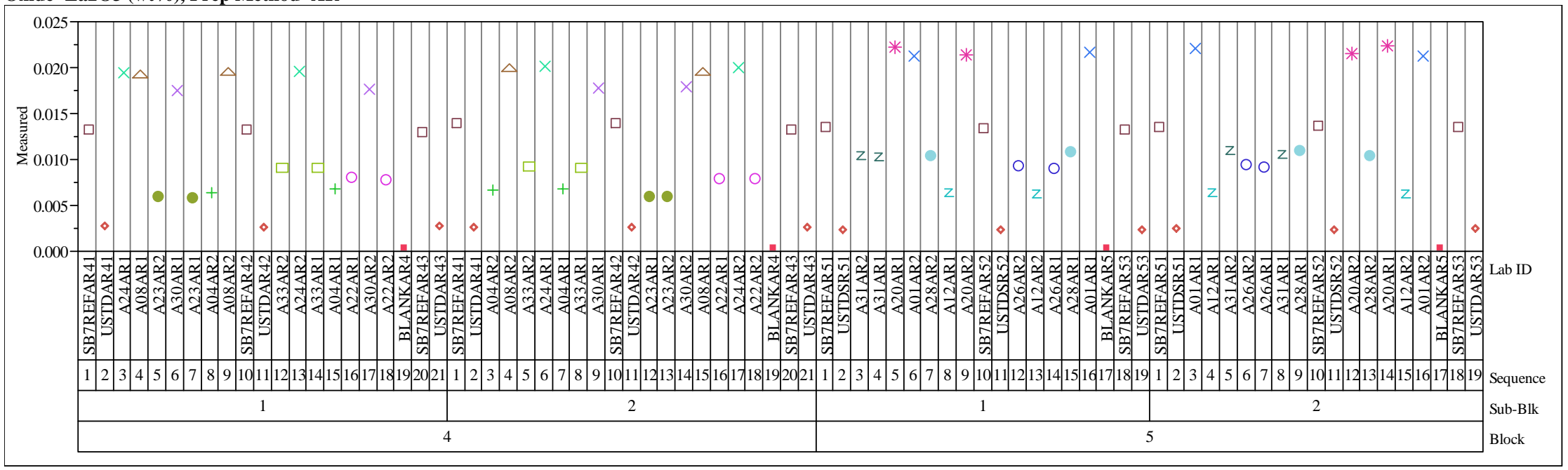


Exhibit A-2. Measurements of Initial Glasses in Analytical Sequence for Samples by Oxide and Preparation Method for Analytical Blocks 4 and 5

Oxide=La2O3 (wt \%), Prep Method=PF

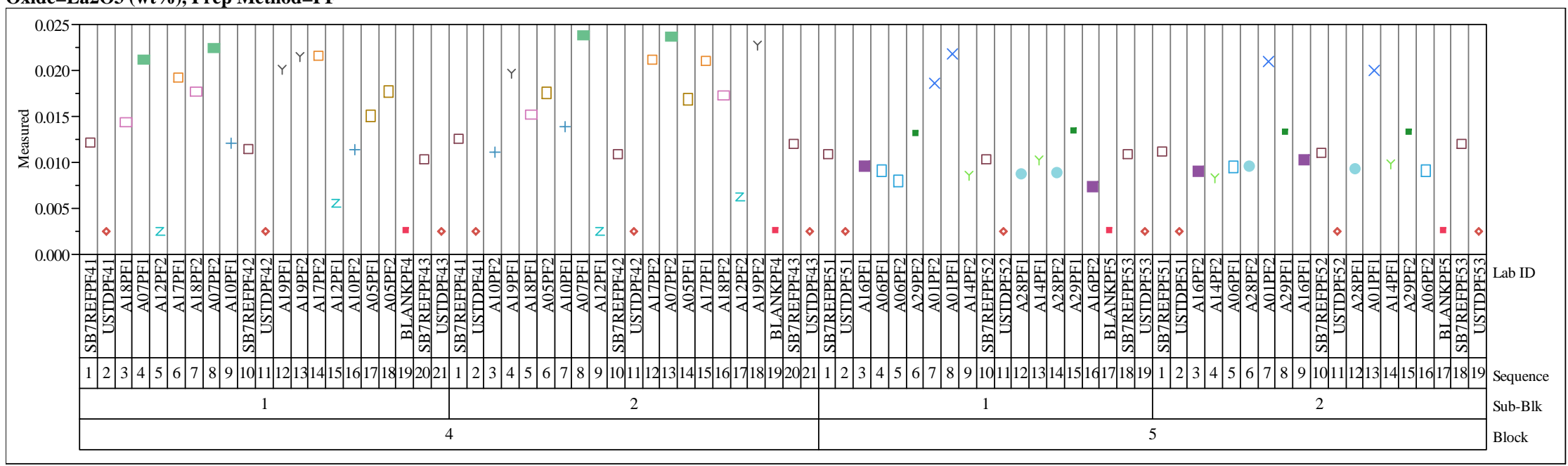

Oxide=Li2O (wt\%), Prep Method=AR

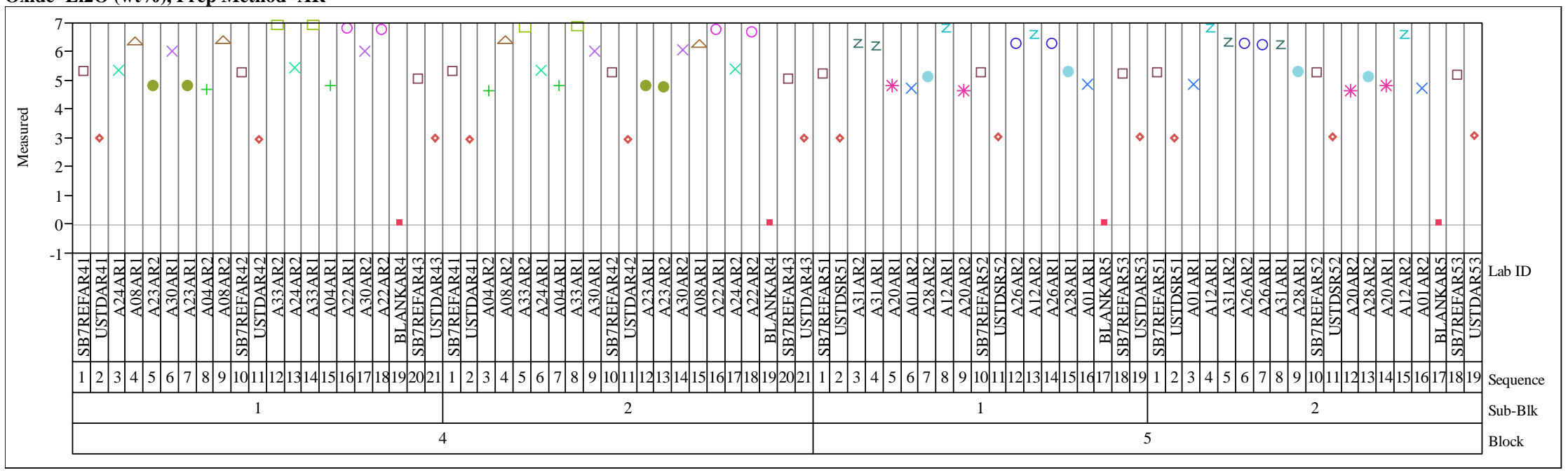


Exhibit A-2. Measurements of Initial Glasses in Analytical Sequence for Samples by Oxide and Preparation Method for Analytical Blocks 4 and 5

Oxide=Li2O (wt\%), Prep Method=PF

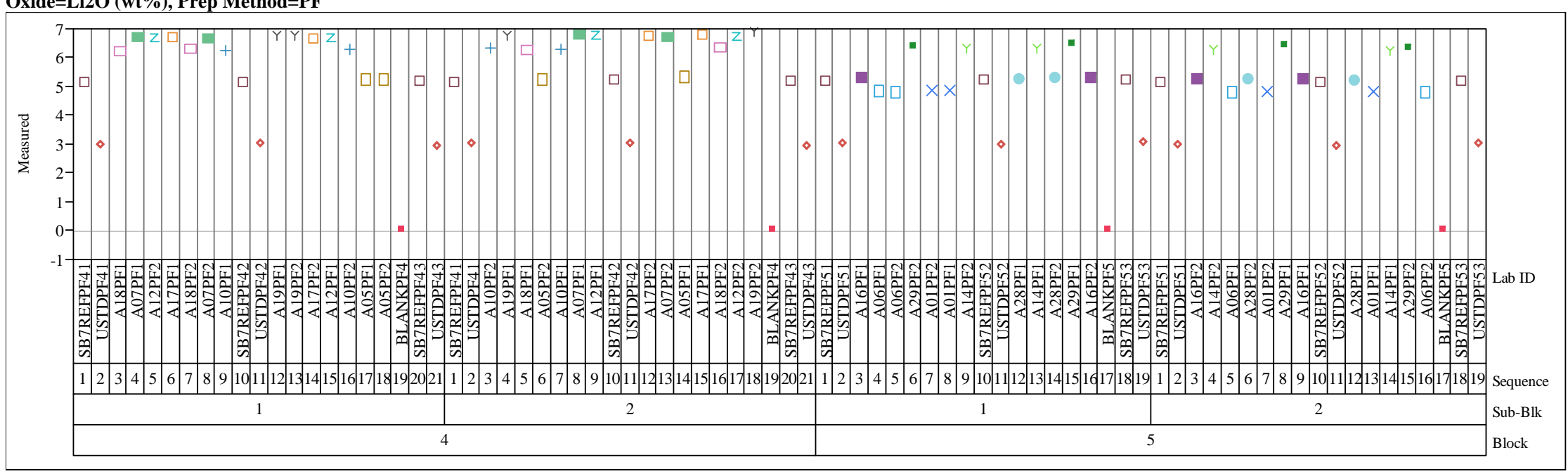

Oxide=MgO (wt\%), Prep Method=AR

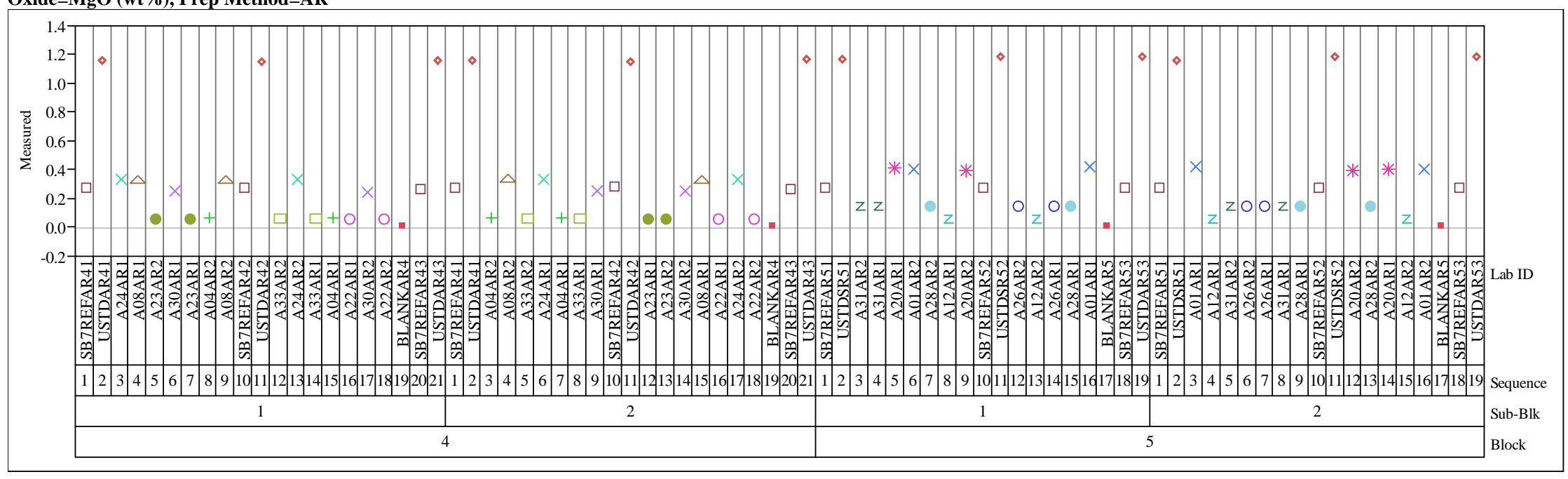


Exhibit A-2. Measurements of Initial Glasses in Analytical Sequence for Samples by Oxide and Preparation Method for Analytical Blocks 4 and 5

Oxide=MgO (wt\%), Prep Method $=$ PF

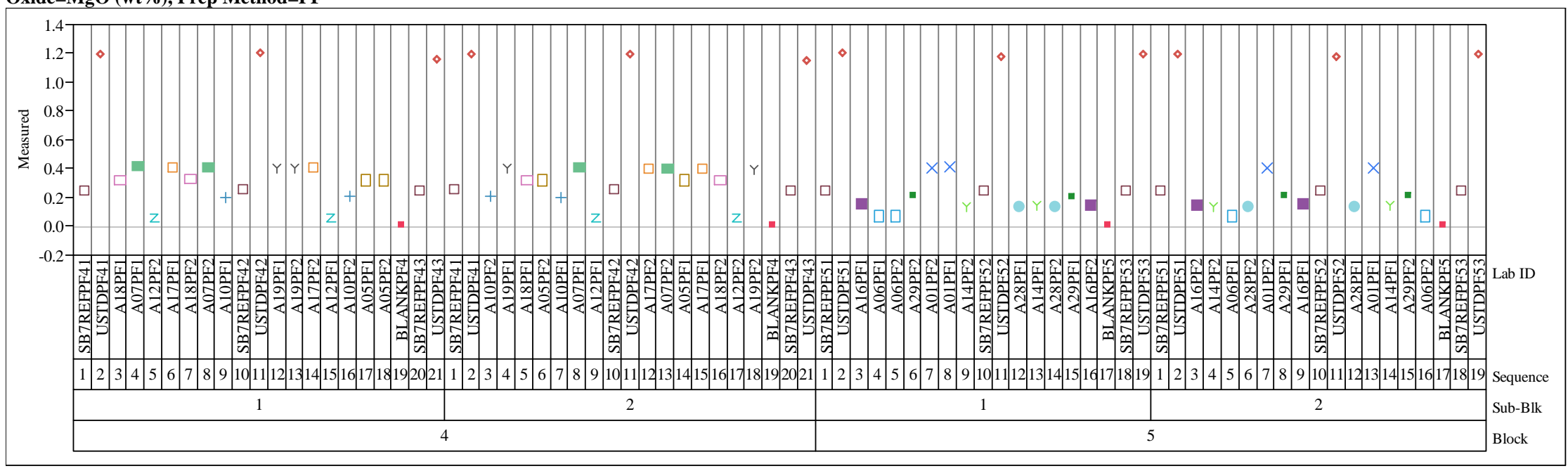

Oxide=MnO (wt \%), Prep Method=AR

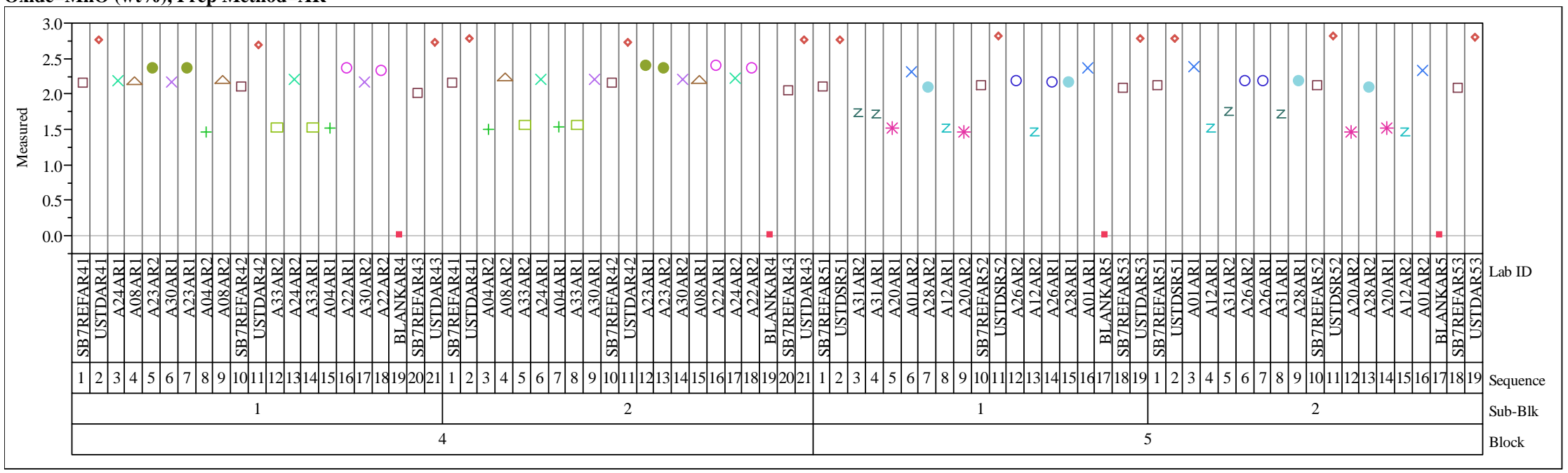


Exhibit A-2. Measurements of Initial Glasses in Analytical Sequence for Samples by Oxide and Preparation Method for Analytical Blocks 4 and 5

Oxide=MnO (wt\%), Prep Method=PF

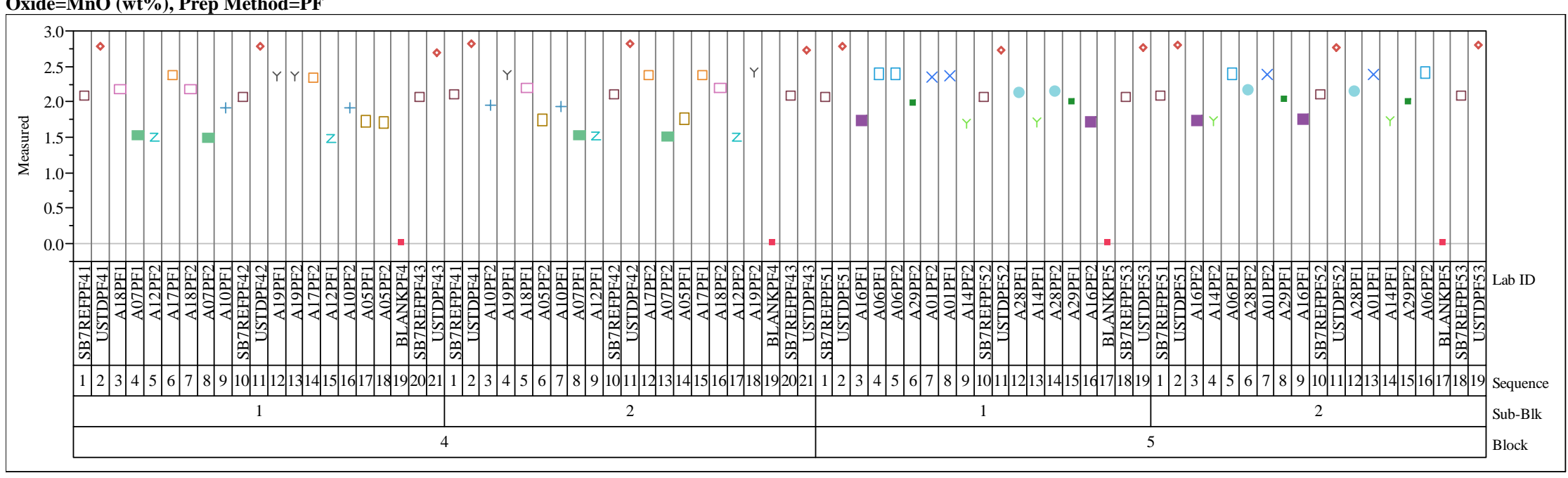

Oxide=Na2O (wt \%), Prep Method=AR

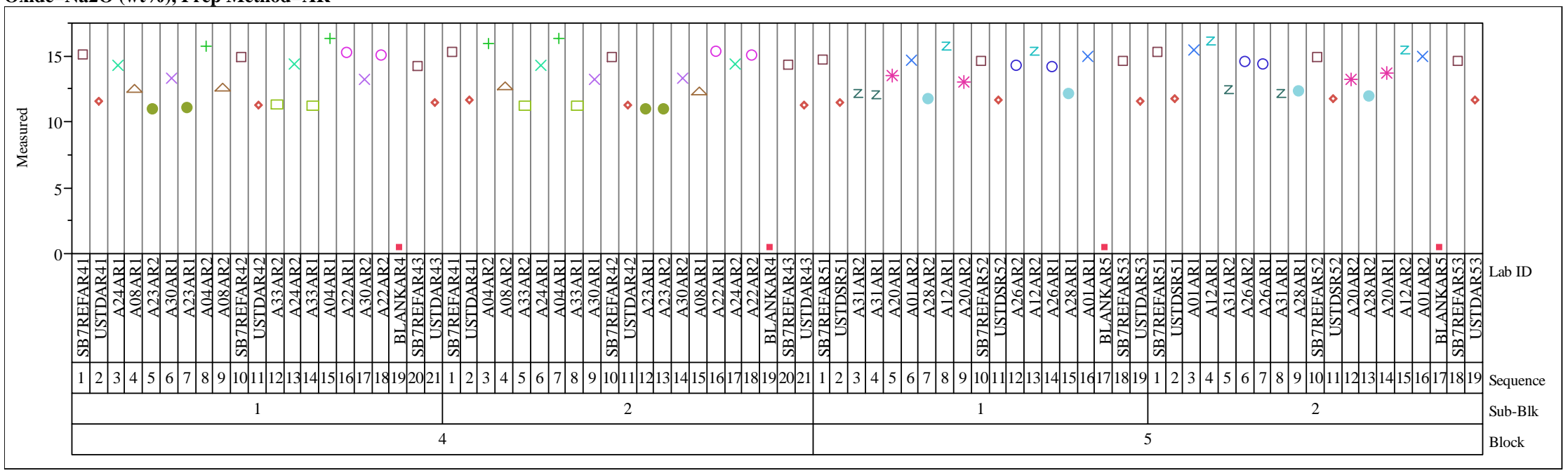


Exhibit A-2. Measurements of Initial Glasses in Analytical Sequence for Samples by Oxide and Preparation Method for Analytical Blocks 4 and 5 Oxide=NiO (wt\%), Prep Method=AR

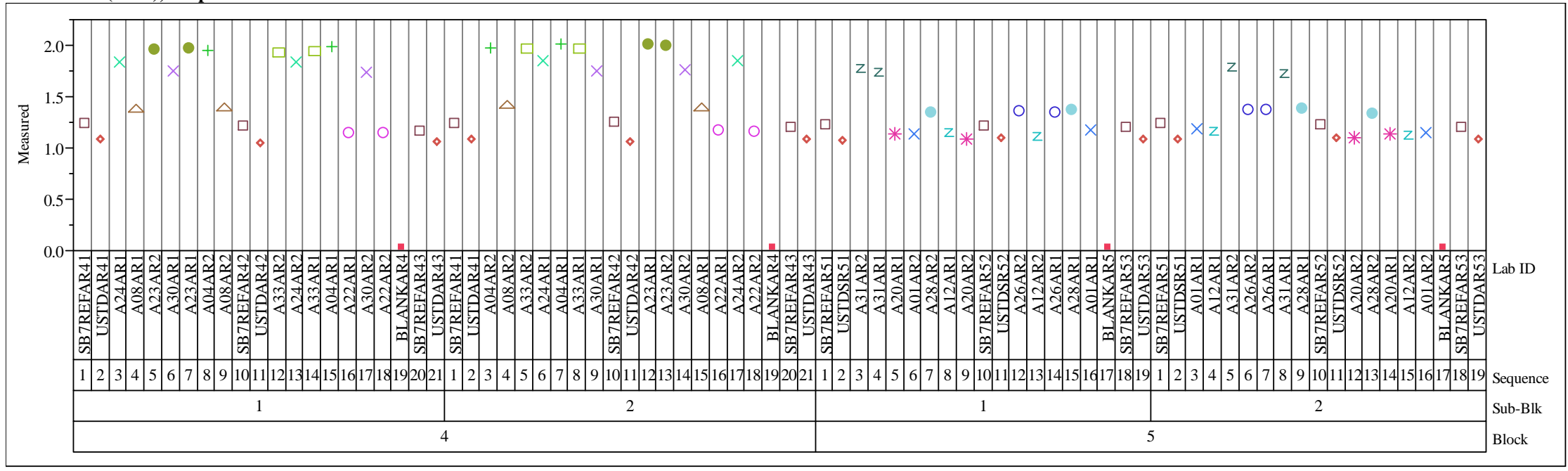

Oxide=NiO (wt \%), Prep Method=PF

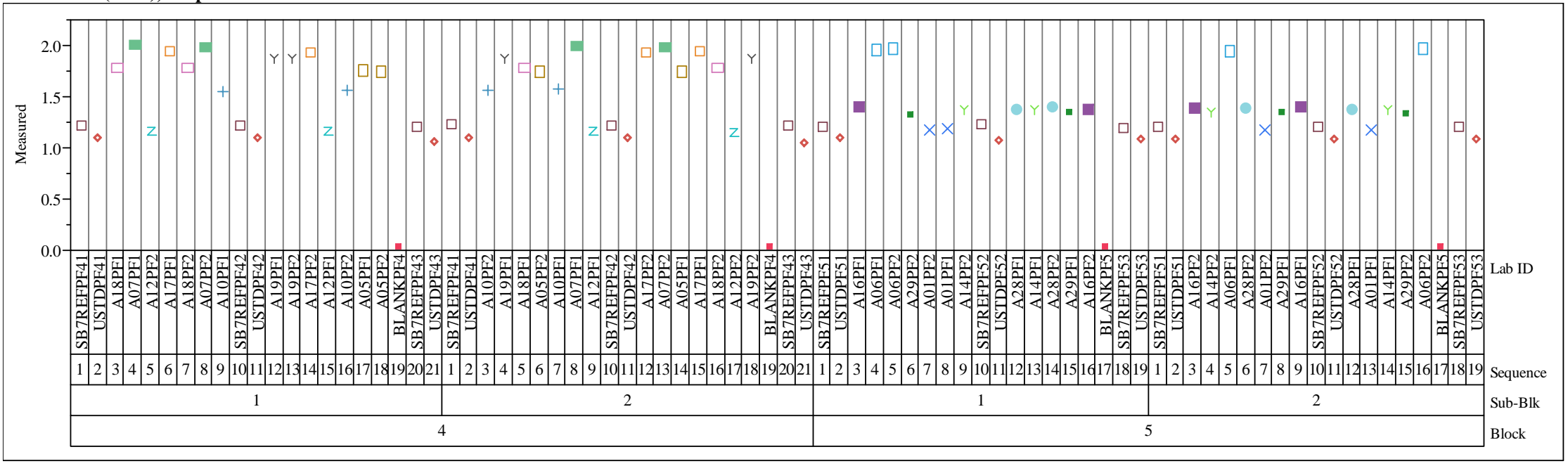


Exhibit A-2. Measurements of Initial Glasses in Analytical Sequence for Samples by Oxide and Preparation Method for Analytical Blocks 4 and 5 Oxide=PbO (wt \%), Prep Method=AR

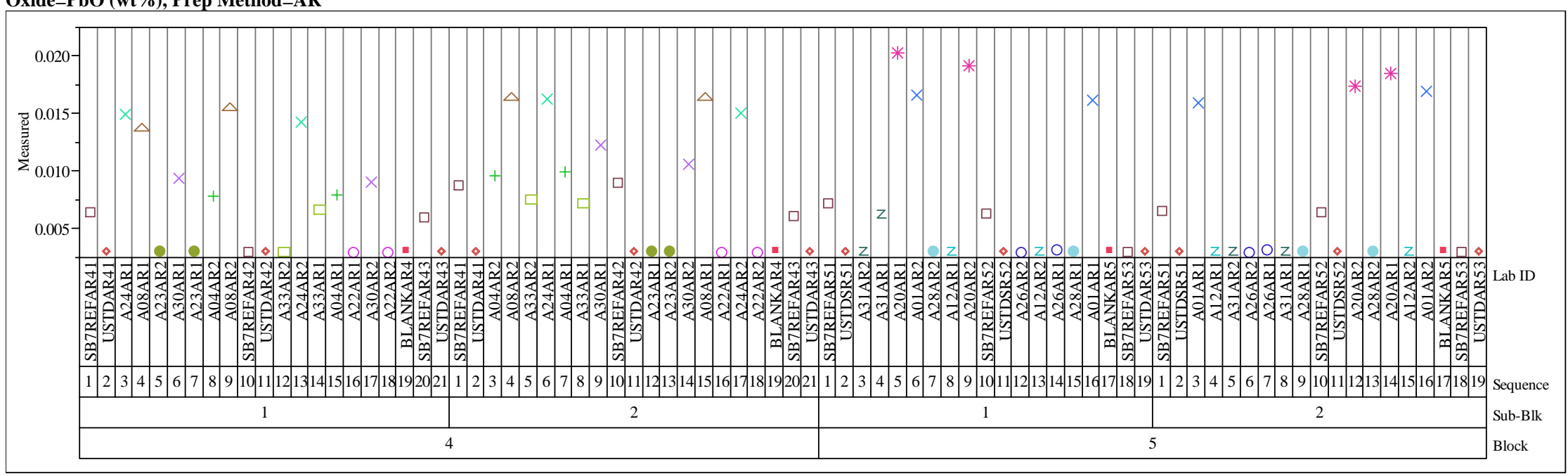

\section{Oxide=PbO (wt\%), Prep Method=PF}

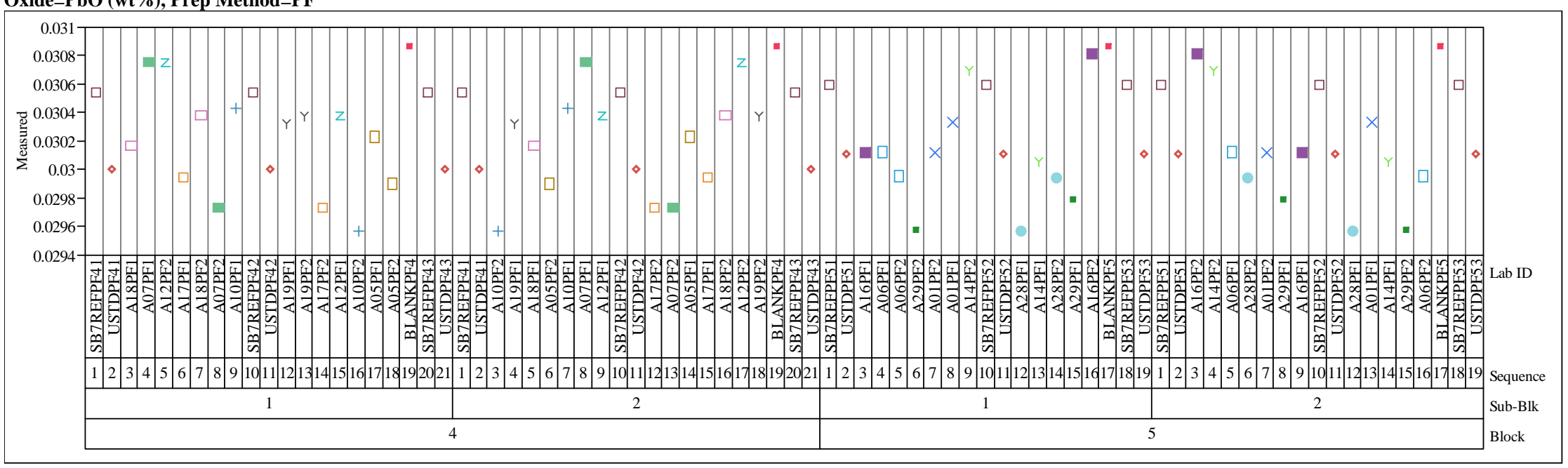


Exhibit A-2. Measurements of Initial Glasses in Analytical Sequence for Samples by Oxide and Preparation Method for Analytical Blocks 4 and 5

Oxide=SiO2 ( $w t \%)$, Prep Method=PF

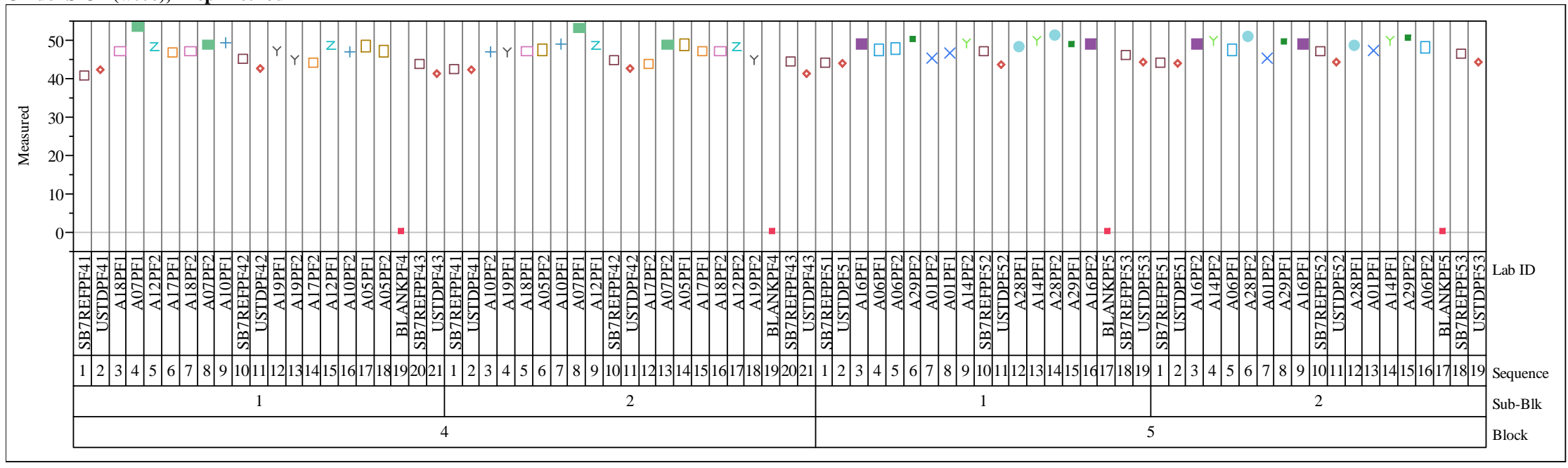

Oxide=SO4 (wt\%), Prep Method=AR

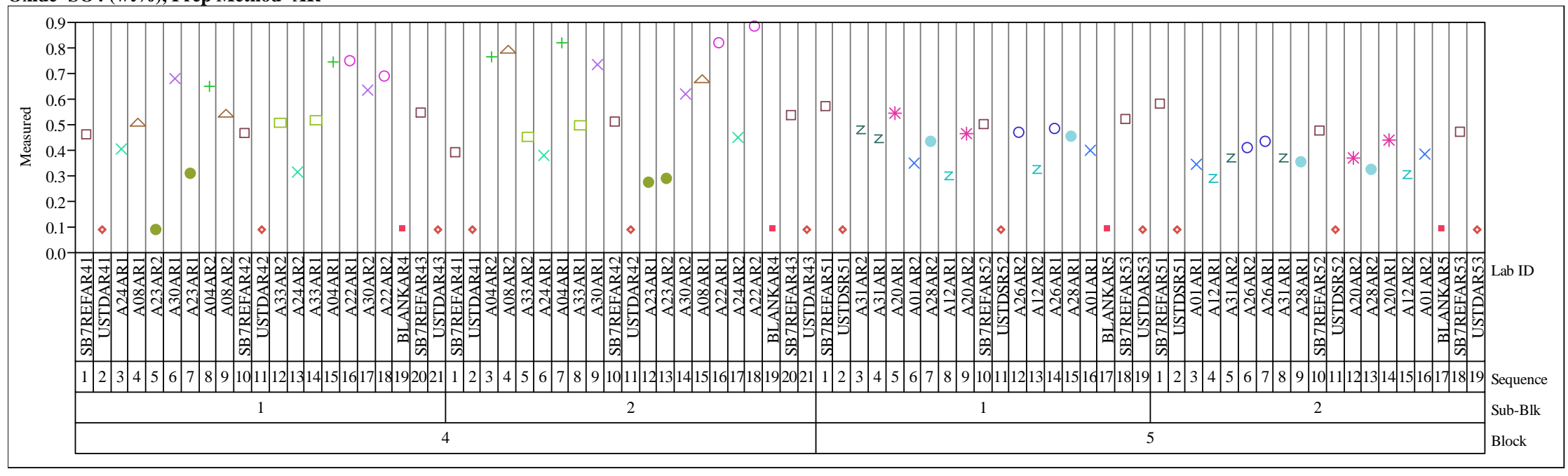


Exhibit A-2. Measurements of Initial Glasses in Analytical Sequence for Samples by Oxide and Preparation Method for Analytical Blocks 4 and 5

Oxide=SO4 (wt \%), Prep Method=PF

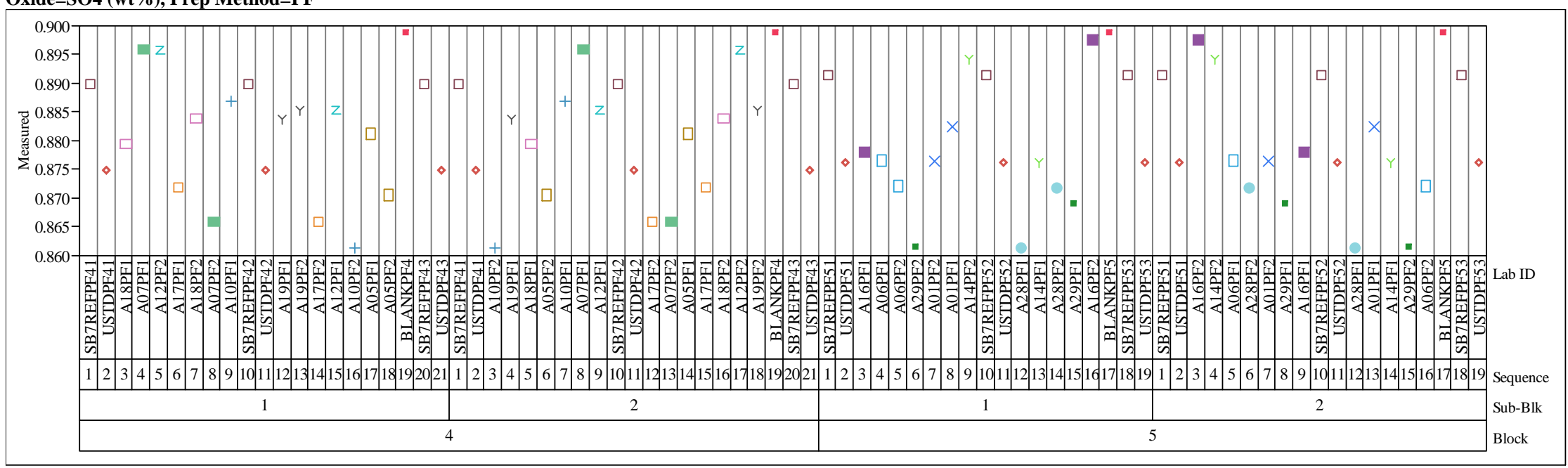

Oxide=ThO2 (wt \%), Prep Method=AR

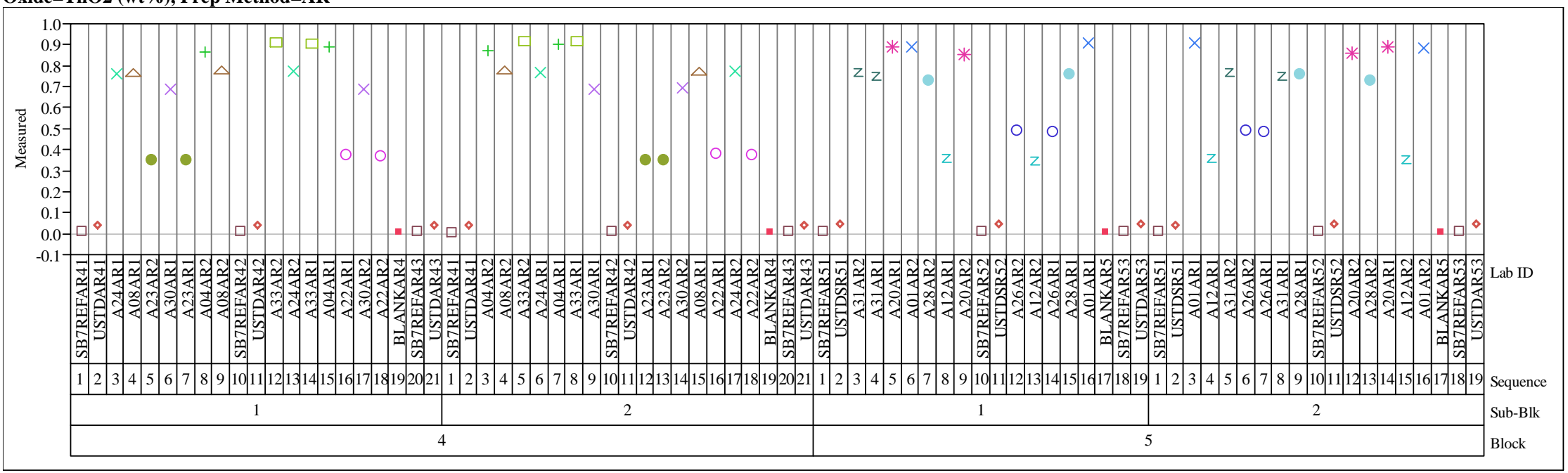


Exhibit A-2. Measurements of Initial Glasses in Analytical Sequence for Samples by Oxide and Preparation Method for Analytical Blocks 4 and 5

Oxide=ThO2 (wt\%), Prep Method=PF

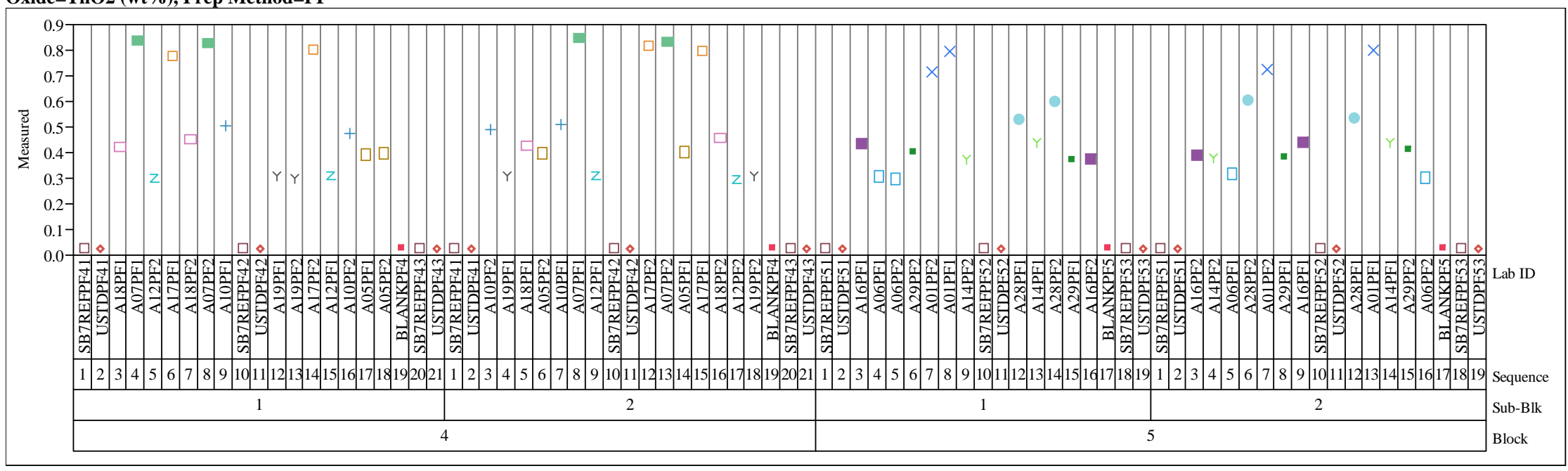

Oxide=TiO2 (wt\%), Prep Method=AR

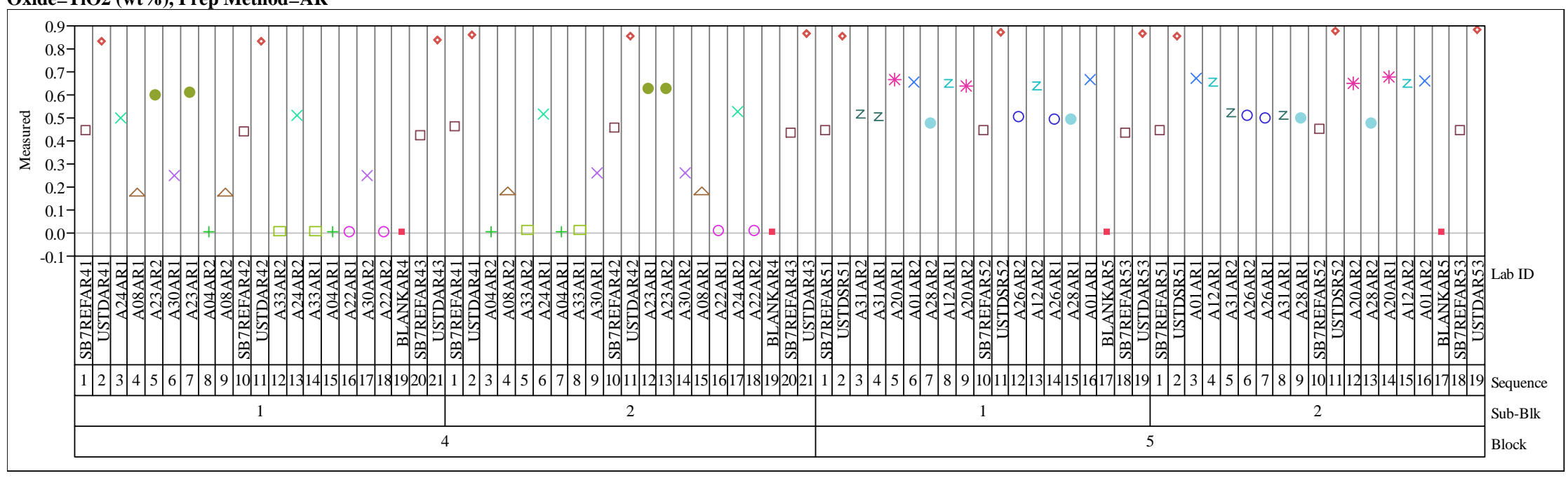


Exhibit A-2. Measurements of Initial Glasses in Analytical Sequence for Samples by Oxide and Preparation Method for Analytical Blocks 4 and 5

Oxide=TiO2 (wt\%), Prep Method=PF

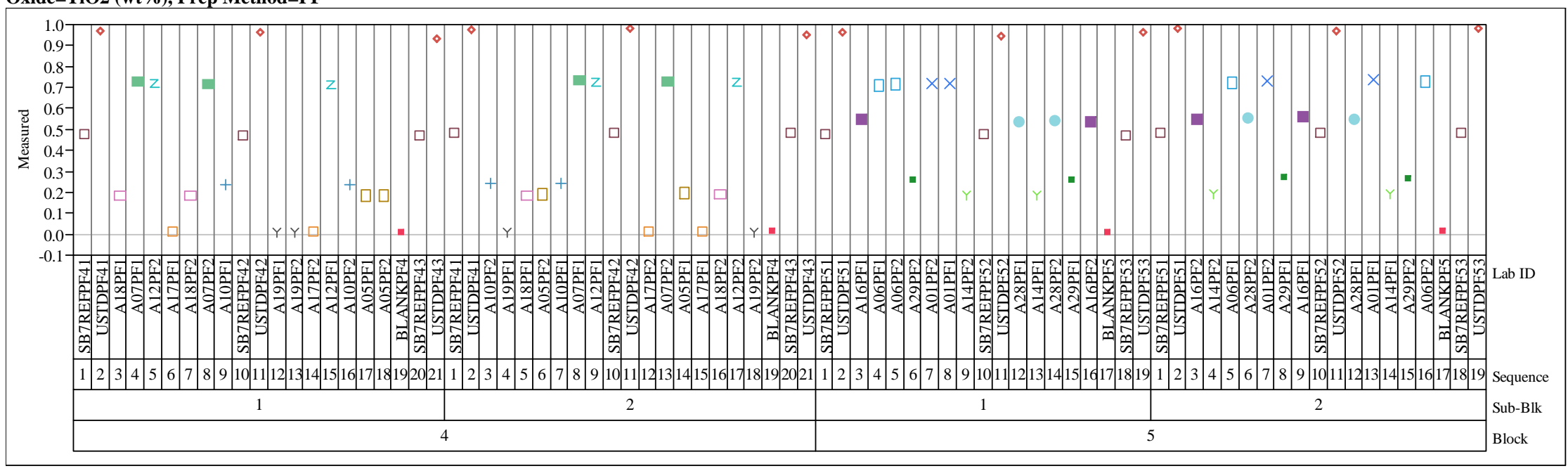

Oxide=U3O8 (wt \%), Prep Method=AR

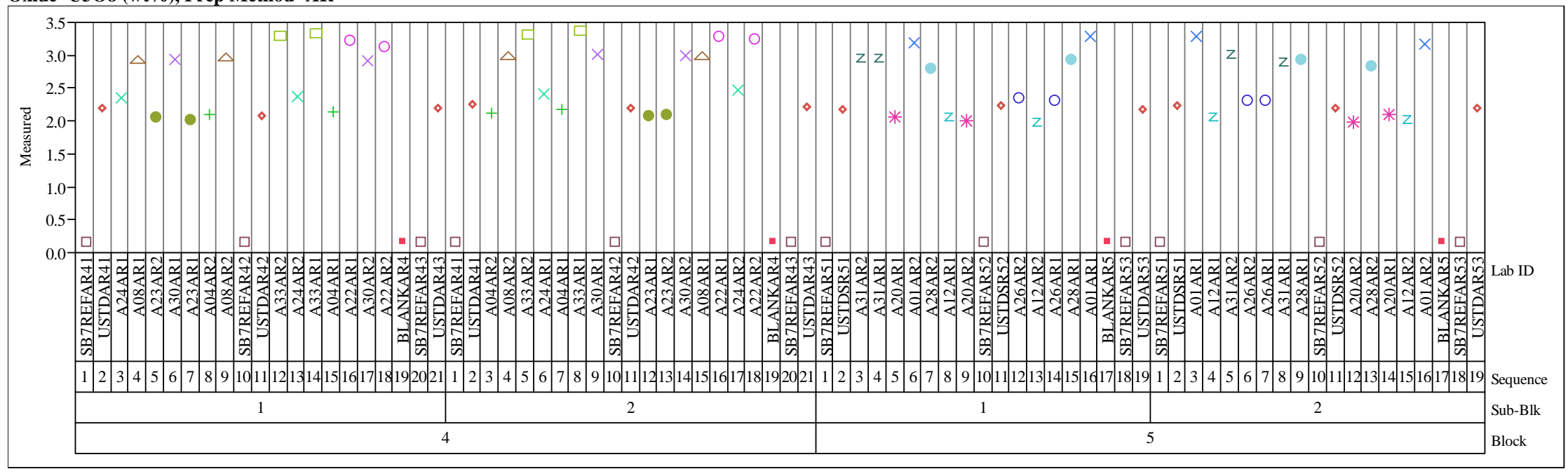


Exhibit A-2. Measurements of Initial Glasses in Analytical Sequence for Samples by Oxide and Preparation Method for Analytical Blocks 4 and 5

Oxide=U3O8 (wt \%), Prep Method=PF

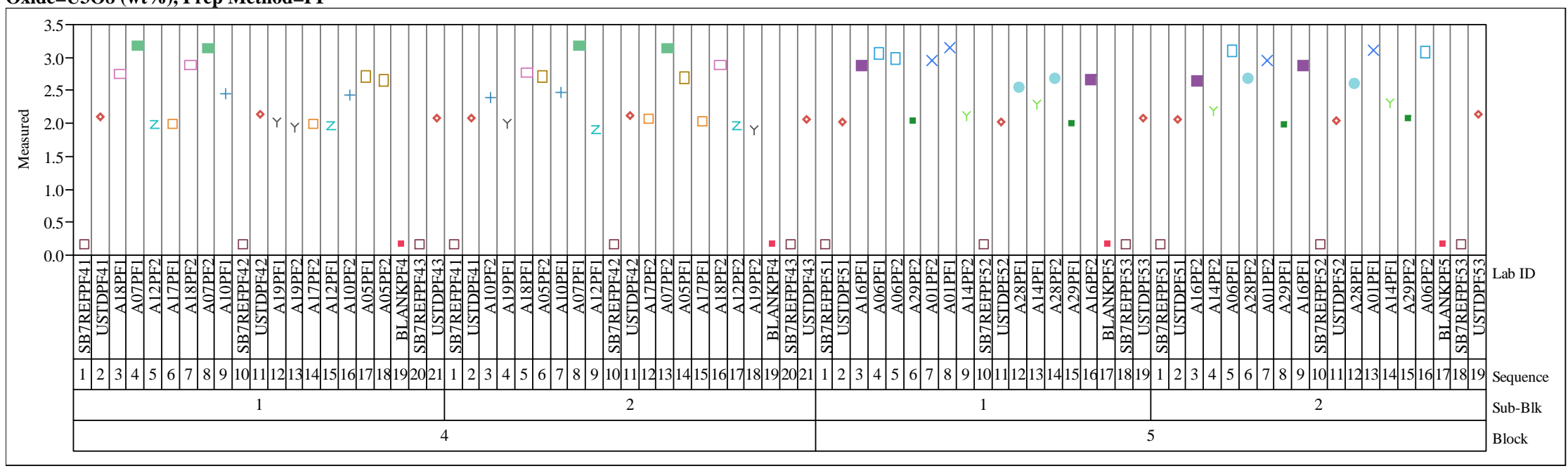

Oxide $=\mathrm{ZnO}$ (wt \%), Prep Method $=$ AR

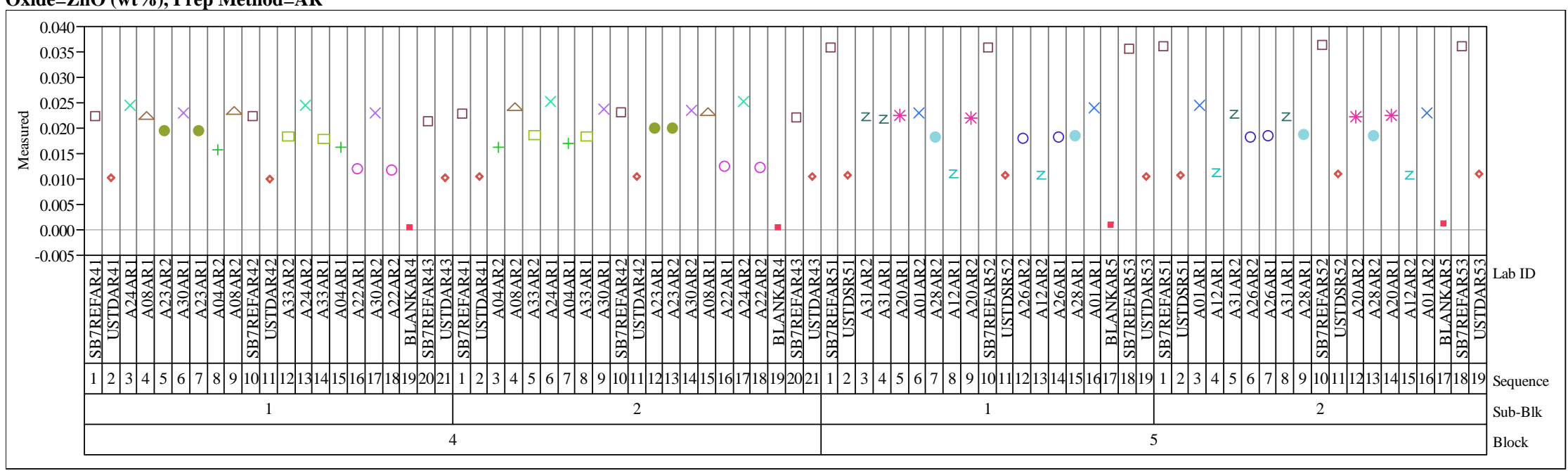


Exhibit A-2. Measurements of Initial Glasses in Analytical Sequence for Samples by Oxide and Preparation Method for Analytical Blocks 4 and 5

Oxide $=\mathrm{ZnO}$ (wt\%), Prep Method=PF

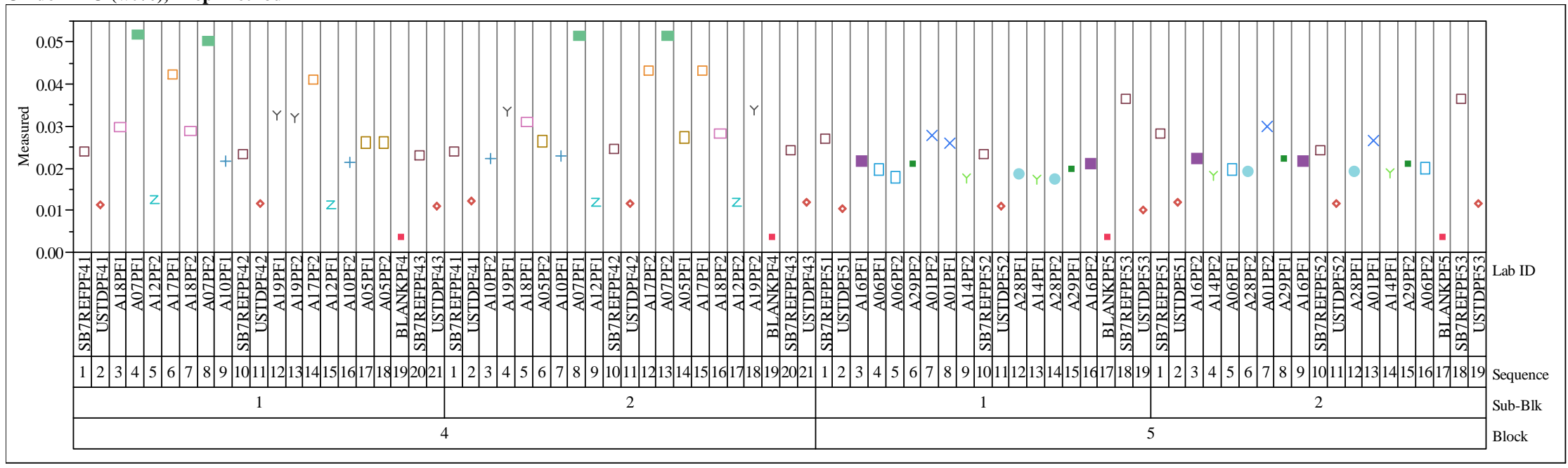

Oxide=ZrO2 (wt \%), Prep Method=AR

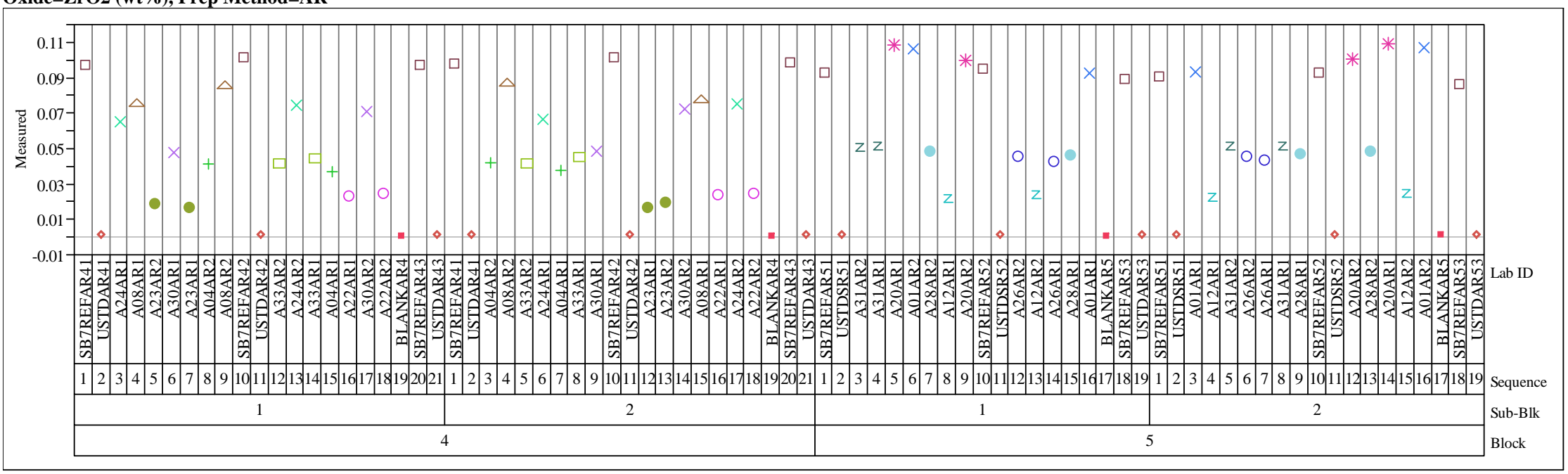


Exhibit A-3. Measurements from by Lab ID within Glass ID by Target Concentration for Each Oxide for Each Prep

Oxide=Al2O3 (wt\%), Prep Method=AR
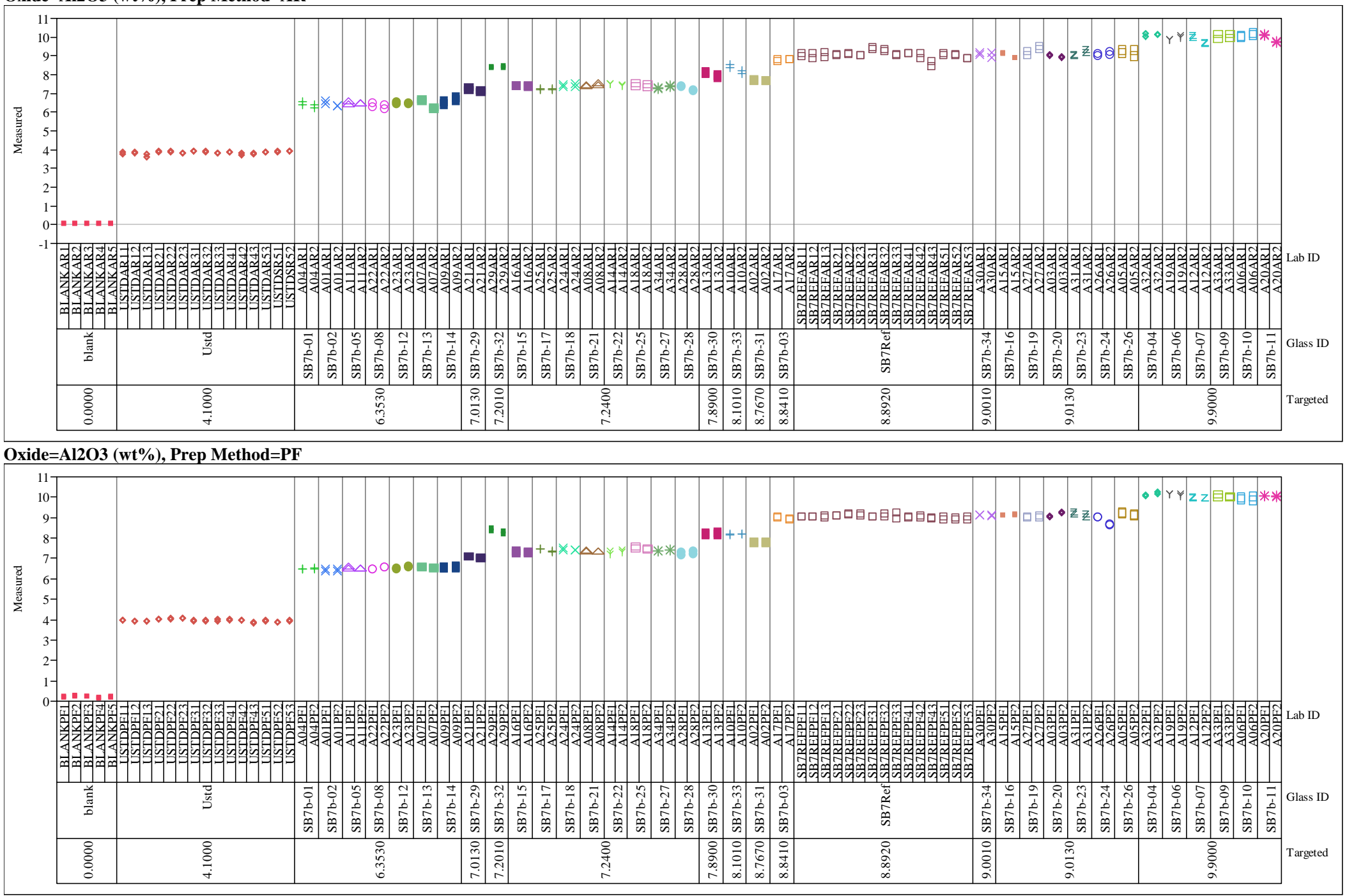
Exhibit A-3. Measurements from by Lab ID within Glass ID by Target Concentration for Each Oxide for Each Prep

Oxide $=$ B2O3 (wt \%), Prep Method $=$ AR

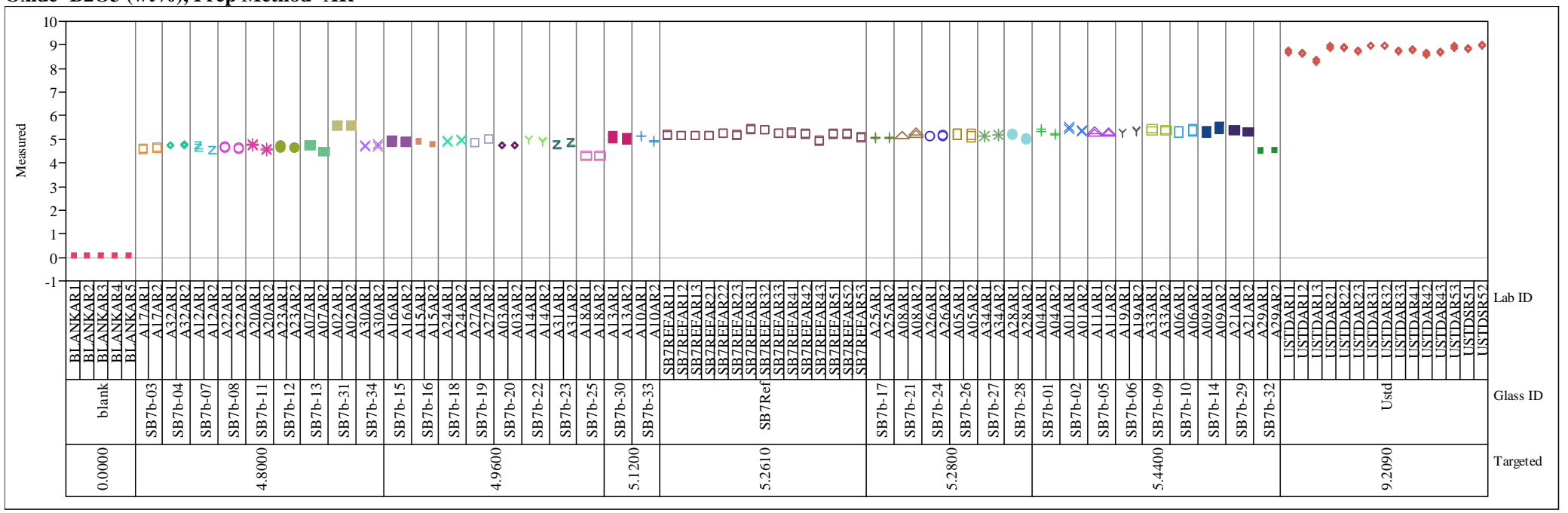

Oxide=B2O3 (wt \%), Prep Method=PF

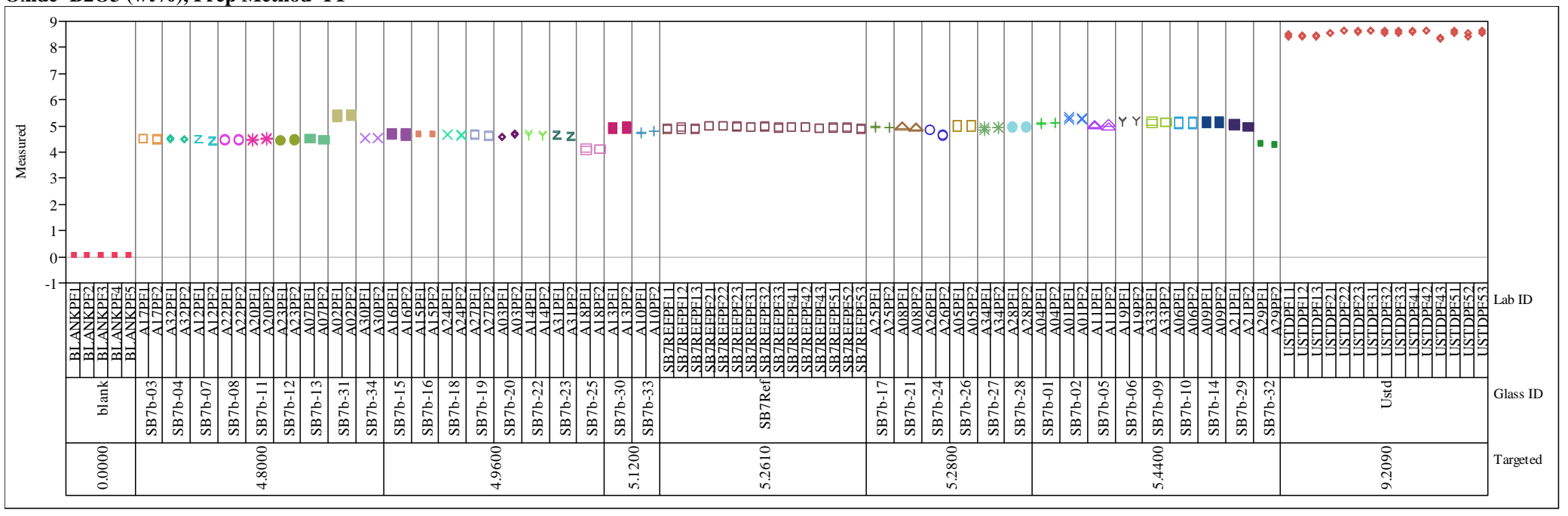


Exhibit A-3. Measurements from by Lab ID within Glass ID by Target Concentration for Each Oxide for Each Prep
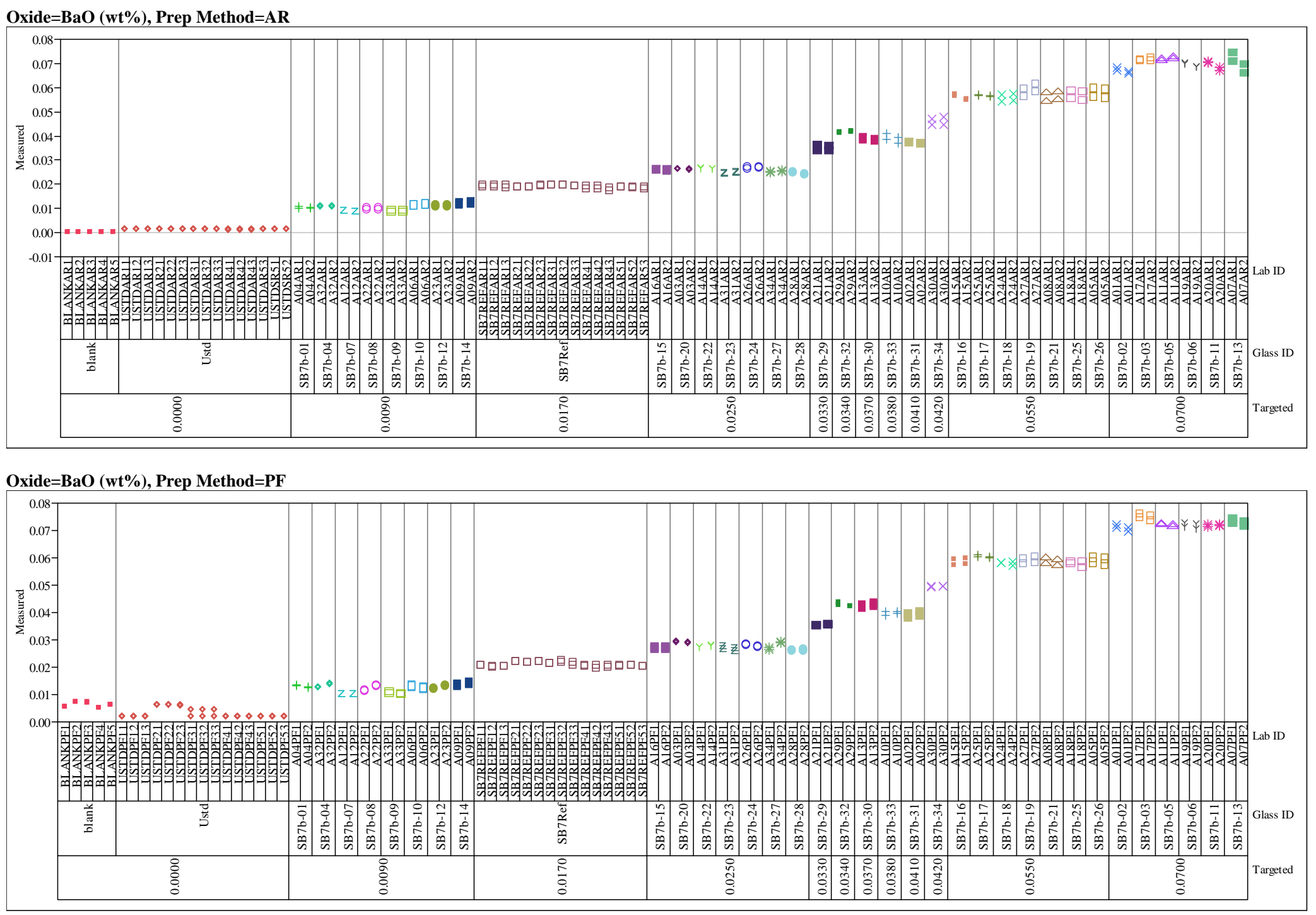
Exhibit A-3. Measurements from by Lab ID within Glass ID by Target Concentration for Each Oxide for Each Prep Oxide $=\mathbf{C a O}(w t \%)$, Prep Method $=$ AR
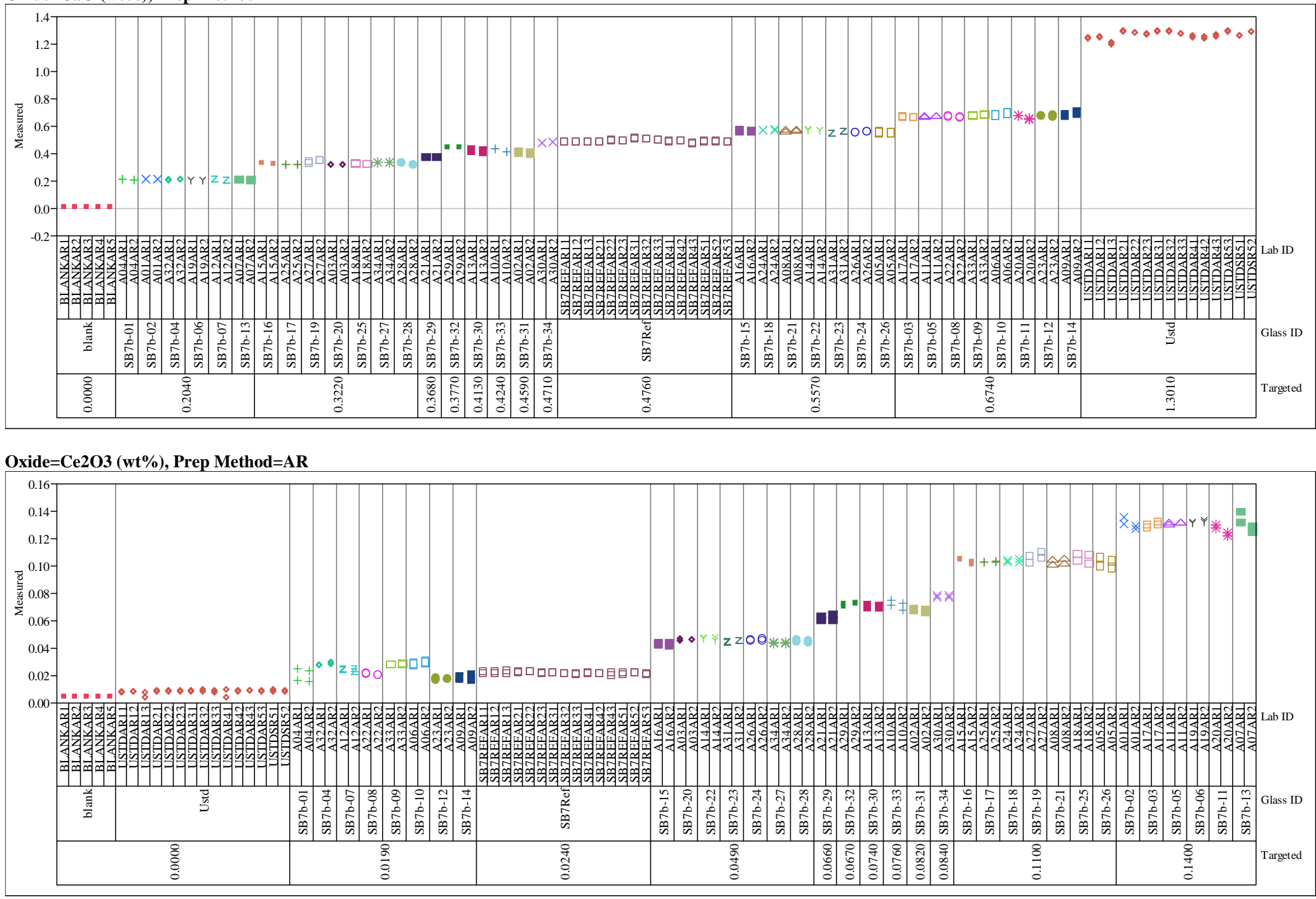
Exhibit A-3. Measurements from by Lab ID within Glass ID by Target Concentration for Each Oxide for Each Prep

Oxide $=\mathrm{Ce} 2 \mathrm{O} 3(\mathrm{wt} \%)$, Prep Method $=\mathrm{PF}$

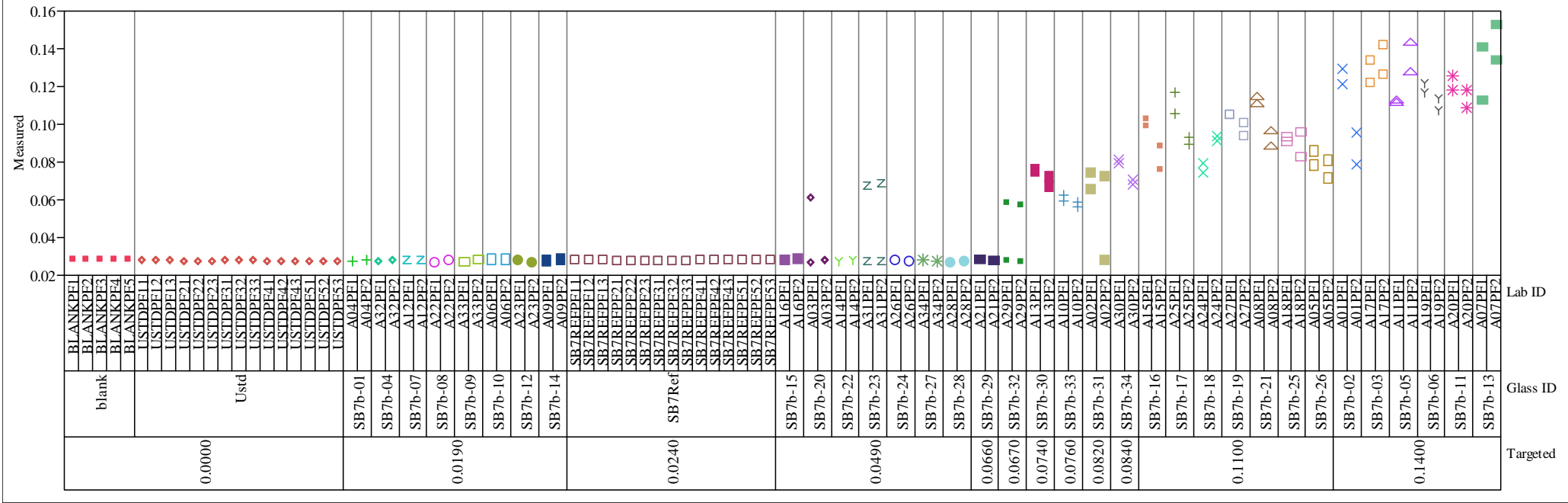

Oxide $=\mathrm{Cr} 2 \mathrm{O} 3(\mathrm{wt} \%)$, Prep Method $=$ AR
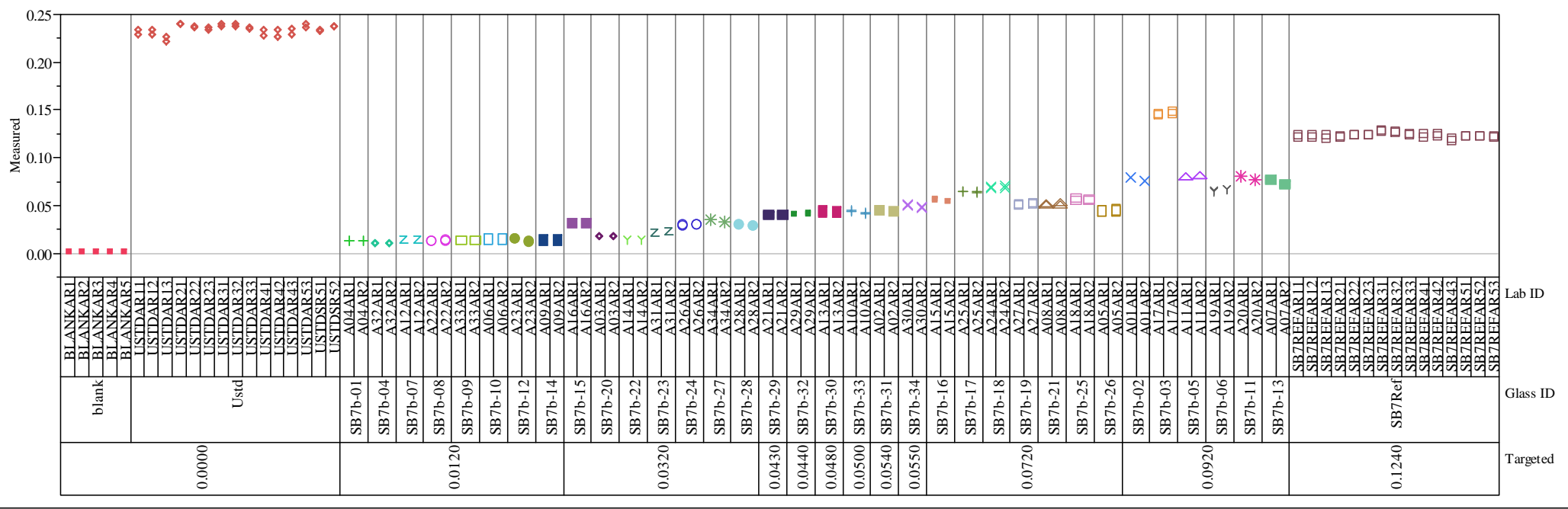
Exhibit A-3. Measurements from by Lab ID within Glass ID by Target Concentration for Each Oxide for Each Prep

Oxide $=\mathrm{Cr} 2 \mathrm{O} 3$ (wt \%), Prep Method $=$ P
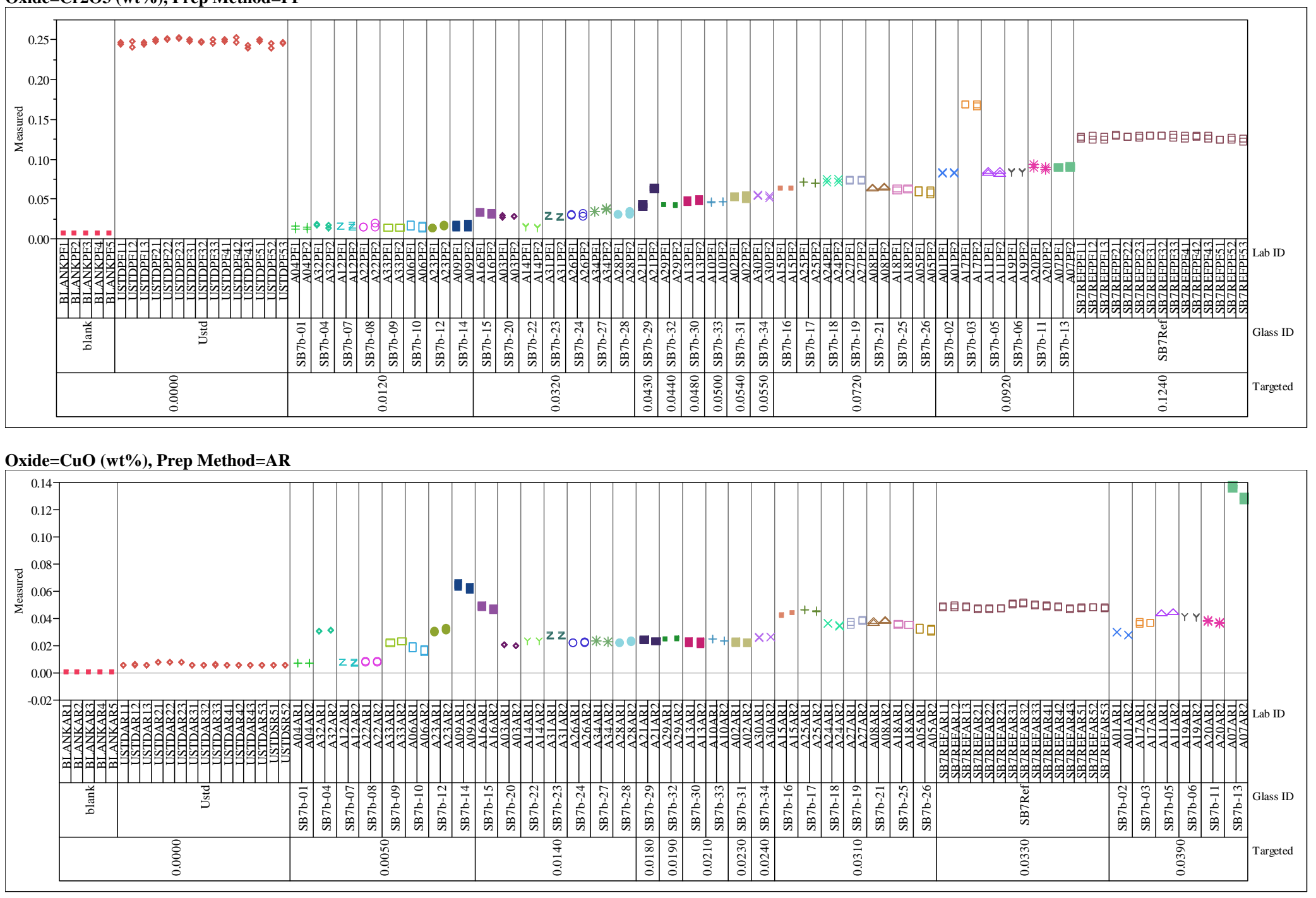
Exhibit A-3. Measurements from by Lab ID within Glass ID by Target Concentration for Each Oxide for Each Prep

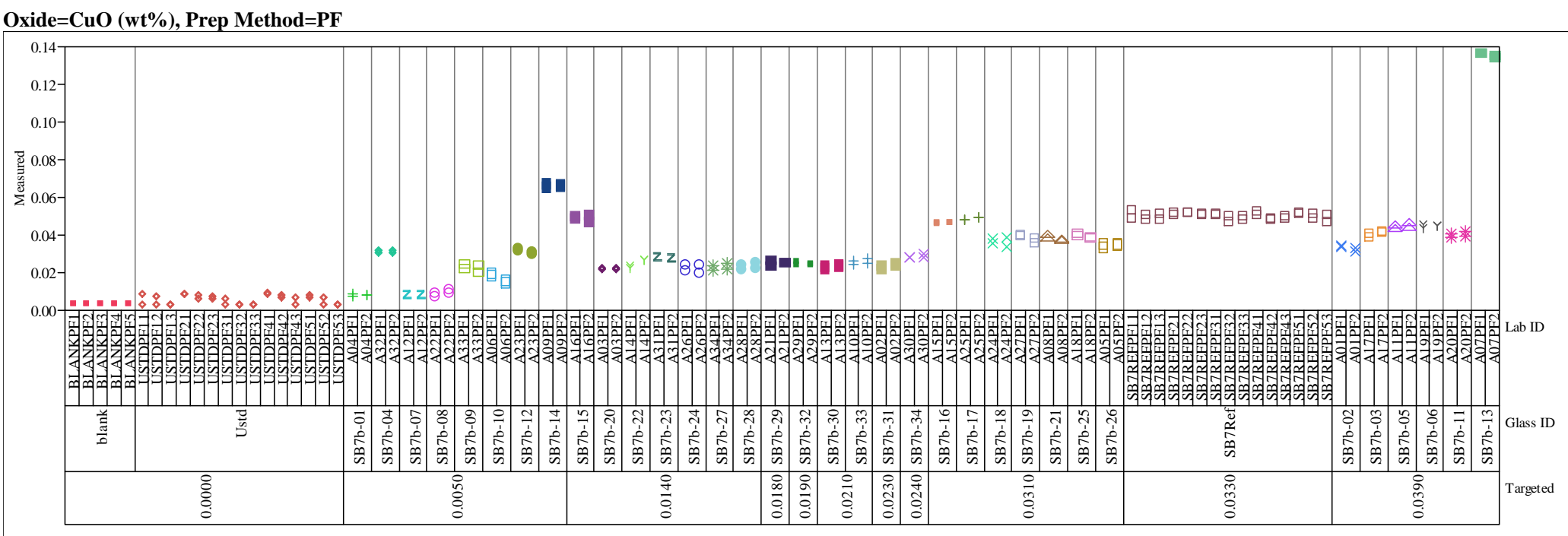

\section{Oxide=Fe2O3 (wt\%), Prep Method=AR}

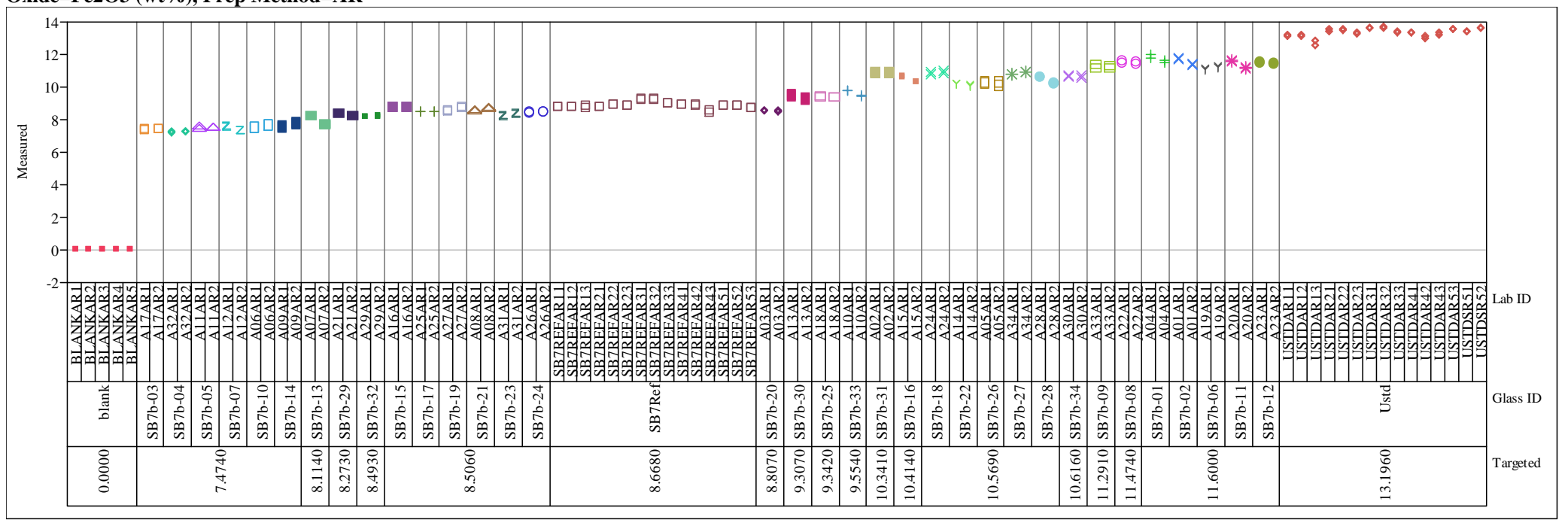


Exhibit A-3. Measurements from by Lab ID within Glass ID by Target Concentration for Each Oxide for Each Prep Oxide $=$ Fe2O3 (wt \%), Prep Method $=$ PF
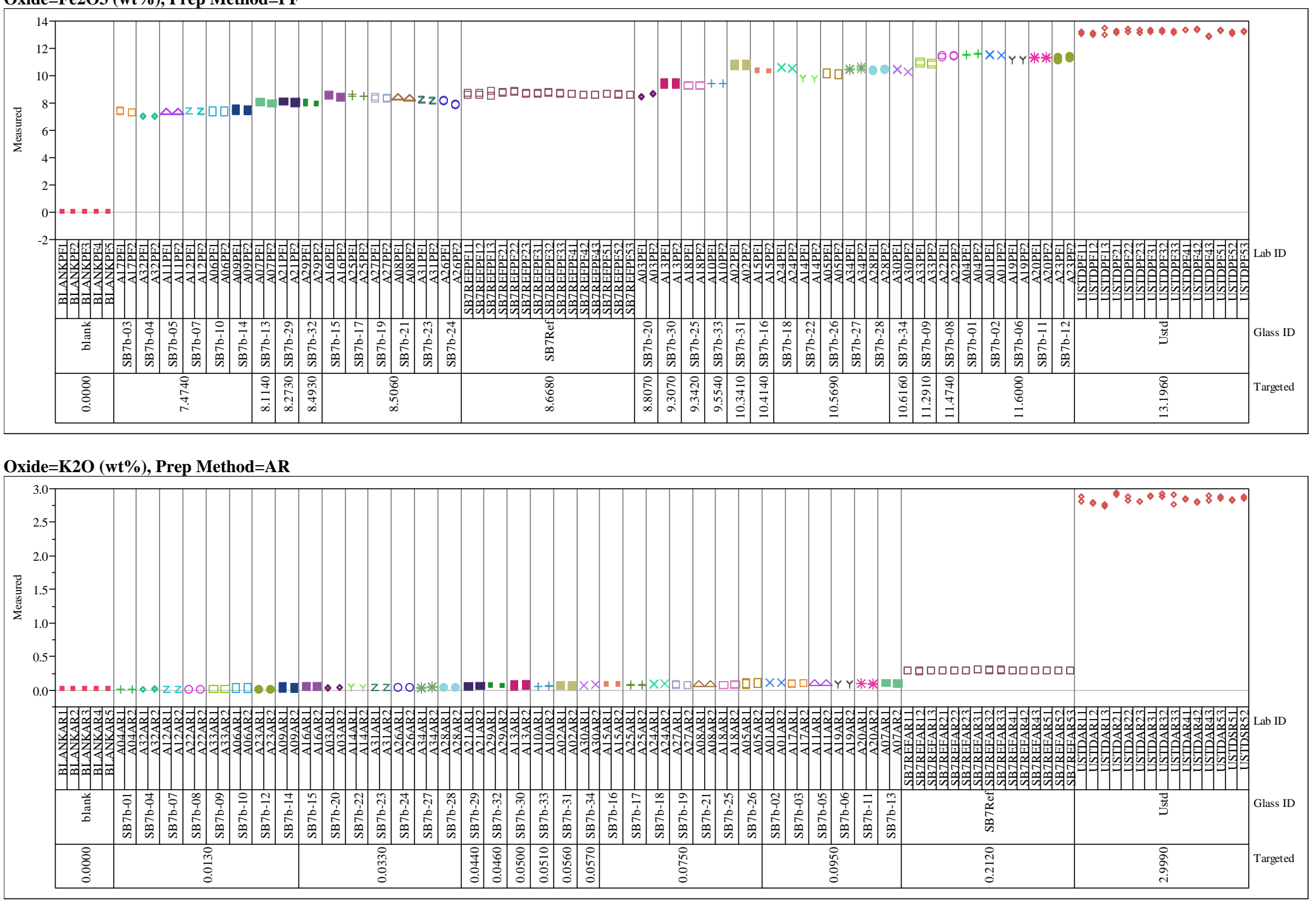
Exhibit A-3. Measurements from by Lab ID within Glass ID by Target Concentration for Each Oxide for Each Prep Oxide=K2O (wt\%), Prep Method=PF
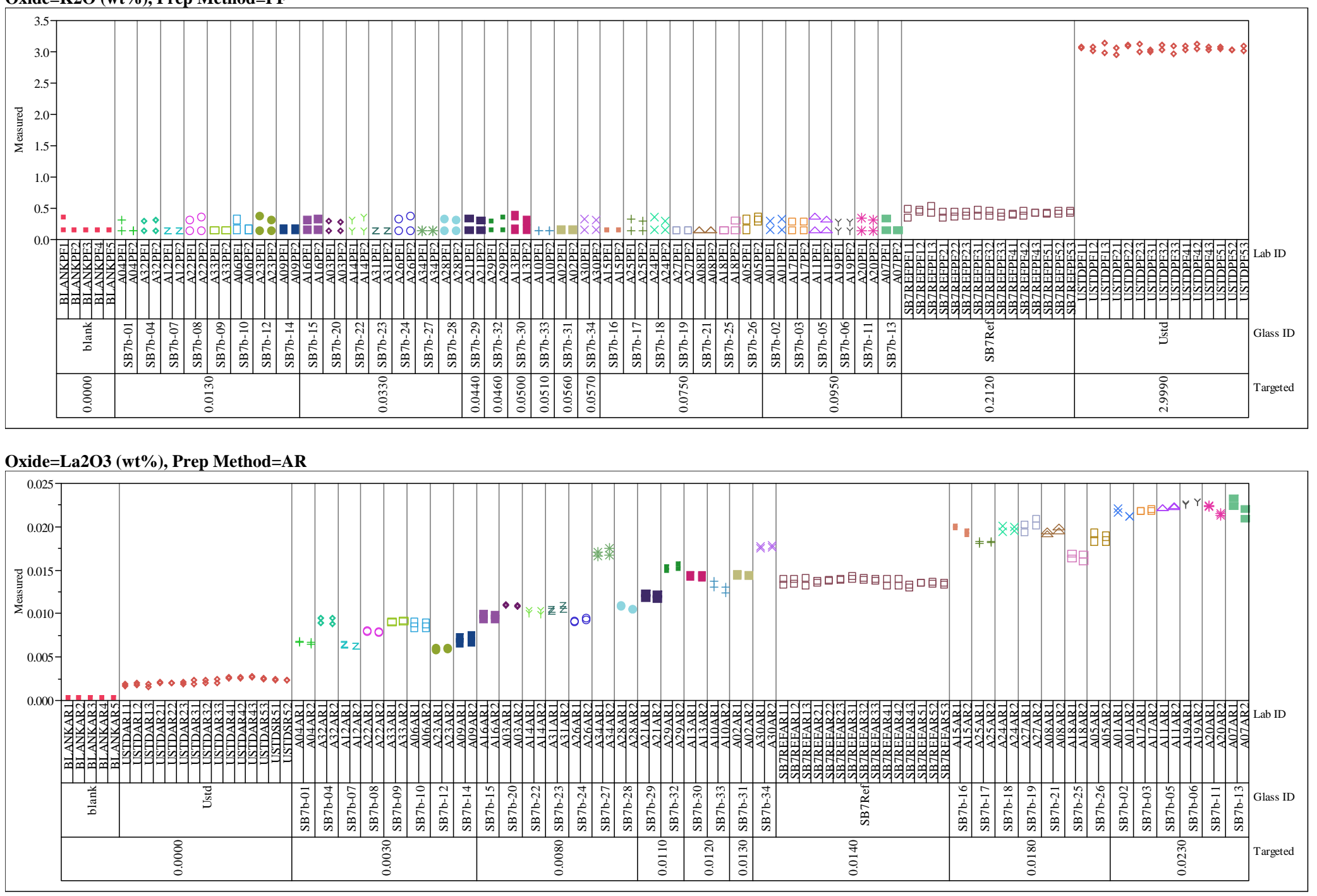
Exhibit A-3. Measurements from by Lab ID within Glass ID by Target Concentration for Each Oxide for Each Prep

Oxide=La2O3 (wt \%), Prep Method=PF

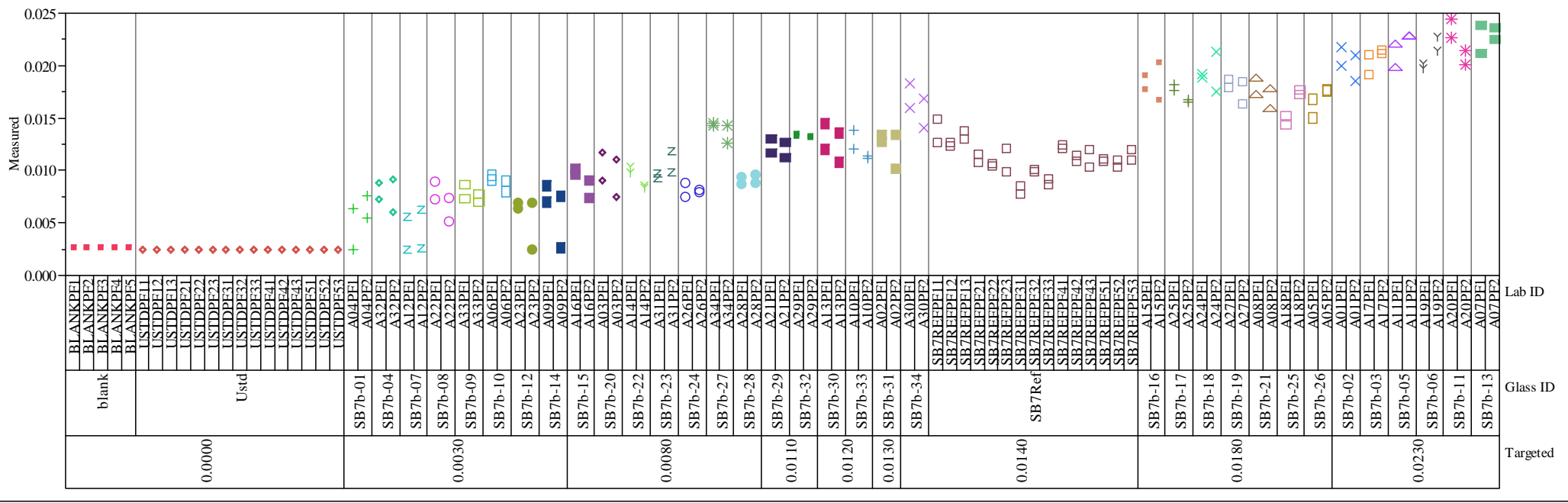

Oxide=Li2O (wt \%), Prep Method=AR

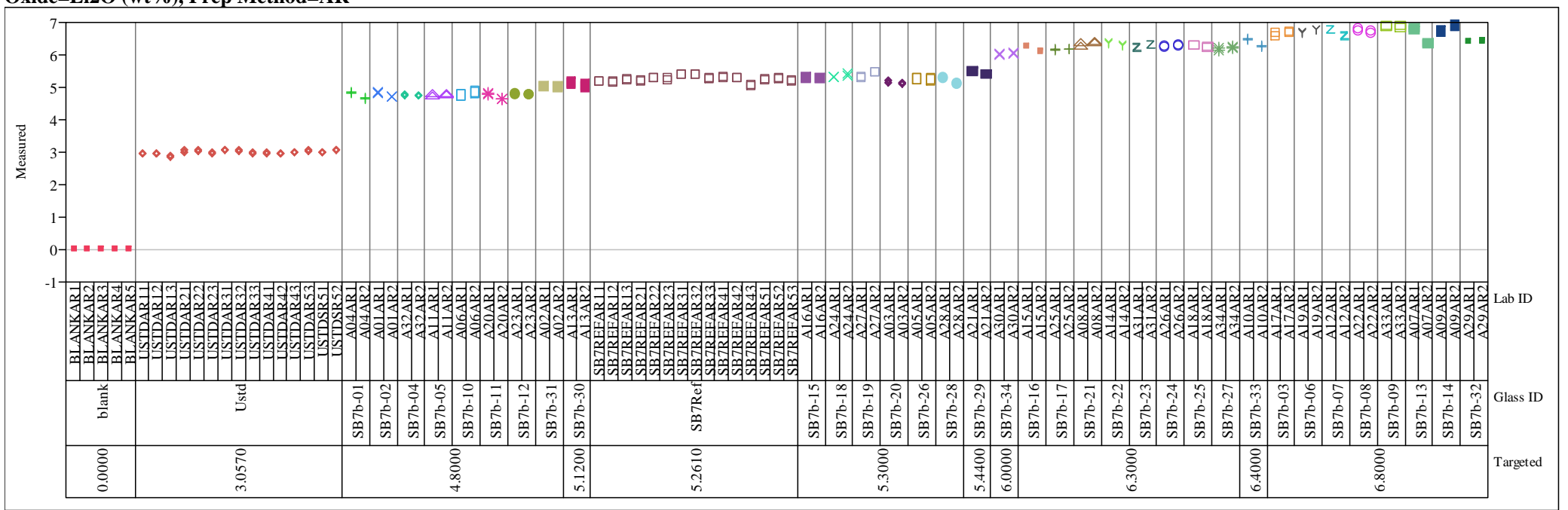


Exhibit A-3. Measurements from by Lab ID within Glass ID by Target Concentration for Each Oxide for Each Prep

Oxide=Li2O (wt\%), Prep Method=PF

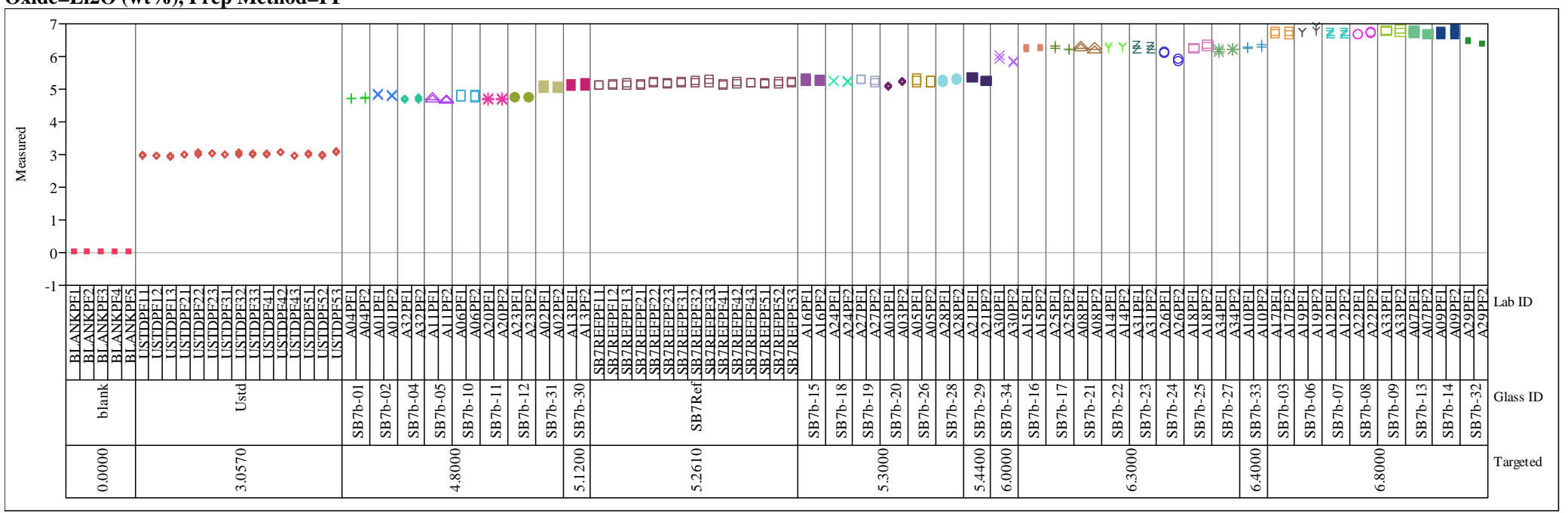

Oxide=MgO (wt\%), Prep Method=AR

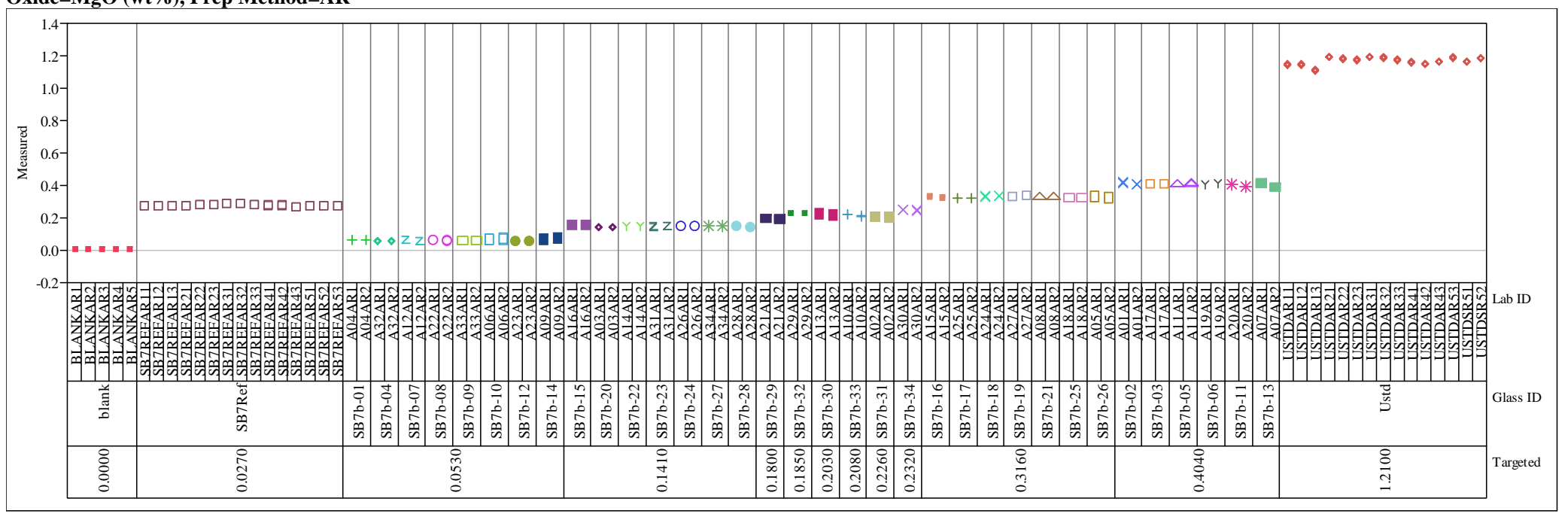


Exhibit A-3. Measurements from by Lab ID within Glass ID by Target Concentration for Each Oxide for Each Prep

Oxide=MgO (wt \%), Prep Method=PF

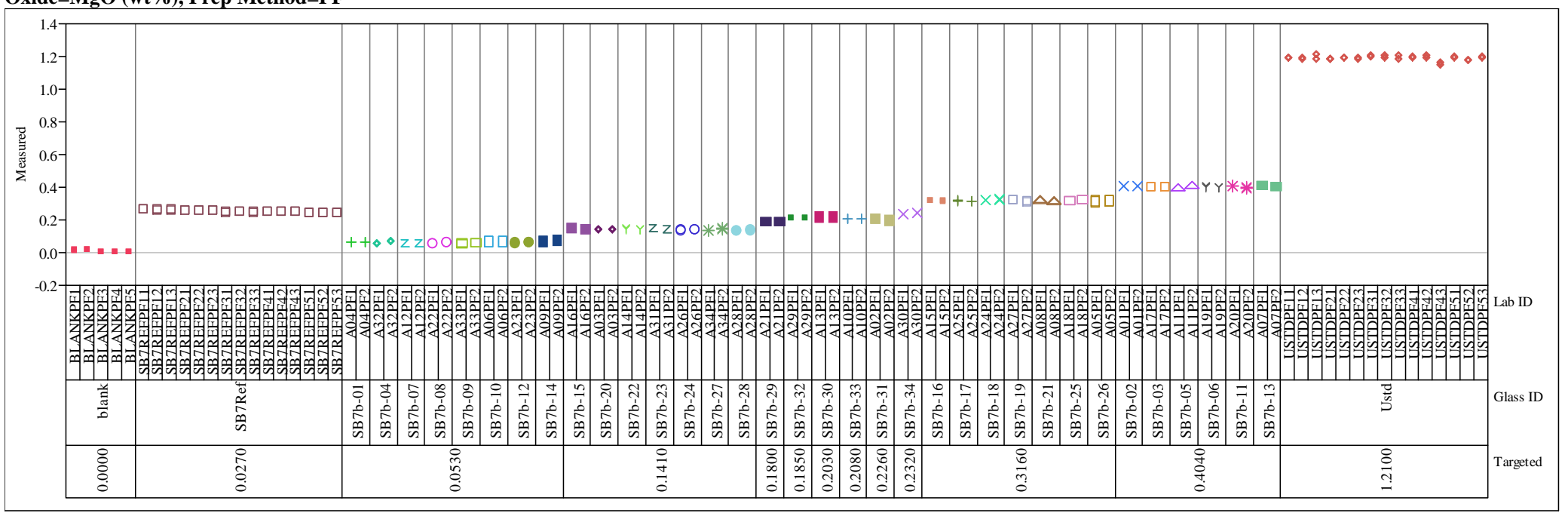

Oxide=MnO (wt\%), Prep Method=AR

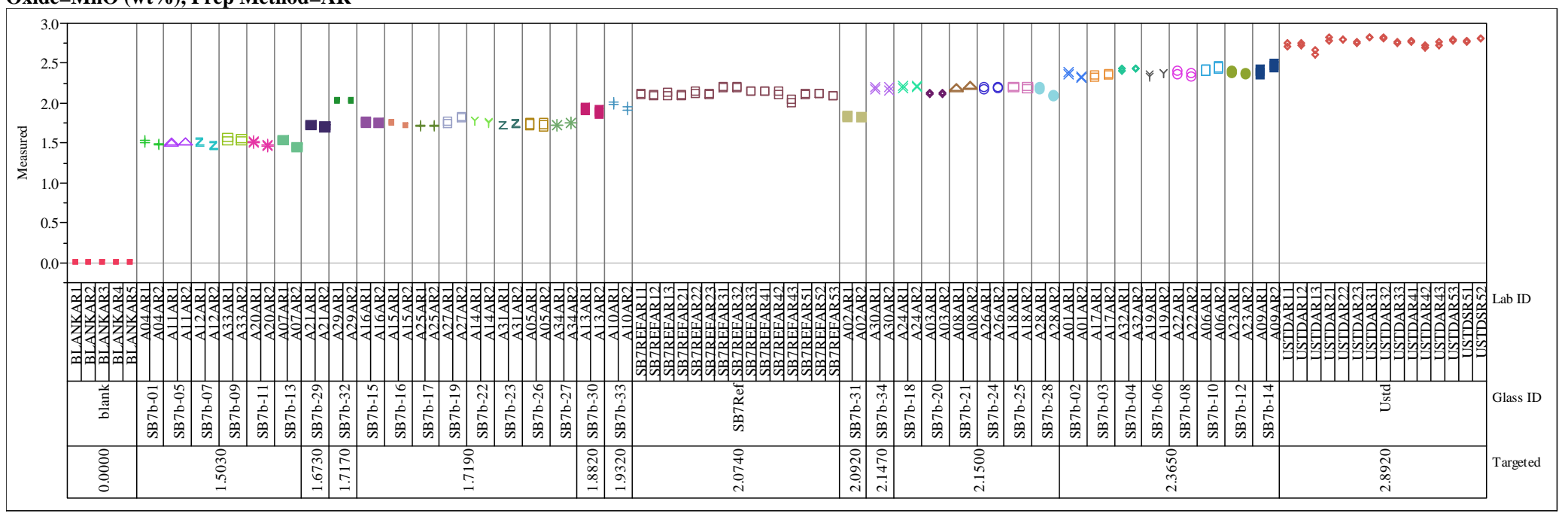


Exhibit A-3. Measurements from by Lab ID within Glass ID by Target Concentration for Each Oxide for Each Prep

Oxide $=\mathrm{MnO}$ (wt \%), Prep Method=PF

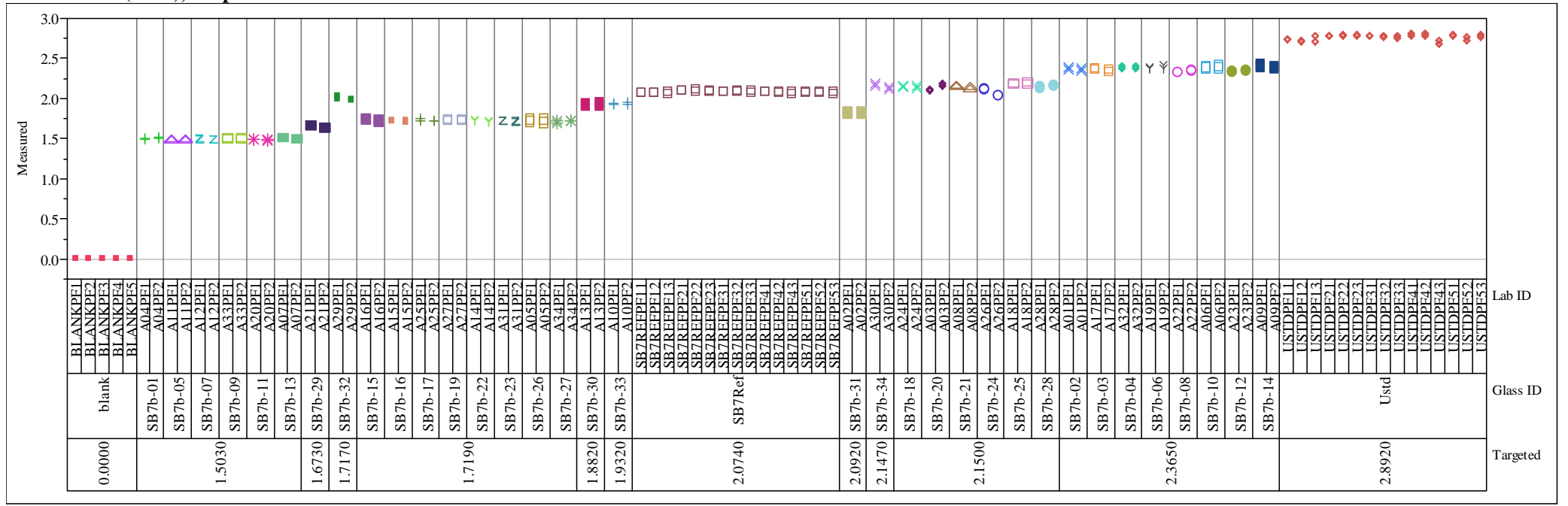

Oxide=Na2O (wt \%), Prep Method=AR

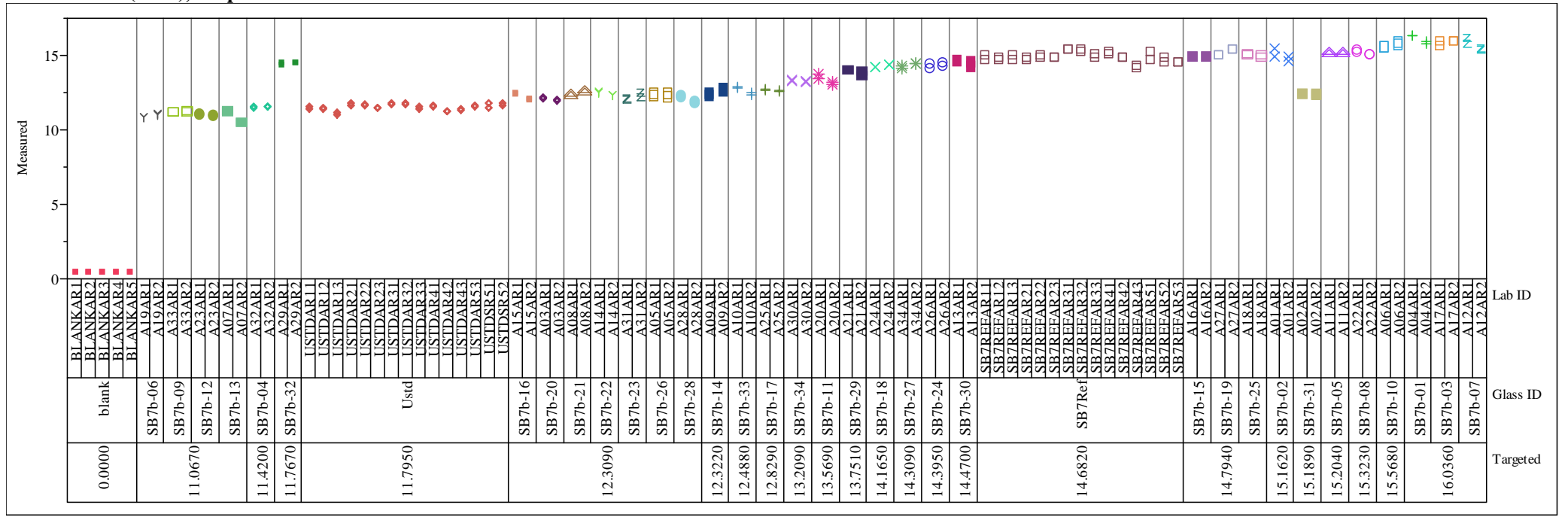


Exhibit A-3. Measurements from by Lab ID within Glass ID by Target Concentration for Each Oxide for Each Prep

Oxide $=\mathrm{NiO}(\mathrm{wt} \%)$, Prep Method $=$ AR

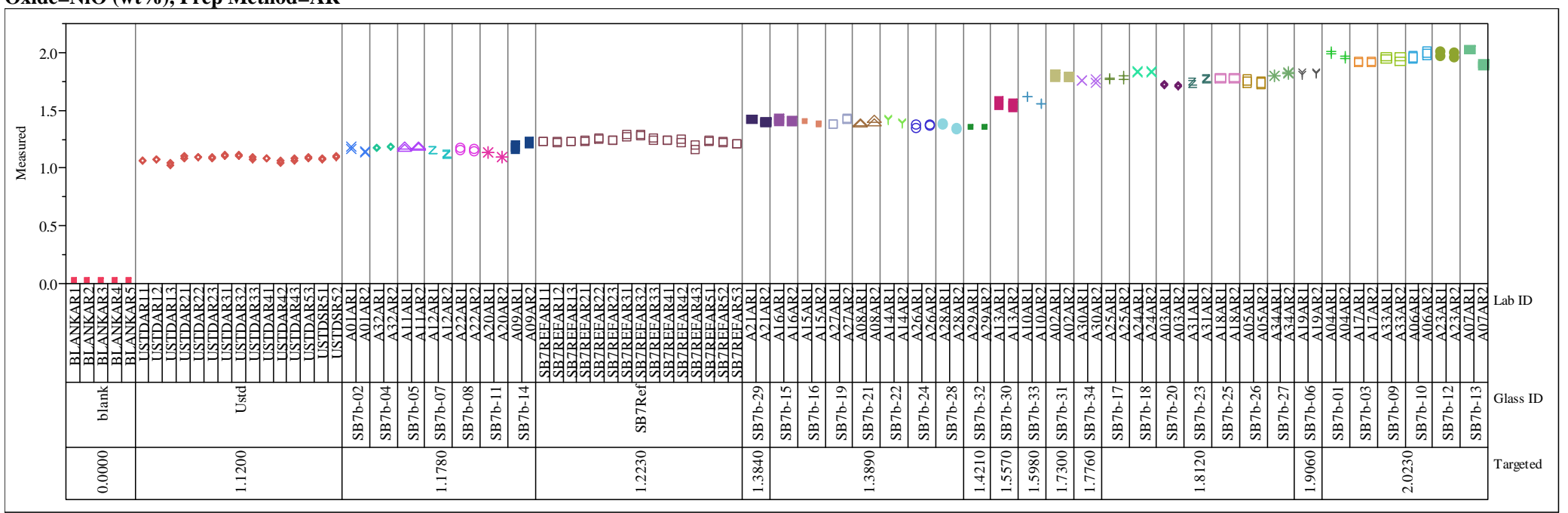

Oxide=NiO (wt \%), Prep Method=PF

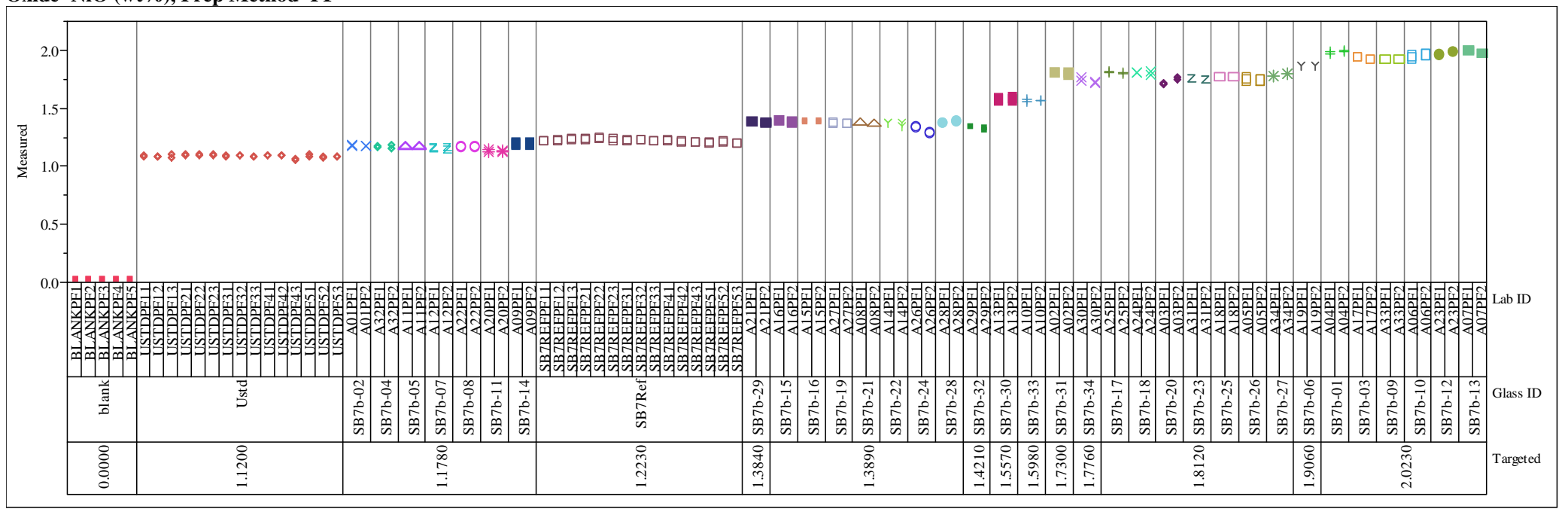


Exhibit A-3. Measurements from by Lab ID within Glass ID by Target Concentration for Each Oxide for Each Prep
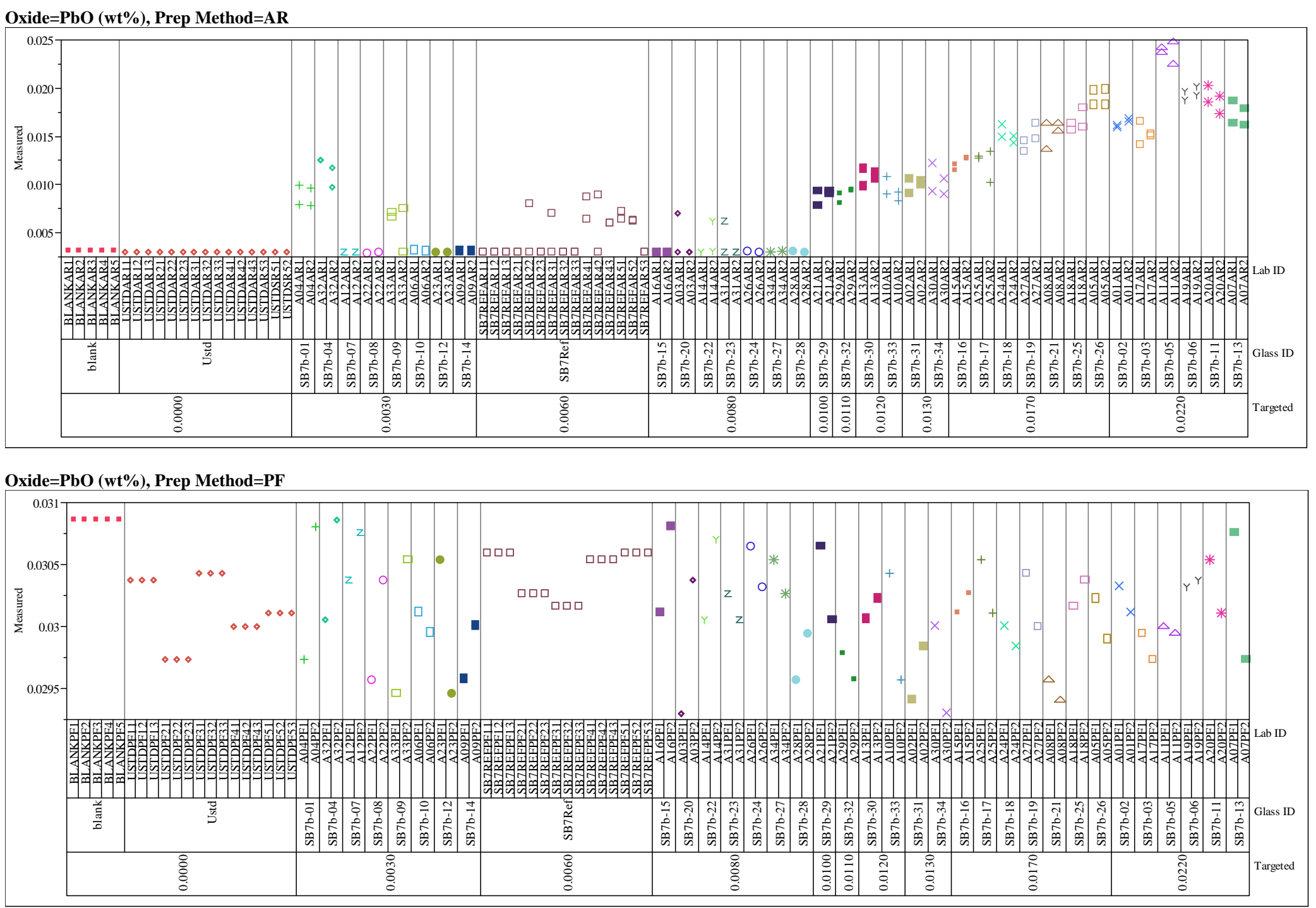
Exhibit A-3. Measurements from by Lab ID within Glass ID by Target Concentration for Each Oxide for Each Prep

Oxide=SiO2 (wt\%), Prep Method=PF

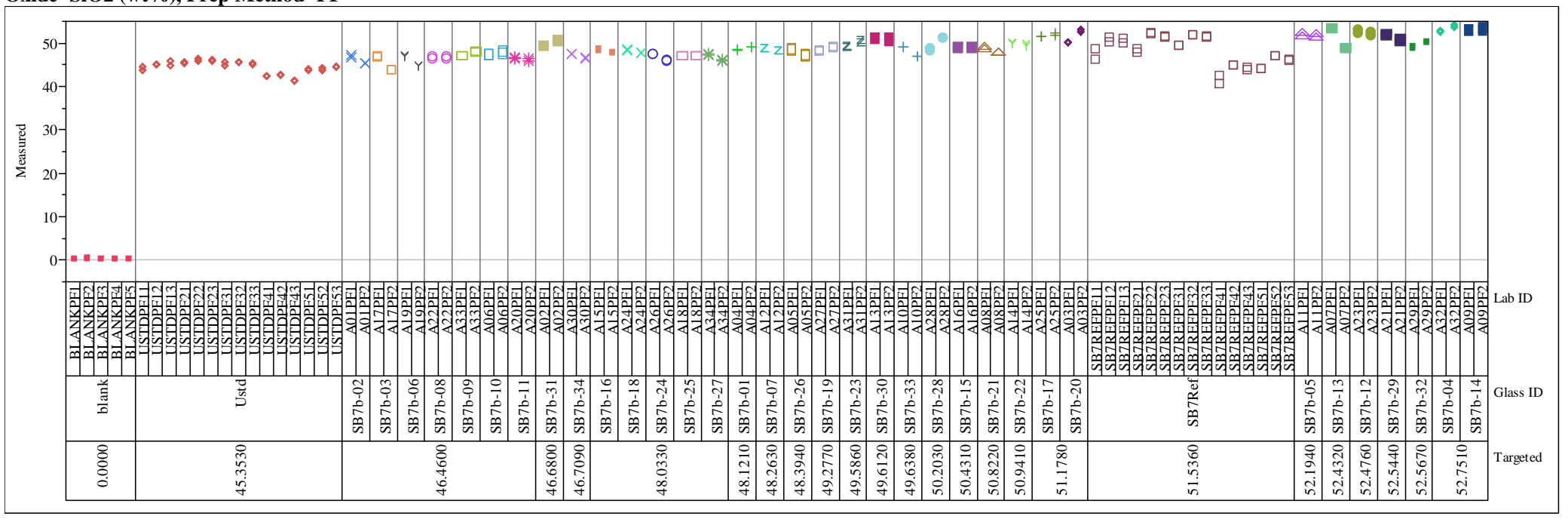

Oxide=SO4 (wt\%), Prep Method=AR

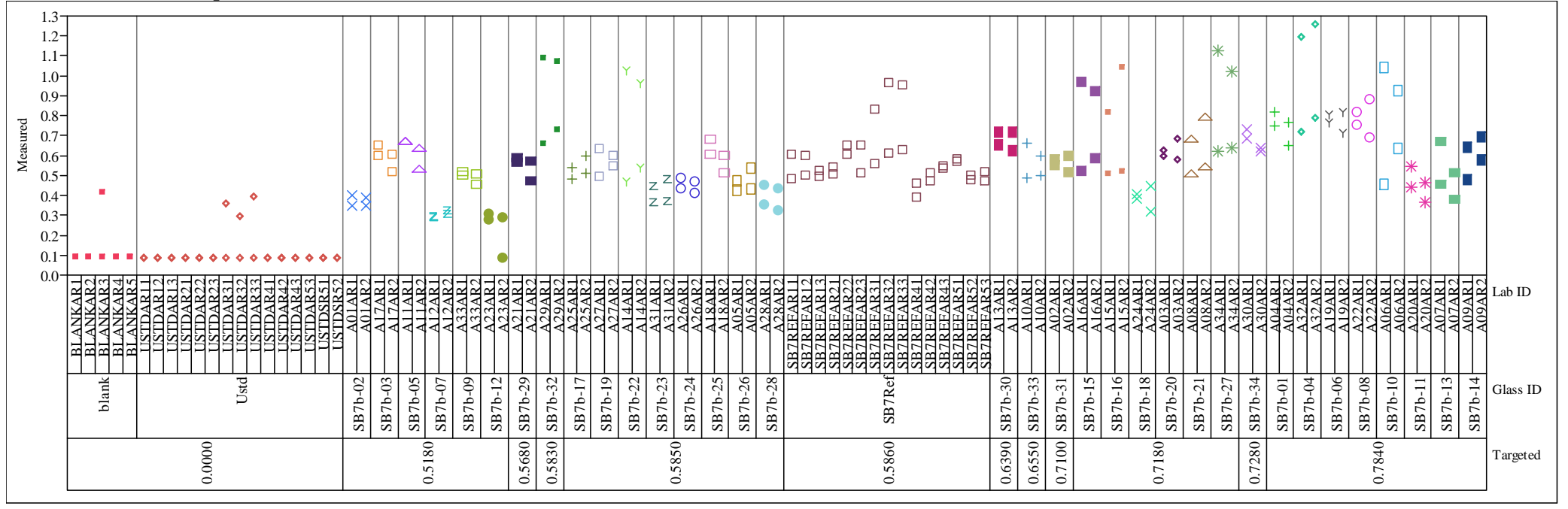


Exhibit A-3. Measurements from by Lab ID within Glass ID by Target Concentration for Each Oxide for Each Prep

Oxide $=\mathrm{SO} 4$ (wt \%), Prep Method=PF

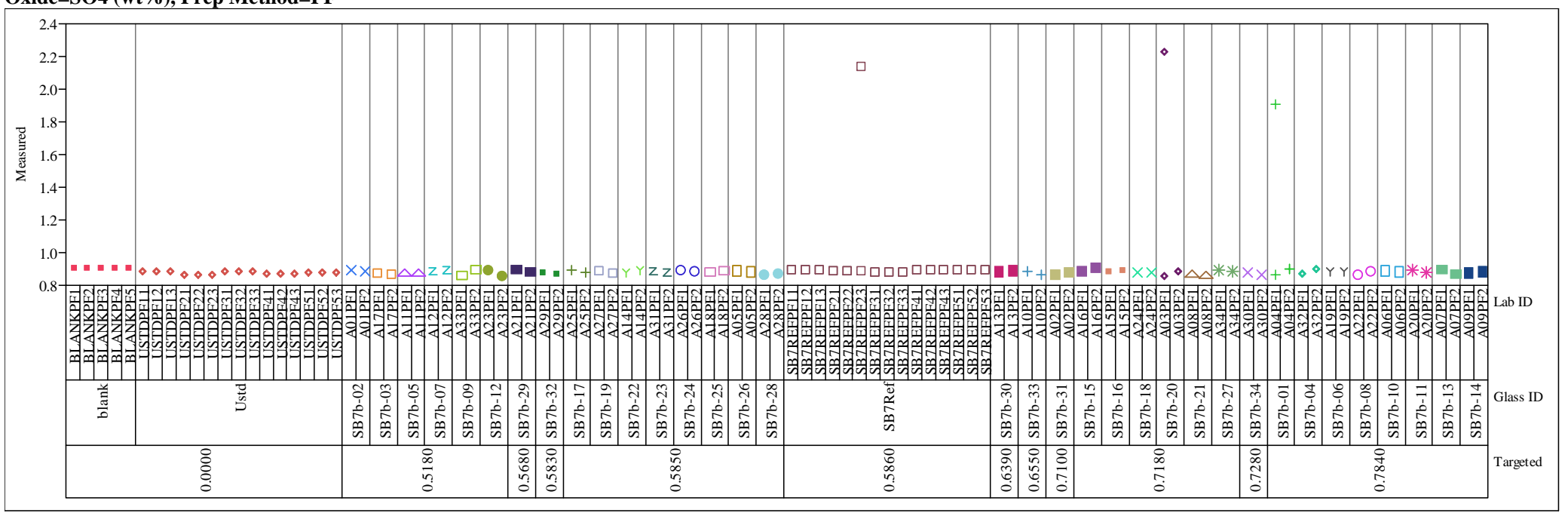

Oxide=ThO2 (wt \%), Prep Method=AR

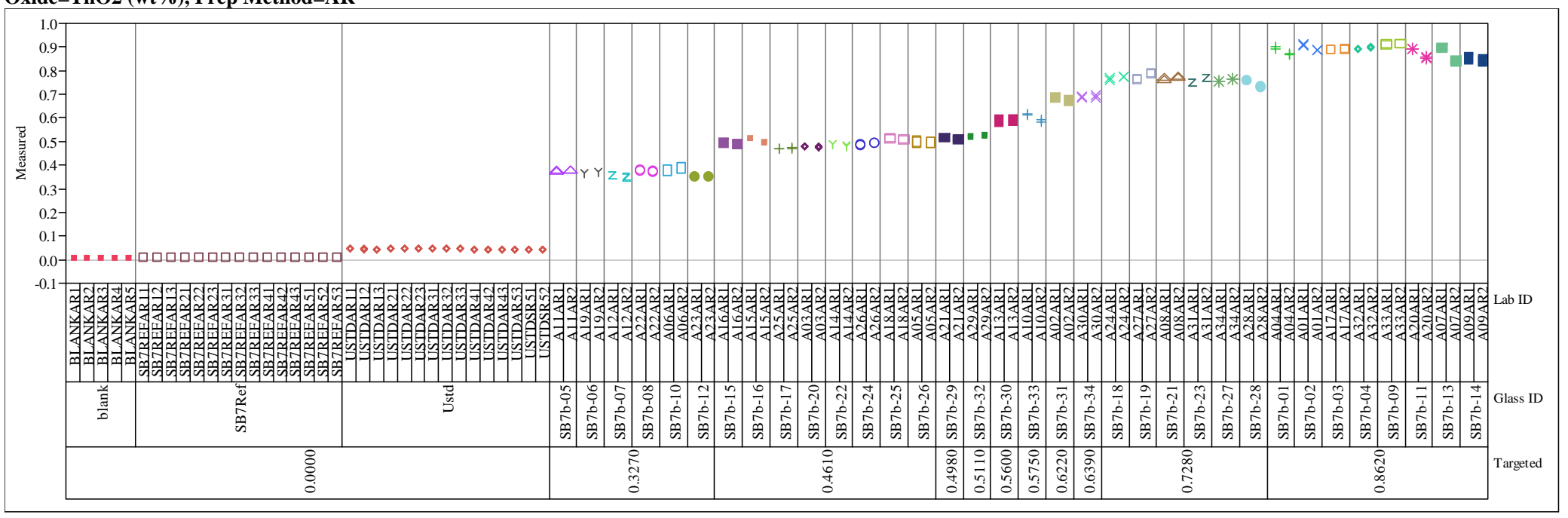


Exhibit A-3. Measurements from by Lab ID within Glass ID by Target Concentration for Each Oxide for Each Prep

Oxide=ThO2 (wt\%), Prep Method=PF

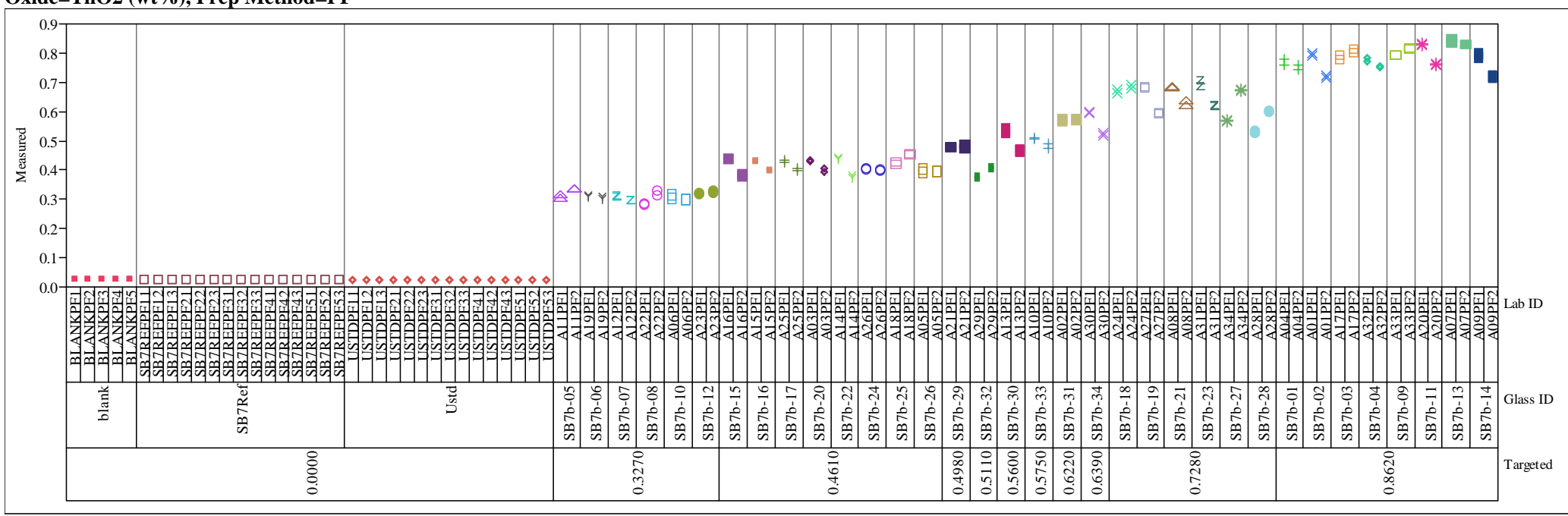

Oxide=TiO2 (wt\%), Prep Method=AR

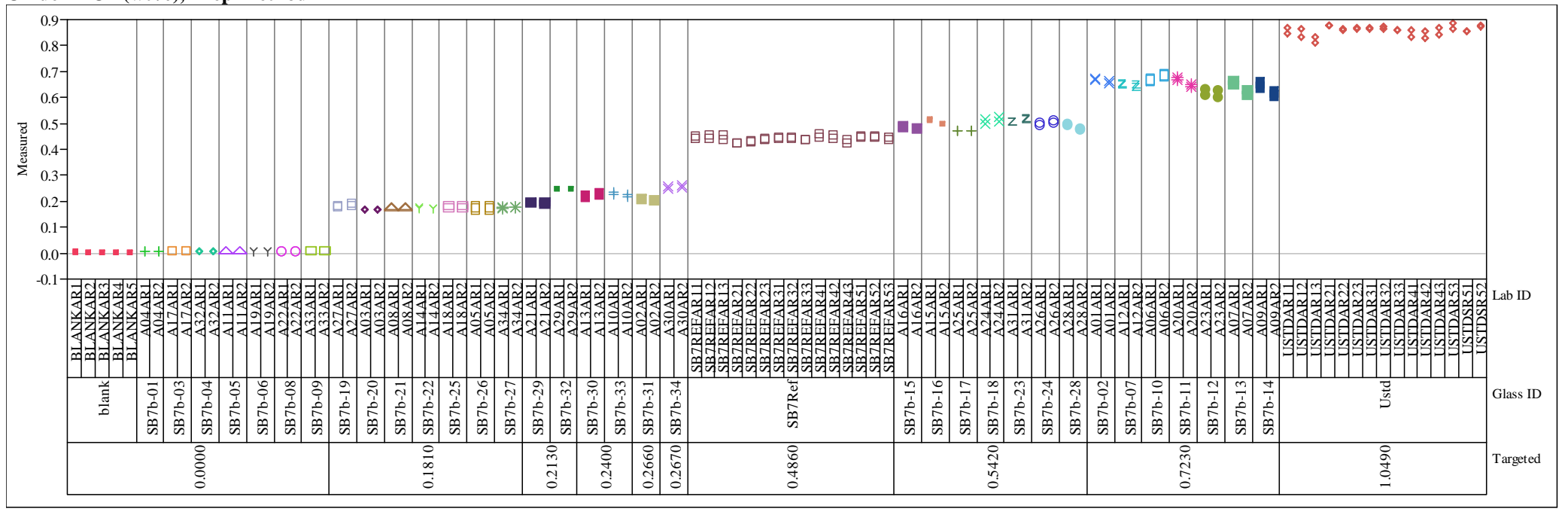


Exhibit A-3. Measurements from by Lab ID within Glass ID by Target Concentration for Each Oxide for Each Prep Oxide=TiO2 (wt $\%)$, Prep Method $=$ PF

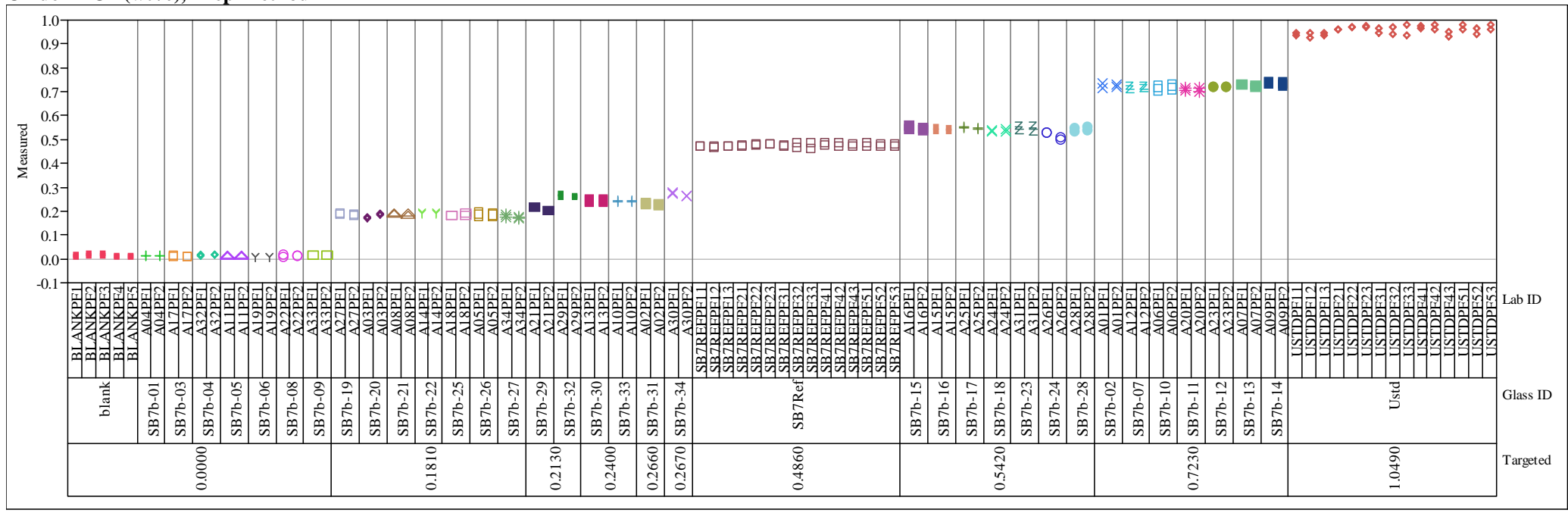

Oxide=U3O8 (wt \%), Prep Method $=$ AR

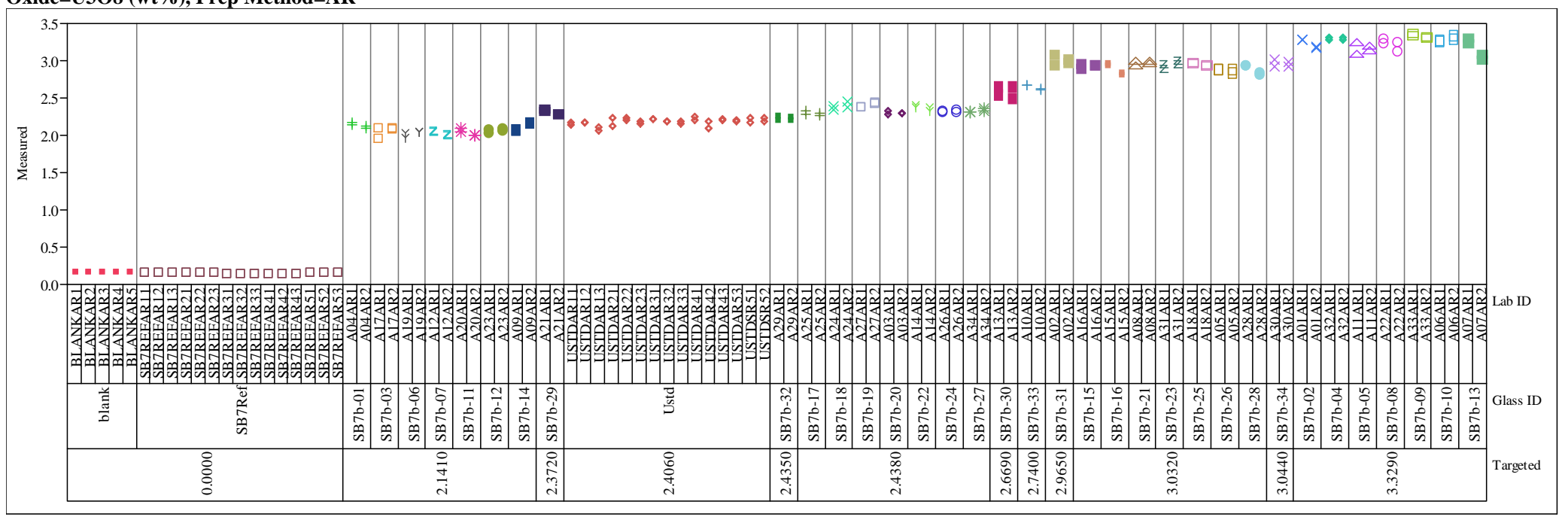


Exhibit A-3. Measurements from by Lab ID within Glass ID by Target Concentration for Each Oxide for Each Prep

Oxide=U3O8 (wt \%), Prep Method $=$ PF
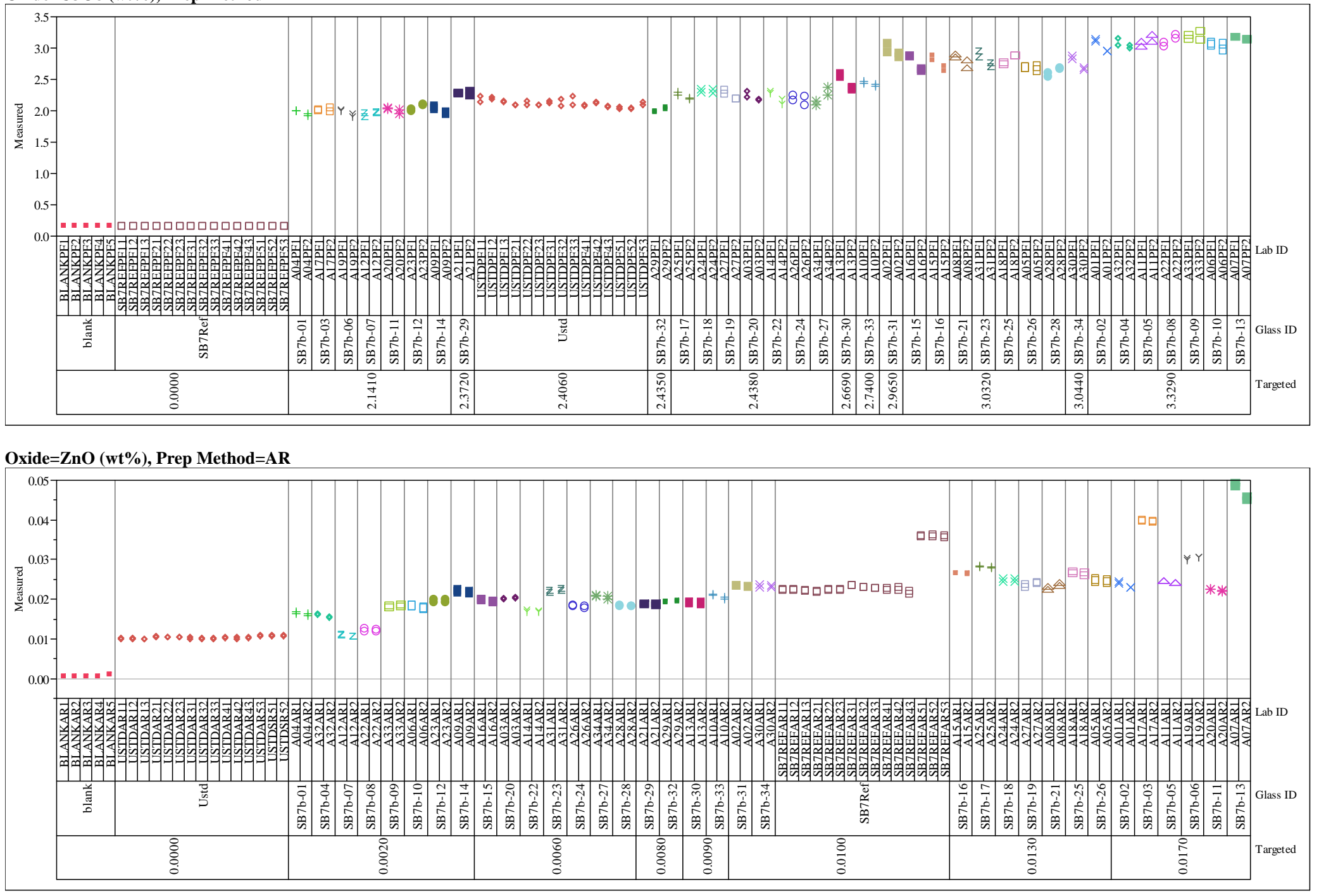
Exhibit A-3. Measurements from by Lab ID within Glass ID by Target Concentration for Each Oxide for Each Prep
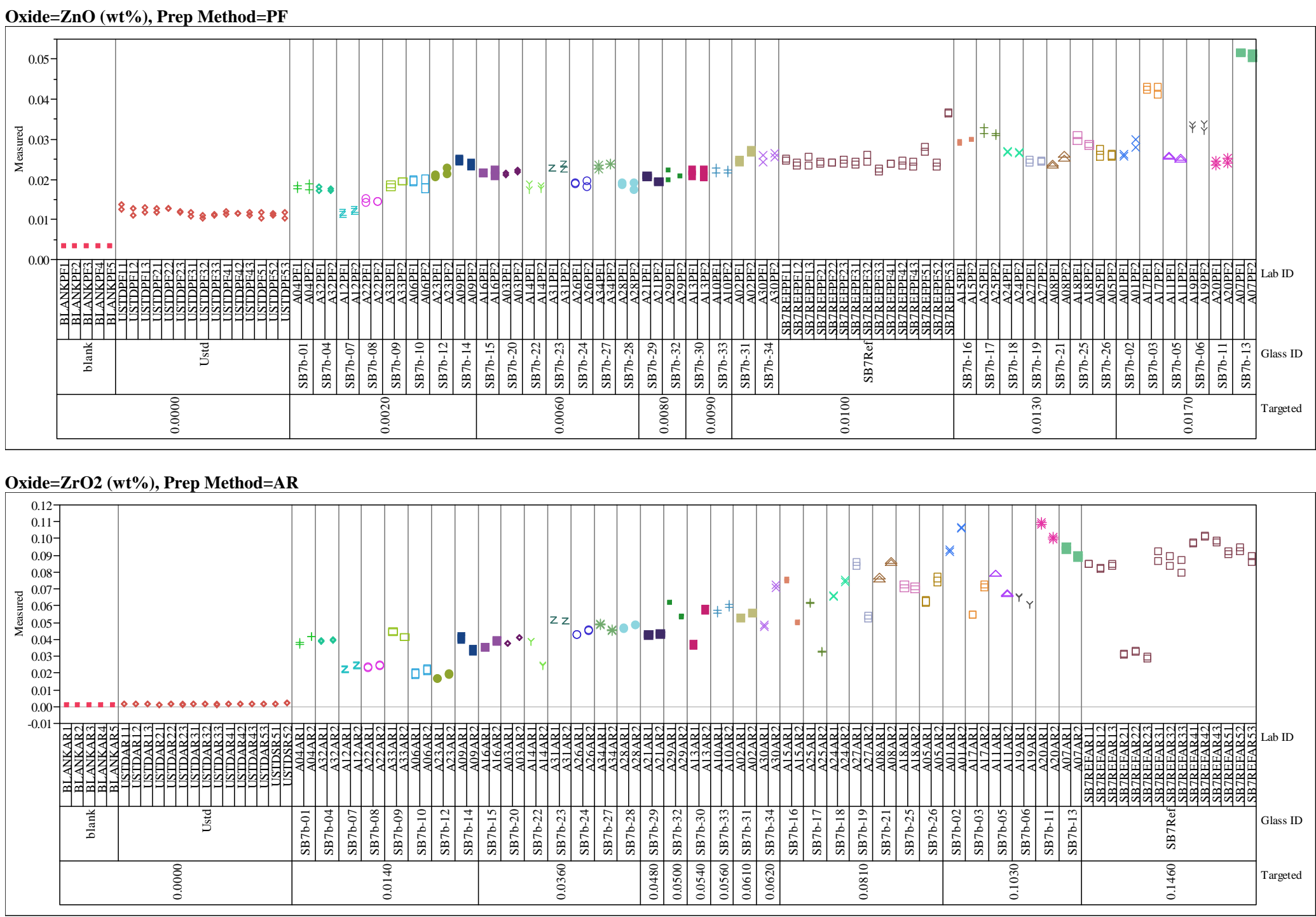
Exhibit A-4. Measurements from by Prep within Glass ID by Target Concentration for Each Oxide

Oxide=Al2O3 (wt\%)

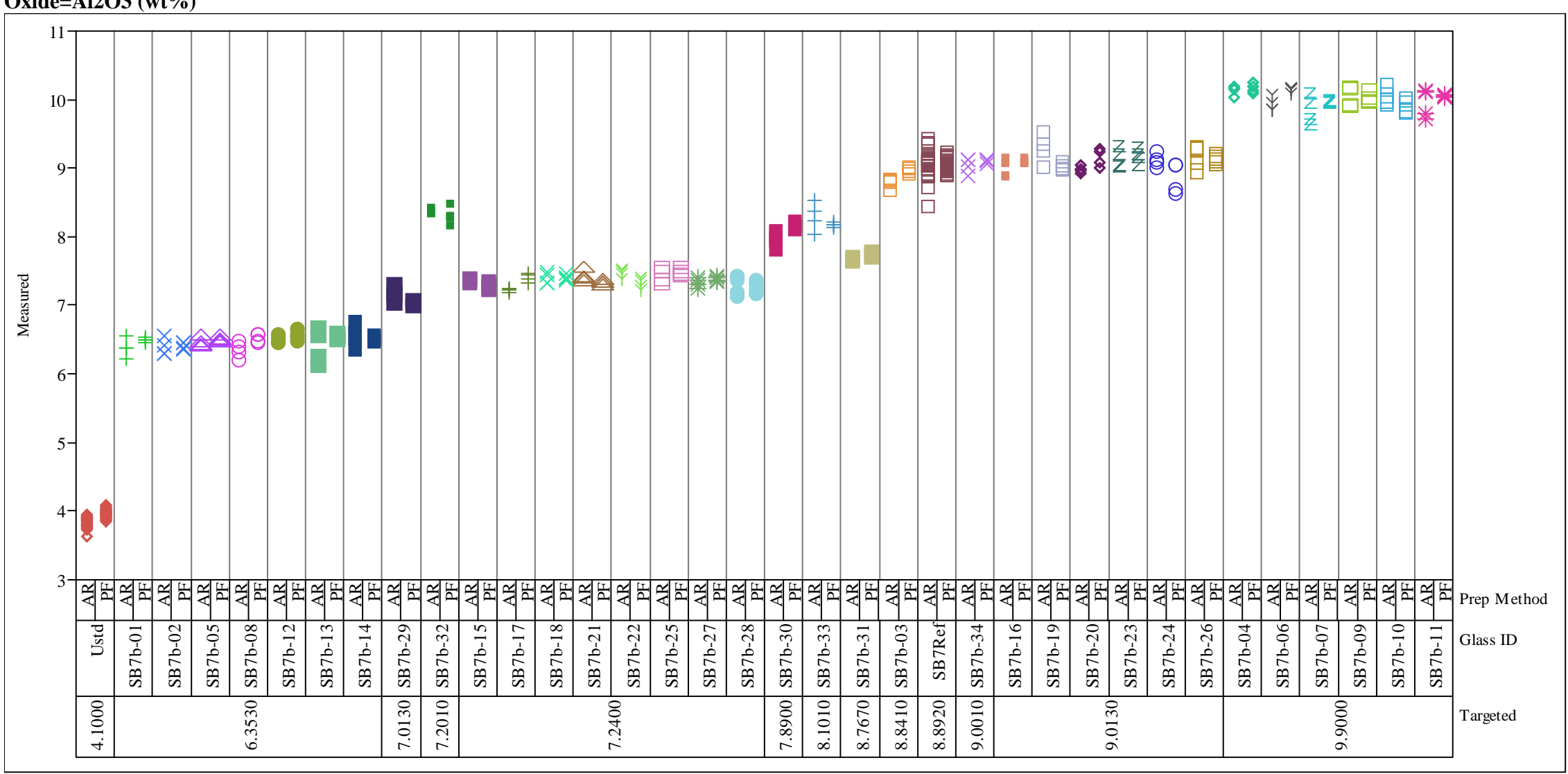


Exhibit A-4. Measurements from by Prep within Glass ID by Target Concentration for Each Oxide

Oxide=B2O3 (wt \%)

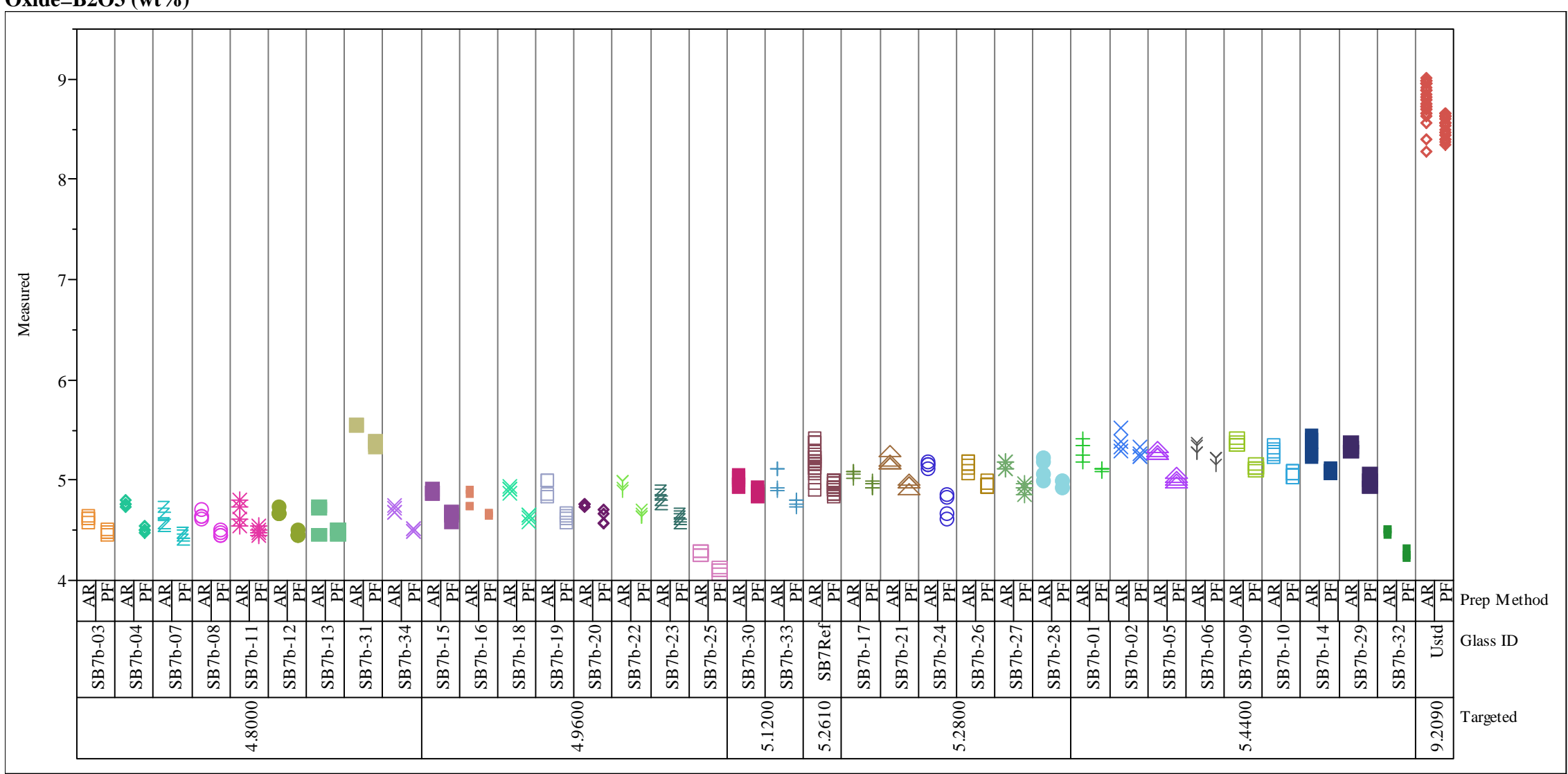


Exhibit A-4. Measurements from by Prep within Glass ID by Target Concentration for Each Oxide

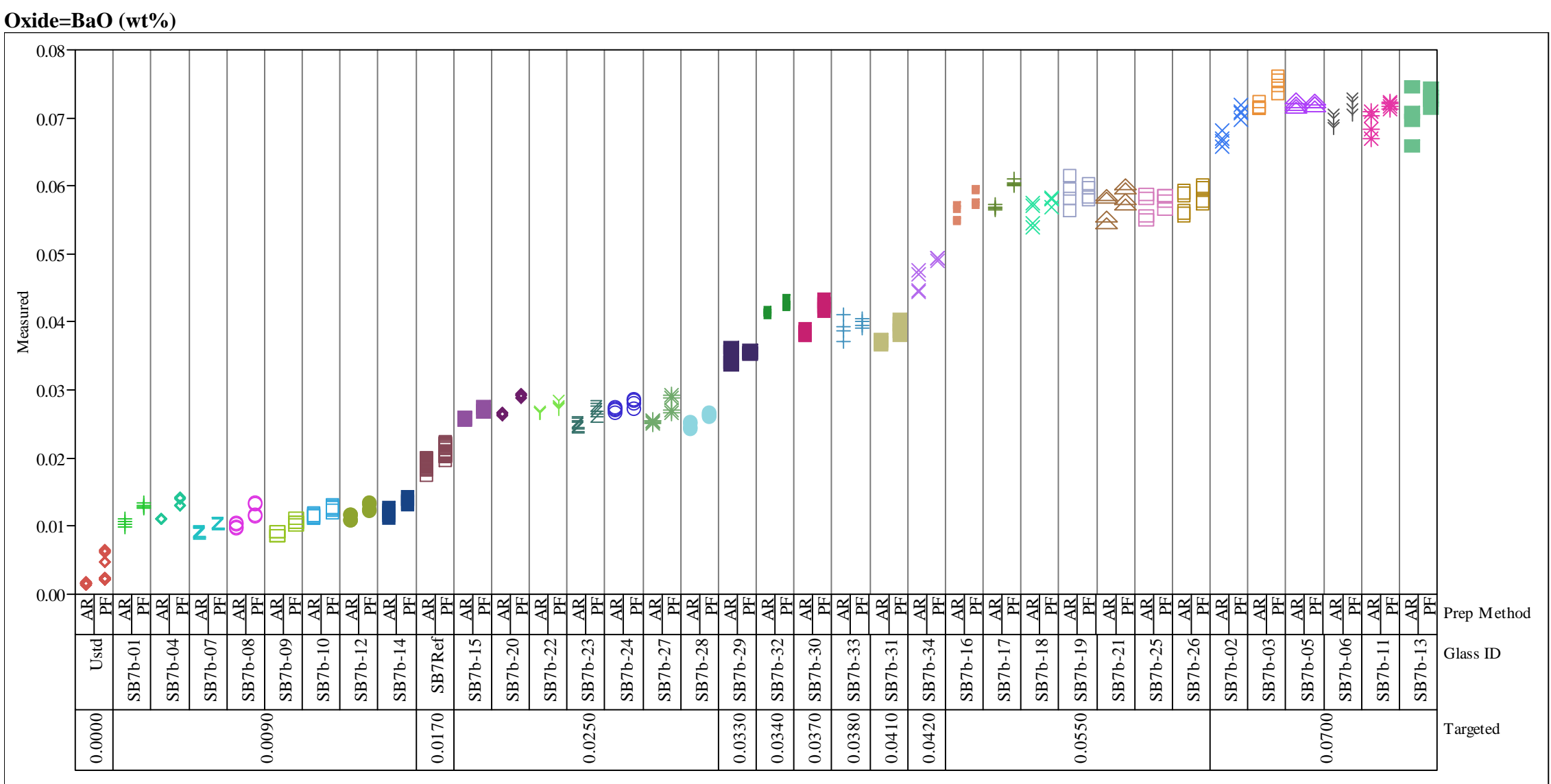


Exhibit A-4. Measurements from by Prep within Glass ID by Target Concentration for Each Oxide

Variability Gauge Oxide $=\mathrm{CaO}$ (wt \%)

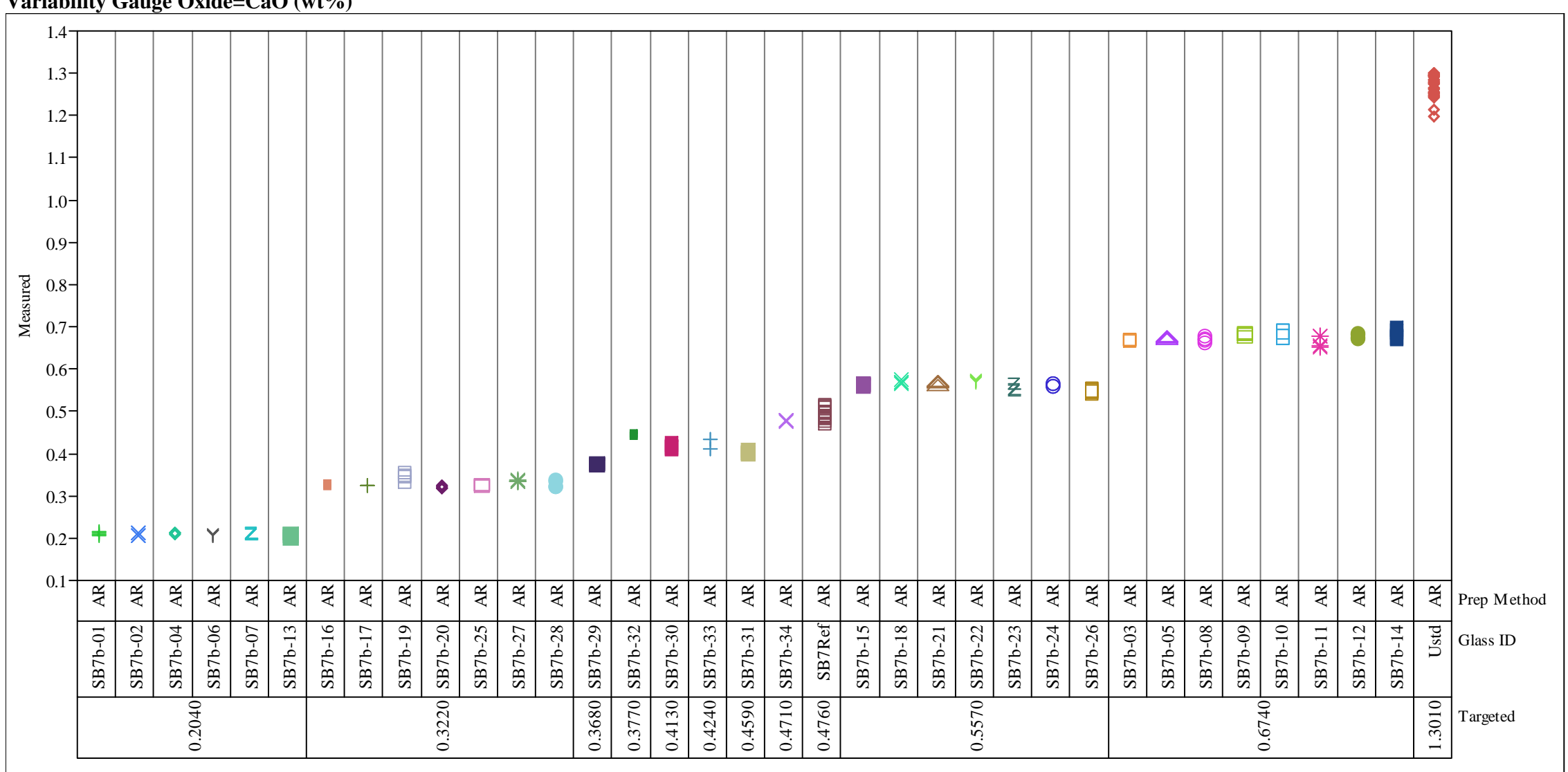


Exhibit A-4. Measurements from by Prep within Glass ID by Target Concentration for Each Oxide

Oxide $=\mathrm{Ce} 2 \mathrm{O} 3(\mathrm{wt} \%)$

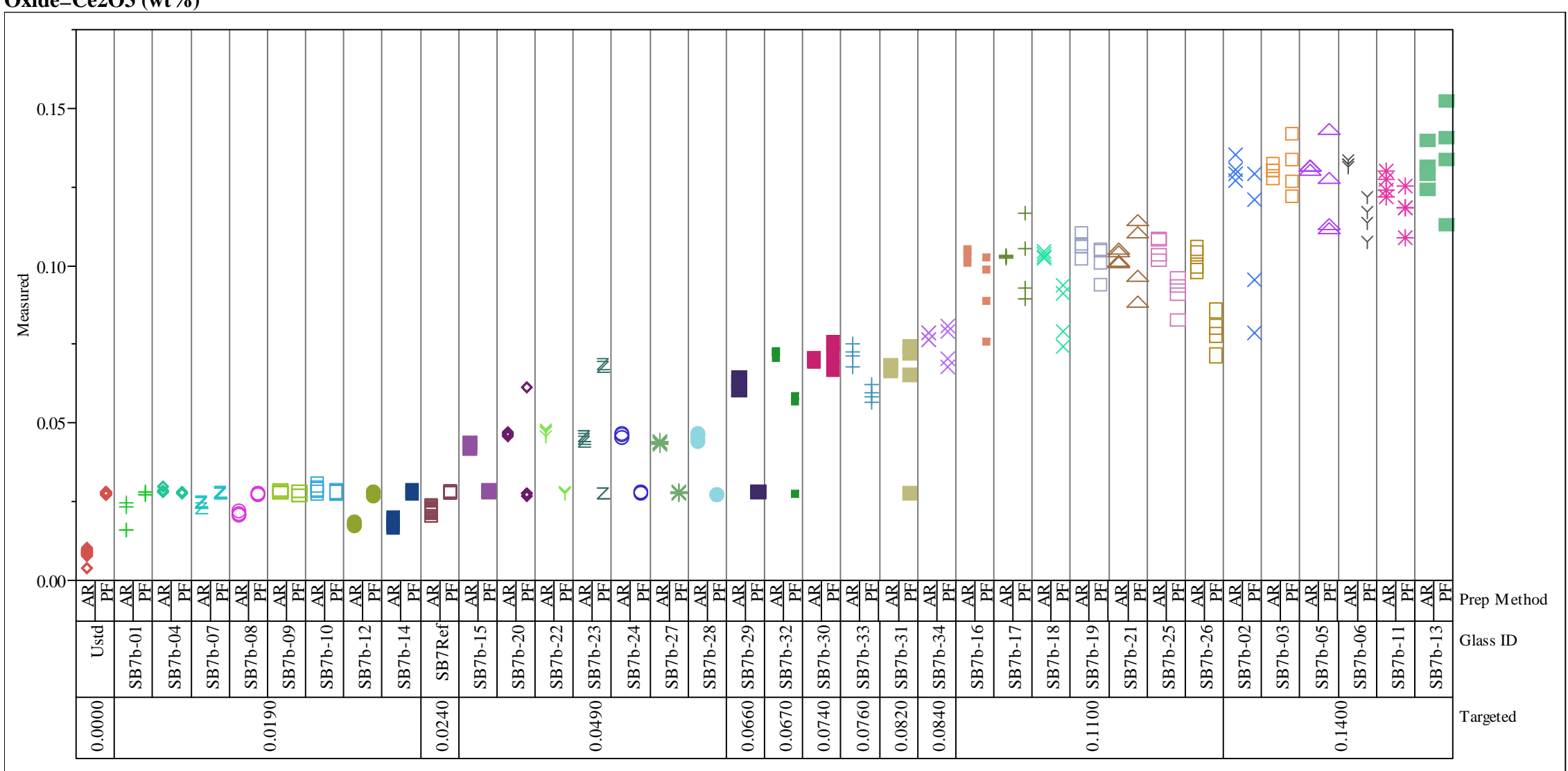


Exhibit A-4. Measurements from by Prep within Glass ID by Target Concentration for Each Oxide

Oxide $=\mathrm{Cr} 2 \mathrm{O} 3(\mathrm{wt} \%)$

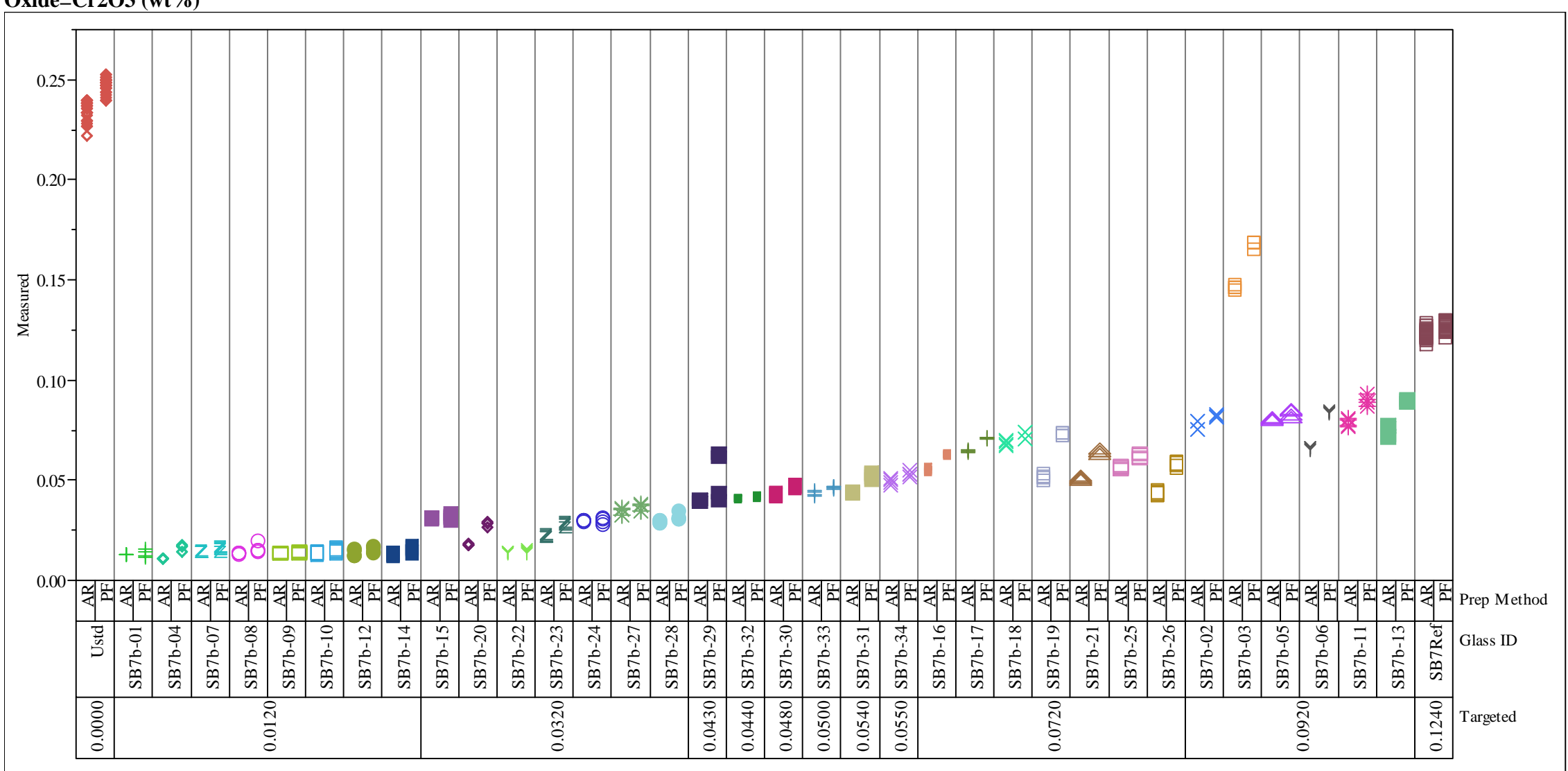


Exhibit A-4. Measurements from by Prep within Glass ID by Target Concentration for Each Oxide

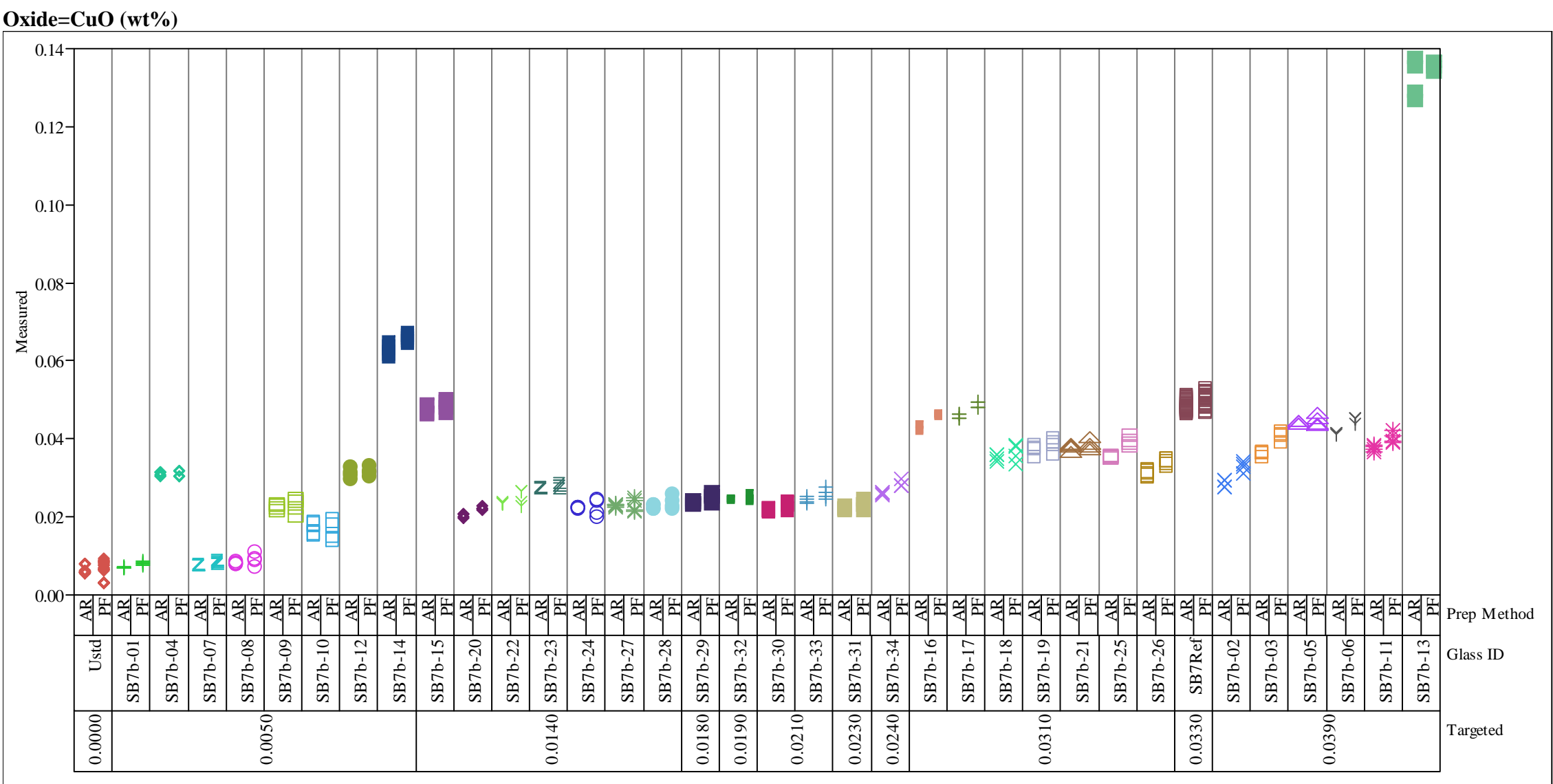


Exhibit A-4. Measurements from by Prep within Glass ID by Target Concentration for Each Oxide

Oxide=Fe2O3 (wt\%)

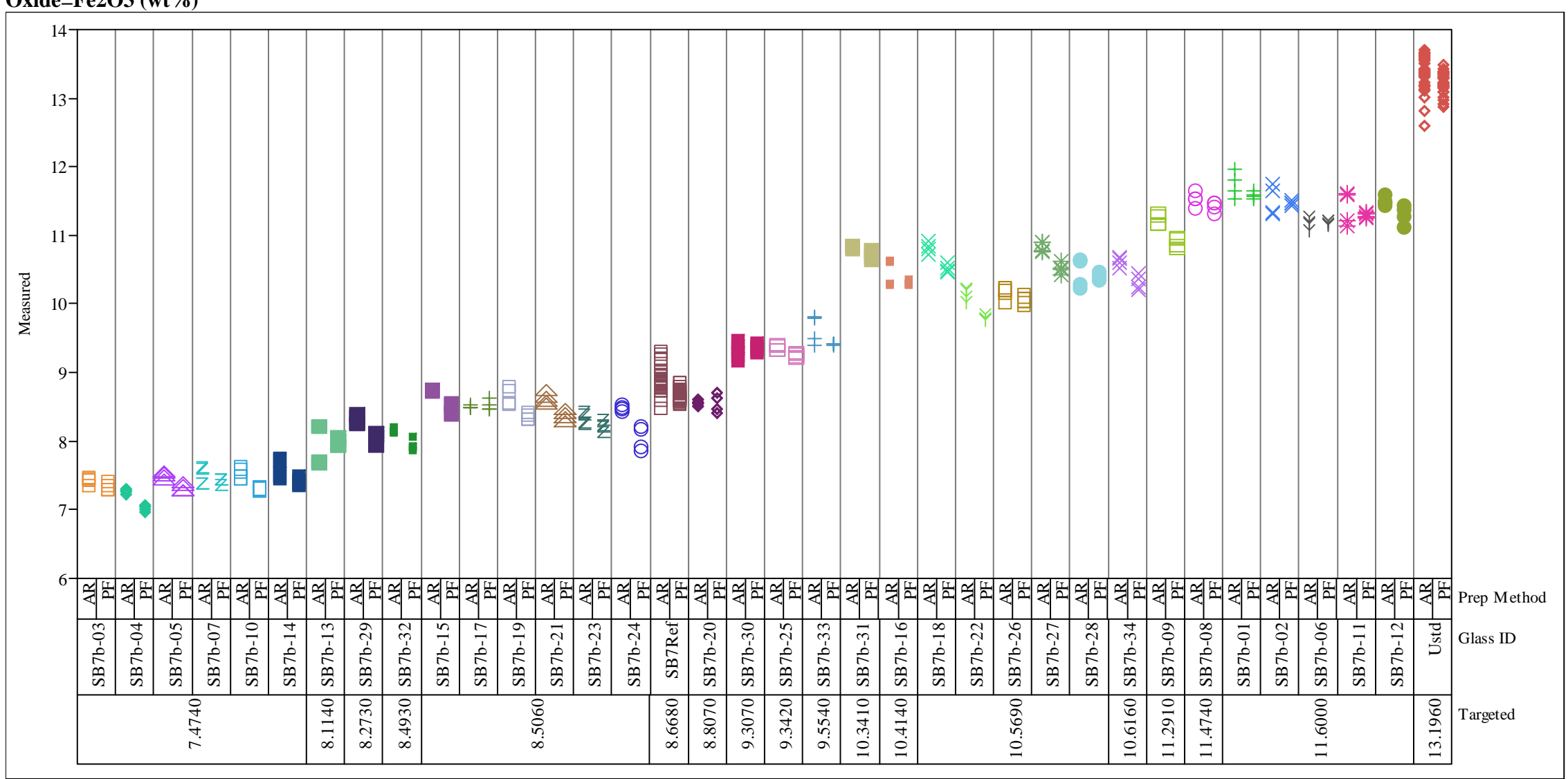


Exhibit A-4. Measurements from by Prep within Glass ID by Target Concentration for Each Oxide

Oxide=K2O (wt\%)

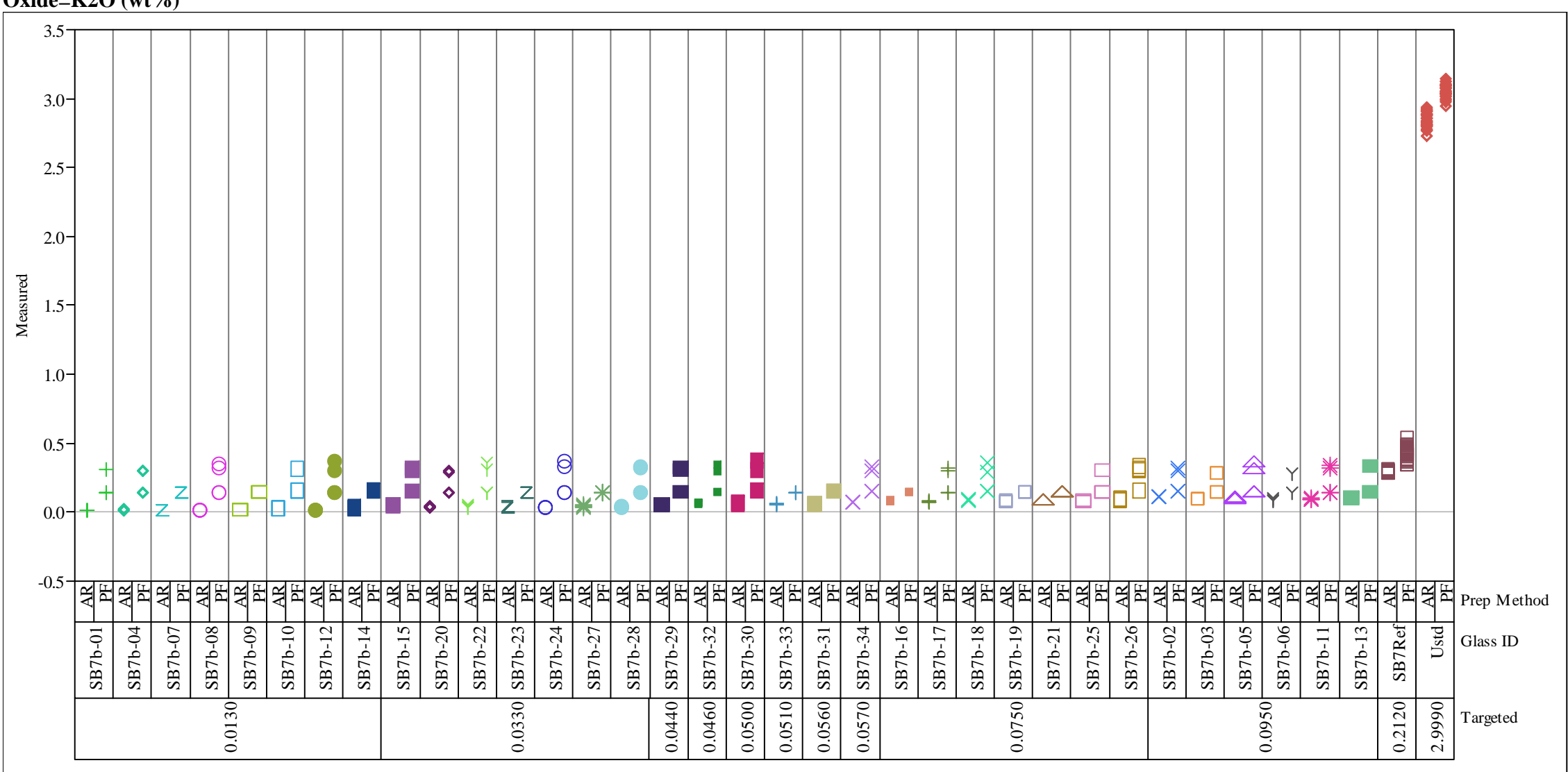


Exhibit A-4. Measurements from by Prep within Glass ID by Target Concentration for Each Oxide

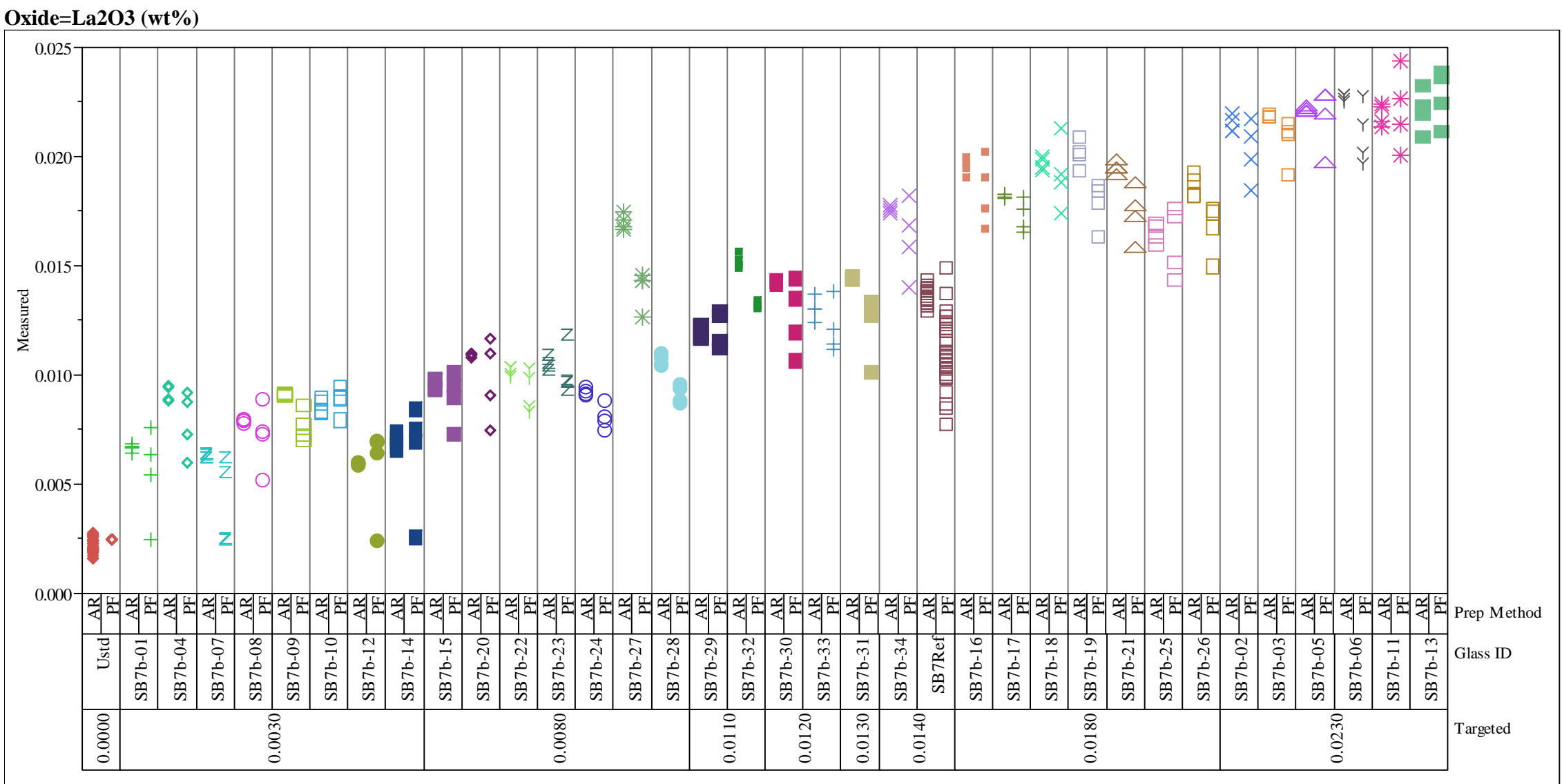


Exhibit A-4. Measurements from by Prep within Glass ID by Target Concentration for Each Oxide

Oxide=Li2O (wt \%)

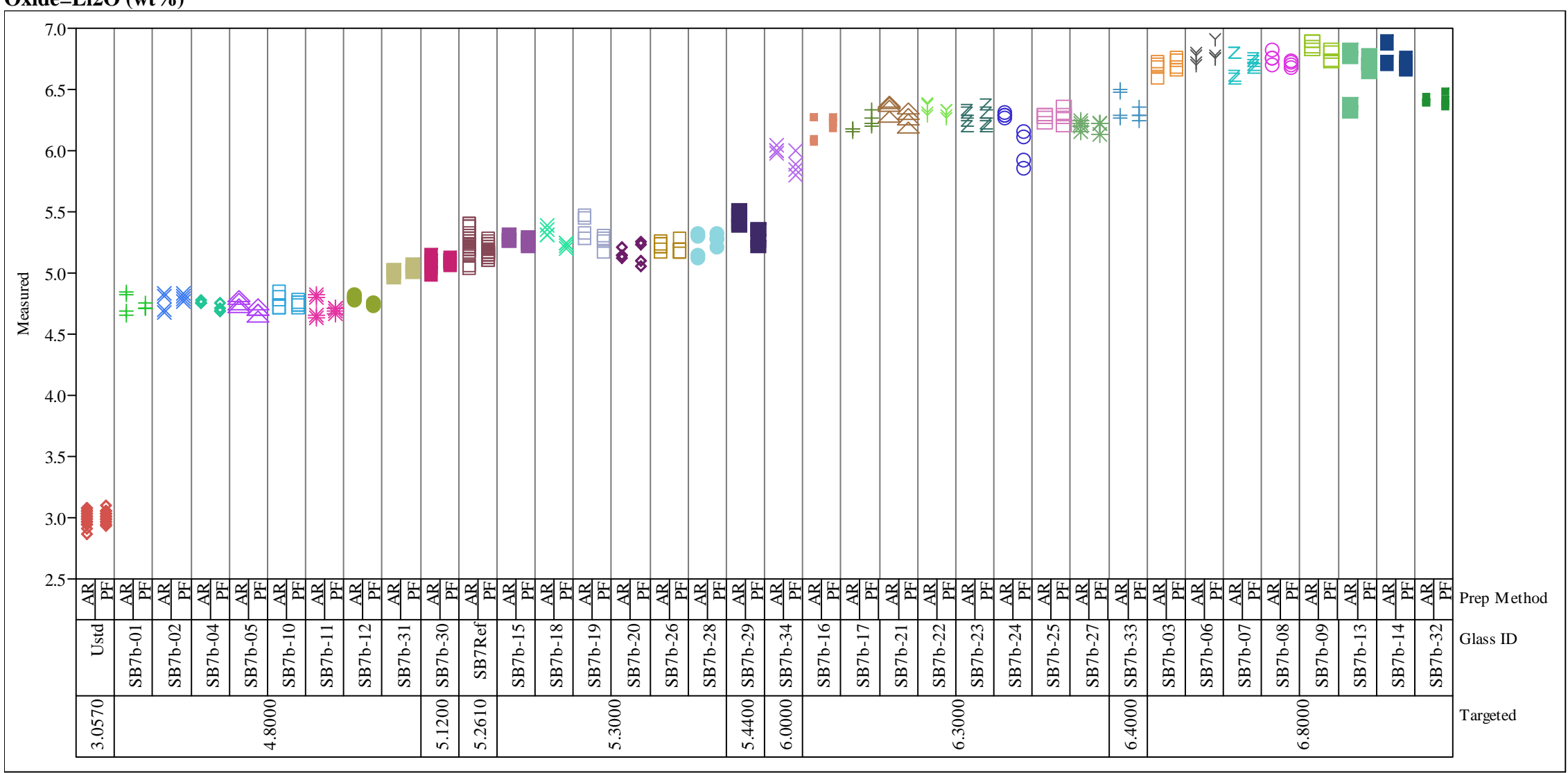


Exhibit A-4. Measurements from by Prep within Glass ID by Target Concentration for Each Oxide

Oxide $=\mathrm{MgO}(\mathrm{wt} \%)$

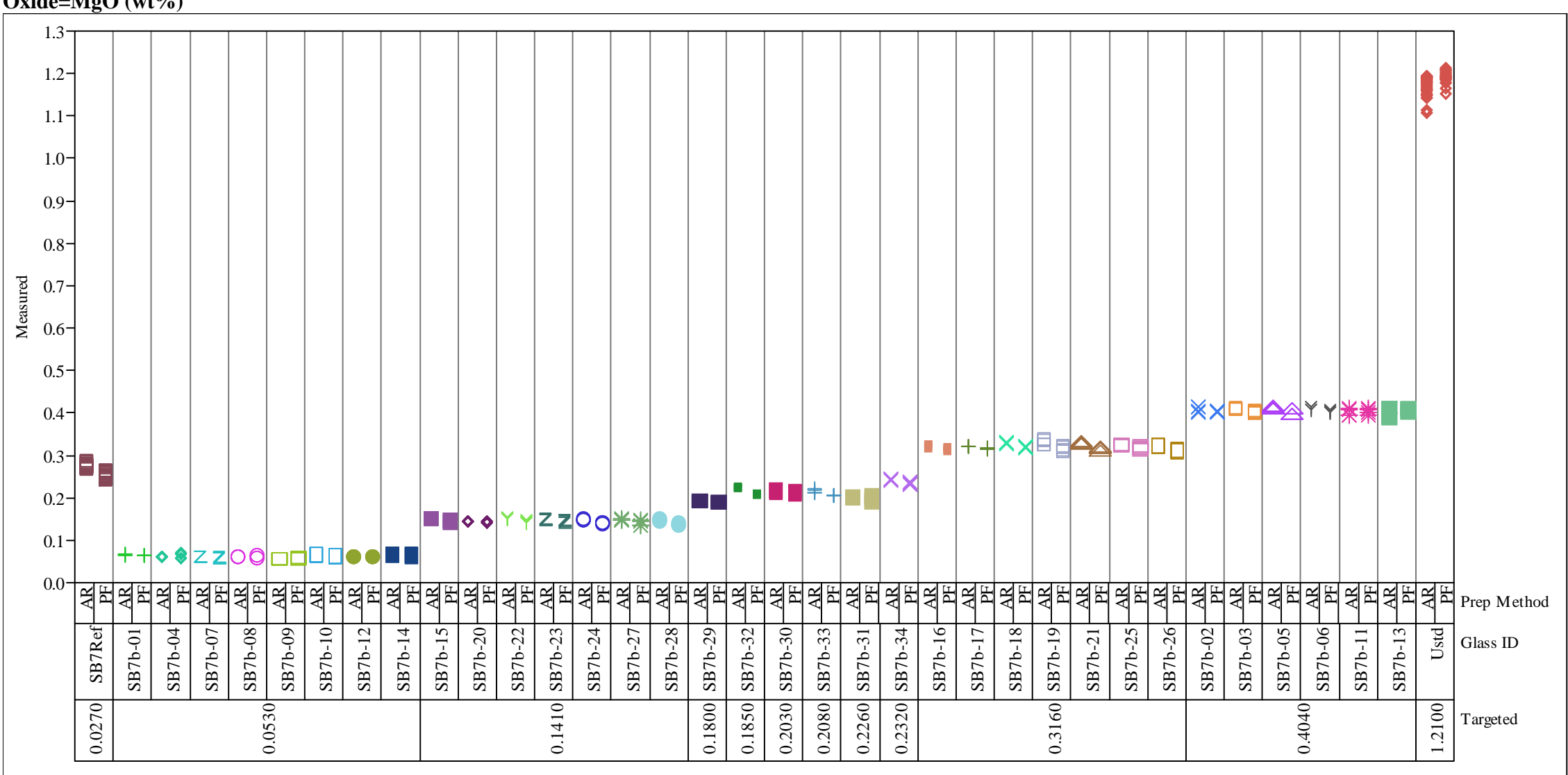


Exhibit A-4. Measurements from by Prep within Glass ID by Target Concentration for Each Oxide

Oxide $=\mathrm{MnO}(\mathrm{wt} \%)$

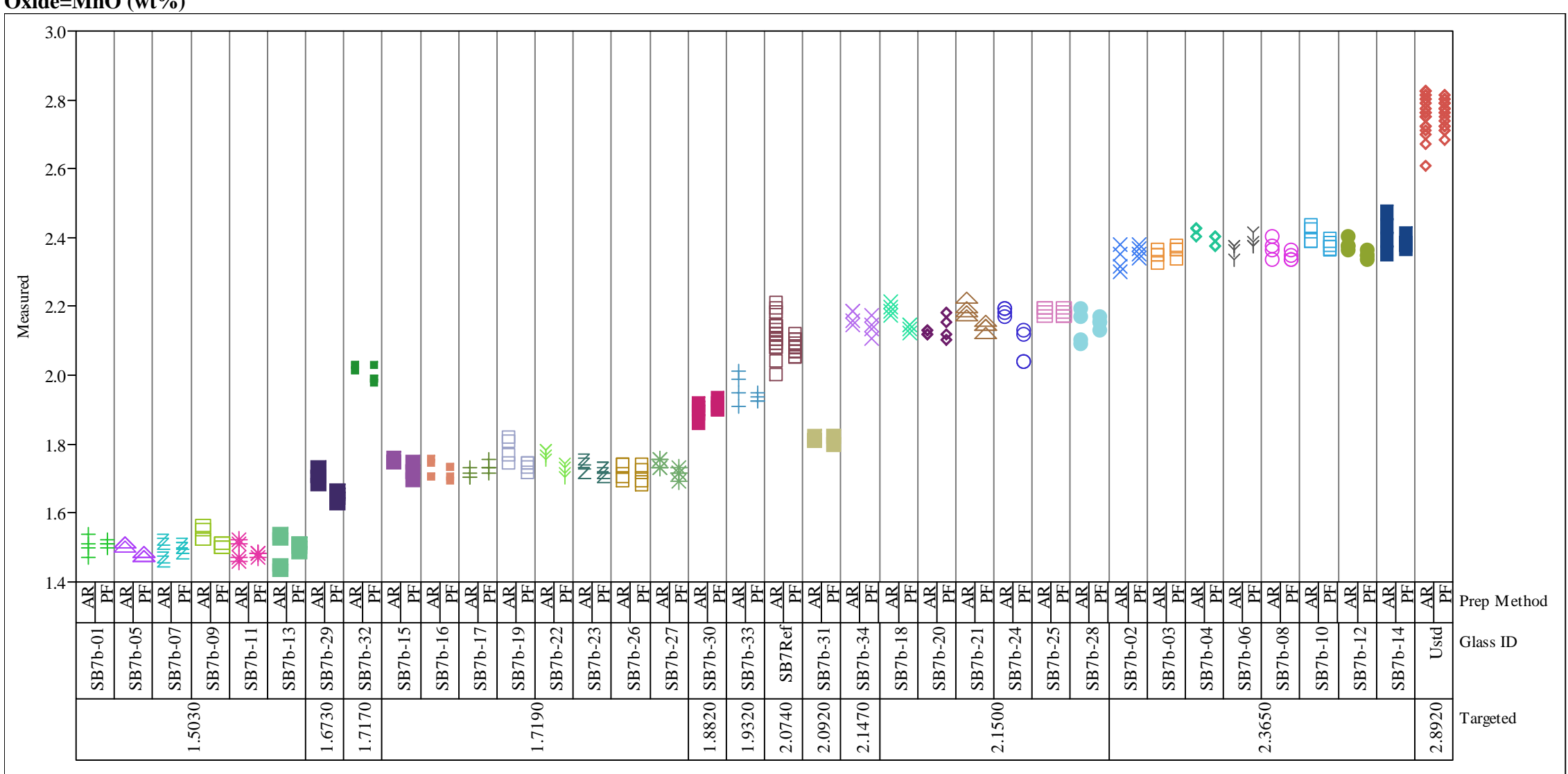


Exhibit A-4. Measurements from by Prep within Glass ID by Target Concentration for Each Oxide

Oxide=Na2O (wt \%)

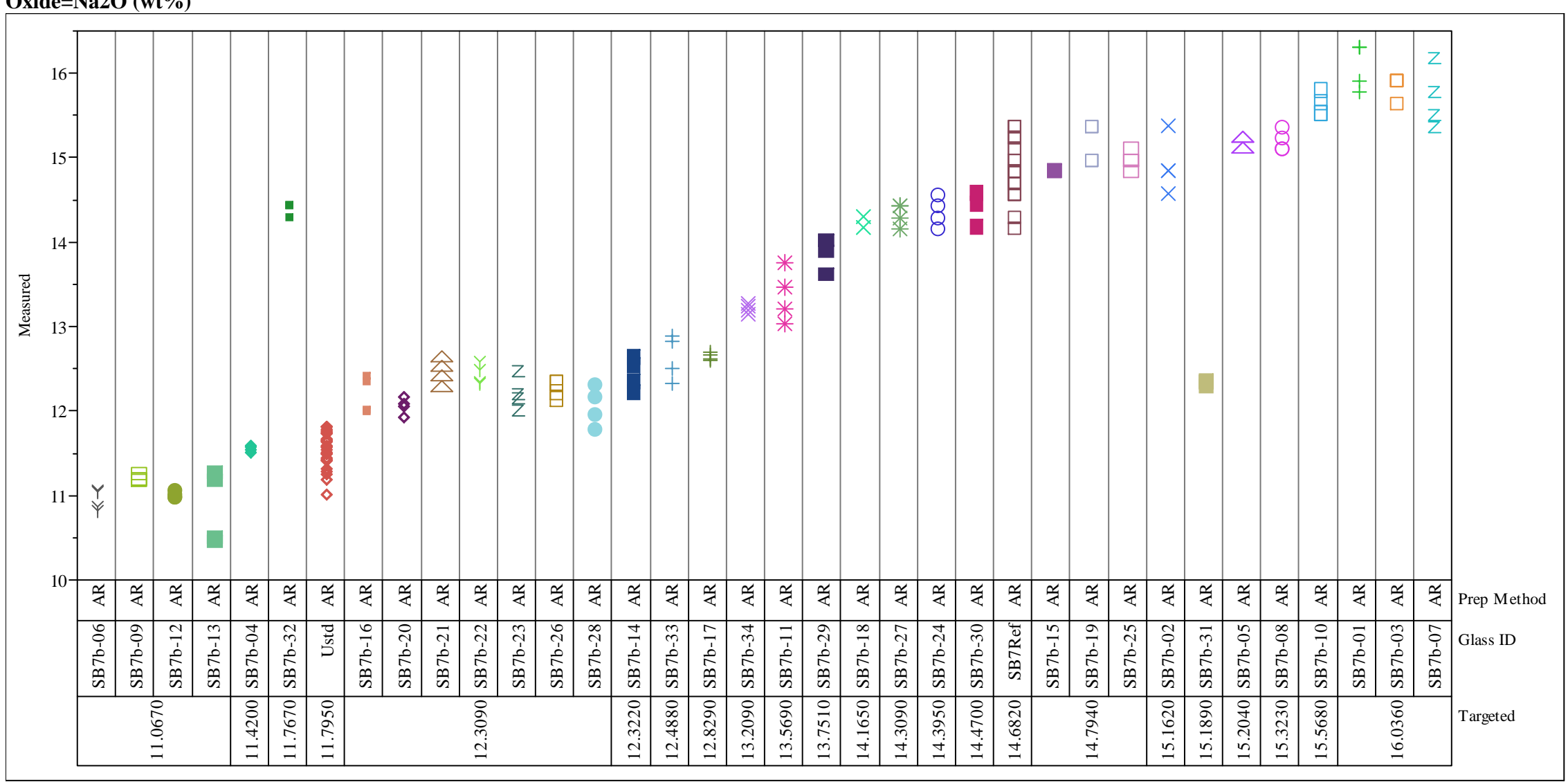


Exhibit A-4. Measurements from by Prep within Glass ID by Target Concentration for Each Oxide

Oxide $=\mathrm{NiO}(\mathrm{wt} \%)$

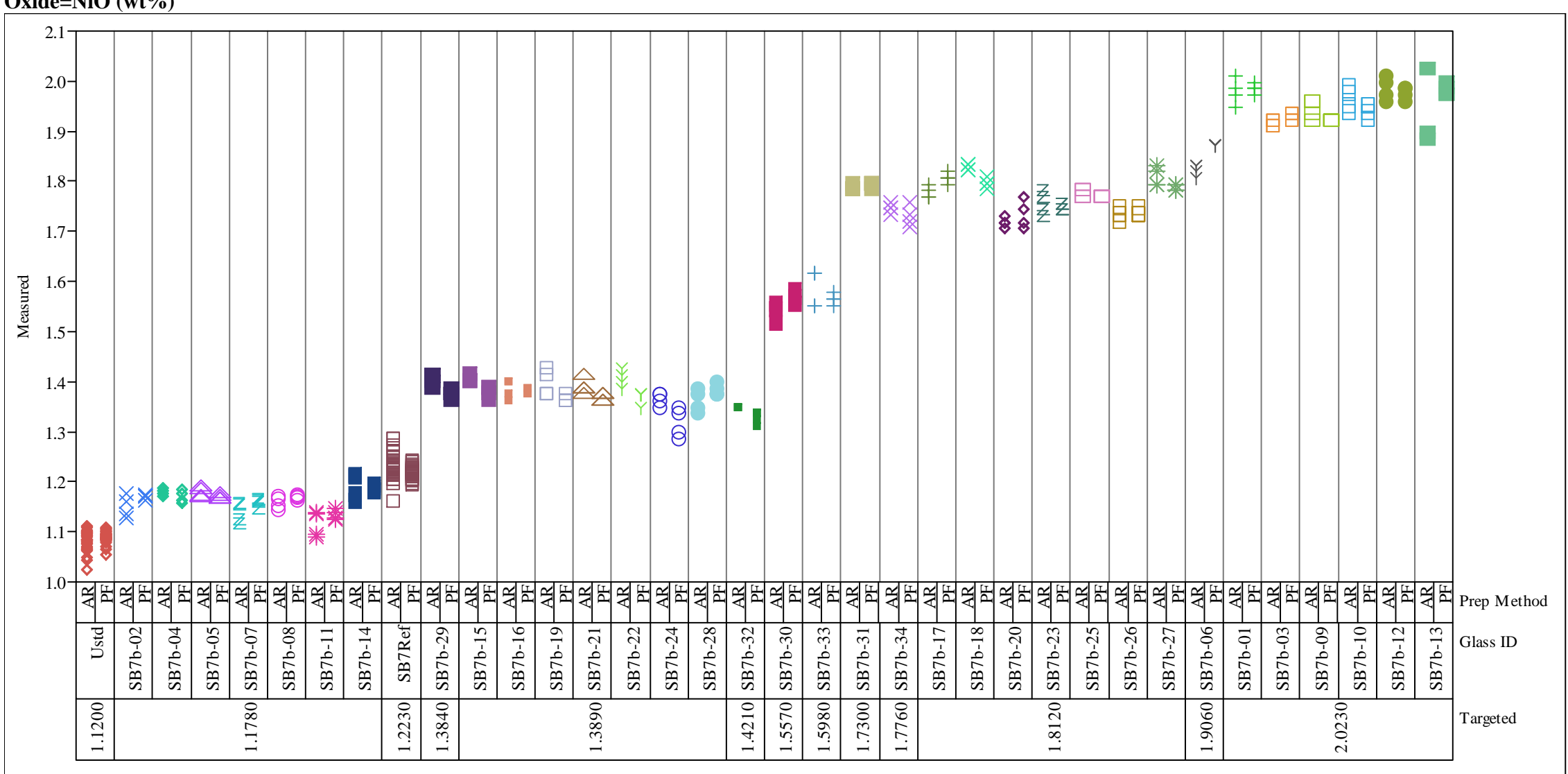


Exhibit A-4. Measurements from by Prep within Glass ID by Target Concentration for Each Oxide

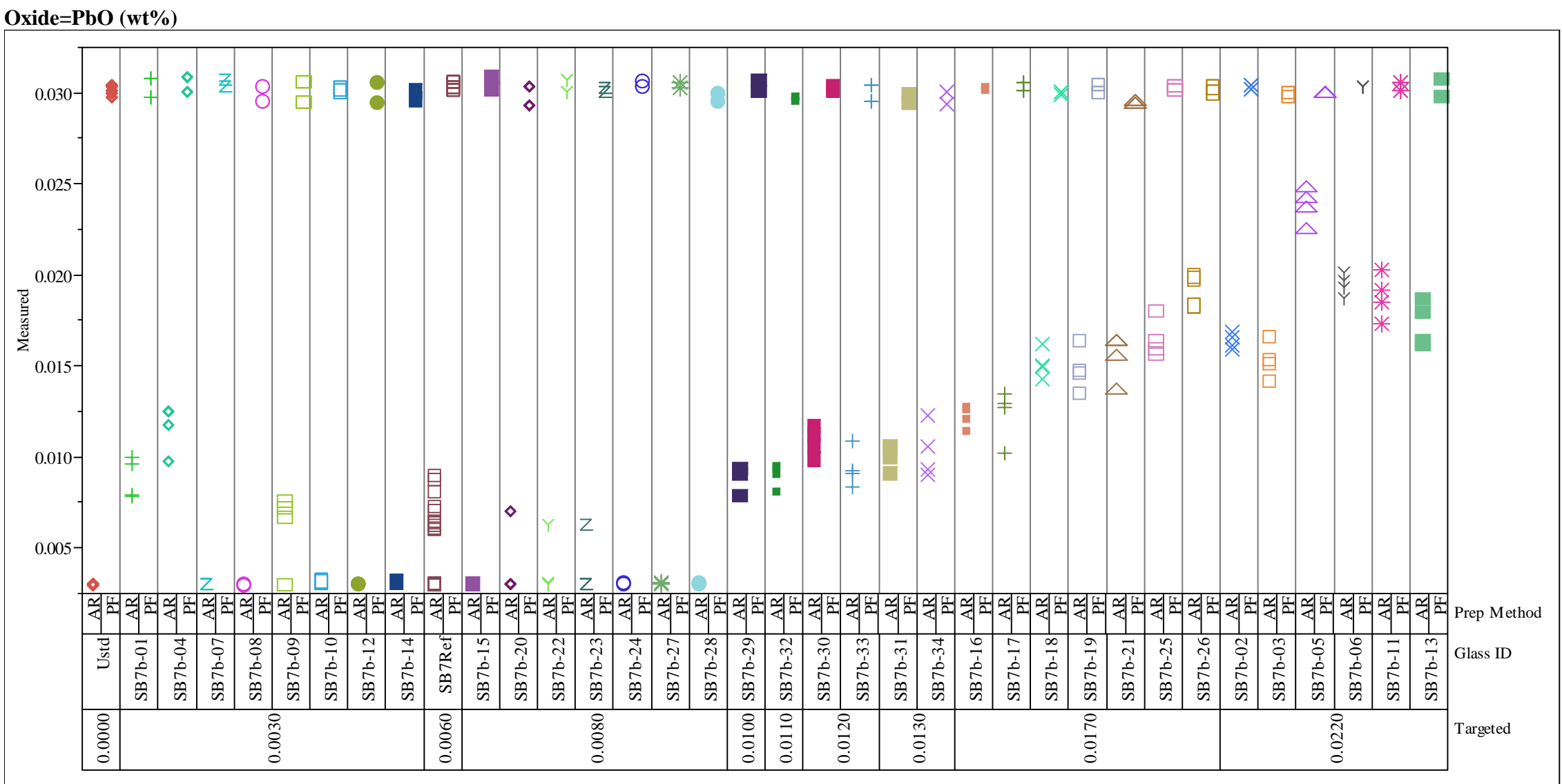


Exhibit A-4. Measurements from by Prep within Glass ID by Target Concentration for Each Oxide

Oxide $=\mathrm{SiO} 2(\mathrm{wt} \%)$

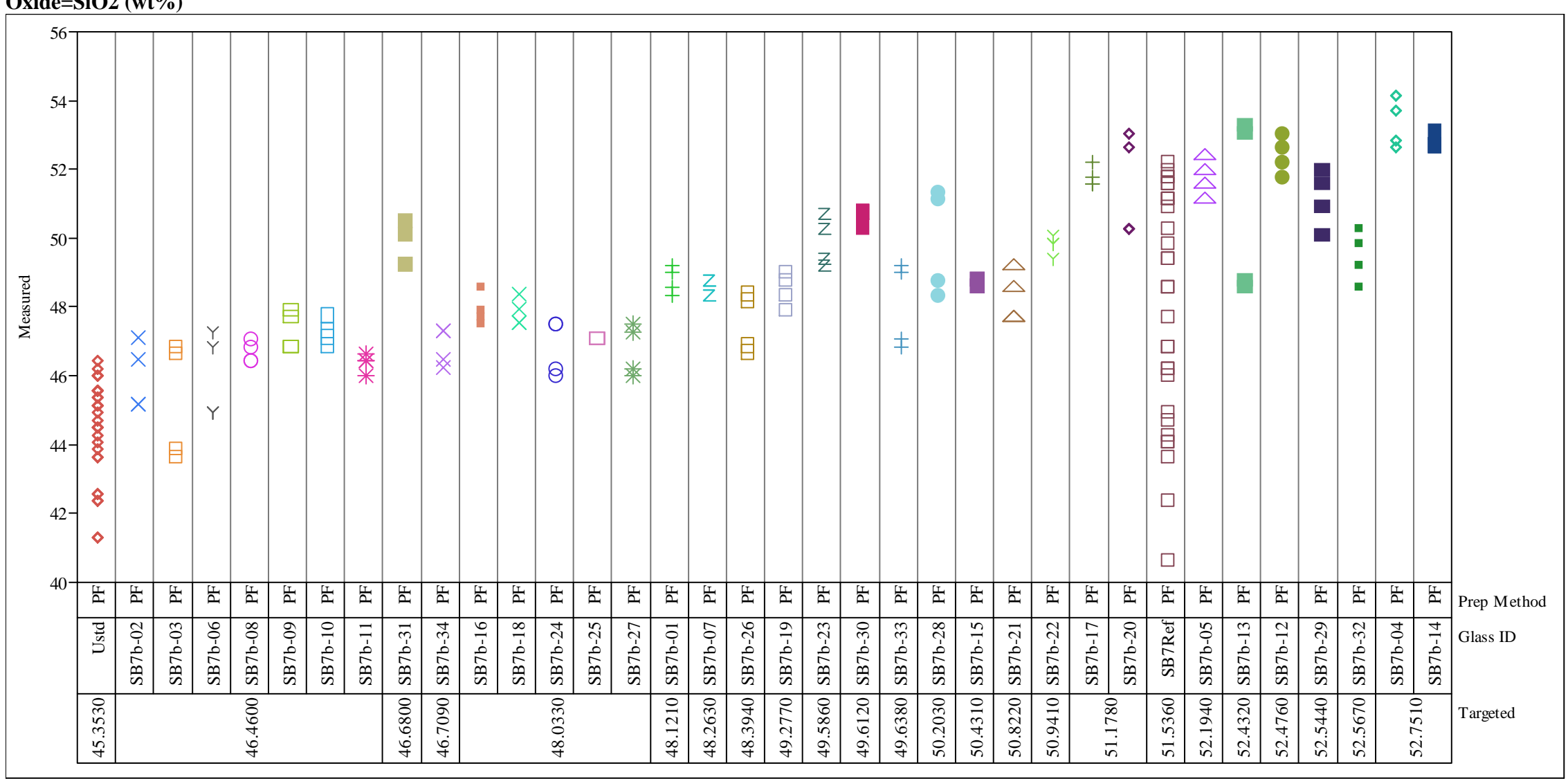


Exhibit A-4. Measurements from by Prep within Glass ID by Target Concentration for Each Oxide

Oxide=SO4 (wt\%)

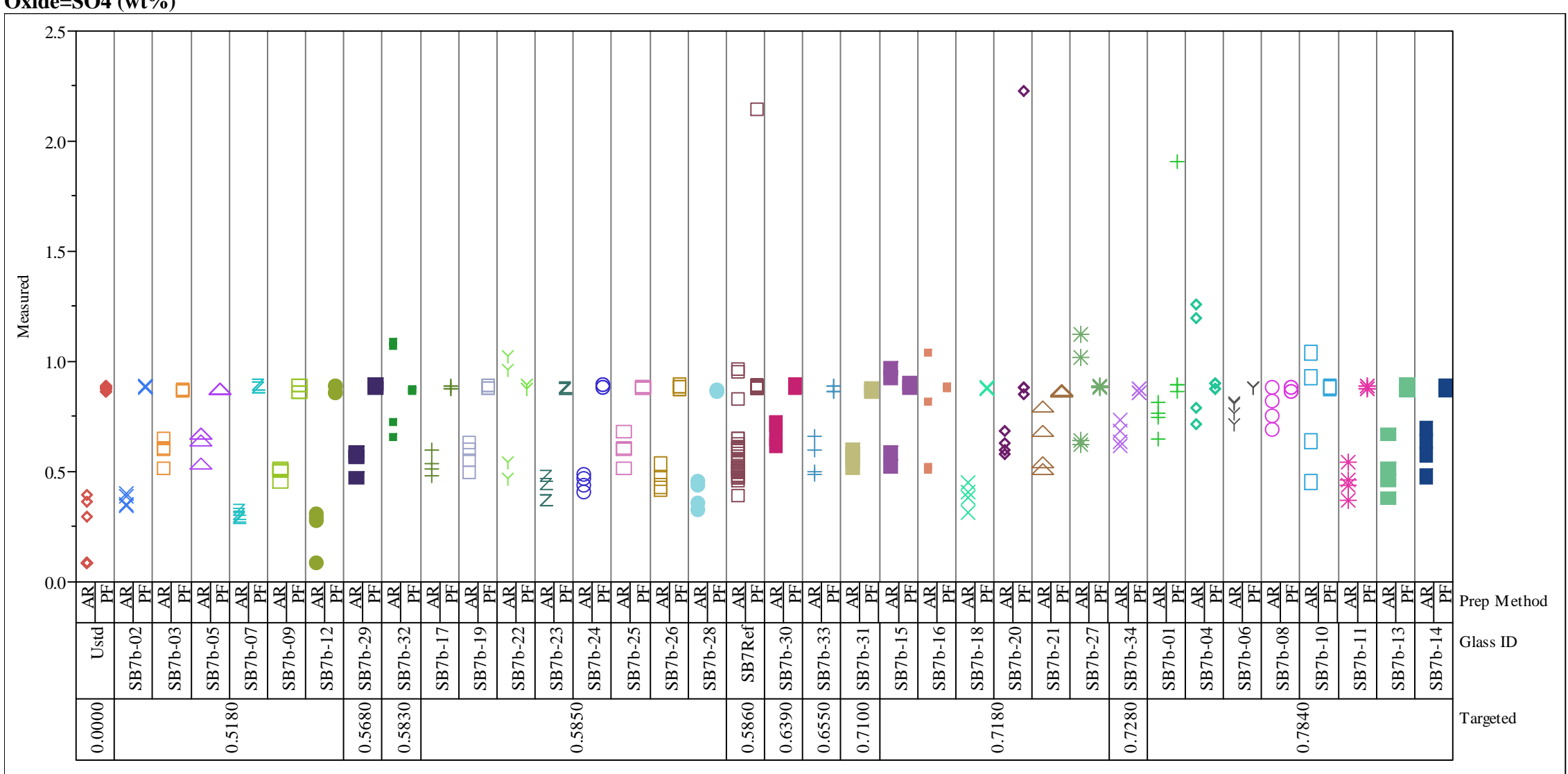


Exhibit A-4. Measurements from by Prep within Glass ID by Target Concentration for Each Oxide

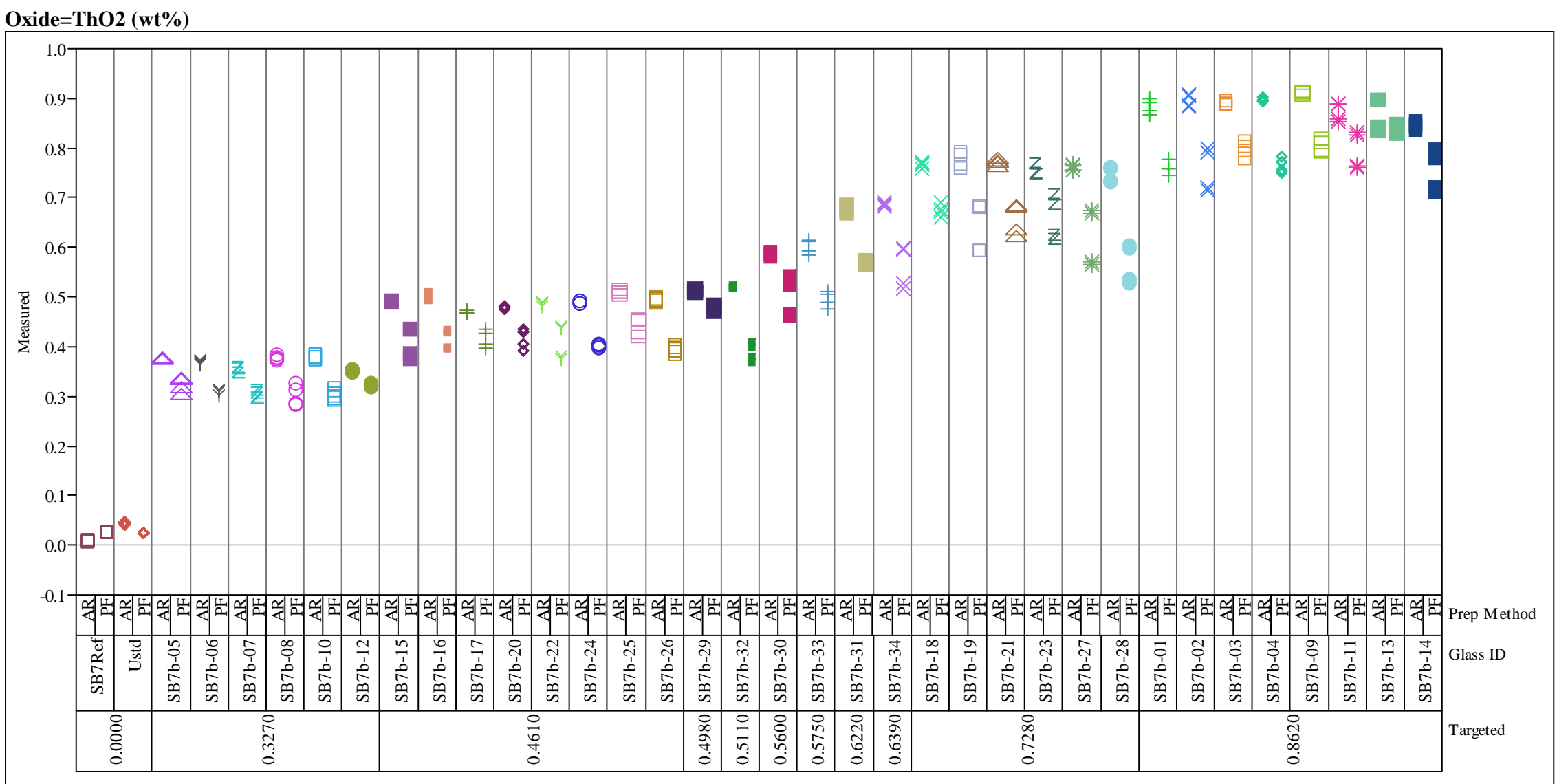


Exhibit A-4. Measurements from by Prep within Glass ID by Target Concentration for Each Oxide

Oxide=TiO2 (wt\%)

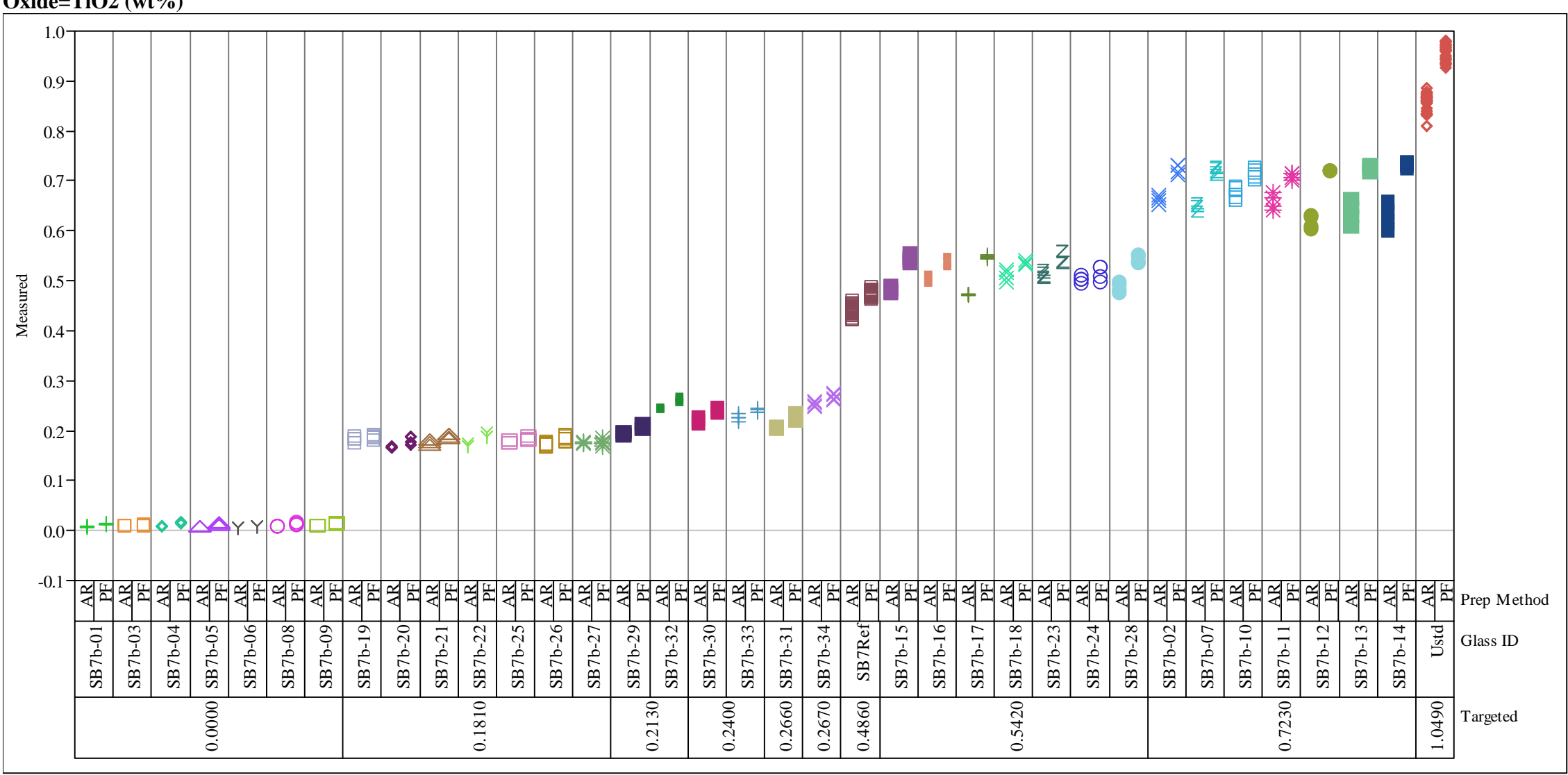


Exhibit A-4. Measurements from by Prep within Glass ID by Target Concentration for Each Oxide

Oxide=U3O8 ( $w$ t \%)

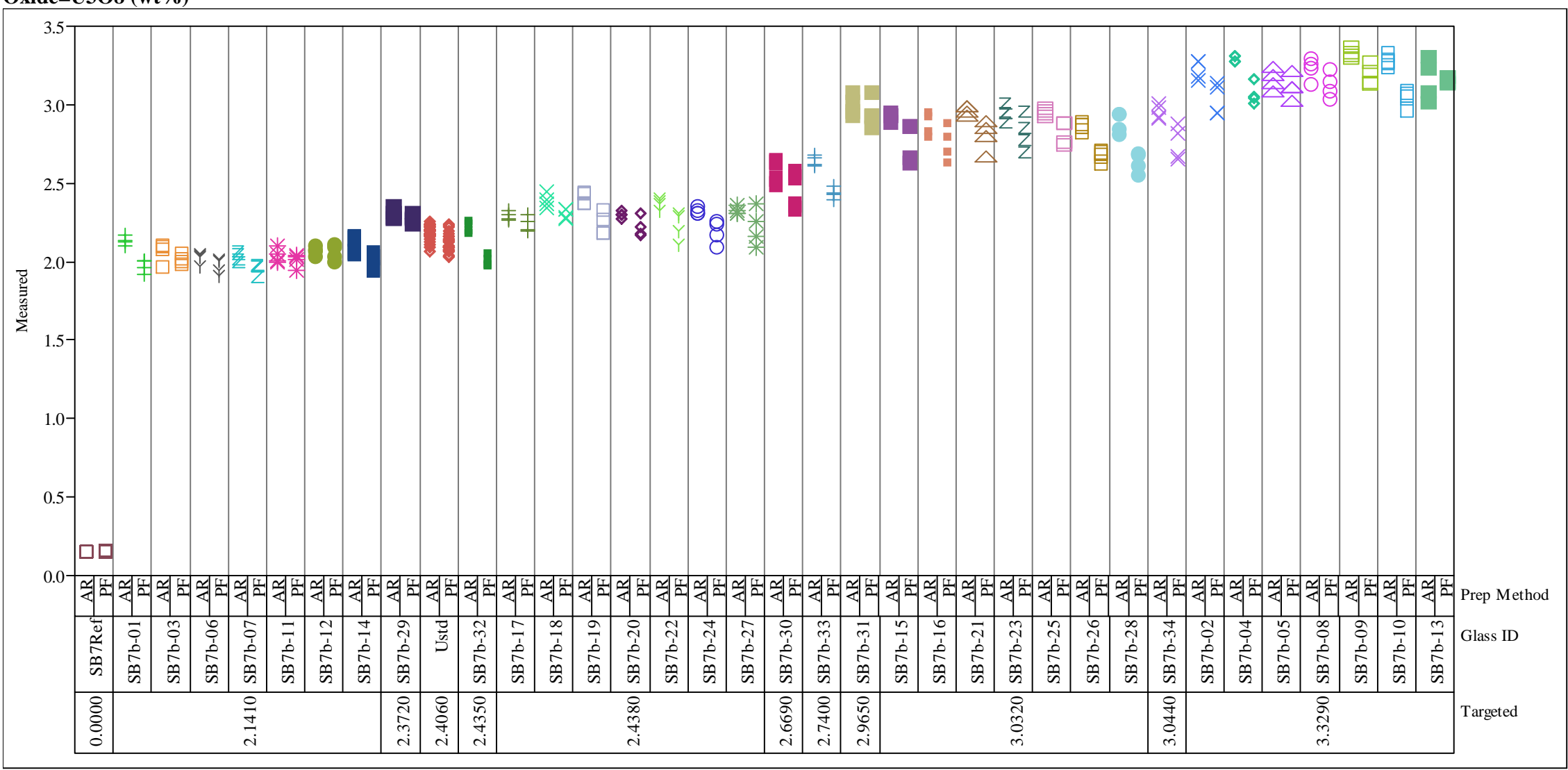


Exhibit A-4. Measurements from by Prep within Glass ID by Target Concentration for Each Oxide

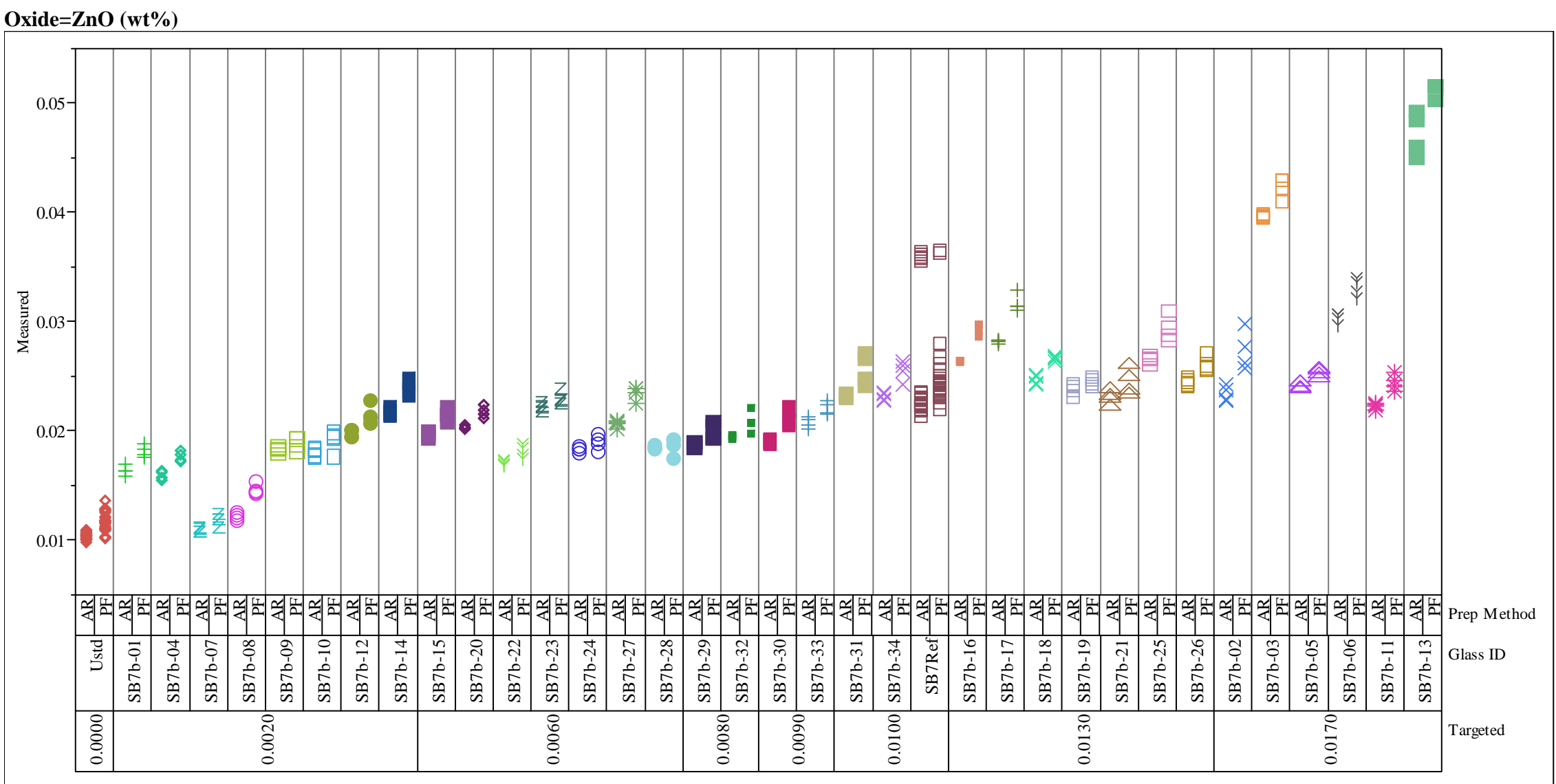


Exhibit A-4. Measurements from by Prep within Glass ID by Target Concentration for Each Oxide

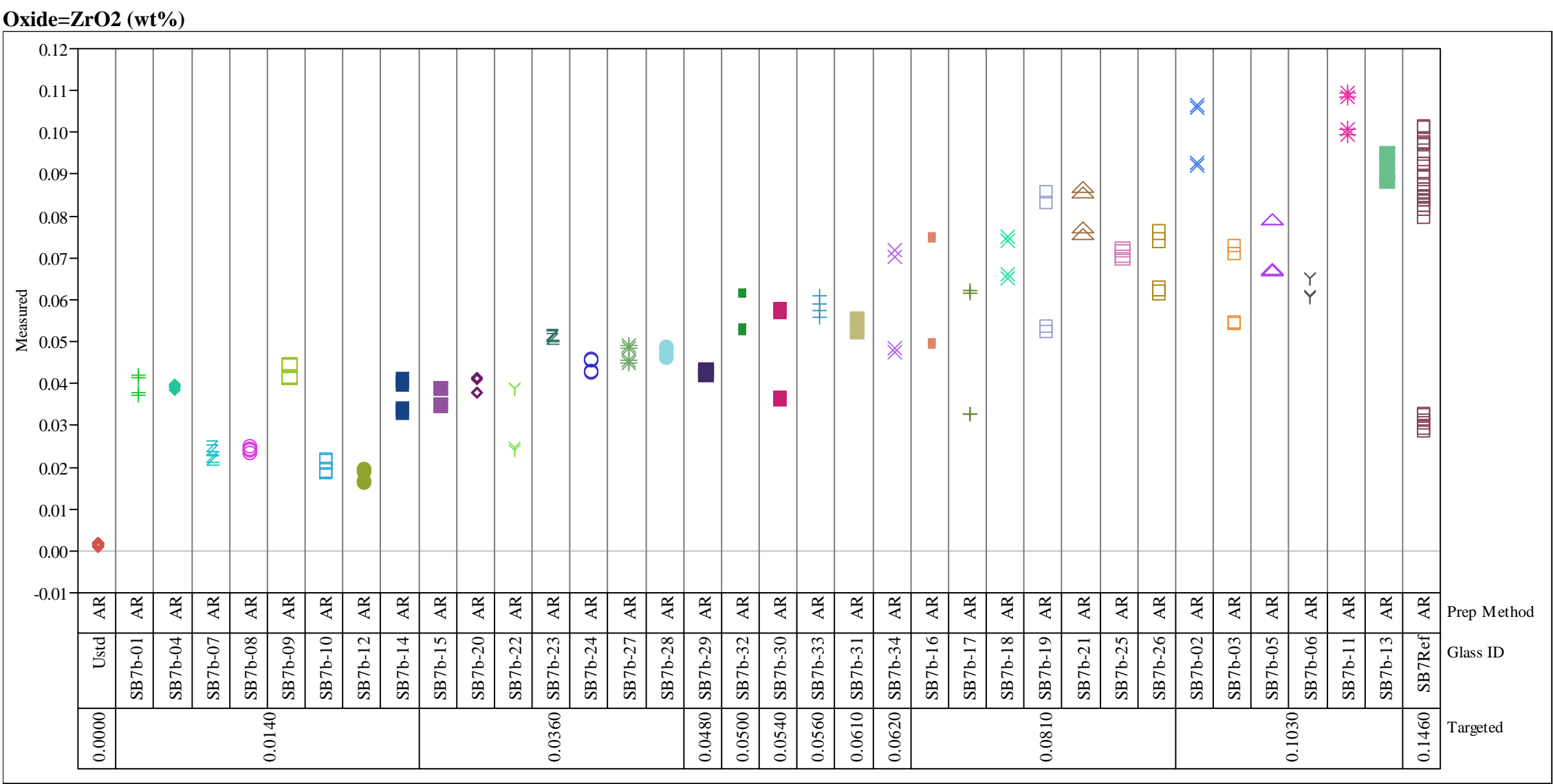


Exhibit A-5. Measurements by Block and Sub-Block for Samples of the Sb7ref Glass and the Ustd Standard Glass by Oxide by Prep

Oneway Analysis of Measured By Blk/Sub-Blk Glass ID=SB7Ref, Oxide=Al2O3 (wt\%), Prep Method=AR, Target $=\mathbf{8 . 8 9 2 0}$

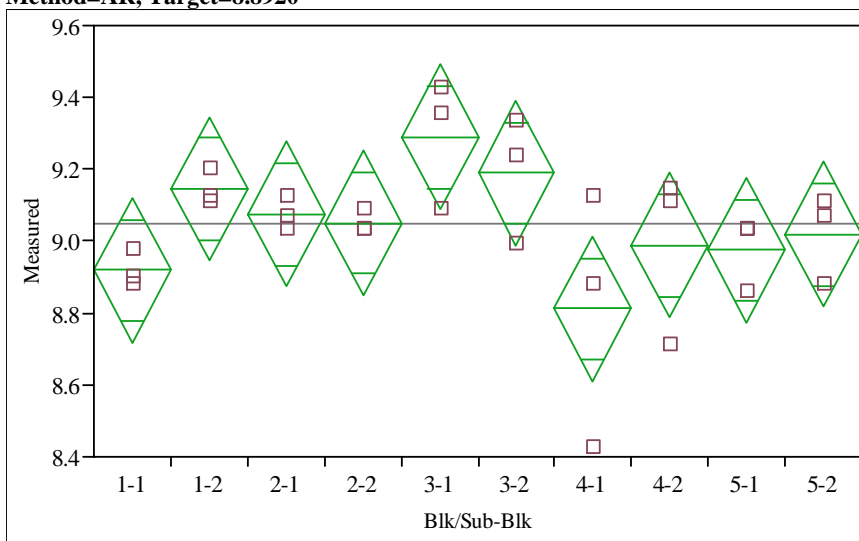

Oneway Anova

Summary of Fit

Adj Rsquare

0.479251

Theror 0.244914

Mean of Response

9.046296

Observations (or Sum Wgts) $\quad 30$

Analysis of Variance

Source DF Sum of Squares Mean Square F Ratio Prob $>$ F

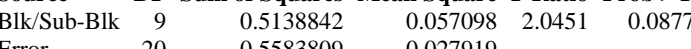

Error $\quad 20-0.5583809$

.027919

\section{Means for Oneway Anova}

Level Number Mean Std Error Lower 95\% Upper 95\%

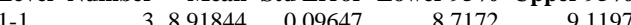

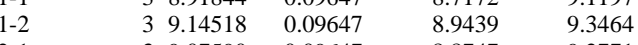

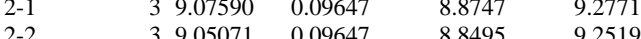

$\begin{array}{llllll}2-2 & 3 & 9.05071 & 0.09647 & 8.8495 & 9.2519 \\ 3-1 & 3 & 9.29004 & 0.09647 & 9.0888 & 9.4913\end{array}$

$\begin{array}{llllll}3-2 & 3 & 9.18927 & 0.09647 & 8.9880 & 9.3905\end{array}$

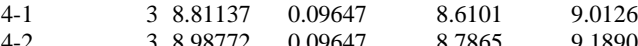

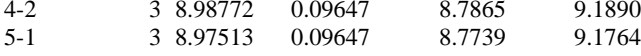

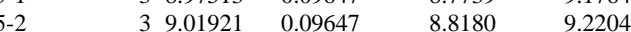

Std Error uses a pooled estimate of error variance
Oneway Analysis of Measured By Blk/Sub-Blk Glass ID=SB7Ref, Oxide=Al2O3 (wt\%), Prep Method $=$ PF, Target $=\mathbf{8 . 8 9 2 0}$

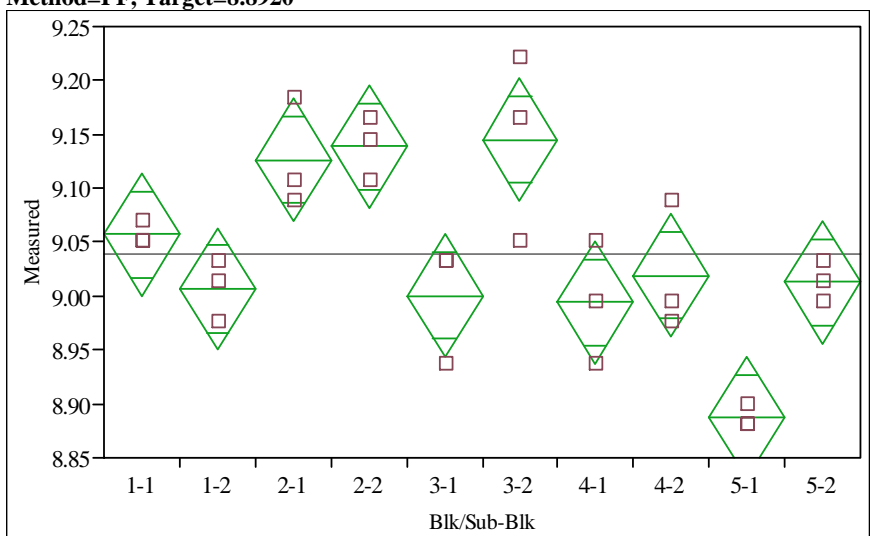

Oneway Anova

Summary of Fit

$\begin{array}{ll}\text { Rsquare } & 0.797043 \\ \text { Adj Rsquare } & 0.705712\end{array}$

Adj Rsquare $\quad 0.705712$

Root Mean Square Error $\quad 0.047048$

Mean of Response

9.038738

Analysis of Variance

Source DF Sum of Squares Mean Square F Ratio Prob $>$ F

$0.17385734-0.019317 \quad 8.7270<0001$

$\begin{array}{llll}\text { Error } & 20 & 0.04427061 & 0.002214\end{array}$

Means for Oneway Anova

Level Number Mean Std Error Lower 95\% Upper 95\%

$\begin{array}{lrrrrr}1-1 & 3 & 9.05700 & 0.02716 & 9.0003 & 9.1137 \\ 1-2 & 3 & 9.00662 & 0.02716 & 8.9500 & 9.0633\end{array}$

$\begin{array}{llllll}1-2 & 3 & 9.00662 & 0.02716 & 8.9500 & 9.0633 \\ 2-1 & 3 & 9.12629 & 0.02716 & 9.0696 & 9.1829\end{array}$

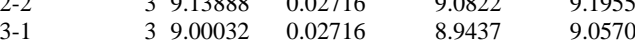

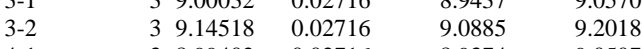

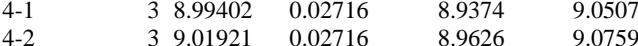

$\begin{array}{llllll}4-2 & 3 & 9.01921 & 0.02716 & 8.9626 & 9.0759 \\ 5-1 & 3 & 8.88695 & 0.02716 & 8.8303 & 8.9436\end{array}$

$\begin{array}{llllll}5-1 & 3 & 8.88695 & 0.02716 & 8.8303 & 8.9436 \\ 5-2 & 3 & 9.01292 & 0.02716 & 8.9563 & 9.0696\end{array}$

Std Error uses a pooled estimate of error variance 
Exhibit A-5. Measurements by Block and Sub-Block for Samples of the Sb7ref Glass and the Ustd Standard Glass by Oxide by Prep

Oneway Analysis of Measured By Blk/Sub-Blk Glass ID=SB7Ref, Oxide=B2O3 (wt\%), Prep Method=AR, Target $=5.2610$

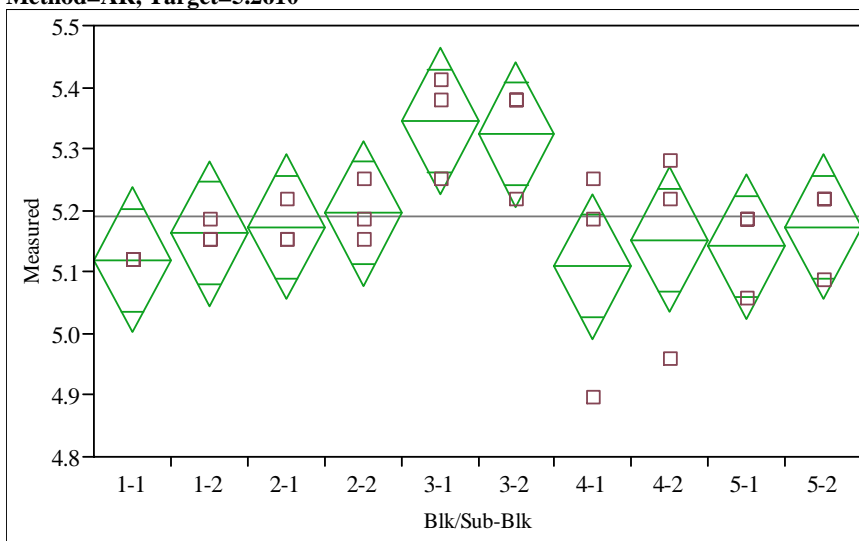

Oneway Anova

Summary of Fit

Adj Rsquare

\subsection{8}

$-0.243953$

Mean of Response 5.189405

Observations (or Sum Wgts)

30

Analysis of Variance

Source DF Sum of Squares Mean Square F Ratio Prob $>$ F

\begin{tabular}{llllll} 
Blk/Sub-Blk & 0.17573346 & 0.019526 & 2.0397 & 0.0885 \\
\hline
\end{tabular}

Error 200.19145789

\section{Means for Oneway Anova}

Level Number Mean Std Error Lower 95\% Upper 95\%

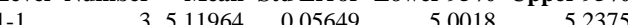

$\begin{array}{llllll}1-2 & 3 & 5.16257 & 0.05649 & 5.0447 & 5.2804\end{array}$

$\begin{array}{llllll}2-1 & 3 & 5.17331 & 0.05649 & 5.0555 & 5.2911\end{array}$

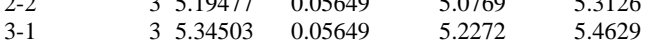

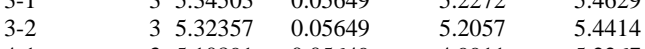

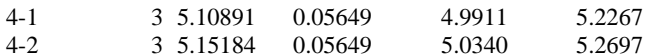

$\begin{array}{llllll}4-2 & 3 & 5.15184 & 0.05649 & 5.0340 & 5.2697 \\ 5-1 & 3 & 5.14111 & 0.05649 & 5.0233 & 5.2589\end{array}$

Std Error uses a pooled estimate of error variance
Oneway Analysis of Measured By Blk/Sub-Blk Glass ID=SB7Ref, Oxide=B2O3 (wt\%), Prep Method $=$ PF, Target $=5.2610$

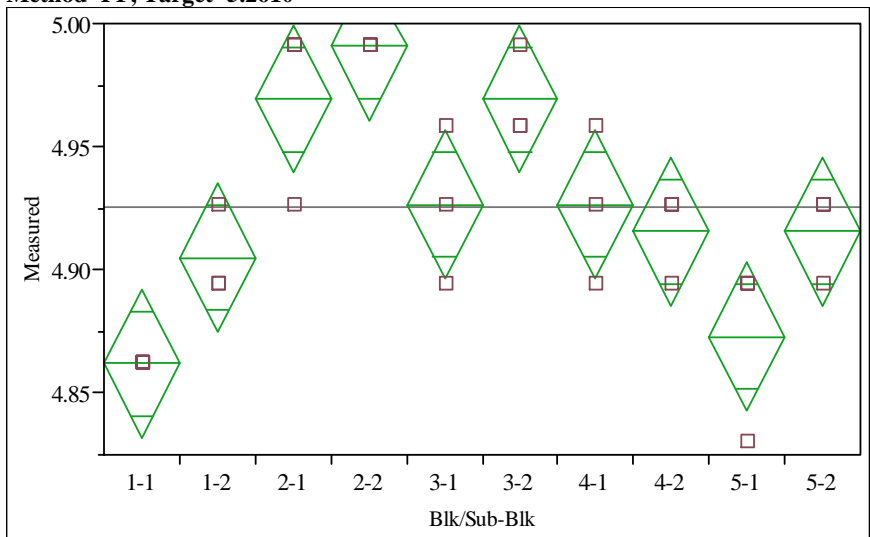

Oneway Anova

Summary of Fit

Rsquare

Adj Rsquare $\quad 0.694558$

Root Mean Square Error $\quad 0.02494$

Mean of Response 4.925374

Analysis of Variance

Source DF Sum of Squares Mean Square F Ratio Prob $>$ F

$\begin{array}{llllll} & 9 & 0.04662034 & 0.005180 & 8.3272 & <.0001\end{array}$

$\begin{array}{llll}\text { Error } & 20 & 0.01244131 & 0.000622\end{array}$

Means for Oneway Anova

Level Number Mean Std Error Lower 95\% Upper 95\%

$\begin{array}{rrrrrr}1-1 & 3 & 4.86205 & 0.01440 & 4.8320 & 4.8921\end{array}$

$\begin{array}{llllll}1-2 & 3 & 4.90498 & 0.01440 & 4.8749 & 4.9350 \\ 2-1 & 3 & 4.96938 & 0.01440 & 4.9393 & 4.9994\end{array}$

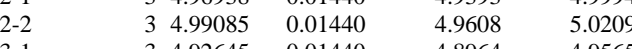

$\begin{array}{llllll}3-1 & 3 & 4.92645 & 0.01440 & 4.8964 & 4.9565 \\ 3-2 & 3 & 4.96938 & 0.01440 & 4.9393 & 4.9994\end{array}$

$\begin{array}{llllll}3-2 & 3 & 4.96938 & 0.01440 & 4.9393 & 4.9994 \\ 4-1 & 3 & 4.92645 & 0.01440 & 4.8964 & 4.9565\end{array}$

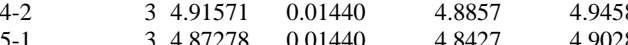

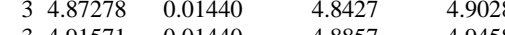

Std Error uses a pooled estimate of error variance 
Exhibit A-5. Measurements by Block and Sub-Block for Samples of the Sb7ref Glass and the Ustd Standard Glass by Oxide by Prep

Oneway Analysis of Measured By Blk/Sub-Blk Glass ID=SB7Ref, Oxide=BaO (wt\%), Prep Method $=$ AR, Target $=\mathbf{0 . 0 1 7 0}$

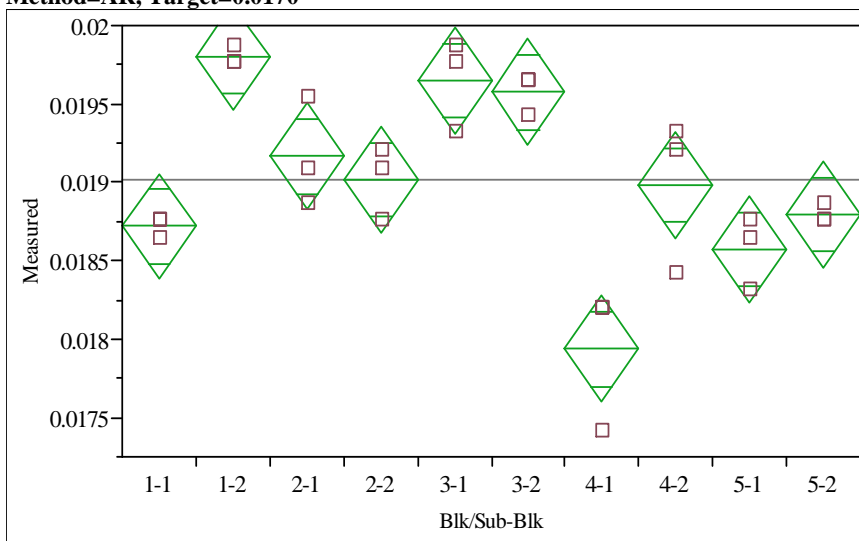

Oneway Anova

Summary of Fit

Adj Rsquare

0.845452

Square Error $\quad 0.775905$

Mean of Response

0.000279

Observations (or Sum Wgts)

30

Analysis of Variance

Source DF Sum of Squares Mean Square F Ratio Prob $>$ F

$\begin{array}{llllll} & \text { Blk/Sub-Blk } & 0.00000855 & 9.4966 \mathrm{e}-7 & 12.1566 & <.0001\end{array}$

Error 200.000156

Means for Oneway Anova

Level Number Mean Std Error Lower 95\% Upper 95\%

$\begin{array}{llllll}1-1 & 3 & 0.018720 & 0.00016 & 0.01838 & 0.01906\end{array}$

$\begin{array}{lllll}3 & 0.019799 & 0.00016 & 0.01946 & 0.02014\end{array}$

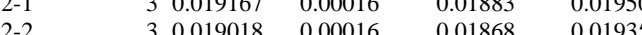

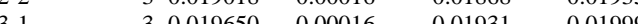

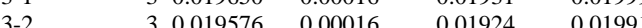

$\begin{array}{llllll}4-1 & 3 & 0.017938 & 0.00016 & 0.01760 & 0.01828\end{array}$

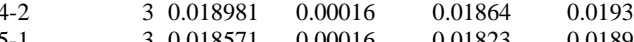

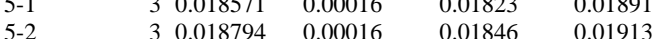

Std Error uses a pooled estimate of error variance
Oneway Analysis of Measured By Blk/Sub-Blk Glass ID=SB7Ref, Oxide=BaO (wt\%), Prep Method $=\mathbf{P F}$, Target $=\mathbf{0 . 0 1 7 0}$

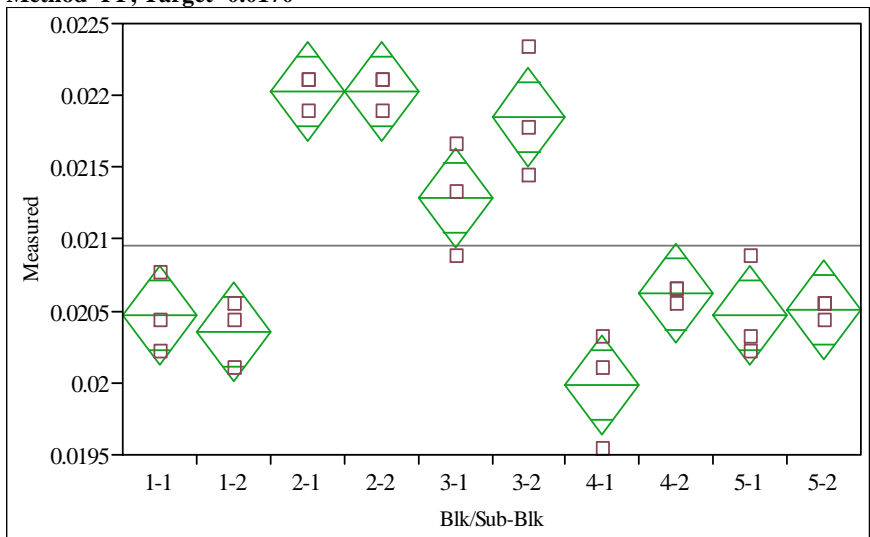

Oneway Anova

Summary of Fit

Rsquare $\quad 0.906374$

Adj Rsquare $\quad 0.864242$

Mean of Response

Mean of Response

0.02096

Analysis of Variance

Source DF Sum of Squares Mean Square F Ratio Prob $>$ F

$0.00001593 \quad 1.7699 \mathrm{e}-6 \quad 21.5129<0001$

$\begin{array}{llll}\text { Error } & 20 & 0.00000165 & 8.2274 \mathrm{e}-8\end{array}$

\section{Means for Oneway Anova}

Level Number Mean Std Error Lower 95\% Upper 95\%

$\begin{array}{rrrrrr}1-1 & 3 & 0.020469 & 0.00017 & 0.02012 & 0.02081 \\ 1-2 & 3 & 0.020358 & 0.00017 & 0.02001 & 0.02070\end{array}$

$\begin{array}{llllll}1-2 & 3 & 0.020358 & 0.00017 & 0.02001 & 0.02070 \\ 2-1 & 3 & 0.022032 & 0.00017 & 0.02169 & 0.02238\end{array}$

$\begin{array}{llllll}2-2 & 3 & 0.022032 & 0.00017 & 0.02169 & 0.02238\end{array}$

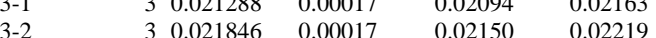

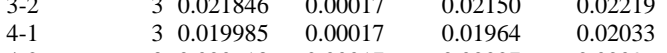

$\begin{array}{llllll}4-2 & 3 & 0.020618 & 0.00017 & 0.02027 & 0.02096 \\ 5-1 & 3 & 0.020469 & 0.00017 & 0.02012 & 0.02081\end{array}$

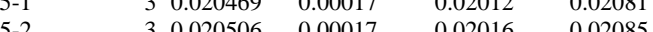

Std Error uses a pooled estimate of error variance 
Exhibit A-5. Measurements by Block and Sub-Block for Samples of the Sb7ref Glass and the Ustd Standard Glass by Oxide by Prep

Oneway Analysis of Measured By Blk/Sub-Blk Glass ID=SB7Ref, Oxide=CaO (wt\%), Prep Method $=$ AR, Target $=\mathbf{0 . 4 7 6 0}$

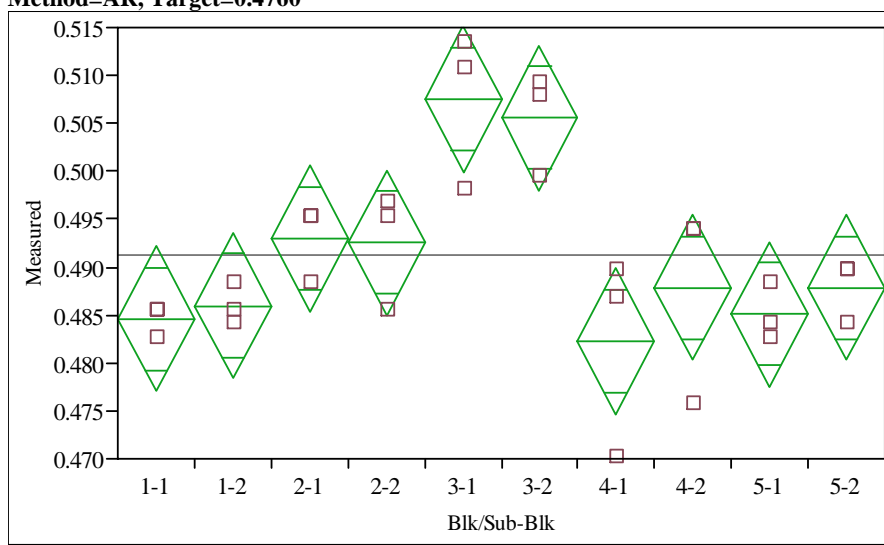

Oneway Anova

Summary of Fit

Adj Rsquare

0.721511

De.596191

0.006304

Observations (or Sum Wgts) $\quad 30$

Analysis of Variance

Source DF Sum of Squares Mean Square F Ratio Prob $>$ F

$\begin{array}{llllll} & 0.00205930 & 0.000229 & 5.7573 & 0.0006\end{array}$

$\begin{array}{lll}\text { Error } & 20 & 0.00079485 \\ \text { C. Total } & 29 & 0.00285415\end{array}$

Means for Oneway Anova

Level Number Mean Std Error Lower 95\% Upper 95\%

$\begin{array}{llllll}1-1 & 3 & 0.484590 & 0.00364 & 0.47700 & 0.49218\end{array}$

$\begin{array}{lllll}3 & 0.485989 & 0.00364 & 0.47840 & 0.49358\end{array}$

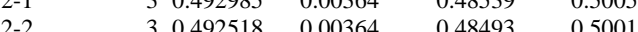

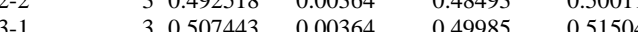

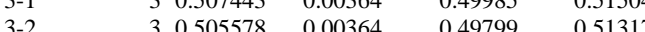

$\begin{array}{llllll}4-1 & 3 & 0.482258 & 0.00364 & 0.47467 & 0.48985\end{array}$

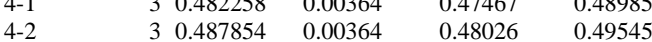

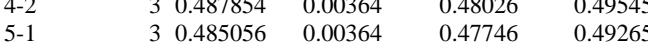

$\begin{array}{llllll}5-2 & 3 & 0.487854 & 0.00364 & 0.48026 & 0.49545\end{array}$

Std Error uses a pooled estimate of error variance
Oneway Analysis of Measured By Blk/Sub-Blk Glass ID=SB7Ref, Oxide=Ce2O3 (wt\%), Prep Method $=$ AR, Target $=0.0240$

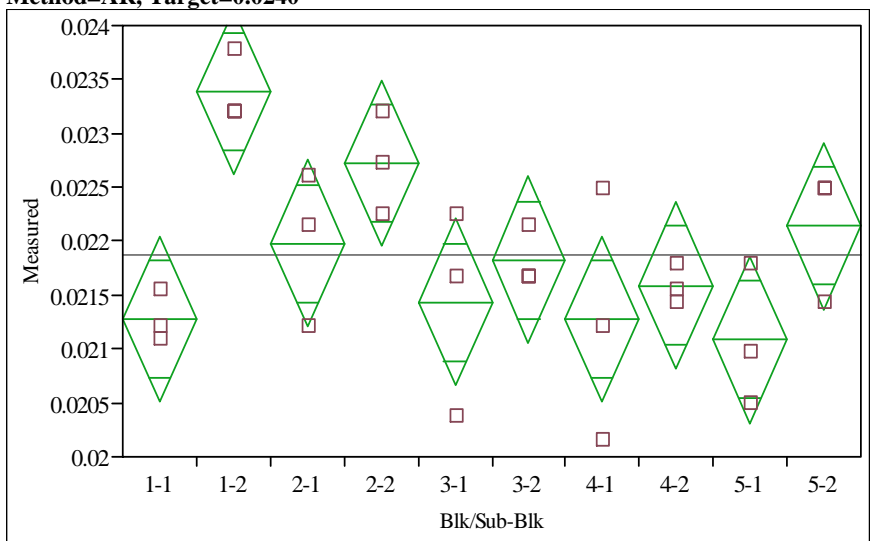

Oneway Anova

Summary of Fit

Rsquare $\quad 0.632196$

Adj Rsquare $\quad 0.466684$

Root Mean Square Error $\quad 0.001872$

Mean of Response

0.021872

Analysis of Variance

Source DF Sum of Squares Mean Square F Ratio Prob $>$ F

$\begin{array}{llll}0.00001410 & 1.5669 \mathrm{e}-6 & 3.8196 & 0.0060\end{array}$

$\begin{array}{llll}\text { Error } & 20 & 0.00000820 & 4.1021 \mathrm{e}-7\end{array}$

\section{Means for Oneway Anova}

Level Number Mean Std Error Lower 95\% Upper 95\%

$\begin{array}{rrrrrr}1-1 & 3 & 0.021279 & 0.00037 & 0.02051 & 0.02205 \\ 1-2 & 3 & 0.023387 & 0.00037 & 0.02262 & 0.02416\end{array}$

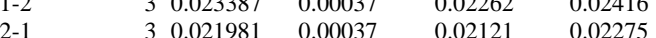

$\begin{array}{llllll}2-2 & 3 & 0.022723 & 0.00037 & 0.02195 & 0.02349\end{array}$

$\begin{array}{llllll}3-1 & 3 & 0.021435 & 0.00037 & 0.02066 & 0.02221 \\ 3-2 & 3 & 0.021825 & 0.00037 & 0.02105 & 0.02260\end{array}$

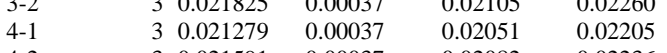

$\begin{array}{llllll}4-2 & 3 & 0.021591 & 0.00037 & 0.02082 & 0.02236 \\ 5-1 & 3 & 0.021083 & 0.00037 & 0.02031 & 0.02185\end{array}$

$\begin{array}{llllll}5-1 & 3 & 0.021083 & 0.0037 & 0.02031 & 0.02185 \\ 5-2 & 3 & 0.022138 & 0.00037 & 0.02137 & 0.02291\end{array}$

Std Error uses a pooled estimate of error variance 
Exhibit A-5. Measurements by Block and Sub-Block for Samples of the Sb7ref Glass and the Ustd Standard Glass by Oxide by Prep

Oneway Analysis of Measured By Blk/Sub-Blk Glass ID=SB7Ref, Oxide=Ce2O3 (wt\%), Prep Method $=$ PF, Target $=0.0240$

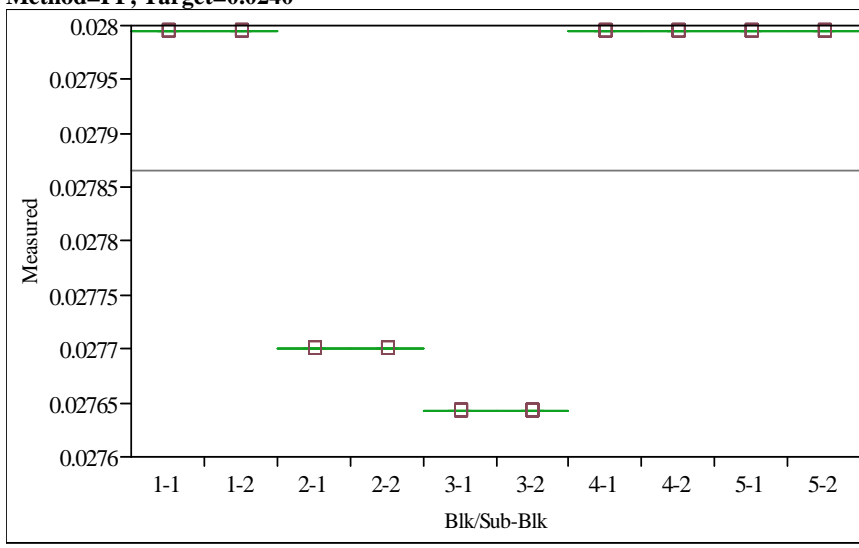

Oneway Anova

ummary of Fit

Rsquare

Adj Rsquare

Root Mean Square Error $\quad 3.99 \mathrm{e}-12$

$\begin{array}{r}0.027865 \\ \hline\end{array}$

Analysis of Variance

Source DF Sum of Squares Mean Square F Ratio Prob $>$ F

Blk/Sub-Blk 9

$\begin{array}{lll}\text { Error } & 20 & 3.1764 \mathrm{e}-22 \\ \text { C. Total } & 29 & 7.57313 \mathrm{e}-7\end{array}$

\section{Means for Oneway Anova}

Level Number Mean Std Error Lower 95\% Upper 95\%

$\begin{array}{llllll}1-1 & 3 & 0.027994 & 2.301 \mathrm{e}-12 & 0.02799 & 0.02799\end{array}$

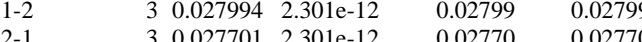

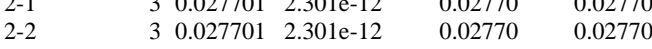

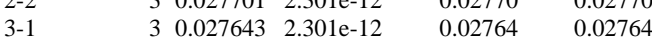

$\begin{array}{llllll}3-2 & 3 & 0.027643 & 2.301 \mathrm{e}-12 & 0.02764 & 0.02764\end{array}$

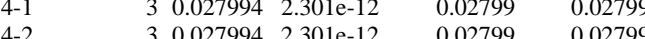

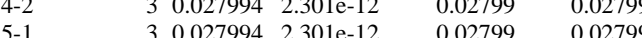

$\begin{array}{llllll}5-1 & 3 & 0.027994 & 2.301 \mathrm{e}-12 & 0.02799 & 0.02799 \\ 5-2 & 3 & 0.027994 & 2.301 \mathrm{e}-12 & 0.02799 & 0.02799\end{array}$

Std Error uses a pooled estimate of error variance
Oneway Analysis of Measured By Blk/Sub-Blk Glass ID=SB7Ref, Oxide=Cr2O3 (wt\%), Prep Method $=$ AR, Target $=\mathbf{0 . 1 2 4 0}$

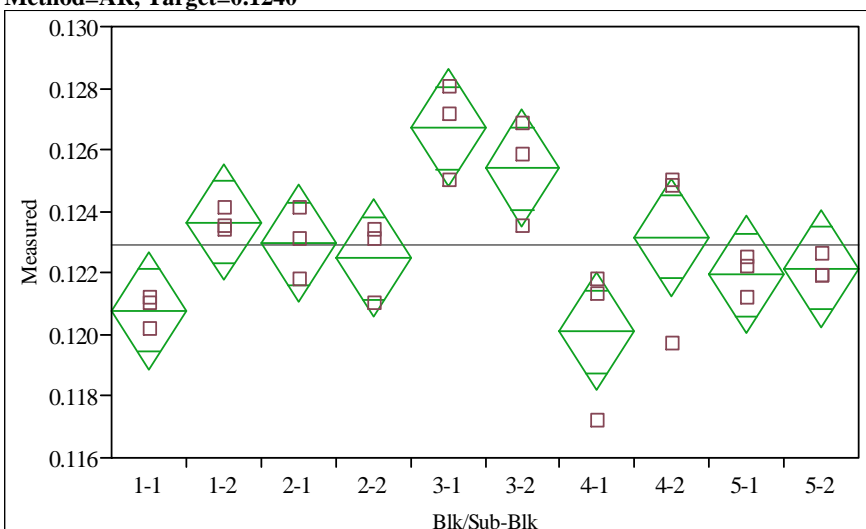

Oneway Anova

Summary of Fi

$\begin{array}{lr}\text { Rsquare } & 0.68247\end{array}$

Adj Rsquare $\quad 0.539582$

Root Mean Square Error $\quad 0.001575$

Mean of Response 0.122935

Analysis of Variance

Source DF Sum of Squares Mean Square F Ratio Prob $>$ F

$\begin{array}{lll}\text { Error } & 20 & 0.00004960 \\ \text { C. Total } & 29 & 0.00015622\end{array}$

\section{Means for Oneway Anova}

Level Number Mean Std Error Lower 95\% Upper 95\%

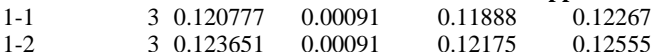

$\begin{array}{lllllll}1-2 & & 3 & 0.123651 & 0.00091 & 0.12175 & 0.12555 \\ 2-1 & & 3 & 0.122969 & 0.00091 & 0.12107 & 0.12487\end{array}$

$\begin{array}{llllll}2-2 & 3 & 0.122482 & 0.00091 & 0.12059 & 0.12438 \\ 3-1 & 3 & 0.126721 & 0.00091 & 0.12482 & 0.12862\end{array}$

$\begin{array}{llllll}3-1 & 3 & 0.126721 & 0.00091 & 0.12482 & 0.12862 \\ 3-2 & 3 & 0.125405 & 0.00091 & 0.12351 & 0.12730\end{array}$

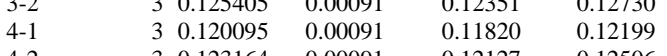

$\begin{array}{llllll}4-2 & 3 & 0.123164 & 0.00091 & 0.12127 & 0.12506 \\ 5-1 & 3 & 0.121946 & 0.00091 & 0.12005 & 0.12384\end{array}$

$\begin{array}{llllll}5-1 & 3 & 0.12194 & 0.00091 & 0.12127 & 0.12506\end{array}$

Std Error uses a pooled estimate of error variance 
Exhibit A-5. Measurements by Block and Sub-Block for Samples of the Sb7ref Glass and the Ustd Standard Glass by Oxide by Prep

Oneway Analysis of Measured By Blk/Sub-Blk Glass ID=SB7Ref, Oxide=Cr2O3 (wt\%), Prep Method=PF, Target $=0.1240$

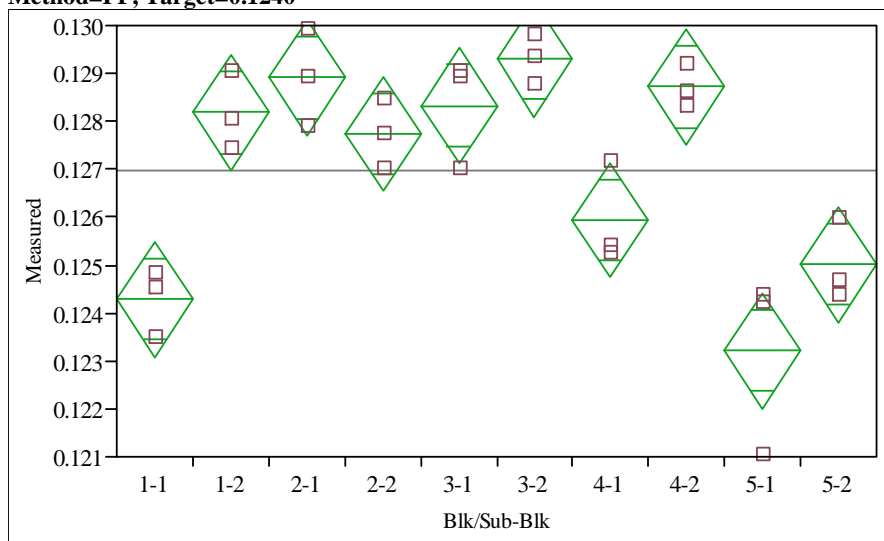

Oneway Anova

Summary of Fit

Adj Rsquare

\subsection{4}

Root

Mean of Respose

Observations (or Sum Wgts) $\quad 30$

Analysis of Variance

Source DF Sum of Squares Mean Square F Ratio Prob $>$ F

$\begin{array}{lllllll} & 0.00012718 & 0.000014 & 14.2050<.0001\end{array}$

Error $20-0.00001090$

000001

Means for Oneway Anova

Level Number Mean Std Error Lower 95\% Upper 95\%

$\begin{array}{llllll}1-1 & 3 & 0.124285 & 0.00058 & 0.12308 & 0.12549\end{array}$

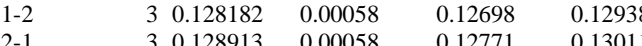

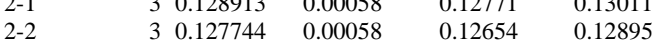

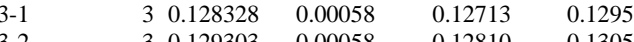

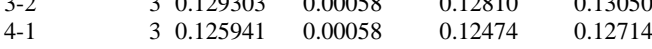

$\begin{array}{llllll}4-2 & 3 & 0.128718 & 0.00058 & 0.12752 & 0.12992 \\ 5-1 & 3 & 0.123213 & 0.00058 & 0.12201 & 0.12441\end{array}$

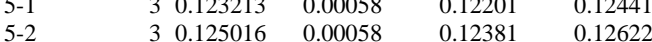

Std Error uses a pooled estimate of error variance
Oneway Analysis of Measured By Blk/Sub-Blk Glass ID=SB7Ref, Oxide=CuO (wt\%), Prep Method $=$ AR, Target $=0.0330$

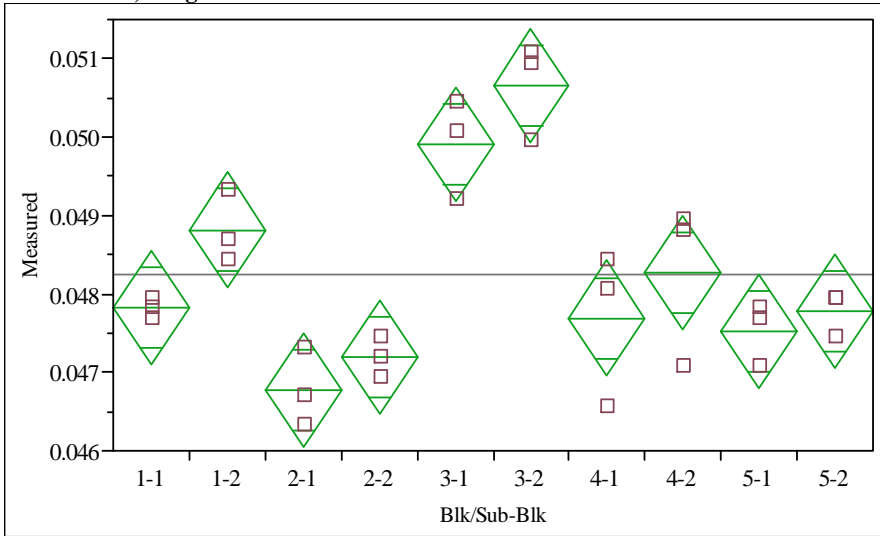

Oneway Anova

Summary of Fi

Rsquare

$\begin{array}{ll}\text { Adj Rsquare } & 0.845606 \\ & 0.776128\end{array}$

Root Mean Square Error $\quad 0.000606$

Mean of Response

0.048244

Analysis of Variance

Source DF Sum of Squares Mean Square F Ratio Prob $>$ F

$0.00004017 \quad 4.4628 \mathrm{e}-6$

$\begin{array}{lll}\text { Error } & 20 & 0.00000733 \\ \text { C. Total } & 29 & 0.00004750\end{array}$

\section{Means for Oneway Anova}

Level Number Mean Std Error Lower 95\% Upper 95\%

$\begin{array}{llllll}1-1 & 3 & 0.047819 & 0.00035 & 0.04709 & 0.04855 \\ 1-2 & 3 & 0.048820 & 0.00035 & 0.04809 & 0.04955\end{array}$

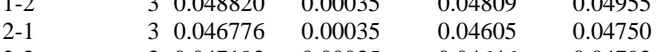

$\begin{array}{llllll}2-2 & 3 & 0.047193 & 0.00035 & 0.04646 & 0.04792 \\ 3-1 & 3 & 0.049905 & 0.00035 & 0.04918 & 0.05063\end{array}$

$\begin{array}{llllll}3-1 & 3 & 0.04905 & 0.00035 & 0.04918 & 0.05063 \\ 3-2 & 3 & 0.050656 & 0.00035 & 0.04993 & 0.05139\end{array}$

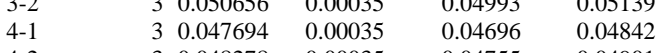

$\begin{array}{llllll}4-2 & 3 & 0.048278 & 0.00035 & 0.04755 & 0.04901 \\ 5-1 & 3 & 0.047527 & 0.00035 & 0.04680 & 0.04826\end{array}$

$\begin{array}{llllll}5-1 & 3 & 0.047527 & 0.00035 & 0.04680 & 0.04826\end{array}$

Std Error uses a pooled estimate of error variance 
Exhibit A-5. Measurements by Block and Sub-Block for Samples of the Sb7ref Glass and the Ustd Standard Glass by Oxide by Prep

Oneway Analysis of Measured By Blk/Sub-Blk Glass ID=SB7Ref, Oxide=CuO (wt\%), Prep Method=PF, Target $=\mathbf{0 . 0 3 3 0}$

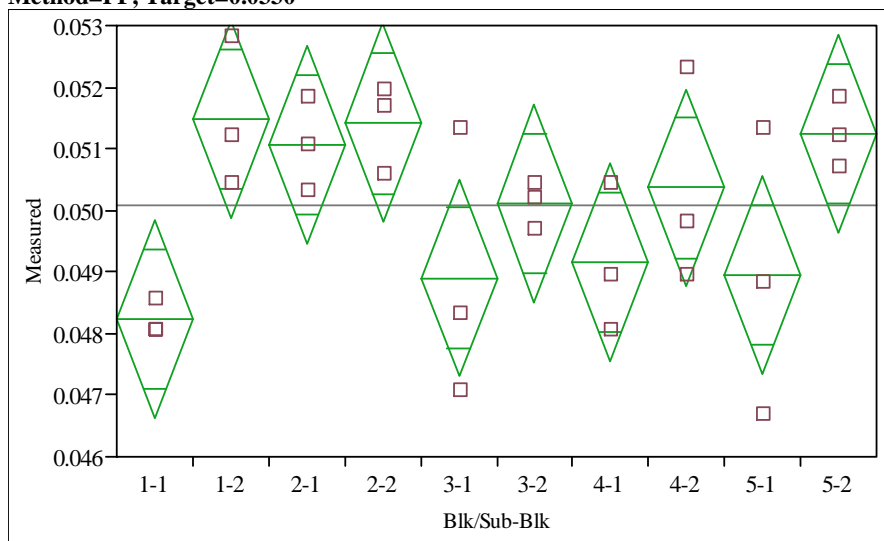

Oneway Anova

Summary of Fit

Adj Rsquare

\subsection{4}

0.310415
0.001355

Mean of Response

0.001335

Observations (or Sum Wgts)

30

Analysis of Variance

Source DF Sum of Squares Mean Square F Ratio Prob > F

$\begin{array}{llllll}\text { Blk/Sub-Blk } & 0.00003928 & 4.3647 \mathrm{e}-6 & 2.4505 & 0.0456\end{array}$

$\begin{array}{lll}\text { Error } & 20 & 0.00003562 \\ \text { C. Total } & 29 & 0.00007491\end{array}$

Means for Oneway Anova

Level Number Mean Std Error Lower 95\% Upper 95\%

$\begin{array}{llllll}1-1 & 3 & 0.048236 & 0.00077 & 0.04663 & 0.04984\end{array}$

$\begin{array}{lllll}3 & 0.051491 & 0.00077 & 0.04988 & 0.05310\end{array}$

$\begin{array}{llllll}2-1 & 3 & 0.051073 & 0.00077 & 0.04947 & 0.05268 \\ 2-2 & 3 & 0.051407 & 0.00077 & 0.04980 & 0.05301\end{array}$

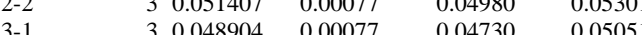

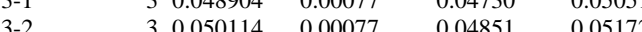

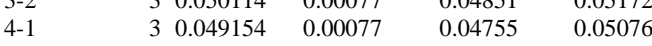

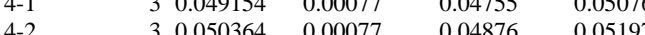

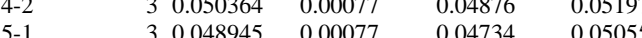

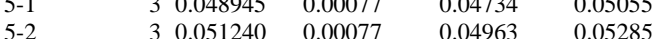

Std Error uses a pooled estimate of error variance
Oneway Analysis of Measured By Blk/Sub-Blk Glass ID=SB7Ref, Oxide=Fe2O3 (wt\%), Prep Method $=$ AR, Target $=\mathbf{8 . 6 6 8 0}$

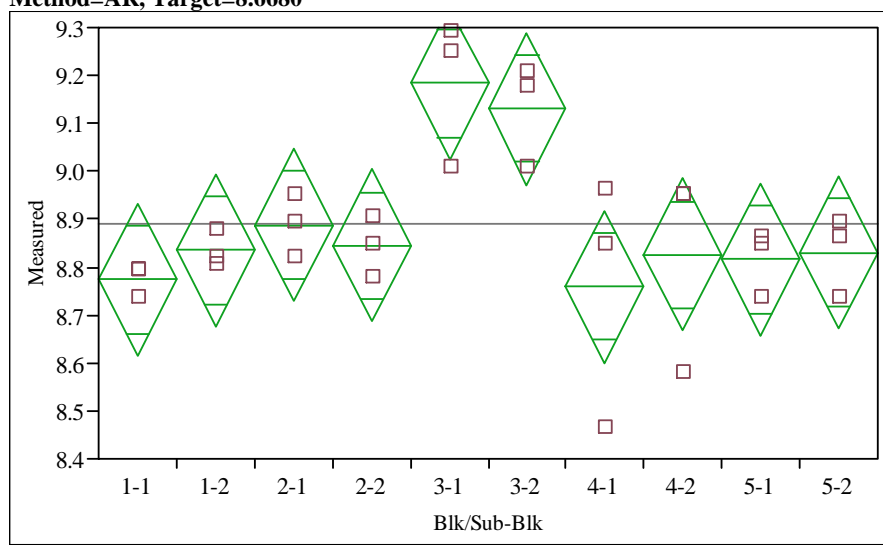

Oneway Anova

Summary of Fit

Rsquare

Adj Rsquare $\quad 0.457035$

Root Mean Square Error $\quad 0.131579$

Mean of Response

8.888921
$\quad 30$

Analysis of Variance

Source DF Sum of Squares Mean Square F Ratio Prob $>$ F

$\begin{array}{llrrrr} & & & & \\ \text { Bll/Sub-Blk } & 9 & 0.57843666 & 0.064271 & 3.7123 & 0.0070\end{array}$

$\begin{array}{llll}\text { Error } & 20 & 0.34626073 & 0.017313\end{array}$

0.92469739

Means for Oneway Anova

Level Number Mean Std Error Lower 95\% Upper 95\%

$\begin{array}{lrrrrr}1-1 & 3 & 8.77359 & 0.07597 & 8.6151 & 8.9321\end{array}$

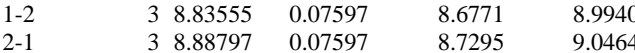

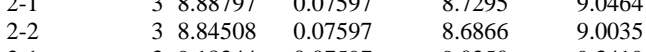

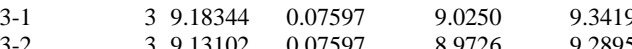

$\begin{array}{llllll}3-2 & 3 & 9.13102 & 0.07597 & 8.9726 & 9.2895 \\ 4-1 & 3 & 8.75930 & 0.07597 & 8.6008 & 8.9178\end{array}$

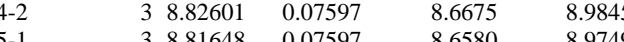

$5-2$

Std Error uses a pooled estimate of error variance 
Exhibit A-5. Measurements by Block and Sub-Block for Samples of the Sb7ref Glass and the Ustd Standard Glass by Oxide by Prep

Oneway Analysis of Measured By Blk/Sub-Blk Glass ID=SB7Ref, Oxide=Fe2O3 (wt\%), Prep Method=PF, Target $=8.6680$

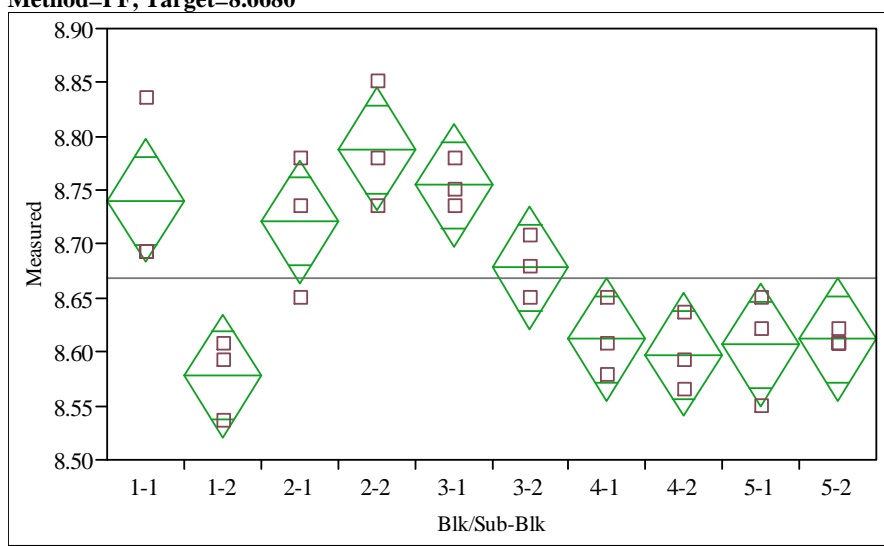

Oneway Anova

Summary of Fit

Adj Rsquare

\subsection{7}

0.681213

Mean of Response

0.047418

Observations (or Sum Wgts)

30

Analysis of Variance

Source DF Sum of Squares Mean Square F Ratio Prob $>$ F

$\begin{array}{llll}0.15957155 & 0.017730 & 7.8855 & <.0001\end{array}$

$\begin{array}{lll}\text { Error } & 20 & 0.04496893 \\ \text { C. Total } & 29 & 0.20454048\end{array}$

\section{Means for Oneway Anova}

Level Number Mean Std Error Lower 95\% Upper 95\%

$\begin{array}{llllrr}1-1 & 3 & 8.74023 & 0.02738 & 8.6831 & 8.7973\end{array}$

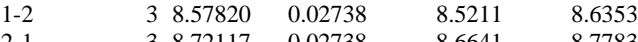

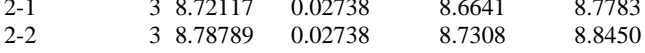

$\begin{array}{llllll}2-2 & 3 & 8.78789 & 0.02738 & 8.7308 & 8.8450 \\ 3-1 & 3 & 8.75453 & 0.02738 & 8.6974 & 8.8116\end{array}$

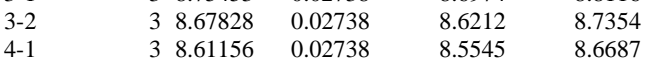

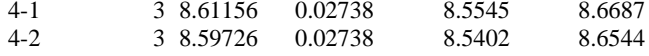

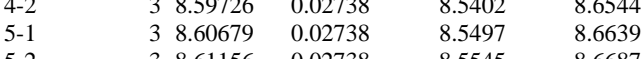

Std Error uses a pooled estimate of error variance
Oneway Analysis of Measured By Blk/Sub-Blk Glass ID=SB7Ref, Oxide=K2O (wt\%), Prep Method $=$ AR, Target $=\mathbf{0 . 2 1 2 0}$

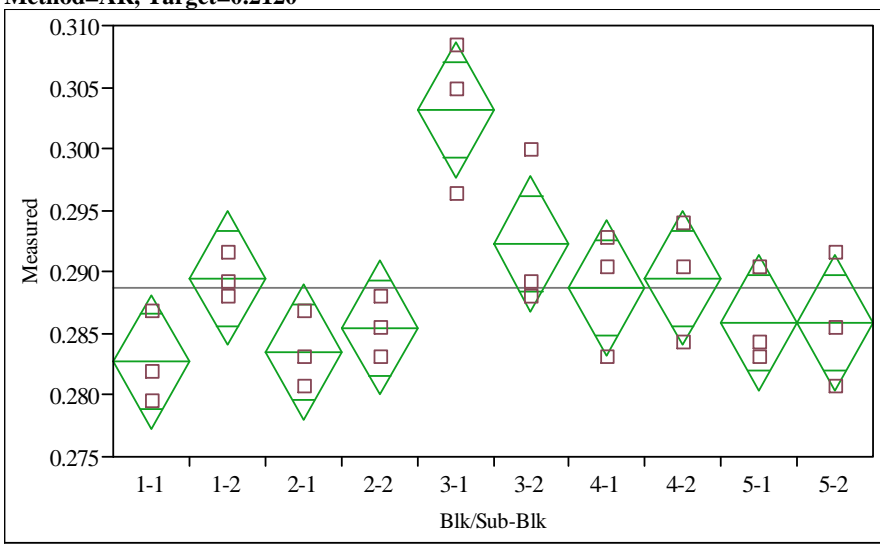

Oneway Anova

Summary of Fit

$\begin{array}{ll}\text { Rsquare } & 0.693451 \\ \text { Adj Rsquare } & 0.555504\end{array}$

Adj Rsquare $\quad 0.555504$

Reot Mean Square Eror 0.004555

Mean of Response 0.288662

Analysis of Variance

Source DF Sum of Squares Mean Square F Ratio Prob $>$ F

$\begin{array}{lllll}9 & 0.00093879 & 0.000104 & 5.0269 & 0.0013\end{array}$

$\begin{array}{lll}\text { Error } & 20 & 0.00041500\end{array}$

00002

\section{Means for Oneway Anova}

Level Number Mean Std Error Lower 95\% Upper 95\%

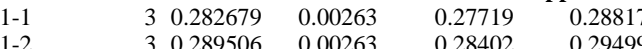

$\begin{array}{llllll}1-2 & 3 & 0.289506 & 0.00263 & 0.28402 & 0.29499 \\ 2-1 & 3 & 0.283483 & 0.00263 & 0.27800 & 0.28897\end{array}$

$\begin{array}{llllll}2-2 & 3 & 0.285490 & 0.00263 & 0.28000 & 0.29098\end{array}$

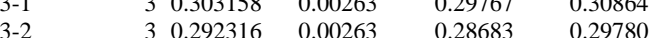

$\begin{array}{llllll}3-2 & 3 & 0.292316 & 0.00263 & 0.28683 & 0.29780 \\ 4-1 & 3 & 0.288702 & 0.00263 & 0.28322 & 0.29419\end{array}$

$\begin{array}{llllll}4-2 & 3 & 0.289506 & 0.00263 & 0.28402 & 0.29499 \\ 5-1 & 3 & 0.285892 & 0.00263 & 0.28041 & 0.29138\end{array}$

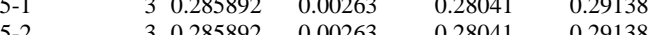

Std Error uses a pooled estimate of error variance 
Exhibit A-5. Measurements by Block and Sub-Block for Samples of the Sb7ref Glass and the Ustd Standard Glass by Oxide by Prep

Oneway Analysis of Measured By Blk/Sub-Blk Glass ID=SB7Ref, Oxide=K20 (wt\%), Prep Method $=$ PF, Target $=\mathbf{0 . 2 1 2 0}$

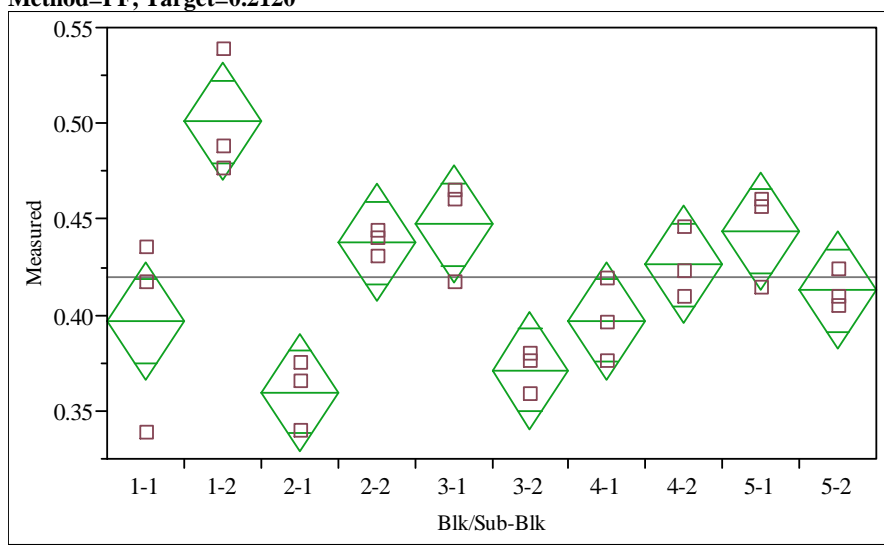

Oneway Anova

Summary of Fit

Rsquare

Adj Rsquare $\quad 0.779543$

Root Mean Square Eror $\quad 0.080337$

0.025452

Observations (or Sum Wgts)

30

Analysis of Variance

Source DF Sum of Squares Mean Square F Ratio Prob $>$ F

$\begin{array}{llll}0.04581295 & 0.005090 & 7.8578 & <.0001\end{array}$

Error $20-0.01295604$

0.000648

Means for Oneway Anova

Level Number Mean Std Error Lower 95\% Upper 95\%

$\begin{array}{llllll}1-1 & 3 & 0.396715 & 0.01469 & 0.36606 & 0.42737\end{array}$

$\begin{array}{llllll}1-2 & 3 & 0.500712 & 0.01469 & 0.47006 & 0.53136\end{array}$

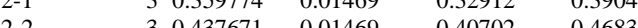

$\begin{array}{llllll}2-2 & 3 & 0.437671 & 0.01469 & 0.40702 & 0.46832 \\ 3-1 & 3 & 0.447308 & 0.01469 & 0.41666 & 0.47796\end{array}$

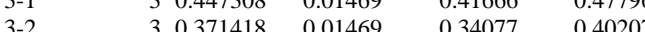

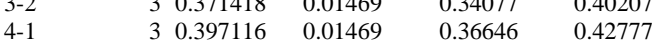

$\begin{array}{llllll}4-2 & 3 & 0.426027 & 0.01469 & 0.39537 & 0.45668\end{array}$

$\begin{array}{llllll}5-1 & 3 & 0.443694 & 0.01469 & 0.41304 & 0.47435\end{array}$

Std Error uses a pooled estimate of error variance
Oneway Analysis of Measured By Blk/Sub-Blk Glass ID=SB7Ref, Oxide=La2O3 (wt\%), Prep Method $=$ AR, Target $=\mathbf{0 . 0 1 4 0}$

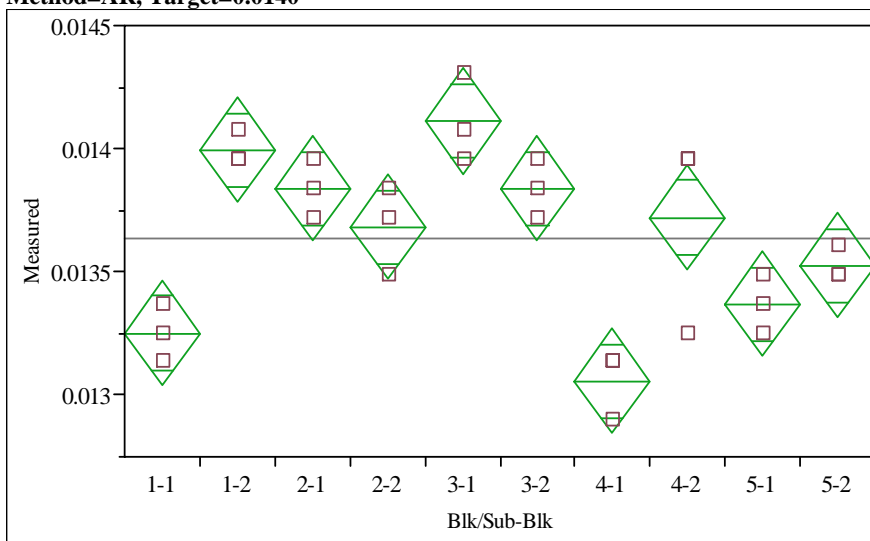

Oneway Anova

Summary of Fit

Rsquare

Adj Rsquare $\quad 0.753161$

Root Mean Square Error $\quad 0.00017$

Mean of Response

0.01364

Analysis of Variance

Source DF Sum of Squares Mean Square F Ratio Prob $>$ F

$\begin{array}{llll}3.03931 \mathrm{e}-6 & 3.377 \mathrm{e}-7 & 10.8317<0001\end{array}$

$\begin{array}{llll}\text { Error } & 20 & 6.23542 \mathrm{e}-7 & 3.1177 \mathrm{e}-8\end{array}$

Means for Oneway Anova

Level Number Mean Std Error Lower 95\% Upper 95\%

$\begin{array}{llllll}1-1 & 3 & 0.013253 & 0.00010 & 0.01304 & 0.01347 \\ 1-2 & 3 & 0.013995 & 0.00010 & 0.01378 & 0.01421\end{array}$

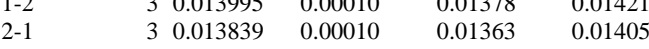

$\begin{array}{llllll}2-2 & 3 & 0.013683 & 0.00010 & 0.01347 & 0.01390 \\ 3-1 & 3 & 0.014113 & 0.00010 & 0.01390 & 0.01433\end{array}$

$\begin{array}{llllll}3-1 & 3 & 0.014133 & 0.00010 & 0.01390 & 0.01433 \\ 3-2 & 3 & 0.013839 & 0.00010 & 0.01363 & 0.01405\end{array}$

$\begin{array}{llllll}3-2 & 3 & 0.013839 & 0.00010 & 0.01363 & 0.01405 \\ 4-1 & 3 & 0.013057 & 0.00010 & 0.01284 & 0.01327\end{array}$

$\begin{array}{llllll}4-2 & 3 & 0.013722 & 0.00010 & 0.01351 & 0.01393 \\ 5-1 & 3 & 0.013370 & 0.00010 & 0.01316 & 0.01358\end{array}$

$\begin{array}{llllll}5-1 & 3 & 0.01332 & 0.00010 & 0.01316 & 0.01358 \\ 5-2 & 3 & 0.013526 & 0.00010 & 0.01331 & 0.01374\end{array}$

Std Error uses a pooled estimate of error variance 
Exhibit A-5. Measurements by Block and Sub-Block for Samples of the Sb7ref Glass and the Ustd Standard Glass by Oxide by Prep

Oneway Analysis of Measured By Blk/Sub-Blk Glass ID=SB7Ref, Oxide=La2O3 (wt\%), Prep Method $=$ PF, Target $=0.0140$

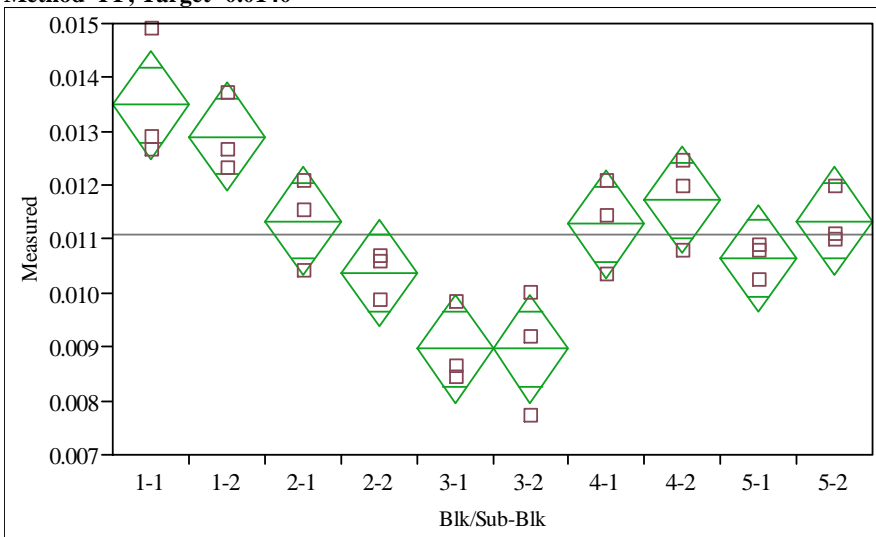

Oneway Anova

Summary of Fit

Adj Rsquare

0.809909

0.000826

Mean of Response

0.000826

Observations (or Sum Wgts)

30

Analysis of Variance

Source DF Sum of Squares Mean Square F Ratio Prob $>$ F

$\begin{array}{llll}0.00005811 & 6.4567 \mathrm{e}-6 & 9.4681 & <.0001\end{array}$

Error $20-0.00001364$

Means for Oneway Anova

Level Number Mean Std Error Lower 95\% Upper 95\%

$\begin{array}{llllll}1-1 & 3 & 0.013487 & 0.00048 & 0.01249 & 0.01448\end{array}$

$\begin{array}{llllll}1-2 & 3 & 0.012901 & 0.00048 & 0.01191 & 0.01390\end{array}$

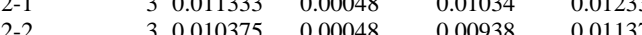

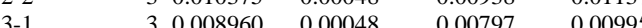

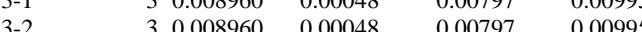

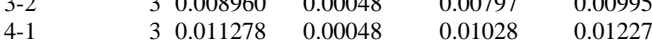

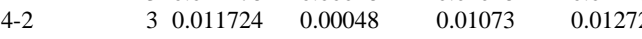

$\begin{array}{llllll}5-1 & 3 & 0.010637 & 0.00048 & 0.00964 & 0.01163\end{array}$

Std Error uses a pooled estimate of error variance
Oneway Analysis of Measured By Blk/Sub-Blk Glass ID=SB7Ref, Oxide=Li2O (wt\%), Prep Method=AR, Target $=5.2610$

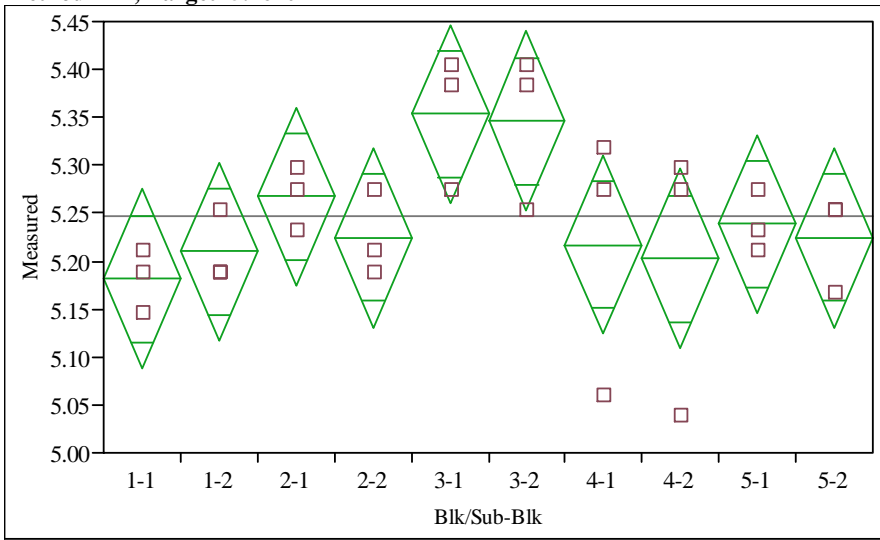

Oneway Anova

Summary of Fi

Rsquare

0.437595

$\begin{array}{ll}\text { Adj Rsquare } & 0.184512 \\ \text { Root Mean Square Error } & 0.077624\end{array}$

Mean of Response $\quad 5.246617$

Mean of Response

30

Analysis of Variance

Source DF Sum of Squares Mean Square F Ratio Prob $>$ F

$\begin{array}{llllll} & & 0.09376561 & 0.010418 & 1.7291 & 0.1474\end{array}$

$\begin{array}{llll}\text { Error } & 20 & 0.12050944 & 0.006025\end{array}$

Means for Oneway Anova

Level Number Mean Std Error Lower 95\% Upper 95\%

$\begin{array}{rrrrrr}1-1 & 3 & 5.18131 & 0.04482 & 5.0878 & 5.2748\end{array}$

$\begin{array}{llllll}1-2 & 3 & 5.21002 & 0.04482 & 5.1165 & 5.3035 \\ 2-1 & 3 & 5.26743 & 0.04482 & 5.1739 & 5.3609\end{array}$

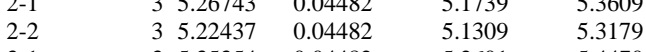

$\begin{array}{llllll}3-1 & 3 & 5.35354 & 0.04482 & 5.2601 & 5.4470 \\ 3-2 & 3 & 5.34637 & 0.04482 & 5.2529 & 5.4399\end{array}$

$\begin{array}{llllll}3-2 & 3 & 5.34637 & 0.04482 & 5.2529 & 5.4399 \\ 4-1 & 3 & 5.21719 & 0.04482 & 5.1237 & 5.3107\end{array}$

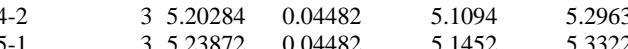

$\begin{array}{llllll}5-1 & 3 & 5.23872 & 0.04482 & 5.1452 & 5.3322 \\ 5-2 & 3 & 5.22437 & 0.04482 & 5.1309 & 5.3179\end{array}$

Std Error uses a pooled estimate of error variance 
Exhibit A-5. Measurements by Block and Sub-Block for Samples of the Sb7ref Glass and the Ustd Standard Glass by Oxide by Prep

Oneway Analysis of Measured By Blk/Sub-Blk Glass ID=SB7Ref, Oxide=Li2O (wt\%), Prep Method=PF, Target $=5.2610$

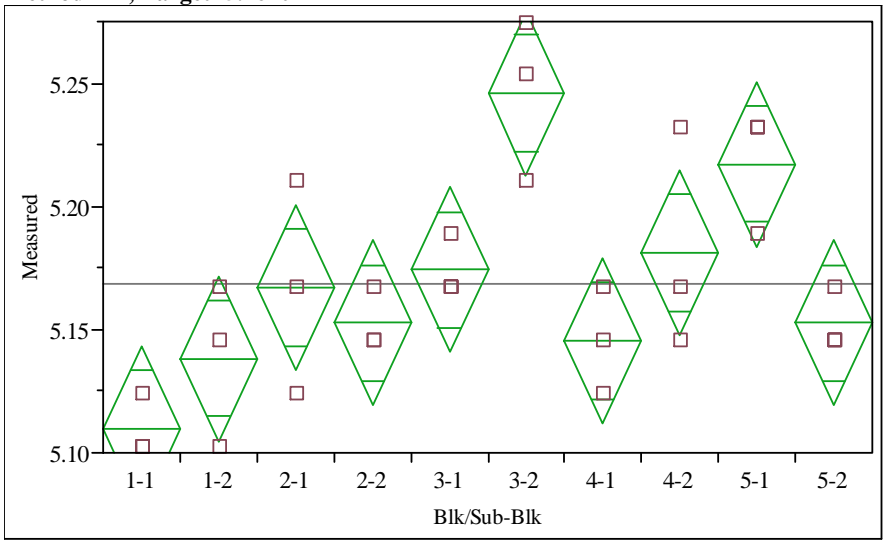

Oneway Anova

Summary of Fit

Adj Rsquare

0.730893

0.609795

Mean of Response

0.027794

Observations (or Sum Wgts)

30

Analysis of Variance

Source DF Sum of Squares Mean Square F Ratio Prob $>$ F

$\begin{array}{llllll} & 0.04196200 & 0.004662 & 6.0356 & 0.0004\end{array}$

Error $20-0.01544993$

\section{Means for Oneway Anova}

Level Number Mean Std Error Lower 95\% Upper 95\%

$1-1$ - $35.10955-0.01605$

$\begin{array}{llllll}1-2 & 3 & 5.13825 & 0.01605 & 5.1048 & 5.1430 \\ 2 & 3 & 5.16696 & 0.0165 & 5.1335 & 5.1717\end{array}$

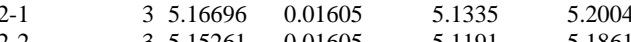

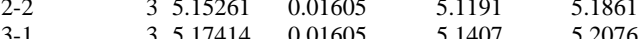

$\begin{array}{llllll}3-1 & 3 & 5.17414 & 0.01605 & 5.1407 & 5.2076 \\ 3-2 & 3 & 5.24590 & 0.01605 & 5.2124 & 5.2794\end{array}$

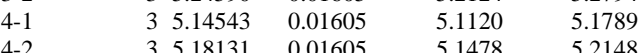

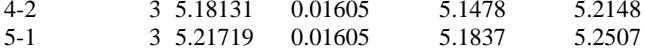

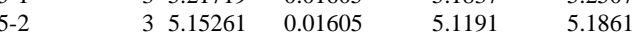

Std Error uses a pooled estimate of error variance
Oneway Analysis of Measured By Blk/Sub-Blk Glass ID=SB7Ref, Oxide=MgO (wt\%), Prep Method $=$ AR, Target $=0.0270$

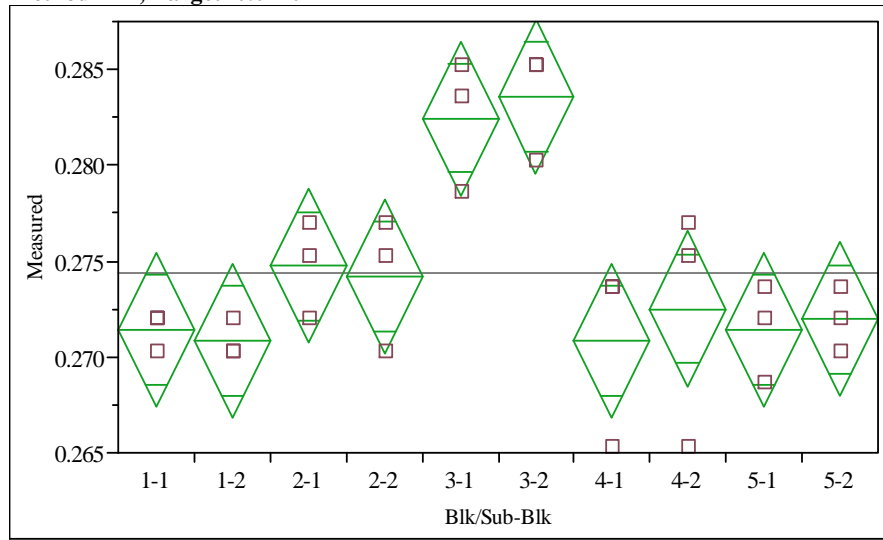

Oneway Anova

Summary of Fit

Rsquare

0.730208

Adj Rsquare $\quad 0.608801$

Root Mean Square Error $\quad 0.003344$

Mean of Response

0.274393

Analysis of Variance

Source DF Sum of Squares Mean Square F Ratio Prob $>$ F

$\begin{array}{llll}0.00060536 & 0.000067 & 6.0146 & 0.0004\end{array}$

$\begin{array}{llll}\text { Error } & 20 & 0.00022366 & 0.000011\end{array}$

\section{Means for Oneway Anova}

Level Number Mean Std Error Lower 95\% Upper 95\%

$\begin{array}{llllll}1-1 & 3 & 0.271408 & 0.00193 & 0.26738 & 0.27544 \\ 1-2 & 3 & 0.270856 & 0.00193 & 0.26683 & 0.27488\end{array}$

$\begin{array}{llllll}1-2 & 3 & 0.270856 & 0.00193 & 0.26683 & 0.27488 \\ 2-1 & 3 & 0.274725 & 0.00193 & 0.27070 & 0.27875\end{array}$

$\begin{array}{llllll}2-2 & 3 & 0.274172 & 0.00193 & 0.27014 & 0.27820 \\ 3-1 & 3 & 0.282464 & 0.00193 & 0.27844 & 0.28649\end{array}$

$\begin{array}{llllll}3-1 & 3 & 0.282464 & 0.00193 & 0.27844 & 0.28649 \\ 3-2 & 3 & 0.283569 & 0.00193 & 0.27954 & 0.28760\end{array}$

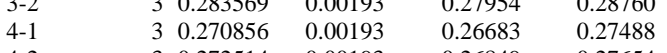

$\begin{array}{llllll}4-2 & 3 & 0.272514 & 0.00193 & 0.26849 & 0.27654 \\ 5-1 & 3 & 0.271408 & 0.00193 & 0.26738 & 0.27544\end{array}$

$\begin{array}{lllll}3 & 0.27148 & 0.0193 & 0.26849 & 0.27654 \\ 3 & 0.271961 & 0.00193 & 0.26738 & 0.27544\end{array}$

Std Error uses a pooled estimate of error variance 
Exhibit A-5. Measurements by Block and Sub-Block for Samples of the Sb7ref Glass and the Ustd Standard Glass by Oxide by Prep

Oneway Analysis of Measured By Blk/Sub-Blk Glass ID=SB7Ref, Oxide=MgO (wt\%), Prep Method $=$ PF, Target $=\mathbf{0 . 0 2 7 0}$

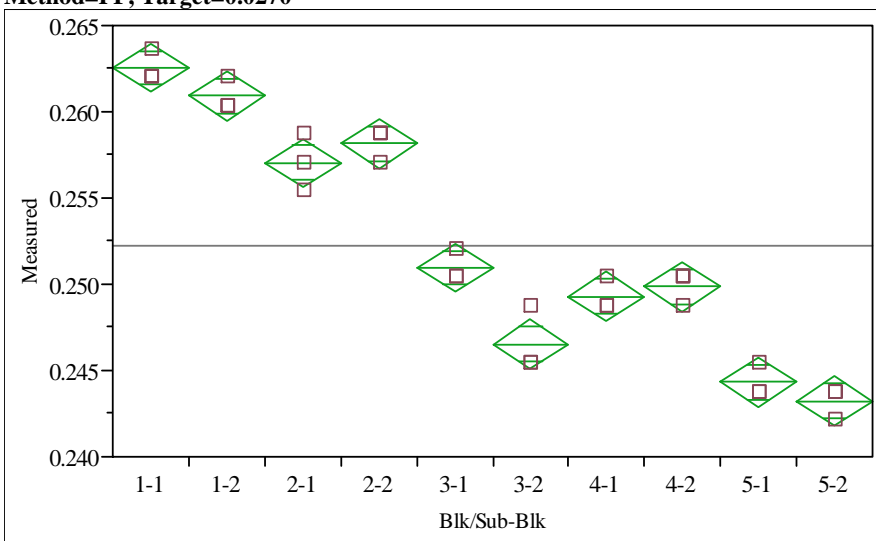

Oneway Anova

Summary of Fit

Rsquare

Adj Rsquare $\quad 0.97923$

Root Mean Square Error $\quad 0.001173$

Mean of Response

0.252283

Observations (or Sum Wgts) 30

Analysis of Variance

Source DF Sum of Squares Mean Square F Ratio Prob > F

$\begin{array}{llllll} & & 0.00129651 & 0.000144 & 104.7704 & <.0001\end{array}$

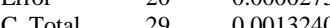

Means for Oneway Anova

Level Number Mean Std Error Lower 95\% Upper 95\%

$\begin{array}{lllllr}1-1 & 3 & 0.262564 & 0.00068 & 0.26115 & 0.26398\end{array}$

$\begin{array}{llllll}1-2 & 3 & 0.260906 & 0.00068 & 0.25949 & 0.26232\end{array}$

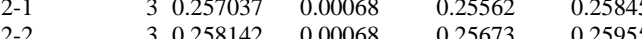

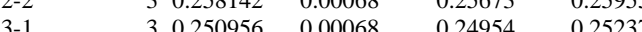

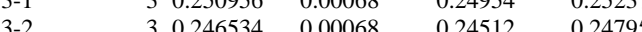

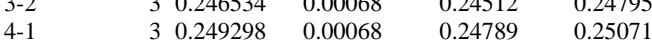

$\begin{array}{llllll}4-2 & 3 & 0.249851 & 0.00068 & 0.24844 & 0.2512\end{array}$

$\begin{array}{llllll}5-1 & 3 & 0.244323 & 0.00068 & 0.24291 & 0.24574\end{array}$

Std Error uses a pooled estimate of error variance
Oneway Analysis of Measured By Blk/Sub-Blk Glass ID=SB7Ref, Oxide=MnO (wt\%), Prep Method=AR, Target $=2.0740$

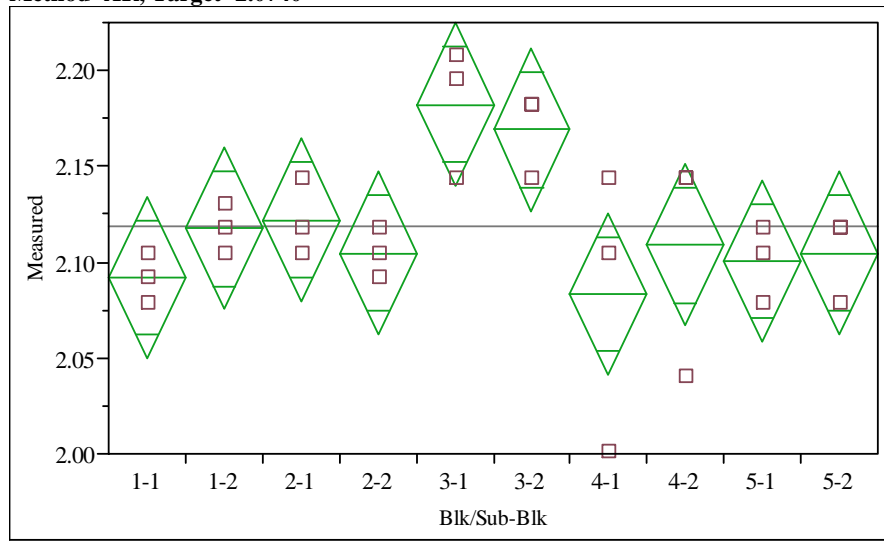

Oneway Anova

Summary of Fi

Rsquare

$\begin{array}{ll}\text { Adj Rsquare } & 0.3321833 \\ \text { Root Mean Square Error } & 0.035203\end{array}$

Root Mean Square Error $\quad 0.035203$

Mean of Response

2.118429

Analysis of Variance

Source DF Sum of Squares Mean Square F Ratio Prob $>$ F

$0.02820898-0.003134 \quad 25291-0.0403$

$\begin{array}{lll}\text { Error } & 20 & 0.02478567 \\ \text { C. Total } & 29 & 0.05299465\end{array}$

Means for Oneway Anova

Level Number Mean Std Error Lower 95\% Upper 95\%

$\begin{array}{llllrr}1-1 & 3 & 2.09174 & 0.02032 & 2.0493 & 2.1341 \\ 1-2 & & 3 & 2.11757 & 0.02032 & 2.0752\end{array}$

$\begin{array}{llllll}1-2 & 3 & 2.11757 & 0.02032 & 2.0752 & 2.1600 \\ 2-1 & 3 & 2.12187 & 0.02032 & 2.0795 & 2.1643\end{array}$

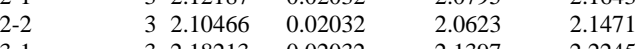

$\begin{array}{llllll}3-1 & 3 & 2.18213 & 0.02032 & 2.1397 & 2.2245 \\ 3-2 & 3 & 2.16922 & 0.02032 & 2.1268 & 2.2116\end{array}$

\begin{tabular}{llllll}
$3-2$ & 3 & 2.16922 & 0.02032 & 2.1268 & 2.2116 \\
$4-1$ & 3 & 2.08314 & 0.02032 & 2.0407 & 2.1255 \\
\hline-2 & 3 & 2.10896 & 0.02032 & 2.0666 & 2.1514
\end{tabular}

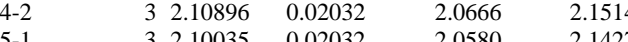

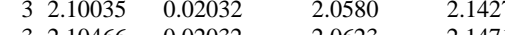

Std Error uses a pooled estimate of error variance 
Exhibit A-5. Measurements by Block and Sub-Block for Samples of the Sb7ref Glass and the Ustd Standard Glass by Oxide by Prep

Oneway Analysis of Measured By Blk/Sub-Blk Glass ID=SB7Ref, Oxide=MnO (wt\%), Prep Method $=$ PF, Target $=2.0740$

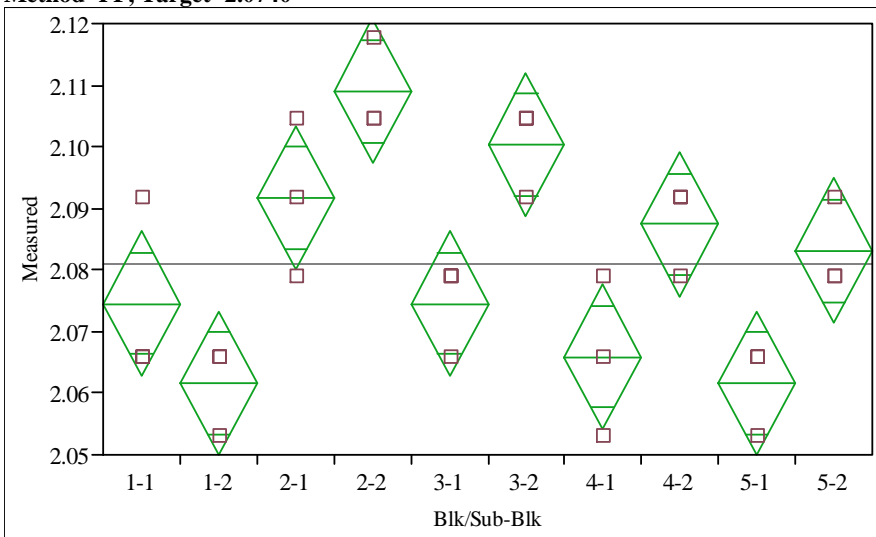

Oneway Anova

Summary of Fit

0.790769

Root Mean Square Error -0.696615

Mean of Response

0.00972

Observations (or Sum Wgts) $\quad 30$

Analysis of Variance

Source DF Sum of Squares Mean Square F Ratio Prob $>$ F

$\begin{array}{llllll}\text { Blk/Sub-Blk } & 9 & 0.00714116 & 0.000793 & 8.3987 & <.0001\end{array}$

Error 200.00188949

\section{Means for Oneway Anova}

Level Number Mean Std Error Lower 95\% Upper 95\%

$\begin{array}{lllrrr}1-1 & 3 & 2.07453 & 0.00561 & 2.0628 & 2.0862\end{array}$

$\begin{array}{llllll}1-2 & 3 & 2.06162 & 0.00561 & 2.0499 & 2.0733 \\ 2-1 & 3 & 2.09174 & 0.00561 & 2.0800 & 2.1034\end{array}$

$\begin{array}{llllll}2-1 & 3 & 2.09174 & 0.00561 & 2.0800 & 2.1034 \\ 2-2 & 3 & 2.10896 & 0.00561 & 2.0973 & 2.1207\end{array}$

$\begin{array}{llllll}2-2 & 3 & 2.10896 & 0.00561 & 2.0973 & 2.1207 \\ 3-1 & 3 & 2.07453 & 0.00561 & 2.0628 & 2.0862\end{array}$

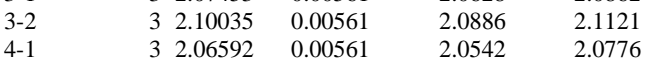

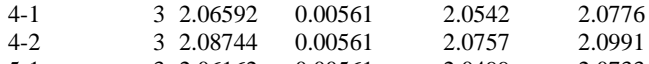

$\begin{array}{llllll}4-2 & 3 & 2.08744 & 0.00561 & 2.0757 & 2.0991 \\ 5-1 & 3 & 2.06162 & 0.00561 & 2.0499 & 2.0733\end{array}$

Std Error uses a pooled estimate of error variance
Oneway Analysis of Measured By Blk/Sub-Blk Glass ID=SB7Ref, Oxide=Na2O (wt\%), Prep Method=AR, Target $=14.6820$

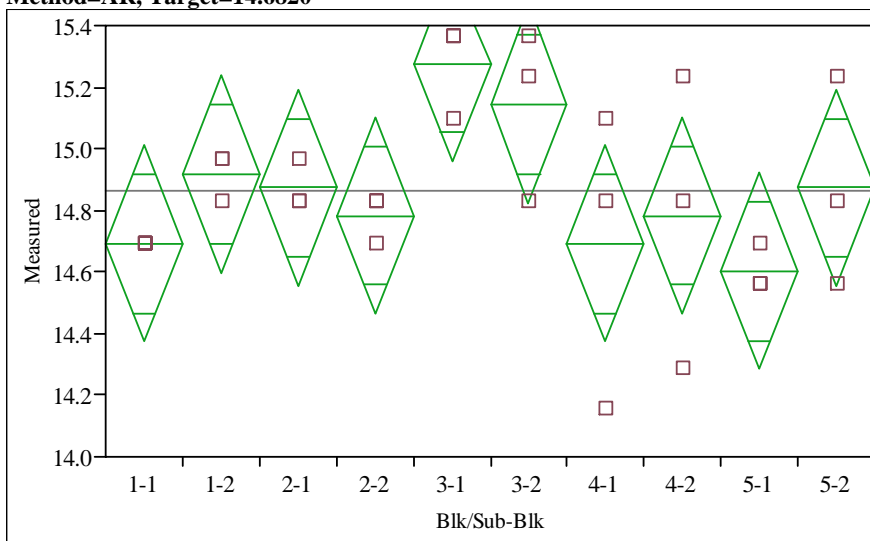

Oneway Anova

Summary of Fit

Rsquare

0.454887

Adj Rsquare $\quad 0.209586$

Root Mean Square Error $\quad 0.265069$

Mean of Response 14.8639

Analysis of Variance

Source DF Sum of Squares Mean Square F Ratio Prob > F

$\begin{array}{lrrrrr}\text { Blk/Sublk } & 9 & 1.1726378 & 0.130293 & 1.8544 & 0.1199\end{array}$

$\begin{array}{llll}\text { Error } & 20 & 1.4052271 & 0.070261\end{array}$

Means for Oneway Anova

Level Number Mean Std Error Lower 95\% Upper 95\%

$\begin{array}{llrrrr}1-1 & 3 & 14.6932 & 0.15304 & 14.374 & 15.012\end{array}$

$\begin{array}{llllll}1-2 & 3 & 14.9179 & 0.15304 & 14.599 & 15.237 \\ 2-1 & 3 & 14.8729 & 0.15304 & 14.554 & 15.192\end{array}$

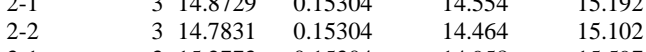

$\begin{array}{llllll}3-1 & 3 & 15.2773 & 0.15304 & 14.958 & 15.597 \\ 3-2 & 3 & 15.1425 & 0.15304 & 14.823 & 15.462\end{array}$

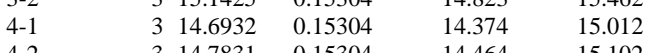

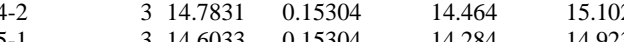

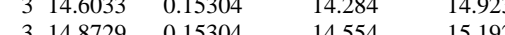

Std Error uses a pooled estimate of error variance 
Exhibit A-5. Measurements by Block and Sub-Block for Samples of the Sb7ref Glass and the Ustd Standard Glass by Oxide by Prep

Oneway Analysis of Measured By Blk/Sub-Blk Glass ID=SB7Ref, Oxide=NiO (wt\%), Prep Method=AR, Target $=\mathbf{1 . 2 2 3 0}$

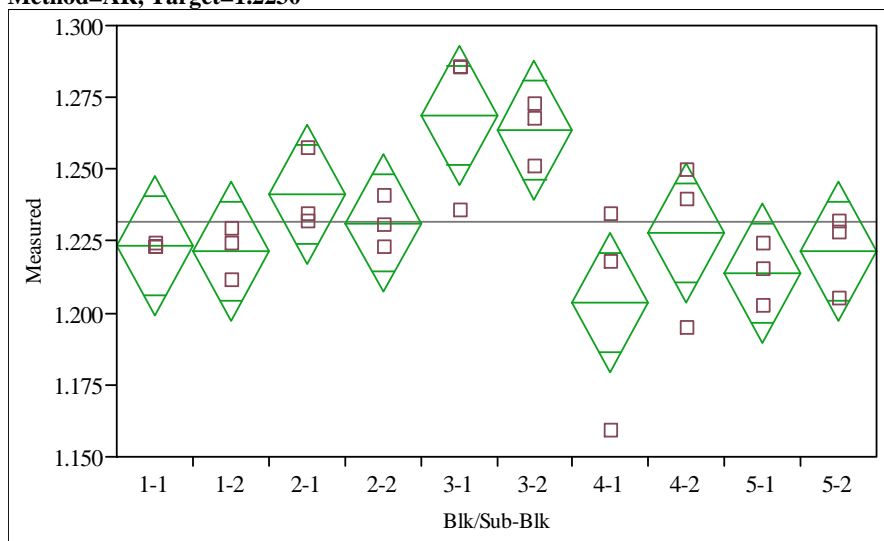

Oneway Anova

Summary of Fit

Adj Rsquare

0.588754

A 0.403694

Mean of Response

1.231695

Analysis of Variance

Source DF Sum of Squares Mean Square F Ratio Prob $>$ F

$\begin{array}{lrrrrr}\text { Blk/Sub-Blk } & 9 & 0.01156775 & 0.001285 & 3.1814 & 0.0149\end{array}$

Error $\quad 20 \quad 0.00808009$

0.000404

$\begin{array}{lll}\text { C. Total } & 29 & 0.01964784\end{array}$

Means for Oneway Anova

Level Number Mean Std Error Lower 95\% Upper 95\%

$\begin{array}{lllrrr}1-1 & 3 & 1.22330 & 0.01160 & 1.1991 & 1.2475\end{array}$

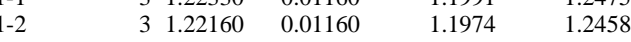

$\begin{array}{llllll}2-1 & 3 & 1.24111 & 0.01160 & 1.2169 & 1.2653\end{array}$

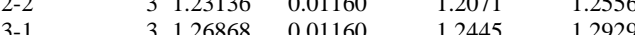

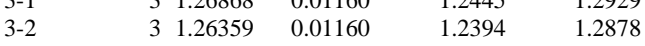

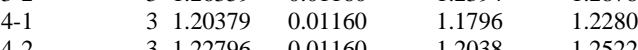

$\begin{array}{llllll}4-2 & 3 & 1.22796 & 0.01160 & 1.2038 & 1.2522 \\ 5-1 & 3 & 1.21397 & 0.01160 & 1.1898 & 1.2382\end{array}$

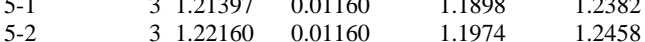

Std Error uses a pooled estimate of error variance
Oneway Analysis of Measured By Blk/Sub-Blk Glass ID=SB7Ref, Oxide=NiO (wt\%), Prep Method=PF, Target $=1.2230$

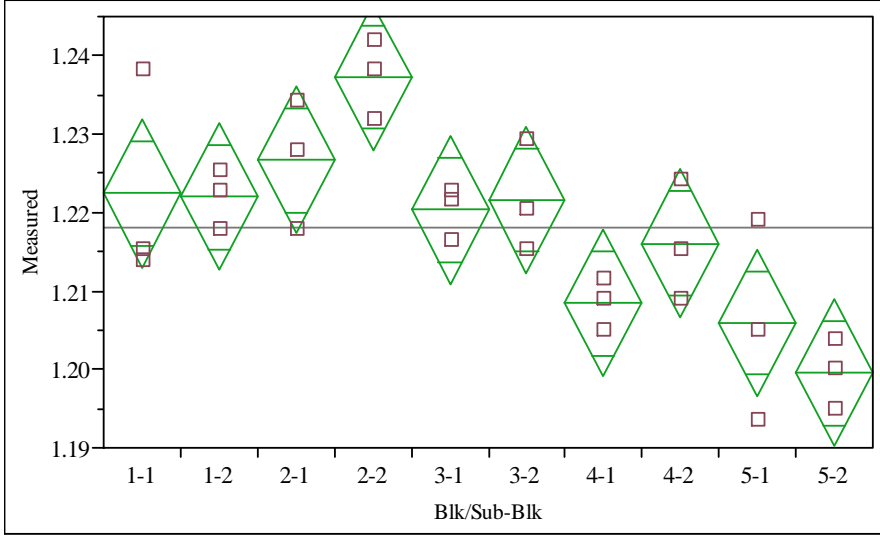

Oneway Anova

Summary of Fit

Rsquare

0.727374

Adj Rsquare $\quad 0.604692$

Root Mean Square Error $\quad 0.007806$

Mean of Response 1.218037

Analysis of Variance

Source DF Sum of Squares Mean Square F Ratio Prob $>$ F

$\begin{array}{lllllll}\text { Bll/S } & & 0.00325168 & 0.000361 & 5.9289 & 0.0005\end{array}$

$\begin{array}{llll}\text { Error } & 20 & 0.00121876 & 0.000061\end{array}$

Means for Oneway Anova

Level Number Mean Std Error Lower 95\% Upper 95\%

$\begin{array}{lrrrrr}1-1 & 3 & 1.22245 & 0.00451 & 1.2130 & 1.2318\end{array}$

$\begin{array}{llllll}1-2 & 3 & 1.22202 & 0.00451 & 1.2126 & 1.2314 \\ 2-1 & 3 & 1.22669 & 0.00451 & 1.2173 & 1.2361\end{array}$

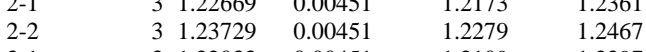

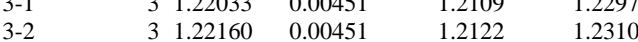

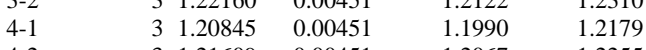

$\begin{array}{llllll}4-2 & 3 & 1.21609 & 0.00451 & 1.2067 & 1.2255 \\ 5-1 & 3 & 1.20591 & 0.00451 & 1.1965 & 1.2153\end{array}$

$\begin{array}{llll}3 & 1.20954 & 0.00451- & 1.1901\end{array}$

Std Error uses a pooled estimate of error variance 
Exhibit A-5. Measurements by Block and Sub-Block for Samples of the Sb7ref Glass and the Ustd Standard Glass by Oxide by Prep

Oneway Analysis of Measured By Blk/Sub-Blk Glass ID=SB7Ref, Oxide=PbO (wt\%), Prep Method=AR, Target $=0.0060$

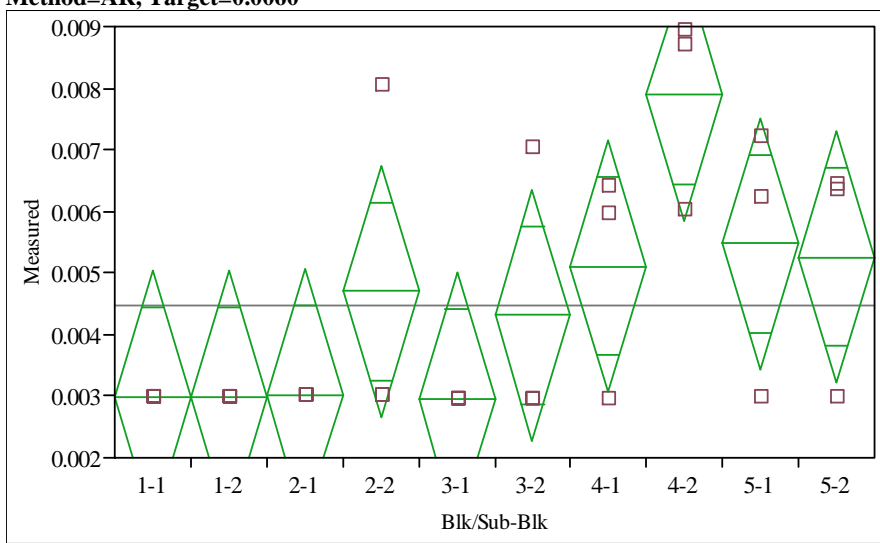

Oneway Anova

Summary of Fit

Adj Rsquare

0.539643

Root Mean Square Erro

0.332482

Mean of Response

0.004473

Analysis of Variance

ource DF Sum of Squares Mean Square F Ratio Prob $>$ F

$\begin{array}{lrrr}0.00006772 & 7.5247 \mathrm{e}-6 & 2.6049 & 0.0358\end{array}$

Error $\quad 20-0.00005777$

$2.8886 \mathrm{e}-6$

Means for Oneway Anova

Level Number Mean Std Error Lower 95\% Upper 95\%

$\begin{array}{llllll}1-1 & 3 & 0.002995 & 0.00098 & 0.00095 & 0.00504\end{array}$

$\begin{array}{lllll}3 & 0.002995 & 0.00098 & 0.00095 & 0.00504\end{array}$

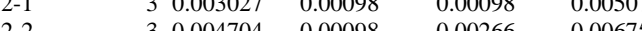

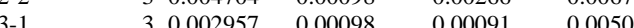

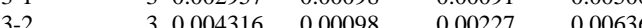

$\begin{array}{llllll}4-1 & 3 & 0.005111 & 0.00098 & 0.00306 & 0.00716\end{array}$

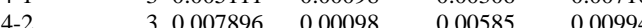

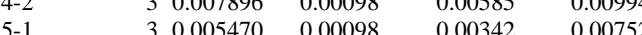

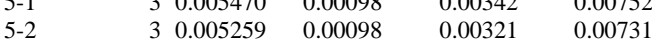

Std Error uses a pooled estimate of error variance
Oneway Analysis of Measured By Blk/Sub-Blk Glass ID=SB7Ref, Oxide=PbO (wt\%), Prep Method $=\mathbf{P F}$, Target $=\mathbf{0 . 0 0 6 0}$

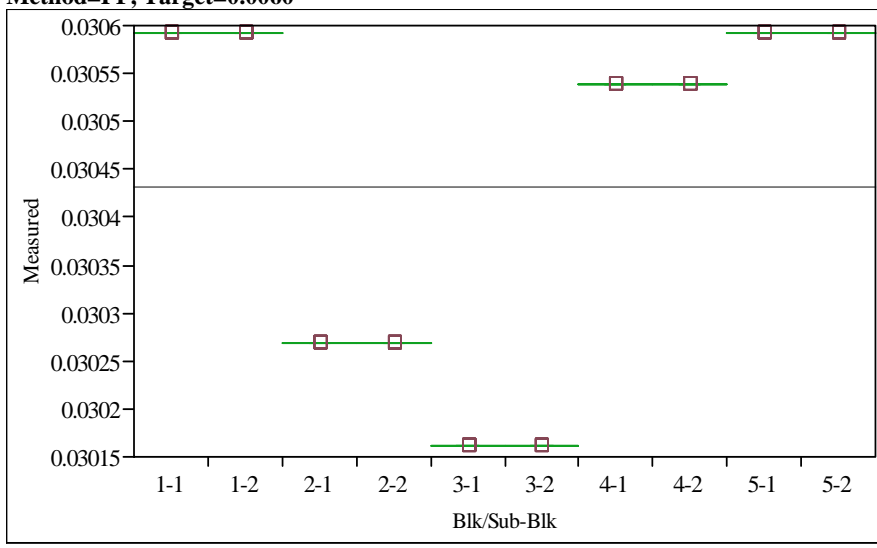

Oneway Anova

Summary of Fit

Rsquare

Adj Rsquare

Root Mean Square E

Analysis of Variance

Source DF Sum of Squares Mean Square F Ratio Prob $>$ F

9.74702e-7 $\quad 1.083 \mathrm{e}-7$

\begin{tabular}{llll} 
Error & 20 & $1.3553 \mathrm{e}-20$ & $6.776 \mathrm{e}-22$ \\
\hline
\end{tabular}

\section{Means for Oneway Anova}

Level Number Mean Std Error Lower 95\% Upper 95\%

$\begin{array}{llllll}1-1 & 3 & 0.030592 & 1.503 \mathrm{e}-11 & 0.03059 & 0.03059 \\ 1-2 & 3 & 0.030592 & 1.503 \mathrm{e}-11 & 0.03059 & 0.03059\end{array}$

$\begin{array}{llllll}1-2 & 3 & 0.030592 & 1.503 \mathrm{e}-11 & 0.03059 & 0.03059 \\ 2-1 & 3 & 0.030269 & 1.503 \mathrm{e}-11 & 0.03027 & 0.03027\end{array}$

$\begin{array}{llllll}2-2 & 3 & 0.030269 & 1.503 \mathrm{e}-11 & 0.03027 & 0.03027\end{array}$

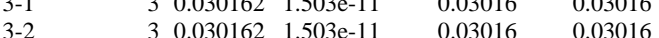

$\begin{array}{llllll}3-2 & 3 & 0.030152 & 1.503 \mathrm{e}-11 & 0.03016 & 0.03016 \\ 4-1 & 3 & 0.030539 & 1.503 \mathrm{e}-11 & 0.03054 & 0.03054\end{array}$

$\begin{array}{llllll}4-2 & 3 & 0.030539 & 1.503 \mathrm{e}-11 & 0.03054 & 0.03054\end{array}$

30.030592 1.503e-11

Std Error uses a pooled estimate of error variance 
Exhibit A-5. Measurements by Block and Sub-Block for Samples of the Sb7ref Glass and the Ustd Standard Glass by Oxide by Prep

Oneway Analysis of Measured By Blk/Sub-Blk Glass ID=SB7Ref, Oxide=SiO2 (wt\%), Prep Method $=$ PF, Target $=51.5360$

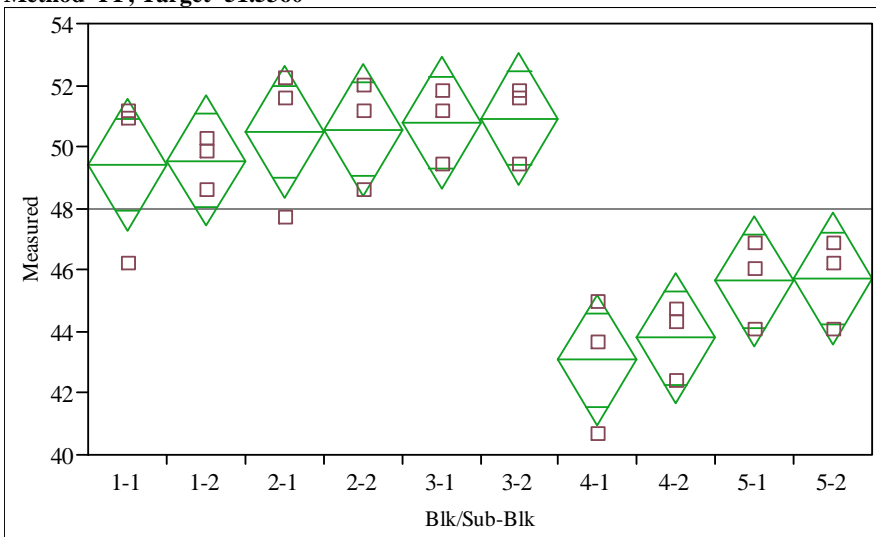

Oneway Anova

Summary of Fit

Adj Rsquare

0.805107

0.717406

Mean of Response

1.769724

Observations (or Sum Wgts)

30

Analysis of Variance

Source DF Sum of Squares Mean Square F Ratio Prob $>$ F

$\begin{array}{llrrrr}\text { Blk/Sub-Blk } & 258.76122 & 28.7512 & 9.1801 & <.0001\end{array}$

$\begin{array}{llr}\text { Error } & 20 & 62.63846 \\ \text { C. Total } & 29 & 321.39968\end{array}$

Means for Oneway Anova

Level Number Mean Std Error Lower 95\% Upper 95\%

$\begin{array}{llrrrr}1-1 & 3 & 49.4178 & 1.0218 & 47.286 & 51.549\end{array}$

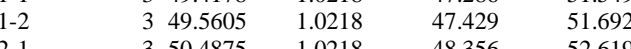

$\begin{array}{llllll}2-1 & 3 & 50.4875 & 1.0218 & 48.356 & 52.619 \\ 2-2 & 3 & 50.5588 & 1.0218 & 48.427 & 52.690\end{array}$

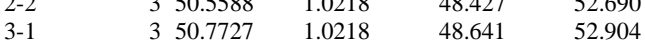

$\begin{array}{llllll}3-2 & 3 & 50.9153 & 1.0218 & 48.784 & 53.047\end{array}$

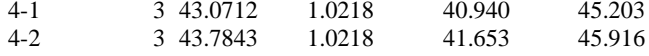

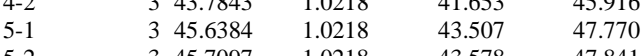

Std Error uses a pooled estimate of error variance
Oneway Analysis of Measured By Blk/Sub-Blk Glass ID=SB7Ref, Oxide=SO4 (wt\%), Prep Method $=$ AR, Target $=0.5860$

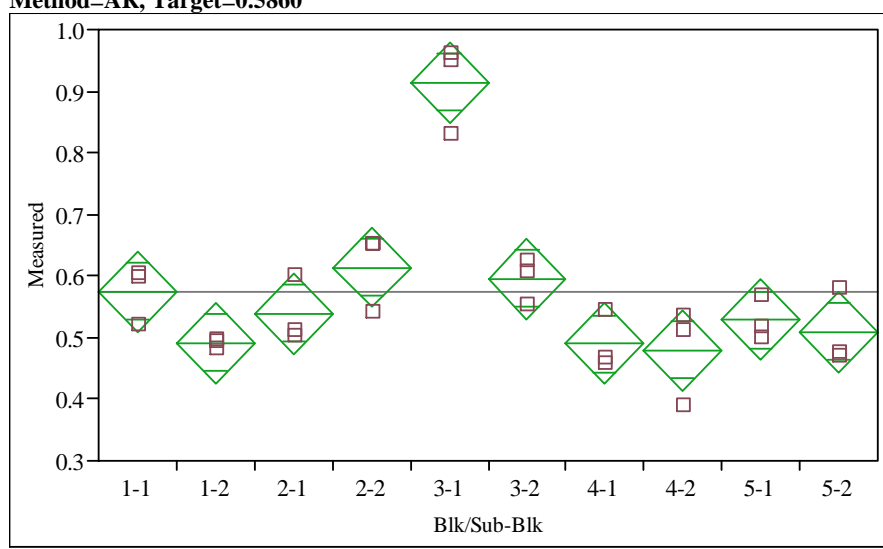

Oneway Anova

Summary of Fi

Rsquare

Adj Rsquare $\quad 0.82576$

Root Mean Square Error $\quad 0.054297$

Mean of Response 0.57381

Analysis of Variance

Source DF Sum of Squares Mean Square F Ratio Prob $>$ F

$\begin{array}{lll}9 & 0.44317138 & 0.049241 \\ & & \end{array}$

$\begin{array}{lll}\text { Error } & 20 & 0.05896250 \\ \text { C. Total } & 29 & 0.50213388\end{array}$

\section{Means for Oneway Anova}

Level Number Mean Std Error Lower 95\% Upper 95\%

$\begin{array}{llllrr}1-1 & 3 & 0.575213 & 0.03135 & 0.50982 & 0.64060 \\ 1-2 & 3 & 0.491328 & 0.03135 & 0.42594 & 0.55672\end{array}$

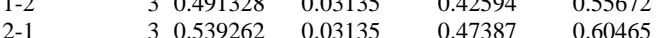

$\begin{array}{llllll}2-2 & 3 & 0.614160 & 0.03135 & 0.54877 & 0.67955\end{array}$

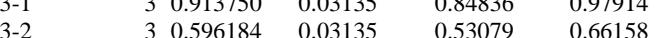

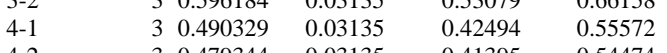

$\begin{array}{llllll}4-2 & 3 & 0.479344 & 0.03135 & 0.41395 & 0.54474 \\ 5-1 & 3 & 0.529276 & 0.03135 & 0.46388 & 0.59467\end{array}$

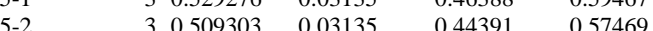

Std Error uses a pooled estimate of error variance 
Exhibit A-5. Measurements by Block and Sub-Block for Samples of the Sb7ref Glass and the Ustd Standard Glass by Oxide by Prep

Oneway Analysis of Measured By Blk/Sub-Blk Glass ID=SB7Ref, Oxide=SO4 (wt\%), Prep Method $=$ PF, Target $=\mathbf{0 . 5 8 6 0}$

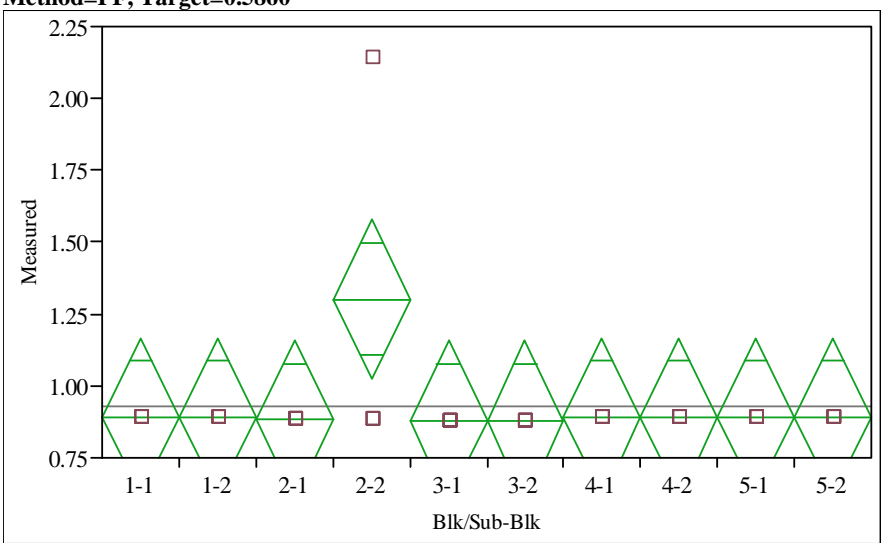

Oneway Anova

Summary of Fit

Adj Rsquare

0.30555

-0.00695
0.22956

Mean of Repronse 0.229456

Observations (or Sum Wgts) $\quad 30$

Analysis of Variance

Source DF Sum of Squares Mean Square F Ratio Prob $>$ F

$\begin{array}{llllll} & & & \\ \text { Blk/Sub-Blk } & 9 & 0.4633072 & 0.051479 & 0.9778 & 0.4866\end{array}$

Error $\quad 20-1.0529974$

0.052650

Means for Oneway Anova

Level Number Mean Std Error Lower 95\% Upper 95\%

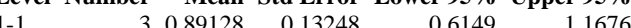

$\begin{array}{llllll}1-2 & 3 & 0.89128 & 0.13248 & 0.6149 & 1.1676\end{array}$

$\begin{array}{llllll}2-1 & 3 & 0.88229 & 0.13248 & 0.6060 & 1.1586 \\ 2-2 & 3 & 1.30122 & 0.13248 & 1.0249 & 1.5776\end{array}$

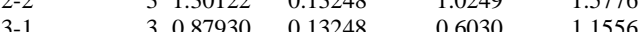

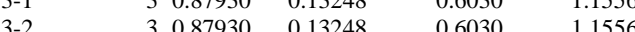

$\begin{array}{llllll}3-2 & 3 & 0.87930 & 0.13248 & 0.6030 & 1.1556 \\ 4-1 & 3 & 0.88978 & 0.13248 & 0.6134 & 1.1661\end{array}$

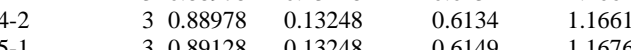

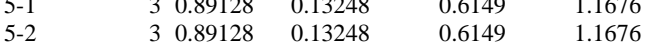

Std Error uses a pooled estimate of error variance
Oneway Analysis of Measured By Blk/Sub-Blk Glass ID=SB7Ref, Oxide=TiO2 (wt\%), Prep Method $=$ AR, Target $=\mathbf{0 . 4 8 6 0}$

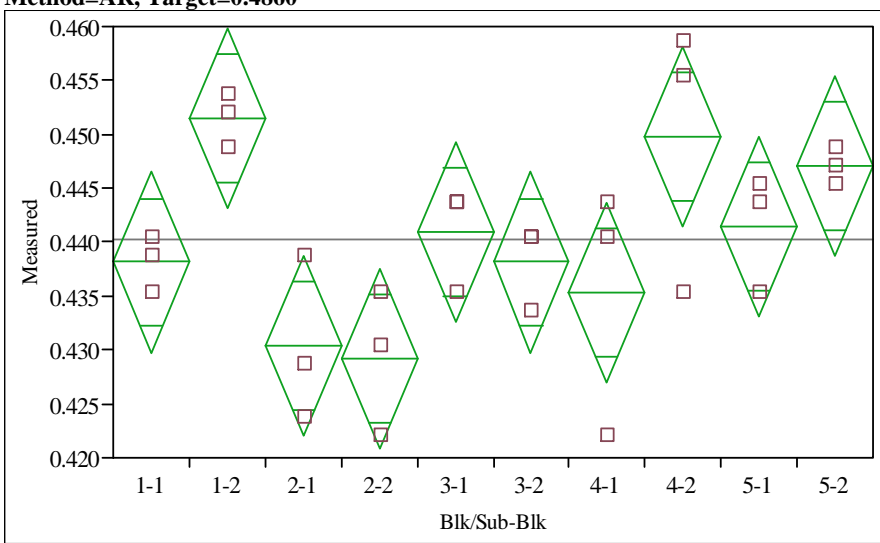

Oneway Anova

Summary of Fit

Rsquare

Adj Rsquare $\quad 0.616416$

Root Mean Square Error $\quad 0.00694$

Mean of Response

0.440185

Analysis of Variance

Source DF Sum of Squares Mean Square F Ratio Prob $>$ F

$\begin{array}{lllll}0.00155257 & 0.000173 & 3.5771 & 0.0084\end{array}$

$\begin{array}{lll}\text { Error } & 20 & 0.00096450\end{array}$

0.00004

\section{Means for Oneway Anova}

Level Number Mean Std Error Lower 95\% Upper 95\%

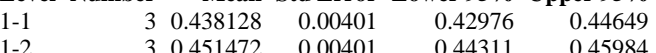

$\begin{array}{llllll}1-2 & 3 & 0.451472 & 0.00401 & 0.44311 & 0.45984 \\ 2-1 & 3 & 0.430344 & 0.00401 & 0.42198 & 0.43871\end{array}$

$\begin{array}{llllll}2-2 & 3 & 0.429232 & 0.00401 & 0.42087 & 0.43760 \\ 3-1 & 3 & 0.440908 & 0.00401 & 0.43254 & 0.44927\end{array}$

$\begin{array}{llllll}3-1 & 3 & 0.440008 & 0.00401 & 0.43254 & 0.44927 \\ 3-2 & 3 & 0.438128 & 0.00401 & 0.42976 & 0.44649\end{array}$

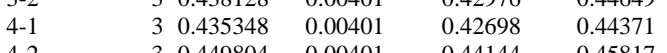

$\begin{array}{llllll}4-2 & 3 & 0.449804 & 0.00401 & 0.44144 & 0.45817 \\ 5-1 & 3 & 0.441464 & 0.00401 & 0.43310 & 0.44983\end{array}$

$\begin{array}{lllll}3 & 0.441464 & 0.00401 & 0.43310 & 0.44983\end{array}$

Std Error uses a pooled estimate of error variance 
Exhibit A-5. Measurements by Block and Sub-Block for Samples of the Sb7ref Glass and the Ustd Standard Glass by Oxide by Prep

Oneway Analysis of Measured By Blk/Sub-Blk Glass ID=SB7Ref, Oxide=TiO2 (wt\%), Prep Method=PF, Target $=0.4860$

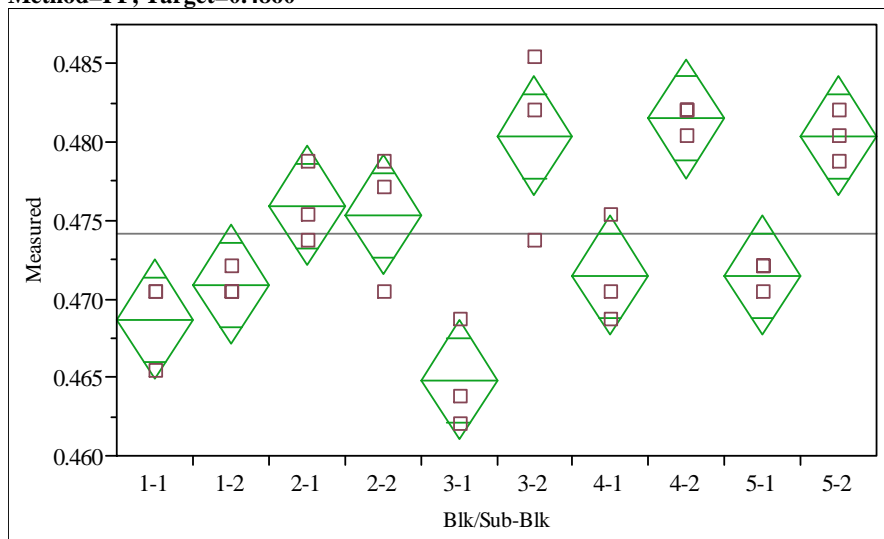

Oneway Anova

Summary of Fit

Adj Rsquare

\subsection{2}

\begin{tabular}{ll}
0.718876 \\
\hline
\end{tabular}

Mean of Response

0.003165

Observations (or Sum Wgts)

30

Analysis of Variance

Source DF Sum of Squares Mean Square F Ratio Prob $>$ F

$\begin{array}{llllll}\text { Blk/Sub-Blk } & 0.00083291 & 0.000093 & 9.2397 & <.0001\end{array}$

$\begin{array}{lll}\text { Crror } & 20 & 0.00020032 \\ \text { Total } & 29 & 0.00103323\end{array}$

\section{Means for Oneway Anova}

Level Number Mean Std Error Lower 95\% Upper 95\%

$\begin{array}{llllll}1-1 & 3 & 0.468708 & 0.00183 & 0.46490 & 0.47252\end{array}$

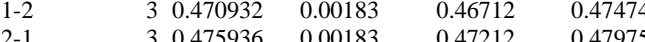

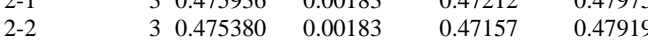

$\begin{array}{llllll}3-1 & 3 & 0.464816 & 0.00183 & 0.46100 & 0.46863\end{array}$

$\begin{array}{llllll}3-2 & 3 & 0.480384 & 0.00183 & 0.47657 & 0.48420\end{array}$

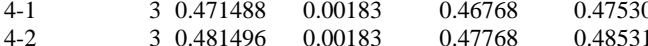

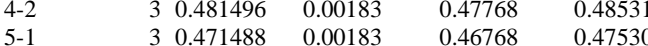

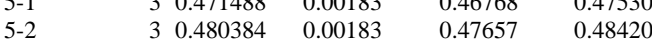

Std Error uses a pooled estimate of error variance
Oneway Analysis of Measured By Blk/Sub-Blk Glass ID=SB7Ref, Oxide=ZnO (wt\%), Prep Method $=$ AR, Target $=0.0100$

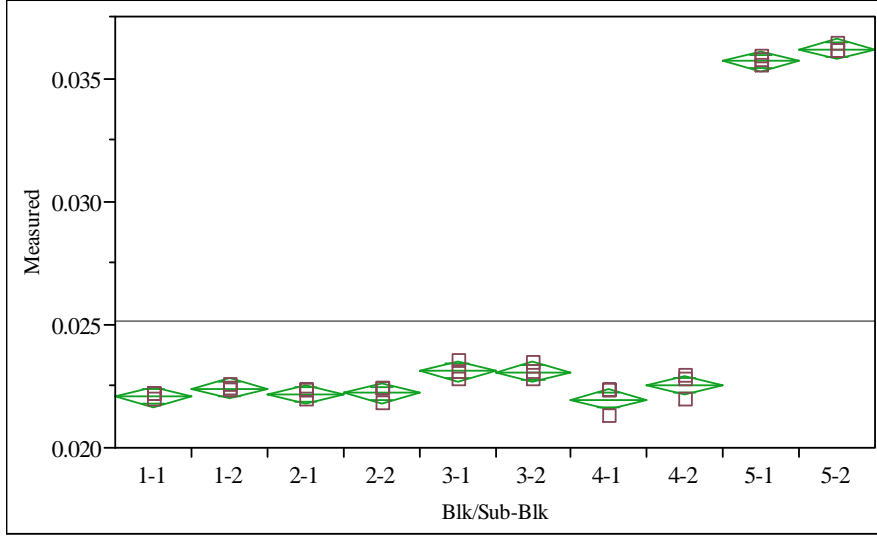

Oneway Anova

Summary of Fi

Rsquare

Adj Rsquare $\quad 0.99626$

Root Mean Square Error $\quad 0.000337$

Mean of Response

0.025137

Analysis of Variance

Source DF Sum of Squares Mean Square F Ratio Prob $>$ F

$0.00087872-0.000098 \quad 859.2303<0001$

$\begin{array}{lll}\text { Error } & 20 & 0.00000227 \\ \text { C. Total } & 29 & 0.00088100\end{array}$

\section{Means for Oneway Anova}

Level Number Mean Std Error Lower 95\% Upper 95\%

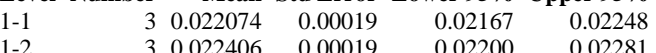

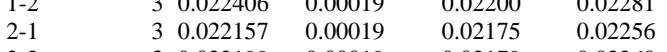

$\begin{array}{llllll}2-2 & 3 & 0.022199 & 0.00019 & 0.02179 & 0.02260 \\ 3-1 & 3 & 0.023112 & 0.00019 & 0.02271 & 0.02352\end{array}$

$\begin{array}{llllll}3-1 & 3 & 0.023112 & 0.00019 & 0.02271 & 0.02352 \\ 3-2 & 3 & 0.023070 & 0.00019 & 0.02266 & 0.02348\end{array}$

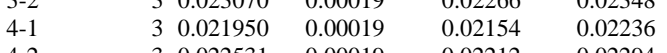

$\begin{array}{llllll}4-2 & 3 & 0.022531 & 0.00019 & 0.02212 & 0.02294 \\ 5-1 & 3 & 0.03584 & 0.00019 & 0.02528 & 0.03609\end{array}$

$\begin{array}{llllll}5-1 & 3 & 0.035684 & 0.00019 & 0.03528 & 0.03609 \\ 5-2 & 3 & 0.036182 & 0.00019 & 0.03578 & 0.03659\end{array}$

Std Error uses a pooled estimate of error variance 
Exhibit A-5. Measurements by Block and Sub-Block for Samples of the Sb7ref Glass and the Ustd Standard Glass by Oxide by Prep

Oneway Analysis of Measured By Blk/Sub-Blk Glass ID=SB7Ref, Oxide=ZnO (wt\%), Prep Method=PF, Target $=0.0100$

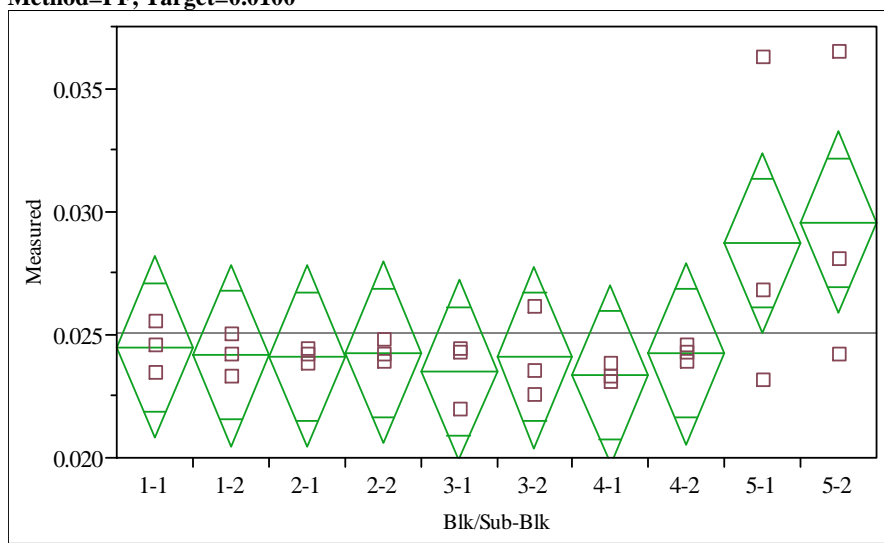

Oneway Anova

Summary of Fit

Adj Rsquare

0.409363

Tevere Error $\quad 0.143576$

Mean of Respons Enor 0.003052

Observations (or Sum Wgts) $\quad 30$

Analysis of Variance

Source DF Sum of Squares Mean Square F Ratio Prob $>$ F

$\begin{array}{llllll}\text { Blk/Sub-Blk } & 0.00012914 & 0.000014 & 1.5402 & 0.2011\end{array}$

Error $20-0.00018633$

Means for Oneway Anova

Level Number Mean Std Error Lower 95\% Upper 95\%

$\begin{array}{llllll}1-1 & 3 & 0.024481 & 0.00176 & 0.02081 & 0.02816\end{array}$

$\begin{array}{lllll}3 & 0.024149 & 0.00176 & 0.02047 & 0.02783\end{array}$

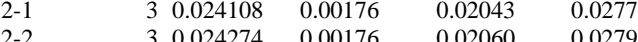

$\begin{array}{llllll}2-2 & 3 & 0.024274 & 0.00176 & 0.02060 & 0.02795 \\ 3-1 & 3 & 0.023527 & 0.00176 & 0.01985 & 0.02720\end{array}$

$\begin{array}{llllll}3-1 & 3 & 0.023527 & 0.00176 & 0.01985 & 0.02720 \\ 3-2 & 3 & 0.024066 & 0.00176 & 0.02039 & 0.02774\end{array}$

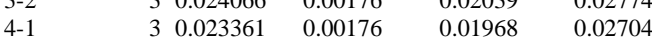

$\begin{array}{llllll}3-2 & 3 & 0.023361 & 0.00176 & 0.02039 & 0.02774 \\ 4-2 & 3 & 0.024232 & 0.00176 & 0.02056 & 0.02704 \\ 5-1 & 3 & 0.02873 & 0.00796\end{array}$

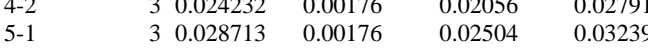

$\begin{array}{llllll}5-2 & 3 & 0.029543 & 0.00176 & 0.02587 & 0.03322\end{array}$

Std Error uses a pooled estimate of error variance
Oneway Analysis of Measured By Blk/Sub-Blk Glass ID=SB7Ref, Oxide=ZrO2 (wt\%), Prep Method $=$ AR, Target $=0.1460$

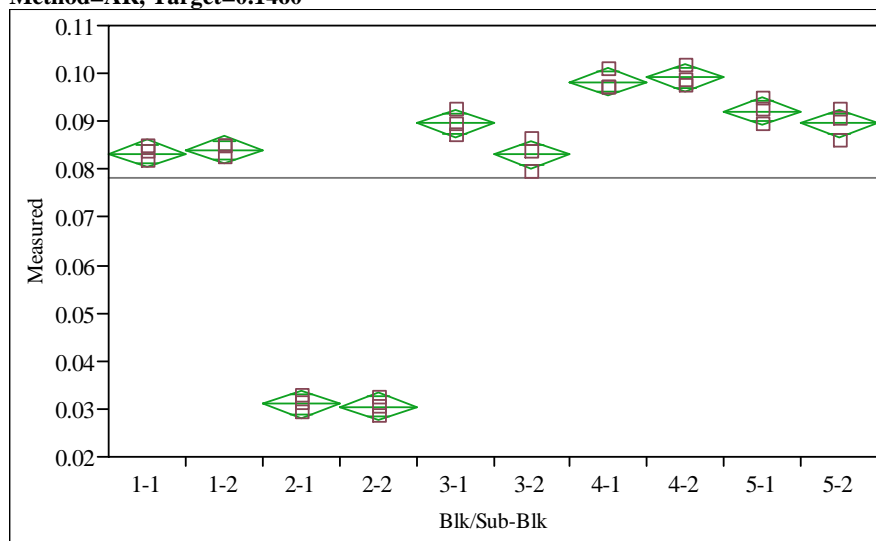

Oneway Anova

Summary of Fit

Rsquare

0.993523

Adj Rsquare $\quad 0.990609$

Root Mean Square Error $\quad 0.002399$

Mean of Response

0.078031

Analysis of Variance

Source DF Sum of Squares Mean Square F Ratio Prob $>$ F

$0.01765293-0.001961340 .8964<.0001$

$\begin{array}{lll}\text { Error } & 20 & 0.00011508 \\ \text { C. Total } & 29 & 0.01776801\end{array}$

\section{Means for Oneway Anova}

Level Number Mean Std Error Lower 95\% Upper 95\%

$\begin{array}{llllll}1-1 & 3 & 0.083254 & 0.00138 & 0.08037 & 0.08614 \\ 1-2 & 3 & 0.084020 & 0.00138 & 0.08113 & 0.08691\end{array}$

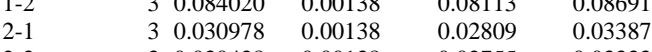

$\begin{array}{llllll}2-2 & 3 & 0.030438 & 0.00138 & 0.02755 & 0.03333 \\ 3-1 & 3 & 0.089558 & 0.00138 & 0.08667 & 0.09245\end{array}$

$\begin{array}{llllll}3-1 & 3 & 0.089558 & 0.00138 & 0.08667 & 0.09245 \\ 3-2 & 3 & 0.083074 & 0.00138 & 0.08019 & 0.08596\end{array}$

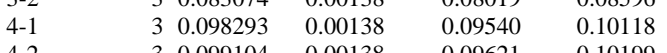

$\begin{array}{llllll}4-2 & 3 & 0.099104 & 0.00138 & 0.09621 & 0.10199 \\ 5-1 & 3 & 0.092080 & 0.00138 & 0.08919 & 0.09497\end{array}$

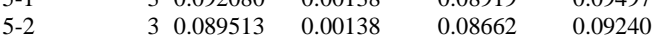

Std Error uses a pooled estimate of error variance 
Exhibit A-5. Measurements by Block and Sub-Block for Samples of the Sb7ref Glass and the Ustd Standard Glass by Oxide by Prep

Oneway Analysis of Measured By Blk/Sub-Blk Glass ID=Ustd, Oxide=Al2O3 (wt\%), Prep Method $=$ AR, Target $=4.1000$

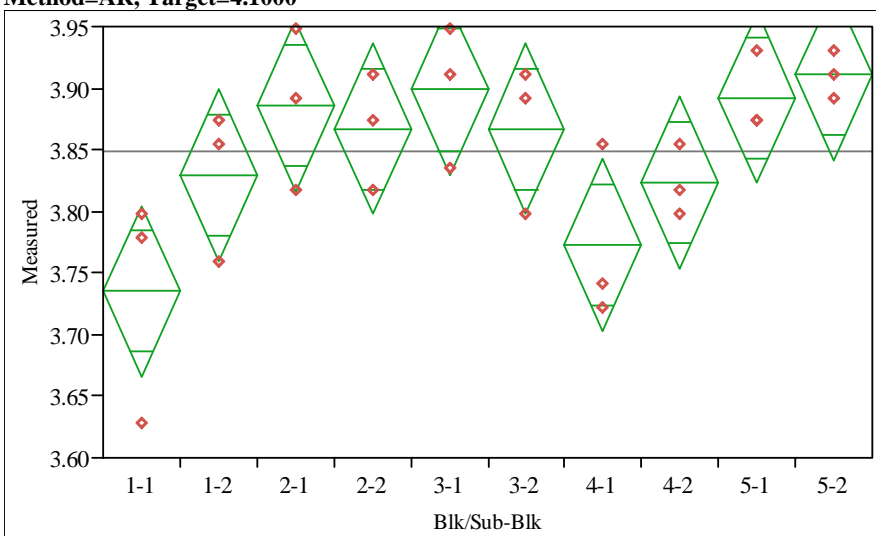

Oneway Anova

Summary of Fit

0.574887

Root Mean Square Enor 0.383585

Mean of Response 3.848282

Observations (or Sum Wgts) $\quad 30$

Analysis of Variance

Source DF Sum of Squares Mean Square F Ratio Prob $>$ F

$\begin{array}{llrrrr}\text { Blk/Sub-Blk } & 9 & 0.09044533 & 0.010049 & 3.0051 & 0.0194\end{array}$

\begin{tabular}{lll} 
Error $\quad 20$ & 0.06688194 \\
\hline
\end{tabular}

0.003344

C. Total $29 \quad 0.15732727$

Means for Oneway Anova

Level Number Mean Std Error Lower 95\% Upper 95\%

$\begin{array}{rrrrrr}1-1 & 3 & 3.73491 & 0.03339 & 3.6653 & 3.8046\end{array}$

$\begin{array}{llll}1-2 & 3 & 3.82939 & 0.03339 \\ 2-1 & 3 & 3.8807 & 0.03339\end{array}$

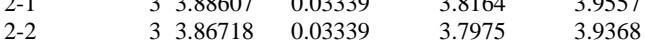

$\begin{array}{llllll}3-1 & 3 & 3.89867 & 0.03339 & 3.8290 & 3.9683\end{array}$

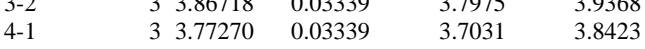

$\begin{array}{llllll}4-2 & 3 & 3.82309 & 0.03339 & 3.7534 & 3.8927\end{array}$

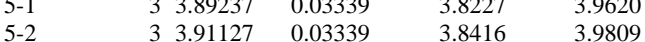

Std Error uses a pooled estimate of error variance
Oneway Analysis of Measured By Blk/Sub-Blk Glass ID=Ustd, Oxide=Al2O3 (wt\%), Prep Method=PF, Target $=4.1000$

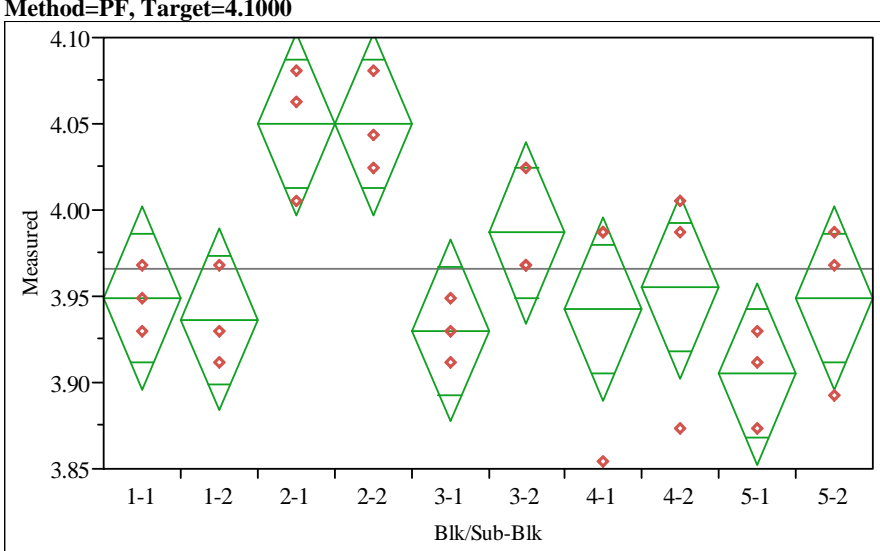

Oneway Anova

Summary of Fit

Rsquare

Adj Rsquare $\quad 0.459005$

Root Mean Square Error $\quad 0.04390$

Mean of Response

3.965431

Analysis of Variance

Source DF Sum of Squares Mean Square F Ratio Prob $>$ F

$\begin{array}{llll}0.06478742 & 0.007199 & 3.7339 & 0.0068\end{array}$

$\begin{array}{lll}\text { Error } & 20 & 0.03855827\end{array}$

0.001928

Means for Oneway Anova

Level Number Mean Std Error Lower 95\% Upper 95\%

$\begin{array}{llrrrr}1-1 & 3 & 3.94906 & 0.02535 & 3.8962 & 4.0019 \\ 1-2 & 3 & 3.93646 & 0.02535 & 3.8836 & 3.9893\end{array}$

$\begin{array}{llllll}1-2 & 3 & 3.93646 & 0.02535 & 3.8836 & 3.9893 \\ 2-1 & 3 & 4.04983 & 0.02535 & 3.9969 & 4.1027\end{array}$

$\begin{array}{llllll}2-2 & 3 & 4.04983 & 0.02535 & 3.9969 & 4.1027 \\ 3-1 & 3 & 3.93016 & 0.02535 & 3.8773 & 3.9830\end{array}$

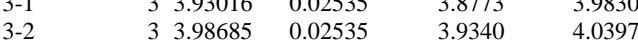

$\begin{array}{llllll}3-2 & 3 & 3.98685 & 0.02535 & 3.9340 & 4.0397 \\ 4-1 & 3 & 3.94276 & 0.02535 & 3.8899 & 3.9956\end{array}$

$\begin{array}{llllll}4-2 & 3 & 3.95535 & 0.02535 & 3.9025 & 4.0082 \\ 5-1 & 3 & 3.90497 & 0.02535 & 3.8521 & 3.9578\end{array}$

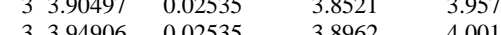

Std Error uses a pooled estimate of error variance 
Exhibit A-5. Measurements by Block and Sub-Block for Samples of the Sb7ref Glass and the Ustd Standard Glass by Oxide by Prep

Oneway Analysis of Measured By Blk/Sub-Blk Glass ID=Ustd, Oxide=B2O3 (wt\%), Prep Method=AR, Target $=9.2090$

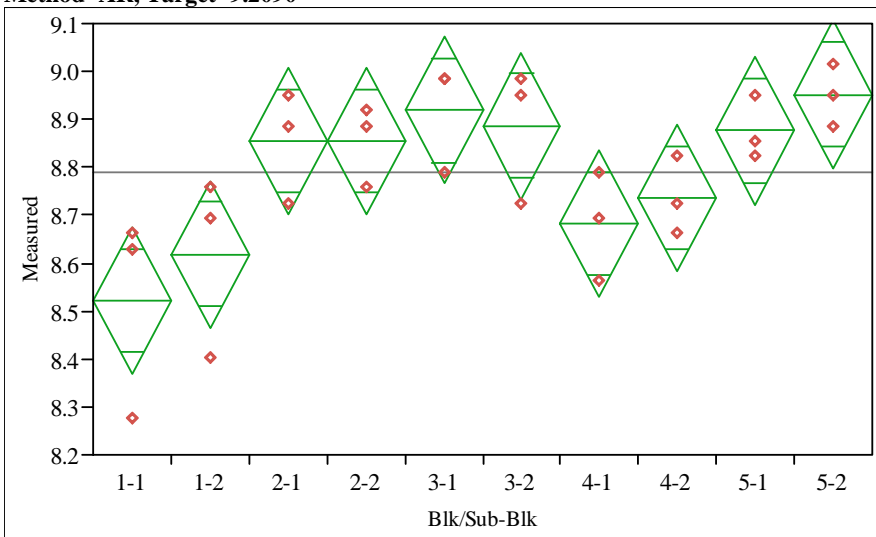

Oneway Anova

Summary of Fit

Adj Rsquare

Root Mean Square Error

\subsection{2}

0.46169

Mean of Respons

8.790327

Observations (or Sum Wgts)

30

Analysis of Variance

Source DF Sum of Squares Mean Square F Ratio Prob $>$ F

$\begin{array}{llllll}\text { Blk/Sub-Blk } & 0.55018225 & 0.061131 & 3.7636 & 0.0065\end{array}$

Error $20-0.32485635$

0.016243

\section{Means for Oneway Anova}

Level Number Mean Std Error Lower 95\% Upper 95\%

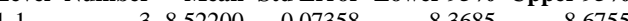

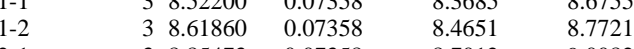

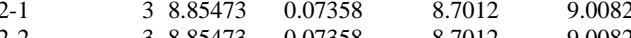

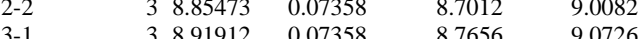

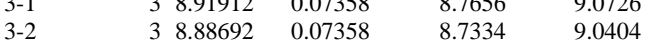

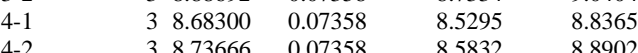

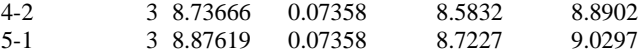

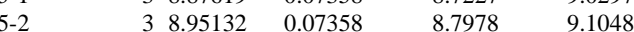

Std Error uses a pooled estimate of error variance
Oneway Analysis of Measured By Blk/Sub-Blk Glass ID=Ustd, Oxide=B2O3 (wt\%), Prep Method=PF, Target $=\mathbf{9 . 2 0 9 0}$

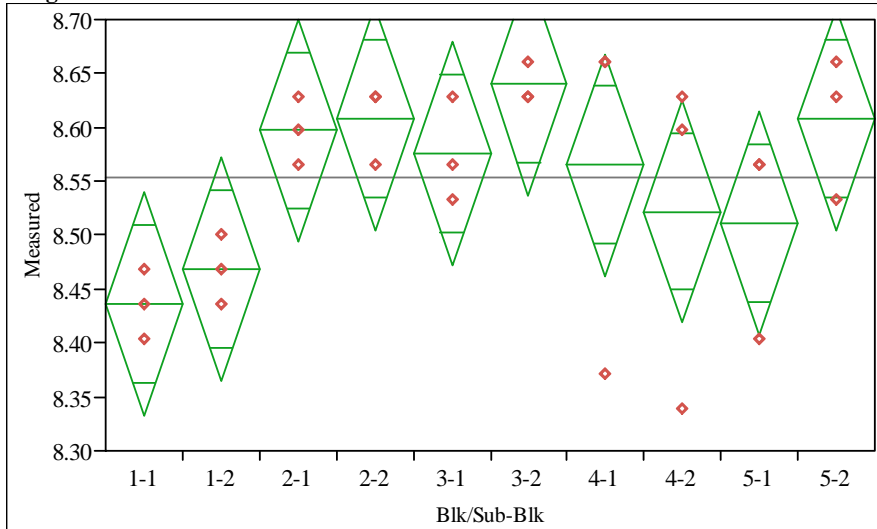

Oneway Anova

Summary of Fi

Rsquare

Adj Rsquare $\quad 0.198729$

Root Mean Square Error $\quad 0.085797$

Mean of Response

8.553128

Analysis of Variance

Source DF Sum of Squares Mean Square F Ratio Prob $>$ F

$\begin{array}{llll}0.11919463 & 0.013244 & 1.7992 & 0.1313\end{array}$

$\begin{array}{lll}\text { Error } & 20 & 0.14722214\end{array}$

Means for Oneway Anova

Level Number Mean Std Error Lower 95\% Upper 95\%

$\begin{array}{rrrrrr}1-1 & 3 & 8.43614 & 0.04953 & 8.3328 & 8.5395 \\ 1-2 & & 3 & 8.46834 & 0.04953 & 8.3650\end{array}$

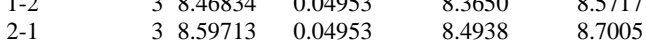

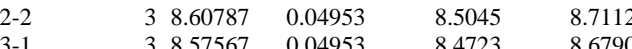

$\begin{array}{llllll}3-1 & 3 & 8.57567 & 0.04953 & 8.4723 & 8.6790 \\ 3-2 & 3 & 8.64007 & 0.04953 & 8.5367 & 8.7434\end{array}$

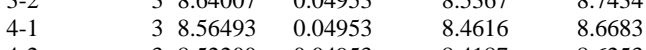

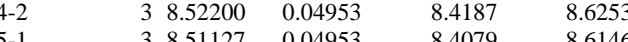

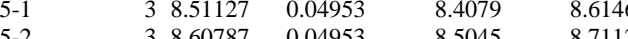

Std Error uses a pooled estimate of error variance 
Exhibit A-5. Measurements by Block and Sub-Block for Samples of the Sb7ref Glass and the Ustd Standard Glass by Oxide by Prep

Oneway Analysis of Measured By Blk/Sub-Blk Glass ID=Ustd, Oxide=CaO (wt\%), Prep Method=AR, Target $\mathbf{1 . 3 0 1 0}$

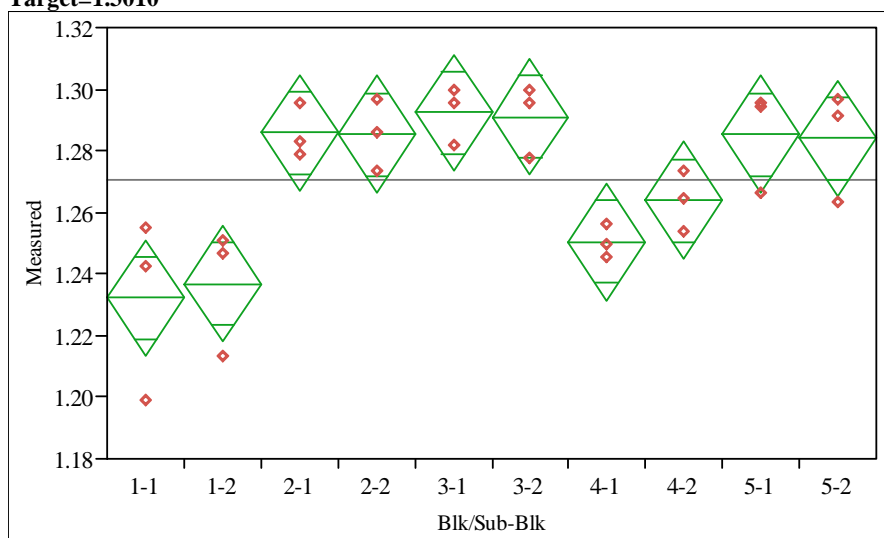

Oneway Anova

Summary of Fit

Adj Rsquare

\subsection{3}

0.629921

Root Mean Square Error $\quad 0.01571$

Mean of Respons

1.270753
$\quad 30$

Analysis of Variance

Source DF Sum of Squares Mean Square F Ratio Prob $>$ F

$\begin{array}{llll}0.01440416 & 0.001600 & 6.4846 & 0.0003\end{array}$

$\begin{array}{lll}\text { Error } & 20 & 0.00493617 \\ & & \end{array}$

0.000247

\section{Means for Oneway Anova}

Level Number Mean Std Error Lower 95\% Upper 95\%

$\begin{array}{llllrr}1-1 & 3 & 1.23223 & 0.00907 & 1.2133 & 1.2511\end{array}$

$\begin{array}{llllll}1-2 & 3 & 1.23689 & 0.00907 & 1.2180 & 1.2558 \\ 2-1 & 3 & 1.28586 & 0.00907 & 1.2669 & 1.3048\end{array}$

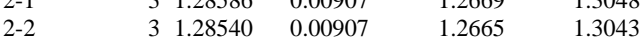

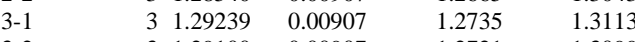

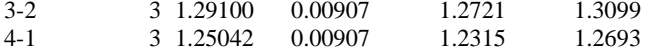

$\begin{array}{llllll}4-2 & 3 & 1.26394 & 0.00907 & 1.2450 & 1.2829\end{array}$

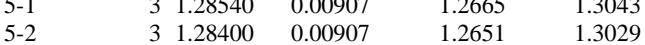

Std Error uses a pooled estimate of error variance
Oneway Analysis of Measured By Blk/Sub-Blk Glass ID=Ustd, Oxide=Fe2O3 (wt\%), Prep Method=AR, Target $=13.1960$

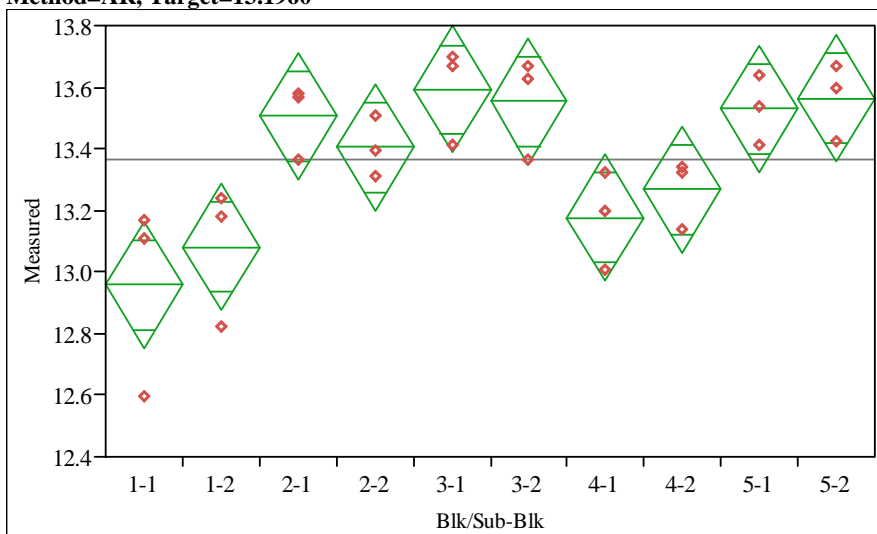

Oneway Anova

Summary of Fi

Rsquare

$\begin{array}{ll}0.705495 \\ \text { Adj Rsquare } & 0.572967\end{array}$

Root Mean Square Error $\quad 0.170748$

Mean of Response

13.36341

Analysis of Variance

Source DF Sum of Squares Mean Square F Ratio Prob $>$ F

$\begin{array}{lllll}9 & 1.3968234 & 0.155203 & 5.3234 & 0.0009\end{array}$

$\begin{array}{llll}\text { Error } & 20 & 0.5830971 & 0.029155\end{array}$

Means for Oneway Anova

Level Number Mean Std Error Lower 95\% Upper 95\%

$\begin{array}{rrrrrr}1-1 & 3 & 12.9578 & 0.09858 & 12.752 & 13.163 \\ 1-2 & 3 & 13.0818 & 0.09858 & 12.876 & 13.287\end{array}$

$\begin{array}{llllll}1-2 & 3 & 13.0818 & 0.09858 & 12.876 & 13.287 \\ 2-1 & 3 & 13.5059 & 0.09858 & 13.300 & 13.712\end{array}$

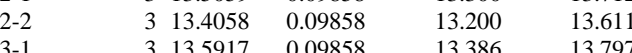

$\begin{array}{llllll}3-1 & 3 & 13.5917 & 0.09858 & 13.386 & 13.797 \\ 3-2 & 3 & 13.5536 & 0.09858 & 13.348 & 13.759\end{array}$

$\begin{array}{llllll}3-2 & 3 & 13.5536 & 0.09858 & 13.348 & 13.759 \\ 4-1 & 3 & 13.1771 & 0.09858 & 12.971 & 13.383\end{array}$

$\begin{array}{llllll}4-2 & 3 & 13.2676 & 0.09858 & 13.062 & 13.473 \\ 5-1 & 3 & 13.5297 & 0.09858 & 13.324 & 13.735\end{array}$

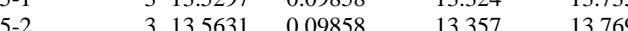

Std Error uses a pooled estimate of error variance 
Exhibit A-5. Measurements by Block and Sub-Block for Samples of the Sb7ref Glass and the Ustd Standard Glass by Oxide by Prep

Oneway Analysis of Measured By Blk/Sub-Blk Glass ID=Ustd, Oxide=Fe2O3 (wt\%), Prep Method=PF, Target $=13.1960$

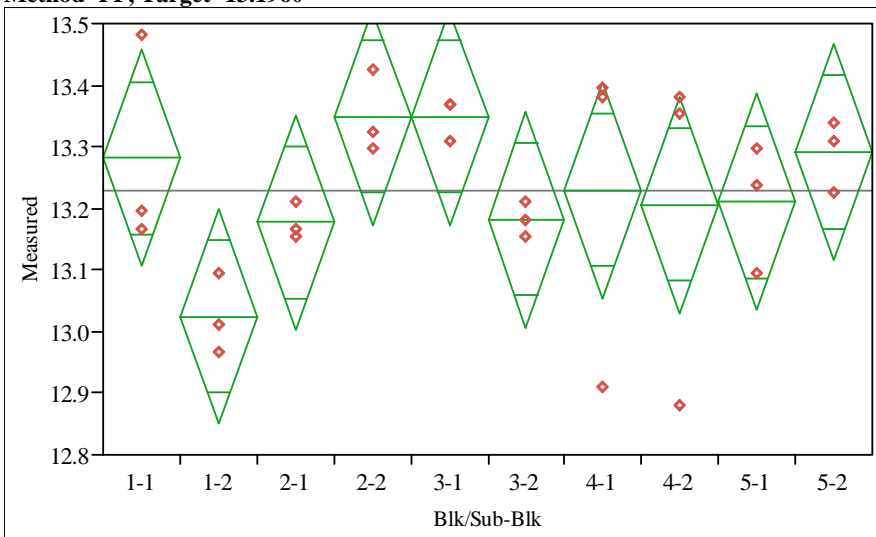

Oneway Anova

Summary of Fit

Adj Rsquare

0.37024

- 0.086848

Mean of Response

0.14545

Observations (or Sum Wgts) $\quad 30$

Analysis of Variance

Source DF Sum of Squares Mean Square F Ratio Prob $>$ F

$\begin{array}{lrrrrr}\text { Blk/Sub-Blk } & 9 & 0.24875311 & 0.027639 & 1.3065 & 0.2939\end{array}$

Error $\quad 20 \quad 0.42311671$

0.021156

Means for Oneway Anova

Level Number Mean Std Error Lower 95\% Upper 95\%

$\begin{array}{rrrrrr}1-1 & 3 & 13.2819 & 0.08398 & 13.107 & 13.457\end{array}$

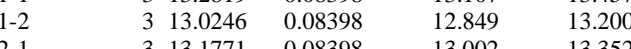

$\begin{array}{llllll}2-1 & 3 & 13.1771 & 0.08398 & 13.002 & 13.352 \\ 2-2 & 3 & 13.3486 & 0.08398 & 13.173 & 13.524 \\ 3-1 & 3 & 13.3486 & 0.08398 & 13.173 & 13.524\end{array}$

$\begin{array}{llllll}2-2 & 3 & 13.3486 & 0.08398 & 13.173 & 13.524 \\ 3-1 & 3 & 13.3486 & 0.08398 & 13.173 & 13.524 \\ 3-2 & 3 & 13.1818 & 0.08388 & 13.007 & 13.357\end{array}$

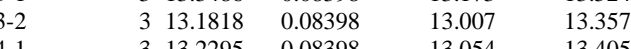

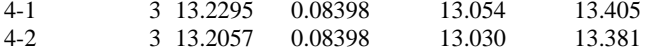

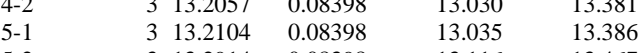

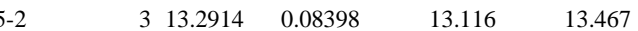

Std Error uses a pooled estimate of error variance
Oneway Analysis of Measured By Blk/Sub-Blk Glass ID=Ustd, Oxide=K2O (wt\%), Prep Method=AR, Oneway Analysis
Target $=2.9990$

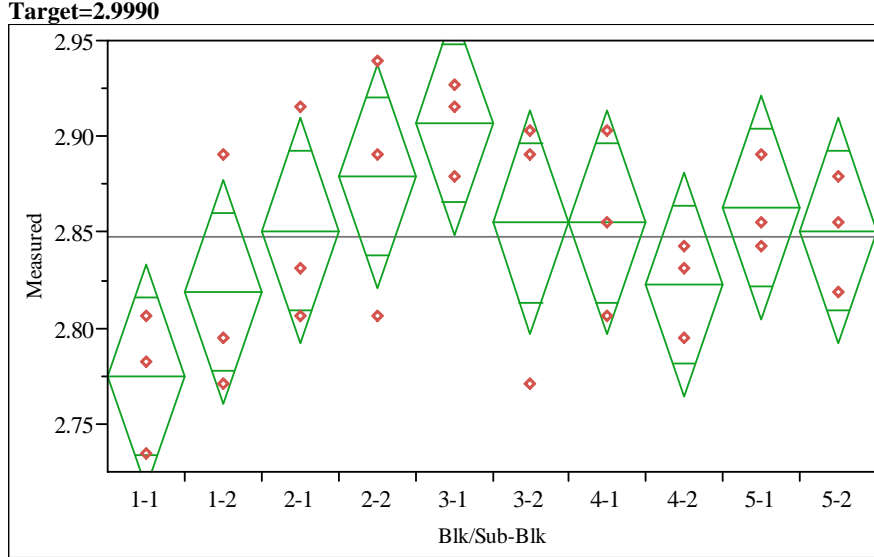

Oneway Anova

Summary of Fi

Rsquare

Adj Rsquare $\quad 0.166608$

Root Mean Square Error $\quad 0.048634$

Mean of Response

2.847674

Analysis of Variance

Source DF Sum of Squares Mean Square F Ratio Prob $>$ F

$\begin{array}{lllll}9 & 0.03499960 & 0.003889 & 1.6442 & 0.1695\end{array}$

$\begin{array}{llll}\text { Error } & 20 & 0.04730459 & 0.002365\end{array}$

Means for Oneway Anova

Level Number Mean Std Error Lower 95\% Upper 95\%

$\begin{array}{rrrrrr}1-1 & 3 & 2.77460 & 0.02808 & 2.7160 & 2.8332 \\ 1-2 & 3 & 2.81876 & 0.02808 & 2.7602 & 2.8773\end{array}$

$\begin{array}{llllll}1-2 & 3 & 2.81876 & 0.02808 & 2.7602 & 2.8773 \\ 2-1 & 3 & 2.85089 & 0.02808 & 2.7923 & 2.9095\end{array}$

$\begin{array}{llllll}2-2 & 3 & 2.87899 & 0.02808 & 2.8204 & 2.9376\end{array}$

$\begin{array}{llllll}3-1 & 3 & 2.90710 & 0.02808 & 2.8485 & 2.9657 \\ 3-2 & 3 & 2.85490 & 0.02808 & 2.7963 & 2.9135 \\ 4-1 & 3 & 2.8540 & 0.02808 & 2.7963 & 2.9135\end{array}$

$\begin{array}{llllll}3-2 & 3 & 2.85490 & 0.02808 & 2.7963 & 2.9135 \\ 4-1 & 3 & 2.85490 & 0.02808 & 2.7963 & 2.9135 \\ 4-2 & 3 & 2.82278 & 0.02808 & 2.7642 & 2.8814\end{array}$

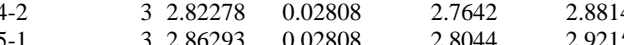

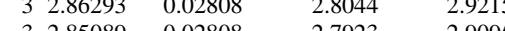

Std Error uses a pooled estimate of error variance 
Exhibit A-5. Measurements by Block and Sub-Block for Samples of the Sb7ref Glass and the Ustd Standard Glass by Oxide by Prep

Oneway Analysis of Measured By Blk/Sub-Blk Glass ID=Ustd, Oxide=K2O (wt\%), Prep Method=PF, Target $=\mathbf{2 . 9 9 9 0}$

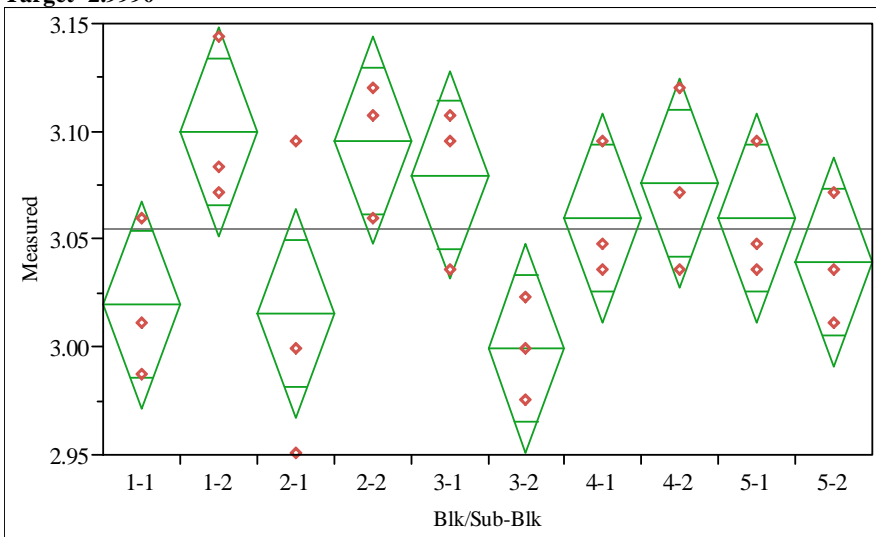

Oneway Anova

Summary of Fit

Adj Rsquare

0.503763

- 0.280456

Mean of Response

0.040133

Observations (or Sum Wgts)

30

Analysis of Variance

Source DF Sum of Squares Mean Square F Ratio Prob $>$ F

$\begin{array}{llllll}\text { Blk/Sub-Blk } & 0.03270208 & 0.003634 & 2.2559 & 0.0623\end{array}$

Error 200.03221356

Means for Oneway Anova

Level Number Mean Std Error Lower 95\% Upper 95\%

$\begin{array}{lllllr}1-1 & 3 & 3.01953 & 0.02317 & 2.9712 & 3.0679\end{array}$

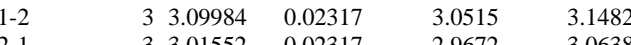

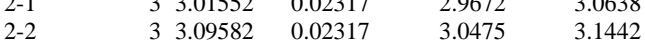

$\begin{array}{llllll}2-2 & 3 & 3.09582 & 0.02317 & 3.0475 & 3.1442 \\ 3-1 & 3 & 3.07976 & 0.02317 & 3.0314 & 3.1281 \\ 3-2 & 3 & 2.9995 & 0.02317 & 2.9511 & 3.0478\end{array}$

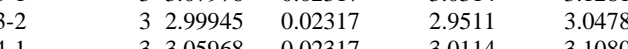

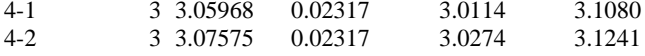

$\begin{array}{llllll}4-2 & 3 & 3.07575 & 0.02317 & 3.0274 & 3.1241 \\ 5-1 & 3 & 3.05968 & 0.02317 & 3.0114 & 3.1080\end{array}$

Std Error uses a pooled estimate of error variance
Oneway Analysis of Measured By Blk/Sub-Blk Glass ID=Ustd, Oxide=Li2O (wt\%), Prep Method=AR, Target $=3.0570$

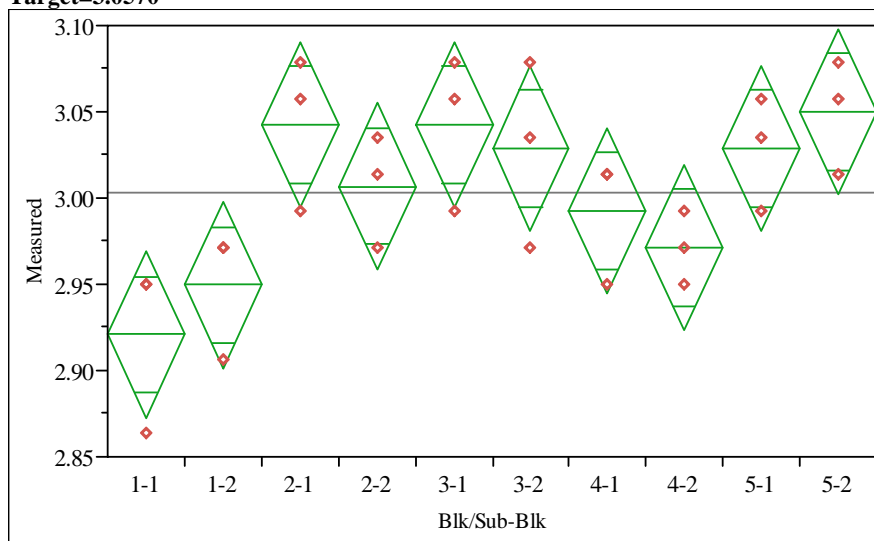

Oneway Anova

Summary of Fit

Rsquare

0.621671

Adj Rsquare $\quad 0.451423$

Root Mean Square Error

Mean of Response

3.003296

Analysis of Variance

Source DF Sum of Squares Mean Square F Ratio Prob $>$ F

$\begin{array}{llll}0.05229801 & 0.005811 & 3.6516 & 0.0076\end{array}$

$\begin{array}{lll}\text { Error } & 20 & 0.03182685 \\ \text { C. Total } & 29 & 0.08412486\end{array}$

Means for Oneway Anova

Level Number Mean Std Error Lower 95\% Upper 95\%

$\begin{array}{llrrrr}1-1 & 3 & 2.92077 & 0.02303 & 2.8727 & 2.9688 \\ 1-2 & 3 & 2.94947 & 0.02303 & 2.9014 & 2.9975\end{array}$

$\begin{array}{llllll}1-2 & 3 & 2.94947 & 0.02303 & 2.9014 & 2.9975 \\ 2-1 & 3 & 3.04277 & 0.02303 & 2.9947 & 3.0908\end{array}$

$\begin{array}{llllll}2-2 & 3 & 3.00688 & 0.02303 & 2.9588 & 3.0549 \\ 3-1 & 3 & 3.04277 & 0.02303 & 2.9947 & 3.0908\end{array}$

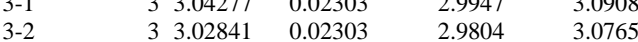

$\begin{array}{llllll}3-2 & 3 & 3.02841 & 0.02303 & 2.9804 & 3.0765 \\ 4-1 & 3 & 2.99253 & 0.02303 & 2.9445 & 3.0406\end{array}$

$\begin{array}{llllll}4-2 & 3 & 2.97100 & 0.02303 & 2.9230 & 3.0190 \\ 5-1 & 3 & 3.02841 & 0.02303 & 2.9804 & 3.0765\end{array}$

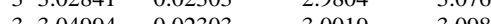

Std Error uses a pooled estimate of error variance 
Exhibit A-5. Measurements by Block and Sub-Block for Samples of the Sb7ref Glass and the Ustd Standard Glass by Oxide by Prep

Oneway Analysis of Measured By Blk/Sub-Blk Glass ID=Ustd, Oxide=Li2O (wt\%), Prep Method=PF, Target $=3.0570$

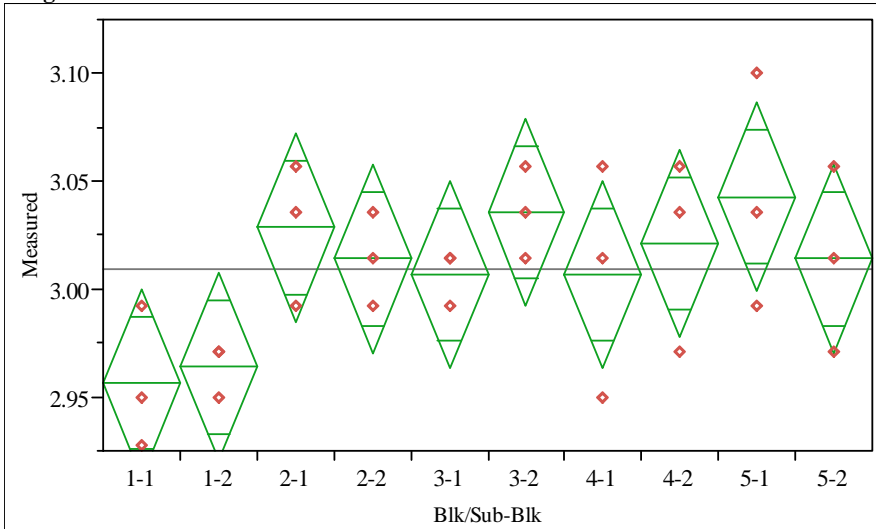

Oneway Anova

Summary of Fit

Rsquare

Adj Rsquare $\quad 0.45179$

Root Men Square Enor -0.205095

Mean of Response

0.036239

Observations (or Sum Wgts)

30

Analysis of Variance

Source DF Sum of Squares Mean Square F Ratio Prob $>$ F

$\begin{array}{llllll}\text { Blk/Sub-Blk } & 0.02164535 & 0.002405 & 1.8314 & 0.1245\end{array}$

$\begin{array}{lll}\text { Error } & 20 & 0.02626488 \\ \text { C. Total } & 29 & 0.04791023\end{array}$

\section{Means for Oneway Anova}

Level Number Mean Std Error Lower 95\% Upper 95\%

$\begin{array}{lllrrr}1-1 & 3 & 2.95665 & 0.02092 & 2.9130 & 3.0003\end{array}$

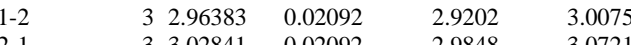

$\begin{array}{llllll}2-1 & 3 & 3.02841 & 0.02092 & 2.9848 & 3.0721 \\ 2-2 & 3 & 3.01406 & 0.02092 & 2.9704 & 3.0577\end{array}$

$\begin{array}{llllll}2-2 & 3 & 3.01406 & 0.02092 & 2.9704 & 3.0577 \\ 3-1 & 3 & 3.00688 & 0.02092 & 2.9632 & 3.0505\end{array}$

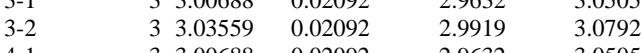

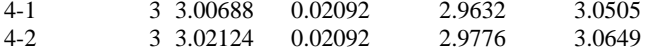

$\begin{array}{llllll}4-2 & 3 & 3.02124 & 0.02092 & 2.9776 & 3.0649 \\ 5-1 & 3 & 3.04277 & 0.02092 & 2.9991 & 3.0864\end{array}$

Std Error uses a pooled estimate of error variance
Oneway Analysis of Measured By Blk/Sub-Blk Glass ID=Ustd, Oxide=MgO (wt\%), Prep Method=AR, Target $\mathbf{1 . 2 1 0 0}$

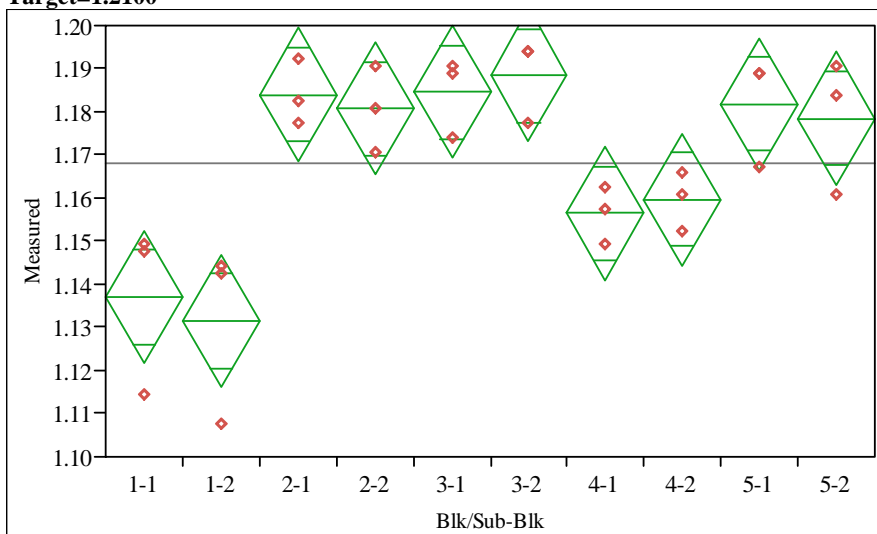

Oneway Anova

Summary of Fit

Rsquare

Adj Rsquare $\quad 0.782041$

Root Mean Square Error $\quad 0.012777$

Mean of Response

1.168272

Analysis of Variance

Source DF Sum of Squares Mean Square F Ratio Prob $>$ F

$\begin{array}{lllllll}\text { Blk/Sub-Blk } & 9 & 0.01171528 & 0.001302 & 7.9734 & <.0001\end{array}$

$\begin{array}{llll}\text { Error } & 20 & 0.00326512 & 0.000163\end{array}$

0.01498040

Means for Oneway Anova

Level Number Mean Std Error Lower 95\% Upper 95\%

$\begin{array}{rrrrrr}1-1 & 3 & 1.13704 & 0.00738 & 1.1217 & 1.1524\end{array}$

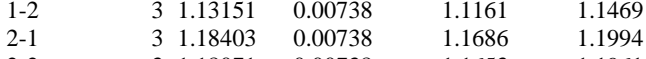

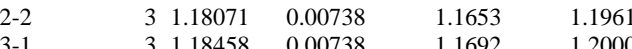

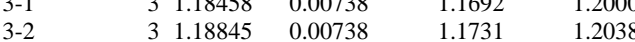

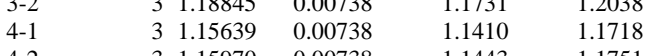

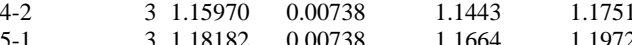

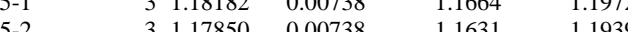

Std Error uses a pooled estimate of error variance 
Exhibit A-5. Measurements by Block and Sub-Block for Samples of the Sb7ref Glass and the Ustd Standard Glass by Oxide by Prep

Oneway Analysis of Measured By Blk/Sub-Blk Glass ID=Ustd, Oxide=MgO (wt\%), Prep Method=PF, Target $=1.2100$

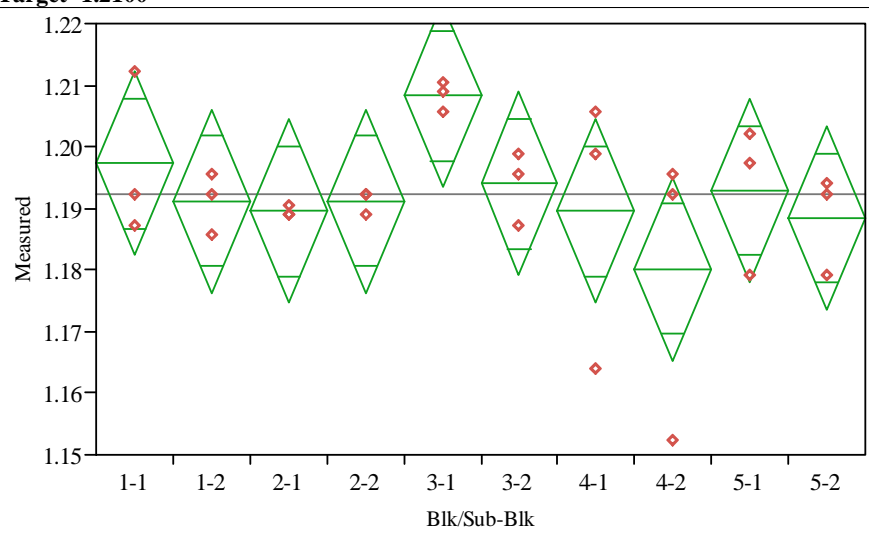

Oneway Anova

Summary of Fit

Adj Rsquare

0.312781

0.003533

Mean of Response Eror

Observations (or Sum Wgts) $\quad 30$

Analysis of Variance

Source DF Sum of Squares Mean Square F Ratio Prob $>$ F

$\begin{array}{llllll} & \text { Blk/Sub-Blk } & 0.00139597 & 0.000155 & 1.0114 & 0.4633\end{array}$

Error $20-0.00306712$

000153

Means for Oneway Anova

Level Number Mean Std Error Lower 95\% Upper 95\%

$\begin{array}{lllrrr}1-1 & 3 & 1.19729 & 0.00715 & 1.1824 & 1.2122\end{array}$

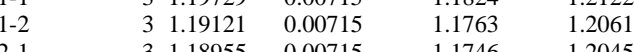

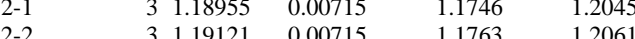

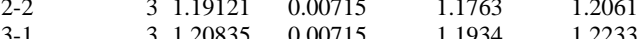

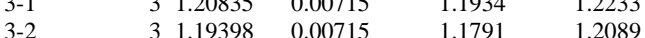

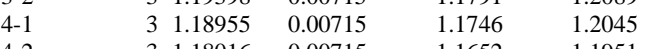

$\begin{array}{llllll}4-2 & 3 & 1.18016 & 0.00715 & 1.1652 & 1.1951 \\ 5-1 & 3 & 1.19287 & 0.00715 & 1.1780 & 1.2078\end{array}$

$\begin{array}{llllll}5-1 & 3 & 1.19287 & 0.0075 & 1.1780 & 1.2078 \\ 5-2 & 3 & 1.18845 & 0.00715 & 1.1735 & 1.2034\end{array}$

Std Error uses a pooled estimate of error variance
Oneway Analysis of Measured By Blk/Sub-Blk Glass ID=Ustd, Oxide=MnO (wt\%), Prep Method=AR, Target $=\mathbf{2 . 8 9 2 0}$

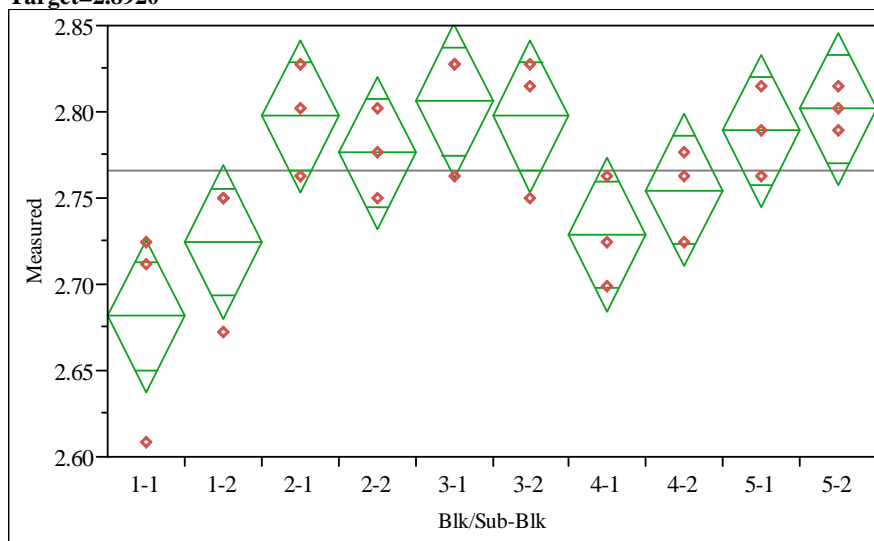

Oneway Anova

Summary of Fit

Rsquare

Adj Rsquare $\quad 0.476604$

Root Mean Square Error $\quad 0.036748$

Mean of Response

2.76575

Analysis of Variance

Source DF Sum of Squares Mean Square F Ratio Prob $>$ F

$\begin{array}{lllll}9 & 0.04781522 & 0.005313 & 3.9342 & 0.0052\end{array}$

$\begin{array}{lll}\text { Error } & 20 & 0.02700860\end{array}$

0.001350

Means for Oneway Anova

Level Number Mean Std Error Lower 95\% Upper 95\%

$\begin{array}{lllrrr}1-1 & 3 & 2.68139 & 0.02122 & 2.6371 & 2.7256\end{array}$

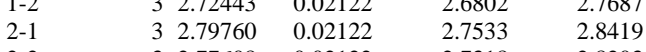

$\begin{array}{llllll}2-2 & 3 & 2.77608 & 0.02122 & 2.7318 & 2.8203 \\ 3-1 & 3 & 2.80621 & 0.02122 & 2.7620 & 2.8505\end{array}$

$\begin{array}{llllll}3-1 & 3 & 2.80621 & 0.02122 & 2.7620 & 2.8505 \\ 3-2 & 3 & 2.79760 & 0.02122 & 2.7533 & 2.8419\end{array}$

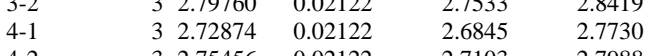

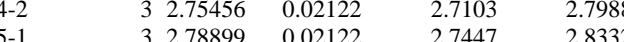

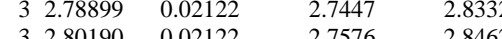

Std Error uses a pooled estimate of error variance 
Exhibit A-5. Measurements by Block and Sub-Block for Samples of the Sb7ref Glass and the Ustd Standard Glass by Oxide by Prep

Oneway Analysis of Measured By Blk/Sub-Blk Glass ID=Ustd, Oxide=MnO (wt\%), Prep Method=PF, Target $=\mathbf{2 . 8 9 2 0}$

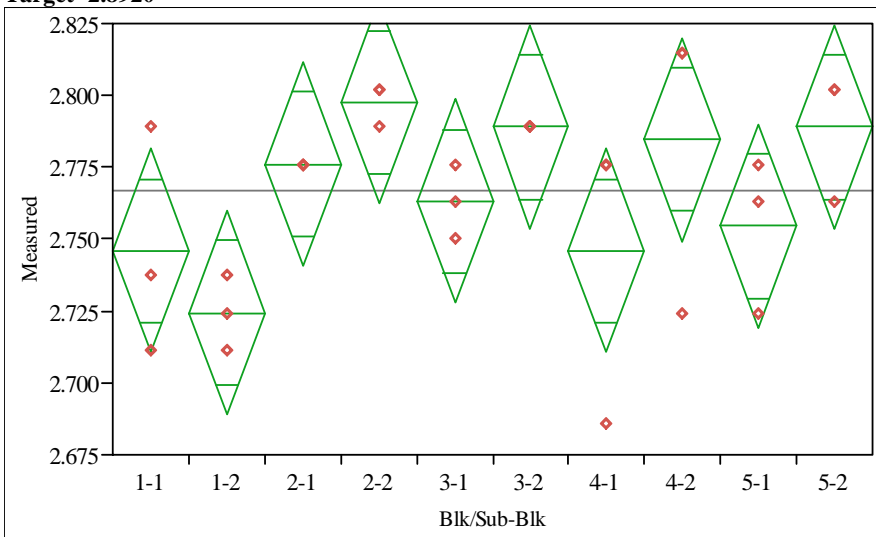

Oneway Anova

Summary of Fit

0.473595

Root Mean Square Error $\quad 0.029349$

Mean of Response 2.767042

Observations (or Sum Wgts) $\quad 30$

Analysis of Variance

Source DF Sum of Squares Mean Square F Ratio Prob $>$ F

$\begin{array}{llllll}\text { Blk/Sub-Blk } & 0.01549938 & 0.001722 & 1.9993 & 0.0945\end{array}$

$\begin{array}{lll}\text { Error } & 20 & 0.01722771 \\ \text { C. Total } & 29 & 0.03272709\end{array}$

Means for Oneway Anova

Level Number Mean Std Error Lower 95\% Upper 95\%

$\begin{array}{llll}3 & 2.74595 & 0.01694 & 0\end{array}$

$\begin{array}{llllll}1-2 & 3 & 2.72443 & 0.01694 & 2.6891 & 2.7898\end{array}$

$\begin{array}{llllll}2-1 & 3 & 2.77608 & 0.01694 & 2.7407 & 2.8114\end{array}$

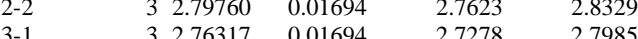

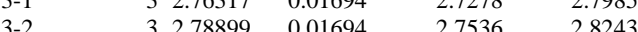

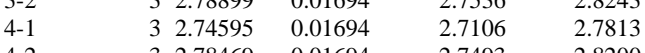

$\begin{array}{llllll}4-2 & 3 & 2.78469 & 0.01694 & 2.7493 & 2.8200 \\ 5-1 & 3 & 2.75456 & 0.01694 & 2.7192 & 2.7899\end{array}$

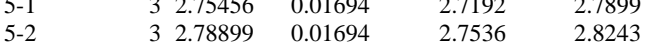

Std Error uses a pooled estimate of error variance
Oneway Analysis of Measured By Blk/Sub-Blk Glass ID=Ustd, Oxide=Na2O (wt\%), Prep Method $=$ AR, Target $=11.7950$

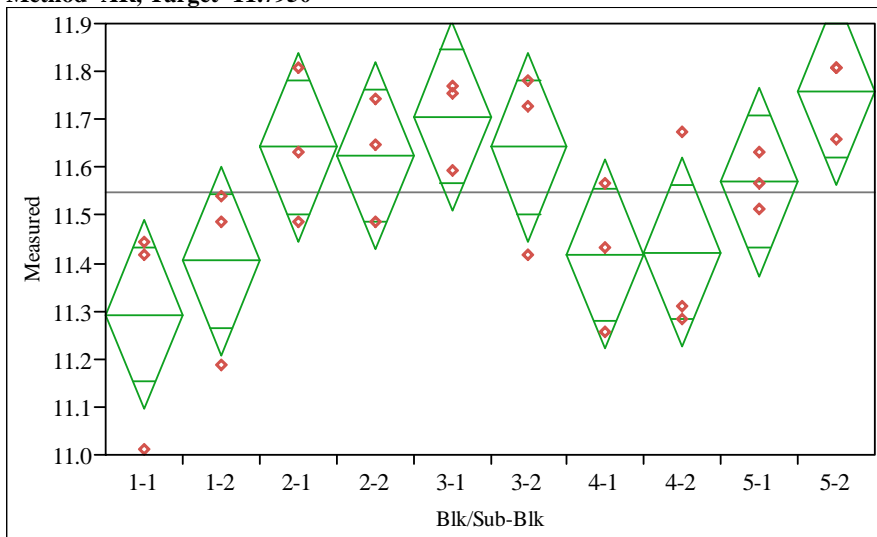

Oneway Anova

Summary of Fit

Rsquare

0.543353

Adj Rsquare $\quad 0.337861$

Root Mean Square Error $\quad 0.163696$

Mean of Response 11.54787

Analysis of Variance

Source DF Sum of Squares Mean Square F Ratio Prob $>$ F

$\begin{array}{llllll} & 9 & 0.6376824 & 0.070854 & 2.6442 & 0.0336\end{array}$

$\begin{array}{llll}\text { Error } & 20 & 0.5359245 & 0.026796\end{array}$

Means for Oneway Anova

Level Number Mean Std Error Lower 95\% Upper 95\%

$\begin{array}{llrrrr}1-1 & 3 & 11.2917 & 0.09451 & 11.095 & 11.489\end{array}$

$\begin{array}{llllll}1-2 & 3 & 11.4041 & 0.09451 & 11.207 & 11.601 \\ 2-1 & 3 & 11.6422 & 0.09451 & 11.445 & 11.839\end{array}$

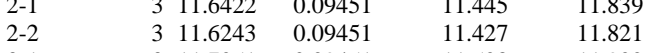

$\begin{array}{llllll}3-1 & 3 & 11.7051 & 0.09451 & 11.508 & 11.902 \\ 3-2 & 3 & 11.6422 & 0.09451 & 11.445 & 11.839\end{array}$

$\begin{array}{llllll}3-2 & 3 & 11.6422 & 0.09451 & 11.445 & 11.839 \\ 4-1 & 3 & 11.4176 & 0.09451 & 11.220 & 11.615\end{array}$

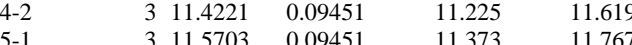

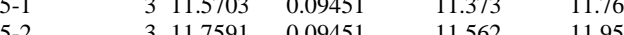

Std Error uses a pooled estimate of error variance 
Exhibit A-5. Measurements by Block and Sub-Block for Samples of the Sb7ref Glass and the Ustd Standard Glass by Oxide by Prep

Oneway Analysis of Measured By Blk/Sub-Blk Glass ID=Ustd, Oxide=NiO (wt\%), Prep Method=AR, Target $=1.1200$

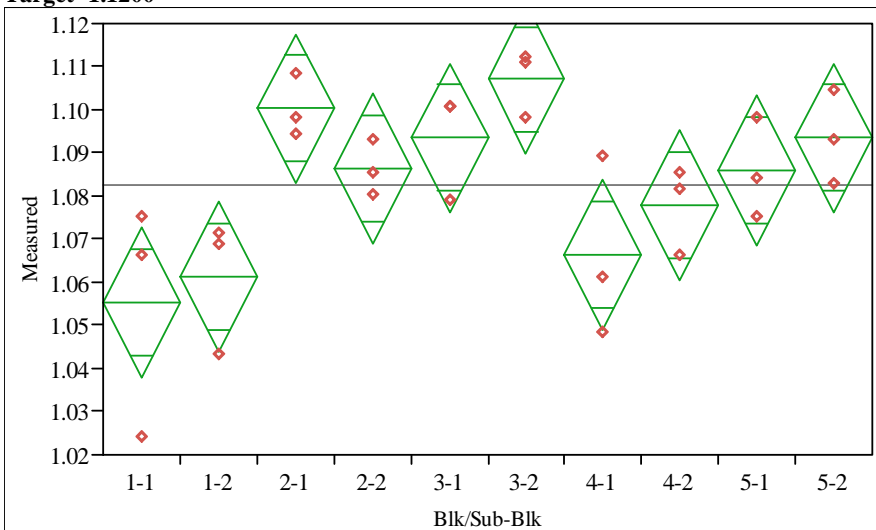

Oneway Anova

Summary of Fit

Adj Rsquare

\subsection{2}

Ther 0.503983

Mean of Response

0.014402

Observations (or Sum Wgts) $\quad 30$

Analysis of Variance

Source DF Sum of Squares Mean Square F Ratio Prob $>$ F

$\begin{array}{llllll}\text { Blk/Sub-Blk } & 0.00797883 & 0.000887 & 4.2740 & 0.0033\end{array}$

$\begin{array}{lll}\text { Error } & 20 & 0.00414853 \\ \text { C. Total } & 29 & 0.01212737\end{array}$

\section{Means for Oneway Anova}

Level Number Mean Std Error Lower 95\% Upper 95\%

$\begin{array}{rrrrrr}1-1 & 3 & 1.05533 & 0.00832 & 1.0380 & 1.0727\end{array}$

$\begin{array}{llllll}1-2 & 3 & 1.06127 & 0.00832 & 1.0439 & 1.0786 \\ 2-1 & 3 & 1.10029 & 0.00832 & 1.0829 & 1.1176\end{array}$

$\begin{array}{llllll}2-1 & 3 & 1.10029 & 0.00832 & 1.0829 & 1.1176 \\ 2-2 & 3 & 1.08629 & 0.00832 & 1.0689 & 1.1036\end{array}$

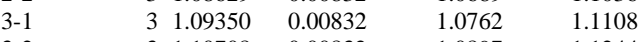

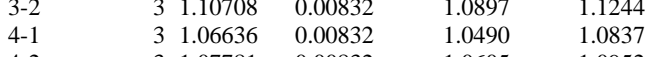

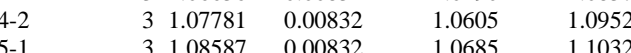

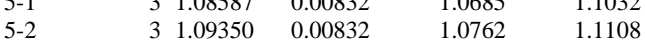

Std Error uses a pooled estimate of error variance
Oneway Analysis of Measured By Blk/Sub-Blk Glass ID=Ustd, Oxide=NiO (wt\%), Prep Method=PF, Target $=1.1200$

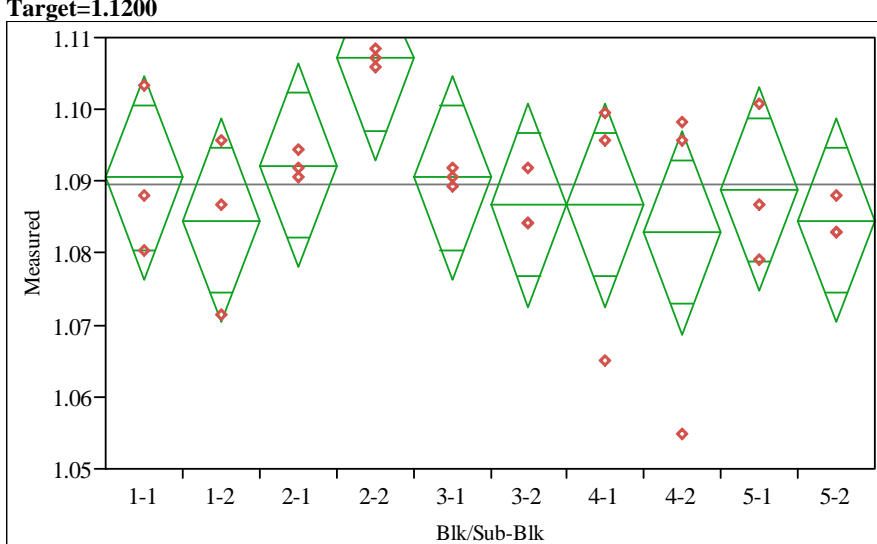

Oneway Anova

Summary of Fit

Rsquare

Adj Rsquare $\quad 0.007879$

Root Mean Square Error $\quad 0.01176$

Mean of Response

1.089472

Analysis of Variance

Source DF Sum of Squares Mean Square F Ratio Prob $>$ F

$\begin{array}{lllll}0.00127840 & 0.000142 & 1.0256 & 0.4537\end{array}$

$\begin{array}{lll}\text { Error } & 20 & 0.00277001 \\ \text { C. Total } & 29 & 0.00404841\end{array}$

Means for Oneway Anova

Level Number Mean Std Error Lower 95\% Upper 95\%

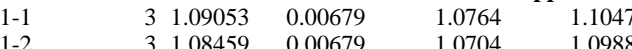

$\begin{array}{llllll}1-2 & 3 & 1.08459 & 0.00679 & 1.0704 & 1.0988 \\ 2-1 & 3 & 1.09223 & 0.00679 & 1.0781 & 1.1064\end{array}$

$\begin{array}{llllll}2-2 & 3 & 1.10708 & 0.00679 & 1.0929 & 1.1212 \\ 3-1 & 3 & 1.09053 & 0.00679 & 1.0764 & 1.1047\end{array}$

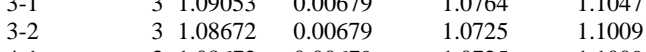

$\begin{array}{llllll}4-1 & 3 & 1.08672 & 0.00679 & 1.0725 & 1.1009 \\ 4-2 & 3 & 1.08290 & 0.00679 & 1.0687 & 1.0971\end{array}$

$\begin{array}{llllll}4-2 & 3 & 1.08290 & 0.00679 & 1.0687 & 1.0971 \\ 5-1 & 3 & 1.08884 & 0.00679 & 1.0747 & 1.1030\end{array}$

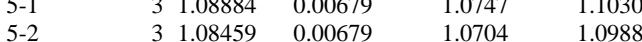

Std Error uses a pooled estimate of error variance 
Exhibit A-5. Measurements by Block and Sub-Block for Samples of the Sb7ref Glass and the Ustd Standard Glass by Oxide by Prep

Oneway Analysis of Measured By Blk/Sub-Blk Glass ID=Ustd, Oxide=SiO2 (wt\%), Prep Method=PF, Target $=45.3530$

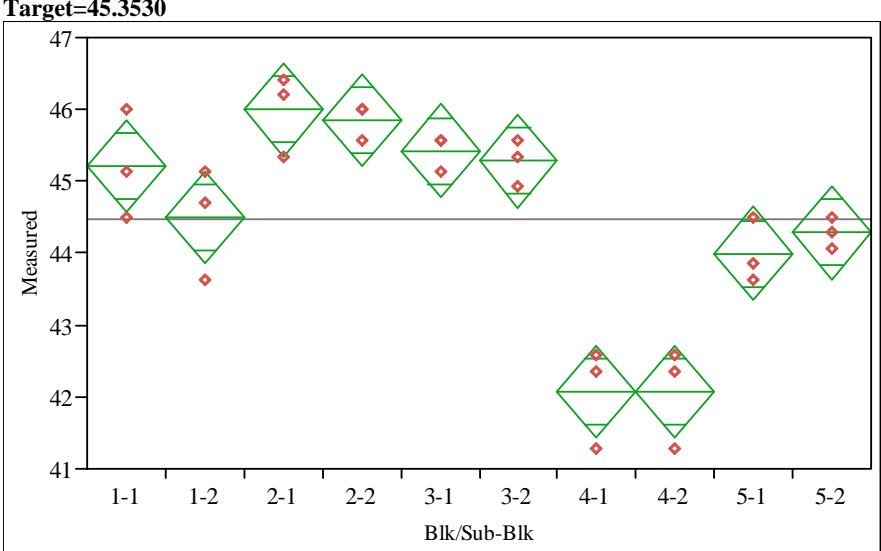

Oneway Anova

Summary of Fit

Adj Rsquare

0.903563

Root 0.860166

Mean of Response

44.46892

Observations (or Sum Wgts) $\quad 30$

Analysis of Variance

Source DF Sum of Squares Mean Square F Ratio Prob $>$ F

$\begin{array}{llllll}\text { Blk/Sub-Blk } & 9 & 54.315142 & 6.03502 & 20.8211 & <.0001\end{array}$

$\begin{array}{llr}\text { Error } & 20 & 5.797032 \\ \text { C. Total } & 29 & 60.112174\end{array}$

Means for Oneway Anova

Level Number Mean Std Error Lower 95\% Upper 95\%

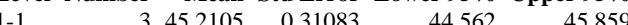

$\begin{array}{llllll}1-2 & 3 & 44.4974 & 0.31083 & 43.849 & 45.146\end{array}$

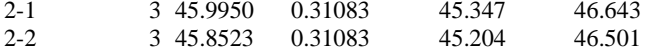

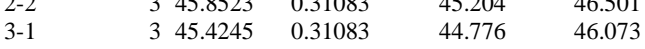

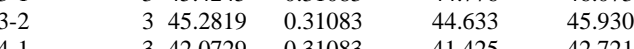

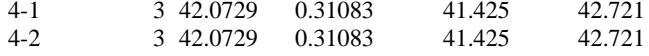

$\begin{array}{llllll}4-2 & 3 & 42.0729 & 0.31083 & 41.425 & 42.721 \\ 5-1 & 3 & 43.9983 & 0.31083 & 43.350 & 44.647\end{array}$

Std Error uses a pooled estimate of error variance
Oneway Analysis of Measured By Blk/Sub-Blk Glass ID=Ustd, Oxide=TiO2 (wt\%), Prep Method=AR, Oneway Analys
Target $=1.0490$

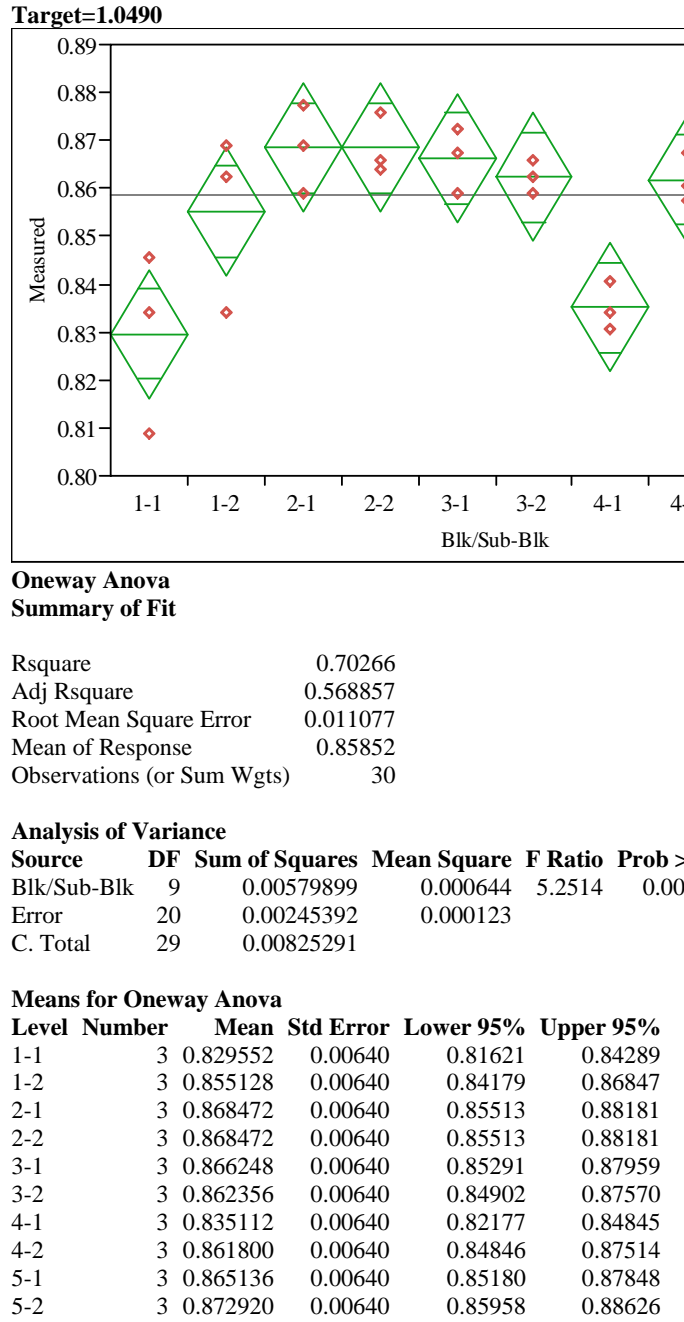

Std Error uses a pooled estimate of error variance 
Exhibit A-5. Measurements by Block and Sub-Block for Samples of the Sb7ref Glass and the Ustd Standard Glass by Oxide by Prep

Oneway Analysis of Measured By Blk/Sub-Blk Glass ID=Ustd, Oxide=TiO2 (wt\%), Prep Method=PF, Target $=1.0490$

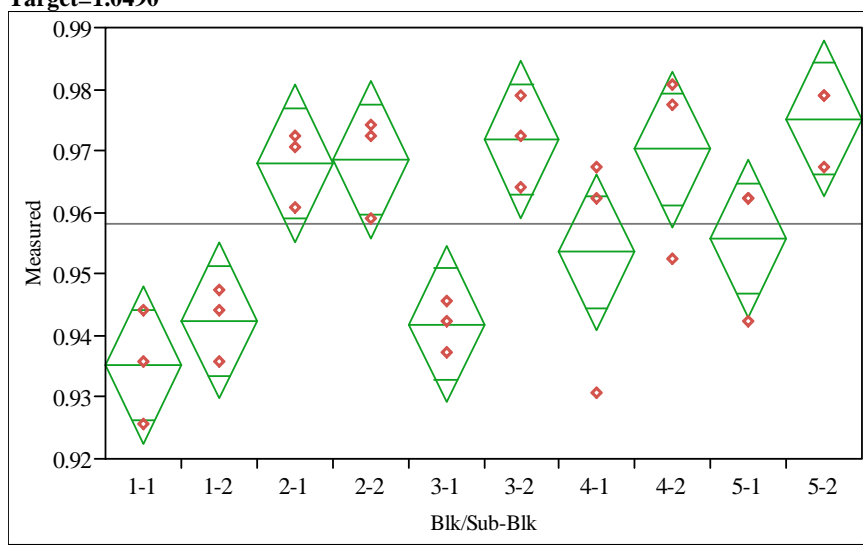

Oneway Anova

Summary of Fit

0.718079

0.591215

Mean of Response Eror 0.958266

Observations (or Sum Wgts) $\quad 30$

Analysis of Variance

Source DF Sum of Squares Mean Square F Ratio Prob $>$ F

$\begin{array}{llllll} & 0.00569289 & 0.000633 & 5.6602 & 0.0006\end{array}$

$\begin{array}{lll}\text { Error } & 20 & 0.00223505 \\ \text { C. Total } & 29 & 0.00792795\end{array}$

Means for Oneway Anova

Level Number Mean Std Error Lower 95\% Upper 95\%

$\begin{array}{lllllr}1-1 & 3 & 0.935192 & 0.00610 & 0.92246 & 0.94792\end{array}$

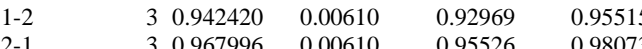

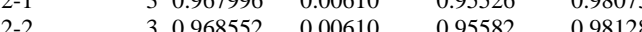

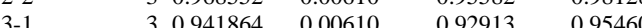

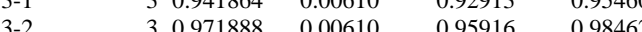

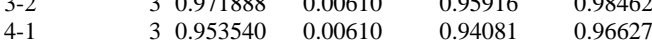

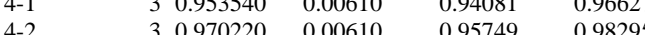

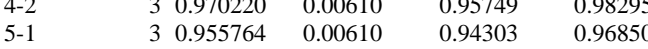

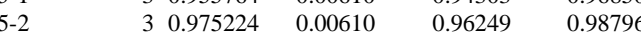

Std Error uses a pooled estimate of error variance
Oneway Analysis of Measured By Blk/Sub-Blk Glass ID=Ustd, Oxide=U308 (wt\%), Prep Method=AR, Target $=2.4060$

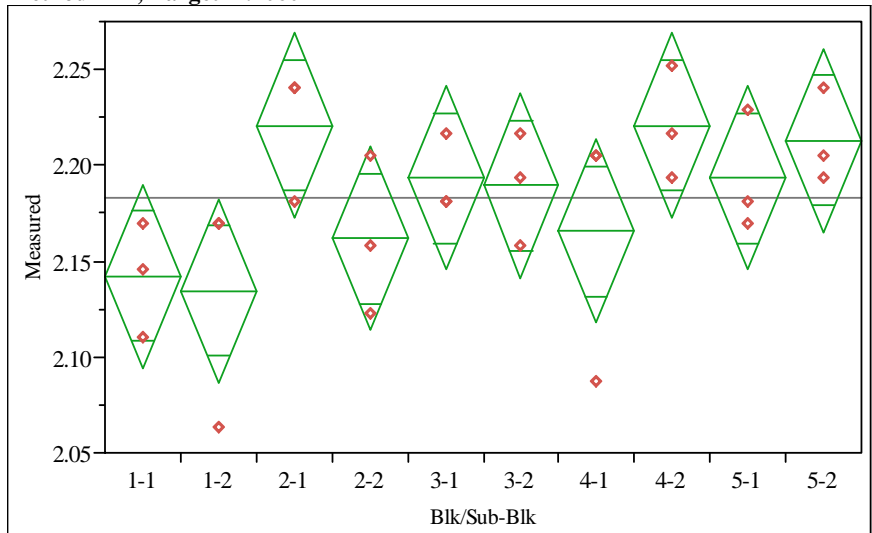

Oneway Anova

Summary of Fi

Rsquare

Adj Rsquare $\quad 0.207094$

Root Mean Square Error $\quad 0.039873$

Mean of Response

2.183485

Analysis of Variance

Source DF Sum of Squares Mean Square F Ratio Prob $>$ F

$\begin{array}{llll}0.02635021 & 0.002928 & 1.8416 & 0.1225\end{array}$

$\begin{array}{lll}\text { Error } & 20 & 0.03179639 \\ \text { C. Total } & 29 & 0.05814660\end{array}$

Means for Oneway Anova

Level Number Mean Std Error Lower 95\% Upper 95\%

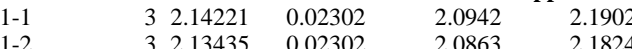

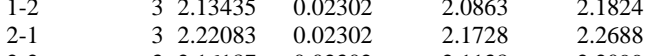

$\begin{array}{llllll}2-2 & 3 & 2.16187 & 0.02302 & 2.1138 & 2.2099 \\ 3-1 & 3 & 2.19331 & 0.02302 & 2.1453 & 2.2413\end{array}$

$\begin{array}{llllll}3-1 & 3 & 2.19331 & 0.02302 & 2.1453 & 2.2413 \\ 3-2 & 3 & 2.18938 & 0.02302 & 2.1414 & 2.2374\end{array}$

$\begin{array}{llllll}3-2 & 3 & 2.18938 & 0.02302 & 2.1414 & 2.2374 \\ 4-1 & 3 & 2.16580 & 0.02302 & 2.1178 & 2.2138\end{array}$

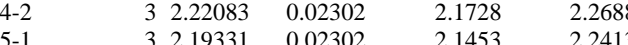

$\begin{array}{llllll}5-1 & 3 & 2.1933 & 0.02302 & 2.1453 & 2.2413 \\ 5-2 & 3 & 2.21297 & 0.02302 & 2.1649 & 2.2610\end{array}$

Std Error uses a pooled estimate of error variance 
Exhibit A-5. Measurements by Block and Sub-Block for Samples of the Sb7ref Glass and the Ustd Standard Glass by Oxide by Prep

Oneway Analysis of Measured By Blk/Sub-Blk Glass ID=Ustd, Oxide=U3O8 (wt\%), Prep Method=PF,

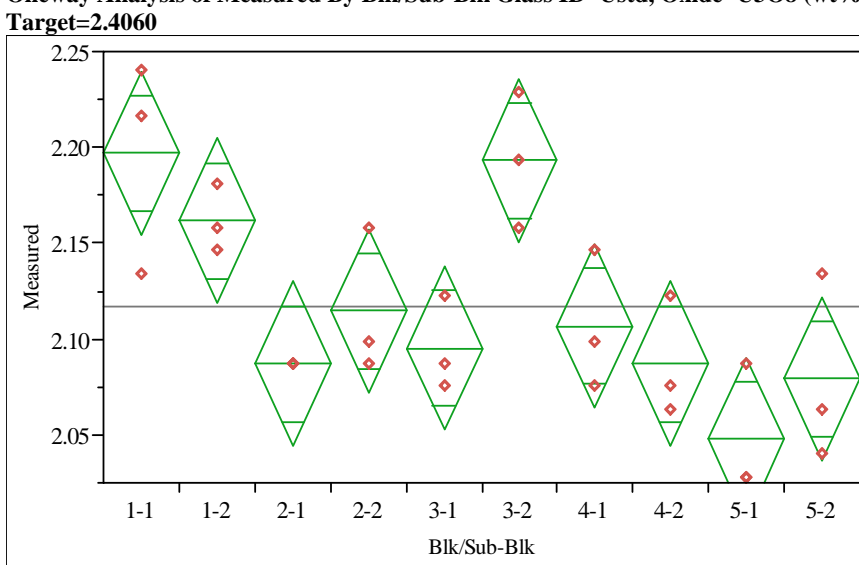

Oneway Anova

Summary of Fit

0.731736

0.611018

Mean of Response 2.117057

Observations (or Sum Wgts) $\quad 30$

Analysis of Variance

Source DF Sum of Squares Mean Square F Ratio Prob $>$ F

$\begin{array}{llll}0.06852446 & 0.007614 & 6.0615 & 0.0004\end{array}$

$\begin{array}{lll}\text { Error } & 20 & 0.02512193 \\ \text { C. Total } & 29 & 0.09364639\end{array}$

\section{Means for Oneway Anova}

Level Number Mean Std Error Lower 95\% Upper 95\%

$\begin{array}{llllrr}1-1 & 3 & 2.19724 & 0.02046 & 2.1546 & 2.2399\end{array}$

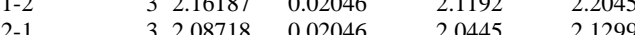

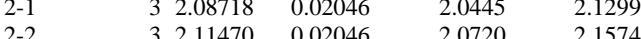

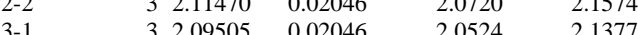

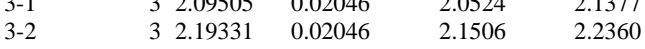

$\begin{array}{llllll}3-2 & 3 & 2.19331 & 0.02046 & 2.1506 & 2.2360 \\ 4-1 & 3 & 2.10684 & 0.02046 & 2.0642 & 2.1495\end{array}$

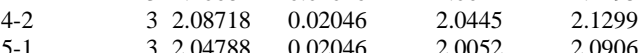

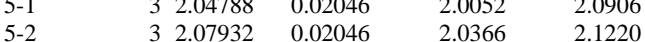

Std Error uses a pooled estimate of error variance 
Exhibit A-6. Average Measured Versus Target Compositions by Glass ID by Oxide
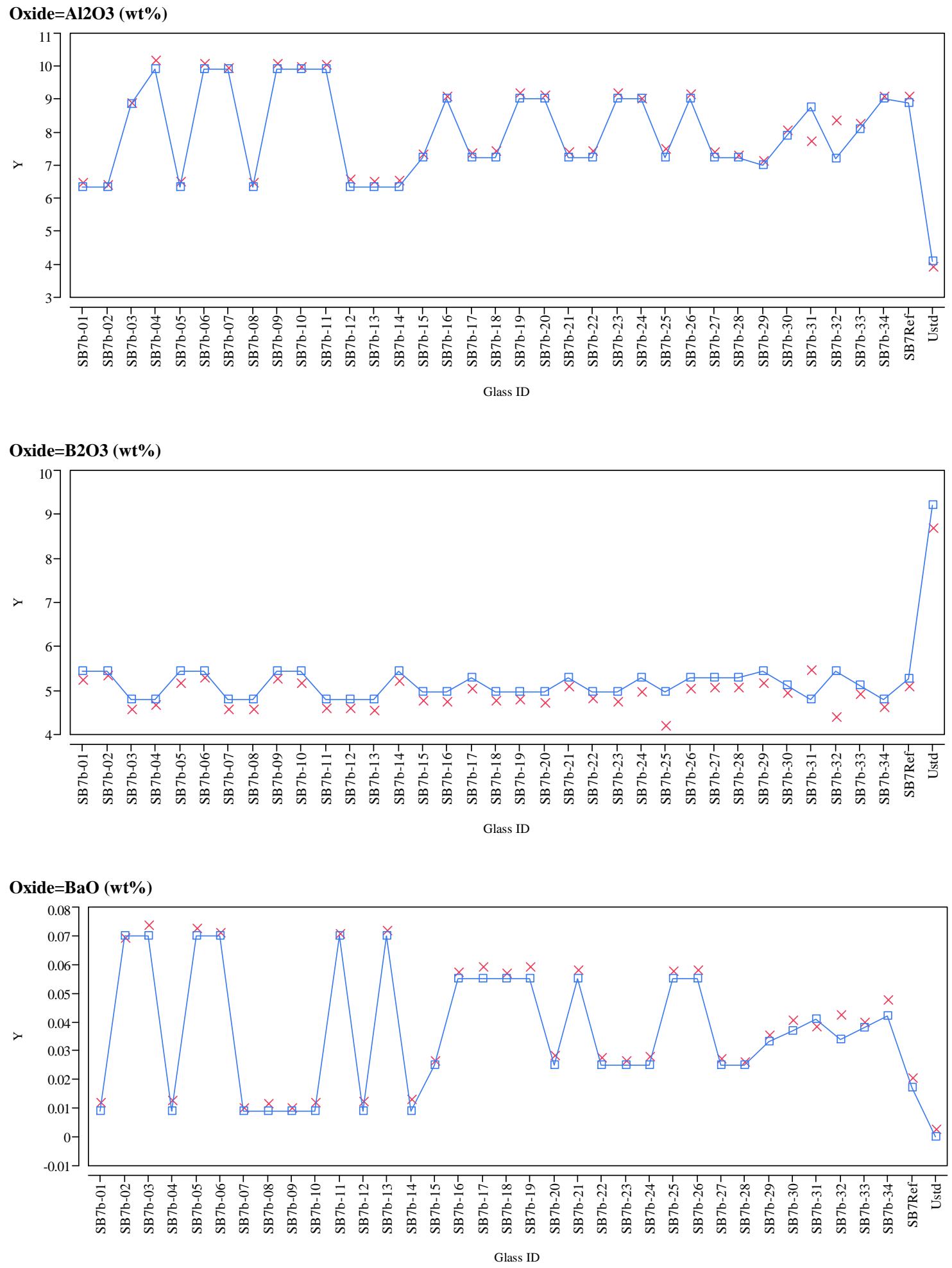

Y X Measured $\square$ - Targeted 
Exhibit A-6. Average Measured Versus Target Compositions by Glass ID by Oxide
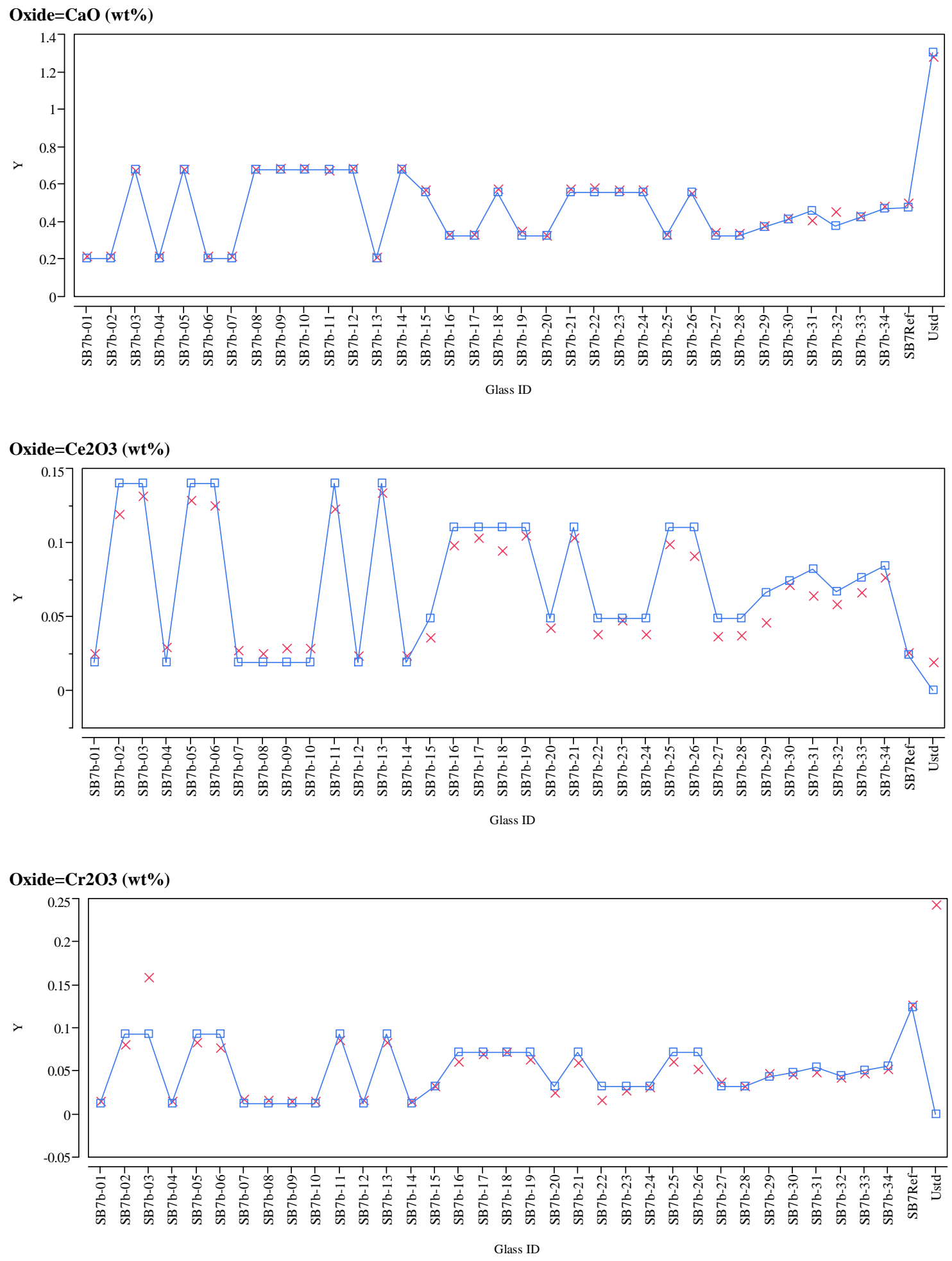

Y $\times$ Measured घ — Targeted 
Exhibit A-6. Average Measured Versus Target Compositions by Glass ID by Oxide
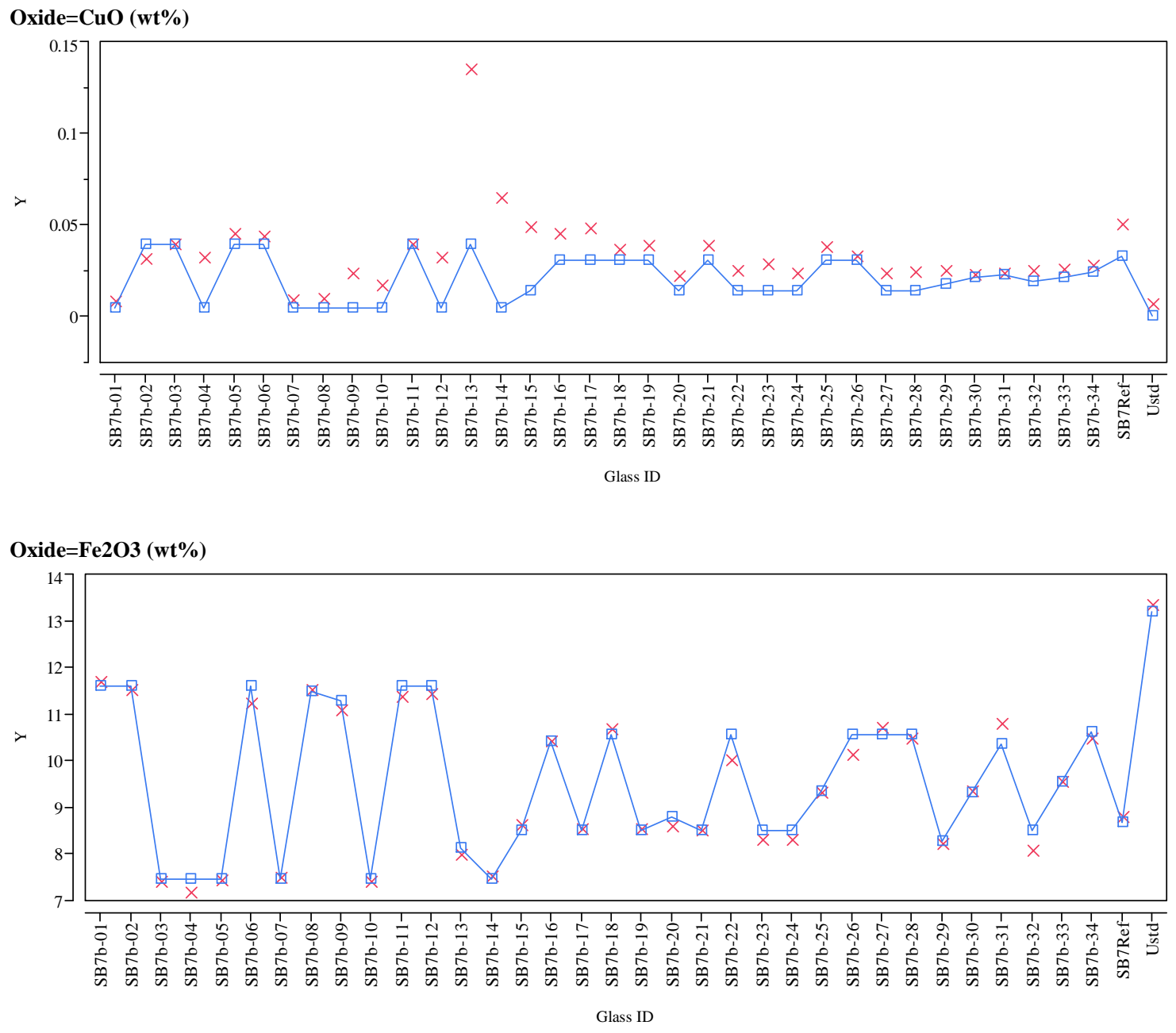

Oxide=K2O ( $w$ t $\%)$

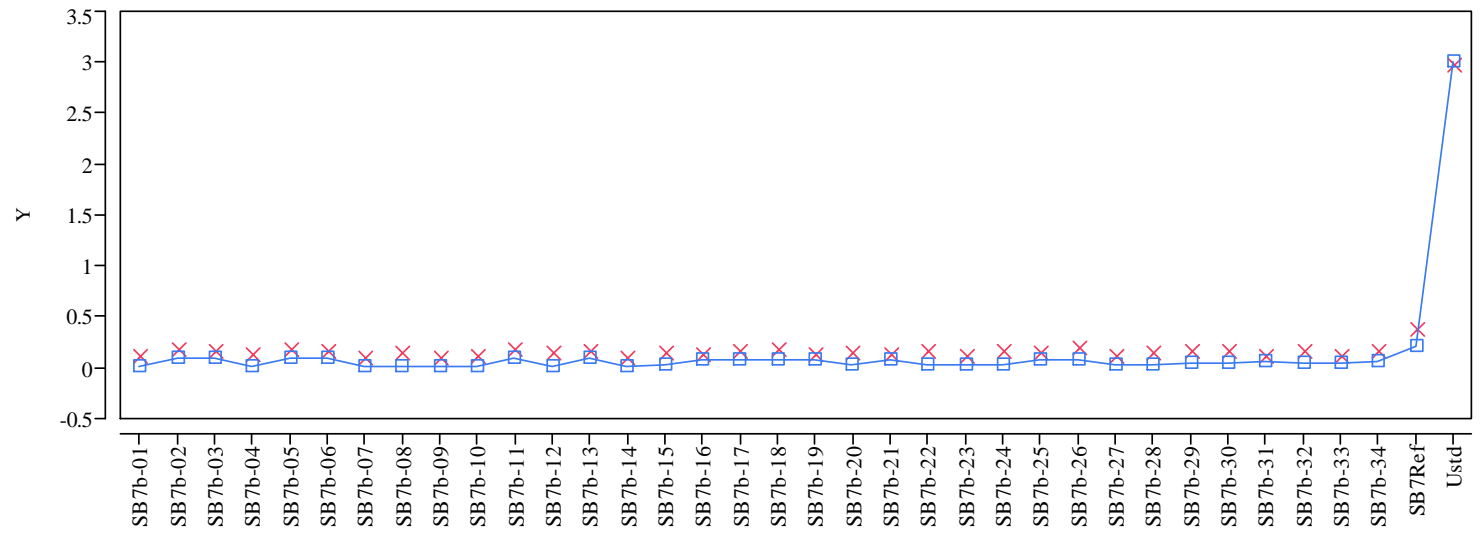

Glass ID

Y $\times$ Measured $\square$ - Targeted 
Exhibit A-6. Average Measured Versus Target Compositions by Glass ID by Oxide
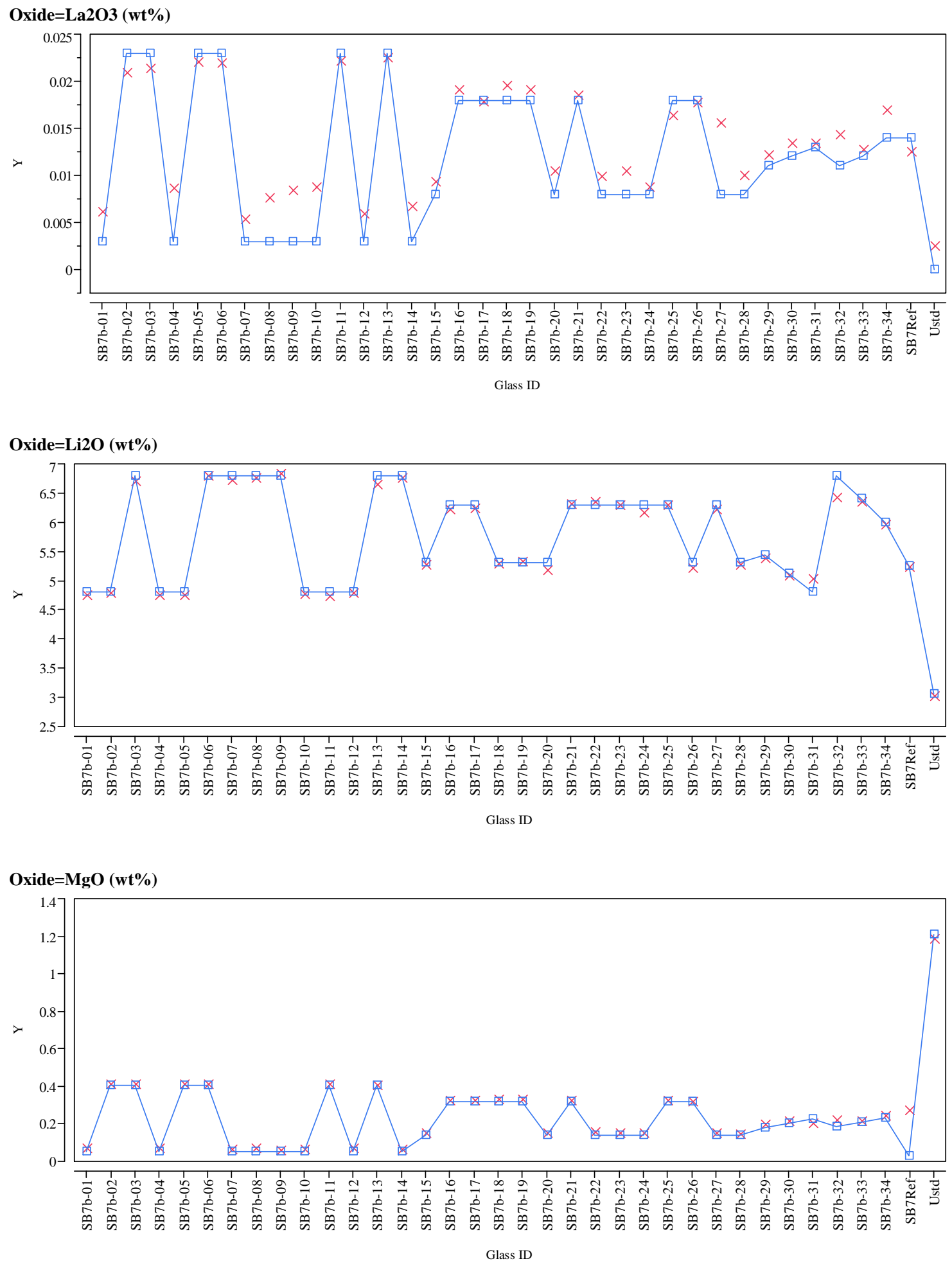

Y $\times$ Measured $\square$ - Targeted 
Exhibit A-6. Average Measured Versus Target Compositions by Glass ID by Oxide
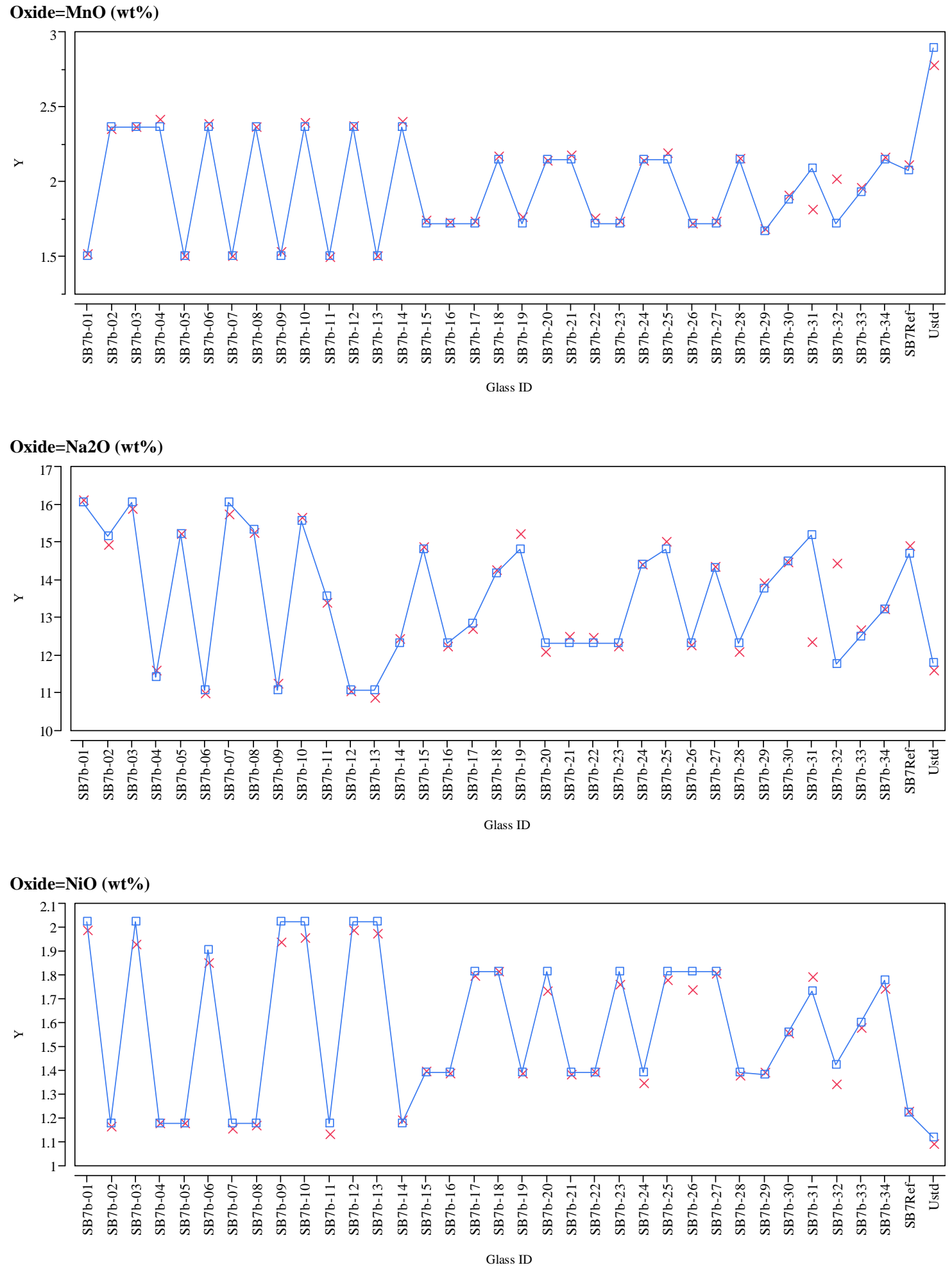

Y $\times$ Measured $\square$ - Targeted 
Exhibit A-6. Average Measured Versus Target Compositions by Glass ID by Oxide
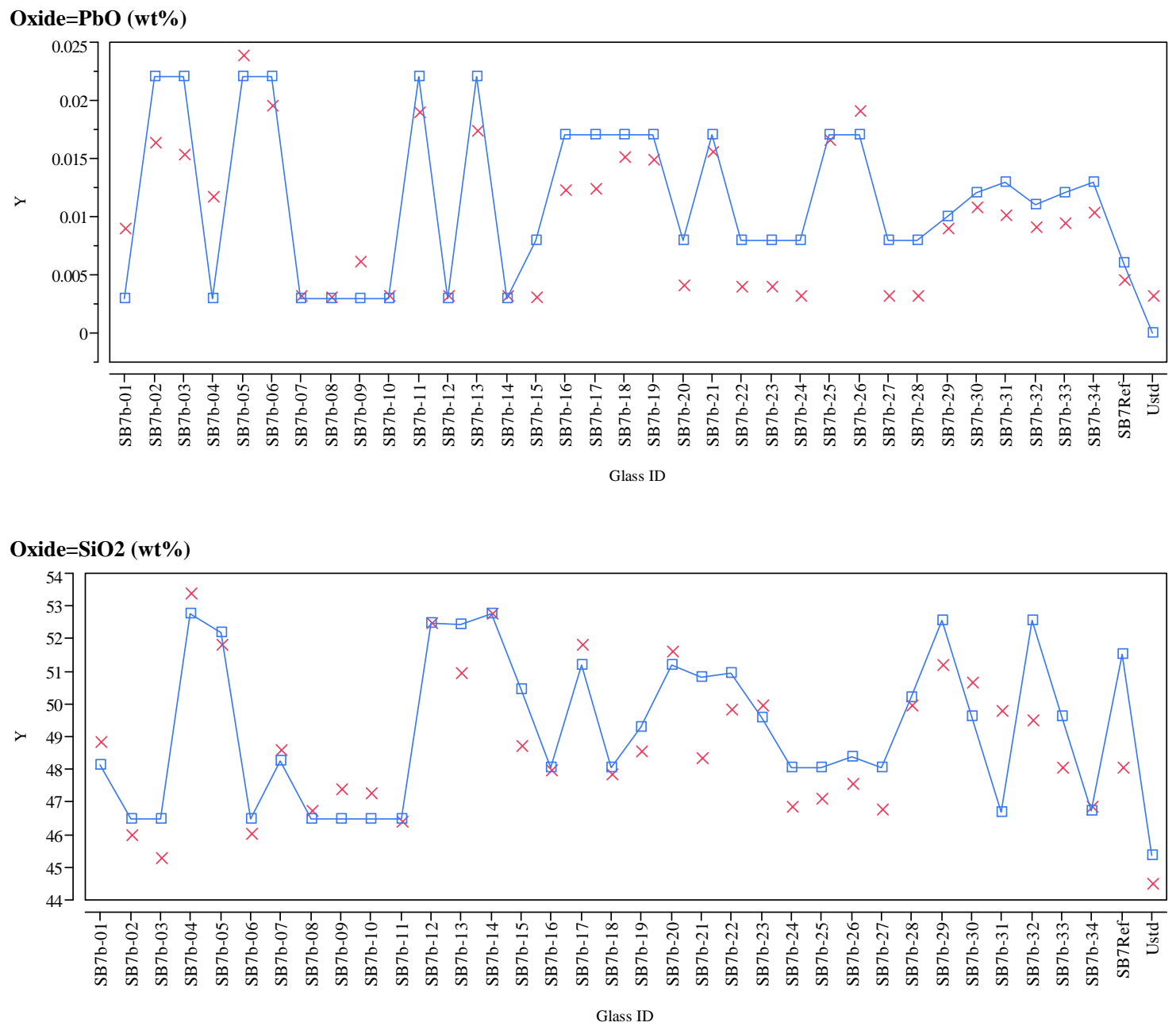

Oxide $=\mathrm{SO} 4$ ( $\mathrm{wt} \%)$

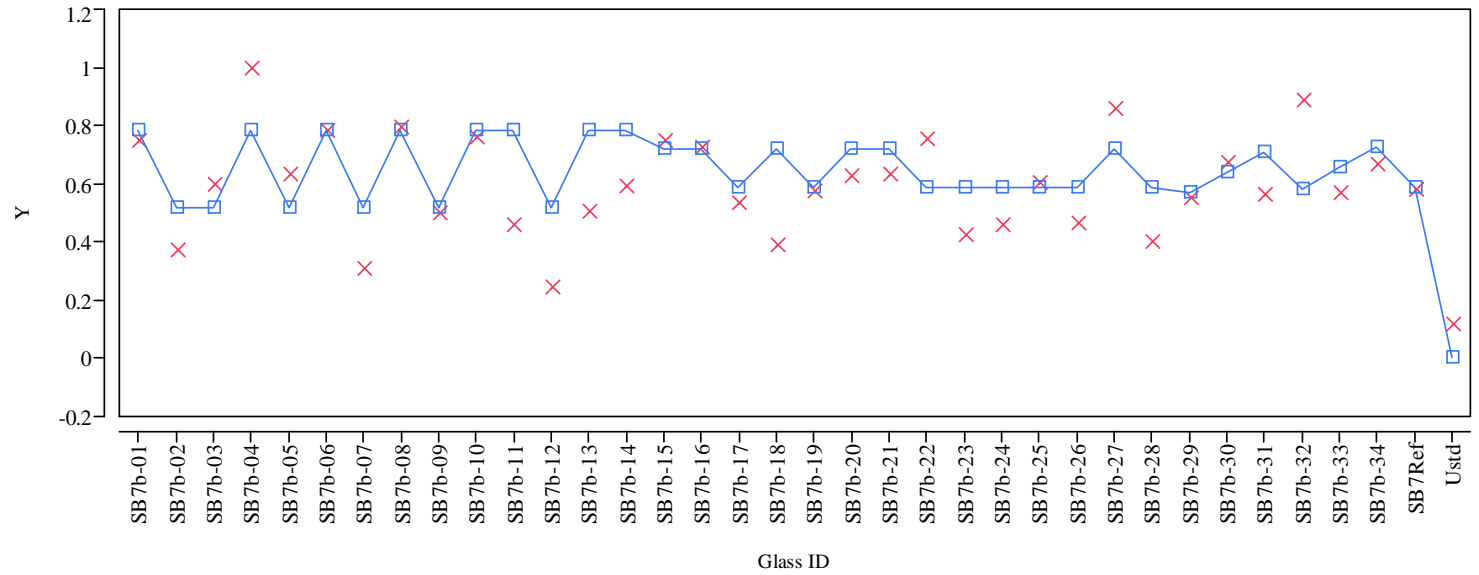

Y $\times$ Measured $\square$ - Targeted 
Exhibit A-6. Average Measured Versus Target Compositions by Glass ID by Oxide
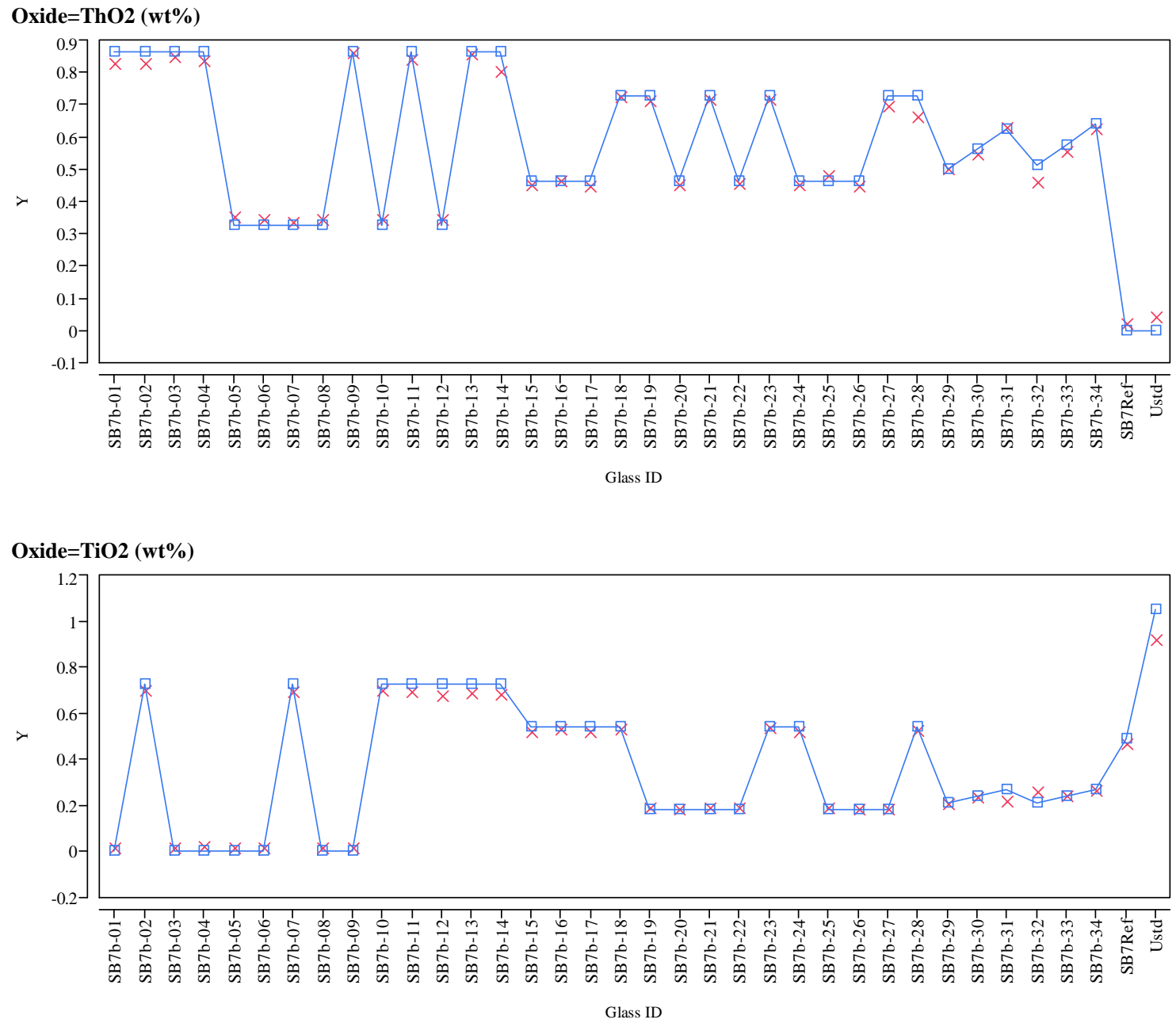

Oxide $=$ U3O8 (wt \%)

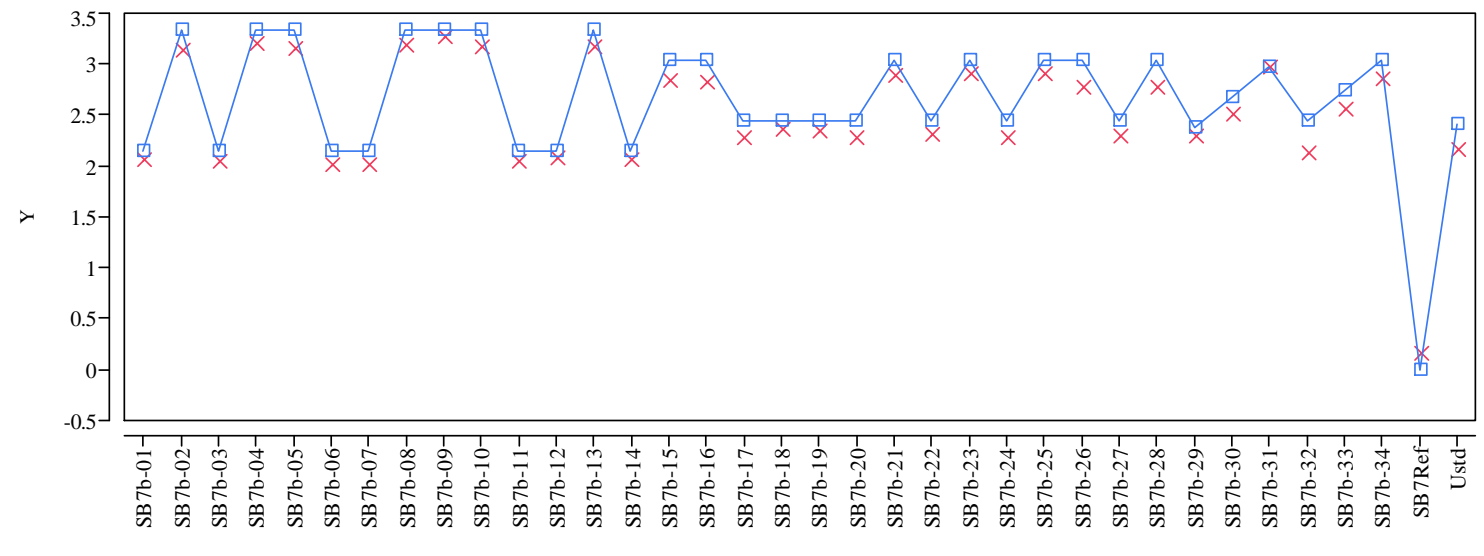

Glass ID

Y $\times$ Measured $\quad$ - Targeted 
Exhibit A-6. Average Measured Versus Target Compositions by Glass ID by Oxide
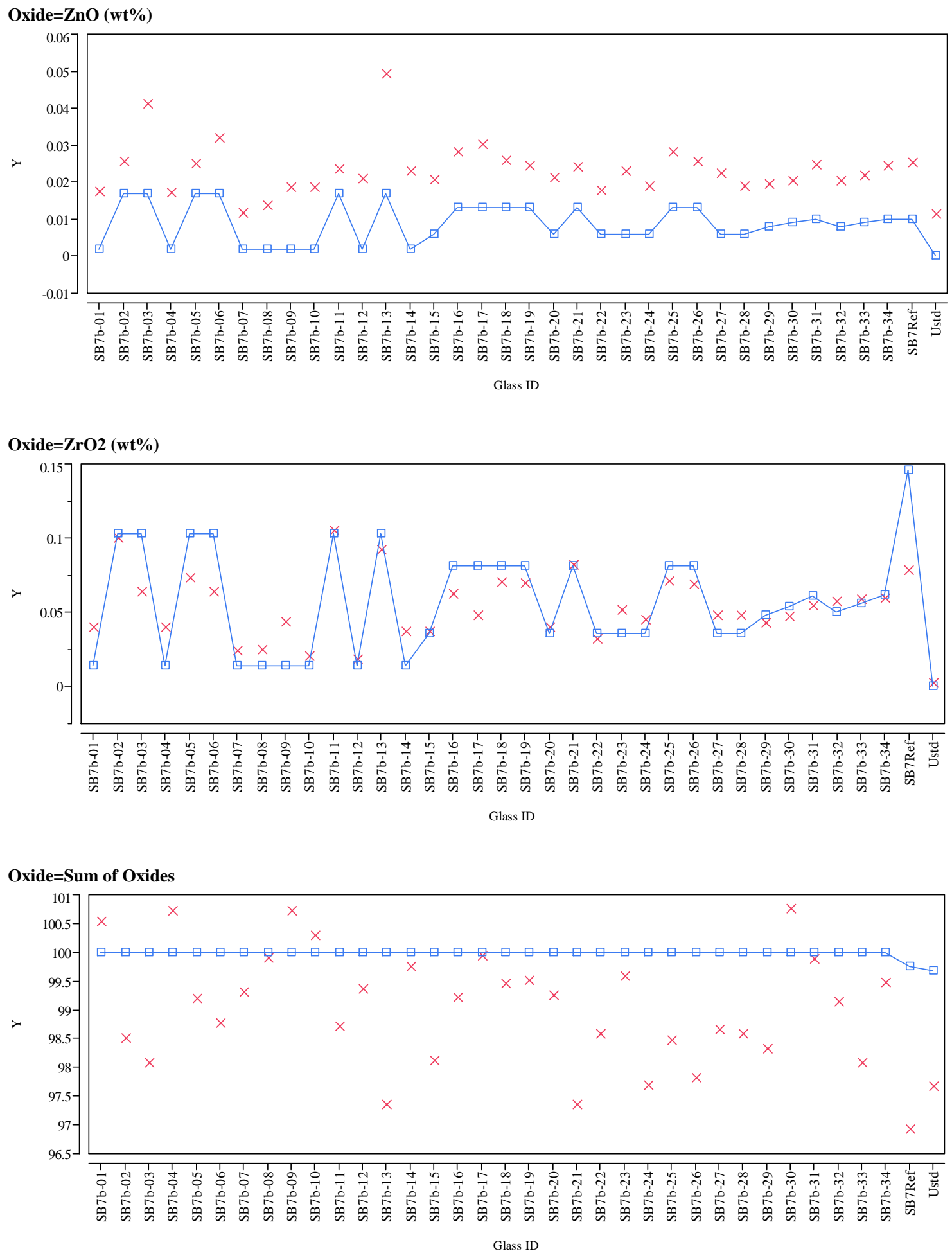

Y x Measured $\square$ - Targeted 
SRNL-STI-2011-00440

Revision 0

Appendix B. Tables and Exhibits Supporting the Analysis of the PCT Results 
Table B-1. As-Received (ar) and Adjusted Measurements of the PCT Solutions (in ppm)

\begin{tabular}{|c|c|c|c|c|c|c|c|c|c|c|c|c|}
\hline Set & Glass ID (w HT) & Block & Seq & Lab ID & B ar & Li ar & Na ar & Si ar & B (ppm) & Li (ppm) & Na (ppm) & Si (ppm) \\
\hline 1 & Soln Std & 1 & 1 & STD_11 & 19.9 & 10 & 82.9 & 52.9 & 19.900 & 10.000 & 82.900 & 52.900 \\
\hline 1 & SB7b-01ccc & 1 & 2 & B084 & 9.25 & 13 & 82.4 & 97.6 & 15.417 & 21.667 & 137.336 & 162.670 \\
\hline 1 & SB7b-09 & 1 & 3 & B071 & 8.14 & 17.1 & 40.4 & 77.5 & 13.567 & 28.501 & 67.335 & 129.169 \\
\hline 1 & SB7b-08ccc & 1 & 4 & B098 & 16.7 & 51.7 & 137 & 196 & 27.834 & 86.168 & 228.338 & 326.673 \\
\hline 1 & SB7b-07 & 1 & 5 & B067 & 8.14 & 20.7 & 107 & 108 & 13.567 & 34.501 & 178.337 & 180.004 \\
\hline 1 & SB7b-14ccc & 1 & 6 & B047 & 9.6 & 30.2 & 59.1 & 144 & 16.000 & 50.334 & 98.502 & 240.005 \\
\hline 1 & blank & 1 & 7 & B072 & $<0.0808$ & $<0.0268$ & $<0.212$ & $<0.176$ & 0.067 & 0.022 & 0.177 & \begin{tabular}{|l|}
0.147 \\
\end{tabular} \\
\hline 1 & SB7b-17ccc & 1 & 8 & B017 & \begin{tabular}{|l|}
7.92 \\
\end{tabular} & \begin{tabular}{|l|}
18.3 \\
\end{tabular} & \begin{tabular}{|l|}
52.8 \\
\end{tabular} & \begin{tabular}{|l|}
95.7 \\
\end{tabular} & 13.200 & 30.501 & 88.002 & \begin{tabular}{|l|}
159.503 \\
\end{tabular} \\
\hline 1 & ARM-1 & 1 & 9 & B079 & 10.4 & 8.32 & 22 & 38.6 & 17.334 & 13.867 & 36.667 & 64.335 \\
\hline 1 & SB7b-01 & 1 & 10 & B021 & 9.23 & 11.7 & 86.8 & 97.4 & 15.384 & 19.500 & 144.670 & 162.337 \\
\hline 1 & Soln Std & 1 & 11 & STD_12 & 19.8 & 9.83 & 82.4 & 52.4 & 19.800 & 9.830 & 82.400 & 52.400 \\
\hline 1 & SB7b-09ccc & 1 & 12 & B089 & 9.33 & 30.5 & 47.6 & 111 & 15.550 & 50.834 & 79.335 & 185.004 \\
\hline 1 & SB7b-04 & 1 & 13 & B038 & 4.39 & 9.08 & 25.3 & 67.8 & 7.317 & 15.134 & 42.168 & 113.002 \\
\hline 1 & SB7b-06ccc & 1 & 14 & B092 & 8.3 & 23 & 44.9 & 98 & 13.834 & 38.334 & 74.835 & 163.337 \\
\hline 1 & SB7b-14 & 1 & 15 & B082 & 8.08 & 18 & 53 & 91.1 & 13.467 & 30.001 & 88.335 & 151.836 \\
\hline 1 & SB7b-06 & 1 & 16 & B020 & 8.22 & 16.6 & 40.7 & 79.1 & 13.700 & 27.667 & 67.835 & 131.836 \\
\hline 1 & SB7b-07ccc & 1 & 17 & B035 & 10.3 & 40.2 & 109 & 165 & 17.167 & 67.001 & 181.670 & 275.006 \\
\hline 1 & SB7b-17 & 1 & 18 & B004 & 7.44 & 15.1 & 53.1 & 90.6 & 12.400 & 25.167 & 88.502 & 151.003 \\
\hline 1 & SB7b-08 & 1 & 19 & B008 & 12.7 & 27.6 & 122 & 126 & 21.167 & 46.001 & 203.337 & 210.004 \\
\hline 1 & SB7b-04ccc & 1 & 20 & B024 & 4.39 & 8.88 & 25.5 & 67.3 & \begin{tabular}{|l|}
7.317 \\
\end{tabular} & 14.800 & 42.501 & 112.169 \\
\hline 1 & EA & 1 & 21 & B046 & 28.4 & 9.37 & 81 & 49.2 & 473.334 & 156.167 & 1350.003 & 820.002 \\
\hline 1 & Soln Std & 1 & 22 & STD_13 & 19.8 & 9.79 & 82.4 & 52.1 & 19.800 & 9.790 & 82.400 & 52.100 \\
\hline 1 & Soln Std & 2 & 1 & STD_21 & 19.7 & 9.91 & 83.3 & 52.1 & 19.700 & 9.910 & 83.300 & 52.100 \\
\hline 1 & SB7b-08ccc & 2 & 2 & \begin{tabular}{|l|} 
B060 \\
\end{tabular} & 17 & 51.9 & 140 & 188 & 28.334 & 86.502 & 233.338 & 313.340 \\
\hline 1 & SB7b-01ccc & 2 & 3 & B009 & 9.38 & 13.1 & 84.6 & 94.7 & 15.634 & 21.834 & 141.003 & 157.836 \\
\hline 1 & SB7b-04 & 2 & 4 & B039 & 4.45 & 9.23 & 25.6 & 68.7 & 7.417 & 15.384 & 42.668 & 114.502 \\
\hline 1 & SB7b-04ccc & 2 & 5 & B016 & 4.44 & 9.06 & 26 & 68.5 & 7.400 & 15.100 & 43.334 & 114.169 \\
\hline 1 & ARM-1 & 2 & 6 & B068 & 10.6 & 8.53 & 22.9 & 38.9 & 17.667 & 14.217 & 38.167 & 64.835 \\
\hline 1 & SB7b-09ccc & 2 & 7 & B053 & 9.38 & 30.9 & 48.4 & 113 & 15.634 & 51.501 & 80.668 & \begin{tabular}{|l|}
188.337 \\
\end{tabular} \\
\hline 1 & SB7b-06 & 2 & 8 & B096 & 8.21 & 16.6 & 40.9 & 78.7 & 13.684 & 27.667 & 68.168 & \begin{tabular}{|l|}
131.169 \\
\end{tabular} \\
\hline 1 & SB7b-14 & 2 & 9 & B003 & 8.26 & 19.1 & 55.3 & 93.9 & 13.767 & 31.834 & 92.169 & 156.503 \\
\hline 1 & EA & 2 & 10 & B051 & 29.4 & 9.45 & 85.1 & 49.7 & \begin{tabular}{|l|}
490.001 \\
\end{tabular} & 157.500 & 1418.336 & 828.335 \\
\hline 1 & Soln Std & 2 & 11 & STD_22 & 19.9 & 9.84 & 83.2 & 52.3 & \begin{tabular}{|l|}
19.900 \\
\end{tabular} & 9.840 & 83.200 & 52.300 \\
\hline 1 & SB7b-09 & 2 & 12 & B010 & 8.11 & 16.9 & 40.7 & 76.5 & 13.517 & 28.167 & 67.835 & 127.503 \\
\hline 1 & SB7b-07ccc & 2 & 13 & B011 & 10.3 & 40.4 & 109 & 176 & 17.167 & 67.335 & 181.670 & \begin{tabular}{|l|}
293.339 \\
\end{tabular} \\
\hline 1 & SB7b-07 & 2 & 14 & B018 & 8.13 & 20.6 & 108 & 108 & 13.550 & 34.334 & 180.004 & 180.004 \\
\hline 1 & SB7b-14ccc & 2 & 15 & B076 & 9.58 & 30.2 & 59.8 & 134 & 15.967 & 50.334 & 99.669 & 223.338 \\
\hline 1 & SB7b-08 & 2 & 16 & $\mathrm{~B} 102$ & 12.7 & 27.7 & 123 & 124 & 21.167 & 46.168 & 205.004 & \begin{tabular}{|l|}
206.671 \\
\end{tabular} \\
\hline 1 & SB7b-17ccc & 2 & 17 & B081 & 7.89 & 18.4 & 53.1 & 92.5 & 13.150 & 30.667 & 88.502 & \begin{tabular}{|l|}
154.170 \\
\end{tabular} \\
\hline 1 & SB7b-01 & 2 & 18 & B056 & 9.39 & 12.3 & 90.2 & 91.7 & 15.650 & 20.500 & 150.336 & 152.836 \\
\hline 1 & SB7b-17 & 2 & 19 & B026 & 7.35 & 15.2 & 53.2 & 84.7 & 12.250 & 25.334 & 88.668 & \begin{tabular}{|l|}
141.169 \\
\end{tabular} \\
\hline 1 & SB7b-06ccc & 2 & 20 & B057 & 8.15 & 22.7 & 44.5 & 96.3 & 13.584 & 37.834 & 74.168 & 160.503 \\
\hline 1 & Soln Std & 2 & 21 & STD_23 & 19.8 & 9.87 & 83.5 & 52.3 & 19.800 & 9.870 & 83.500 & 52.300 \\
\hline 1 & Soln Std & 3 & 1 & STD_31 & 19.9 & 9.87 & 83.5 & 52.2 & 19.900 & 9.870 & 83.500 & 52.200 \\
\hline 1 & SB7b-01 & 3 & 2 & \begin{tabular}{|l|} 
B001 \\
\end{tabular} & 9.45 & 12.4 & 91.1 & 93.3 & 15.750 & 20.667 & 151.836 & 155.503 \\
\hline 1 & SB7b-09 & 3 & 3 & B041 & 8 & 16.8 & 40.1 & 75.7 & 13.334 & 28.001 & 66.835 & 126.169 \\
\hline 1 & SB7b-09ccc & 3 & 4 & B027 & 15.6 & 51 & 80.6 & 199 & 26.001 & 85.002 & 134.336 & 331.673 \\
\hline 1 & ARM-1 & 3 & 5 & B097 & 10.6 & 8.53 & 22.9 & 38.9 & 17.667 & 14.217 & 38.167 & 64.835 \\
\hline 1 & SB7b-14ccc & 3 & 6 & B058 & 9.53 & 30.1 & 59.5 & 124 & 15.884 & 50.168 & 99.169 & \begin{tabular}{|l|}
206.671 \\
\end{tabular} \\
\hline 1 & SB7b-08ccc & 3 & 7 & B028 & 17 & 51.7 & 141 & 192 & 28.334 & 86.168 & 235.005 & 320.006 \\
\hline 1 & SB7b-14 & 3 & 8 & B014 & 8.32 & 19 & 55.3 & 93.9 & 13.867 & 31.667 & 92.169 & \begin{tabular}{|l|}
156.503 \\
\end{tabular} \\
\hline 1 & EA & 3 & 9 & B012 & 29.5 & 9.57 & 85.3 & 49.9 & \begin{tabular}{|l|}
491.668 \\
\end{tabular} & 159.500 & \begin{tabular}{|l|}
1421.670 \\
\end{tabular} & \begin{tabular}{|l|}
831.668 \\
\end{tabular} \\
\hline 1 & SB7b-17ccc & 3 & 10 & B049 & 7.96 & 18.5 & 53.4 & 96 & 13.267 & 30.834 & 89.002 & 160.003 \\
\hline 1 & Soln Std & 3 & 11 & STD_32 & 19.9 & 9.97 & 83.7 & 52.4 & 19.900 & 9.970 & 83.700 & 52.400 \\
\hline 1 & SB7b-06ccc & 3 & 12 & B043 & 8.14 & 22.7 & 44.1 & 93.9 & 13.567 & 37.834 & 73.501 & 156.503 \\
\hline 1 & SB7b-06 & 3 & 13 & B044 & 8.21 & 16.8 & 41.4 & 77.5 & 13.684 & 28.001 & 69.001 & \begin{tabular}{|l|}
129.169 \\
\end{tabular} \\
\hline 1 & SB7b-07 & 3 & 14 & B073 & 7.94 & 20.3 & 105 & 103 & 13.234 & 33.834 & 175.004 & 171.670 \\
\hline 1 & SB7b-08 & 3 & 15 & B103 & 12.7 & 27.8 & 123 & 124 & 21.167 & 46.334 & 205.004 & \begin{tabular}{|l|}
206.671 \\
\end{tabular} \\
\hline 1 & SB7b-01ccc & 3 & 16 & B036 & 9.39 & 13.2 & 85.4 & 91.8 & 15.650 & 22.000 & 142.336 & 153.003 \\
\hline 1 & SB7b-07ccc & 3 & 17 & B029 & 10.2 & 40.1 & 110 & 144 & 17.000 & 66.835 & 183.337 & 240.005 \\
\hline 1 & SB7b-17 & 3 & 18 & B109 & 7.33 & 15.1 & 53.2 & 84.5 & 12.217 & 25.167 & 88.668 & 140.836 \\
\hline 1 & SB7b-04 & 3 & 19 & B074 & 4.3 & 9.2 & 25.5 & 65.8 & 7.167 & 15.334 & 42.501 & \begin{tabular}{|l|}
109.669 \\
\end{tabular} \\
\hline 1 & SB7b-04ccc & 3 & 20 & B104 & 4.36 & 8.97 & 25.8 & 66.7 & 7.267 & 14.950 & 43.001 & \begin{tabular}{|l|}
111.169 \\
\end{tabular} \\
\hline 1 & Soln Std & 3 & 21 & STD_33 & 19.7 & 9.87 & 83.5 & 52.1 & 19.700 & 9.870 & 83.500 & 52.100 \\
\hline 1 & Soln Std & 4 & 1 & STD_41 & 19.7 & 10.1 & 82.9 & 51.7 & 19.700 & 10.100 & 82.900 & 51.700 \\
\hline 1 & SB7b-03 & 4 & 2 & \begin{tabular}{|l|} 
B088 \\
\end{tabular} & 9.91 & 24 & 121 & 103 & 16.517 & 40.001 & 201.671 & 171.670 \\
\hline 1 & SB7b-15 & 4 & 3 & B013 & 7.04 & 13.6 & 71.4 & 87.6 & 11.734 & 22.667 & 119.002 & 146.003 \\
\hline 1 & SB7b-10 & 4 & 4 & B075 & 7.1 & 9.91 & 68.6 & 72.7 & 11.834 & 16.517 & 114.336 & 121.169 \\
\hline 1 & SB7b-15ccc & 4 & 5 & B090 & 6.9 & 13.8 & 65.3 & 85.8 & 11.500 & 23.000 & 108.836 & \begin{tabular}{|l|}
143.003 \\
\end{tabular} \\
\hline 1 & SB7b-13 & 4 & 6 & B087 & 6.76 & 17 & 40.9 & 85.5 & 11.267 & 28.334 & 68.168 & 142.503 \\
\hline 1 & SB7b-10ccc & 4 & 7 & B007 & 7.25 & 11 & 66.5 & 72.8 & 12.084 & 18.334 & 110.836 & \begin{tabular}{|l}
121.336 \\
\end{tabular} \\
\hline 1 & SB7b-16ccc & 4 & 8 & B050 & 6.71 & 15.6 & 44.3 & 79.5 & 11.184 & 26.001 & 73.835 & 132.503 \\
\hline
\end{tabular}


Table B-1. As-Received (ar) and Adjusted Measurements of the PCT Solutions (in ppm)

\begin{tabular}{|c|c|c|c|c|c|c|c|c|c|c|c|c|}
\hline Set & Glass ID (w HT) & Block & Seq & Lab ID & B ar & Li ar & Na ar & Si ar & B (ppm) & Li (ppm) & Na (ppm) & Si (ppm) \\
\hline 1 & SB7b-12 & 4 & 9 & B065 & 5.61 & 10.4 & 31.9 & 72.9 & 9.350 & \begin{tabular}{|l|}
17.334 \\
\end{tabular} & 53.168 & 121.502 \\
\hline 1 & SB7b-05 & 4 & 10 & B019 & 7.3 & 11.3 & 69.7 & 81.3 & 12.167 & 18.834 & 116.169 & 135.503 \\
\hline 1 & Soln Std & 4 & 11 & STD_42 & 19.5 & 9.9 & 82.4 & 51 & 19.500 & 9.900 & 82.400 & 51.000 \\
\hline 1 & SB7b-02ccc & 4 & 12 & B062 & 9.24 & 13.1 & 71.9 & 81.4 & 15.400 & 21.834 & 119.836 & 135.669 \\
\hline 1 & SB7b-05ccc & 4 & 13 & B083 & 7.22 & 11.5 & 65.4 & 80.7 & 12.034 & 19.167 & 109.002 & 134.503 \\
\hline 1 & SB7b-12ccc & 4 & 14 & B094 & 5.64 & 10.3 & 31.9 & 72.3 & 9.400 & 17.167 & 53.168 & 120.502 \\
\hline 1 & SB7b-02 & 4 & 15 & B037 & 9.36 & 12.7 & 78.1 & 82.1 & 15.600 & 21.167 & 130.169 & 136.836 \\
\hline 1 & SB7b-11ccc & 4 & 16 & B078 & 5.67 & 9.83 & 44.5 & 63.1 & 9.450 & 16.384 & 74.168 & 105.169 \\
\hline 1 & SB7b-16 & 4 & 17 & B033 & 6.54 & 14.1 & 44.7 & 75.6 & 10.900 & 23.500 & 74.501 & 126.003 \\
\hline 1 & SB7b-11 & 4 & 18 & B069 & 5.9 & 10.1 & 47.1 & 63.6 & 9.834 & 16.834 & 78.502 & 106.002 \\
\hline 1 & SB7b-03ccc & 4 & 19 & B059 & 14.4 & 44.8 & 128 & 146 & 24.000 & 74.668 & 213.338 & 243.338 \\
\hline 1 & SB7b-13ccc & 4 & 20 & B093 & 8.52 & 27 & 48.9 & 116 & 14.200 & 45.001 & 81.502 & 193.337 \\
\hline 1 & Soln Std & 4 & 21 & STD_43 & 19.5 & 9.95 & 82.5 & 51.2 & 19.500 & 9.950 & 82.500 & 51.200 \\
\hline 1 & Soln Std & 5 & 1 & STD_51 & 19.8 & 9.82 & 83 & 52.2 & 19.800 & 9.820 & 83.000 & 52.200 \\
\hline 1 & SB7b-03ccc & 5 & 2 & \begin{tabular}{|l|} 
B040 \\
\end{tabular} & 14.6 & 46.8 & 128 & 152 & 24.334 & 78.002 & 213.338 & 253.338 \\
\hline 1 & SB7b-10ccc & 5 & 3 & B095 & 7.46 & 11.1 & 68 & 75.2 & 12.434 & 18.500 & 113.336 & 125.336 \\
\hline 1 & SB7b-15ccc & 5 & 4 & B077 & 6.89 & 13.3 & 64.6 & 86.5 & 11.484 & 22.167 & 107.669 & 144.170 \\
\hline 1 & SB7b-10 & 5 & 5 & B031 & 7.34 & 10.3 & 71.4 & 73.9 & 12.234 & 17.167 & 119.002 & 123.169 \\
\hline 1 & SB7b-11 & 5 & 6 & B105 & 5.99 & 10 & 47.9 & 65.9 & 9.984 & 16.667 & 79.835 & 109.836 \\
\hline 1 & SB7b-15 & 5 & 7 & B064 & 6.95 & 13.2 & 71.2 & 88.6 & 11.584 & 22.000 & 118.669 & 147.670 \\
\hline 1 & SB7b-16ccc & 5 & 8 & B005 & 7 & 16.3 & 46 & 82.7 & 11.667 & 27.167 & 76.668 & 137.836 \\
\hline 1 & SB7b-05 & 5 & 9 & B070 & 7.59 & 11.4 & 72.1 & 86 & 12.650 & 19.000 & 120.169 & 143.336 \\
\hline 1 & SB7b-13ccc & 5 & 10 & B055 & 8.65 & 27.6 & 49.6 & 117 & 14.417 & 46.001 & 82.668 & 195.004 \\
\hline 1 & Soln Std & 5 & 11 & \begin{tabular}{|l|} 
STD_52 \\
\end{tabular} & 19.6 & 9.75 & 82.6 & 52.1 & 19.600 & 9.750 & 82.600 & 52.100 \\
\hline 1 & SB7b-02 & 5 & 12 & B100 & 9.59 & 12.7 & 79.4 & 84.9 & 15.984 & 21.167 & 132.336 & 141.503 \\
\hline 1 & SB7b-11ccc & 5 & 13 & B030 & 5.52 & 9.31 & 43.5 & 62.5 & 9.200 & 15.517 & 72.501 & 104.169 \\
\hline 1 & SB7b-05ccc & 5 & 14 & B032 & 7.01 & 10.9 & 62.9 & 80.2 & 11.684 & 18.167 & 104.835 & 133.669 \\
\hline 1 & SB7b-16 & 5 & 15 & B061 & 6.69 & 14.2 & 45.2 & 76.1 & 11.150 & 23.667 & 75.335 & 126.836 \\
\hline 1 & SB7b-12 & 5 & 16 & B080 & 5.77 & 10.3 & 32.5 & 74.6 & 9.617 & 17.167 & 54.168 & 124.336 \\
\hline 1 & SB7b-13 & 5 & 17 & B048 & 6.96 & 17.8 & 42.2 & 90.6 & 11.600 & 29.667 & 70.335 & 151.003 \\
\hline 1 & SB7b-12ccc & 5 & 18 & B042 & 5.64 & 10.2 & 32.1 & 74.8 & 9.400 & 17.000 & 53.501 & 124.669 \\
\hline 1 & SB7b-02ccc & 5 & 19 & B091 & 8.89 & 12.3 & 69.7 & 80.9 & 14.817 & 20.500 & 116.169 & 134.836 \\
\hline 1 & SB7b-03 & 5 & 20 & B066 & 9.98 & 24.4 & 123 & 105 & 16.634 & 40.667 & 205.004 & 175.004 \\
\hline 1 & Soln Std & 5 & 21 & STD_53 & 19.8 & 9.94 & 83.6 & 52.6 & 19.800 & 9.940 & 83.600 & 52.600 \\
\hline 1 & Soln Std & 6 & 1 & STD_61 & 19.7 & 9.79 & 82.8 & 52 & 19.700 & 9.790 & 82.800 & 52.000 \\
\hline 1 & SB7b-15ccc & 6 & 2 & \begin{tabular}{|l|} 
B099 \\
\end{tabular} & 6.9 & 13.4 & 64.9 & 102 & 11.500 & 22.334 & 108.169 & 170.003 \\
\hline 1 & SB7b-02 & 6 & 3 & B106 & 9.42 & 12.5 & 78.3 & 86.6 & 15.700 & 20.834 & 130.503 & 144.336 \\
\hline 1 & SB7b-16 & 6 & 4 & B107 & 6.62 & 14 & 44.9 & 82.3 & 11.034 & 23.334 & 74.835 & 137.169 \\
\hline 1 & SB7b-05 & 6 & 5 & B054 & 7.38 & 11.1 & 70.6 & 87.5 & 12.300 & 18.500 & 117.669 & 145.836 \\
\hline 1 & SB7b-10 & 6 & 6 & B063 & 7.34 & 10.4 & 71.6 & 76.2 & 12.234 & 17.334 & 119.336 & 127.003 \\
\hline 1 & SB7b-10ccc & 6 & 7 & B101 & 7.28 & 10.9 & 66.7 & 74.7 & 12.134 & 18.167 & 111.169 & 124.502 \\
\hline 1 & SB7b-03 & 6 & 8 & B034 & 10.2 & 25 & 127 & 109 & 17.000 & 41.668 & 211.671 & 181.670 \\
\hline 1 & SB7b-16ccc & 6 & 9 & B006 & 7.12 & 16.5 & 46.8 & 82.6 & 11.867 & 27.501 & 78.002 & 137.669 \\
\hline 1 & SB7b-15 & 6 & 10 & B045 & 6.99 & 13.1 & 71.5 & 90.1 & 11.650 & 21.834 & 119.169 & 150.170 \\
\hline 1 & Soln Std & 6 & 11 & \begin{tabular}{|l|} 
STD_62 \\
\end{tabular} & 19.7 & 9.76 & 82.4 & 52 & 19.700 & 9.760 & 82.400 & 52.000 \\
\hline 1 & SB7b-05ccc & 6 & 12 & B108 & 7.1 & 11 & 63.4 & 81 & 11.834 & 18.334 & 105.669 & 135.003 \\
\hline 1 & SB7b-02ccc & 6 & 13 & B086 & 9.01 & 12.3 & 70.6 & 82.2 & 15.017 & 20.500 & 117.669 & 137.003 \\
\hline 1 & SB7b-11ccc & 6 & 14 & B002 & 5.62 & 9.53 & 44 & 63.7 & 9.367 & 15.884 & 73.335 & 106.169 \\
\hline 1 & SB7b-13 & 6 & 15 & B052 & 6.92 & 17.6 & 42.1 & 89.4 & 11.534 & 29.334 & 70.168 & 149.003 \\
\hline 1 & SB7b-12 & 6 & 16 & B015 & 5.81 & 10.3 & 32.7 & 74.6 & 9.684 & 17.167 & 54.501 & 124.336 \\
\hline 1 & SB7b-13ccc & 6 & 17 & B085 & 8.51 & 27.2 & 49.2 & 120 & 14.184 & 45.334 & 82.002 & 200.004 \\
\hline 1 & SB7b-03ccc & 6 & 18 & B110 & 14.6 & 46.4 & 130 & 159 & 24.334 & 77.335 & 216.671 & 265.005 \\
\hline 1 & SB7b-12ccc & 6 & 19 & B025 & 5.77 & \begin{tabular}{|l|}
10.2 \\
\end{tabular} & 32.2 & \begin{tabular}{|l|}
74.3 \\
\end{tabular} & 9.617 & 17.000 & 53.668 & 123.836 \\
\hline 1 & blank & 6 & 20 & B023 & $<0.0808$ & \begin{tabular}{|c|}
$<0.0268$ \\
\end{tabular} & $<0.212$ & $<0.176$ & 0.067 & 0.022 & 0.177 & 0.147 \\
\hline 1 & SB7b-11 & 6 & 21 & B022 & 5.97 & 9.98 & \begin{tabular}{|l|}
47.9 \\
\end{tabular} & \begin{tabular}{|l|}
65.6 \\
\end{tabular} & 9.950 & 16.634 & 79.835 & 109.336 \\
\hline 1 & Soln Std & 6 & 22 & \begin{tabular}{|l|} 
STD_63 \\
\end{tabular} & 19.7 & 9.84 & 83.4 & 52.4 & 19.700 & 9.840 & 83.400 & 52.400 \\
\hline 2 & Soln Std & 1 & 1 & STD_11 & 19.8 & 9.98 & 82.9 & 52.1 & 19.800 & 9.980 & 82.900 & 52.100 \\
\hline 2 & SB7b-28 & 1 & 2 & \begin{tabular}{|l|}
$\mathrm{C} 036$ \\
\end{tabular} & 7.68 & 12.7 & 46.7 & 78.6 & 12.800 & 21.167 & 77.835 & 131.003 \\
\hline 2 & ARM-1 & 1 & 3 & $\mathrm{C} 022$ & 11.5 & 9.16 & 24.6 & 40.8 & 19.167 & 15.267 & 41.001 & 68.001 \\
\hline 2 & SB7b-31 & 1 & 4 & C067 & 7.58 & 11.5 & 43.7 & 72.8 & 12.634 & 19.167 & 72.835 & 121.336 \\
\hline 2 & EA & 1 & 5 & C057 & 19.6 & 7.16 & 58.7 & 38.5 & 326.667 & 119.334 & 978.335 & 641.668 \\
\hline 2 & SB7b-32 & 1 & 6 & C088 & 7.73 & 20.4 & 86 & 102 & 12.884 & 34.001 & 143.336 & 170.003 \\
\hline 2 & SB7b-30 & 1 & 7 & $\mathrm{C} 080$ & 7.5 & \begin{tabular}{|l|}
12.9 \\
\end{tabular} & \begin{tabular}{|l|}
69.5 \\
\end{tabular} & \begin{tabular}{|l|}
87.5 \\
\end{tabular} & 12.500 & 21.500 & 115.836 & 145.836 \\
\hline 2 & blank & 1 & 8 & C055 & $<0.0808$ & $<0.0268$ & $<0.212$ & $<0.176$ & 0.067 & 0.022 & 0.177 & 0.147 \\
\hline 2 & SB7b-33 & 1 & 9 & C056 & 7.69 & \begin{tabular}{|l|}
17.1 \\
\end{tabular} & 54.1 & \begin{tabular}{|l|}
86.9 \\
\end{tabular} & 12.817 & 28.501 & 90.168 & 144.836 \\
\hline 2 & SB7b-28ccc & 1 & 10 & \begin{tabular}{|l|}
$\mathrm{C} 079$ \\
\end{tabular} & 7.31 & 13.8 & 44.7 & 81.8 & 12.184 & 23.000 & 74.501 & 136.336 \\
\hline 2 & Soln Std & 1 & 11 & STD_12 & 19.7 & 10 & 82.9 & 52 & 19.700 & 10.000 & 82.900 & 52.000 \\
\hline 2 & SB7b-22ccc & 1 & 12 & C070 & 7.54 & 19.8 & 51.6 & 95.7 & 12.567 & 33.001 & 86.002 & 159.503 \\
\hline 2 & SB7b-22 & 1 & 13 & $\mathrm{C} 013$ & 7.59 & 18 & 55.5 & 95 & 12.650 & 30.001 & 92.502 & 158.337 \\
\hline 2 & SB7b-25 & 1 & 14 & $\mathrm{C} 031$ & 10.7 & 40.8 & 105 & 147 & 17.834 & 68.001 & 175.004 & 245.005 \\
\hline 2 & SB7b-25ccc & 1 & 15 & C012 & 10.1 & 24.7 & 113 & 121 & 16.834 & 41.167 & 188.337 & 201.671 \\
\hline 2 & SB7b-20ccc & 1 & 16 & C104 & 5.89 & 12.4 & 40.6 & 78.5 & 9.817 & 20.667 & 67.668 & 130.836 \\
\hline
\end{tabular}


Table B-1. As-Received (ar) and Adjusted Measurements of the PCT Solutions (in ppm)

\begin{tabular}{|c|c|c|c|c|c|c|c|c|c|c|c|c|}
\hline Set & Glass ID (w HT) & Block & Seq & Lab ID & B ar & Li ar & Na ar & Si ar & B (ppm) & Li (ppm) & Na (ppm) & Si (ppm) \\
\hline 2 & SB7b-31ccc & 1 & 17 & C021 & 7.61 & 11.8 & 42.7 & 73.3 & 12.684 & 19.667 & 71.168 & 122.169 \\
\hline 2 & SB7b-33ccc & 1 & 18 & C008 & 8.78 & 36.2 & 87.2 & 133 & 14.634 & 60.335 & 145.336 & 221.671 \\
\hline 2 & SB7b-20 & 1 & 19 & C101 & 5.88 & 12.1 & 41.7 & 77.7 & 9.800 & 20.167 & 69.501 & 129.503 \\
\hline 2 & SB7b-30ccc & 1 & 20 & C037 & 7.15 & 12.9 & 61.8 & 82.2 & 11.917 & 21.500 & 103.002 & 137.003 \\
\hline 2 & SB7b-32ccc & 1 & 21 & C038 & 8.4 & 22 & 54.6 & 97.7 & 14.000 & 36.667 & 91.002 & 162.837 \\
\hline 2 & Soln Std & 1 & 22 & STD_13 & 20 & 10.2 & 84.1 & 52.8 & 20.000 & 10.200 & 84.100 & 52.800 \\
\hline 2 & Soln Std & 2 & 1 & STD_21 & 20.2 & 10.1 & 84.7 & 52.8 & 20.200 & 10.100 & 84.700 & 52.800 \\
\hline 2 & SB7b-20ccc & 2 & 2 & C082 & 5.87 & 12.1 & 40.1 & 78.7 & 9.784 & 20.167 & 66.835 & 131.169 \\
\hline 2 & SB7b-32 & 2 & 3 & C027 & 7.67 & 20.2 & 85.3 & 102 & 12.784 & 33.667 & 142.170 & 170.003 \\
\hline 2 & SB7b-33ccc & 2 & 4 & C092 & 7.66 & 15.7 & 60.4 & 80.7 & 12.767 & 26.167 & 100.669 & 134.503 \\
\hline 2 & EA & 2 & 5 & $\mathrm{C} 020$ & 19.9 & 7.12 & 59.3 & 38.8 & 331.667 & 118.667 & 988.335 & 646.668 \\
\hline 2 & SB7b-33 & 2 & 6 & C028 & 7.88 & 17.8 & 58.8 & 85.9 & 13.134 & 29.667 & 98.002 & 143.170 \\
\hline 2 & SB7b-31ccc & 2 & 7 & C052 & 7.64 & 11.7 & 41.8 & 73.8 & 12.734 & 19.500 & 69.668 & \begin{tabular}{|l|}
123.002 \\
\end{tabular} \\
\hline 2 & SB7b-25 & 2 & 8 & C107 & 10.8 & 40.6 & 105 & 148 & 18.000 & 67.668 & 175.004 & 246.672 \\
\hline 2 & SB7b-31 & 2 & 9 & C087 & 7.7 & 11.6 & 43.9 & 73.8 & 12.834 & 19.334 & 73.168 & 123.002 \\
\hline 2 & SB7b-30ccc & 2 & 10 & C091 & 7.26 & 12.8 & 61.9 & 82.3 & 12.100 & 21.334 & 103.169 & 137.169 \\
\hline 2 & Soln Std & 2 & 11 & \begin{tabular}{|l|} 
STD_22 \\
\end{tabular} & 20.1 & 10.1 & 84.3 & 52.8 & 20.100 & 10.100 & 84.300 & 52.800 \\
\hline 2 & SB7b-22 & 2 & 12 & \begin{tabular}{|l|} 
C049 \\
\end{tabular} & 7.21 & 17 & 52.9 & 89.7 & 12.017 & 28.334 & 88.168 & 149.503 \\
\hline 2 & SB7b-28ccc & 2 & 13 & C018 & 7.4 & 13.8 & 44.8 & 81.4 & 12.334 & 23.000 & 74.668 & 135.669 \\
\hline 2 & SB7b-20 & 2 & 14 & C034 & 5.95 & 12.2 & 41.8 & 78.6 & 9.917 & 20.334 & 69.668 & 131.003 \\
\hline 2 & SB7b-32ccc & 2 & 15 & C058 & 8.5 & 22.2 & 55.5 & 99 & 14.167 & 37.001 & 92.502 & 165.003 \\
\hline 2 & SB7b-25ccc & 2 & 16 & C060 & 9.88 & 24.2 & 111 & 119 & 16.467 & 40.334 & 185.004 & \begin{tabular}{|l|}
198.337 \\
\end{tabular} \\
\hline 2 & SB7b-30 & 2 & 17 & C062 & 7.45 & 12.9 & 68.8 & 87.1 & 12.417 & 21.500 & 114.669 & 145.170 \\
\hline 2 & SB7b-28 & 2 & 18 & C105 & 7.63 & 13 & 46.9 & 80.6 & 12.717 & 21.667 & 78.168 & 134.336 \\
\hline 2 & SB7b-22ccc & 2 & 19 & $\mathrm{C} 007$ & 7.63 & 20.1 & 52.2 & 98.1 & 12.717 & 33.501 & 87.002 & \begin{tabular}{|l|}
163.503 \\
\end{tabular} \\
\hline 2 & ARM-1 & 2 & 20 & C094 & 11.1 & 9.07 & 23.9 & 40.3 & 18.500 & 15.117 & 39.834 & 67.168 \\
\hline 2 & Soln Std & 2 & 21 & \begin{tabular}{|l|} 
STD_23 \\
\end{tabular} & 19.9 & 10.1 & 83.4 & 52.8 & 19.900 & 10.100 & 83.400 & 52.800 \\
\hline 2 & Soln Std & 3 & 1 & STD_31 & 20 & 10.1 & 83.7 & 52.7 & 20.000 & 10.100 & 83.700 & 52.700 \\
\hline 2 & SB7b-25ccc & 3 & 2 & \begin{tabular}{|l|} 
C090 \\
\end{tabular} & 8.59 & 20.5 & 93.6 & 106 & 14.317 & 34.167 & 156.003 & \begin{tabular}{|l|}
176.670 \\
\end{tabular} \\
\hline 2 & SB7b-20 & 3 & 3 & C095 & 5.83 & 11.9 & 41.1 & 77.3 & 9.717 & 19.834 & 68.501 & 128.836 \\
\hline 2 & ARM-1 & 3 & 4 & C086 & 11 & 8.88 & 23.7 & 40.4 & 18.334 & 14.800 & 39.501 & 67.335 \\
\hline 2 & SB7b-32ccc & 3 & 5 & $\mathrm{C} 024$ & 8.57 & 22 & 55.3 & 101 & 14.284 & 36.667 & 92.169 & \begin{tabular}{|l|}
168.337 \\
\end{tabular} \\
\hline 2 & SB7b-28 & 3 & 6 & C074 & 7.46 & 12.8 & 46 & 79.6 & 12.434 & 21.334 & 76.668 & 132.669 \\
\hline 2 & SB7b-28ccc & 3 & 7 & C109 & 7.49 & 13.9 & 44.9 & 83.5 & 12.484 & 23.167 & 74.835 & 139.169 \\
\hline 2 & SB7b-31ccc & 3 & 8 & C023 & 7.58 & 11.8 & 41.5 & 73.5 & 12.634 & 19.667 & 69.168 & \begin{tabular}{|l|}
122.502 \\
\end{tabular} \\
\hline 2 & SB7b-33ccc & 3 & 9 & C064 & 7.74 & 17.8 & 57.7 & 85.4 & 12.900 & 29.667 & 96.169 & 142.336 \\
\hline 2 & SB7b-22ccc & 3 & 10 & C071 & 7.64 & 20.1 & 52.5 & 96.7 & 12.734 & 33.501 & 87.502 & 161.170 \\
\hline 2 & Soln Std & 3 & 11 & STD_32 & 19.7 & 10 & 82.6 & 51.8 & 19.700 & 10.000 & 82.600 & 51.800 \\
\hline 2 & SB7b-33 & 3 & 12 & \begin{tabular}{|l|}
$\mathrm{C} 054$ \\
\end{tabular} & 7.68 & 17.1 & 53.3 & 86 & 12.800 & 28.501 & 88.835 & \begin{tabular}{|l}
143.336 \\
\end{tabular} \\
\hline 2 & SB7b-22 & 3 & 13 & C006 & 7.53 & 17.9 & 54.8 & 95.1 & 12.550 & 29.834 & 91.335 & 158.503 \\
\hline 2 & SB7b-25 & 3 & 14 & C081 & 10.6 & 40.5 & 103 & 147 & 17.667 & 67.501 & 171.670 & 245.005 \\
\hline 2 & SB7b-32 & 3 & 15 & C001 & 7.81 & 20.8 & 86.4 & 105 & 13.017 & 34.667 & 144.003 & 175.004 \\
\hline 2 & SB7b-30ccc & 3 & 16 & C098 & 7.27 & 12.9 & 62.1 & 82.7 & 12.117 & 21.500 & 103.502 & \begin{tabular}{|l}
137.836 \\
\end{tabular} \\
\hline 2 & SB7b-20ccc & 3 & 17 & C041 & 5.83 & 12.1 & 39.7 & 77.7 & 9.717 & 20.167 & 66.168 & 129.503 \\
\hline 2 & EA & 3 & 18 & C072 & 19.1 & 6.93 & 56.6 & 37.5 & \begin{tabular}{|l|}
318.334 \\
\end{tabular} & 115.500 & 943.335 & \begin{tabular}{|l}
625.001 \\
\end{tabular} \\
\hline 2 & SB7b-30 & 3 & 19 & C096 & 7.49 & 12.9 & 68.6 & 86.8 & 12.484 & 21.500 & 114.336 & 144.670 \\
\hline 2 & SB7b-31 & 3 & 20 & C029 & 7.52 & 11.5 & 42.7 & 72.5 & 12.534 & 19.167 & 71.168 & 120.836 \\
\hline 2 & Soln Std & 3 & 21 & STD_33 & 19.9 & 10.1 & 83.3 & 52.5 & 19.900 & 10.100 & 83.300 & 52.500 \\
\hline 2 & Soln Std & 4 & 1 & STD_41 & 20 & 10.1 & 84.5 & 52.7 & 20.000 & 10.100 & 84.500 & 52.700 \\
\hline 2 & SB7b-29 & 4 & 2 & \begin{tabular}{|l|} 
C099 \\
\end{tabular} & 7.69 & 14.3 & 63.2 & 91.7 & 12.817 & 23.834 & 105.335 & 152.836 \\
\hline 2 & SB7b-21ccc & 4 & 3 & C106 & 8.15 & 19.3 & 51.5 & 90.9 & 13.584 & 32.167 & 85.835 & 151.503 \\
\hline 2 & SB7b-23ccc & 4 & 4 & C014 & 7.07 & 18.5 & 48.5 & 88.8 & 11.784 & 30.834 & 80.835 & 148.003 \\
\hline 2 & SB7b-23 & 4 & 5 & C042 & 6.44 & 15 & 46.2 & 78.1 & 10.734 & 25.001 & 77.002 & \begin{tabular}{|l|}
130.169 \\
\end{tabular} \\
\hline 2 & SB7b-27 & 4 & 6 & C089 & 10.7 & 20.7 & 91.4 & 107 & 17.834 & 34.501 & 152.336 & 178.337 \\
\hline 2 & SB7b-18ccc & 4 & 7 & C069 & 8.23 & 14.5 & 67.7 & 85.2 & 13.717 & 24.167 & 112.836 & 142.003 \\
\hline 2 & SB7b-27ccc & 4 & 8 & C065 & 12.9 & 38.8 & 99.1 & 146 & 21.500 & 64.668 & 165.170 & \begin{tabular}{|l}
243.338 \\
\end{tabular} \\
\hline 2 & SB7b-19ccc & 4 & 9 & C050 & 6.85 & 13.4 & 66.1 & 83.7 & 11.417 & 22.334 & 110.169 & 139.503 \\
\hline 2 & SB7b-21 & 4 & 10 & C051 & 7.89 & 17 & 53.1 & 86.8 & 13.150 & 28.334 & 88.502 & 144.670 \\
\hline 2 & Soln Std & 4 & 11 & STD_42 & 19.6 & 9.98 & 83.2 & 51.9 & 19.600 & 9.980 & 83.200 & 51.900 \\
\hline 2 & SB7b-24ccc & 4 & 12 & \begin{tabular}{|l|}
$\mathrm{C} 030$ \\
\end{tabular} & 9.04 & 23.9 & 74.7 & 104 & 15.067 & 39.834 & 124.502 & \begin{tabular}{|l}
173.337 \\
\end{tabular} \\
\hline 2 & SB7b-29ccc & 4 & 13 & C102 & 7.57 & 14.8 & 59.3 & 91.9 & 12.617 & 24.667 & 98.835 & 153.170 \\
\hline 2 & SB7b-18 & 4 & 14 & C032 & 8.06 & 14 & 70.1 & 83.3 & 13.434 & 23.334 & 116.836 & 138.836 \\
\hline 2 & SB7b-19 & 4 & 15 & C059 & 6.81 & 12.8 & 71 & 83 & 11.350 & 21.334 & 118.336 & \begin{tabular}{|l}
138.336 \\
\end{tabular} \\
\hline 2 & SB7b-34 & 4 & 16 & C061 & 7.78 & 17.3 & 55 & 87.7 & 12.967 & 28.834 & 91.669 & \begin{tabular}{|l|}
146.170 \\
\end{tabular} \\
\hline 2 & SB7b-26ccc & 4 & 17 & C100 & 7.15 & 13 & 43.9 & 74.8 & 11.917 & 21.667 & 73.168 & \begin{tabular}{|l}
124.669 \\
\end{tabular} \\
\hline 2 & SB7b-24 & 4 & 18 & C035 & 7.83 & 16.6 & 71.8 & 87.8 & 13.050 & 27.667 & 119.669 & 146.336 \\
\hline 2 & SB7b-34ccc & 4 & 19 & C044 & 8.84 & 36.3 & 88.5 & 133 & 14.734 & 60.501 & 147.503 & 221.671 \\
\hline 2 & SB7b-26 & 4 & 20 & C043 & 6.88 & 11.7 & 42.8 & 70.3 & 11.467 & 19.500 & 71.335 & \begin{tabular}{|l|l|}
117.169 \\
\end{tabular} \\
\hline 2 & Soln Std & 4 & 21 & STD_43 & 19.6 & 9.99 & 83.8 & 51.9 & 19.600 & 9.990 & 83.800 & 51.900 \\
\hline 2 & Soln Std & 5 & 1 & \begin{tabular}{|l|} 
STD_51 \\
\end{tabular} & 19.7 & 9.79 & 82.5 & 51.7 & 19.700 & 9.790 & 82.500 & 51.700 \\
\hline 2 & SB7b-26ccc & 5 & 2 & \begin{tabular}{|l|} 
C085 \\
\end{tabular} & 7.22 & 12.6 & 43 & 74.1 & 12.034 & 21.000 & 71.668 & \begin{tabular}{|l|}
123.502 \\
\end{tabular} \\
\hline 2 & SB7b-18ccc & 5 & 3 & C033 & 8.28 & 14.2 & 67.2 & 85.4 & 13.800 & 23.667 & 112.002 & 142.336 \\
\hline
\end{tabular}


SRNL-STI-2011-00440

Revision 0

Table B-1. As-Received (ar) and Adjusted Measurements of the PCT Solutions (in ppm)

\begin{tabular}{|c|c|c|c|c|c|c|c|c|c|c|c|c|}
\hline Set & Glass ID (w HT) & Block & Seq & Lab ID & B ar & Li ar & Na ar & Si ar & B (ppm) & $\mathbf{L i}(\mathrm{ppm})$ & $\mathrm{Na}$ (ppm) & Si (ppm) \\
\hline 2 & SB7b-27 & 5 & 4 & C010 & 11.2 & 21.2 & 94.7 & 107 & 18.667 & 35.334 & 157.836 & 178.337 \\
\hline 2 & SB7b-18 & 5 & 5 & C045 & 8.33 & 14 & 71.9 & 85.4 & 13.884 & 23.334 & 119.836 & 142.336 \\
\hline 2 & SB7b-21ccc & 5 & 6 & C068 & 8.16 & 19.1 & 51.4 & 92.4 & 13.600 & 31.834 & 85.668 & 154.003 \\
\hline 2 & SB7b-21 & 5 & 7 & C103 & 7.76 & 16.5 & 51.8 & 84.9 & 12.934 & 27.501 & 86.335 & 141.503 \\
\hline 2 & SB7b-23ccc & 5 & 8 & C040 & 6.74 & 17.4 & 46 & 83.9 & 11.234 & 29.001 & 76.668 & 139.836 \\
\hline 2 & SB7b-29ccc & 5 & 9 & C053 & 7.44 & 14.2 & 58.1 & 91.7 & 12.400 & 23.667 & 96.835 & 152.836 \\
\hline 2 & SB7b-29 & 5 & 10 & C016 & 7.28 & 13.2 & 60.3 & 86.9 & 12.134 & 22.000 & 100.502 & 144.836 \\
\hline 2 & Soln Std & 5 & 11 & STD_52 & 19.7 & 9.87 & 82.8 & 51.8 & 19.700 & 9.870 & 82.800 & 51.800 \\
\hline 2 & SB7b-24ccc & 5 & 12 & \begin{tabular}{|l|} 
C108 \\
\end{tabular} & 8.92 & 23.2 & 73.5 & 105 & 14.867 & 38.667 & 122.502 & 175.004 \\
\hline 2 & SB7b-23 & 5 & 13 & C046 & 6.42 & 14.6 & 46.1 & 78.5 & 10.700 & 24.334 & 76.835 & 130.836 \\
\hline 2 & SB7b-26 & 5 & 14 & C048 & 7.09 & 11.9 & 43.9 & 72.9 & 11.817 & 19.834 & 73.168 & 121.502 \\
\hline 2 & SB7b-19ccc & 5 & 15 & $\mathrm{C} 011$ & 6.74 & 13.1 & 65.2 & 83.7 & 11.234 & 21.834 & 108.669 & 139.503 \\
\hline 2 & SB7b-34 & 5 & 16 & $\mathrm{C} 075$ & 7.57 & 15.5 & 60.4 & 80.1 & 12.617 & 25.834 & 100.669 & 133.503 \\
\hline 2 & SB7b-19 & 5 & 17 & C093 & 6.81 & 12.8 & 71.4 & 83.8 & 11.350 & 21.334 & 119.002 & 139.669 \\
\hline 2 & SB7b-24 & 5 & 18 & C004 & 7.92 & 16.5 & 72.2 & 88 & 13.200 & 27.501 & 120.336 & 146.670 \\
\hline 2 & SB7b-27ccc & 5 & 19 & C015 & 12.6 & 37.2 & 96.4 & 145 & 21.000 & 62.001 & 160.670 & 241.672 \\
\hline 2 & SB7b-34ccc & 5 & 20 & C063 & 8.7 & 35.5 & 87 & 132 & 14.500 & 59.168 & 145.003 & 220.004 \\
\hline 2 & Soln Std & 5 & 21 & STD_53 & 19.8 & 9.93 & 83.7 & 52.4 & 19.800 & 9.930 & 83.700 & 52.400 \\
\hline 2 & Soln Std & 6 & 1 & STD_61 & 19.8 & 9.86 & 83 & 52.2 & 19.800 & 9.860 & 83.000 & 52.200 \\
\hline 2 & SB7b-26ccc & 6 & 2 & \begin{tabular}{|l|}
$\mathrm{C} 047$ \\
\end{tabular} & 7.12 & 12.5 & 42.7 & 74.1 & 11.867 & 20.834 & 71.168 & 123.502 \\
\hline 2 & SB7b-23ccc & 6 & 3 & C025 & 6.92 & 17.8 & 46.8 & 96.3 & 11.534 & 29.667 & 78.002 & 160.503 \\
\hline 2 & SB7b-34ccc & 6 & 4 & C083 & 7.83 & 17.8 & 59.1 & 85.6 & 13.050 & 29.667 & 98.502 & 142.670 \\
\hline 2 & SB7b-21 & 6 & 5 & C019 & 7.88 & 16.7 & 52.6 & 86.3 & 13.134 & 27.834 & 87.668 & 143.836 \\
\hline 2 & SB7b-18ccc & 6 & 6 & C078 & 8 & 13.9 & 65.2 & 83.3 & 13.334 & 23.167 & 108.669 & 138.836 \\
\hline 2 & SB7b-29 & 6 & 7 & C003 & 7.3 & 13.3 & 60.5 & 87.8 & 12.167 & 22.167 & 100.835 & 146.336 \\
\hline 2 & SB7b-27ccc & 6 & 8 & C002 & 12.6 & 37.5 & 96.8 & 143 & 21.000 & 62.501 & 161.337 & 238.338 \\
\hline 2 & SB7b-29ccc & 6 & 9 & C005 & 7.12 & 13.6 & 55.9 & 86.8 & 11.867 & 22.667 & 93.169 & 144.670 \\
\hline 2 & SB7b-24ccc & 6 & 10 & C039 & 8.67 & 22.8 & 71.9 & 102 & 14.450 & 38.001 & 119.836 & 170.003 \\
\hline 2 & Soln Std & 6 & 11 & STD_62 & 19.7 & 9.77 & 82.7 & 52 & 19.700 & 9.770 & 82.700 & 52.000 \\
\hline 2 & SB7b-26 & 6 & 12 & \begin{tabular}{|l|}
$\mathrm{C} 066$ \\
\end{tabular} & 7.03 & 11.8 & 43.5 & 71.8 & 11.717 & 19.667 & 72.501 & 119.669 \\
\hline 2 & blank & 6 & 13 & C077 & $<0.0808$ & $<0.601$ & $<0.212$ & $<0.176$ & 0.067 & 0.501 & 0.177 & 0.147 \\
\hline 2 & SB7b-18 & 6 & 14 & C076 & 8.05 & 13.6 & \begin{tabular}{l|l|}
69.6 \\
\end{tabular} & 83.8 & 13.417 & 22.667 & 116.002 & 139.669 \\
\hline 2 & SB7b-19ccc & 6 & 15 & C097 & 6.53 & 12.6 & 62.9 & 84.1 & 10.884 & 21.000 & 104.835 & 140.169 \\
\hline 2 & SB7b-24 & 6 & 16 & C084 & 8 & 16.1 & 71.9 & 86.7 & 13.334 & 26.834 & 119.836 & 144.503 \\
\hline 2 & SB7b-19 & 6 & 17 & C073 & 6.83 & 12.7 & 70.8 & 83.6 & 11.384 & 21.167 & 118.002 & 139.336 \\
\hline 2 & SB7b-23 & 6 & 18 & C110 & 6.44 & 14.8 & 46.4 & 80.1 & 10.734 & 24.667 & 77.335 & 133.503 \\
\hline 2 & SB7b-21ccc & 6 & 19 & $\mathrm{C} 017$ & 8.21 & 19.4 & 52.2 & 95.2 & 13.684 & 32.334 & 87.002 & 158.670 \\
\hline 2 & SB7b-34 & 6 & 20 & $\mathrm{C} 026$ & 7.55 & 15.3 & 60 & 80.7 & 12.584 & 25.501 & 100.002 & 134.503 \\
\hline 2 & SB7b-27 & 6 & 21 & $\mathrm{C} 009$ & 10.6 & 20.1 & 90 & 103 & 17.667 & 33.501 & 150.003 & 171.670 \\
\hline 2 & Soln Std & 6 & 22 & STD_63 & 19.9 & 9.93 & 83.4 & 52.4 & 19.900 & 9.930 & 83.400 & 52.400 \\
\hline
\end{tabular}


Exhibit B-1. PCT Measurements (as Common Logarithms) in Analytical Sequence for Blocks 1- 3 for Analytical Set 1

Set $=1$

Variability Chart for $\log [B \mathrm{ppm}]$

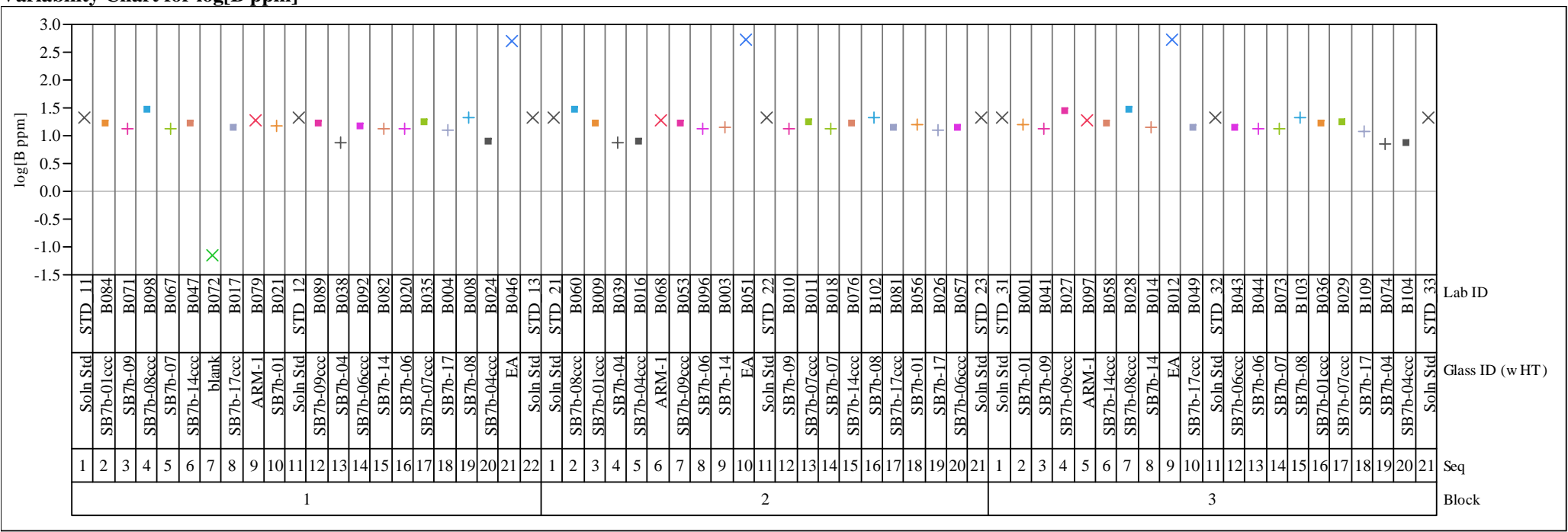

Set $=1$

Variability Chart for $\log [\mathrm{Li}$ ppm $]$

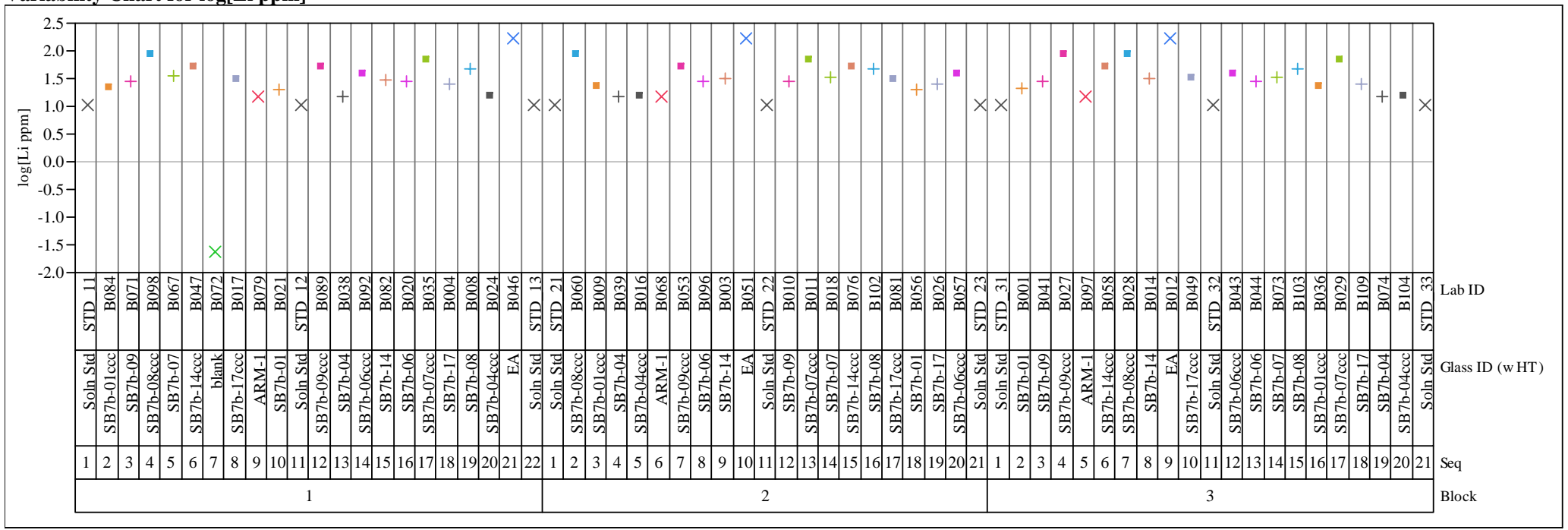


Exhibit B-1. PCT Measurements (as Common Logarithms) in Analytical Sequence for Blocks 1- 3 for Analytical Set 1

Set $=1$

Variability Chart for log[Na ppm]

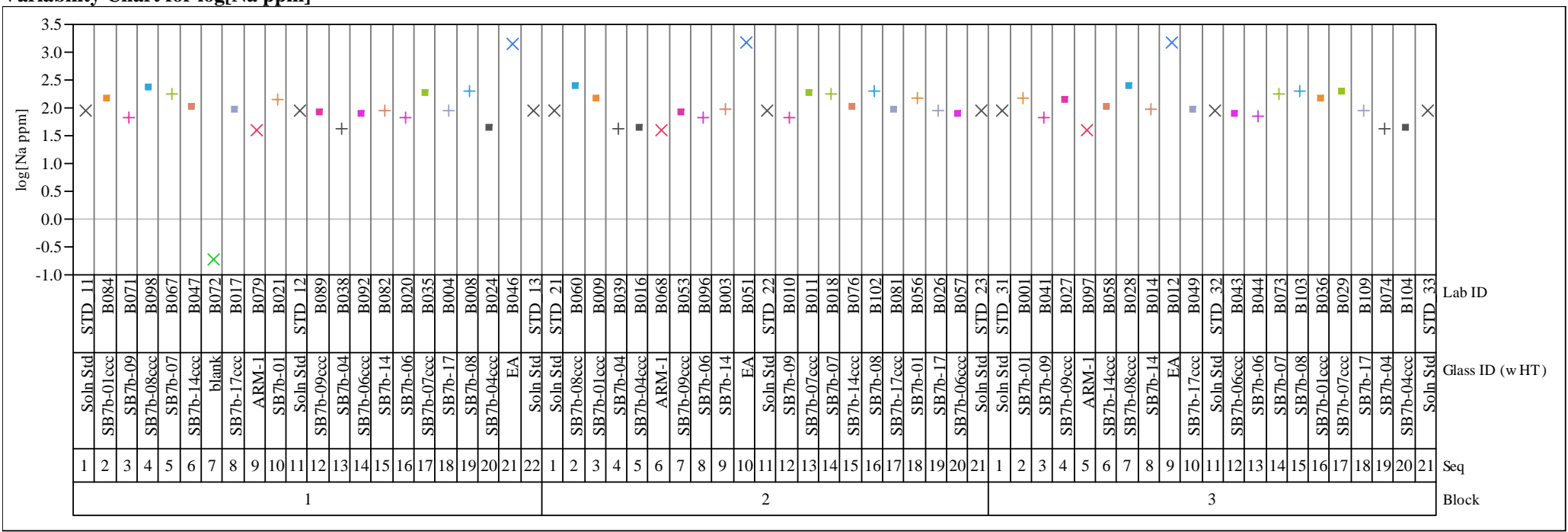

Set $=1$

Variability Chart for $\log [\mathrm{Si}$ ppm $]$

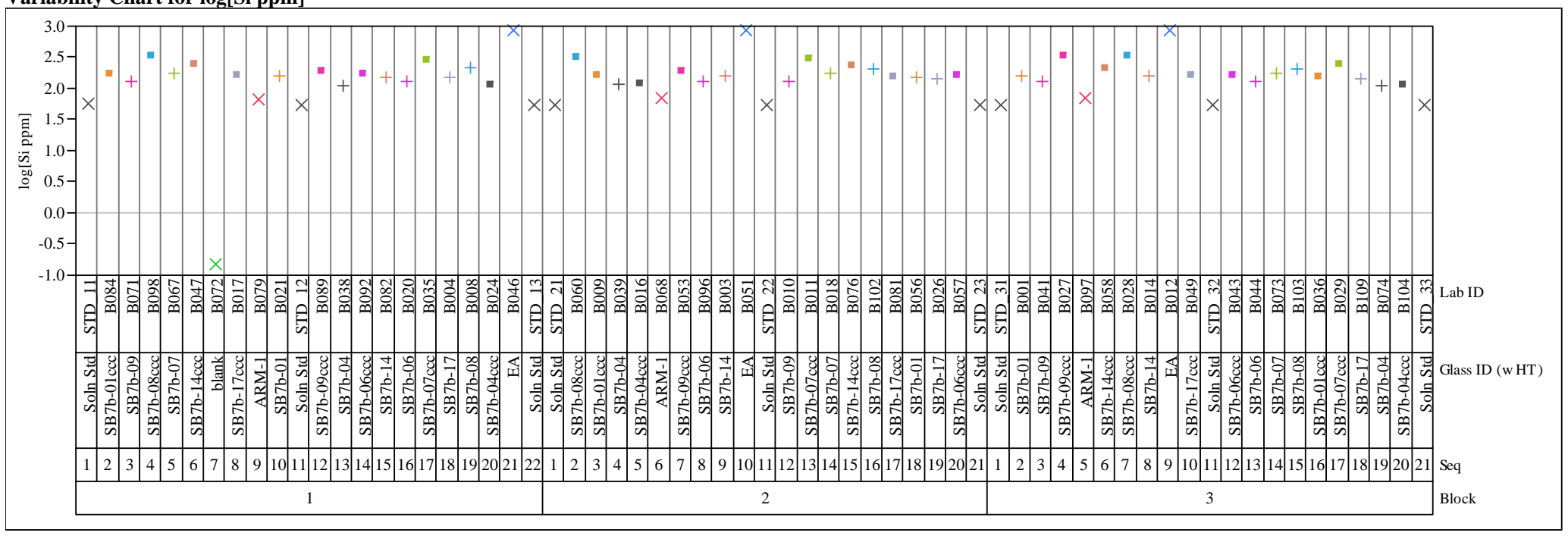


Exhibit B-2. PCT Measurements (as Common Logarithms) in Analytical Sequence for Blocks 4- 6 for Analytical Set 1

Set $=1$

Variability Chart for $\log [\mathrm{B}$ ppm]

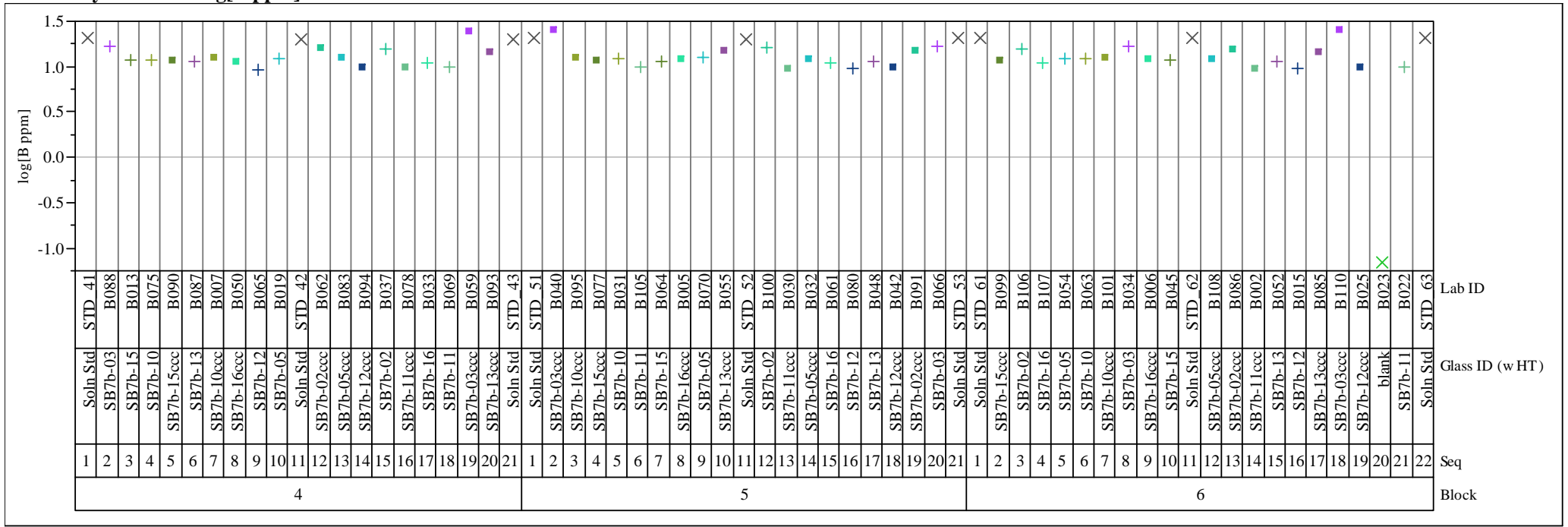

Set $=1$

Variability Chart for log[Li ppm]

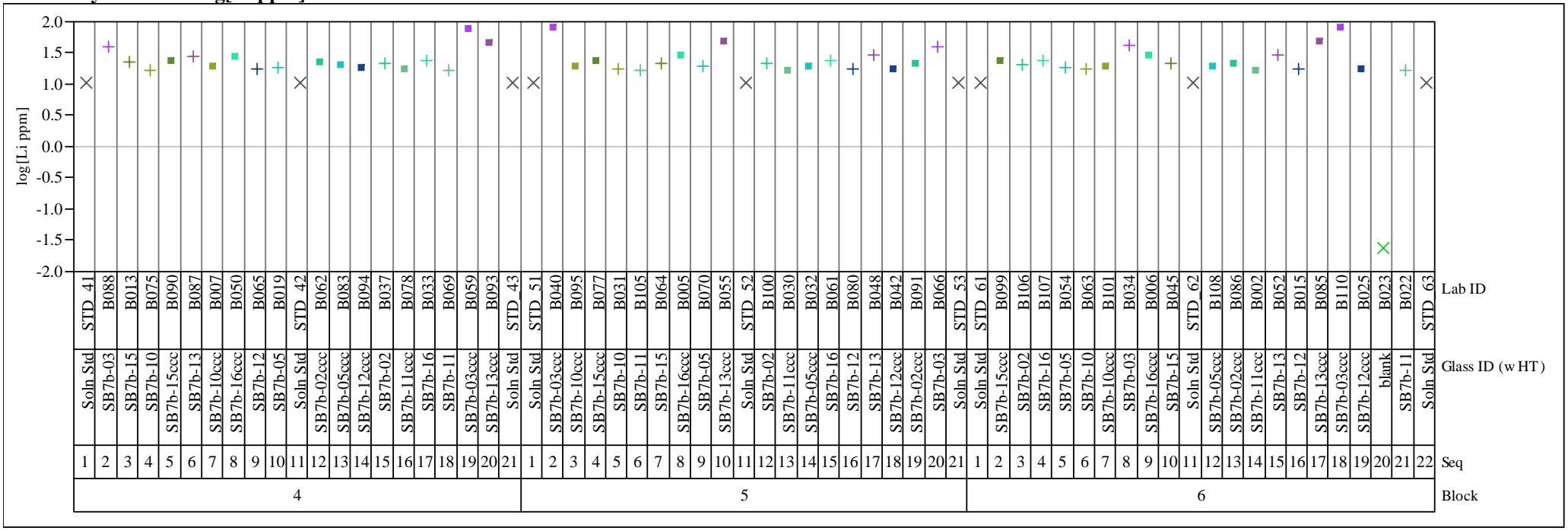


Exhibit B-2. PCT Measurements (as Common Logarithms) in Analytical Sequence for Blocks 4- 6 for Analytical Set 1

Set $=1$

Variability Chart for log[Na ppm]

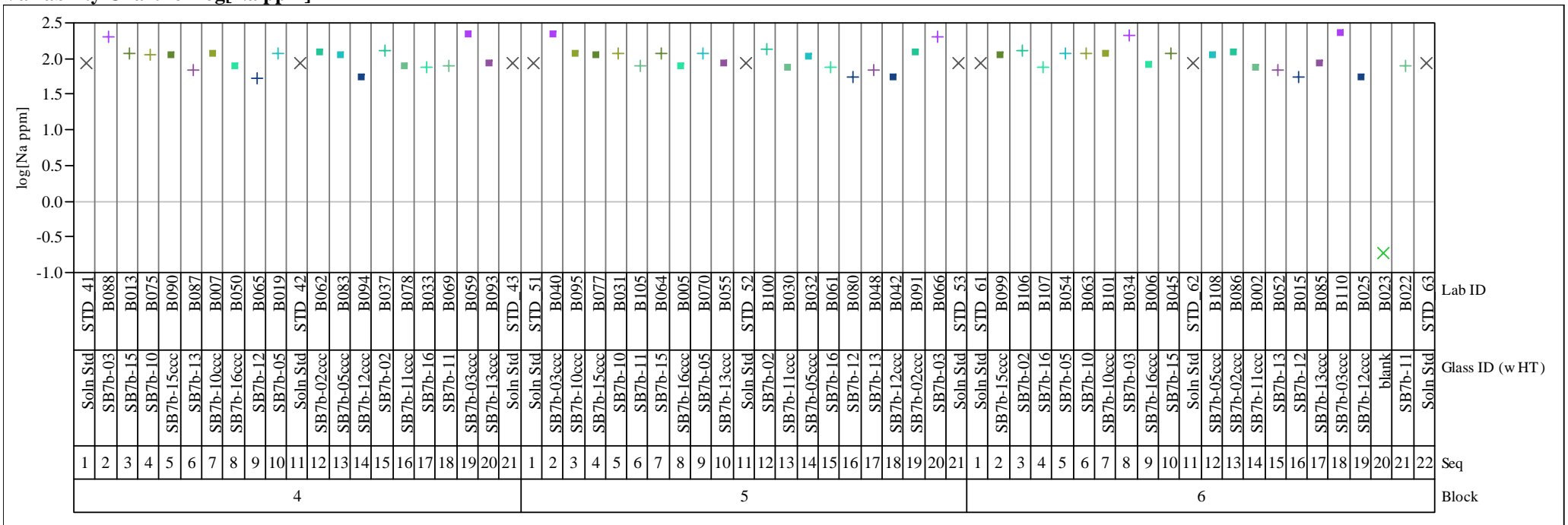

Set $=1$

Variability Chart for log[Si ppm]

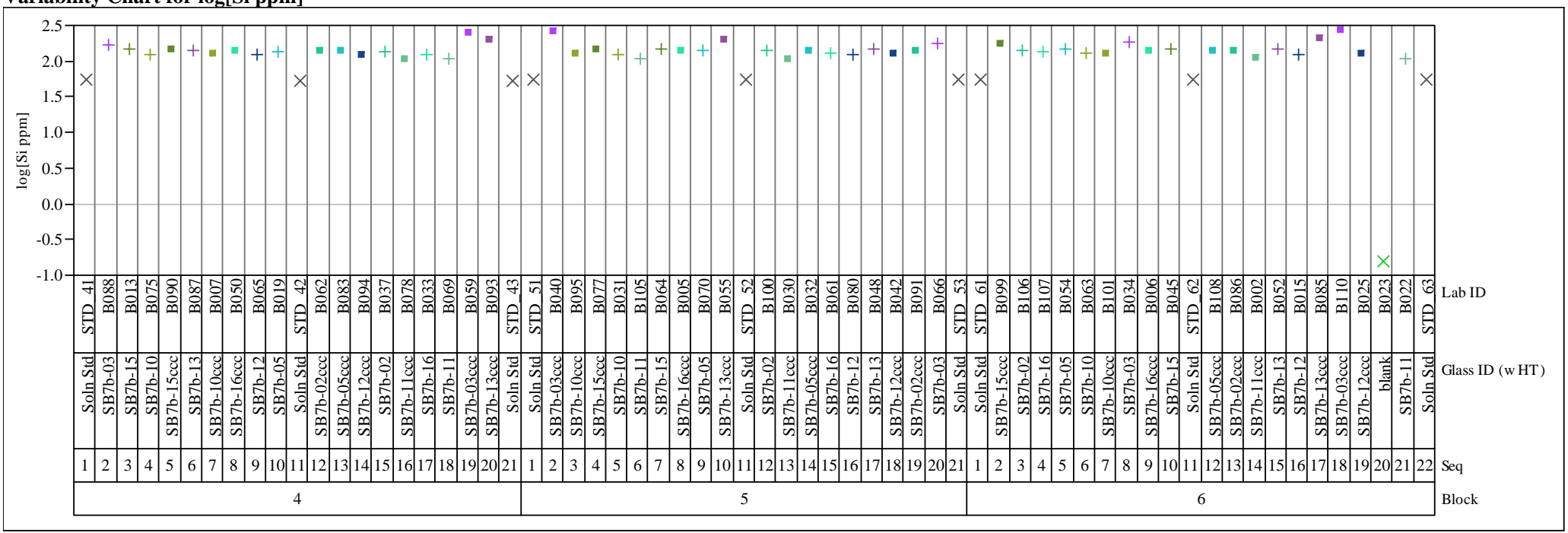


Exhibit B-3. PCT Measurements (as Common Logarithms) in Analytical Sequence for Blocks 1- 3 for Analytical Set 2

Set $=2$

Variability Chart for $\log [\mathrm{B}$ ppm]

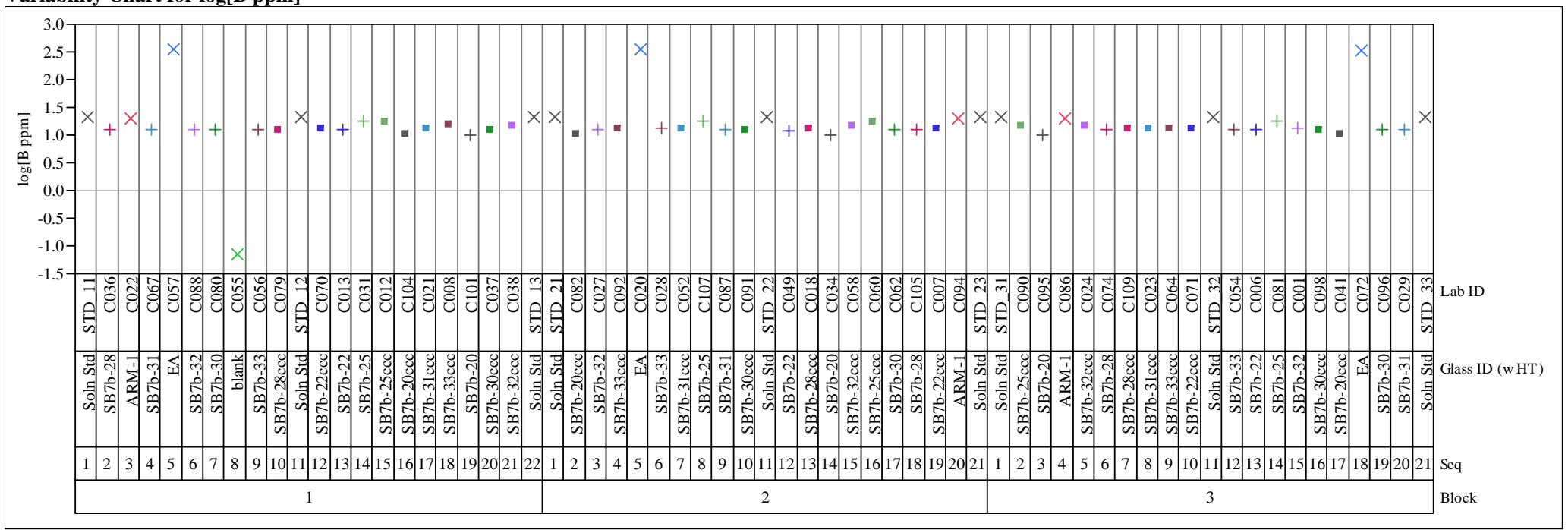

Set $=2$

Variability Chart for $\log [\mathrm{Li}$ ppm]

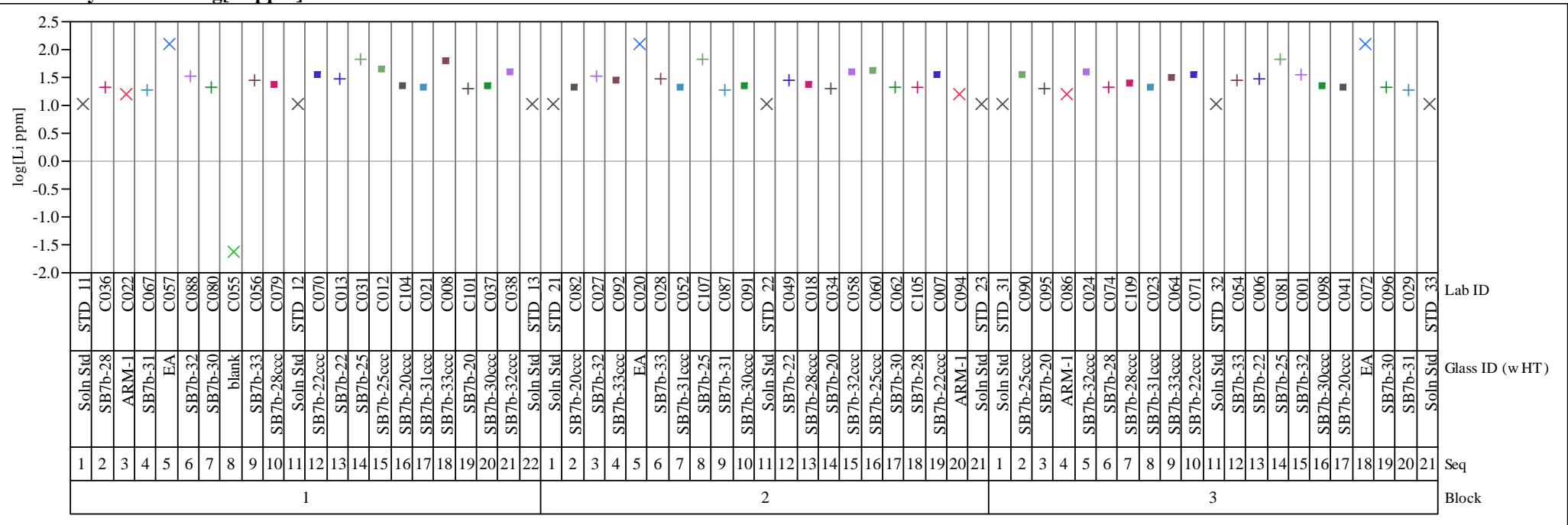


Exhibit B-3. PCT Measurements (as Common Logarithms) in Analytical Sequence for Blocks 1- 3 for Analytical Set 2

Set $=2$

Variability Chart for log[Na ppm]

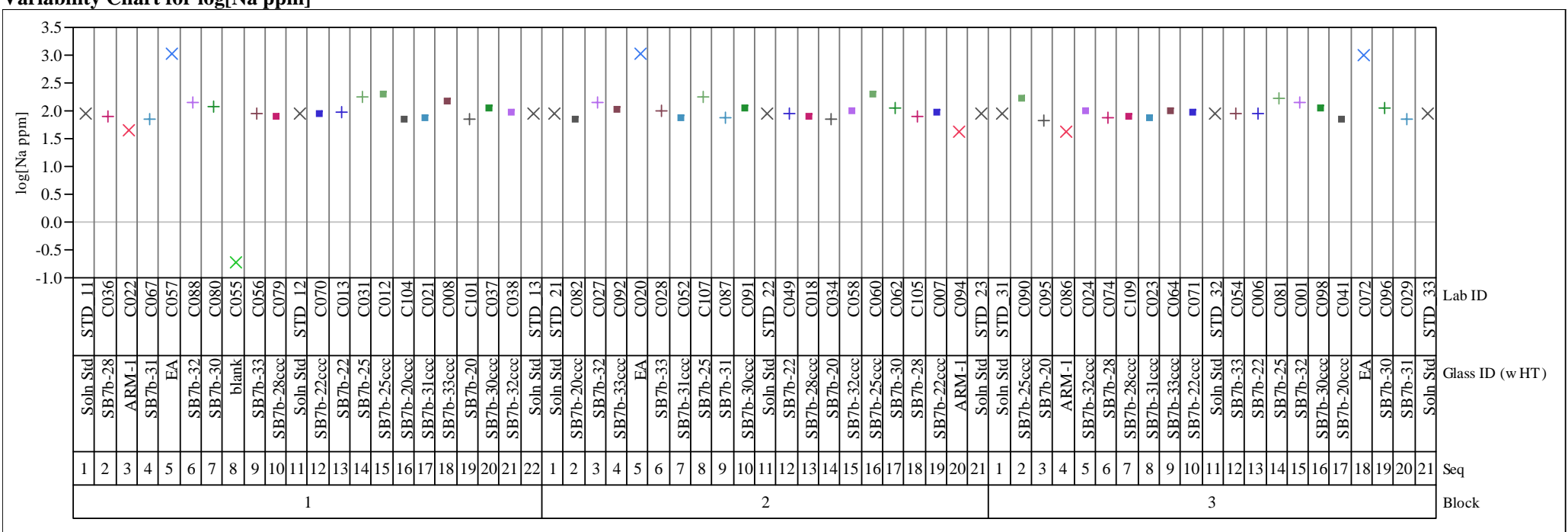

Set $=2$

Variability Chart for log[Si ppm]

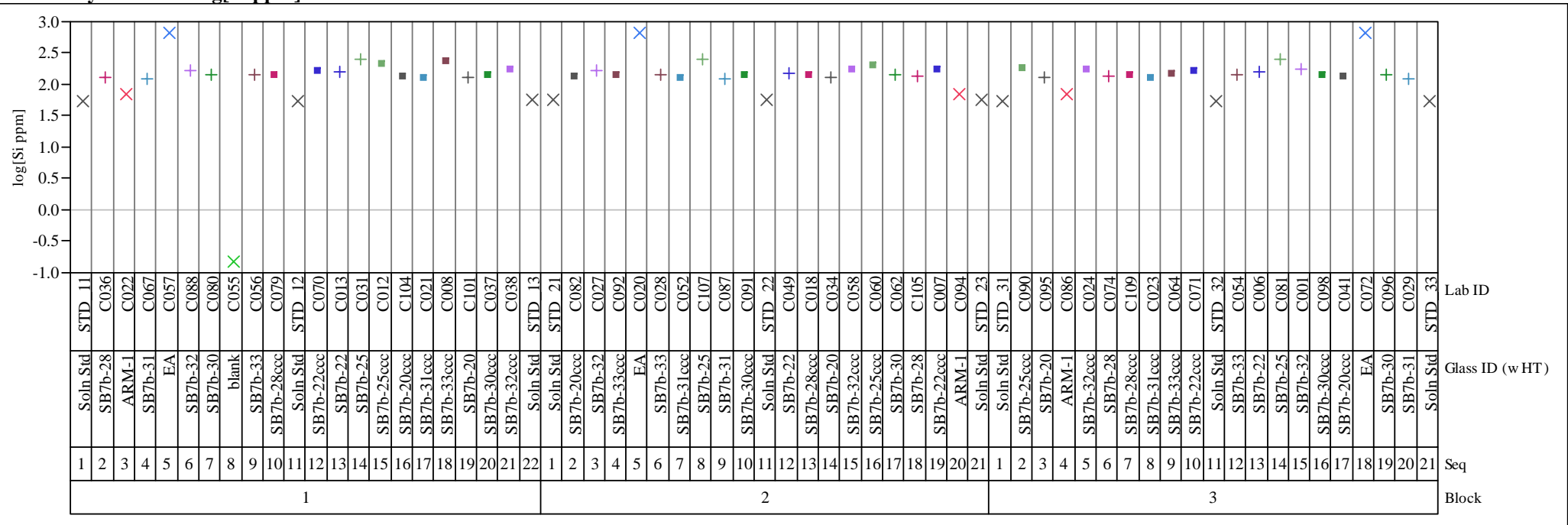


Exhibit B-4. PCT Measurements (as Common Logarithms) in Analytical Sequence for Blocks 4- 6 for Analytical Set 2

Set $=2$

Variability Chart for $\log [\mathrm{B}$ ppm $]$

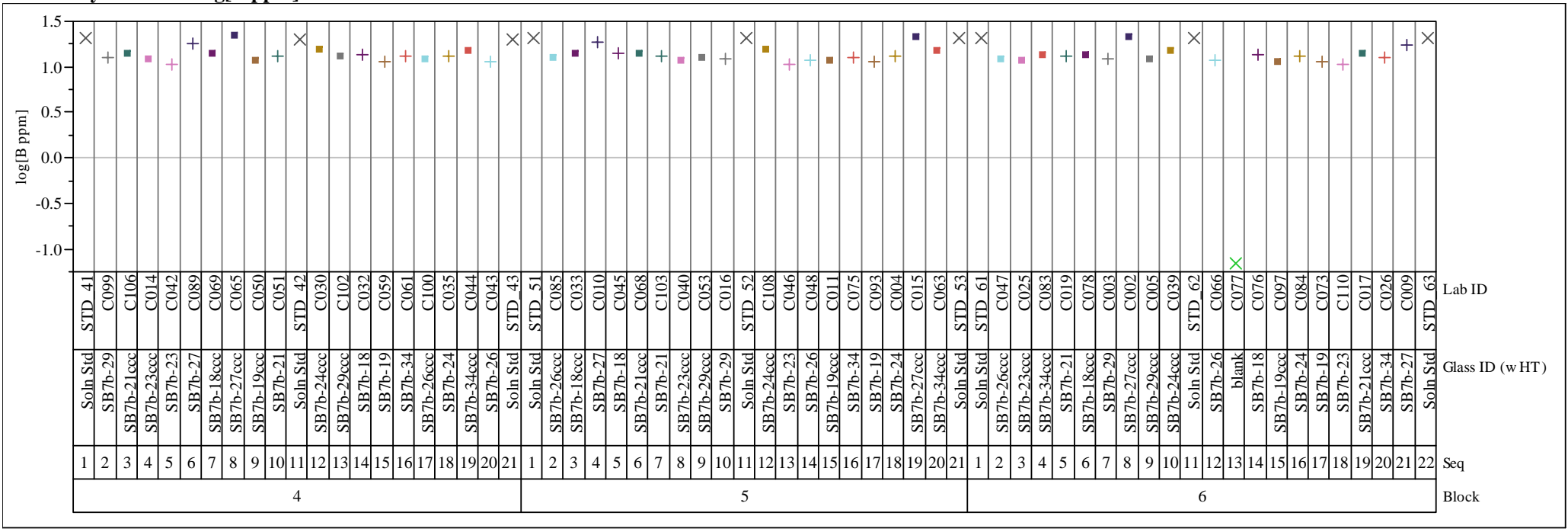

Set $=2$

Variability Chart for $\log [\mathrm{Li} \mathrm{ppm}]$

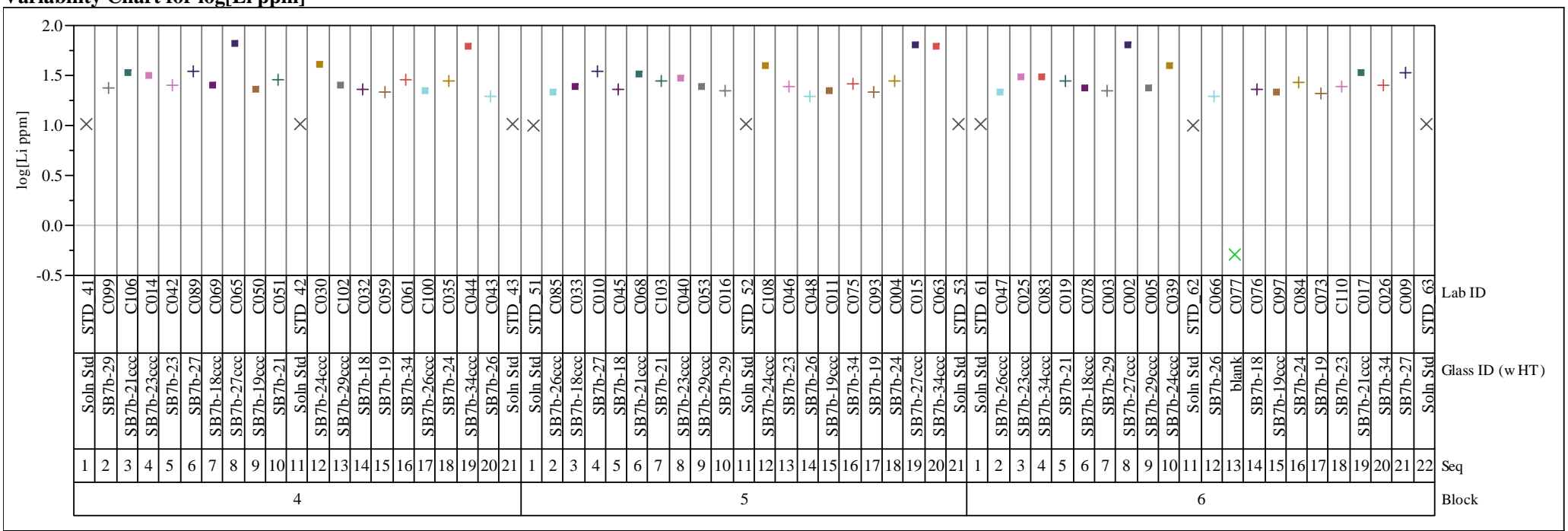


Exhibit B-4. PCT Measurements (as Common Logarithms) in Analytical Sequence for Blocks 4- 6 for Analytical Set 2

Set $=2$

Variability Chart for $\log [\mathrm{Na} \mathrm{ppm}]$

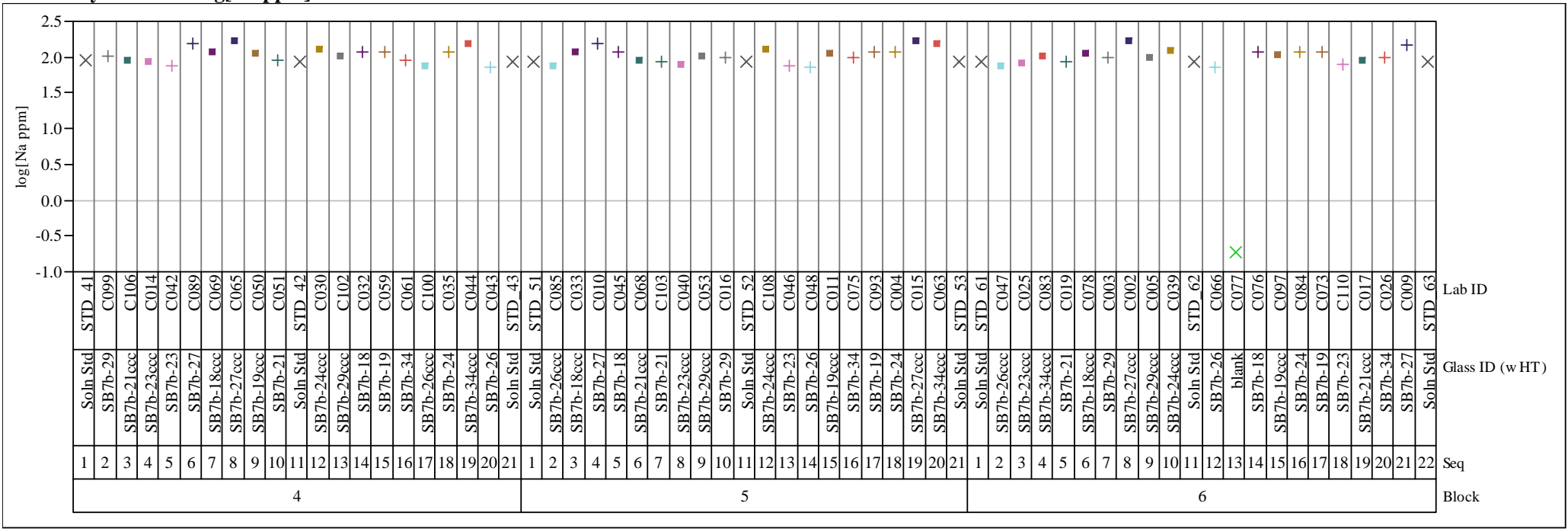

Set $=2$

Variability Chart for $\log [\mathrm{Si}$ ppm]

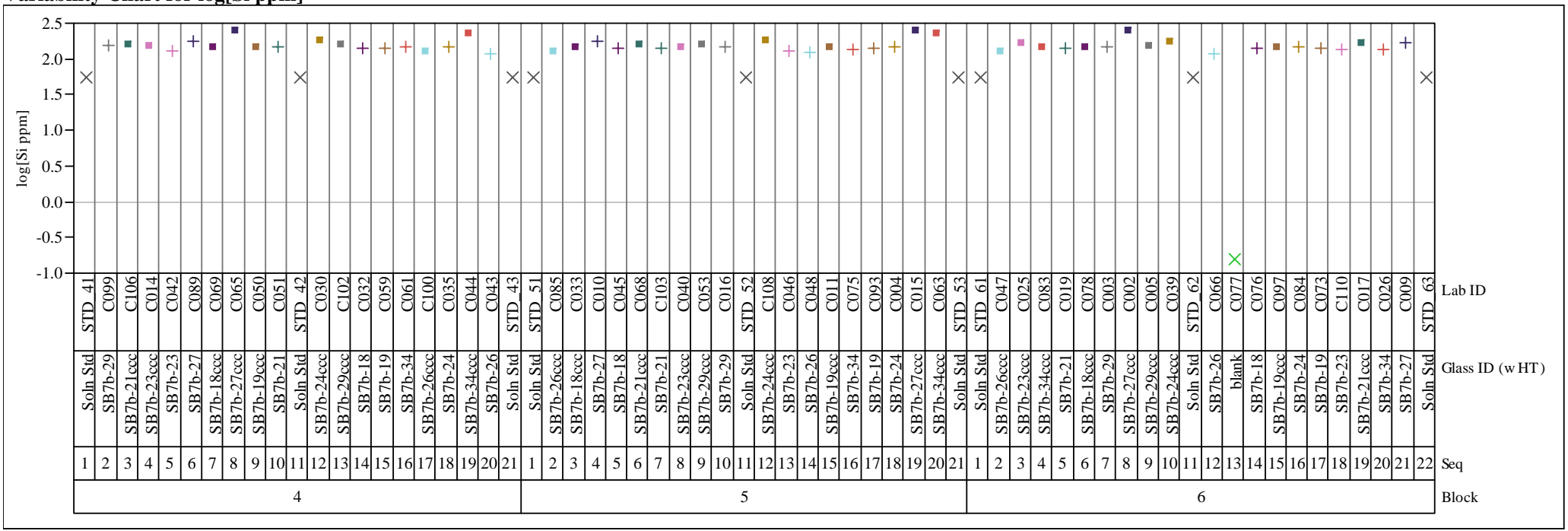


Exhibit B-5. Measurements of the Multi-Element Solution Standard by ICP Block by Analytical Set

Oneway Analysis of B (ppm) By Block Type=Soln Std, Set $=1$.

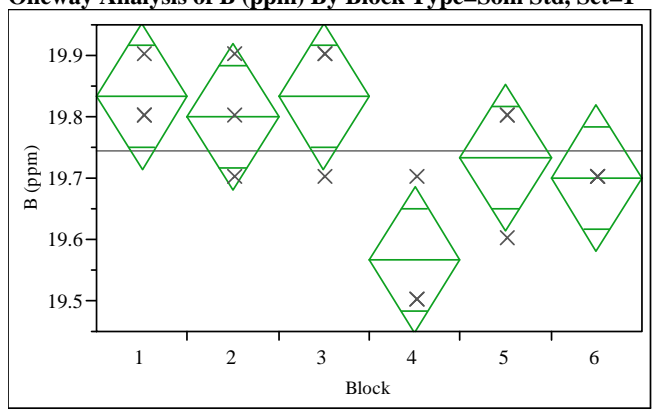

\section{Oneway Anova}

\section{Rsquare \\ 0.596639}

Adj Rsquare

0.596639

Root Mean Square Error $\quad 0.094281$

Mean of Response

19.74444

Analysis of Variance

ource DF Sum of Squares Mean Square F Ratio Prob > F

$\begin{array}{lrllll}\text { Block } & 5 & 0.15777778 & 0.031556 & 3.5500 & 0.033\end{array}$

Error 120.10666667

\section{Means for Oneway Anova}

\begin{tabular}{lrrrrr}
\multicolumn{1}{l}{ Level Number } & Mean & Std Error & Lower 95\% & Upper 95\% \\
1 & 3 & 19.8333 & 0.05443 & 19.715 & 19.952 \\
2 & 3 & 19.8000 & 0.05443 & 19.681 & 19.919 \\
3 & 3 & 19.8333 & 0.05443 & 19.715 & 19.952 \\
4 & 3 & 19.5667 & 0.05443 & 19.448 & 19.685 \\
5 & 3 & 19.7333 & 0.05443 & 19.615 & 19.852 \\
6 & 3 & 19.7000 & 0.05443 & 19.581 & 19.819
\end{tabular}

Std Error uses a pooled estimate of error variance

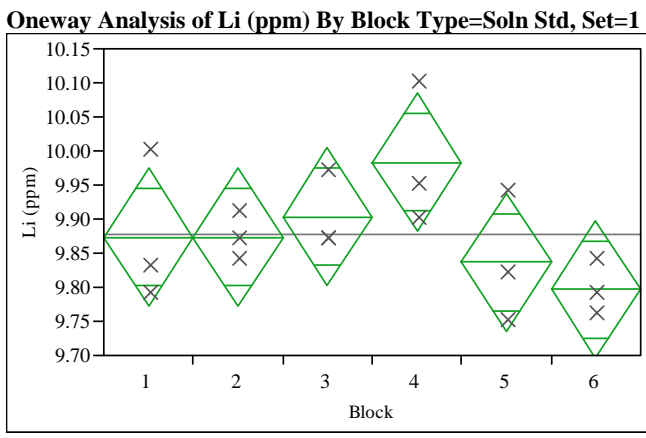

Oneway Anova

Summary of Fi

Rsquare

0.437954

Adj Rsquare

0.203768

Root Mean Squa

Mean of Response 0.087772

Observations (or Sum Wgts)

Analysis of Variance

Source DF Sum of Squares Mean Square F Ratio Prob $>$ F $\begin{array}{lrrrrr}\text { Block } & 5 & 0.06031111 & 0.012062 & 1.8701 & 0.1736\end{array}$ Eror $12 \quad 0.07740000$

\section{Means for Oneway Anova}

Level Number Mean Std Error Lower 95\% Upper 95\%

$\begin{array}{llllll}1 & 3 & 9.87333 & 0.04637 & 9.7723 & 9.974 \\ 2 & 3 & 9.87333 & 0.04637 & 9.7723 & 9.974\end{array}$

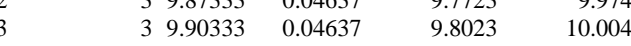

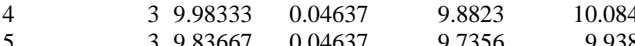

$\begin{array}{llllll}5 & 3 & 9.83667 & 0.04637 & 9.7356 & 9.938 \\ 6 & 3 & 9.79667 & 0.04637 & 9.6956 & 9.898\end{array}$

Std Error uses a pooled estimate of error variance 
Exhibit B-5. Measurements of the Multi-Element Solution Standard by ICP Block by Analytical Set

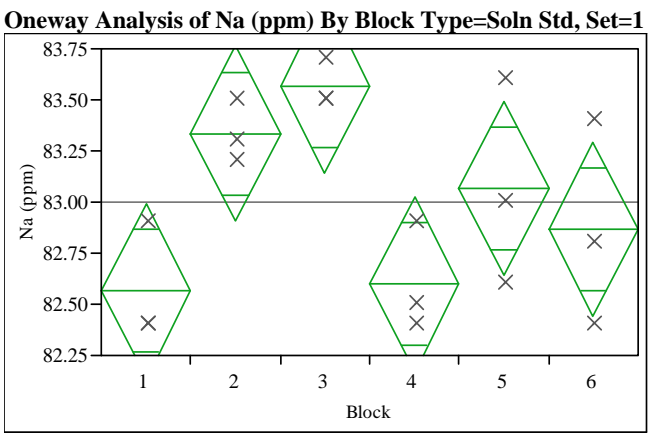

\section{Oneway Anova}

$\begin{array}{ll}\text { Rsquare } & 0.633333 \\ \text { Adj Rsquare } & 0.480556\end{array}$

Root Mean Square Erior

Mean of Response

83

Analysis of Variance

Source DF Sum of Squares Mean Square F Ratio Prob > F

$\begin{array}{llllll}\text { Block } & 5 & 2.4066667 & 0.481333 & 4.1455 & 0.0203\end{array}$

Error $12 \quad 1.3933333$ 0.11611

\section{Means for Oneway Anova}

\begin{tabular}{lrrrrr}
\multicolumn{1}{l}{ Level Number } & Mean & Std Error & Lower 95\% & Upper 95\% \\
1 & 3 & 82.5667 & 0.19673 & 82.138 & 82.995 \\
2 & 3 & 83.3333 & 0.19673 & 82.905 & 83.762 \\
3 & 3 & 83.5667 & 0.19673 & 83.138 & 83.995 \\
4 & 3 & 82.6000 & 0.19673 & 82.171 & 83.029 \\
5 & 3 & 83.0667 & 0.19673 & 82.638 & 83.495 \\
6 & 3 & 82.8667 & 0.19673 & 82.438 & 83.295
\end{tabular}

Std Error uses a pooled estimate of error variance

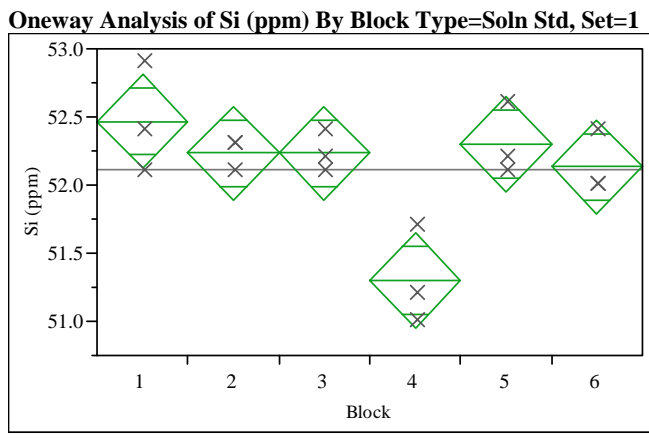

Oneway Anova

Summary of Fi

Rsquare

Adj Rsquare

0.737789

Root Mean Square Error $\quad 0.274874$

Mean of Response

Observations (or Sum Wgts)

Analysis of Variance

DF Sum of Squares Mean Square F Ratio Prob $>$ F

$\begin{array}{lrrrrr}\text { Block } & 5 & 2.5511111 & 0.510222 & 6.7529 & 0.0033\end{array}$

Error $12-0.9066667$

0.075556

\section{Means for Oneway Anova}

Level Number Mean Std Error Lower 95\% Upper 95\%

$\begin{array}{llrrrr}1 & 3 & 52.4667 & 0.15870 & 52.121 & 52.812 \\ 2 & 3 & 52.233 & 0.15870 & 51.888 & 52.579\end{array}$

$\begin{array}{llllll}1 & 3 & 52.2333 & 0.15870 & 51.888 & 52.579 \\ 4 & 3 & 52.2333 & 0.15870 & 51.888 & 52.579\end{array}$

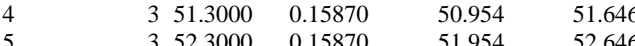

$\begin{array}{llllll}5 & 3 & 52.3000 & 0.15870 & 51.954 & 52.646 \\ 6 & 3 & 52.1333 & 0.15870 & 51.788 & 52.479\end{array}$

Std Error uses a pooled estimate of error variance 
Exhibit B-5. Measurements of the Multi-Element Solution Standard by ICP Block by Analytical Set

Fit Y by X Group

Oneway Analysis of B (ppm) By Block Type=Soln Std, Set=2

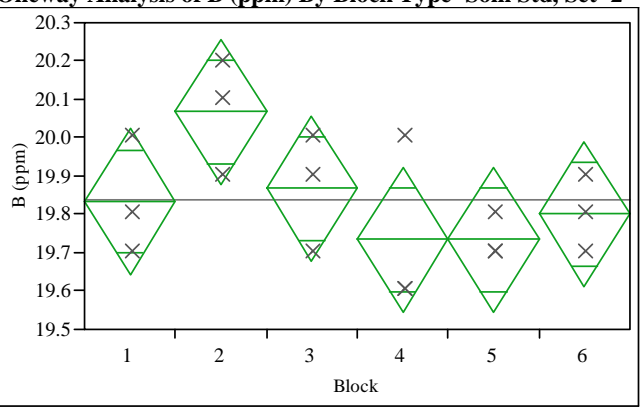

\section{Oneway Anova}

Summary of Fit

Rsquare

0.456354

Adj Rsquare

0.229834

Root Mean Square Error $\quad 0.150923$

Mean of Response

\section{Analysis of Variance}

Source DF Sum of Squares Mean Square F Ratio Prob >

$\begin{array}{llllll}\text { Block } & 5 & 0.22944444 & 0.045889 & 2.0146 & 0.1486\end{array}$

Error $12-0.27333333$ 0.02277

\section{Means for Oneway Anova}

\begin{tabular}{lrrrrr}
\multicolumn{1}{l}{ Level Number } & \multicolumn{2}{c}{ Mean } & Std Error & Lower 95\% & Upper 95\% \\
1 & 3 & 19.8333 & 0.08714 & 19.643 & 20.023 \\
2 & 3 & 20.0667 & 0.08714 & 19.877 & 20.257 \\
3 & 3 & 19.8667 & 0.08714 & 19.677 & 20.057 \\
4 & 3 & 19.7333 & 0.08714 & 19.543 & 19.923 \\
5 & 3 & 19.7333 & 0.08714 & 19.543 & 19.923 \\
6 & 3 & 19.8000 & 0.08714 & 19.610 & 19.990
\end{tabular}

Std Error uses a pooled estimate of error variance

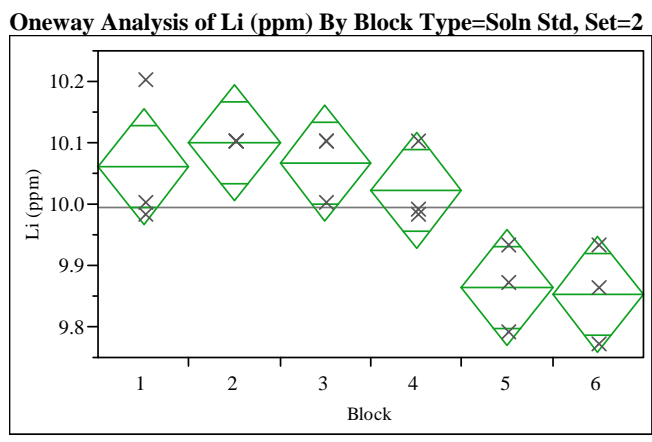

\section{Oneway Anova}

Summary of Fi

Rsquare

Adj Rsquare $\quad 0.721452$

Root Mean Square Error $\quad 0.075203$

Mean of Response

9.994444

Observations (or Sum Wgts)

Analysis of Variance

DF Sum of Squares Mean Square F Ratio Prob > F

$\begin{array}{lrlrll}\text { Block } & 5 & 0.17577778 & 0.035156 & 6.2161 & 0.0046\end{array}$

Error $12-0.06786667$

0.005656

\section{Means for Oneway Anova}

Level Number Mean Std Error Lower 95\% Upper 95\%

$\begin{array}{rrrrrr}1 & 3 & 10.0600 & 0.04342 & 9.965 & 10.155 \\ 2 & 3 & 10.1000 & 0.04342 & 10.005 & 10.195\end{array}$

$\begin{array}{rrrrrr}2 & 3 & 10.1000 & 0.04342 & 10.005 & 10.195 \\ 3 & 3 & 10.0667 & 0.04342 & 9.972 & 10.161\end{array}$

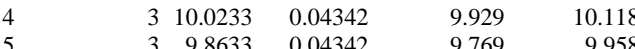

$\begin{array}{llllll}5 & 3 & 9.8633 & 0.04342 & 9.769 & 9.958 \\ 6 & 3 & 9.8533 & 0.04342 & 9.759 & 9.948\end{array}$

Std Error uses a pooled estimate of error variance 
Exhibit B-5. Measurements of the Multi-Element Solution Standard by ICP Block by Analytical Set

Oneway Analysis of Na (ppm) By Block Type=Soln Std, Set=2

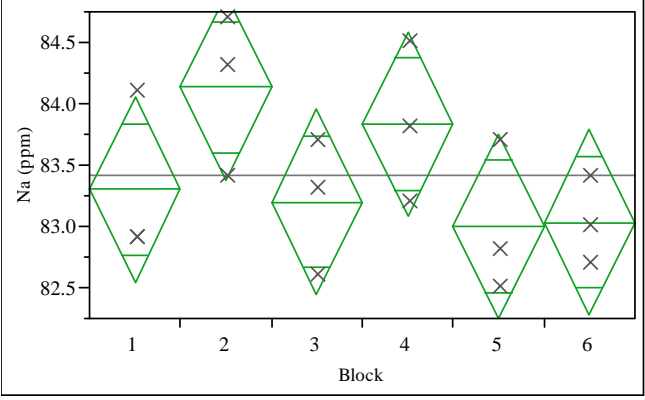

\section{Oneway Anova}

$\begin{array}{lr}\text { Rsquare } & 0.424785 \\ \text { Adj Rsquare } & 0.185112 \\ \text { Root Mean Square Error } & 0.601387 \\ \text { Mean of Response } & 83.41667 \\ \text { Observations (or Sum Wgts) } & 18\end{array}$

Analysis of Variance

Source DF Sum of Squares Mean Square F Ratio Prob > $\begin{array}{llllll}\text { Block } & 5 & 3.2050000 & 0.641000 & 1.7724 & 0.193\end{array}$

Error 124.3400000 0.361667

\section{Means for Oneway Anova}

\begin{tabular}{lrrrrr}
\multicolumn{2}{l}{ Level Number } & Mean & Std Error & Lower 95\% & Upper 95\% \\
1 & 3 & 83.3000 & 0.34721 & 82.543 & 84.057 \\
2 & 3 & 84.1333 & 0.34721 & 83.377 & 84.890 \\
3 & 3 & 83.2000 & 0.34721 & 82.443 & 83.957 \\
4 & 3 & 83.8333 & 0.34721 & 83.077 & 84.590 \\
5 & 3 & 83.0000 & 0.34721 & 82.243 & 83.757 \\
6 & 3 & 83.0333 & 0.34721 & 82.277 & 83.790
\end{tabular}

Std Error uses a pooled estimate of error variance

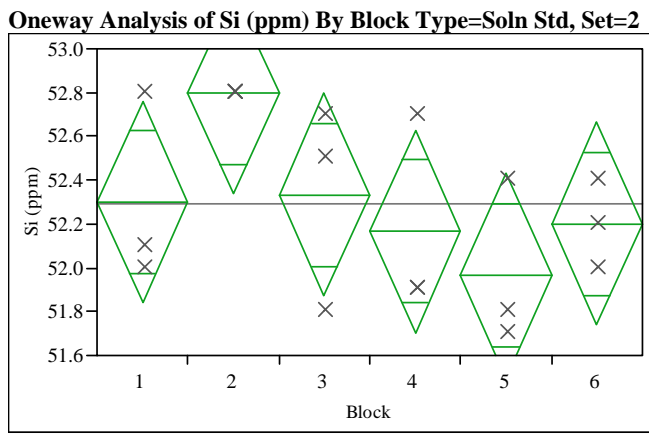

Oneway Anova

Summary of Fi

Rsquare

Adj Rsquare $\quad 0.419239$

$\begin{array}{ll}\text { Adj Rsquare } & 0.177256 \\ \text { Root Mean Square Error } & 0.367423\end{array}$

Mean of Response

Observations (or Sum Wgts)

18

Analysis of Variance

Source DF Sum of Squares Mean Square F Ratio Prob $>$ F

$\begin{array}{lrrrrr}\text { Block } & 5 & 1.1694444 & 0.233889 & 1.7325 & 0.2017\end{array}$

E. Tor $12-1.6200000$

\section{Means for Oneway Anova}

Level Number Mean Std Error Lower 95\% Upper 95\%

$\begin{array}{llrrrr}1 & 3 & 52.3000 & 0.21213 & 51.838 & 52.762 \\ 2 & 3 & 52.8000 & 0.21213 & 52.338 & 53.262\end{array}$

$\begin{array}{llllll}1 & 3 & 52.8000 & 0.21213 & 52.338 & 53.262 \\ & 3 & 52.3333 & 0.21213 & 51.871 & 52.796\end{array}$

$\begin{array}{llllll}4 & 3 & 52.1667 & 0.21213 & 51.704 & 52.629 \\ 5 & 3 & 51.9667 & 0.21213 & 51.504 & 52.429\end{array}$

$\begin{array}{llllll}5 & 3 & 51.9667 & 0.21213 & 51.504 & 52.429 \\ 6 & 3 & 52.2000 & 0.21213 & 51.738 & 52.662\end{array}$

Std Error uses a pooled estimate of error variance 
Exhibit B-6. PCT Measurements by Heat Treatment for Glasses and Standards by Analytical Set

Set $=1$

Variability Chart for B (ppm)

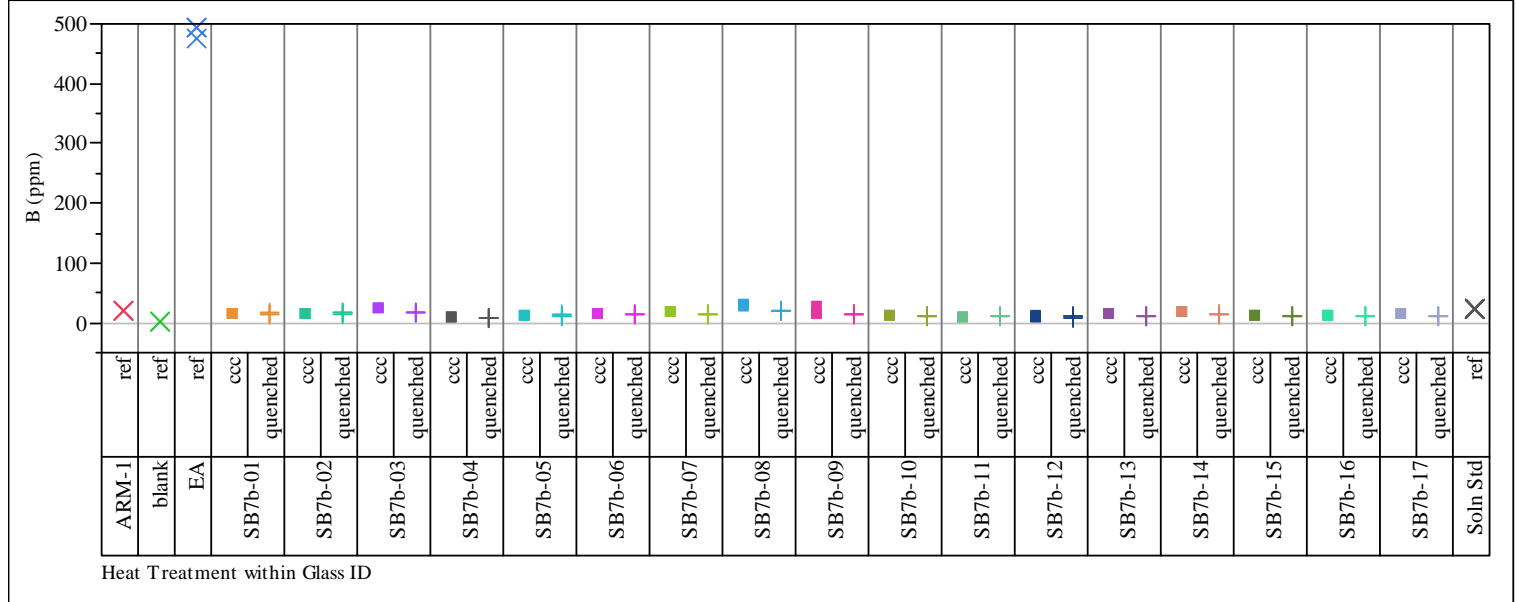

Set $=1$

Variability Chart for Li (ppm)

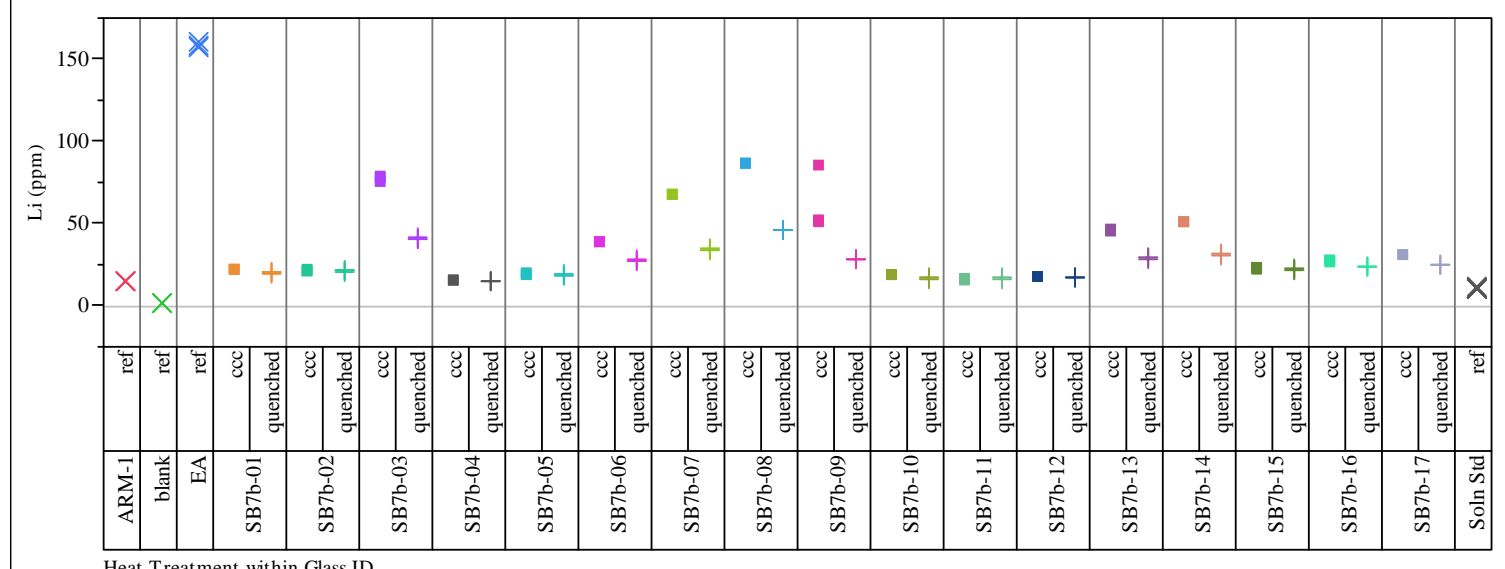

Heat Treatment within Glass ID

Set $=1$

Variability Chart for Na (ppm)

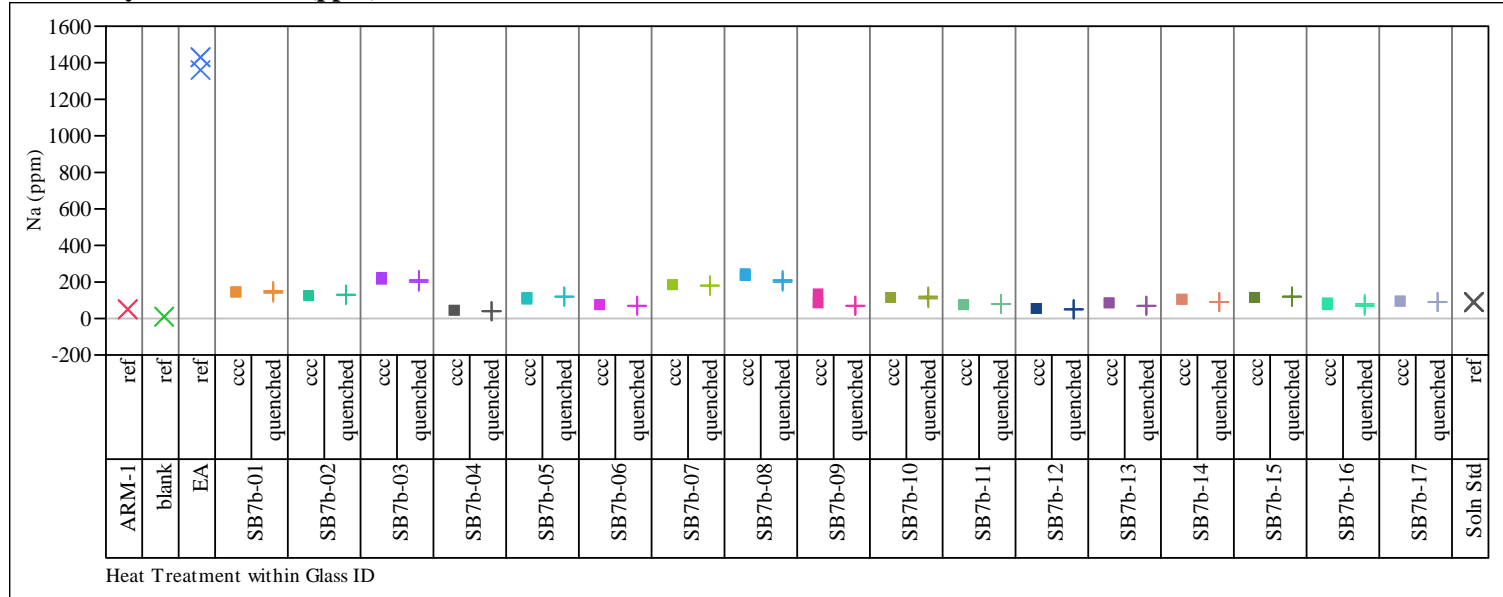


Exhibit B-6. PCT Measurements by Heat Treatment for Glasses and Standards by Analytical Set

Set $=1$

Variability Chart for Si (ppm)

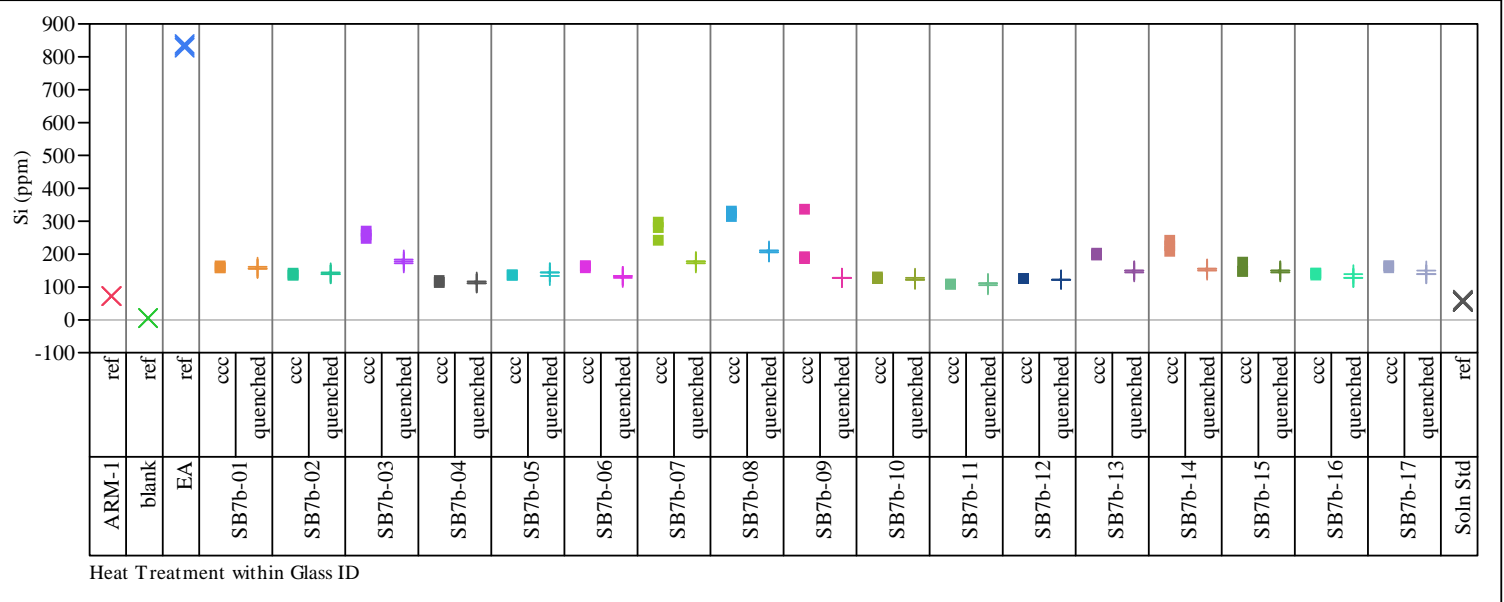

Set $=1$

Variability Chart for $\log [\mathrm{B}$ ppm]

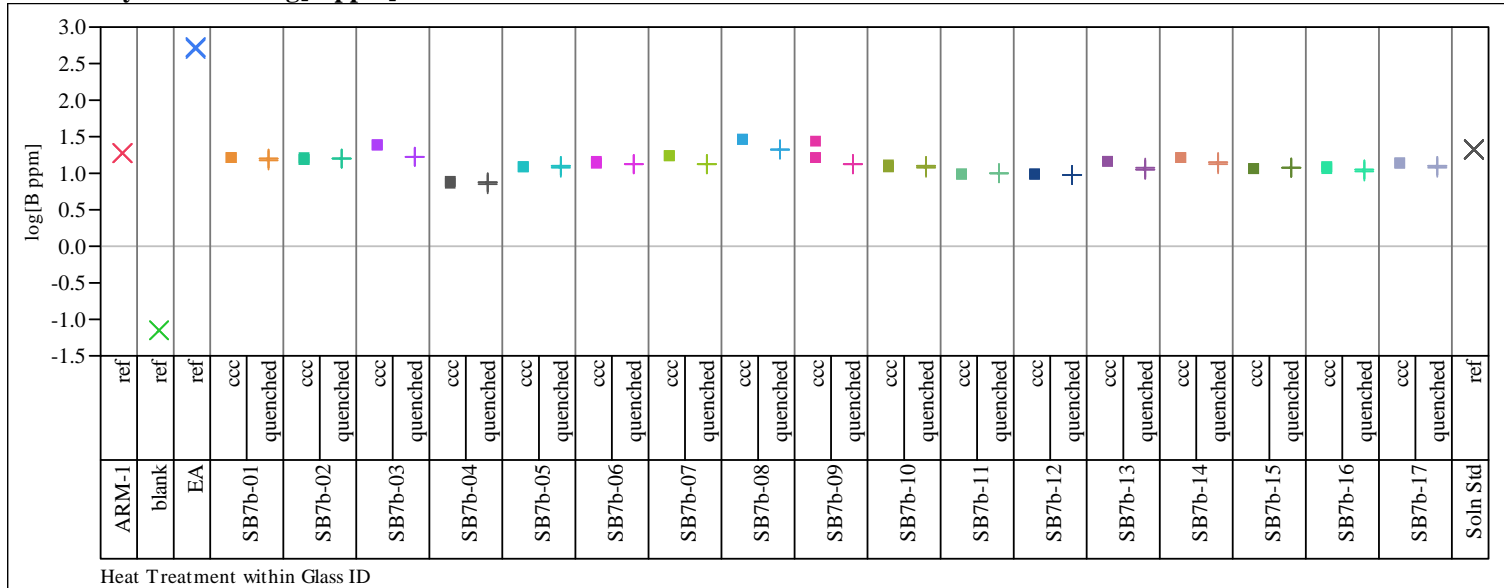

Set $=1$

Variability Chart for $\log [\mathrm{Li}$ ppm]

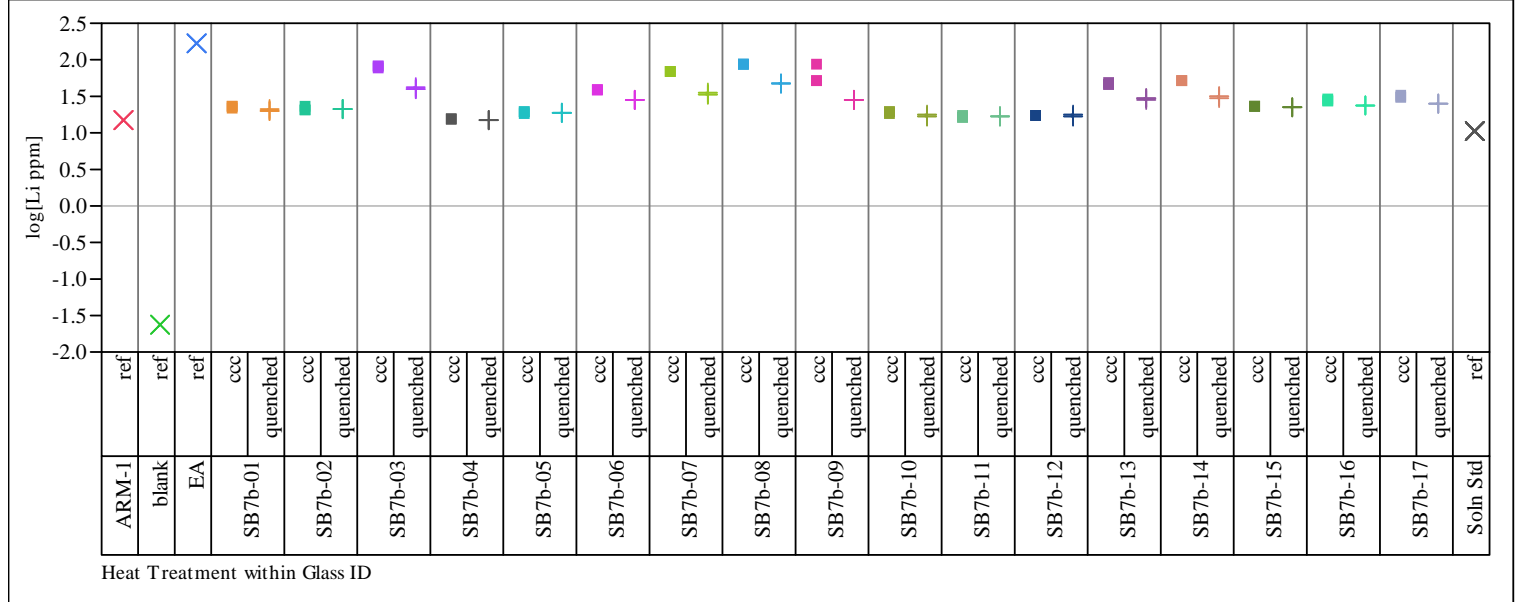


Exhibit B-6. PCT Measurements by Heat Treatment for Glasses and Standards by Analytical Set

Set $=1$

Variability Chart for log[Na ppm]

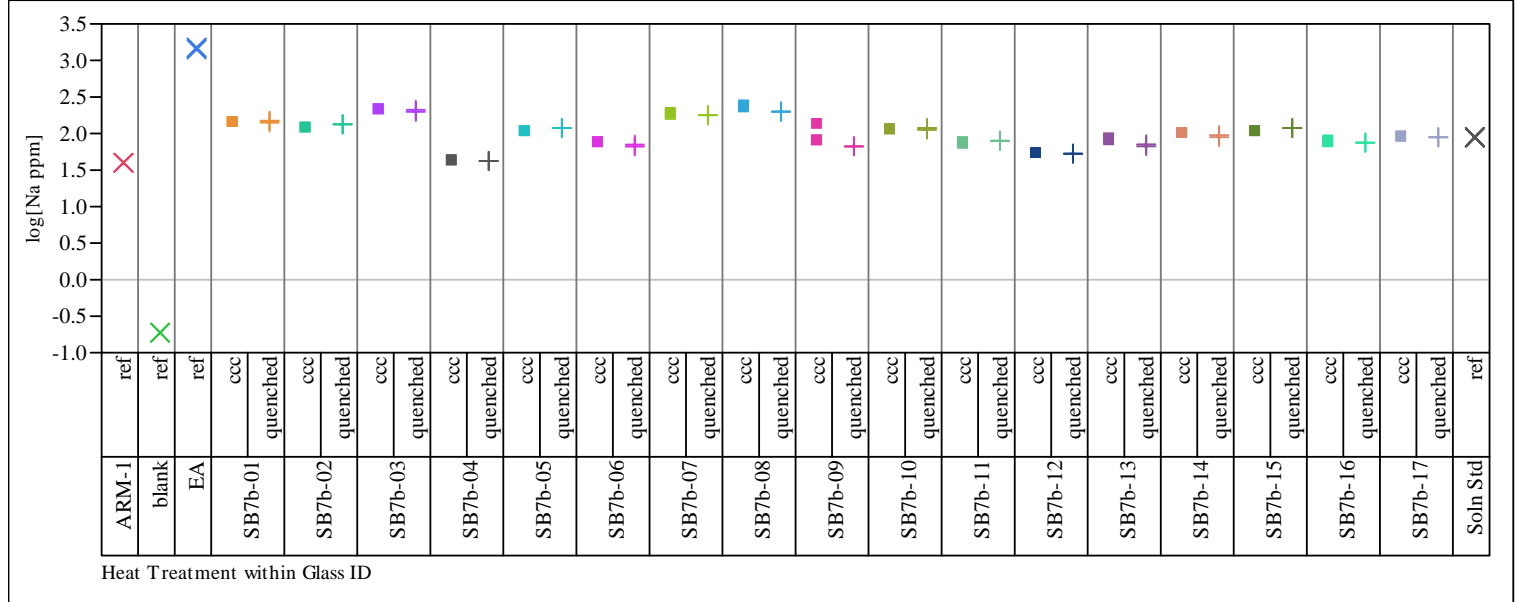

Set $=1$

Variability Chart for log[Si ppm]

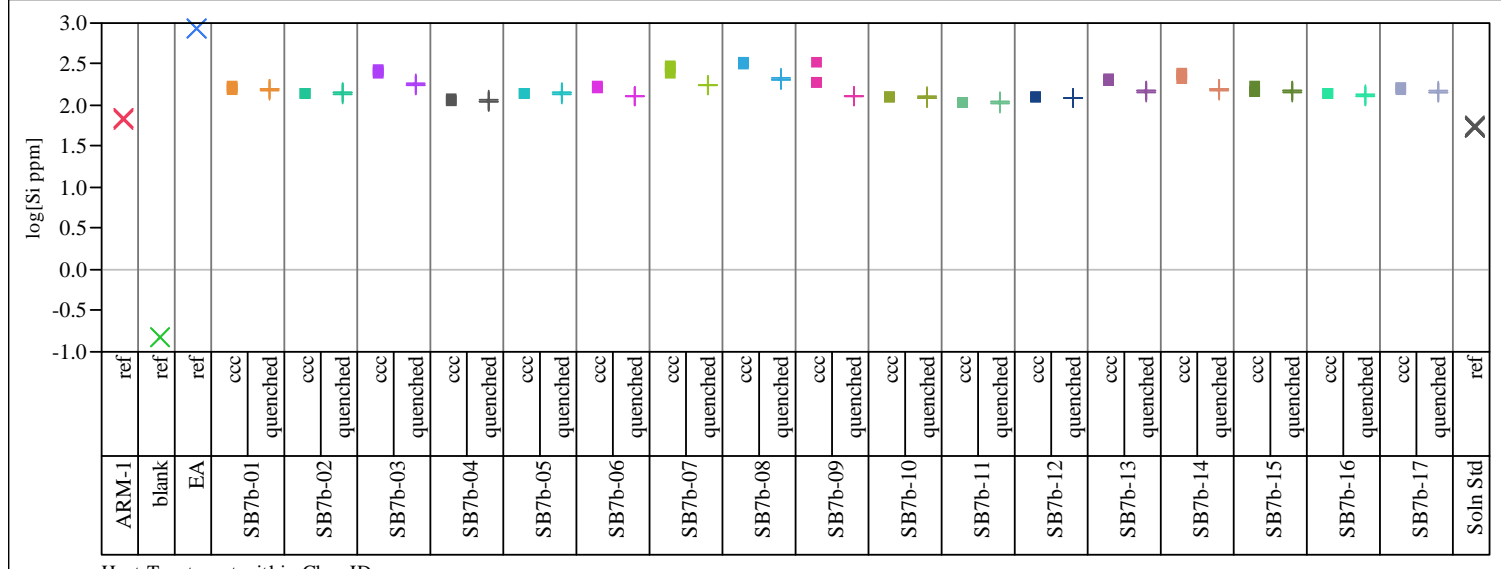

Heat Treatment within Glass ID

Set $=2$

Variability Chart for B (ppm)

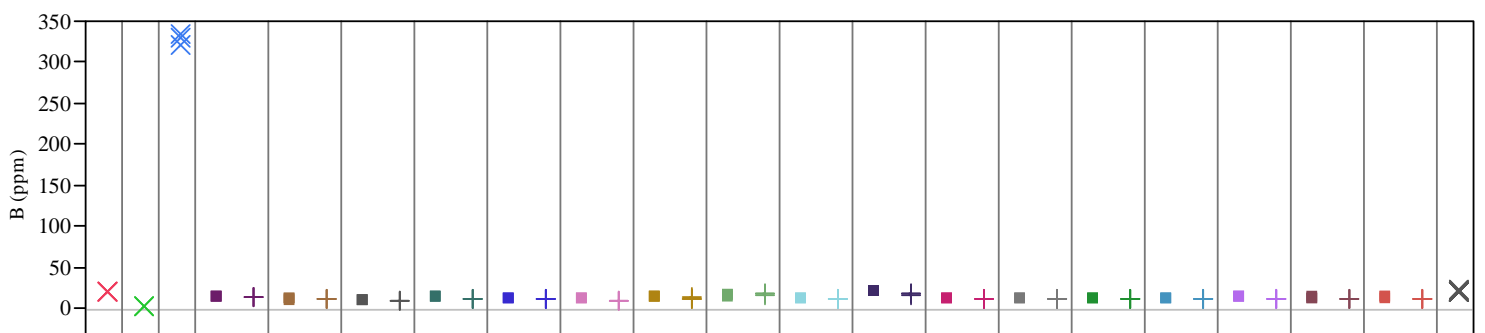

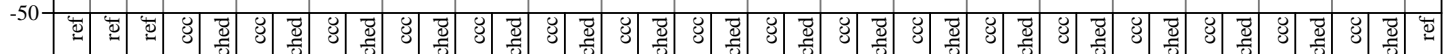

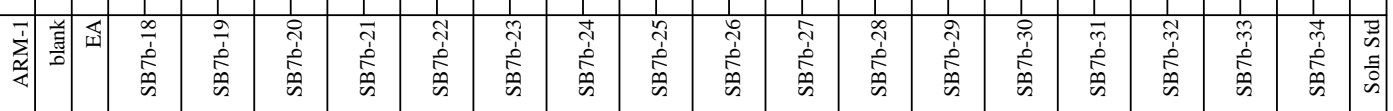


Exhibit B-6. PCT Measurements by Heat Treatment for Glasses and Standards by Analytical Set

Set $=2$

Variability Chart for Li (ppm)

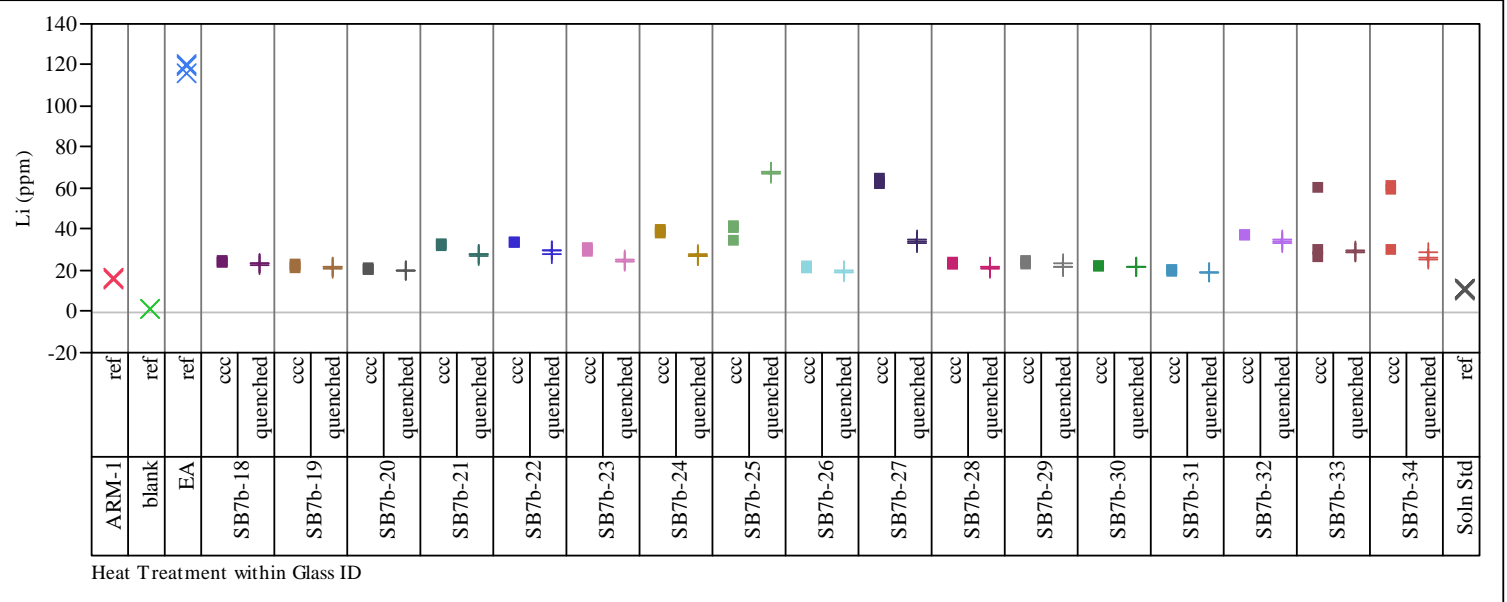

Set $=2$

Variability Chart for Na (ppm)

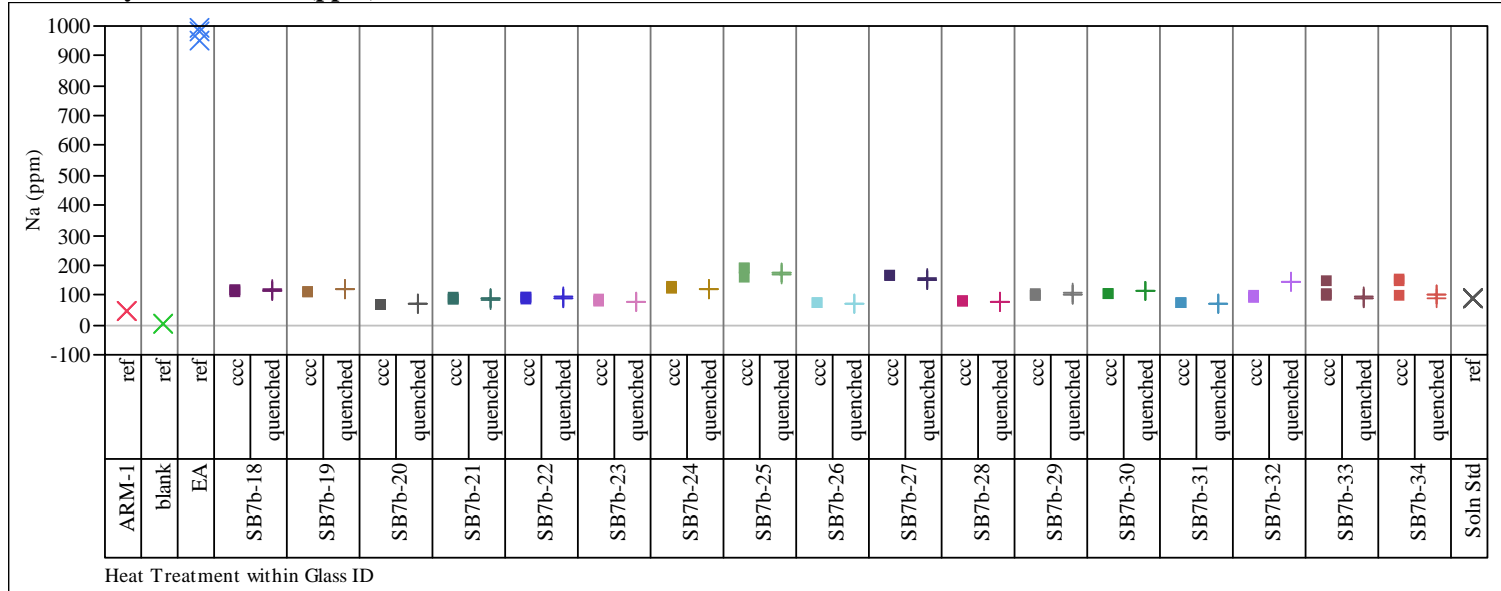

Set $=2$

Variability Chart for Si (ppm)

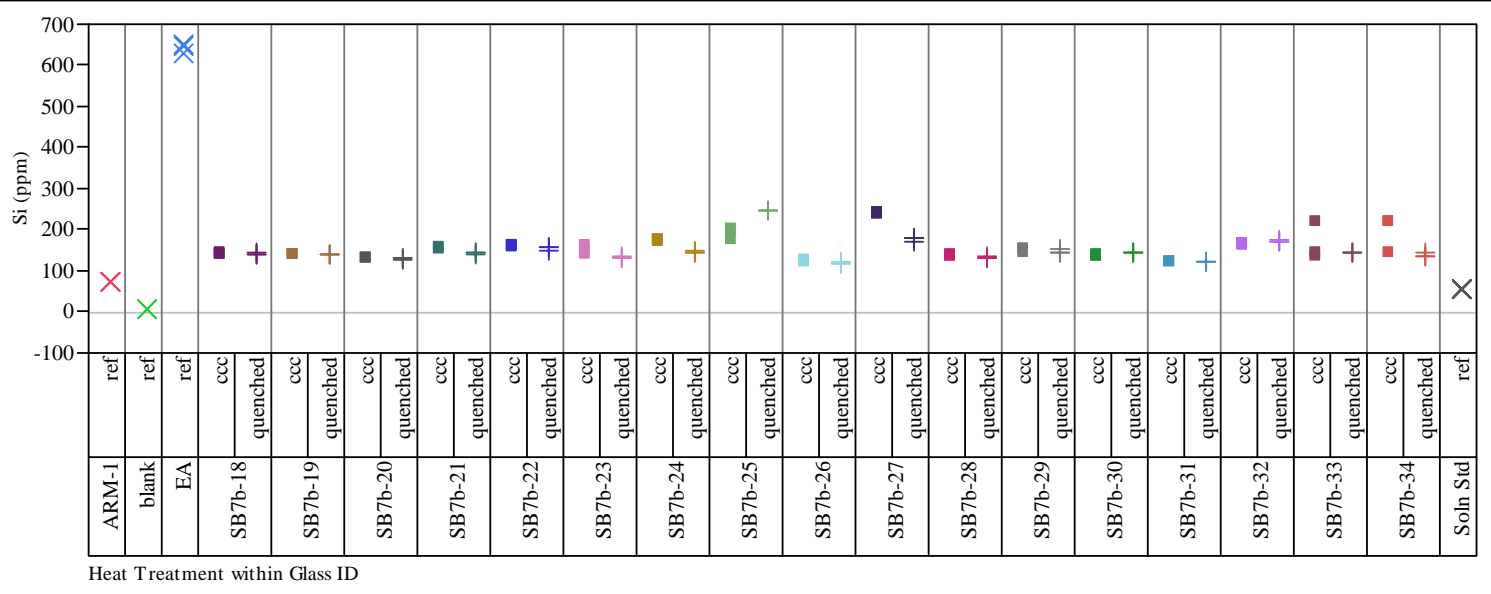


Exhibit B-6. PCT Measurements by Heat Treatment for Glasses and Standards by Analytical Set

Set $=2$

Variability Chart for $\log [\mathrm{B}$ ppm]

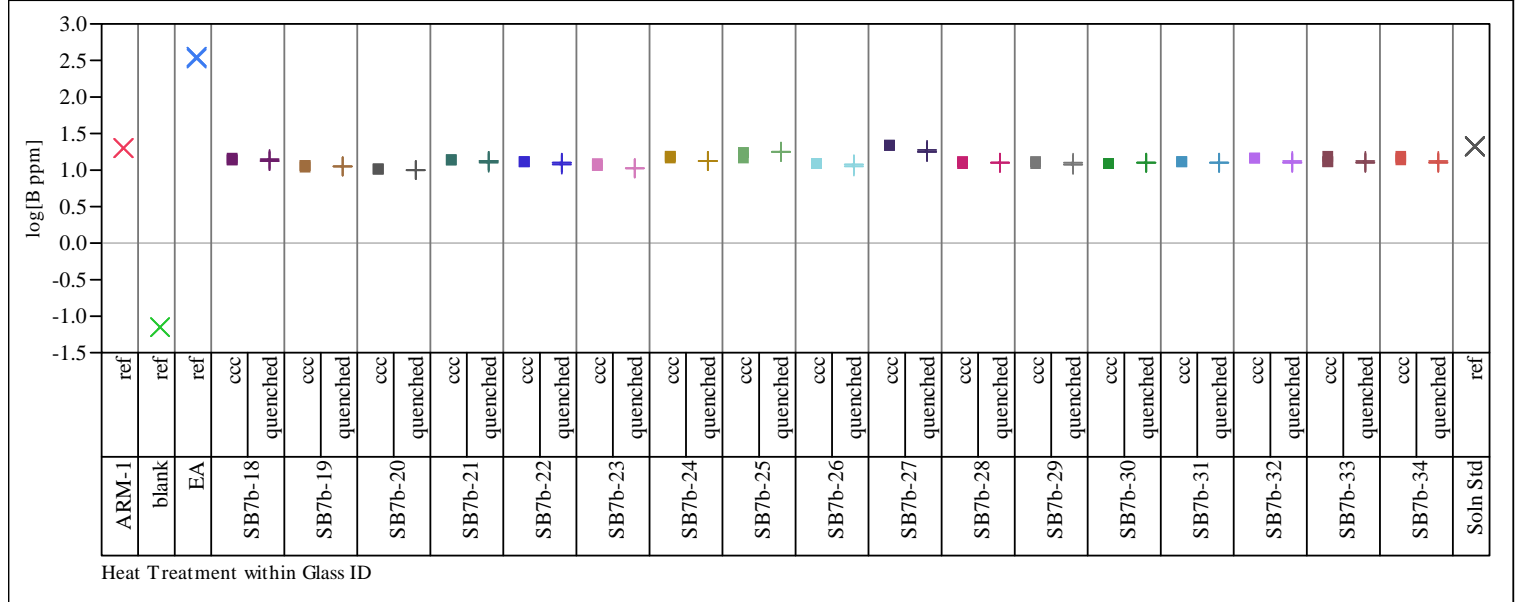

Set $=2$

Variability Chart for $\log [\mathrm{Li} \mathrm{ppm}]$

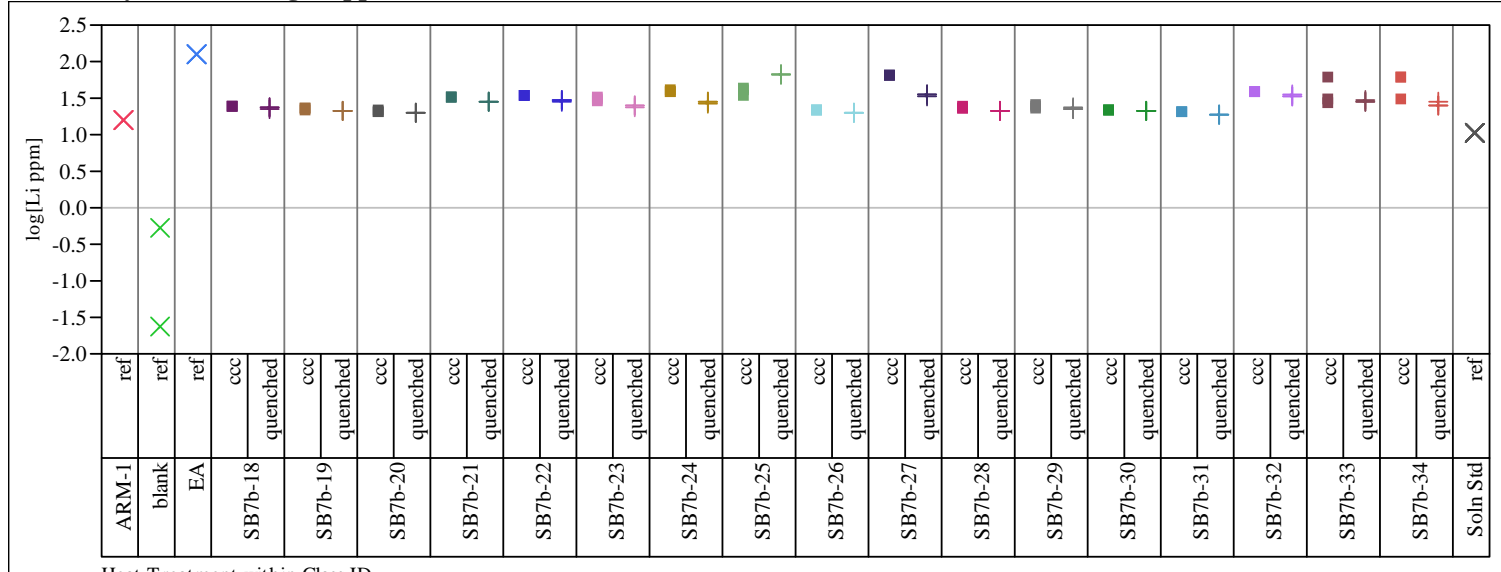

Heat Treatment within Glass ID

Set $=2$

Variability Chart for log[Na ppm]

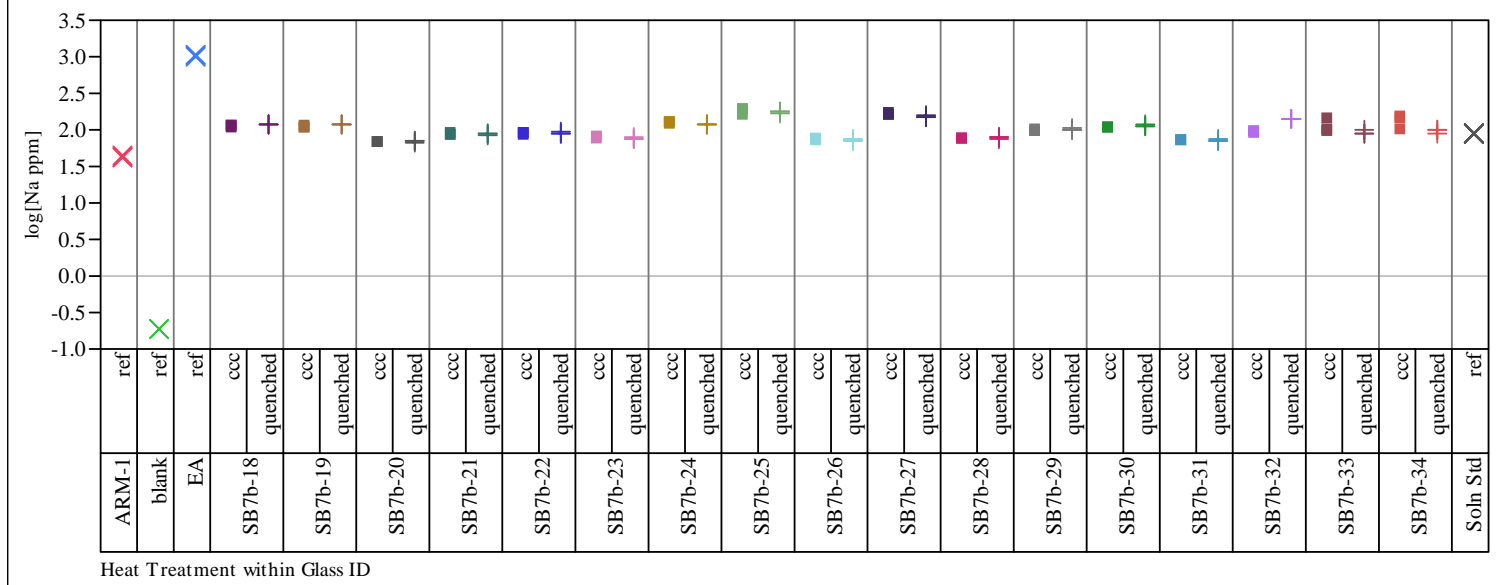


Exhibit B-6. PCT Measurements by Heat Treatment for Glasses and Standards by Analytical Set

Set $=2$

Variability Chart for log[Si ppm]

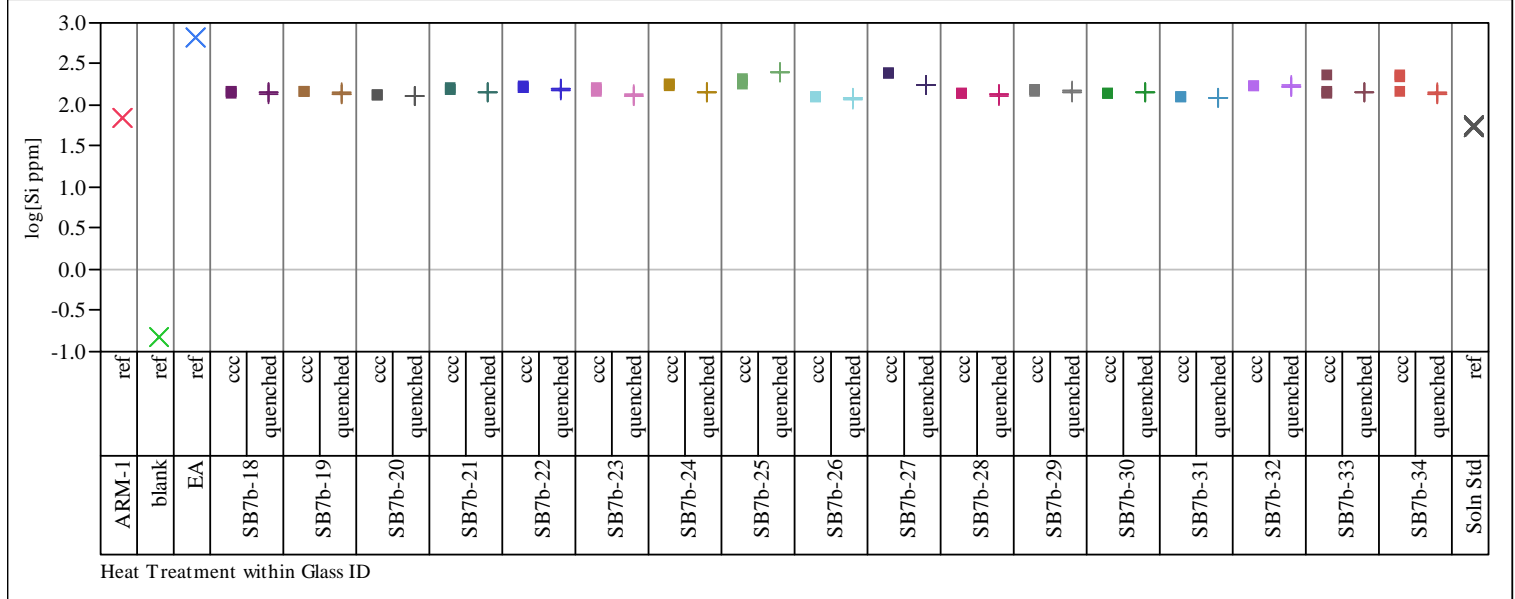


Exhibit B-7. Correlations and Scatter Plots of Normalized PCTs Over Both Compositional Views and Heat Treatments for Glasses and Standards

\section{Correlations}

$\begin{array}{lrrrr} & \begin{array}{rrr}\log \mathbf{N L}[\mathbf{B} \\ (\mathbf{g} / \mathbf{L})]\end{array} & \begin{array}{r}\log \\ \mathbf{N L}[\mathbf{L i}(\mathbf{g} / \mathbf{L})]\end{array} & \log \mathbf{N L [ N a} & \log \mathbf{N L}[\mathbf{S i} \\ \mathbf{( g / L )}] & (\mathbf{g} / \mathbf{L})] \\ \log \mathrm{NL}[\mathrm{B}(\mathrm{g} / \mathrm{L})] & 1.0000 & 0.9058 & 0.9313 & 0.9276 \\ \log \mathrm{NL}[\mathrm{Li}(\mathrm{g} / \mathrm{L})] & 0.9058 & 1.0000 & 0.8620 & 0.9741 \\ \log \mathrm{NL}[\mathrm{Na}(\mathrm{g} / \mathrm{L})] & 0.9313 & 0.8620 & 1.0000 & 0.9152 \\ \log \mathrm{NL}[\mathrm{Si}(\mathrm{g} / \mathrm{L})] & 0.9276 & 0.9741 & 0.9152 & 1.0000\end{array}$

\section{Scatterplot Matrix}

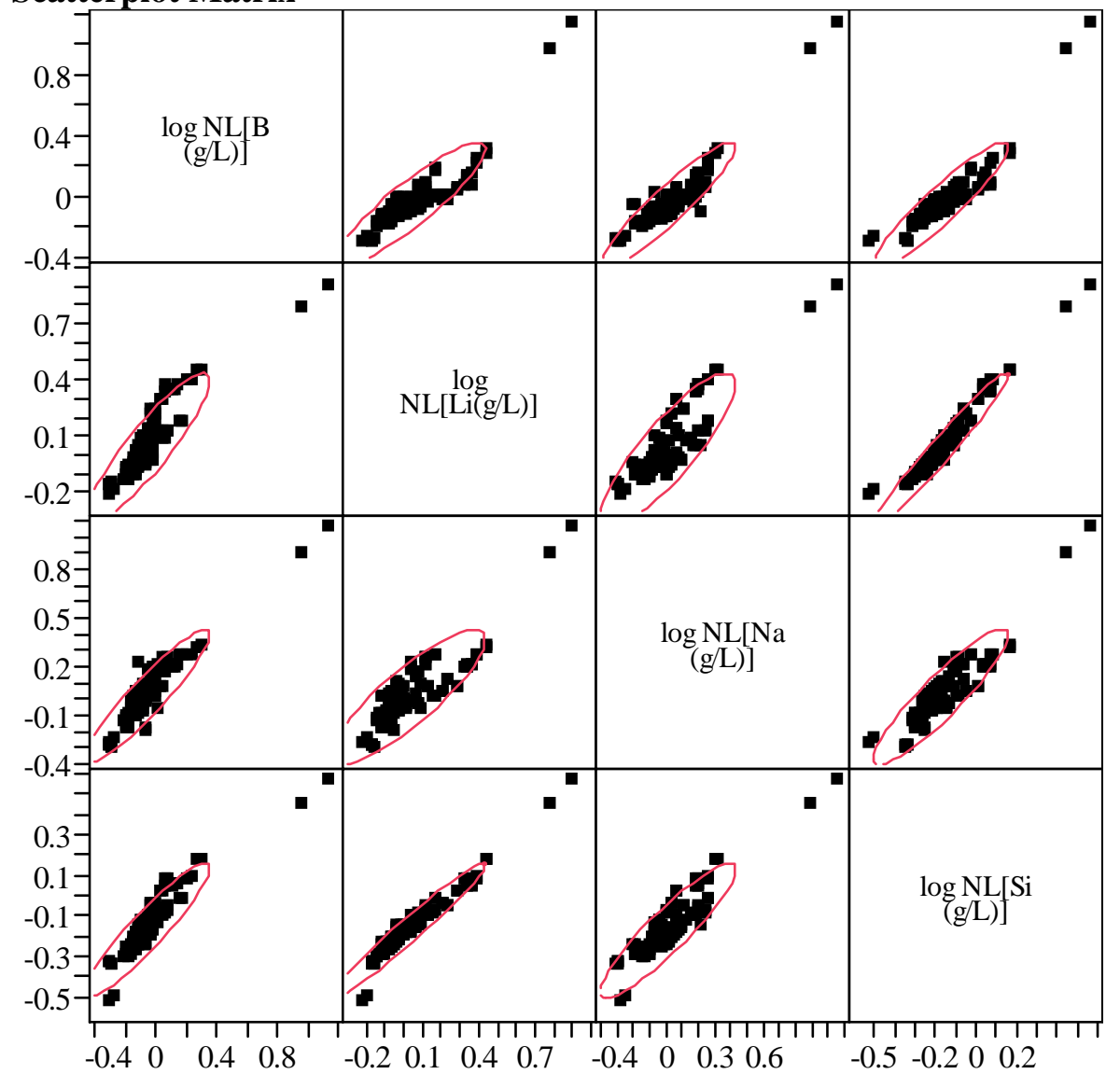


Exhibit B-8. Effects of Heat Treatment for the Glasses by Compositional View

Comp View=measured

Variability Chart for log NL[B (g/L)]

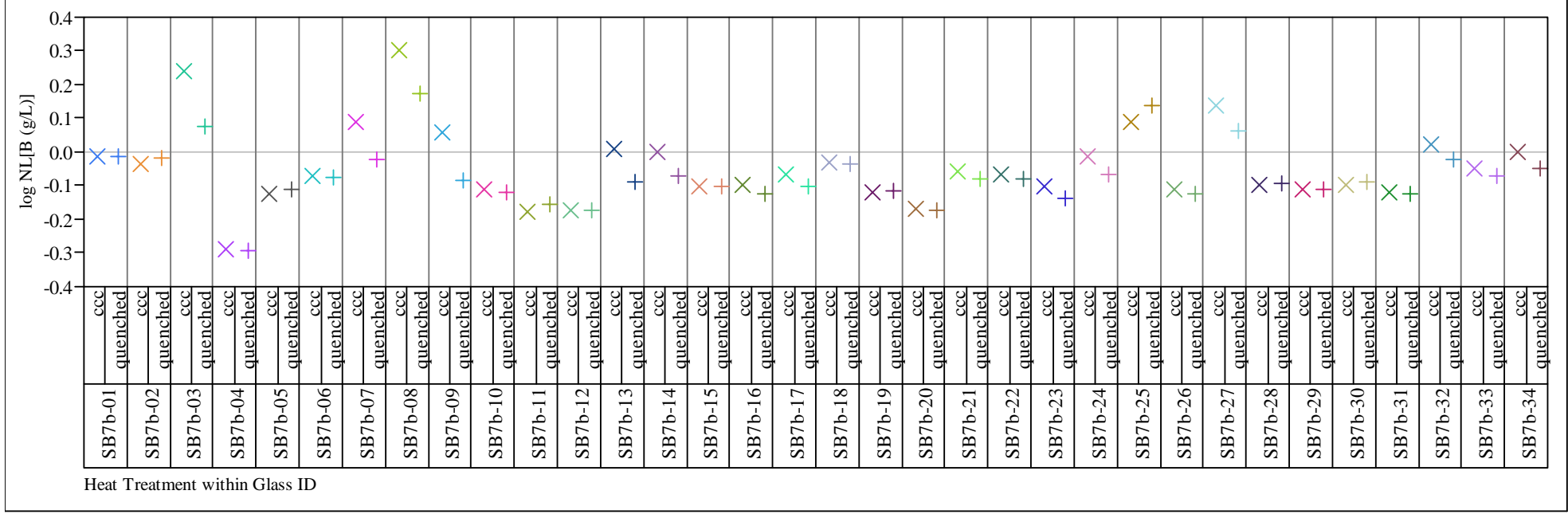

Comp View=measured

Variability Chart for $\log \mathrm{NL}[\mathrm{Li}(\mathrm{g} / \mathrm{L})]$

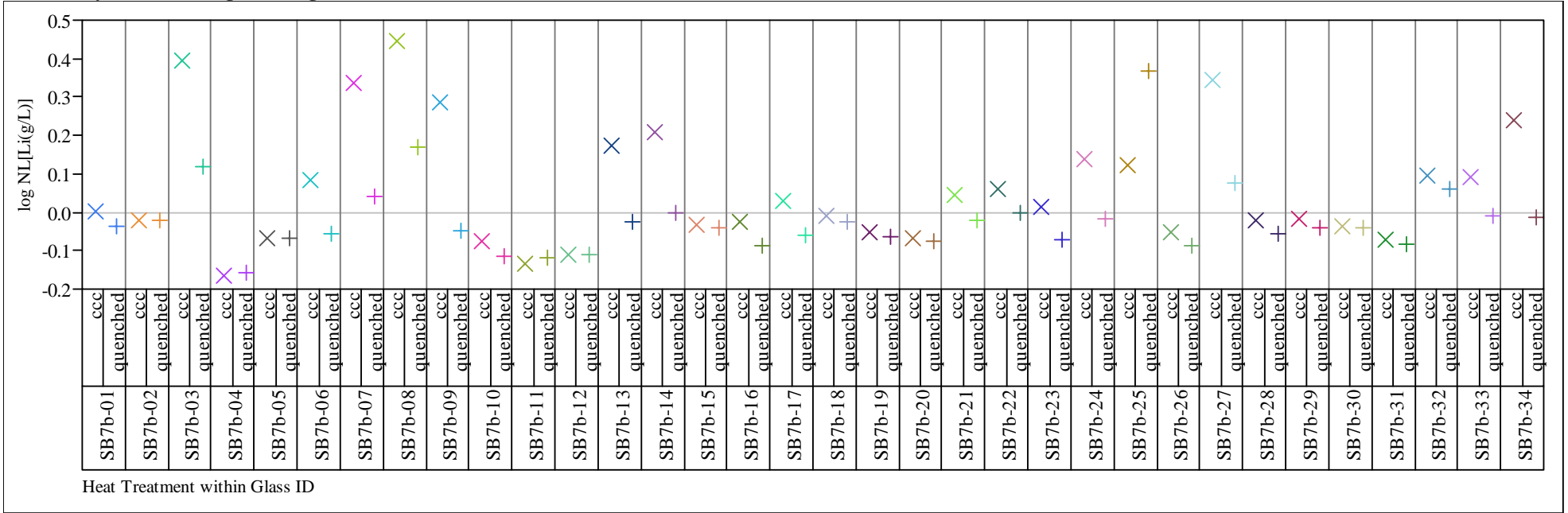


Exhibit B-8. Effects of Heat Treatment for the Glasses by Compositional View

Comp View=measured

Variability Chart for $\log$ NL[Na (g/L)]

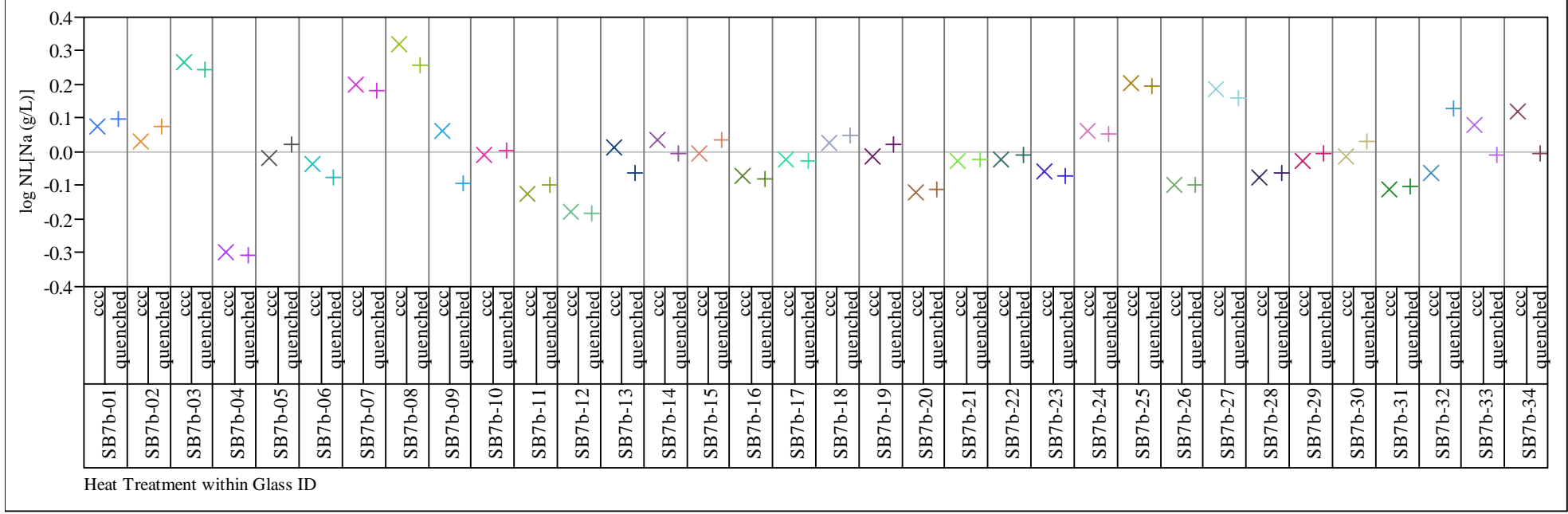

Comp View=measured

Variability Chart for $\log \mathrm{NL}[\mathrm{Si}(\mathrm{g} / \mathrm{L})]$

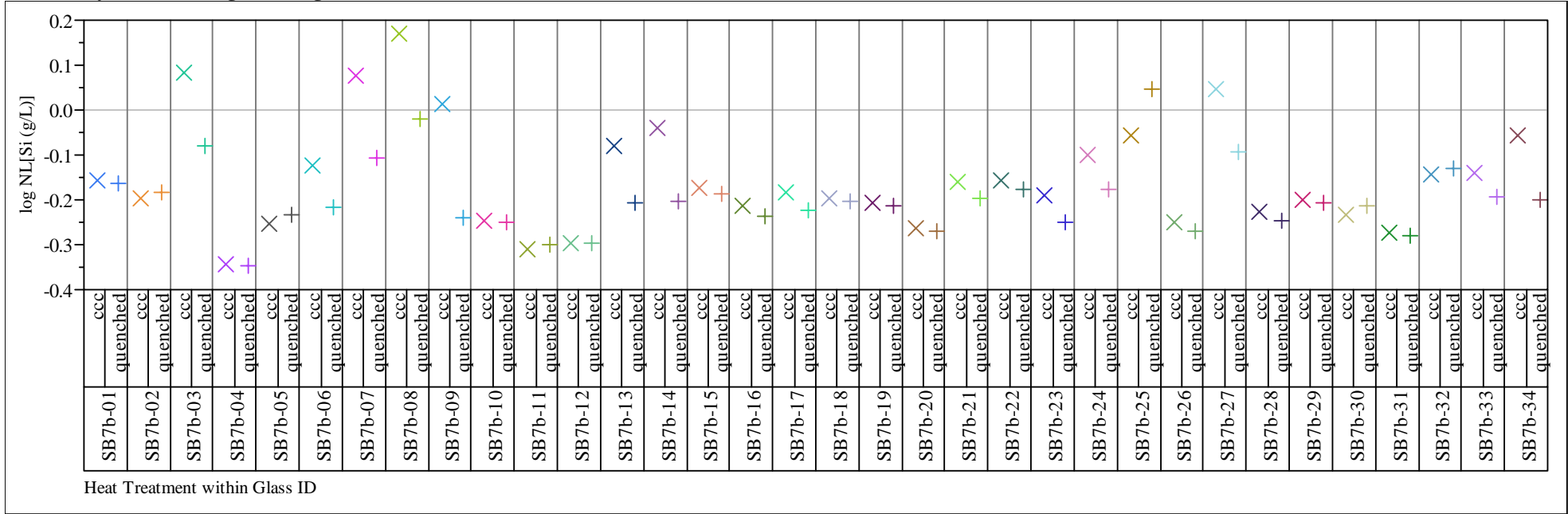


Exhibit B-8. Effects of Heat Treatment for the Glasses by Compositional View

Comp View=Target

Variability Chart for $\log$ NL[B (g/L)]

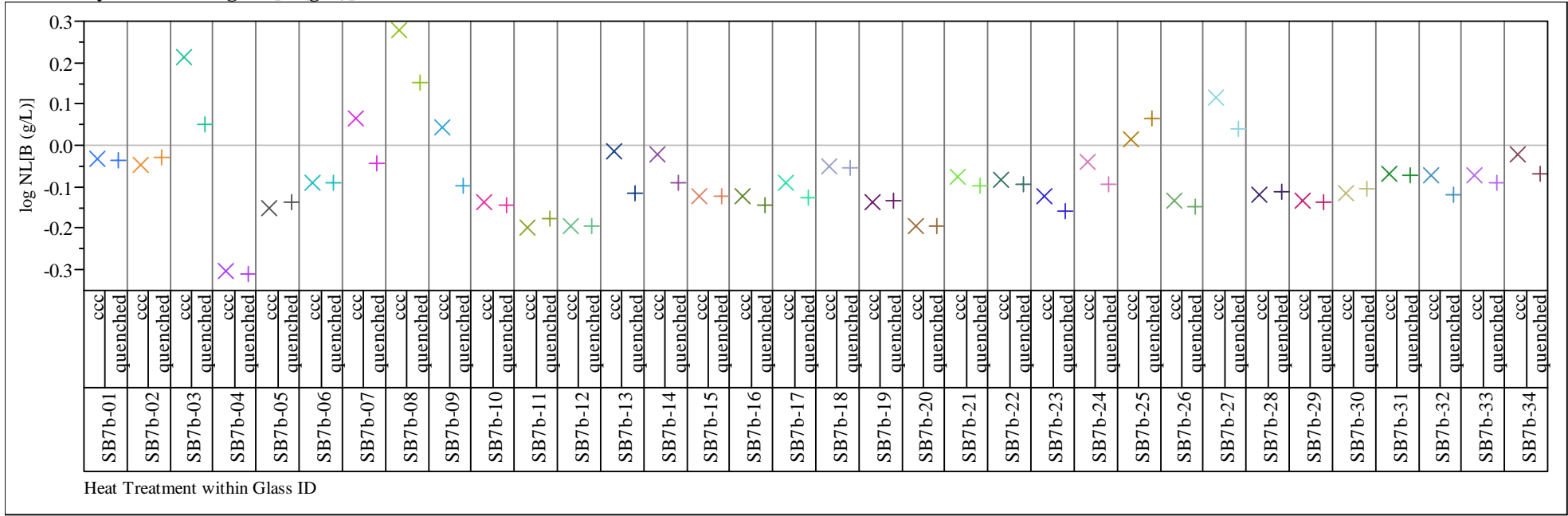

Comp View=Target

Variability Chart for $\log \mathrm{NL}[\mathrm{Li}(\mathrm{g} / \mathrm{L})]$

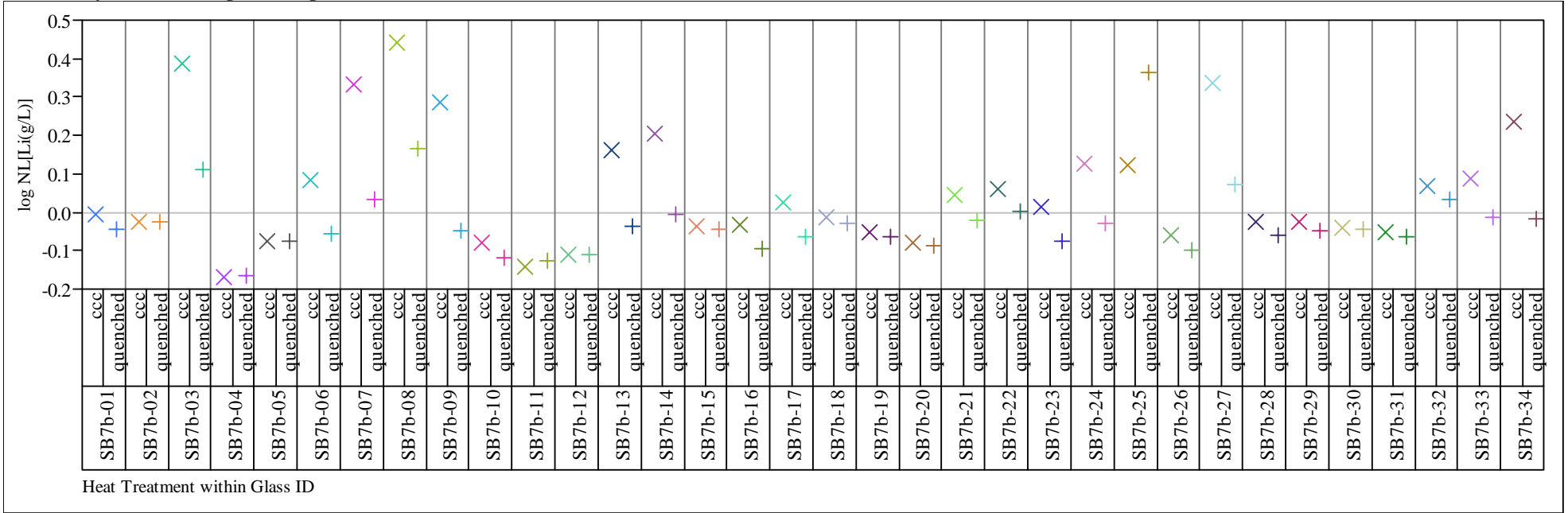


Exhibit B-8. Effects of Heat Treatment for the Glasses by Compositional View

Comp View=Target

Variability Chart for log NL[Na (g/L)]

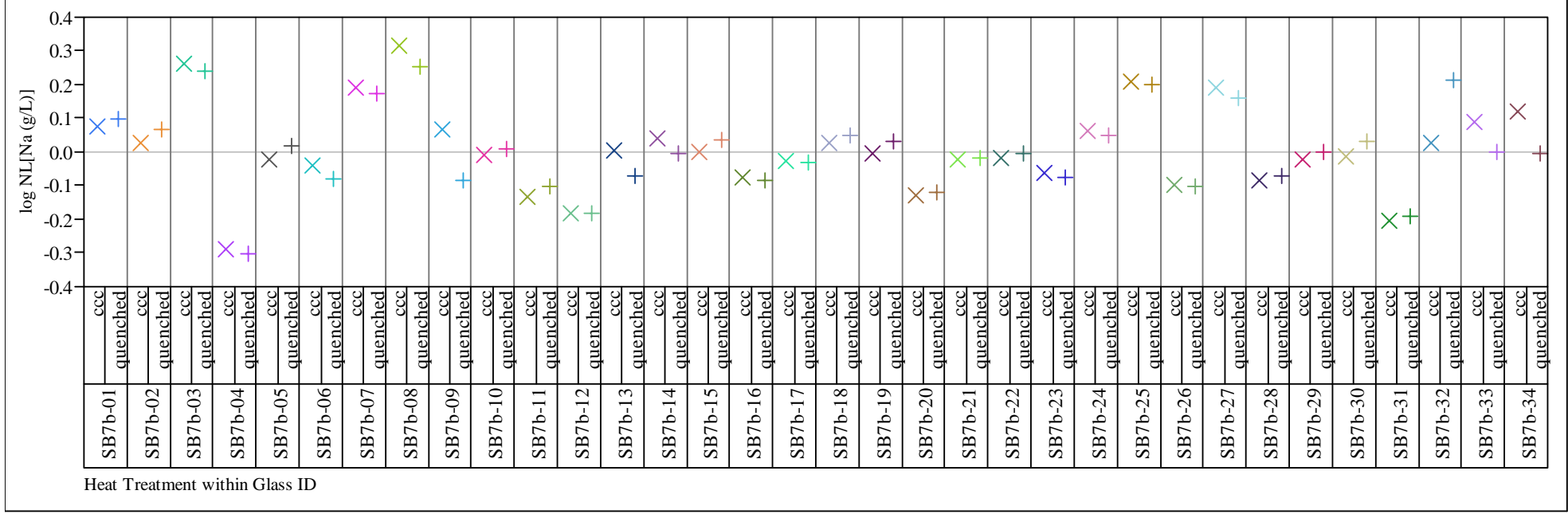

Comp View=Target

Variability Chart for $\log \mathrm{NL}[\mathrm{Si}(\mathrm{g} / \mathrm{L})]$

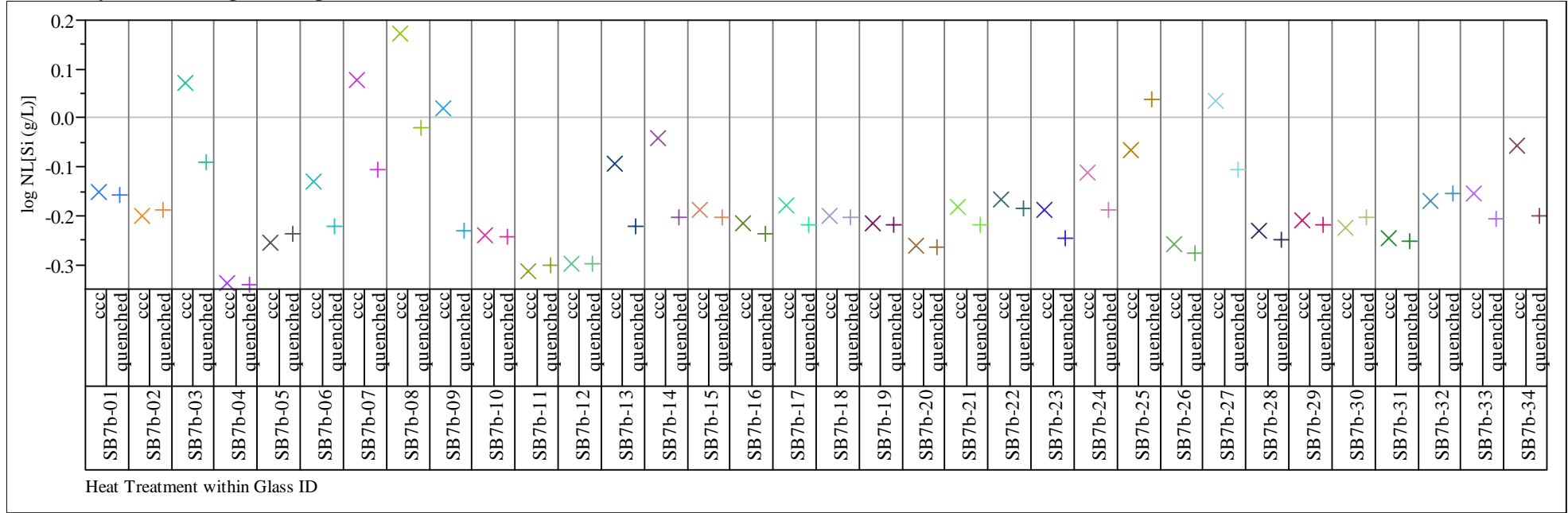


Exhibit B-9. del Gp $\left(\Delta G_{p}\right)$ Predictions versus the Common Logarithm of the Normalized Leachate $(\log \mathrm{NL}[]$.$) for B, Li, Na and Si for Target Compositions for Both Heat Treatments$

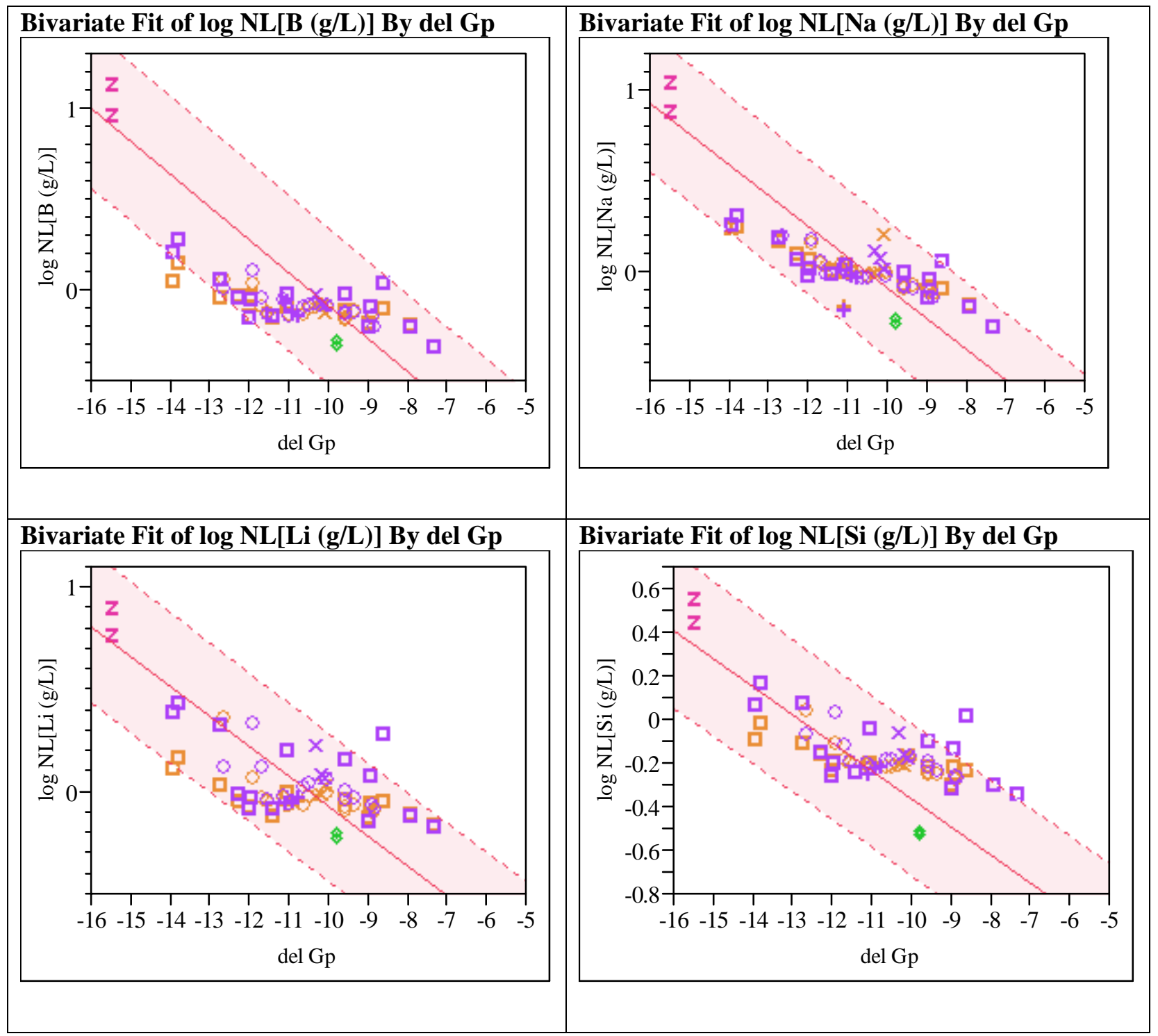

\begin{tabular}{|c|l|c|c|l|c|}
\hline$\diamond$ & ARM & -- & $\square$ & D-Opt EV Outer Layer & quenched \\
\hline z & EA & --- & + & Nominal-Frit 418 & ccc \\
\hline$\bullet$ & D-Opt EV Inner Layer & ccc & + & Nominal-Frit 418 & quenched \\
\hline 0 & D-Opt EV Inner Layer & quenched & $\times$ & Nominal-Frit 702 & ccc \\
\hline$\square$ & D-Opt EV Outer Layer & ccc & $\times$ & Nominal-Frit 702 & quenched \\
\hline
\end{tabular}


Exhibit B-10. del Gp $\left(\Delta G_{p}\right)$ Predictions versus the Common Logarithm of the Normalized Leachate ( $\log \mathrm{NL}[$.$] ) for \mathrm{B}, \mathrm{Li}, \mathrm{Na}$ and $\mathrm{Si}$ for Measured Compositions for Both Heat Treatments

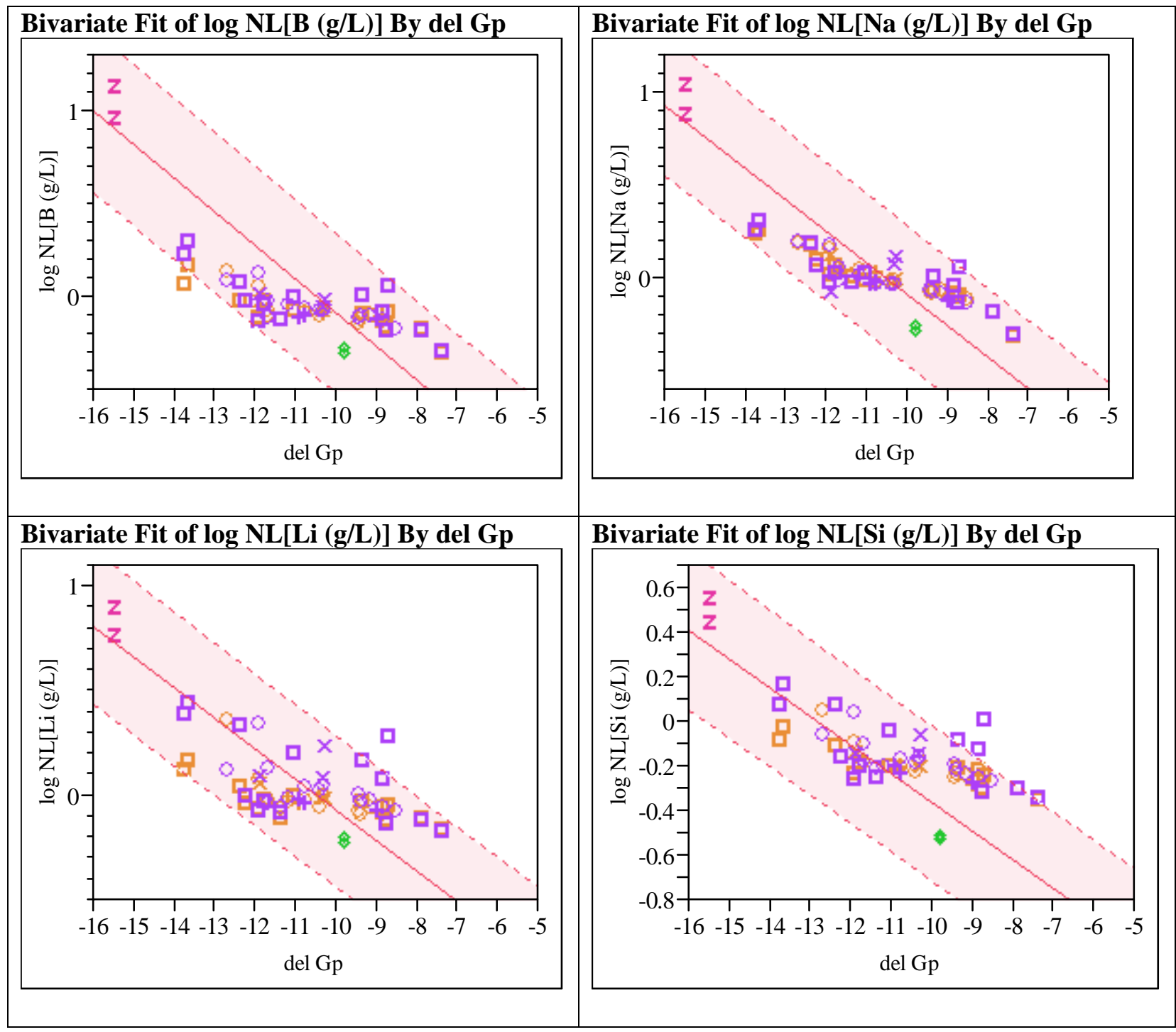

\begin{tabular}{|c|l|c|c|l|c|}
\hline$\diamond$ & ARM & --- & $\square$ & D-Opt EV Outer Layer & quenched \\
\hline z & EA & --- & + & Nominal-Frit 418 & ccc \\
\hline$\diamond$ & D-Opt EV Inner Layer & ccc & + & Nominal-Frit 418 & quenched \\
\hline & D-Opt EV Inner Layer & quenched & $\times$ & Nominal-Frit 702 & ccc \\
\hline$\square$ & D-Opt EV Outer Layer & ccc & $\times$ & Nominal-Frit 702 & quenched \\
\hline
\end{tabular}




\section{Distribution:}

J.W. Amoroso, 999-W

A. B. Barnes, 999-W

H.P. Boyd, 704-27S

J. M. Bricker, 704-27S

M. A. Broome, 704-29S

C.L. Crawford, 773-42A

T.B. Edwards, 999-W

T. L. Fellinger, 704-26S

S. D. Fink, 773-A

K.M. Fox, 999-W

B. J. Giddings, 786-5A

J. M. Gillam, 766-H

C. C. Herman, 999-W

R. N. Hinds, 704-S

E. W. Holtzscheiter, 704-15S

J. F. Iaukea, 704-30S

P. R. Jackson, DOE-SR, 703-46A

F.C. Johnson, 999-W

M. T. Keefer, 766-H

P.L. Lee, 703-41A

S. L. Marra, 773-A

D.W. McIlmoyle, 766-H

J. E. Occhipinti, 704-S

D.K. Peeler, 999-W

F. M. Pennebaker, 773-42A

J. W. Ray, 704-S

H. B. Shah, 766-H

D. C. Sherburne, 704-S

A. V. Staub, 704-27S

M. E. Stone, 999-W

K. H. Subramanian, 766-H

J. P. Vaughan, 773-41A 